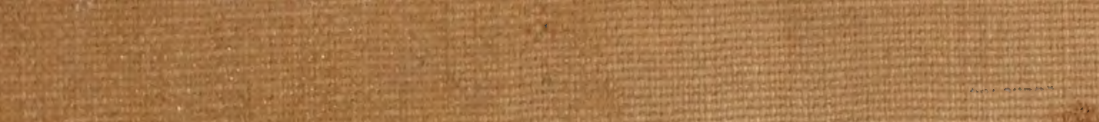

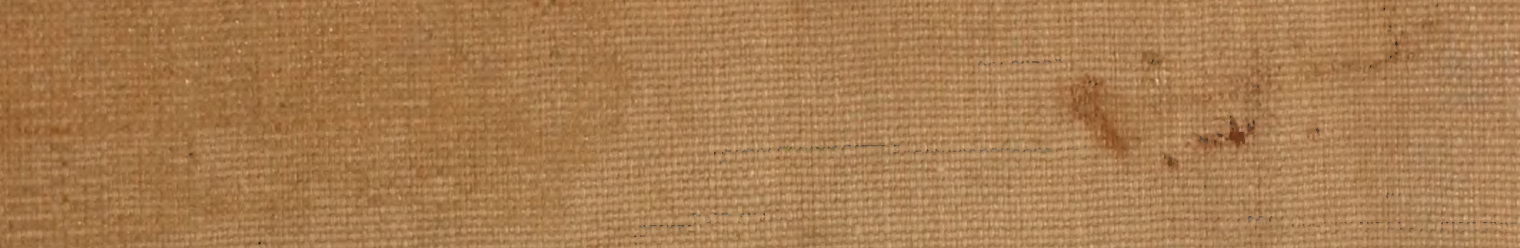

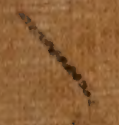

(1)

(1)

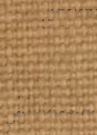

(1)

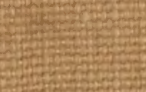

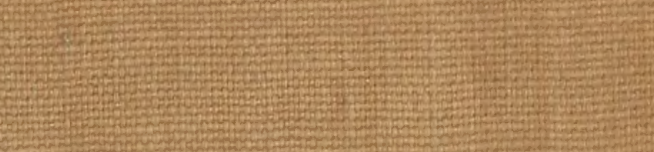

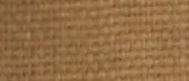

(1)

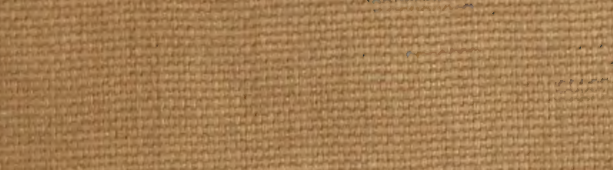

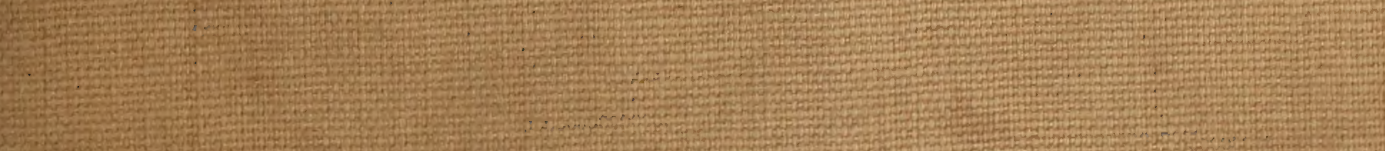

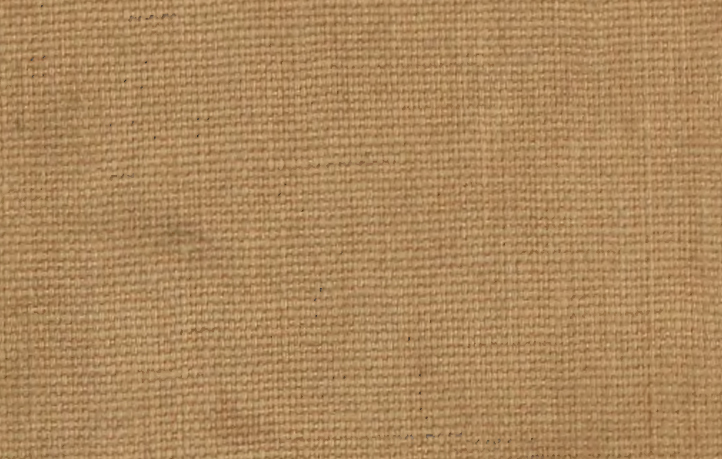

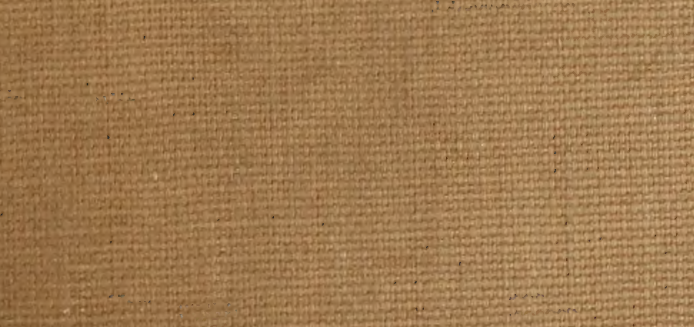

(2)

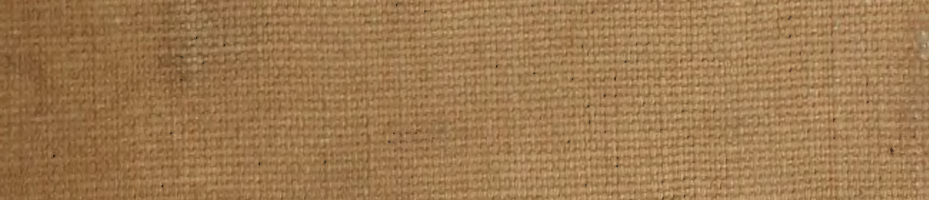

-

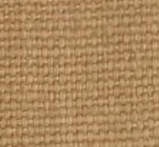



WILSON COPEPOD LIBRARY

Smithsonian Institution

Invertebrate Zoology

(Crustacea) 

WILSON COPEPOD LIBRARY

Smithsonian Institution

Invertebrate Zoology

(Crustacea) 

Maxilliped). Die 1. Antenne kann sehr verschieden lang sein; die Zahl ihrer Glieder schwankt zwischen 3 und 25. Sie trägt Borsten und faden- oder schlauchförmige, blasse Sinnesorgane (Aesthetasken). Die 5 folgenden Paare heißen Mundgliedmaßen. Die 2, Antenne weist meistens ein 2 gliedriges Basipodit, ein 2 gliedriges Endopodit und ein mehrgliedriges Exopodit auf, welch letzteres oft reduziert ist oder auch ganz ausfallen kann. Hinter der 2. Antenne begrenzt ein vorspringender Höcker (Oberlippe) die Mundöffnung nach vorne. Die Mandibel hat am ersten Gliede des Basipoditen einen Kaufortsatz (Kaulade), der an dem der Mundöffnung zugewandten Rande gezähnelt ist; das zweite Glied des Basipoditen mit den beiden Ästen bildet den Palpus, kann aber in hohem Maße rückgebildet oder auch ganz verschwunden sein. Die 1. Maxille ist von gedrungenem Bau, hat kurze Äste und an den Rändern beborstete Auswüchse (Loben). Der Innenrand des Basipoditen der einfachen 2. Maxille zeigt eine Reihe von Loben, welche wie das kurze Endopodit mit langen Hakenund Reusenborsten besetzt sind. Die Beborstung ist jedoch oft reduziert, so daB im extremsten Falle nur ein Endhaken übrig bleibt. Der ebenfalls einfache, aber meistens gestrecktere Maxilliped hat ein 2 gliedriges Basipodit und ein höchstens 5 gliedriges Endopodit und ist mit Borsten mehr oder weniger reich ausgestattet.

Die Thoraxsegmente tragen die Beinpaare. Die 4 vorderen Paare sind gewöhnlich zu Ruderorganen ausgebildet; auf einem 2 gliedrigen Basipodit tragen sie, gelenkig damit verbunden, ein Exopodit und ein Endopodit, deren Gliederzahl höchstens drei ist. Das 5. Paar ist nur sehr selten den vorderen Beinpaaren gleichgestaltet, sondern meistens in beiden Geschlechtern mehr oder weniger abweichend gebaut; in einigen Fällen fehlt es völlig.

Die Geschlechter bei den Copepoden sind getrennt. Die Begattung findet vermittelst Spermatophoren statt, welche entweder direkt oder mit Hilfe des umgebildeten 5. Beinpaares des of an die Genitalöffnung des $q$ geklebt werden. Bei den planktonischen Arten werden die Eier mit wenigen Ausnahmen einzeln abgelegt; bei den übrigen werden sie in einen oder zwei Ballen, Säckchen oder Schnüren an der Genitalöffnung aufgehängt oder sie treten in einen dorsalen Brutraum ein.

Die Männchen unterscheiden sich gewöhnlich deutlich von den Weibchen durch eine Anzahl von sekundären Genitalcharakteren. Bau und Gliederung des Rumpfes weichen häufig in beiden Geschlechtern ab; gewisse Gliedmaßen des $\sigma^{7}$ sind zu Kopulationsorganen umgewandelt: die 1. Antennen werden zu Spür- oder zu Greiforganen, das 5. Beinpaar, die Maxillipeden oder die 2. Antennen sind zu Greif- und Klammerorganen umgebildet, welche bei der Begattung eine Rolle spielen. Sehr oft sind die Mundgliedmaßen des or mehr oder weniger um- oder rückgebildet.

Die jüngsten Larven sind typische Nauplien ohne Stirnhörner. Nach Beendigung der Nauplius- und der Metanaupliusphase werden 5 weitere Copepodid- (oder Cyclopid-) Stadien durchlaufen. Das erste derselben ist gekennzeichnet durch einen zylindrischen, in Vorder- und Hinterrumpf geteilten, 
segmentierten Rumpf und durch den Besitz von 6 Kopfgliedmaßen und 2 Paar Schwimmbeinen. Nach Ablauf des 5: Copepodid-Stadiums häutet sich das Tier zum letzten Mal und ist dann geschlechtsreif.

Fast in jeder Wasseransammlung können Vertreter dieser Crustaceengruppe angetroffen werden. In größter Fülle von Arten aber bewohnen die Copepoden das Meer und die relativ wenigen typischen Genera der Süßwasserbewohner lassen. sich leicht in das System der marinen Copepoden einreihen. Die wärmeren Gebiete sind im allgemeinen reicher an Arten und Genera als die arktischen Gegenden; an Individuenzahl aber stehen letztere den ersteren nicht nach.

Bei den nicht-parasitischen Arten unterscheidet man die freischwimmenden (planktonischen oder pelagischen) von den benthonischen (littoralen), d. h. denjenigen, welche sich an und in dem Boden und dessen Besiedelung an Pflanzen und Tieren aufhalten. Die planktonischen Arten gehören teils dem Küstengebiet an (neritische Arten), teils pflanzen sie sich regelmäßig im offenen Ozean fort (ozeanische Arten).

Alle Zonen des Meeres von der Oberfläche bis in die größten Tiefen hinab werden von den Copepoden bewohnt. Von vielen freilebenden Arten werden gewisse Schichten (obere, mittlere und abyssale) bevorzugt. Die meisten Planktoncopepoden haben eine große räumliche Verbreitung; die Zahl der Arten, die dem Pacifischen, dem Atlantischen und dem Indischen Ozean gemeinschaftlich angehören, vermehrt sich rasch. Einzelne Arten sind sowohl für das arktische wie für das antarktische Gebiet nachgewiesen.

Als Südgrenze der Verbreitung der nordischen Arten ist sowohl im pacifischen wie im atlantischen Ozean die Parallele von $50^{\circ} \mathrm{N}$. B. gewählt worden und ich habe mich ziemlich streng an diese Grenze gehalten. Angesichts der Tatsache, daß man erst in den letzten 10-15 Jahren angefangen hat, der Verbreitung der Copepoden sowie der Planktonorganismen überhaupt genauer nachzugehen, kann es nicht wunder nehmen, daß man sogar noch vor einigen Jahren über die Verbreitung der niarinen Planktoncopepoden sehr lückenhaft unterrichtet war. Es hat zwar Gebiete gegeben, welche mehr und besser als andere den Copepodenforschern die Gelegenheit boten, sich dem Studium dieser Gruppe zu widmen. Solche bevorzugte Areale sind z. B. die Nordsee und das Mittelmeer gewesen. Aus den Ozeanen aber stand den Systematikern hauptsächlich nur das Material zu Gebote, welches als Ausbeute einzelner großer Expeditionen ihnen zur Untersuchung übergeben wurde. Als daher das Studium des Planktons gegen Ende des vergangenen Jahrhunderts in weit größerem Maßstabe als bis dahin in Angriff genommen wurde, machten auch die Kenntnisse von der Verbreitung der Copepoden merkliche Fortschritte. Es stellte sich bald heraus, daß viele anscheinend seltene oder bisher nur in einzelnen Exemplaren bekannte Arten zu den am weitesten verbreiteten und am häufigsten vorkommenden Tierformen gehörten. Wie oben schon erwähnt wurde, ișt die Zahl der Spezies, die den drei wärmeren Ozeanen gemeinsam 
sind, schon eine recht erhebliche. Arten, die man nur aus den Tropen kannte, tauchten in nördlicheren, ja sogar in arktischen Gegenden wieder auf. Aus den kälteren, tieferen Schichten der tropischen und gemäßigten Regionen wurden eine Menge von Formen ans Tageslicht gebracht, die selten oder nie an der Oberfläche erbeutet worden waren.

Was den Nord-Atlantischen Ozean im besonderen betrifft, so hat man jetzt dort das Vorkommen vieler, früher nur aus südlicheren Gegenden bekannten Arten festgestellt, und nicht weniger bedeutend ist die Zahl der in dem betreffenden Gebiet neu entdeckten Arten. Die jüngsten Arbeiten von Sars, Thompson, Wolfenden, Farran haben, insofern sie sich mit den nordatlantischen Copepoden beschäfigten, den Beweis erbracht, daß neue Funde sowohl in systematischer wie in faunistischer Hinsicht auch fernerhin noch zu erwarten sind.

Die Copepodenfauna des Pacifischen Ozeans nördlich des 50. Breitengrades ist fast so gut wie unbekannt.

Die in dieser Arbeit dargebotene Übersicht der nordischen Planktoncopepoden darf aus obigem Grunde nichts weniger als endgültig betrachtet werden. In Anbetracht des in gewisser Hinsicht vorläufigen Charakters dieser Beiträge habe ich es auch unterlassen, solche Formen mit aufzunehmen, welche, obgleich in ihrer Verbreitung das nordische Gebiet berührend, aber bis jetzt noch nicht nördlich vom $50^{\circ} \mathrm{N}$. B. gefunden, dennoch daselbst einheimisch erwartet werden dürfen.

Die ausführliche Begründung der systematischen Einteilung der pelagischen Copepoden findet sich in Giesbrecht (1892). Als Grundlage für meine Arbeit habe ich, insoweit es sich um die Gymnoplea handelt, das Werk von Giesbrecht und Schmeil (1898) benützt; für die Podoplea kam dafür die Monographie Giesbrechts (1892) in Betracht.

Die Bestimmungstabelle für die Gattungen wird am Ende der Arbeit gegeben werden.

In den Diagnosen sind folgende Abkürzungen angewandt worden:

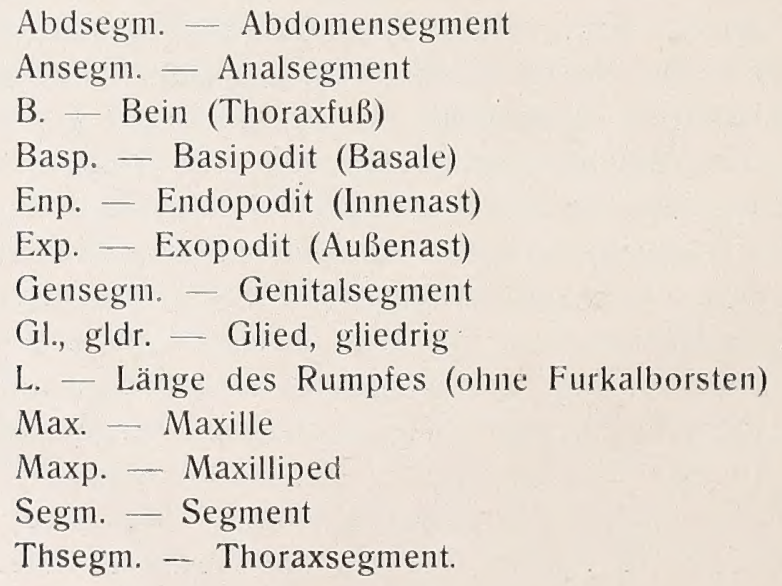




\section{Trib. Gymnoplea.}

Die Genitalöffnungen liegen im ersten Segment des Hinterleibes, die paarigen des $q$ ventral, die unpaarigen des ơ lateral. 5. B. beim $\bigcirc$ den vorhergehenden ähnlich oder rückgebildet oder fehlend, beim ơ ein Kopılationsorgan und stets vorhanden. Das 1. Segment des Hinterleibes trägt keine Beine. Von den 1. Antennen des genikuliert nur eine oder keine. Die Ejer werden meistens einzeln abgelegt, seltener in Ballen oder Säckchen an der Genitalöffnung aufgehängt. Herz meistens vorhanden. Ovarium unpaarig, Oviducte paarig; Hoden und Vasa deferentia unpaarig. Darm meistens einfach, zuweilen mit vorderem und kleinem seitlichen Blindsack. Bauchmark lang. Auge unpaarig, paarig oder fehlend. Fast stets planktonisch.

\section{Calanidae.}

Die 1. Antennen des or sind symmetrisch oder nahezu symmetrisch gebaut und genikulieren nicht; sie sind mit Aesthetasken reicher ausgestattet als beim $O$ (Spürantennen). Das 5. B. des $Q$ ist entweder den vorhergehenden ähnlich oder zeigt alle Stufen der Rückbildung, nicht selten bis zum völligen Ausfall. Die $\sigma^{\pi}$ weichen von den ${ }_{7}$ außer in Segmentierung und Form des Abdomens, in Bau, Gliederung und Anhängen der 1. Antennen und des 5. B., meistens auch durch eine Um- und Rückbildung der Mundgliedmaßen ab. Kopf vom Thorax gewöhnlich getrennt, die beiden letzten Thsegm. gewöhnlich verschmolzen. Rostrum 2'- oder 1-spitzig, zuweilen fehlend. Abdomen des 7 4-, selten 3- oder 2-gldr., des o 5-gldr., mit linker Genitalöffnung und oft mit sehr verkürztem Ansegm. 1. Antenne des $\bigcirc$ 16- bis 25-gldr. Exp. der 2. Antenne 4 bis $1 / 2$ mal so lang wie das Enp., 5- bis 8-gldr., mit verkürzten Mittelgl. und meistens gestrecktem Endgl. Kaulade der Mandibel mit 6 bis 8 Zähnen; Exp. 5-, Enp. 2-gldr. 1. Maxille mit wenigstens 2 Innenrandloben und 1 Außenrandlobus; Exp. stets vorhanden, Enp. 3- bis 1-gldr., selten mit dem 2. Gl. des Basp. verschmolzen. Distale und proximale Reusenborsten der 2. Maxille meistens etwa gleich lang; jene zuweilen in weiche Schläuche verwandelt. Maxilliped langgestreckt, mit 5-gldr. Endabschnitt (Enp.), dessen Länge "1/2 bis 1/3 von der des 2. Gi. des Basp. beträgt. Exp. des 1.-4. B. 3- (nur im 1. B. zuweilen 2-) gldr.; Enp. des 1. und 2. B. 1- bis 3-, des 3. und 4. B. 3-gldr.; Enddorn aller Exp. zuweilen mit glattem Saum. Die Enp. des 5. B. des of sind selten 3-gldr., meistens rudimentär oder fehlend; die Exp. bilden Haken oder Scheren; zuweilen fehlt das ganze B. auf einer Seite. 


\section{Calanus Leach.}

Kopf mit dem 1. Thsegm. verschmolzen oder davon getrennt (Unterschiede in dieser Beziehung können auch zwischen o und $q$ derselben Art bestehen). Die letzten beiden Thsegm. getrennt. Rostrum in zwei weiche Zipfel endigend. Abdomen des $q$ 4-gldr., des $\sigma^{7} 5$-gldr. 1. Antenne beim $q$ 25-gldr. mit einer sehr langen und lang gefiederten Hinterrandborste am 23. und 24. Gl., beim $\sigma^{7}$ mit reduzierter Gliederzahl. Mundteile des $\sigma^{\top}$ von denen des $q$ kaum abweichend oder mehr weniger verkümmert. 1.-4. B. mit 3-gldr. Exp. und Enp.; alle Exp. mit 1, 1, 2 Außenranddornen, der Enddorn skalpellförmig, meistens (die des 2.-4. B. stets) mit glattrandigem Saum; Enp. des 1. B. mit 1, 2, 6 . Borsten; letztes Gl. des Enp. des 2, und 3. B. mit 8 Borsten. 5. B. des $\&$ den vorhergehenden ähnlich. 5 . B. des or ein zuweilen schwimmbeinähnliches, zuweilen scherenartiges Greiforgan; Basp. beiderseits 2-gldr., das rechte B. mit 3-gldr. Exp. und Enp., das linke mit 3-gldr. Exp. (ohne Fiederborsten) und rückgebildetem oder fehlendem Enp.

1. 1. Antenne überragt das Rumpfende "um wenigstens die halbe Rumpflänge

Dieselbe überragt das Rumpfende nicht oder höchstens um wenige Gl. 3

2. Furkalborsten symmetrisch; Furka doppelt so lang wie breit C. tenuicornis Furka links mit einer verlängerten Borste; Furka etwa so lang wie breit; (Ventralfläche des Gensegm. schwach gewölbt) C. gracilis

3. Stirn mit medianer Crista; 1. Gl. des Basp. des 5. B. mit gefiedertem Innenrande

C. cristatus

Stirn ohne Crista; 1 Gi. des Basp. des 5. B. am Innenrande ohne Zähnchen; 1. Antenne fast borstenlos

C. tonsus

Stirn rund, ohne Crista; 1 . Gl. des Basp. des 5. B. mit gezähneltem Innenrande; 1 . Antenne normal beborstet

4. Lateralteile des letzten Thsegm. abgerundet · 5 Dieselbe zugespitzt

5. Stirn von der Seite gesehen schmal gerundet C. helgolandicus Stirn von der Seite geseher breit gerundet

C. finmarchicus

6. Proximales Stück am Außenrande des letzten Gl. des Exp. des 2.-4. B. 2-3 mal so lang wie das distale; distales Stück des 1. Gl. des Basp. des 5. B. ungezähnelt

C. hyperboraeus Proximales Stück am Außenrande des letzten Gl. des Exp. des 2.-4. B. weniger als doppelt so lang wie das distale; Innenrand des 1. Gl. des Basp. des 5. B. in seiner ganzen Länge gezähnelt

7. Gezähnelter Saum am 1. Basalgl. des 5. B. nicht eingebuchtet, flach konvex, distal durch eine Lücke unterbrochen, hinter welcher noch 2-3 Zähnchen stehen

C. simillimus

Gezähnelter Saum distal von der Mitte eingebuchtet; sämtliche Zähnchen bilden eine ununterbrochene Reihe

C. propinquus 
1. 1. Antenne ïberragt das Rumpfende um ca, 6 Gl.

C. tenuicornis

1. Antenne überragt das Rumpfende nicht oder wenig

2

2. Innenrand des 1. Gl. des Basp. des 5. B. ohne Zähnchensaum; Außenrand des Endgl. des Exp. am 2.-4. B. gezähnelt; (Enp. des 5. B. gleich gebaut)

C. gracilis

1. Gl. des Basp. des 5. B. mit gezähneltem Innenrand; Außenrand des Endgl. des Exp. am 2.--4. B. glatt oder gefiedert . 3

3. Linkes Exp. des 5. B. mehr als doppelt so lang wie das rechte 4 Linkes Exp. des 5. B. weniger als doppelt so lang wie das rechte 6

4. Letztes Thsegm. zugespitzt; distales Stück des Innenrandes am 1. Gl: des Basp. des 5. B. ungezähnelt; 5. B. sehr wenig asymmetrisch

C. hyperboraeus

Letztes Thsegm. abgerundet; Innenrand des 1. Gl. des Basp. des 5. B. in seiner ganzen Ausdehnung gezähnelt; 5. B. deutlich asymmetrisch 5

5. Enp. des linken B. die Mitte des 2. Gl. des Exp. erreichend; letztes G1. des Exp. desselben B. wenig kürzer als das vorletzte Gl. C. finmarchicus Enp. des linken B. nur wenig länger als das 1. Gl. des Exp., letztes Gil. des Exp. desselben B. breiter und kürzer als bei finmarchicus, kaum die halbe Länge des vorletzten Gl. erreichend C. helgolandicus

6. Rechter Exp. und linker Enp. ragen nicht bis zum distalen Rand des 1. Gl. des linken Exp. hervor

C. simillimus

Dieselben ragen bis zum distalen Rand des 1. Gl. des linken Exp. hervor

C. propinquus

\section{Calanus finmarchicus Gunner.}

1765, Monuculus f., Ginner, p. 175, f. 20-23. 1892, C. f. (inclus. C. helgolandicus Cls.), Giesbrecht, p. 89 , t. 6 f. $19 ;$ t. 7 f. 32,33 ; t. 8 f. 3 , $15,20,21,31,33.1898$, C. f. (inclus. C. helgolandicus Cls.), Giesbrecht u. Schmeil, p. 14. 1903, C. f., Sars, p. 9, t. 1-3.

f. Kopf vom 1. Thsegm. getrennt; Stirn in Lateralansicht breit gerundet; Lateralteile des letzten Thsegm. abgerundet; Furkalborsten symmetrisch. 1. Antenne das Rumpfende höchstens um 3 Gl. überragend; Aesthetasken an keinem Glied verdoppelt. Distaler Rand des 2. Gl. des Basp. des 2.-4. B. mit Zacke; der Randdorn teilt den Außenrand des letzten Gl. des Exp. des 2.-4. B. nach dem Verhältnisse 2:1, 2:1, 3:1. 1. Gl. des Basp. des 5. B. der ganzen Länge nach am Innenrande mit konkavem, gezähneltem Saum. б. Kopf vom 1. Thsegm. getrennt. 1. Antenne gerade; 1. und 2. Gi. verschmolzen. Mundteile denen des. $\%$ ähnlich. Die Enp. des 5. B. einander gleich; rechtes Exp. ohne Innenrandborsten, mit dornförmiger Endborste; links das Basp. und die proximalen GI. des Exp. verlängert, letztes Gl. des Exp. nur wenig kürzer als das vorhergehende Gl.; linkes Enp. ungefähr die Mitte des 2. Gl. des Exp. erreichend. - L. \& 2.7-5.4 mm, o $2.35-3.6 \mathrm{~mm}$. 
Anmerkung. Nach dem Vorgange von Sars (1903) habe ich hier C. finmarchicus und C. helgolandicus als selbständige Arten aufgeführt, obgleich andere Autoren (Wolfenden 1904, Gough 1905) die Trennung für nicht berechtigt halten. Verbreitung: nach Sars (1. c. p. 11) ist diese Art besonders charakteristisch für die arktische Region und wurde von Grönland im Westen bis nach den Behring-Inseln im Osten durch das ganze Polarbecken hindurch gefunden. An der norwegischen Küste soll sie sich nicht weiter östlich als Vadsö zeigen; in die Nordsee aber dringt sie weit südlich vor.

\section{Calanus helgolandicus Claus.}

1863 Cetochilus h., Claus, p. 171, t. 26 f. 2-9. 1903, C. h., Sars, p. 11, t. 4.

Verwandt mit C. finmarchicus, aber: 9 . Stirn in Lateralansicht schmal gerundet; Furkaläste kürzer, kaum länger als das Analsegment (bei C. finmarchicus etwas länger als dasselbe). - 0 . Exp. des linken 5. B. relativ länger als bei C. finmarchicus, letztes Gl. kürzer und breiter, ungefähr halb so lang wie das vorhergehende Gl. Enp. des linken 5. B. das 1. Gl. des Exp. nur wenig überragend. - L. क $3 \mathrm{~mm}$, o $2.8 \mathrm{~mm}$.

Nordsee, Westkuiste Frankreichs, Mittelmeer, Schwarzes Meer, NeuSeeland (?); Pacif. Ozean.

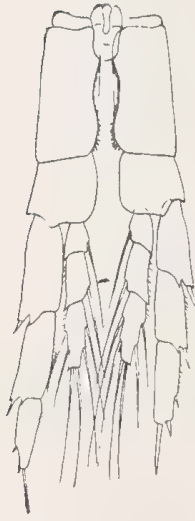

a

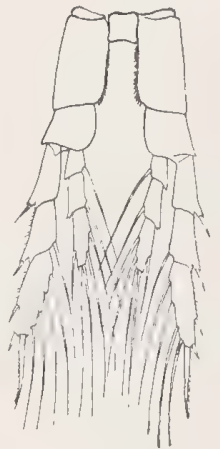

b

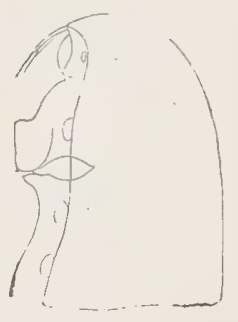

$c$

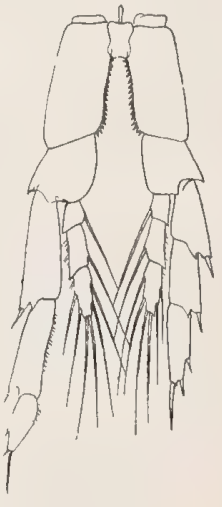

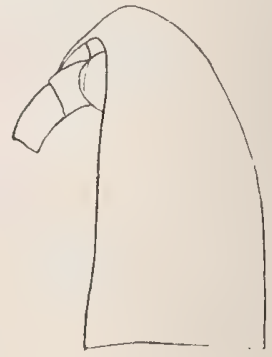

b

Fig. 2. Calanus helgolandicus. a 5. B. 万ั, b Kopf und 1. Thsegm. , lateral. Nach Sars.

Fig. 1. Calanus finmarchicus. a 5. B. or, b 5. B. 9 , c Kopf und 1. Thsegm. O, lateral. Nach Sars.

\section{Calanus hyperboraeus Kröyer.}

1838, C. h., Kröyer, p. 310 , t. $4 . \quad 1892$, C. h., Giesbrecht, p. 91 , t. 6 f. 6 ; t. 7 f. 12 ; t. 8 f. $10,23.1898$, C. h., Giesbrecht und Schmeil, p. 15.1903 , C. h., Sars, p. 12 , t. 5 .

Verwandt mit C. finmarchicus; aber: $\bigcirc$. Lateralecken des letzten Thsegm. zugespitzt; Innenrand des 1. Gl. des Basp. des 5. B. distal ohne 
Zähnchen. - o. 5. Thsegm. und 1. Gl. des Basp. des 5. B. wie beim ๆ; 5. B. nur wenig asymmetrisch, linkes Exp. kaum länger als das rechte, mit etwas verschmälertem Endglied; linkes Enp. die Mitte des Endgl. des linken Exp. erreichend. - L. o bis $9 \mathrm{~mm}$, o bis $6.5 \mathrm{~mm}$.

Atlant. Ozean (nördl. von $60^{\circ} \mathrm{N}$. Br.); nördlicher Teil der Nordsee; Polarmeer von Grönland bis Behringstraße.

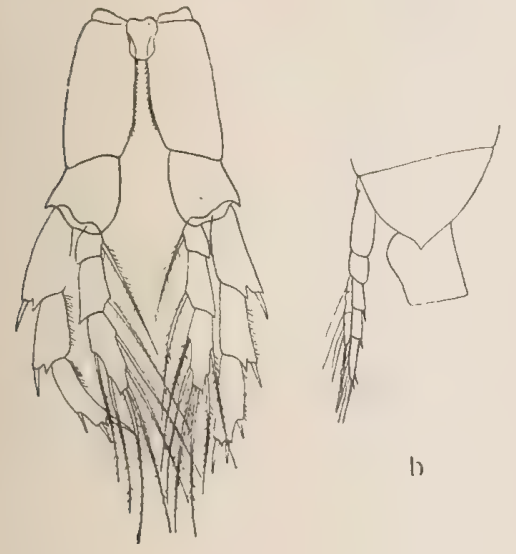

a

Fig. 3. Calanus hyperboraens. a 5. B. ơ, b 5. Thsegm. und Ansegm. , lateral. Nach Sars.

\section{Calanus propinquus Brady.}

1883, C. p., Brady, p. 34 t. 2. 1902 , C. p., Giesbrecht, p. 16 , t. 1 f. $1-8$.

Verwandt mit C. finmarchicus; aber: o mit zugespitzten Lateralecken am letzten Thsegm.; Randdorn teilt den Außenrand des letzten Gl. des Exp. des 2.-4 B. nach dem Verhältnisse $12: 11,5: 4,5: 3$; gezähnelter Saum am Innenrande des 1. Gl. des Basp. des 5. B. ohne Lücke, distal von der Mitte eingebuchtet. - $\sigma$. Rechtes Exp. reicht etwa bis zum distalen Rand des 1. Gl. des linken Exp. und das linke Exp. überragt diesen Rand etwas. L. $\bigcirc 5-5.5 \mathrm{~mm}$.

Die Verbreitung ist nicht ganz sicher wegen Verwechslung mit C. simillimus. Nach Giesbrecht (1902) ist ihr Vorkommen nördlich vom Äquator zweifelhaft.

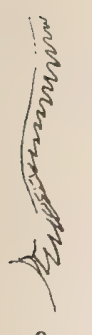

a
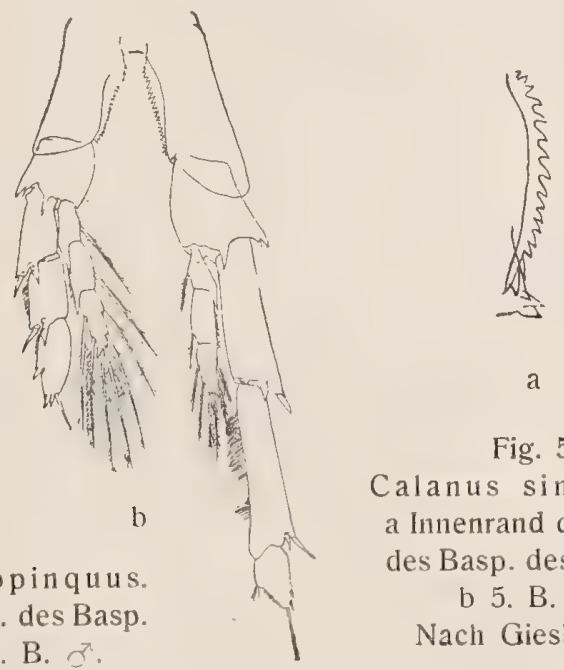

a

Fig. 5.

Calanus simillimus. a Innenrand des 1. Gl. des Basp. des 5. B. 우,

b 5. B. of.

Nach Giesbrecht.

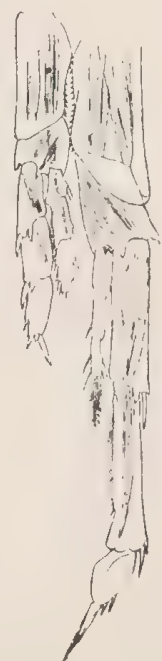

b 


\section{Calanus simillimus Giesbrecht.}

1892, C. propinquus, Giesbrecht, p. 91 , t. 7 f. 31,34 ; t. 8 f. 14,22 , 24, 32. 1902, C. s., Giesbrecht, p. 16, 17 t. 1 f. 9.

Verwandt mit C. propinquus; aber: ${ }^{\text {. }}$. Gezähnelter Saum am Innenrande des 1. Gl. des Basp. des 5. B. nicht eingebuchtet, flach konvex, kurz vor dem distalen Ende mit einer Lücke, hinter welcher noch 2 oder 3 Zähnchen stehen. - $\sigma$. Rechtes Exp. und linkes Enp. ragen nicht bis zum distalen Rand des 1. Gi. des linken Exp. hervor; linkes Enp. schmäler als bei C. propinqu us mit stärker verkümmerten Borsten. - L. $q$ und o ca. $3.5 \mathrm{~mm}$.

Als sicherer Fundort kann nur das SW des Atlant. Ozeans zwischen 37 und $52^{\circ} \mathrm{S}$. genannt werden. Die weitere Verbreitung ist wegen Verwechslung mit C. propinquus unsicher.

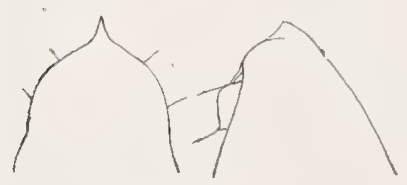

2 b

Fig. 6.

\section{Calanus cristatus Kröyer.}

1848-49, C. c., Kröyer, p. 547, 553, 607. 1892 , C. c. Giesbrecht, p. 91 , t. 6 f. $14,15.1898$, C. c., Giesbrecht und Schmeil, p. 16.

Nur aus weiblichen Jugendstadien bekannt. Stirn mit Crista. - L. (letztes Stadium vor der Calanus cristatus juv. Reife) $8.3 \mathrm{~mm}$.

a Kopf dorsal, b Kopf lateral. Nach Giesbrecht.

Pacif. Ozean (53-60 ${ }^{\circ} \mathrm{N}$. Br.); Atlant. Ozean $\left(52^{0} \mathrm{~N}\right.$. Br. $)$ in Tiefen von $560-1510$ Faden.

\section{Calanus gracilis Dana.}

1849, C. g., Cyclopsina longicornis, Dana, p. 18, $24 . \quad 1863$, Cetochilus longiremis, Claus, p. 171, t. 26 f 1. 1892, C. g., Giesbrecht, p. 90 , t. 1 f. 1 ; t. 6 f. 1 ; t. 7 f. 26 ; t. 8 f. $2,4,6-8,12,16,26.1898$, C. g. Giesbr. und Schmeil, p. 17.

q. Kopf mit dem 1. Thsegm. verschmolzen; Stirn und Lateralteile des letzten Thsegm. abgerundet; Furka links mit einer verlängerten Borste. 1. Antenne wenigstens $1 \frac{1}{2} / 2$ mal so lang wie der Rumpf; Aesthetasken an keinem Gl. verdoppelt. Am Grunde der Innenrandborste des 2. G1. des Basp. des 1. B. ein Fortsatz; Randdorn teilt den Außenrand des letzten Gl. des Exp. des 2.-4. B. nach dem Verhältnis $1: 1,4: 3,4: 4$; besondere Eigentümlichkeiten am Enddorn des Exp. des 1., und am Außenrande der Gl. des Enp. des 2. B. 1. Gl. des Basp. des 5. B. mit gefiedertem Innenrande. - ot. Kopf vom Thsegm. getrennt. 1. Antenne gerade, 1. mit 2., 24. mit 25. Gl. verschmolzen. Mundteile stark rückgebildet. Letztes Gl. des Exp. des 2.-4. B. 


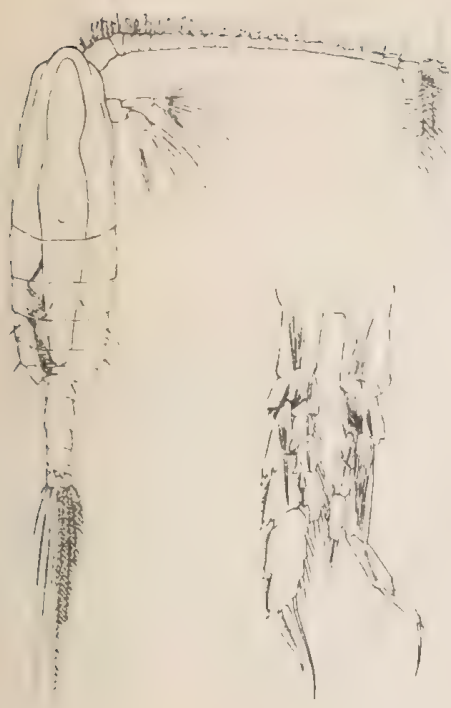

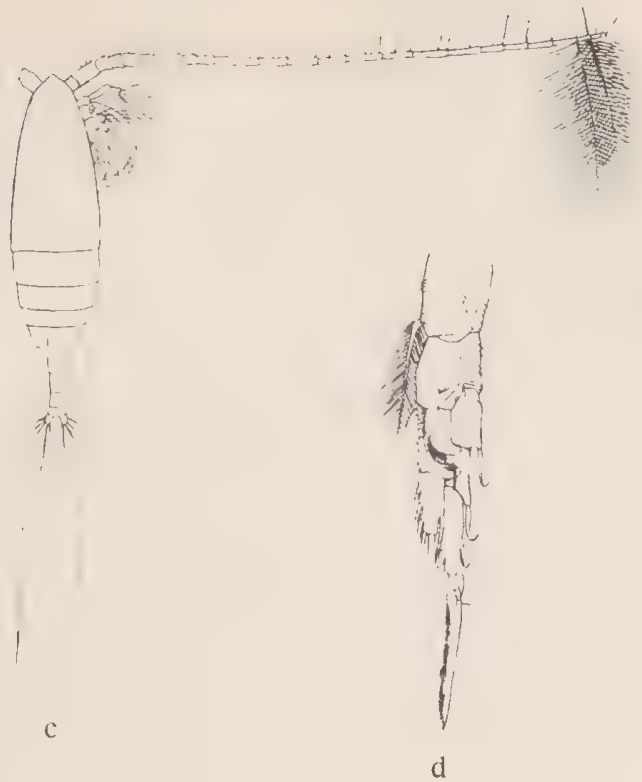

Fig. 7.

Calanus gracilis.

a o dorsal, b 5. B. ơ, c $q$ dorsal, d 1. B. 9 , Vorderfläche.

Nach Giesbrecht.

mit gezähneltem Außenrande. Rechtes 5. B. und Enp. des linken wie an den vorhergehenden B.; links das Basp. und 1. und 2. Gl. des Exp. verlängert, 3. verkürzt; linkes Exp. ohne Innenrandborsten. - L. o $2,4-3.25 \mathrm{~mm}$, o $2.5-2.8 \mathrm{~mm}$.

Mittelmeer; Atlant., Indischer und Pacif. Ozean.

\section{Calanus tenuicornis Dana.}

1849 , C. t., Dana, p. 15.1892 , C. t., Giesbrecht, p. 90 , t. 6 f. 12,13 ; t. 7 f. $5,16,23$; t. 8 f. $18,27,1898$, C. t., Giesbr. und Schmeil, p. 18.

Q. Kopf vom 1. Thsegm. getrennt; Stirn und Lateralteile des letzten Thsegm. abgerundet; Furkalborsten symmetrisch. 1. Antenne wenigstens $11 / 2$ mal so lang wie der Rumpf, Aesthetasken an keinem Gi. verdoppelt. Randdorn teilt den Außenrand des letzten Gl. des Exp. des 2._4. B. nach dem Verhältnis $5: 4,10: 7,7: 4 ; 1$. Gl. des Basp. des 5. B. am Innenrande gefiedert. - $\sigma^{\zeta}$. Kopf vom 1. Thsegm. getrennt; 1 . Antenne ähnlich wie beim \%, aber das 1. mit 2., 3.-5., 7. mit 8., 9. mit 10., 24. mit 25. Gl. verschmolzen. Mundteile stark rückgebildet. Die Exp. des 5. B. ohne 


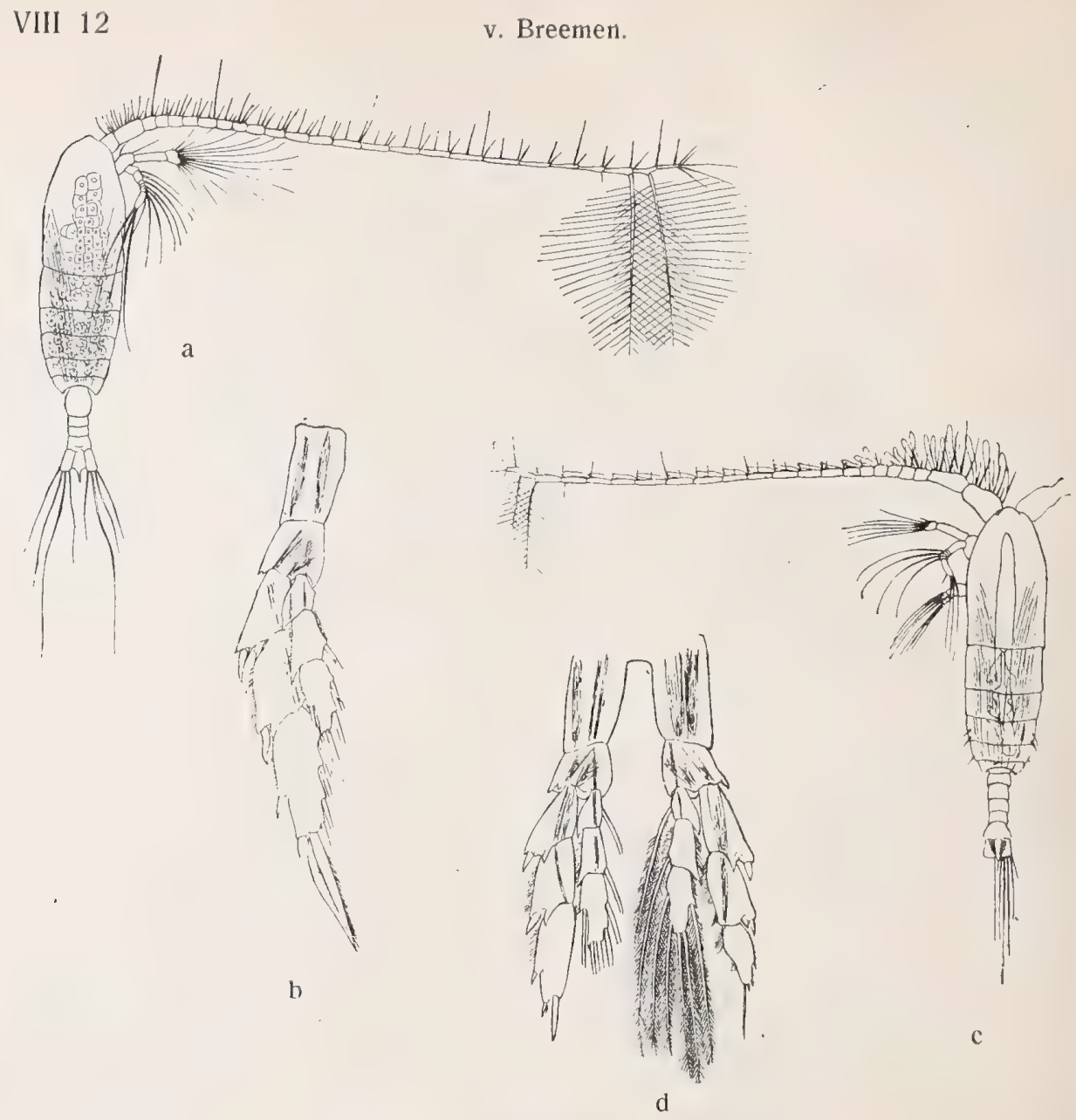

Fig. 8. Calanus tenuicornis.

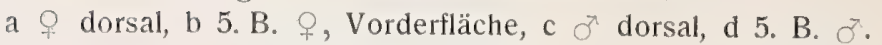
Nach Giesbrecht.

Innenrandborsten, das rechte mit dornförmiger, das linke mit dünner Endborste; rechtes Basp. und 1. und 2. Gl. des Exp. etwas verlängert, 3. verkürzt. L. क $1.9-2.5 \mathrm{~mm}$, o $1.85-1.95 \mathrm{~mm}$.

Mittelmeer, Atlant. und Pacif. Ozean.

\section{Calanus tonsus Brady.}

1883, C. t., Brady, p. 34 t. 4 f. 8, 9. 1898, C.t., Giesbr. und Schmeil, p. 19.

7. Verwandt mit C. finmarchicus und C. propinquus, aber die 1. Antenne fast borstenlos, Gensegm. breit und gewölbt, Innenrand des 1. Gl. des Basp. des 5. B. nicht gezähnelt. - L. $\subsetneq 3.6 \mathrm{~mm}$.

Atlant. und Pacif. Ozean. 
Megacalanus Wolfenden.

Verwandt mit Calanus: - Kopf vom 1. Thsegm. getrennt; 4. und 5. Thsegm. gesondert; Mundorgane denen von Calanus sehr ähnlich; 1. Antenue 25-gldr.; Endgl. des Exp. des 2.-4. B. mit drei Außenranddornen; Innenrand des Basp. des 5. B. nicht gezähnelt oder gefiedert.

\section{Megacalanus princeps Brady.}

1883, Calanus p., Brady, p. 36, t. 4. 1905 (non 1904), M. p., Wolfenden.

‥ 1. Antenne länger als der Rumpf; 2. Maxille mit starken, gebogenen Borsten, welche an Innenrande mit zahlreichen, feinen und dichtgestellten Haaren, ausgenommen nach der Basis zu, ausgestattet sind; 2. Gl. des Basp. des 1. B. ohne Auswuchs. L. \& $12.5 \mathrm{~mm}$. - o unbekannt.

Atlant. Ozean.

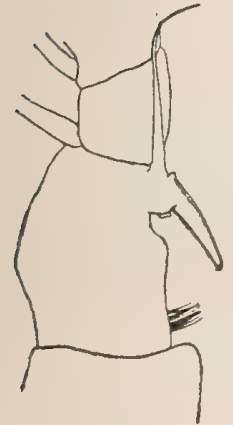

Fig. 9. Megacalanus longicornis.

2. Gil. des Basp. des 1. B. $q$.

Nach Wolfenden.

\section{Megacalanus longicornis G. O. Sars.}

1904 (non 1905), M. princeps, Wolfenden, p. 112, t. 9 f. 1, 2. 1905, Macrocalanus 1., Sars, Nr. 26, p. 7. 1905, M. bradyi, Wolfenden.

Unterscheidet sich von $M$. princeps Brady hauptsächlich durch folgende Merkmale:- - . Körperform schmäler und länger; Rostralfäden gerade nach unten gerichtet; 1. Antenne länger als bei $M$. princeps; Enddornen der 2. Maxille nur mit zerstreuten Haaren; 2. Gl. des Basp. des 1. B. an der inneren Seite mit kräftigem, dornartigem Vorsprung, daneben eine gebogene Fiederborste. L. O $9,5 \mathrm{~mm}$. - o unbekannt.

Nord-Atlantischer Ozean.

\section{Eucalanus Dana.}

Rumpf beim o weit gestreckt, beim o kürzer als beim q; Kopf dreieckig, öfters verlängert, beim $\%$ und $\delta$ mit dem 1 . Thsegm. verschmolzen; Rostrum in dünne Fäden ausgehend. Abdomen kurz, beim $\bigcirc$ 3-oder 4-gldr., beim of 5-gldr. Ansegm. und die asymmetrische Furka verschmolzen ${ }^{1}$ ).

1) Der größere Furkalast und die längere Furkalborste können auf der linken Seite statt auf der rechten vorkommen. 
1. Antenne das Rumpfende überragend, zuweilen von asymmetrischer Länge, beim o 23-gldr., mit langen, z. T. farbig gefiederten Endborsten, beim ot 24-gldr. Enp. der 2. Antenne länger als das 7-oder 8-gldr. Exp. Mandibel des $O$ länger als die 1. Maxille; das 2. Gl. des Basp. bildet mit dem Exp. eine Walze, woran das Enp. proximal vom Exp. artikuliert. Enp. des Maxp. lang, mit langen Borsten versehen. Mundteile des or mehr oder weniger rückgebildet. B. kurz; Exp. 3-gldr., Enp. des 1. B. 2-, des 2.-4. B. 3-gldr.; Endgl. des Exp: des 2.-4. B. mit drei Außenranddornen, ihren Enddorn mit breitem, nicht gezähneltem Saum, der Enddorn des 1. B. denjenigen der folgenden B. ähnlich; Endgl. des Enp. des 1. B. mit 4, des 2.-4. B. mit 5 Borsten. 5. B. beim $\&$ fehlend, beim of beiderseits einästig, links 4-gldr., rechts 1 - bis 4-gldr. oder fehlend.

1 Abdomen 4-gldr.

E. elongatus

Abdomen 3-gldr.

2 Stirn dreieckig, beiderseits eingeknickt, stark verjüngt; Gensegm, länger als breit

E. attenuatus

Stirn flach abgerundet; Gensegm. viel breiter als lang, zwịebelförmig

E. crassus

1 Das rechte 5. B. fehit

E. crassus

Beide B. des 5. Paares vorhanden.

2 Exp. der 2. Antenne erreicht fast den Rand des 1. Gl. des Enp.; linkes 5. B. beträchtlich kürzer als das 4 . B.

E. attenuatus

Exp. der 2. Antenne reicht lange nicht bis zum distalen Rande des 1. G1. des Enp.; linkes 5. B. so lang wie das 4. B. (ohne Enddorn). E. elongatus. (Über Eucalanus atlanticus Wolfenden siehe Anmerkung pag. 15.)

\section{Eucalanus elongatus Dana.}

1849, Calanus e., Dana, p. 18. 1892, E. e., Giesbrecht, p. 131, 149, t. 11 f. $2,7,12,20,25,32,36$; t. 35 f. $1,2,13,23,24$. 1898, E. e., Giesbr. und Schmeil, p. 20.

q. Stirn regelmäßig dreieckig; Gensegm. länger als breit; zwischen ihm und dem Ansegm. 2 freie Segm.. Die beiden ersten Gl. des Exp. der 2. Antenne gesondert; das proximale Gl. des Enp. über 3 mal so lang wie breit und wenig länger als das distale. Mandibel: 2. Gl. des Basp. mit 3 Innenrandborsten; das Enp. bleibt hinter dem Endrande des Basp. um die eigene Länge zurück. 2. Innenrandlobus der 1. Max. vorhanden; 3. Lobus mit 4, 2. Gl. des Basp. 

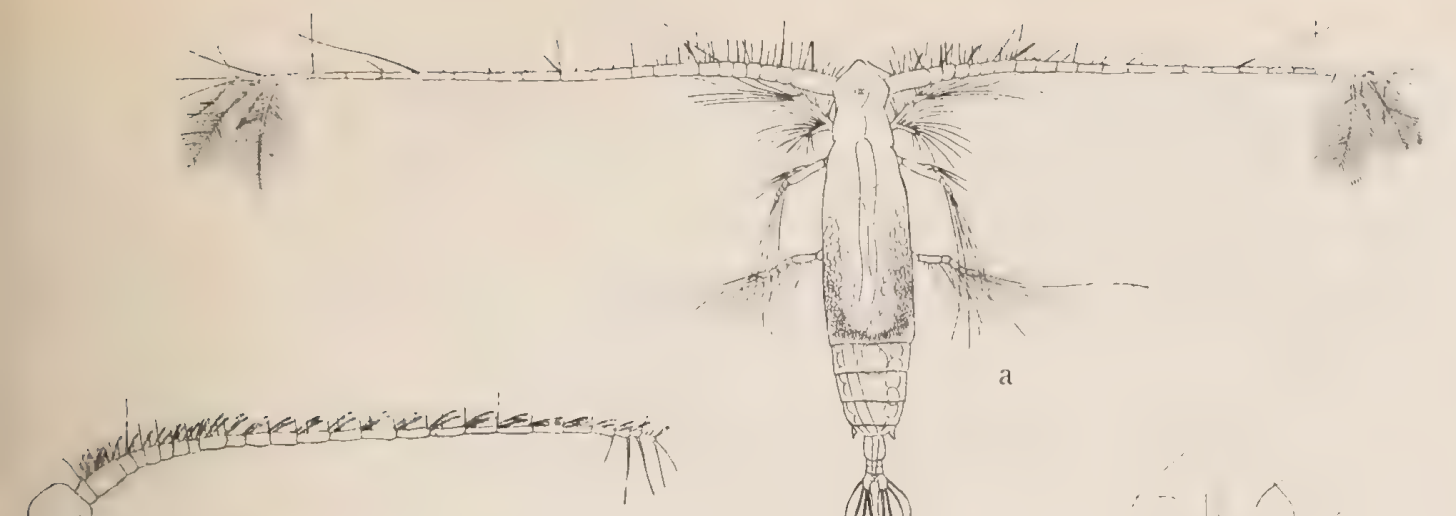

b)
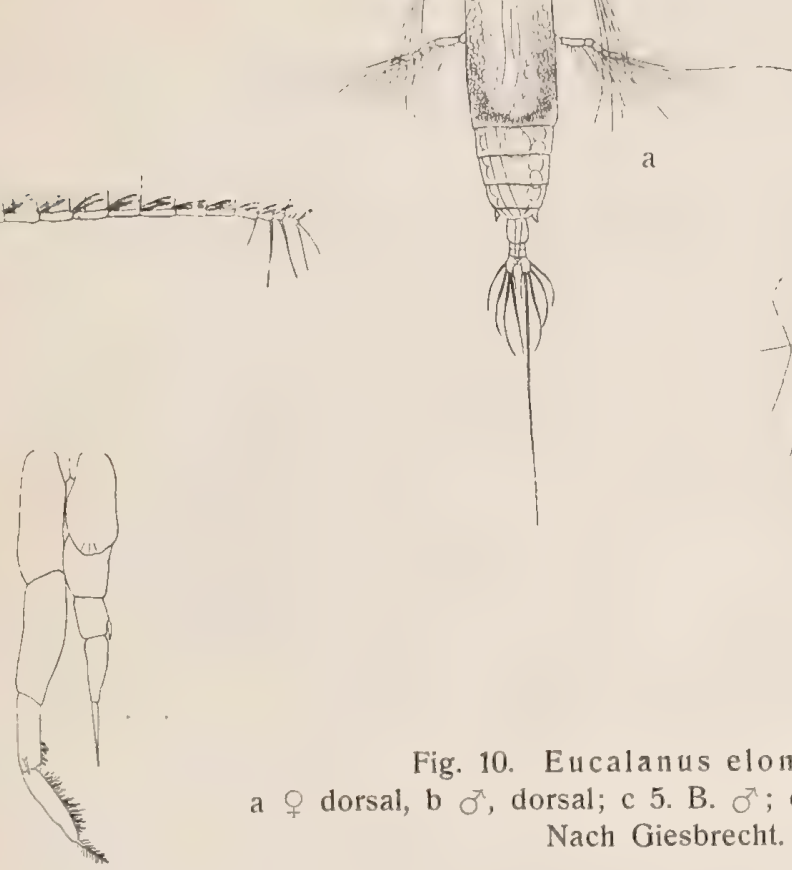

a

Fig. 10. Eucalanus elongatus.

a $O$ dorsal, b $\sigma^{\text {T }}$, dorsal; c 5. B. $\sigma^{\top}$; d Kopf , ventral. Nach Giesbrecht.

mit 5. Borsten. - o . Stark ausgeprägte Sexual-Charaktere; rechtes 5. B. vorhanden, linkes so lang wie das 4. B. (ohne Enddorn). - L. $q 4,4-8,25 \mathrm{~mm}$, o $3,9-4,8 \mathrm{~mm}$.

Mittelmeer, Atlant. Ozean (bis Skagerak im Osten); Pacif. Ozean.

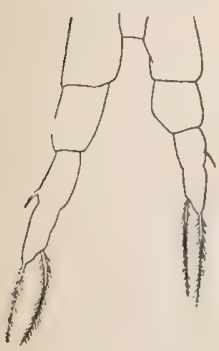

Fig. 11. Eucalanus atlanticus.

5. B. $\sigma^{7}$.

Nach Wolfenden.
Anmerkung. Wolfenden (1904, p. 113 t. 9 f. 3, 4) erwähnt das Vorkommen von Männchen einer $\mathrm{E} u \mathrm{c}$ a 1 a $\mathrm{n}$ u s spezies, bei welchen zusammen mit dem Vorkommen des größeren Furkalastes und der längeren Furkalborste auf der linken statt auf der rechten Seite (welche Unregelmäßigkeit Giesbrecht schon für das o von E. elongatus festgestellt hatte) die Mundorgane nicht zurückgebildet und denèn des o vollkommen gleich sind. Bis der Beweis erbracht wird, daß diese $\sigma^{7}$ nur männliche E. e lo ng a tus vor der letzten Häutung (im letzten Copepodidstadium) sind, betrachtet Wolfenden sie als zu einer neuen Spezies gehörig, die er $E$. atlanticus nennt. Das 5. B. o ist in Fig. 11 abgebildet. - L. o 4,45-4,5 mm.

Nord-Atlant. Ozean. 


\section{Eucalanus attenuatus Dana.}

1849, Calanus a., Dana, p. 18. 1863, Calanella mediterranea, Claus, p. 176 , t. 28 f. $6-11.1892$, E. a., Giesbrecht, p. 131 , t. 3 f. 1 ; t. 11 f. 1 , $11,13,16,18,24,40$; t. 35 f. $3,6,17,25,34,37.1898$, E. a., Giesbr. und Schmeil, p. 20.

ๆ. Stirn dreieckig, beiderseits eingeknickt, stark verjüngt. Gensegm. länger als breit; zwischen ihm und dem Ansegm. 1 freies Segm. Die beiden ersten Gl. des Exp. der 2. Antenne gesondert; das proximale Gl. des Enp. 4 mal so lang wie breit und $11 / 3$ mal so lang wie das distale. Mandibel: 2. Gl. des Basp. mit 2 Innenrandborsten; das Enp. bleibt hinter dem Endrande

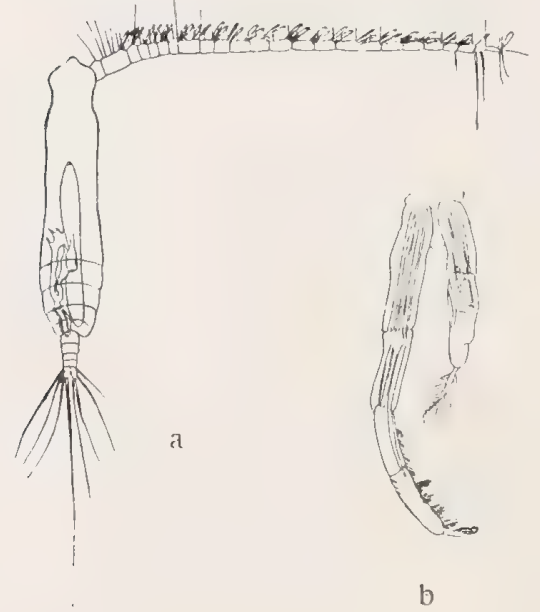

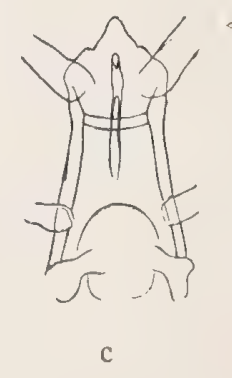

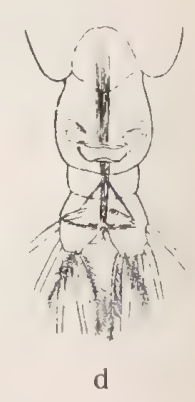

Fig. 12. Eucalanus attenuatus. a ơ dorsal, b 5. B. ఠ, c Kopf \&, ventral, d Abdomen $Q$, ventral. Nach Giesbrecht.

des Basp. um mehr als die eigene Länge zurück. 1. Max. wie bei E. elongatus. 1. Gl. des Enp. des Maxp. mit 3, 2. mit 4 Borsten. - o. Stark ausgeprägte Sexual-Charaktere; rechtes 5. B. vorhanden, linkes um die Länge des letzten Gl. des Exp. des 4. B. kürzer als dieses. - L. O 4,2-4,85 $\mathrm{mm}$, o $3,1-3,25 \mathrm{~mm}$.

Mittelmeer; Atlant. Ozean bis zum Faröerkanal; Pacif. Ozean; Rotes Meer; Mal. Arch.; Ind. Ozean.

\section{Eucalanus crassus Giesbrecht.}

1888, E. c., Giesbrecht, p. 333. 1892, E. c., Giesbrecht, p. 132, t. 4 f. 9 ; t. 11 f. $8,10,17,21,22,38$; t. 35 f. $4,20,26-28$. 1898, E. c., Giesbr. und Schmeil, p. 22.

Q. Zwischen Gensegm. und Ansegm. 1 freies Segm.; Stirn flach abgerundet; Gensegm. viel breiter als lang, zwiebelförmig; Asymmetrie der Furka 

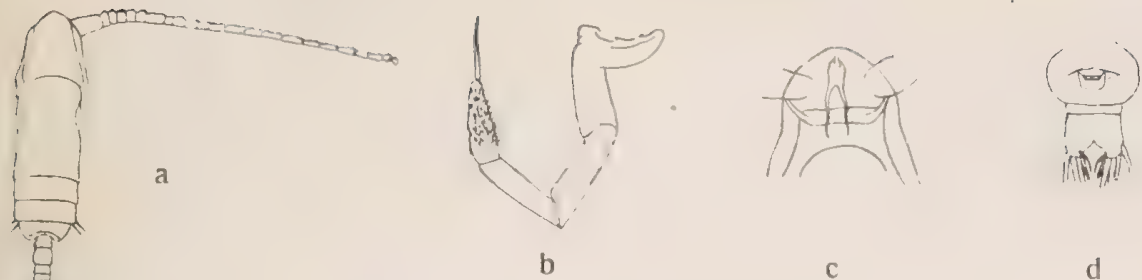

C

d

Fig. 13.

Eucalanus crassus.

a $\varnothing$ dorsal, b 5.B. $\delta$, c Kopf $ᄋ$, ventral, d Abdomen q, ventral.

Nach Giesbrecht.

und ihrer Borsten gering; proximales Gl. des Enp. der 2. Antenne etwa doppelt so lang wie breit. - S. Sexual-Charaktere weniger ausgeprägt; rechtes 5. B. fehlt. - L. $\bigcirc 2,9-3,3 \mathrm{~mm}$, o $2,9 \mathrm{~mm}$.

Mittelmeer, Atlant., Pacif. und Ind. Ozean.

\section{Rhincalanus Dana.}

Rumpf gestreckt, beim ő kürzer als beim $\odot$; Kopf nach vorne zugespitzt, mit dem 1. Thsegm. verschmolzen; Rostrum an das vordere Ende gerückt, mit 2 dünnen Fäden. Abdomen des ${ }_{7}$ 3-, des o 5-gldr. Ansegm. und die

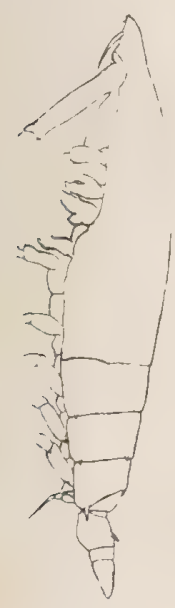

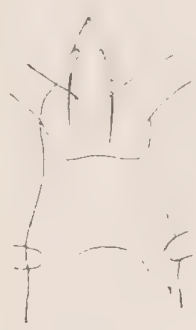

b

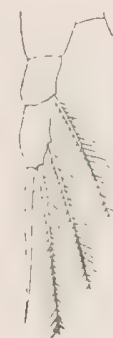

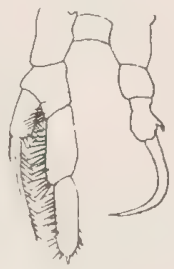

d

Fig. 14. Rhincalanus nasutus.

a $q$ lateral, b Kopf $\circ$, ventral, c 5. B. o, d 5. B. 7 . Nach Giesbrecht.

asymmetrische Furka verschmolzen. Thsegm. und Gensegm. mit Stacheln. 1. Antenne beim ${ }_{T}$ 23-gldr., das Rumpfende weit überragend, beim $\delta$ 24-gldr., verkürzt. Exp. und Enp. der 2. Antenne gleich lang. Mandibel nicht länger als die 1. Maxille. Enp. der Maxp. kürzer als jedes der Gl. des Basp. Kopfgliedmaßen des ơ denen des $q$ ähnlich. B. kurz; Exp. und Enp. des 1. B. Nord. Plankton,

VIII 2 
2-, des 2.-4. B. 3-gldr. Zahl der Außenranddornen der Exp. wie bei Eucalanus; die Außenranddornen des Exp. des 2.-4. B. mit dem Gl. verwachsen; Zahl der Borsten der Enp. wie bei Eucalanus. 5. B. in beiden Geschlechtern vorhanden, beim $q$ einästig, jederseits 3-gldr, beim $\sigma$ mit 2-gldr. Basp., links mit 1-gldr. Exp. und 2-gldr. Enp., rechts mit 1-gldr. Ast.

\section{Rhincalanus cornutus Dana.}

1849, Calanus c. und C. rostrifrons, Dana, p. 19. 1892, R. c., Giesbrecht, p. 153, t. 12 f. 13,15 ; t. 35 f. 45, 48. 1898, R. c., Giesbr. u. Schmeil, p. 23.

Vorderkopf mit stielförmigem Stirnteil; Rostralfäden seitlich ausgebogen, in der Dorsalansicht sichtbar. 5. B. des $q$ ohne Borste am 2, und mit nur

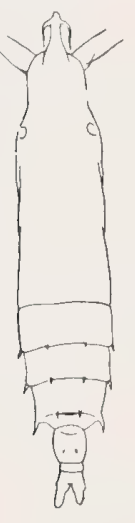

a

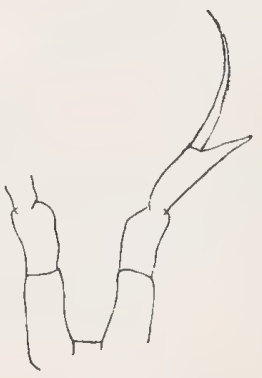

b

Fig. 15.

Rhincalanus cornutus.

a 7 dorsal, b 5. B. q, c 5. B. ð.

Nach Giesbrecht.

1 starken Borste an dem in eine Spitze ausgehenden 3. G1.; 5. B. des o rechts mit fast gerader Endborste, links mit kurzem Exp., der den distalen Rand des 1. Gl. des Enp. nicht erreicht. - L. क $3.6 \mathrm{~mm}$, o $2.68 \mathrm{~mm}$.

Atlant., Ind. und Pacif. Ozean.

\section{Rhincalanus nasutus Giesbrecht.}

1888, R. n., Giesbrecht, p. 334. 1892, R. n., Giesbrecht, p. 152, t. 3, f. 6; t. 9 f. 6,14 ; t. 12 f. $9-12,14,16,17$; t. 35 f. $46,47,49.1898$, R. n., Giesbr. und Schmeil, p. 22. 1903, R. n., Sars, p. 15, t. 6, 7.

Vorderkopf mit verlängerter, an den Seiten eingebuchteter Stirn; Rostralfäden ventral ansitzend, in der Dorsalansicht verdeckt. 5. B. des $q$ mit 
1 Borste am 2. und 3 Borsten am 3. Gl.; 5. B. des o rechts mit stark gekrümmter Endborste, links mit langem, fast bis zum Ende des Enp. reichenden Exp. - L. $ๆ 3.9-5.1 \mathrm{~mm}$, o $3.8 \mathrm{~mm}$.

Mittelmeer, Atlant. Ozean von $6^{n} \mathrm{~N}$. Br. bis nach Island; Pacif. Ozean von der Magellaenstraße bis $6^{\circ} \mathrm{N}$. Br.; Ind. Ozean.

Anmerkung. Die Angabe Th. Scott's über das Vorkommen von Rhincalanus gigas Brady an der Schottischen Küste (19th. Rep. Fish. Board Scotl., III, p. 237, t. 17 f. 1-4) hat nach Giesbrecht (1902) Bezug auf Rh, nasutus.

\section{Mecynocera J. C. Thompson.}

‥ Kopf vom 1. Thsegm. getrennt; Rostralfäden zart. Abdomen symmetrisch, kurz, 3-gldr.; Ansegm. mit der Furka artikulierend, beide symmetrisch. 1. Antenne von asymmetrischer Länge, über doppelt so lang wie der Rumpf, 23-gldr., mit einzelnen sehr langen Borsten. Exp. der 2. Ant. kaum halb so lang wie des Enp. Mandibel kürzer als die 1. Maxille, mit langem Enp. B. kurz; Exp. 3-, Enp. des 1. B. 1-, des 2--4. B. 3-gldr.; 2 Außenranddorne am Endgl. des 2. und 3. B.; Endborste der Exp. mit schmalem, glattem Saum. 5. B. symmetrisch, jederseits 5-gldr., ohne Enp. - ot unbekannt.

\section{Mecynocera clausi J. C. Thompson.}

1888, M. c., Thompson, p. 150, t. 11 . 1892, M. c., Giesbrecht, p. 160, t. 5 f. 1 ; t. 11 f. $43-45$; t. 35 f. 21, 22 . 1898, M. c., Giesbr. u. Schmeil, p. 23.

Mit den Merkmalen der Gattung. - L. क $0.92-1 \mathrm{~mm}$.

Mittelmeer, Atlant. und Pacif. Ozean; Ind. Ozean.
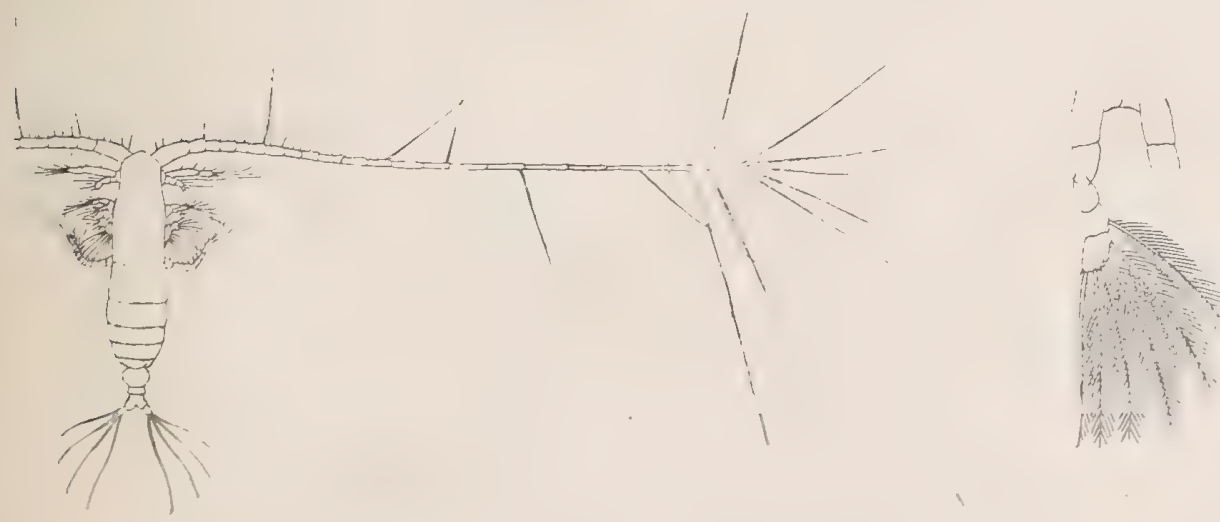

a

b

Fig. 16. Mecynocera clausi. a $q$ lorsal, b 5. B. ㅇ․ Nach Giesbrecht. 


\section{Paracalanus Boeck.}

Kopf mit dem 1. Thsegm., 4. mit 5. Thsegm. verschmolzen; Rostrum mit 2 Fäden. Abdomen des $q$ 4-, des o 5-gldr. 1. Antenne beim $q$ 25-gldr.; letztes Gl. weniger als $1 \frac{1}{2}$ mal so lang wie das vorletzte; beim $\sigma^{7}$ verschmilzt das 1.-6. und das 7. mit 8. Gl.; Endgl. verkürzt, aber gesondert. Exp. der 2. Antenne mit kurzem Endgl., kürzer als das Enp. Mandibel mit breiter Kaulade. Mundteile beim o verkümmert. Exp. des 1.-4. B. und Enp. des 2.-4. B. 3-gldr., Enp. des 1. B. 2-gldr.; Exp. mit 2 Außenranddornen am letzten Gl. und glatt gesäumter Endborste. Proximales Stück des Außenrandes am letzten Gl. des Exp. des 3. und 4. B. gezähnelt, dasjenige des 4. B. über doppelt so lang wie das distale; Enddorn des Exp. des 3. B. länger als das Endgl.; 2. Gl. des Enp. des 1. B. mit 5, 3. Gl. des Enp. des 2. B. mit 7 Borsten. 5. B. beim q kurz, 2-gldr., beim o beiderseits 1-ästig, rechts 2-, links 5-gldr.

\section{Paracalanus parvus Claus.}

1863, Calanus p., Claus, p. 173, t. 26 f. $10-14$; t. 27 f. $1-4.1892$, P. p., Giesbrecht, p. 164 , t. 1 f. 5 ; t. 6 f. $28-30$; t. 9 f. $5,11,25,27,31,32$. 1898, P. p. Giesbr. u. Schmeil, p. 24. 1903, P. p., Sars, p. 17, t. 8, 9.

Rostrum aus 2 schlaffen Fäden bestehend. Innenrandborste der Furka kaum länger als diese. 1. Antenne reicht etwa bis zur Mitte des Abdomens.

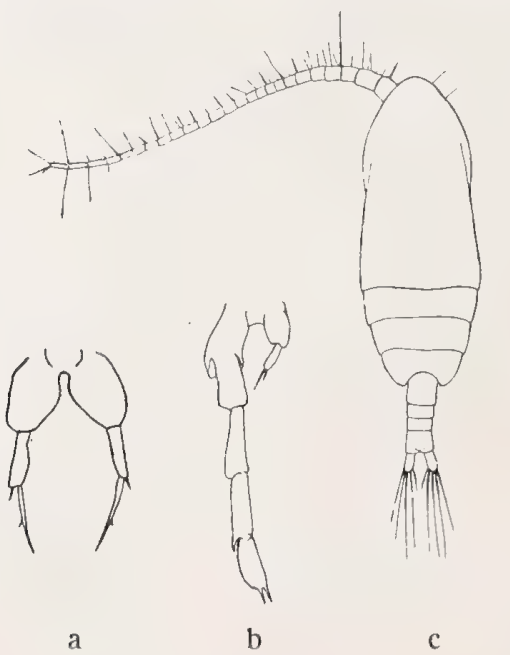

Fig. 17.

Paracalanus parvus.

a 5. B. ., b 5. B. \%, c q dorsal. Nach Giesbrecht.
Flächen des 1. und 2, Gl, des Exp. des 3. B. und des 2. Gl. des Enp. des 4. B. nackt. 5. B. beim $q$ beiderseits einästig, 2-gldr., etwa über halb so lang wie das 1. Gl. des Basp. des 4. B. (ohne Borsten); am distalen Ende des Endgl. eine äußere, kleinere und eine innere, längere Borste, welch' letztere wenigstens so lang wie das Endgl. ist; 5. B. beim or rechts 2 -gldr. und (ohne die Endborsten) viel kürzer als der Basalteil des 5-gldr. linken B. - L. $+0.8-1 \mathrm{~mm}$, o $0.9-1 \mathrm{~mm}$.

Mittelmeer, Schwarzes Meer, Atl. Ozean, westl. Ostsee, Nordsee, Südküste Norwegens, Golf von Guinea; Pacif. Ozean; Ind. Ozean; Rotes und Arab. Meer. 
Trib. Gymnoplea.

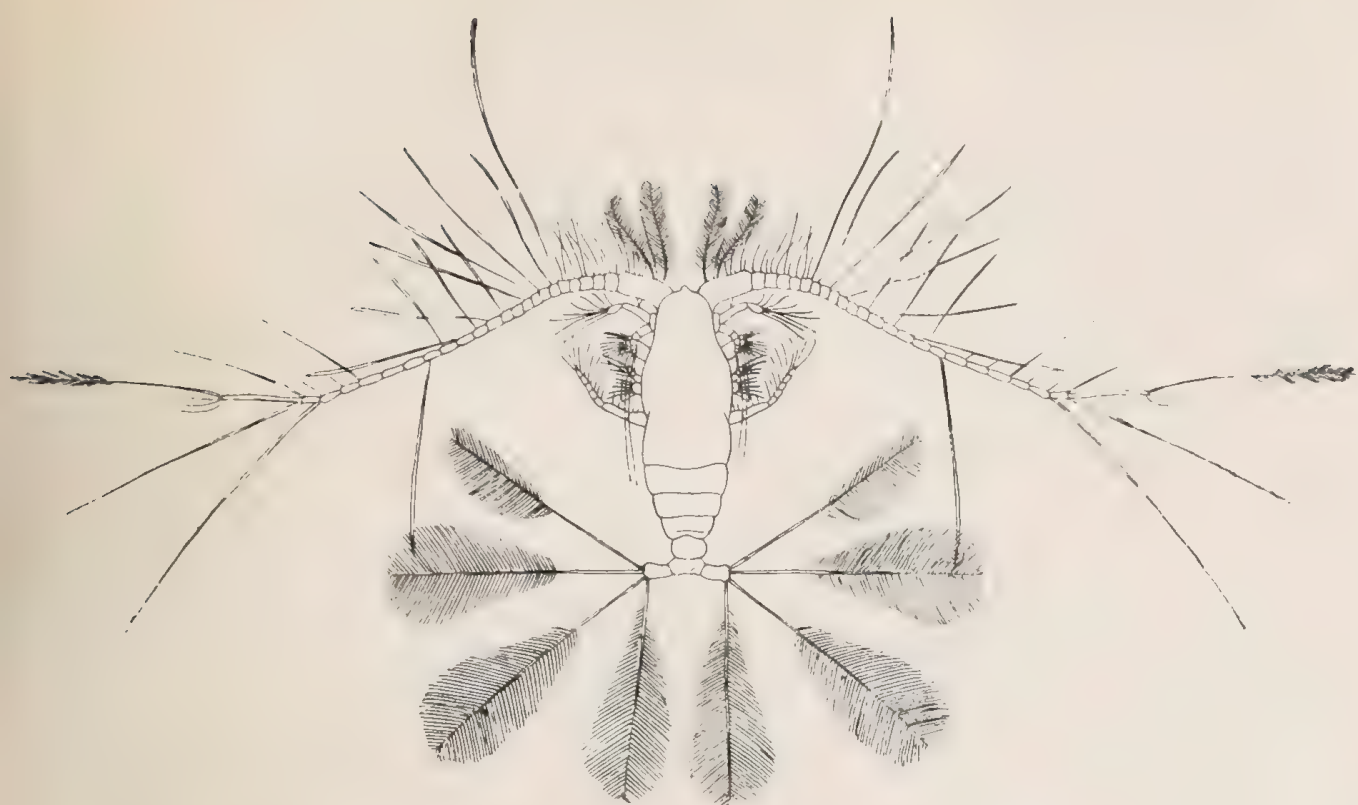

a
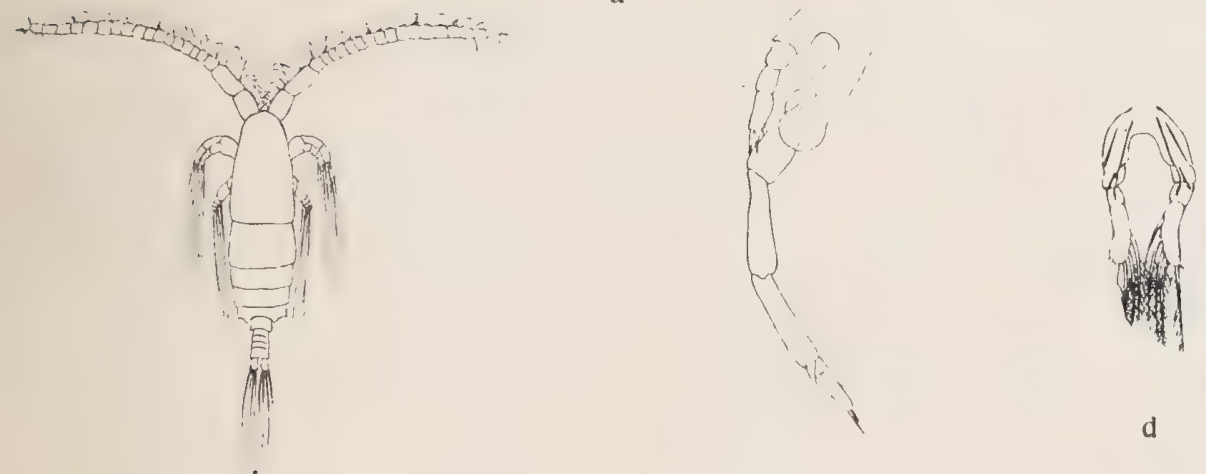

Fig, 18. Calocalanus pavo.

a o dorsal, b o dorsal, c 5. B. đa, d 5. B. f.

Nach Giesbrecht.

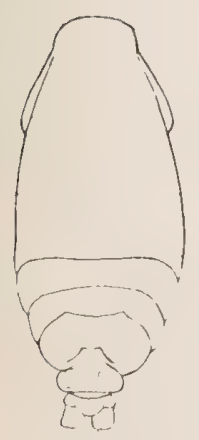

a.

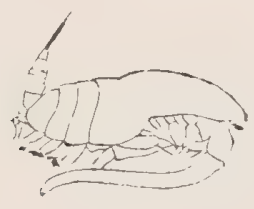

b

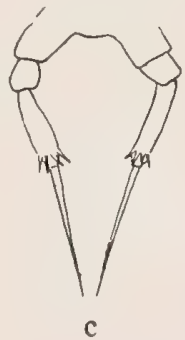

Fig. 19. Calocalanus styliremis.

a $q$ dorsal, b o lateral, c 5. B. ․ Nach Giesbrecht. 


\section{Calocalanus Giesbrecht.}

Weicht von Paracalanus hauptsächlich in Folgendem ab: - Grenze zwischen Kopf und 1. Thsegm. und zwischen 4. und 5. Thsegm. bisweilen ziemlich deutlich vorhanden; 2. Gl. des Basp. des 1. B. ohne Innenrandborste; Außenrand der Außenäste nicht gezähnelt; 2. Gl. des Enp. des 1. B. mit 4 Borsten. - $q$. Abdomen 2- oder 3-gldr.; Furka zuweilen asymmetrisch; letztes G1. der 1. Antenne wenigstens doppelt so lang wie das vorletzte. 5. B. 3bis 4-gldr. - $\sigma^{7}$. An der 1. Antenne verschmilzt das 1. und 2., das 3.-6., 24. und 25. Gl. 5. B. rechts 4-, links 5-gldr.

\section{Calocalanus pavo Dana.}

1849, Calanus. p., Dana, p. 13. 1892, C. p., Giesbrecht, p. 175, t. 1 f. 13 ; t. 4 f. 15 ; t. 9 f. $3,4,13,19$; t. 36 f. $43-45$. 1898, C. p., Giesbr. und Schmeil, p. 26.

१. Abdomen 2-gldr.; Gensegm. zwiebelförmig; Furka und Furkalborsten symmetrisch. Endgl. der 1. Antenne $5 \mathrm{mal}$ so lang wie das vorletzte. 1. Gl. des Basp. des 1. B. mit Innenrandborste; Endgl. des Enp. des 3. und 4. B. mit je 1 Gruppe von Stacheln; proximales Stück des Außenrandes des Endgl. des Exp. des 4. B. etwas über doppelt so lang wie das distale. 5. B. so lang wie das Basp. des 4. B. - $\sigma^{7} \cdot 1 .-4$. B. wie beim $q$; rechtes 5. B. am Ende mit 2 Börstchen. - L. † $0.88-1.2 \mathrm{~mm}$, of $1.04 \mathrm{~mm}$.

Mittelmeer, Atlant. und Pacif. Ozean; Rotes Meer; Mal. Arch.; Ind. Ozean.

\section{Calocalanus styliremis Giesbrecht.}

1888, C. S., Giesbrecht, p. 333. 1892, C. S., Giesbrecht, p. 176, t. 9 f. $15,18,29$; t. 36 f. $46-48$. 1898, C. s., Giesbr. und Schmeil, p. 26.

†. Abdomell 3 gldr.; Gensegm. zwiebelförmig, Furka symmetrisch. Endgl. der 1. Antenne doppelt so lang wie das vorletzte Gl. 1. Gl. des Basp. des 1. B. ohne Innenrandborste; Endgl. des Enp. des 3. und 4. B. mit je 2 Gruppen von Stacheln; proximales Stück des Außenrandes des Endgl. des Enp. des 4. B. $3 \mathrm{mal}$ so lang wie das distale. 5. B. etwa halb so lang wie das Basp. des 4. B. - o. 1.-4. B. wie beim ; rechtes 5. B. am Ende mit 1 Börstchen. - L. q $0.6-0.72 \mathrm{~mm}, \sigma^{\top} 0.55 \mathrm{~mm}$.

Mittelmeer, Nord-Atl. Ozean; Pacif. Ozean.

\section{Clausocalanus Giesbrecht.}

Kopf mit dem 1. Thsegm., 4. mit 5. Thsegm. verschmolzen; Rostrum beim ${ }_{7}$ 2-spitzig, beim ơ verkümmert. Abdomen des of 4-, des ơ 5-gldr., aber mit sehr kurzem Ansegm. 1. Antenne des $q$ den Thorax überragend, 
23-gldr., 8. mit 9., 24. mit 25. Gl. verschmolzen, mit kurzen Aesthetasken, des o mit verschmolzenen 1. und 2., 8.-10., 13.-16., 20. und 21., 24. und 25. Gl. Exp. der 2. Antenne $1 \frac{1}{2}$ mal so lang wie das Enp. Mundteile des or stark verkümmert. Exp. des 1.-4. B. 3-gldr.; Enp. des 1. B. 1-, des 2. B. 2-, des 3. und 4. B. 3-gldr.; 2. Gl. des Basp. des 2. und 3. B. breit, kelchförmig, am distalen Rande gezackt; Exp. des 2. und 3. B. ebenfalls breit; Endgl. des Exp. des 2.-4. B. mit 3 Außenranddornen, fein gezähneltem Enddorn und 4 Innenrandborsten; 1. Gi. des Exp. des 1. B. mit Außenranddorn; Enp. des 1. B. mit 5 Borsten; 5. B. vorhanden, beiderseits 1 -ästig, beim $q 3$-gldr., ohne Anhänge, beim of das lange linke 5-, das kurze rechte 1 - bis 3-gldr.

\section{Clausocalanus arcuicornis Dana.}

1849, Calanus a., Dana, p. 12. 1863; Calanus mastigophorus Claus, p. 173 , t. 27.1892 , C. a., Giesbrecht, p. 186 , t. 1 f. 14 ; t. 2 f. 7 ; t. 10 , f. $3-8,14,16,17,19$; t. 36 f. $29-31,34.1898$, C. a., Giesbr. und Schmeil, p. 27.

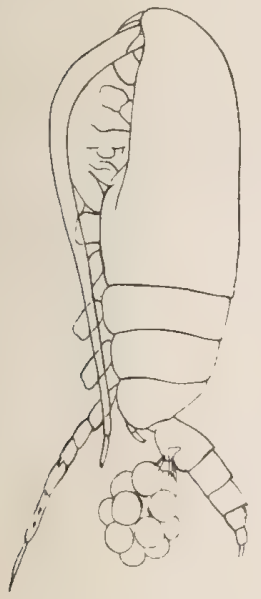

a

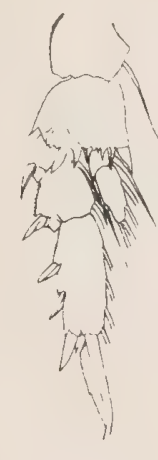

b

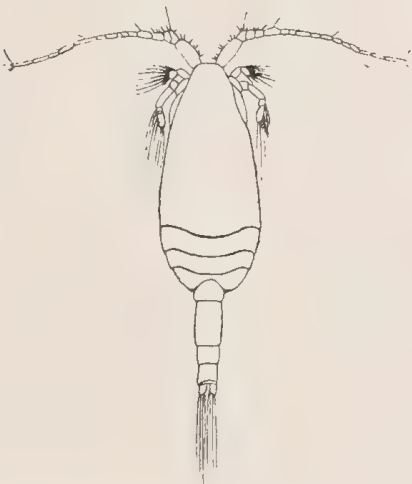

c

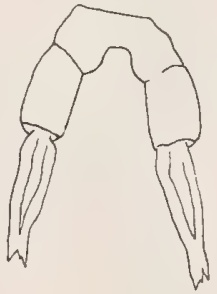

d

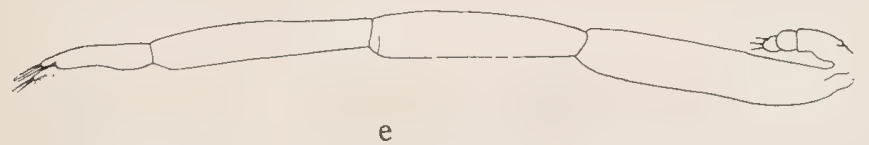

Fig. 20. Clausocalanus arcuicornis.

a $q$ lateral, b 2. B. $q$, c $\sigma^{\top}$ dorsal, d 5. B. 오, e 5. B. $\sigma^{\top}$. Nach Giesbrecht.

○. Gensegm länger als jedes der beiden folgenden Segm.; Furka etwa so lang wie breit. Das 4., 6., 8., 18., 22. Gl. der 1. Antenne ohne Aesthetask. - $0^{7}$. 2. Abdsegm. wenigstens so lang wie die beiden folgenden zusammen; 5. B. rechts 3-gldr. - L. o 1,15-1,6 mm, o 1,12-1,2 mm.

Mittelmeer, Atlant., Pacif., Indischer Ozean, Rotes Meer. 
Ctenocalanus Giesbrecht.

Weicht von Clausocalanus in folgendem ab:

2. und 3. B. nicht verbreitert; Außenranddornen am Endgl. des Exp. des 3. und 4. B. kammartig gezähnelt.

7. Rostralfäden schlaffer; 25. Gl. der 1. Antenne gesondert; Grenzen zwischen dem 1. und 2. und dem 9. und 10. Gl. undeutlich; 5. B. asymmetrisch.

万. 1. Antenne 21-gldr. 5. B. links 5-gidr., rechts nur durch einen Höcker angedeutet.

\section{Ctenocalanus vanus Giesbr.}

1888, C. v., Giesbrecht, p. 335. 1892, C. v., Giesbrecht, p. 194, t. 10, f. $20,21,26$; t. 36 f. 28.1898 , C. v., Giesbr. und Schmeil, p. 28 (q). 1904, C. v., Wolfenden, p. 125 , t. 9 f. $9($ ऊ).

Mit den Merkmalen der Gattung. - L. क 1,1 mm, o 1,25 mm.

Mittelmeer, Atl. Ozean; Pacif. Ozean; Antarkt. Ozean.

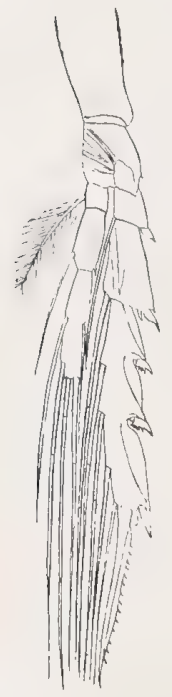

a

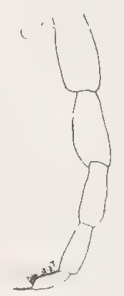

b

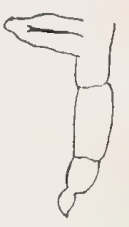

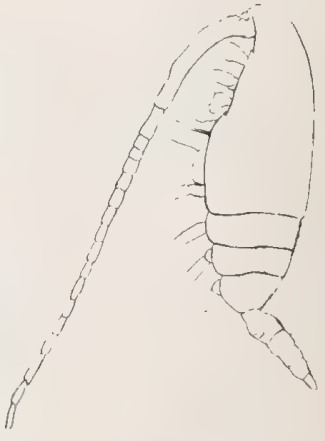

d

Fig. 21. Ctenocalanus vanus.

a 4. B. ㅇ ; b 5. B. ot ; 5 . B. q ; d f lateral. a, c, d nach Giesbrecht, b nach Wolfenden.

Weicht von Clausocalanus in folgendem $a b: 2$. und 3 . B. nicht verbreitert, von gewöhnlichem. Bau; 5. B. beim $q$ fehlend ${ }^{1}$ ).

q. 1. Antenne 24-gldr., 8. mit 9. Gl. verschmolzen, das Endgl. gesondert; 5. B. fehit $\left.{ }^{1}\right)$

7. Rostrum erhalten; an den 1. Antennen verschmelzen das 1. und 2., 8.-12., 20. und 21. Gl.; 5. B. links. 5-, rechts 4-gldr.

1) Ein ganz rudimentäres 5. B. ist bei weiblichen P. elongatus von Boeck und Mrázek angetroffen worden. 


\section{Pseudocalanus elongatus Boeck.}

1864, Clausia elongata, Boeck, p. 10. 1872, P. e., Boeck, p. 37. 1882, Lucullus acuspes, Giesbrecht, p. 163, t. 2 f. 14, 17. 1892, P. e., Giesbrecht, p. 197, t. 10 f. 22, 31-33. 1898, P. e., Giesbr. und Schmeil, p. 28. 1903, P. e., Sars, p. 20, t. 10, 11.

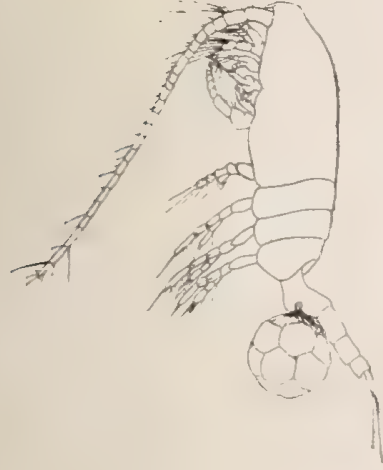

2

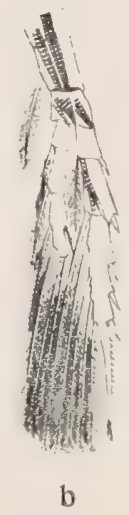

Fig. 22.

Pseudocalanus elongatus.

a $q$ lateral; b 4. B. $q$; c, d 5. B. q;

e ơ dorsal; f 5. B. ơ. a, b, e, f nach

Giesbrecht; c, d nach Mrázek.

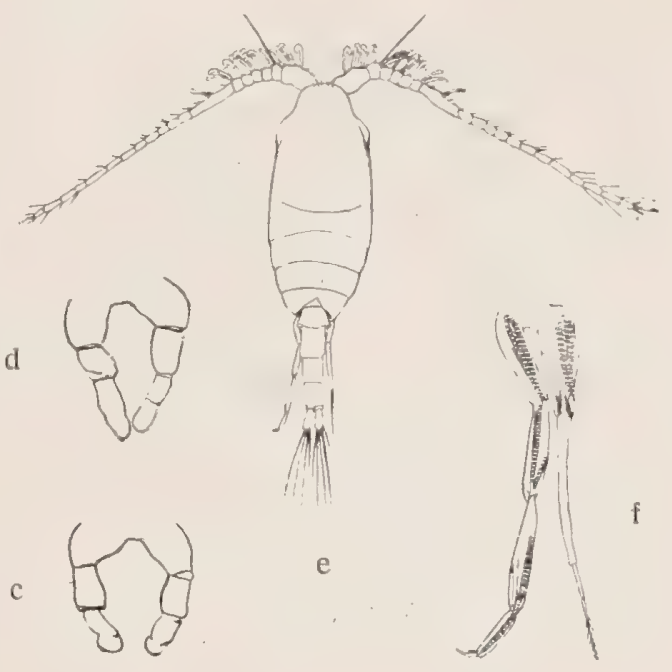

$\sigma^{7}$. Endgl. des rechten 5. B. kürzer als die halbe Länge des B. und so lang wie das vorhergehende Gl., das das distale Ende des 2. Gl. des linken B. überragt. - L. क $1,2-1,6 \mathrm{~mm}$, or $1,25-1,36 \mathrm{~mm}$.

Nord-Atlant. Ozean, Ostsee, Schwarzes Meer; Pacif. Ozean (?); Arkt. Ozean von Baffin's-Bai bis Neu-Sibirische Ins.; Golf von Suez.

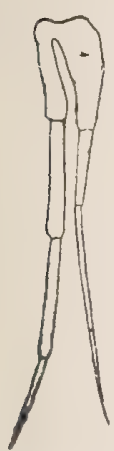

Fig. 23. Pseudocalanus major. 5, B. $\sigma^{7}$.

\section{Pseudocalanus major G. O. Sars.}

1900, P. m., Sars, p. 69, t. 20.

6. Endgl. des rechten 5. B. größer als die halbe Länge des B. und viel länger als das vorletzte Gl., das nicht über das zweite Gl. des linken B. hinausreicht. L. ㅇ $2,4 \mathrm{~mm}$, of $1,8 \mathrm{~mm}$.

Arkt. Ozean.

Anmerkung. Pseudocalanus gracilis G. O. Sars (1903, p. 154, Suppl. t. 1) soll sich von P. elongatus durch schlankere Körperform, buchtig hervorragende Stirn, größere Länge der vorderen Antenne und relativ schmälere und längere Schwimmfüße unterscheiden. Von Sars im Nordmeer gefunden. 
Microcalanus G. O. Sars.

Von Pseudocalanus in folgendem abweichend: 1. G1. des Exp. des 1. B. ohne Außenranddorn; Enp. des 1. B. mit 4 Borsten. - $0^{7}$. 5. B. links schlank, 5- (6-?) gldr., rechts 3-gldr., das rechte Endgl. nicht nadelförmig, kurz.

\section{Microcalanus pygmaeus G. O. Sars.}

1900, Spinocalanus longicornis ơ, Sars, p. 77, t. 22 f. 13, 14. (す). 1900, Pseudocalanus p., Sars, p. 73, t. 21 ( $($ ). 1902, Pseudocalanus p., Mrázek, p. 508, 511, t. 5 f. 2-7a, 11, 12. ? 1902, Pseudocalanus p., Giesbrecht, p. 20, t. 2 f. 1-5. 1903, M. p., Sars, p. 20, 156.

Endgl. und Endborste des Exp. des 2.-4. B. schlank; 1. Antenne des ${ }_{T}$ das Ende der Furka erreichend. Rechtes 5. B. des ot etwa halb so lang wie das linke. - L. q $0,86 \mathrm{~mm}, \sigma^{7} 1 \mathrm{~mm}$.

Arkt. Meer, Nordmeer (?); Antarkt. Meer (?).

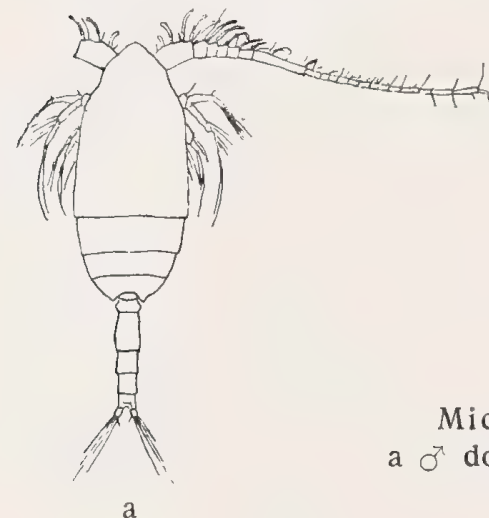

Microcalanus pygmaeus.

Fig. 24 .

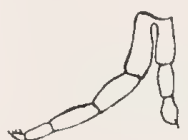

$\mathrm{b}$

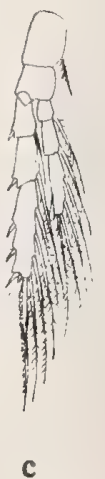

Anmerkung. Die von Giesbrecht (I. c.) im antarktischen Meere gefundenen f Exemplare stimmen in einigen Punkten nicht ganz mit Sars' Beschreibung überein. So vor allem haben die 1. Antennen bei den antarktischen Tieren eine etwas geringere relative Länge als bei den arktischen, da sie höchstens bis zum Vorderrande des Analsegmentes reichen. Auch im Bau der Schwimmfüße machen sich Unterschiede bemerkbar. L. $q 0,65-0,78 \mathrm{~mm}$.

2. Microcalanus pusillus G. O. Sars.

? 1902, Pseudocalanus spec., Mrázek, p. 112 , t. 5 f. $8-10$ (ठ). 1903, M. p., Sars, p. 156, Suppl. t. 2 ; t. 3 f. 1.

Endgl. des Exp. des 2.-4. B. relativ breiter mit auffallend breiter und grob gesägter Endborste; 1. Antenne des $\odot$ kaum das Gensegm. überragend; rechtes 5. B. des ơ kaum ein Drittel so lang wie das linke. - L. $q 0,7 \mathrm{~mm}, \sigma^{7} 0,7 \mathrm{~mm}$. Nordmeer. 

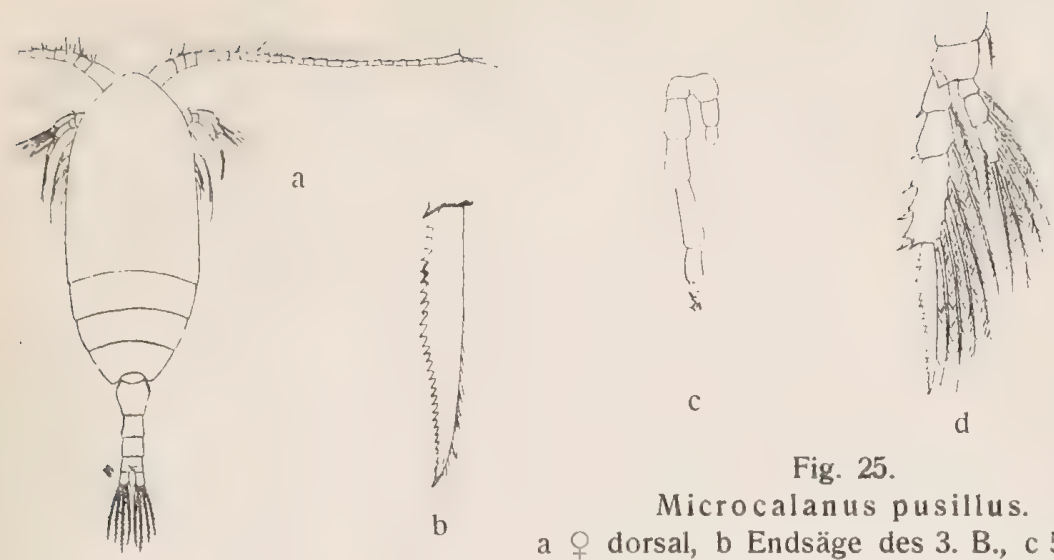

Fig. 25.

Microcalanus pusillus.

a $\bigcirc$ dorsal, b Endsäge des 3. B., c 5. B. ơ, d 3. B. . . Nach Sars.

\section{Drepanopus Brady.}

Von Clausocalanus in folgendem verschieden: - 2. und 3. B. nicht verbreitert, von gewöhnlichem Bau; Enp. des 1. B. 1-, des 2. B. 1- oder 2-, des 3. B. 2- oder 3-, des 4. B. 3-gldr. \%. 1. Antenne 24-gldr, 8. mit 9. Gl. verschmolzen, das Endgl. gesondert; Aesthetasken gut entwickelt; 5. B. 2-gldr. mit kräftig gebogener Borste am Ende. - $\sigma^{7}$. Rostrum erhalten; Verkürzung des Ansegm. und Rückbildung der. Mundgliedmaßen ähnlich, aber weniger stark wie bei Clausocalanus; 5. B. rechts länger, 4-gldr., links kürzer, 5-gldr.

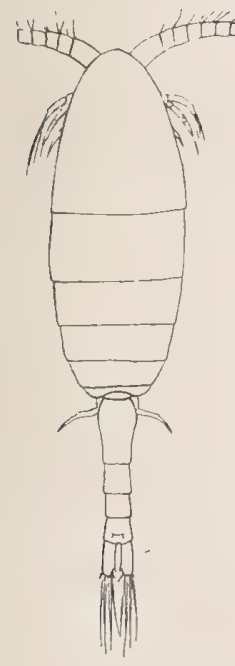

a

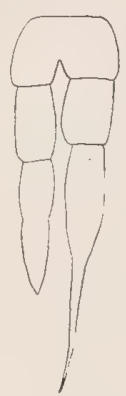

C

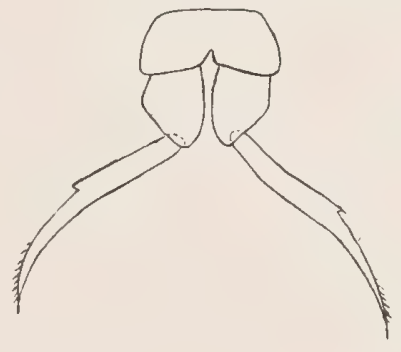

b

Fig. 26.

Drepanopus bungei.

a $\bigcirc$ dorsal, b 5. B. $q$,

c 5. B. o (unreif).

Nach Sars.

Drepanopus bungei G. O. Sars.

1898, D. b., Sars, p. 337, t. 9.

Enp. des 2. B. 1-, des 3. B. 2-gidr.; Endborste des 5. B. des $q$ der ganzen Länge nach gekrümmt. - L. $+1,3 \mathrm{~mm}$. Das $\delta$ nur in Jugendstadien bekannt.

Arktisches Meer. 
Anmerkung. An völlig reifen $q$ Individuen ist die Grenze zwischen Kopf und 1. Thsegm. und zwischen den beiden letzten Thsegm. viel weniger merkbar als in der Figur angegeben ist.

\section{Spinocalanus Giesbrecht.}

Von Clausocalanus in folgendem abweichend: - Verschmelzung vom Kopf mit dem 1. Thsegm. und vom 4. mit dem 5. Thsegm. bisweilen unvollkommen; 2. und 3. B. von gewöhnlichem Bau; Endgl. des Exp. des 1. B. mit 4, des 2.-4. B. mit 5 Innenrandborsten; Enp. des 1. B. mit 4 Borsten; 1. Gl. des Exp. des 1. B. mit oder ohne Außenrandborste; Enp. des 2.-4. B. an der Hinterfläche mit Gruppen von Stacheln besetzt; 5. B. fehlt beim $\bigcirc$.

ๆ. Rostrum fehlt; 1. Antenne das Rumpfende überragend, 24-gldr., mit verschmolzenen 8. und 9. G1. - $\sigma^{7}$. Rostrum fehlt; 5. B. beiderseits 2 ästig mit einfachem Enp. und gegliedertem Exp.

\section{Spinocalanus abyssalis Giesbrecht.}

1888, S. a., Giesbrecht, p. 335. 1892, S. a., Giesbrecht, p. 209, t. 13 f. $42-48$; t. 36 f. 49 . 1898, S. a., Giesbr. und Schmeil, p: 31 (q). 1900, S. longicornis, Sars, p. 75 , t. 22 f. $1-12$ ( 7$)$. 1903, S. longicornis, Sars, p. 22 , t. $12(q)$; S. a., p. 157 , Suppl. t. 3 f. $2\left(\sigma^{7}\right)$.
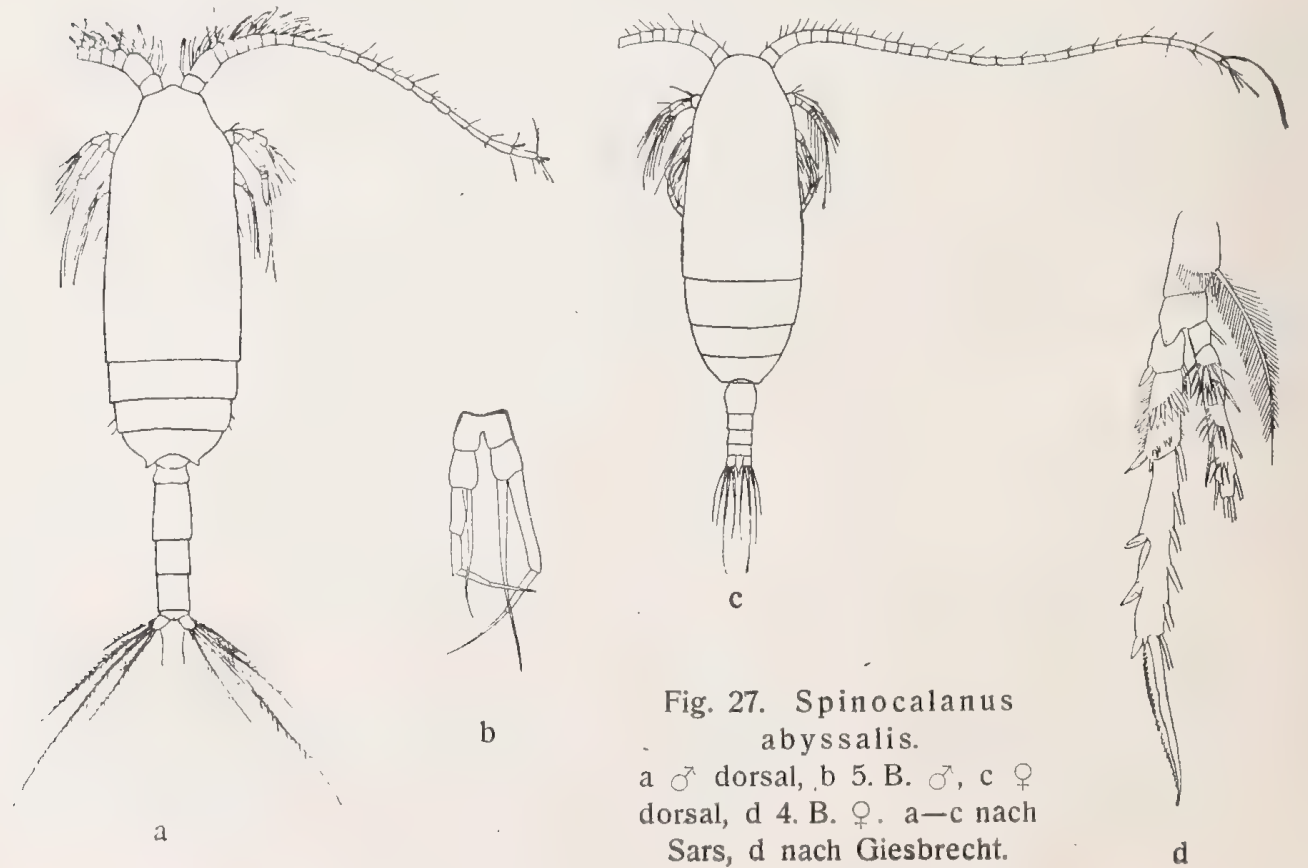
Endgl. des Enp. des 2. B., 2. und 3. Gl. des Enp. des 3. (und 4.) B. jedes mit 2 Reihen von Stacheln an der Hinterseite; Vorderkopf des o in lateraler Ansicht stark verjüngt; Exp. der 2. Antenne 11/5 mal so lang wie das Enp. - L. T 1,25-1,60 mm; ơ 1,6 mm.

Nordatl. Ozean, Nordmeer, Polarmeer; Pacif. Ozean.

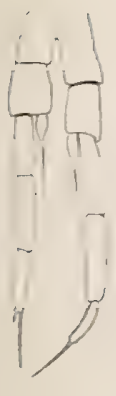

Fig. 28.

Spinocalanus schaudinni.

5. B. $0^{\pi}$.

Nach Mrázek.

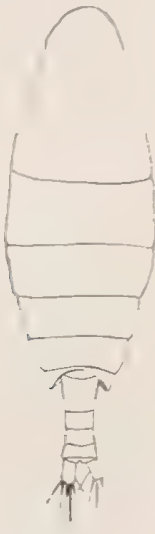

a

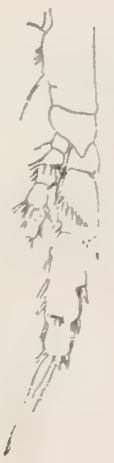

b
Fig. 29. Spinocalanus magnus. a $q$ dorsal, b 3. B. $q$. Nach Farran.

Anmerkung. Spinocalanus schaudinni Mrázek (1902, p. 509, t. 4; t. 5 f. 1) weicht, was das 을 betrifft, spezifisch von S. abysalis (=longicornis) nicht ab; für die Länge des gibt Mrázek $1,19 \mathrm{~mm}$. Zwischen den Männchen besteht ein Unterschied in dem 5. B., wozu die Figuren zu vergleichen sind. $\mathrm{Ob}$ dies ein spezifischer oder nur ein Reifeunterschied ist (vielleicht war das einzige von Mrázek gefundene 3 Exemplar nicht völlig ausgewachsen), ist vorläufig nicht $z u$ entscheiden.

Fundort: Arkt. Meer.

2. Spinocalanus magnus Wolfenden.

1904, S. m., Wolfenden, p. 118 . 1905, S. m., Farran, p. 30, t. 3 f. $1-12$.

Endgl. des Enp. des 2. B., 2. und 3. Gl. des Enp. des 3. B. jedes mit 1 Reihe von Stacheln an der Hinterseite; Vorderkopf des $\bigcirc$ in lateraler Ansicht hoch gewölbt; Enp. der Antenne fast so lang wie das Enp. -- L. C $2,9 \mathrm{~mm}, \sigma^{7}$ (unreif) $2,1 \mathrm{~mm}$.

Nordatl. Ozean.

\section{Aetideus Brady.}

Vorderrumpf 4-gldr.; letztes Thsegm. jederseits in eine Spitze verlängert, Rostrum beim groB, in 2 dicke, stark chitinisierte Zinken ausgehend, beim $\delta$ eingegangen. Abd. beim 0 4-gldr., symmetrisch, beim o 5 -gldr., mit sehr verkürztem Ansegm. 1. Antenne des $\bigcirc$ 23-gldr., 8. und 9. und 24. und 25. G1. verschmolzen. Exp. der 2. Antenne beim 0 länger als das Enp.; die zwei ersten Gl. des Maxp. etwa gleich lang; Mundteile des o verkümmert. Exp. des 1.-4. B. 3-gldr.; Enp. des 1. B. 1-, des 2. B. 1- oder 2-, des 3. und 4. B. 3-gidr. Außenrandborsten am 1. Gl. des Exp. des 1. B. fehlend. 5. B. fehlt beim $q$, beim ơ links 1-ästig, 5-gldr., rechts fehlend. 


\section{Aetideus armatus Boeck.}

1872, Pseudocalanus a., Boeck, p. 6. 1883, Aetidius a., Brady, p. 76 , t. 10. 1903, A. a., Sars, p. 25, t. 13, 14. 1904, A. tenuirostris, Wolfenden, p. 116.

Q. Rostrum an der Basis kaum verdickt, ohne Chitinknöpfchen zwischen den Haken; Spitzen des 5. Thsegm. das Ende des Gensegm. ungefähr er-

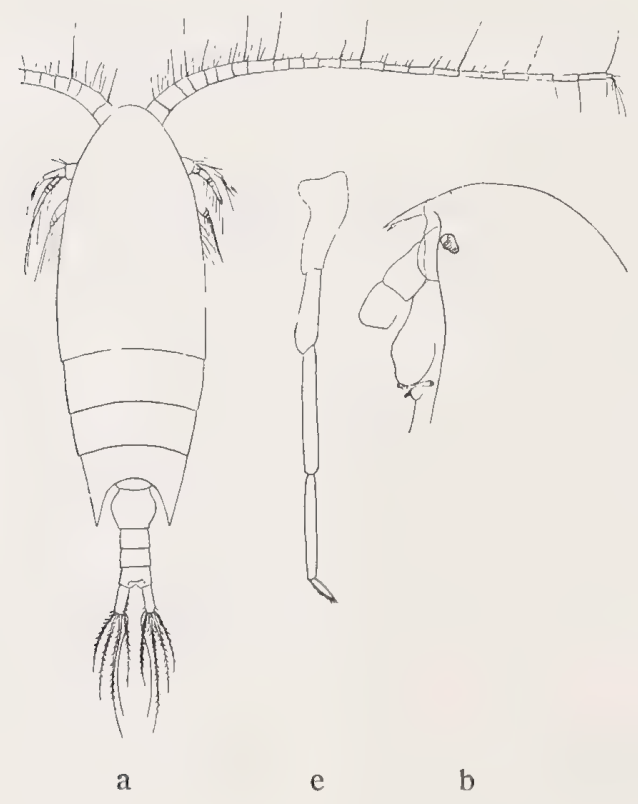

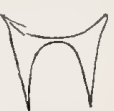

c

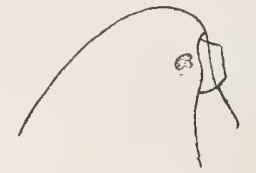

d
Fig. 30 .

Aetideus armatus.

a. $\subsetneq$ dorsal, b $ᄋ$, Kopf lateral,

c Rostrum ㅇ, d $\sigma^{\pi}$, Kopf lateral, e 5. B. $\sigma^{7}$. Nach Sars.

reichend. - $\sigma^{7}$. 3. G1. des linken 5. B. das längste, Endgl. klein und fein behaart. - L. \& $1,8 \mathrm{~mm}$, of $1,45 \mathrm{~mm}$.

Atlant. Ozean, Nordmeer; Pacif. und Ind. Ozean.

Anmerkung. Wolfenden (1904, p. 116) ist der Ansicht, das Aetideus armatus aus dem Faröerkanal und dem Nord-Atl. Ozean nicht identisch ist mit der von Giesbrecht aus dem Mittelmeer beschriebenen Form. Erstere hat einen viel weniger hohen vorderen Kopf, ein kleineres und kürzeres Rostrum ohne sekundäre. Chitinknöpfchen an der Basis der Zinken, während das Endp. des 2. B. 2-gldr. ist und die Spitzen des 5. Thsegm. weniger lang und stark sind (bei der Mittelmeerform ragen sie gewöhnlich über den hinteren Rand des 3. Abdsegm. hinaus). Wolfenden schlägt vor, die atlant. Form A. tenuirostris zu nennen. Da aber von Sars (1903, p. 159), der Exemplare aus dem Challenger-Material mit norwegischen Tieren verglichen hat, $z$ wischen diesen beiden gar keine Unterschiede festgestellt werden konnten, muß der Speziesnamen arma tus für die atlantische Art beibehalten werden und wäre, wenn wirklich die Mittelmeerform eine eigene Art darstellte, dieselbe neu zu benennen.

Für letztere Form siehe: 1892, Aëtidius a., Giesbrecht, p. 213 , t. 2 f. 6 ; t. 14 f. $1-13$; t. 36 f. $6-9$. 


\section{Bradyidius Giesbrecht.}

Weicht von Aetideus in folgendem ab:- - Rostrum in beiden Geschlechtern gleich entwickelt; Enp. des 2. B. 2-gldr.; Außenrandborste am 1. Gl. des Exp. des 1. B. vorhanden.

7. Rostrum klein, 2-spitzig; 1. Antenne 24-gldr., 24. vom 25. Gl. getremnt; Borsten der letzten 6 Gl. dick, lang, quer geringelt; das distale Gl. des Basp. des Maxp. deutlich länger als das proximale. - o. Rostrum klein, 2-spitzig; 5. B. beiderseits 1 - bis 2-ästig.

\section{Bradyidius armatus Brady.}

1878, Pseudocalanus a. $q$ (non Boeck), Brady, p. 46, t. 4 f. $1-9$, 11. 1884, Undinopsis bradyi, Sars. 1898, B. a., Giesbrecht und Schmeil, p. 32. 1898, B. a., Th. Scott, p. 264, t. 12 f. $1-19$. 1903, Undinopsis bradyi, Sars, p. 32, 163, t. 19, 20.

Q. Spitzen des letzten Thsegm. so lang wie das Gensegm.; 1. Antenne so lang wie der Vorderkörper; Exp. der 2. Antenne kaum länger als das Enp., Enp. des 2.-4. B. mit Dörnchen auf der Hinterfläche. - 万. 5. B. einfach, linkes B. schlank, 5-gldr., rechtes B. fehlend oder rudimentär, etwa $1 / 3$ mal so lang wie das linke und 3-gldr. - L. O 2,65 mm, of 2,20 mm.

Nordatlant. Ozean, Nordmeer bis Grönland, Mittelmeer; Ind. Ozean. An der norwegischen Küste dicht am Boden in Tiefen von 20-40 Faden (Sars).

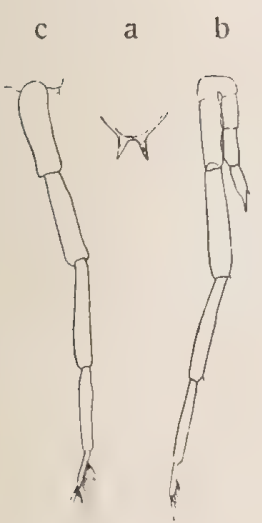

Fig. 31. Bradyidius armatus.

a Rostrum , b 5. B. or, c 5. B. $0^{x}$. a und b nach Sars, c nach Th. Scott.

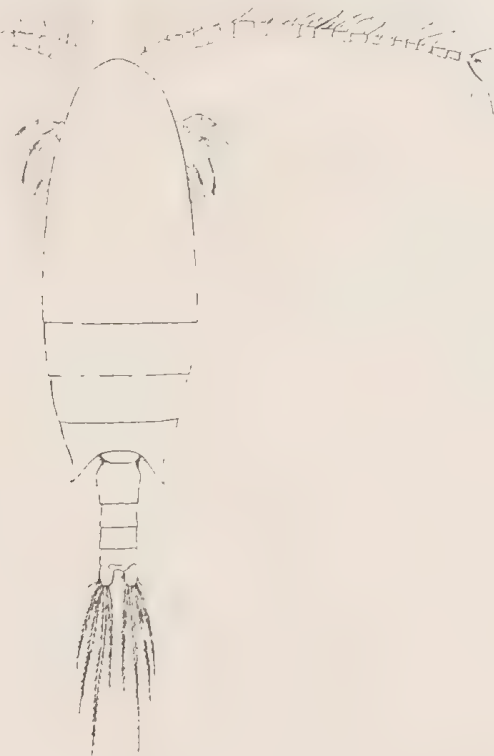

a

Fig. 32. Bradyidius similis. a $q$ dorsal, b 5. B. $\sigma^{7}$. Nach Sars. 


\section{Bradyidius similis G. O. Sars.}

1903, Undinopsis s., Sars, p. 34 t. 21.

7. Spitzen des letzten Thsegm. nur halb so lang wie das Gensegin.; 1. Antenne etwas kürzer als der Vorderkörper; Exp. der 2. Antenne deutlich länger als das Enp.; Enp. des 2.-4. B. ohne Dörnchen auf der Hinterfläche. - - 5. B. beiderseits mit griffelförmigem Enp. und 3-gldr. Exp., rechtes B. länger als das linke. - L. \& 3,0 $\mathrm{mm}$, o $2,4 \mathrm{~mm}$.

Norwegische Küste, dicht am Boden in Tiefen von 50-100 Faden (Sars).

\section{Bradyetes Farran.}

Von Aetideus in folgendem abweichend: - $\bigcirc$. Kopf unvollkommen vom 1. Thsegm. getrennt, letztes Thsegm. gerundet; Rostrum fehlend; Enp. des 2. B. 2-gldr.; Außenrandborste des 1. Gl. des Exp. des 1. B. vorhanden; 1. Antenne 24-gldr., 24. vom 25. Gl. getrennt, die 5 letzten Gl. mit starken, geringelten Borsten.

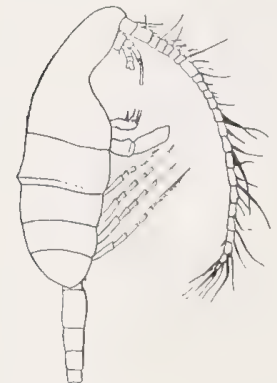

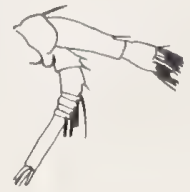

b

Fig. 33. Bradyetes inermis. a lateral, b 2. Antenne 9 . Nach Farran.

\section{Bradyetes inermis Farran.}

1905, B, i., Farran, p. 32 , t. 3 f. $13-20$; t. 4 f. $13-14$.

Mit den Merkmalen der Gattung. L. $.2,57 \mathrm{~mm}$. o unbekannt.

Nord-Atlant. Ozean (w. von Irland).

\section{Pseudaetideus Wolfenden.}

Weicht von Aetideus in folgendem $a b:-$ Rostrum in beiden Geschlechtern vorhanden, klein, 2-spitzig; Enp. des 2. B. 2-gldr.; Außenrandborste des 1. Gl. des Exp. des 1. B. vorhanden. Distales Gl. des Basp. des Maxp. ( $q$ ) viel länger als das proximale; 5 . B. des $\delta$ beiderseits 5 -gldr., mit reduzierten Enp.

\section{Pseudaetideus armatus Boeck.}

1872, Euchaeta armata, Boeck, p. 39. 1903, Chiridius a., Sars, p. 29, t. 15, 16. 1903, Chiridius a., Farran, p. 15, t. 16 f. 1-13. 1903, P. a., Wolfenden, p. 266. 1904, P. a., Wolfenden, p. 115, t. 9 f. $29,30,31$.

Mit den Merkmalen der Gattung. - L. ๆ 3,3-4 $\mathrm{mm}$, o 3,5-4 $\mathrm{mm}$. Nord-Atlant. Ozean (Europa), Nordmeer. 
Anmerkung. Farran (1905, p. 34) erwähnt eine kleinere Varietåt, bei welcher völlig ausgewachsene Individuen von beiden Geschiechtern nur 2,65 mm maßen. (Atl. Ozean, w. von Irland.)

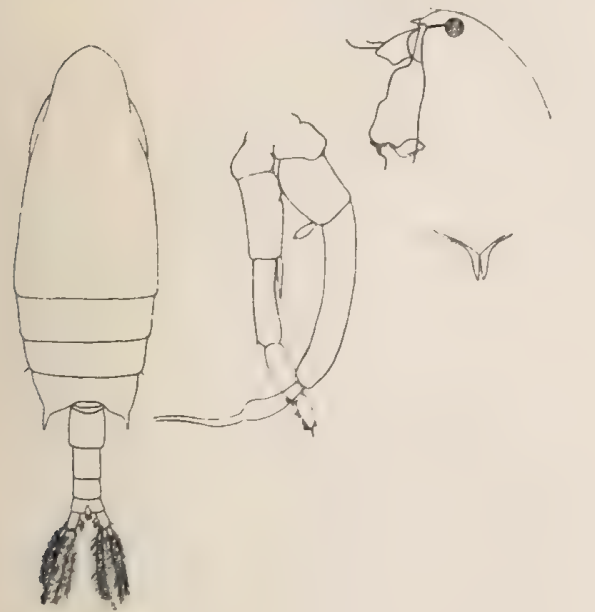

a

Fig. 34. Pseudaetideus armatus. a $\%$ dorsal, b Kopf $ᄋ$, lateral, c Rostrum, d 5. B. ơ. a, d nach Wolfenden, b, c nach Sars.

a
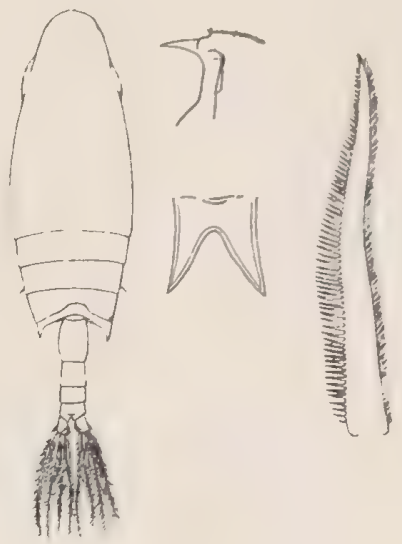

c

Fig. 35. a und b Aetideopsis rostrata. a Kopf 9 , b Rostrum . Nach Sars.

$c$ und $d$ Faroella multiserrata. c $q$ dorsal, d Endsäge eines B. $q$. Nach Wolfenden.

\section{Aetideopsis G. O. Sars.}

Weicht von Aetideus besonders in folgendem ab: -9 . Die beiden letzten Thsegm. getrennt; Rostrum groß, 2-spitzig; 1. Antenne 24-gldr,, 24. vom 25. Gl. getrennt. Distales Gl. des Basp. des Maxp. deutlich länger als das proximale. Enp. des 2. B. 2-gldr.; AuBenrandborste am 2. Gl. des Exp. des 1. B. vorhanden; Endborste des Endgl. des Exp. des 2.-4. B. mit zahlreichen, dichtgereihten, langen Zähnen. - $\sigma$ unbekannt.

\section{Aetideopsis rostrata G. O. Sars.}

1903, A. r., Sars, p. 160, Suppl. t. 4, 5.

Mit den Merkmalen der Gattung. - L. ๆ. 4,4 mm. - ऽ unbekannt.

Nord-Atlant. Ozean (Europa), Nordmeer.

Anm. Faroella multiserrata Wolfenden (1904, p. 117, t. 9 f. 26, 28) wurde später von dem Autor als synonym mit Aetideopsis rostrata Sars betrachtet. Nach Sars aber sind beide Arten nicht identisch (S. Pearson, 1906, p. 13). Aus den von beiden Arten bestehenden Beschreibungen und Abbildungen ist es aber nicht möglich, die Unterschiede zu ersehen. L. 3,04 und mehr, o $3,5 \mathrm{~mm}$. 


\section{Chiridius Giesbrecht.}

Von Aetideus in folgendem abweichend: - Rostrum in beiden Geschlechtern fehlend; Enp. des 2. B. 1-gldr.; Außenrandborste des 1. Gl. des Exp. des 1. B. vorhanden; 5. B. des o beiderseits 5-gldr., 1-ästig.

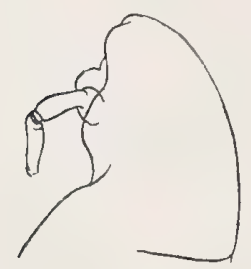

Fig. 36.

Chiridius poppei. Kopf lateral, $q$. Nach Giesbrecht.

\section{Chiridius poppei Giesbrecht.}

1892, Ch. p., Giesbrecht, p. 224, t. 14 f. 14-18; t. 36 f. 10-12. 1898, Ch. p., Giesbr. und Schmeil, p. 33 .

Q. Vorderkörper 3 mal so lang wie der Hinterkörper; die beiden auf das Gensegm. folgenden Abdsegm. breiter als lang. - L. $\supsetneq 1,8(-2,64) \mathrm{mm}, \sigma^{7}$ unbekannt.

Mittelmeer; ? Nord-Atl. Ozean (w. von Irland) (Farran, 1905, p. 135 ).

2. Chiridius obtusifrons G. O. Sars.

1900, C. armatus (non Boeck!), Sars, p. 64, t. 17. 1903, C. 0., Sars, p. 29, t. 17.
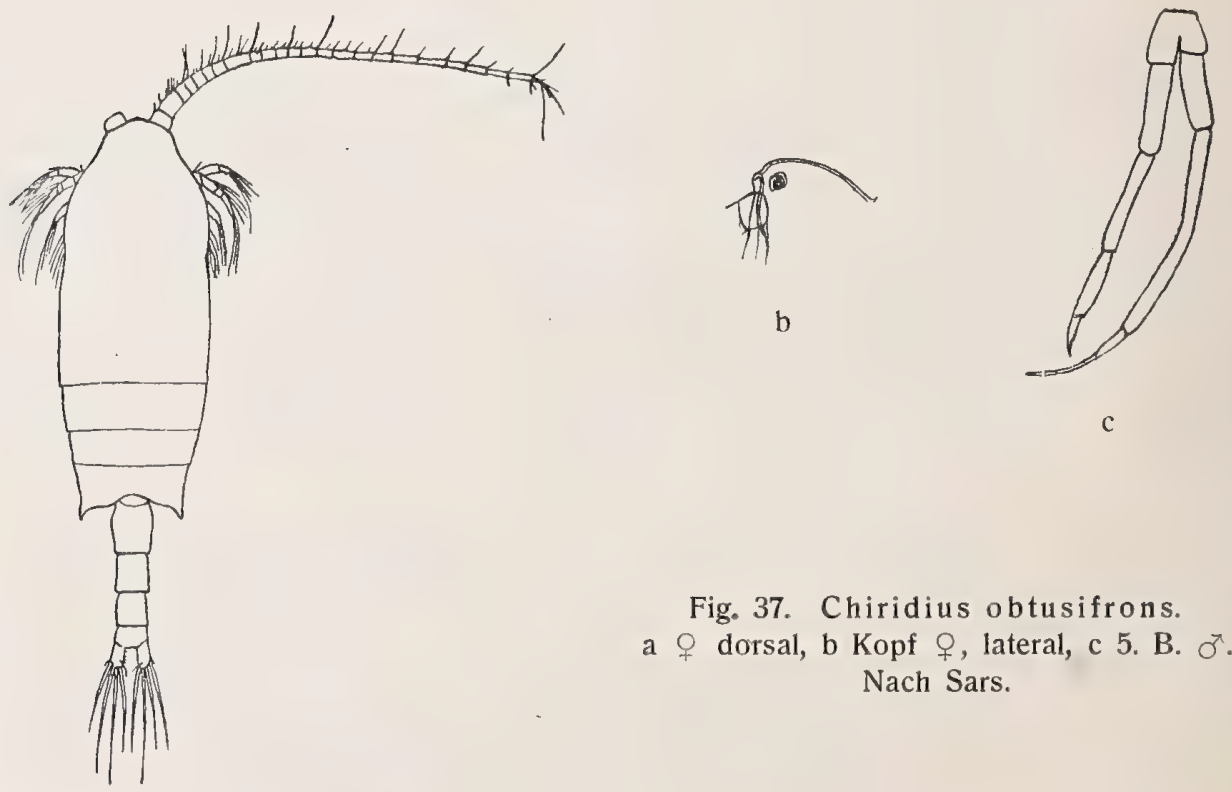

Fig. 37. Chiridius obtusifrons.

a $q$ dorsal, b Kopf $q$, lateral, c 5. B. or. Nach Sars. 
7. Hinterkörper fast halb so lang wie der Vorderkörper; das auf das Gensegm. folgende Abdsegm. länger als breit. - $\sigma .5$. B. beiderseits einästig, (undeutlich) 5-gldr.; rechtes B. länger als das linke. - L. ๆ 4,2 mm, ơ 2,9 mm.

Nordmeer, Polarmeer.

Anmerkung. Wolfenden (1904, p. 117, t. 9 f. 23) erwähnt ein ơ Exemplar einer Chiridius spezies als $\mathrm{Ch}$. vanhöffeni, weil er die Form für identisch hält mit der von V a nhöffen beschriebenen Pseudocalanus armatus (Grönland Expedition, II, 1897) und meint, es könnte das bis dahin unbekannte $\sigma^{x}$ von $\mathrm{Ch}$. obtusifrons sein. Dasselbe ist aber schon 1900 von Sars beschrieben und abgebildet worden. Der kurzen, vorläufigen Beschreibung bei Wolfenden findet sich nur eine Habitusfigur des ganzen Tieres hinzugefügt. Im Bau des 5. B. scheint das $\sigma^{\top}$ mit demjenigen von Ch. obtusifrons übereinzustimmen, aber weicht von diesem ab durch die Zweigliedrigkeit des Enp. des 2. B.; L. 3,1 mm. Fundort: Faröerkanal.

\section{Gaidius Giesbrecht.}

Von Aetideus in folgendem abweichend: - Rostrum 1-spitzig, in beiden Geschlechtern vorhanden; das distale Gl. des Basp. des Maxp. länger als das proximale ( $\supsetneq$ ); Enp. des 2. B. 1- oder 2-gldr.; Exp. des 1. B. 2- oder 3-gldr.; Fiedern am proximalen Stücke des Innenrandes des 1. Gl. des Basp. des 4. B. breiter als an den vorhergehenden B. (q); 5. B. des o beiderseits 5-gldr., mit rudimentärem Enp.

\section{Gaidius brevispinus G. O. Sars.}

1900, Chiridius b., Sars, p. 68, t. 19. 1903, G. b., Sars, p. 162, Suppl. t. 6 f. 2.1904 , G. major, Wolfenden, p. 114.

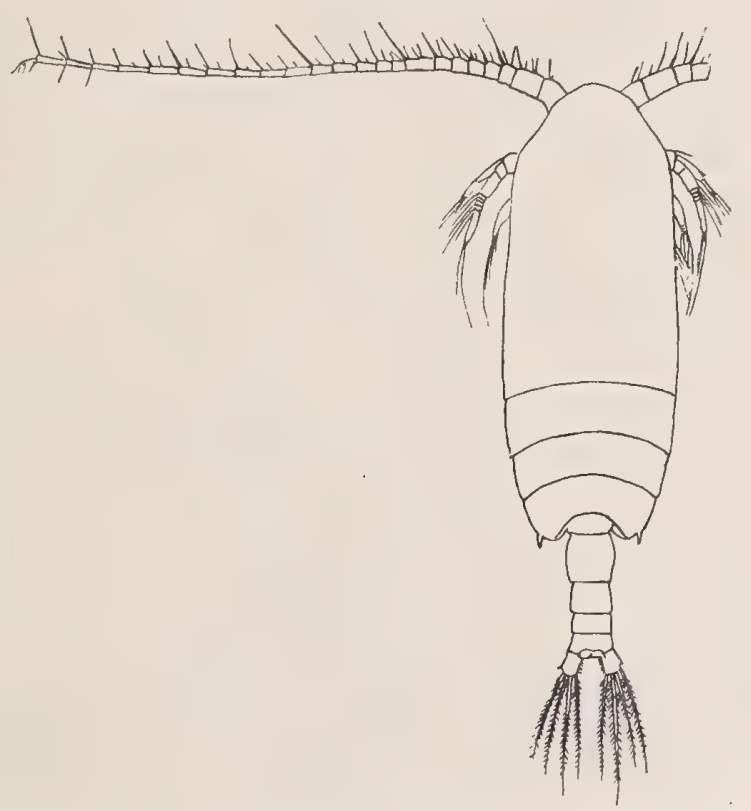

Fig. 38. Gaidius brevis pinus. $\quad$ dorsal. Nach Sars. 
Fortsatz des letzten Thsegm. beim $q$ klein und dornförmig. Exp. des 1. B. 3-gldr.; Enp. des 2. B. 2-gIdr.; 1. Antenne beim $q$ das Ende der Furka erreichend, beim ơ kürzer als der Vorderkörper. - L. ㅇ 4,6-4,8 mm, ठ 3,1 mm.

Nord-Atlant. Ozean (Europa); Nordmeer; Polarmeer.

2. Gaidius tenuispinus G. O. Sars.

1900, Chiridius t., Sars, p. 67, t. 18.1902 , G. t., Mrazek, p. 512, Textf. 7-9. 190\$, Chiridius t., Sars, p. 30, t. 18 ( $)$ ). G. t., p. 162, Suppl. t. 6 f. $1\left(\sigma^{7}\right)$. 1904, G. borealis, Wolfenden, p. 131; G. pungens, p. 111, 112,137, t. 9 f. 43.
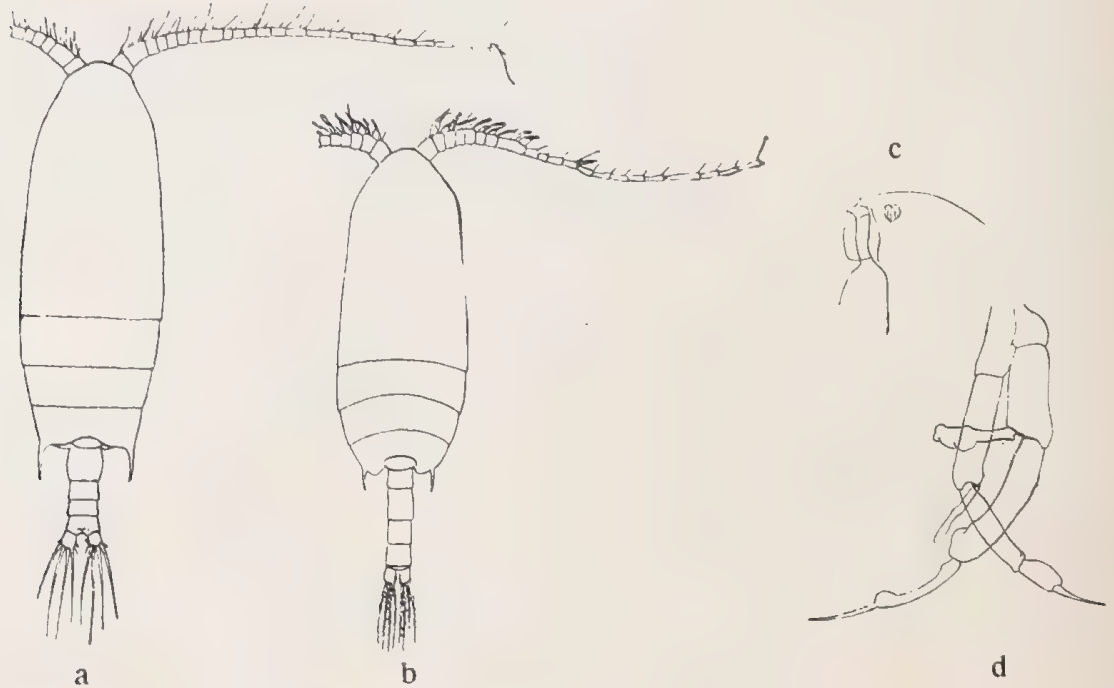

Fig. 39. Gaidius tenuispinus.

a $q$ dorsal, b o dorsal, c Kopf $q$, lateral, d 5. B. or. $a-c$ nach Sars, d nach Wolfenden.

Fortsaiz des letzten Thsegm. des $Q$ schlank, fast so lang wie das Gensegm.; 1. Antenne beim $O$ den Hinterrand des 2. Abdsegm. erreichend, beim ơ länger als der Vorderkörper. Exp. des 1. B. (undeutlich) 3-gldr.; Enp. des 2. B. 2-gldr. - L. ๆ 3,5-3,8 mm, o $2 \mathrm{~mm}$.

Nord-Atlant. Ozean (Europa), Nordmeer, Polarmeer.

Anmerkung. Gaidius te $\mathrm{n}$ u is p in us Sars ist mit Ga idius pungens Giesbrecht sehr nahe verwandt. Die letztere Art unterscheidet sich durch das 2-gidr. Exp. des 1. B. und das 1-gldr. Enp. des 2. B. sowohl von G. te nu is p in us als von G. brevispinus; sie wurde von Giesbrecht nach. $q$ Exx. aus dem Pacif. Ozean $\left(35^{\circ}\right.$ nördl. Br., $125^{\circ}$ westl. L.) beschrieben (1895, p. 349, t. 1 f. 1-4). Gaidius p un ge ns bei Wolfenden (1904, c. 1.) ist mit G. te nu is p in us Sars identisch. 


\section{Bryaxis Boeck M. S.}

Von Aetideus in folgendem abweichend: - 9 . Die beiden letzten Thsegm. bisweilen getrennt; Rostrum fehlend; 1. Antenne 24-gldr., dicht besetzt mit starken, gefiederten Borsten; Exp. der 2. Antenne viel kürzer als das Enp.; Enp. des 2. B. 2-gldr.; Außenrandborste am 1. Gl. des Exp. des 1. B. vorhanden.

\section{Bryaxis brevicornis Boeck M. S.}

1903, B. b., Sars, p. 35, t. 22, 23.

q. Die beiden letzten Thsegm. verschmolzen; Endgl. des Exp. der 2. Antenne kurz, mit 3 schwachen Borsten, das 3.-6. Gl. mit langen, starken Borsten. L. क $2,6 \mathrm{~mm}$. - o unbekannt.

Nord-Atl. Ozean (Europa); Nordmeer.

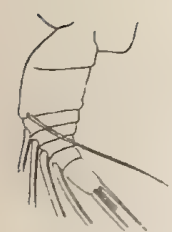

b

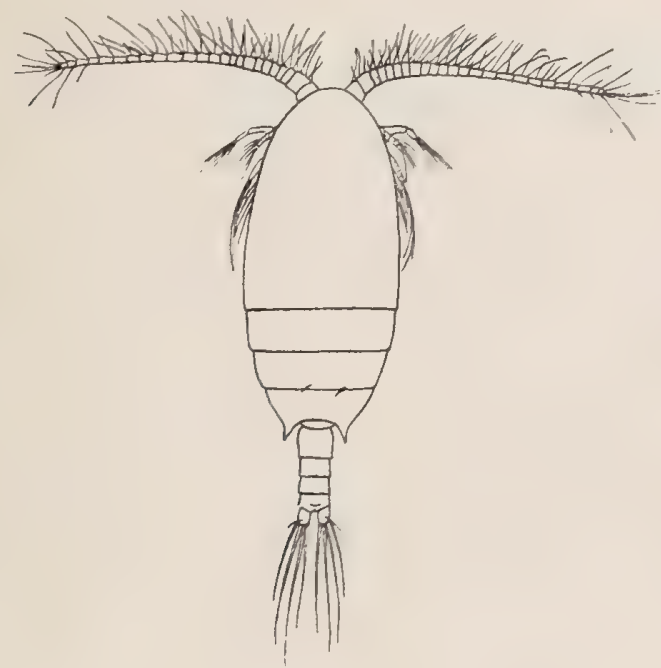

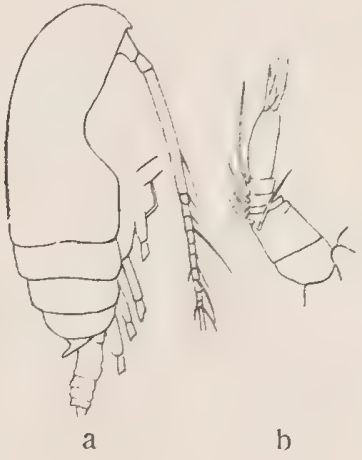

Fig. 40. Bryaxis brevicornis. a ㅇ dorsal, nach Sars:

b Exp. der 2. Antenne 우, nach Farran.

Fig. 41. Bry axis $m$ in or. a o dorsal,

b. Exp. der 2. Antenne Nach Farran.

\section{Bryaxis minor Farran.}

1905, B. m., Farran, p. 32, t. 4 f. $1-5,7-12$.

. Die beiden letzten Thsegm. getrennt; Endgl. des Exp. der 2. Antenne lang, mit 3 starken Borsten, das 3.-6. Gl. mit schwachen Borsten. L. \& 1,6 mm. - o unbekannt.

Nord-Atlant. Ozean (w. von Irland). 


\section{Gaetanus ${ }^{1}$ ) Giesbrecht.}

Von Aetideus in folgendem abweichend: - Rostrum kurz, 1-spitzig (oder bisweilen fehlend?); auf der Rückenfläche des Kopfes ein medianer Stachel. Exp. des 1. B. 2- oder 3-gldr.; Enp. des 2. B. 1-oder 2-gldr.; Außenrandborste am 1. Gl. des Exp. des 1. B. fehlend oder bisweilen vorhanden; Innenrandfiedern des 1. Gl. des Basp. des 4. B. lamellös oder stachelig.

1. Kopfstachel dünn, nach vorn und unten gerichtet 2 Kopfstachel kräftig, nach vorn oder aufrecht nach oben gerichtet 4

2. Exp. des 1. B. 2-gldr.; Enp. des 2. B. 1-gldr. Exp. des 1. B. 3-gldr.; Enp. des 2. B. 2-gldr.

1. Antenne kürzer als der Rumpf

1. Antenne so lang wie oder länger als der Rumpf S. G. major und kruppi

4. 1. Antenne das Körperende nicht überragend; Exp. des 1. B. 3-gldr.; 1. Gl. des Exp. des 1. B. mit rudimentärer Außenrandborste G. latifrons 1. Antenne das Körperende überragend; Exp. des 1. B: 2-gldr., eine Zweiteilung des 1. Gl. höchstens schwach angedeutet; 1. Gil. des Exp. des 1. B. ohne Außenrandborste

5. 1. Antenne $2 \mathrm{mal}$ so lang wie der Rumpf

1. Antenne höchstens $1 \frac{1}{2}$ mal so lang wie der Rumpf

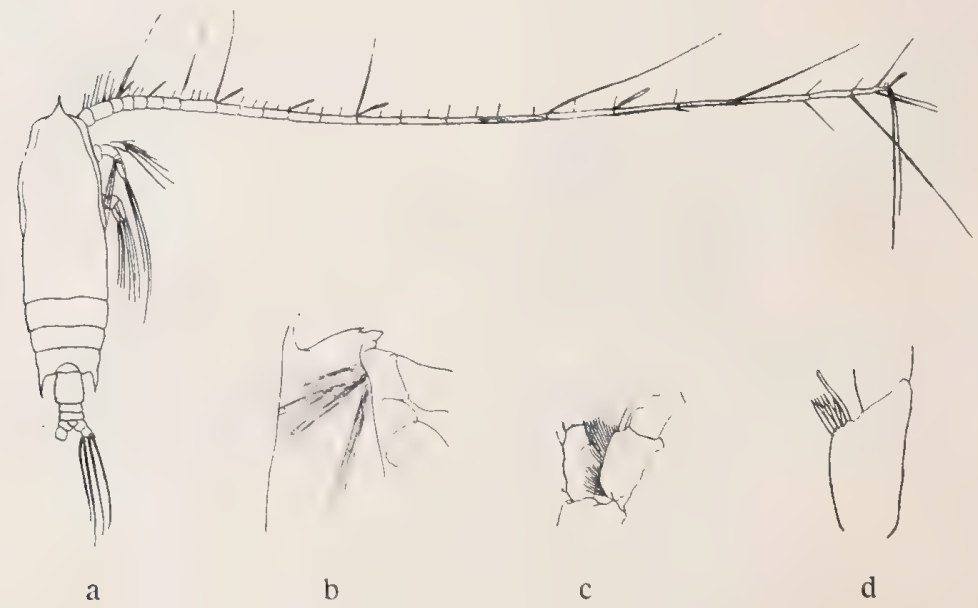

Fig. 42. Gaetanus miles.

a dorsal, b Kopf $q$, lateral, c 1. B. $q$, d 1. Gl. des Basp. des 4. B. $q$ (bei der pacifischen Form). Nach Giesbrecht.

1) Für die Beschreibung von Gaetanus denticulatus Aurivillius siehe Anhang. 


\section{Gaetanus miles Giesbrecht.}

1888, G. m., Giesbrecht, p. 335. . 1892, G. m., Giesbrecht, p. 219, t. 14 f. $21,24,25,27,30$; t. 36 f. 1, 3.1898 , G. m., Giesbr. und Schmeil, p. 32. 1903, G. m., Thompson, p. 17 , t. 1 f. $3-5$.

O. Kopfstachel dick, nach vorn gerichtet. 1. Antenne über doppelt so lang wie der Rumpf. Innenrand des 2. Gi. des Exp. der 2. Antenne ohne Zipfel. Außenrand des 1. Gl. des Basp. des Maxp. mit lamellösem Anhang. Exp. des 1. B. 2-gldr.; 1. Gl. des Basp. des 4. B. mit dornförmigen Borsten am Innenrande. L. $ᄋ 3,5 \mathrm{~mm}$. - o unbekannt.

Nord-Atlant. Ozean (nördl. bis zum Faröerkanal); Pacif. Ozean $\left(3^{0} \mathrm{~S}\right.$. bis $35^{\circ}$ N. B.)

Anmerkung. Bei pacifischen Exemplaren beträgt die Zahi der Stacheln am 1. Gl. des Basp. des 4. B. 5 bis 6 , bei atlantischen Exemplaren ist die Zahl größer. Auch hat die Lamelle am Basp. des Maxp. bei letzteren eine etwas andere Form.

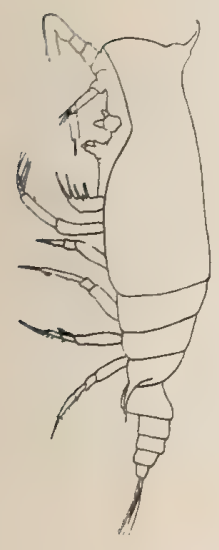

a

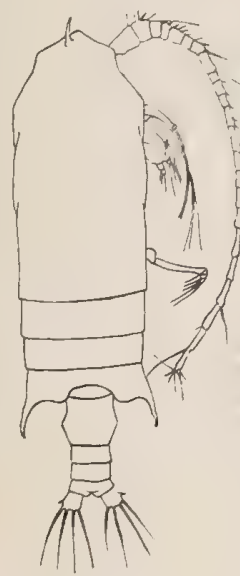

b

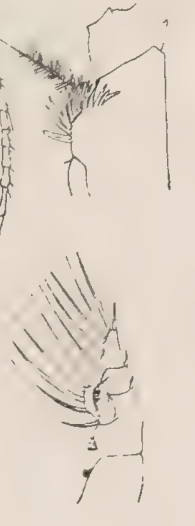

c

Fig. 43. Gaetanus latifrons G. O. Sars. a lateral, b o dorsal, c 1. B. 오, d Basp. des 4. B. \%. Nach Farran.

2. Gaetanus latifrons G. O. Sars.

1905, G. I., Sars, p. 11. 1905, G. holti, Farran, p. 33, t. 6 f. $1-12$.

․ Kopfstachel dick, aufrecht. 1. Antenne etwa so lang wie der Körper. Innenrand des 2. Gl. des Exp. der 2. Antenne mit 2 Zipfel, jede mit einer kurzen Borste. Zipfel des letzten Thsegm. lang. Exp. des 1. B. 3-gldr., 1. Gl. mit Außenrandborste. L. श 4,85-5,1 mm. - o unbekannt.

Nord-Atlant. Ozean.

\section{Gaetanus armiger Giesbrecht.}

1888, G. a., Giesbrecht, p. 335. 1892, G. a., Giesbrecht, p. 219, t. 14 f. $19,20,22,23,26,28,29$; t. 36 f. $2,4,5.1893$, Aetidius a., Th. Scott, p. 71, t. 8. 1898, G. a., Giesbr. und Schmeil, p. 33. 1904, G. atlanticus, Wolfenden, p. 132.

૧. Kopfstachel dünn, nach unten gerichtet. 1. Antenne kürzer als der Rumpf. Innenrand des 2. Gl. des Exp. der 2. Antenne mit einem kleinen Zipfel. 
Lamellöser Anhang am 1: Gl. des Basp. des Maxp. nur angedeutet. Exp. des 1. B. 3-gldr. L. \& 3,2-4,4 mm. - o unbekannt.

Nord-Atlant. Ozean, Golf von Guinea; Pacif. Ozean.

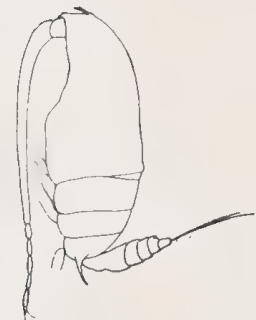

a

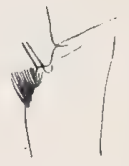

b

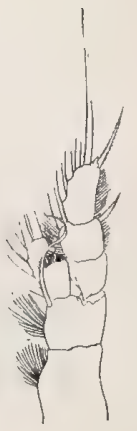

c

Fig. 44. Ga et a $\mathrm{n}$ s a r m iger.

a ㅇ lateral, b 1. Gl. des Basp. des 4. B. , c 1. B. ㅇ. Nach Giesbrecht.

Anmerkung. Wolfenden (1904, p. 132) schlägt vor, die atlantische Form von G. a r m i g e r vorläufig als selbständige Art mit dem Namen C. a $\mathrm{t}$ a a $\mathrm{t}$ i c u s zu belegen, da sie in einigen Merkmalen (Beborstung des 1. Gl. des Basp. des 4. B., Größe) von der typischen Form abzuweichen scheint.

\section{Gaetanus major Wolfenden.}

1904, G. m., Wolfenden, p. 114 , t. 9 f. $7,8$.

Verwandt mit G. armiger:

ๆ. 1. Antenne so lang wie oder länger als der Körper. Lamellöser Anhang des Maxp. fehlt. Exp. des 1. B. 3-gldr.

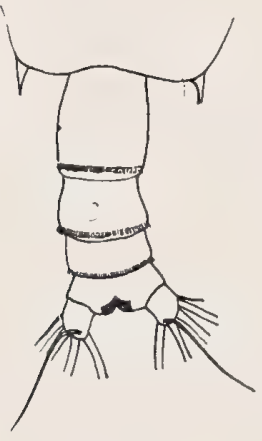

Fig. 45.

Gaetanus major . Nach Wolfenden.
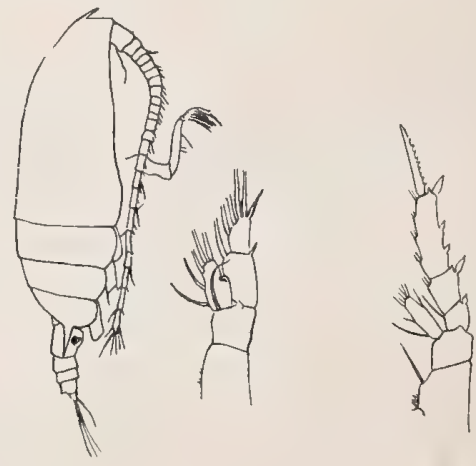

b

Fig. 46. Gaetanus minor. a ㅇ. lateral, b 1. B. , c 2. B. ‥ Nach Farran. 
5. Kopfstachel kurz; Dornen des letzten Thsegm. kurz; 1. Antenne 22-gldr., 1. mit 2., 8. mit 9., 24. mit 25. Gl. verschmolzen, 19. Gl. lang. Mundteile stark zurückgebildet. 5. B. demjenigen von Gaidius (brevispinus) sehr ähnlich. - L. ㅇ 4,7-5 mm, ơ $4,65 \mathrm{~mm}$.

Nord-Atlant. Ozean.

\section{Gaetanus minor Farran.}

1905, G. m., Farran, p. 34 , t. 5 f. $1-11$.

१. Kopfstachel schlank, nach unten gerichtet. 1. Antenne bis an das 2. Abdsegm. reichend; Maxp. mit lamellösem Anhang. Exp. des 1. B. 2-gldr.; Enp. des 2. B. 2-gldr.; 1. Gl. des Basp. des 4. B. am Innenrande mit feinen Borsten. L. 2,4 mm. - (Reifes) or unbekannt.

Nord.-Atlant. Ozean.

Anmerkung. Farran (I. c.) fand außerdem unreife $o$ und $o^{\text {t }}$ Individuen einer Gaetanusspezies, die im allgemeinen $G$. $m$ i n o r sehr ähnlich waren, aber deren 1. Antenne bis an die Furka reichte. Länge $2 \mathrm{~mm}$.

\section{Gaetanus kruppi Giesbrecht.}

1904, G. k., Giesbrecht, p. 202 , t. 7 f. 8 ; t. 8 f. 29.

Verwandt mit armiger: - † Körperlänge gröBer; Fortsatz des letzten Thsegm. ziemlich kurz, $1 / 3$ mal so lang wie der Außenrand des 1. Gl. des Basp. des 4. B. (bei armiger gleich so lang). 1. Antenne das Körperende um die 3 letzten Gl. überragend, das 22. Gl. relativ länger als bei armiger. Maxp. mehr demjenigen von miles als dem von armiger ähnlich, besonders

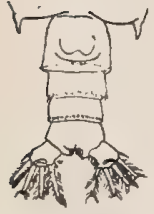

a

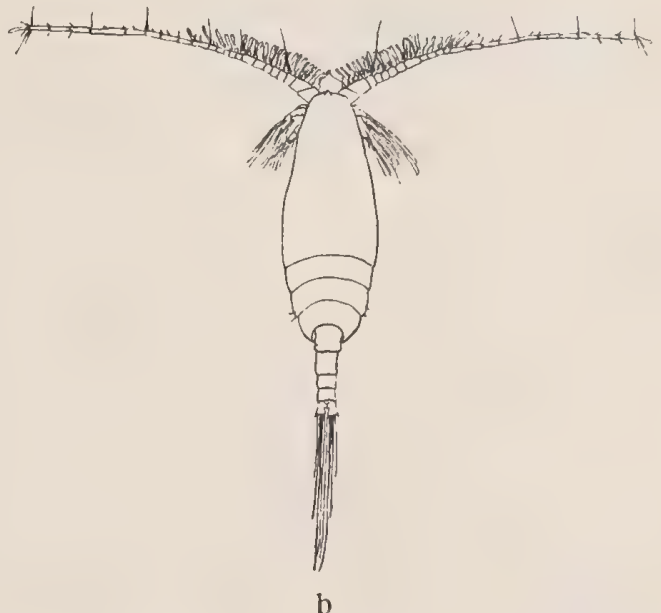

Fig. 47. Gaetanus kruppi. a Abdomen $q$, ventral, b dorsal. Nach Giesbrecht. 
durch die Chitinlamelle am 1. Gl. des Basp. - $\sigma^{7}$. Seitenspitzen des letzten Thsegm. viel kürzer als beim $\odot$; Ansegm. merklich verkürzt. 1. Antenne etwas kürzer als der Körper. Mundgliedmaßen denen des or von Aetideus bis auf wenig ganz ähnlich. Außenranddorn des 2. Gl. des Exp. des 1. B. rudimentär; Innenrand des 1. Gl. des Basp. des 4. B. ohne Borsten. 5. B. beiderseits mit 2-gldr. Basp. und 1-gldr. Enp.; Exp. des rechten B. 2-, des linken 3-gldr. - L. ? $3,6-4$, o $3,7 \mathrm{~mm}$.

Mittelmeer, Nord-Atlant. Ozean.

\section{Gaetanus caudani Canu.}

1896, C. c., Canu, p. 422. 1898, G. c., Giesbr. und Schmeil, p. 33. 1903, G. pile atus, Farran, p. 16, t. 17 f. 1-11. 1902, G. c., Wolfenden, p. 114 , t. 9 f. $20-22$.

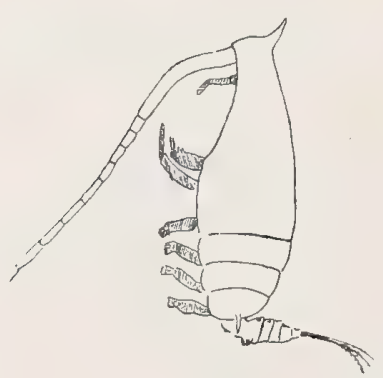

a

Fig. 48. Ga etan us c a d a ni. a

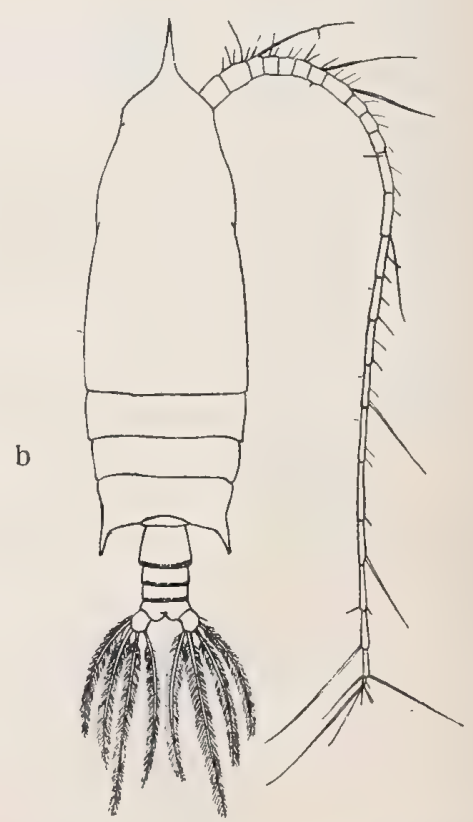

Q. Kopfstachel dick, aufrecht. 1. Antenne das Körperende um die letzten. 5 Gl. überragend. Innenrand des 2. G1. des Exp. der 2. Antenne ohne proximale Borsten. Zipfel des letzten Thsegm. nicht über das Genitalsegment hinausreichend. Exp. des 1. B. 2-gldr. L. क 5,6 mm. - (Reifes) o unbekannt.

Nord-Atlant. Ozean.

Undeuchaeta Giesbrecht.

Q. Vorderrumpf 4- oder 5-gldr., mit Spuren einer Trennung zwischen Kopf und 1. Thsegm.; Lateralecke des letzten Thsegm. abgerundet oder nach 
hinten verlängert. Rostrum 1-spitzig; Abd. 4-gldr.; Gensegm. meist mehr oder weniger asymmetrisch. Enp. der 2. Antenne meist über halb so lang wie das Exp. Exp. der 1. Maxille klein, seine mittleren Borsten oft kürzer als die proximalen und distalen; Außenlobus mit stark verlängerter, mittlerer Borste, Enp. reichlich beborstet; 2. Gl. des Maxp. wenigstens 3 mal so lang wie das breitgliedrige Enp. Exp. des 1. B. 2-gldr. (mit Spuren einer Gliederung des 1. Gl.) oder 3-gldr., des 2.-4. B. 3-gldr.; Enp. des 1. B. 1-, des 2. B. 1- oder 2-, des 3. und 4. B. 3-gldr.; Exp. des 1. B. mit 2 oder 3 Außenranddornen; 1. Gl. des Basp. des 4. B. am Innenrande mit oder ohne Dornen; 5. B. fehlt. -- $0^{7}$. Gliederung des'Rumpfes wie beim 0 ; Abdomen 5-gldr., $\cdot$ mit sehr kurzem Analsegment. Mundteile rückgebildet. 5. B. groB, asymmetrisch, beiderseits 2-ästig mit 1-gldr. Enp. (das des linken B. bisweilen stark reduziert).

1. Gensegm. symmetrisch

U. spectabilis Gensegm. asymmetrisch

2. 1. Gl. des Basp. des 4. B. mit 6 braungefärbten, starken Dornen

1. Gl. des Basp. des 4. B. ohne Dornen

U. pustulifera

Stirn mit medianer Crista 3

Stirn ohne Crista

U. major

$U$. minor.

\section{Undeuchaeta major Giesbrecht.}

1888, U. m., Giesbrecht, p. 335 . 1892, U. m., Giesbrecht, p. 227, t. 37 f. 56, 57, 59. 1898, U. m., Giesbr. und Schmeil, p. 34 . 1905, U. m., Esterly, p. 148 , f. 16 .

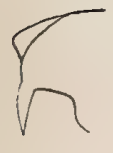

$\mathrm{b}$

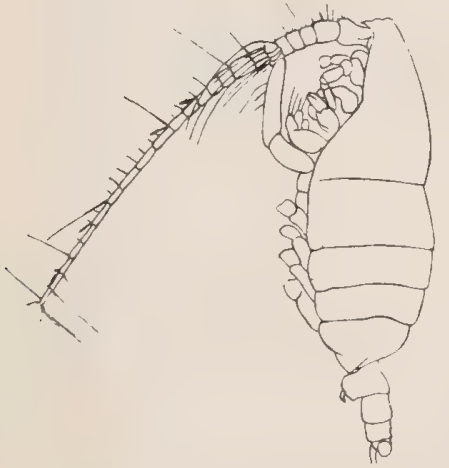

a
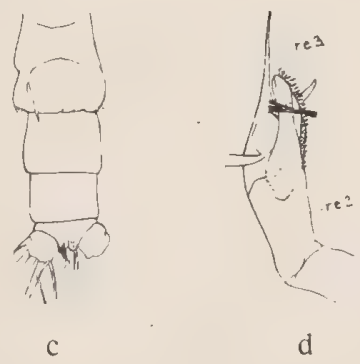

Fig. 49. Undeuchaeta major.

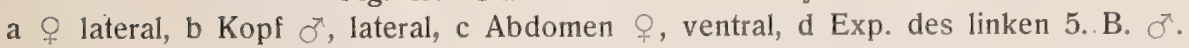
a und $c$ nach Giesbrecht, b und d nach Esterly. 
१. Stirn mit medianer Crista; Gensegm. mit einem Buckel auf der rechten Seite und mit einem hakigen, spitzen Anhang rechts von der Genitalöffnung. Exp. des 1. B. 2-gldr. (mit Spuren einer Gliederung des 1. Gl.); Enp. des 2. B. 1-gldr.; Exp. des 1. B. mit 2 Außenranddornen; 1. Gl. des Basp. des 4. B. ohne Dornen am Innenrande. - $\sigma^{7}$. Stirn mit Crista. Exp. des 1. B. undeutlich 3-gldr.; Exp. des 5. B. beiderseits 3-gldr.; linkes 5. B. mit stark rückgebildetem, stabförmigem Enp., Endgl. des Exp. mit langer Spitze, 2. Gl. des Exp. mit einem gezähnelten und einem artikulierten Fortsatz am Ende; rechtes 5. B. 2-ästig, Enp. lang, stabförmig, Endgl. des Exp. griffelförmig. L. ๆ $4,5-5,5$, o $6-6,5 \mathrm{~mm}$.

Atlant., Pacif. und Ind. Ocean.

\section{Undeuchaeta minor Giesbrecht.}

1888, U. m., Giesbrecht, p. 335. 1892, U. m., Giesbrecht, p. 228, t. 14 f. $31-34$; t. 37 f. $55,58.1898$, U. m., Giesbr. und Schmeil, p. 34 .

ๆ. Stirn ohne Crista; Gensegm. mit einem Dorn auf

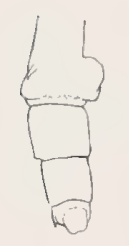

Fig. 50 .

Undeuchaeta minor. Abdomen $q$, lateral. Nach Giesbrecht. der Dorsalfläche. Exp. des 1. B. 2-gldr., (mit Spuren einer Gliederung des 1. Gl.), Enp. des 2. B. 1-gldr.; Exp. des 1. B. mit 2 Außenranddornen; 1. Gl. des Basp. des 4. B. ohne Dornen am Innenrande. L. 3,2-4,2 mm. - o unbekannt.

Atlant., Pacif. und Ind. Ozean.

förmigen, auswärts gerichteten Auswuchs. Exp. des 1. B. deutlich 3-gldr.; Enp. des 2. B. 1-gldr. 1. Gl. des Basp. des 4. B. am Innenrande mit 6 starken, braungefärbten Dornen. L. $6,9 \mathrm{~mm}$. - $\sigma^{\top}$ unbekannt.

Atlant. Ozean.

\section{Undeuchaeta spectabilis G. O. Sars.}

․ Stirn ohne Crista; Vorderrumpf 5-gldr., die beiden letzten Thsegm. getrennt; Lateralecken des letzten Thsegm. kurz zugespitzt; Abdomen 4-gldr., symmetrisch. 1. Antenne so lang wie der Vorderkörper, 24-gldr., 24. vom 25. G1. getrennt. Enp. der 2. Antenne halb so lang wie das Exp. Exp. der 1. Maxille klein, mit 10 fast gleich langen Borsten; Außenlobus mit stark verlängerten mittleren Borsten; Enp. dicht befiedert; 2. Gl. des Maxp. 3 mal so lang wie das Enp. Exp. des 1. B. 2-gldr., (mit Spuren einer Gliederung des 1. Gl.), des 2.-4. B. 3-gldr., Enp. des 1. B. 1-, des 2. B. 2-, des 3. und 4. B. 
3-gldr.; Exp. des 1. B. mit 3 Außenranddornen; 1. Gl. des Basp. des 4. B. am Innenrande mit 5 starken Dornen; 5. B. fehlt. - o Gliederung des Rumpfes wie beim $₹$, Lateralecken des letzten Thsegm. weniger hervorragend; Abdomen 5-gldr., mit sehr kurzem Ansegm. Mundteile rückgebildet. 5. B. groß, asymmetrisch, beiderseits 2-ästig mit 1-gldr. Enp. - L. $q 8 \mathrm{~mm}$, o $6 \mathrm{~mm}$.

\section{Arktischer Ozean.}
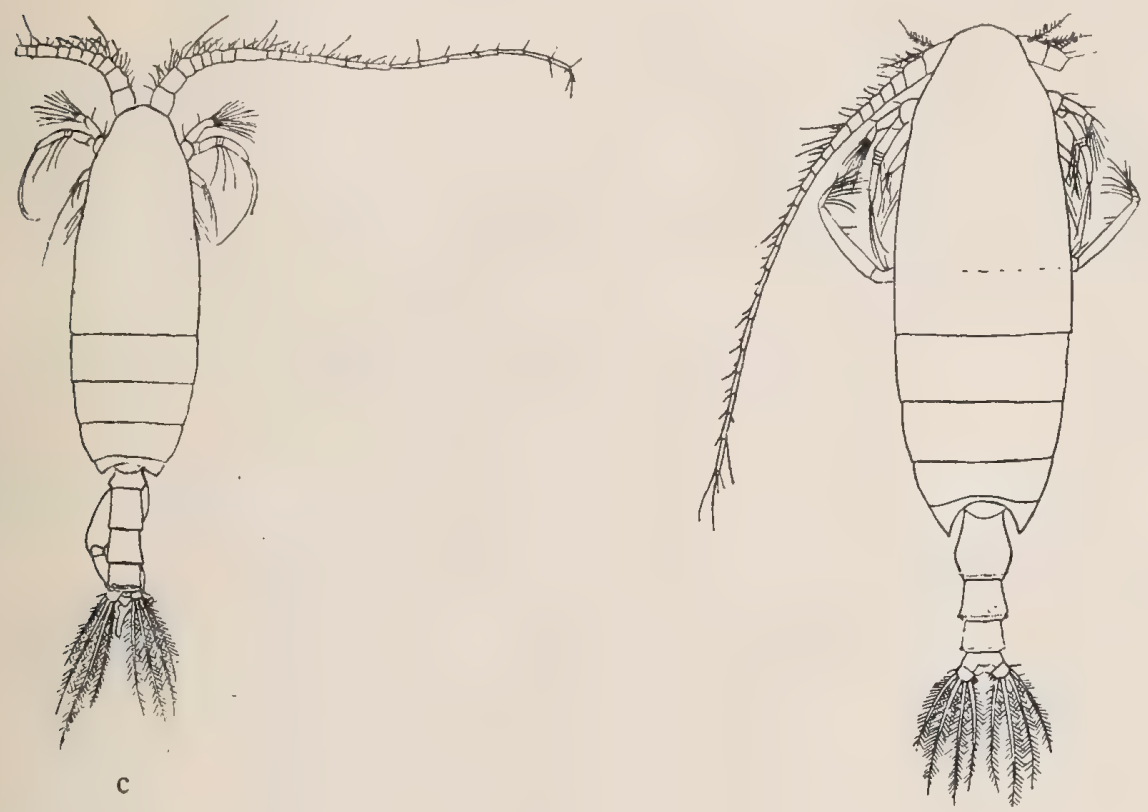

a

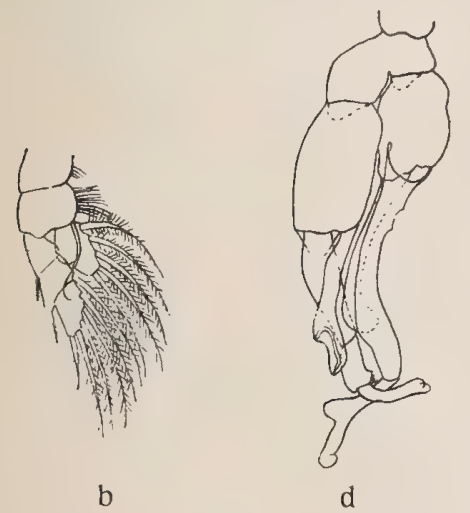

Fig. 51.

Undeuchaeta spectabilis.

a o dorsal, b 1. B. , c o dorsal, d 5. B. $\%$. Nach Sars.

Chirundina Giesbrecht.

Verwandt mit Undeuchaeta und Euchirella: - $q$. Rostrum 1-spitzig; Stirn mit Crista; Lateralecken des letzten Thsegm. in kurze Zipfel ausgehend. 
Gensegm. symmetrisch. Enp. der 2. Antenne etwa halb so lang wie das Exp. 1. Maxille wie bei Euchirella, aber reicher beborstet. Exp. des 1. B. 2-gldr.; Innenrand des 1. Gl. des Basp. des 4. B. ohne Stacheln. - Reifes o unbekannt.

\section{Chirundina streetsi Giesbrecht.}

1895, C. s., Giesbrecht, p. 250, t. 1 f. 5-10. 1902, Euchirella c arinata, Wolfenden, p. 366 ( $($ ). 1904, Euchirell a carinata, Wolfenden, p. $115(q)$.

Q. Stirnseiten ziemlich stark vorspringend; Seitenecken des letzten Thsegm. in kurze, stumpfe Zinken ausgehend; Exp. des 1. B. 2-gldr. mit deutlicher Grenze zwischen den beiden verschmolzenen, proximalen Gl. L. $5-5,3 \mathrm{~mm}$. - (Reifes) ơ unbekannt.

Pacif. und Atlant. Ozean.

\section{Chirundina angulata G. O. Sars. ${ }^{1}$ )}

1905, C. a., Sars, Nr. 26, p. 13.

q. Körper dicker als bei der vorigen Art; Stirncrista wenig vorspringend, fast verschwunden; Seitenecken des letzten Thsegm. in eine dreieckige Spitze verlängert. Exp. des 1. B. 2-gldr. mit völlig verschmolzenen, proximalen Gl. L. $5 \mathrm{~mm}$. - oे unbekannt.

Atlant. Ozean.

\section{Euchirella Giesbrecht.}

Kopf meist vom 1. Thsegm. getrennt; 4. und 5. Thsegm. verschmolzen; Lateralecken des letzten Thsegm. abgerundet; Rostrum 1-spitzig oder fehlend; Abdomen beim o 4-, beim o 5-gldr. mit sehr verkürztem Ansegm.; Gensegm. und Furkalborsten zuweilen asymmetrisch. 1. Antenne beim $q$ 23-gldr. Enp. der 2. Antenne $1 / 2-1 / 4$ so lang wie das Exp.; Äste der 1. Maxille kurz; Enp. mit wenigen Hakenborsten; Mundgliedmaßen des $\sigma^{\pi}$ rückgebildet. Exp. 1. B. 2-, des 2.-4. B. 3-gldr.; Enp. des 1. und 2. B. 1-, des 3. und 4. B. 3-gldr.; Fiederung am Innenrande des 1. Gl. des Basp. des 4. B. meist durch Stacheln ersetzt, die aber beim or ausfallen. 5. B. beim $q$ fehlend, beim $\sigma^{7}$ rechts 2-ästig mit Schere, links griffelförmig mit rudimentärem Enp.

1. Rostrum fehlt E. curticauda

Kopf mit 1-spitzigem Rostrum

2. Stirn ohne Crista

E. rostrata

Stirn mit Crista

E. pulchra

I) = Undeuchaeta major. Vergl. Sars 1907, p. 3. (Anmerkung während der Revision.) 


\section{Euchirella rostrata Claus.}

1866, Undina r., Claus, p. 11 , t. 1 f. 2 . 1892, E. r., Giesbrecht, p. 232, t. 2 f. 11 ; t. 15 f. $6,27,28$; t. 36 f. $16,17,23.1898$, E. r., Giesbr. und Schmeil, p. 36 (). 1900, E. r., Cleve, p. 4, t. 2 f. $1-12$ (ठ)

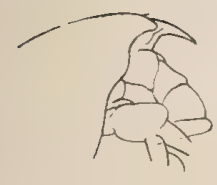

a

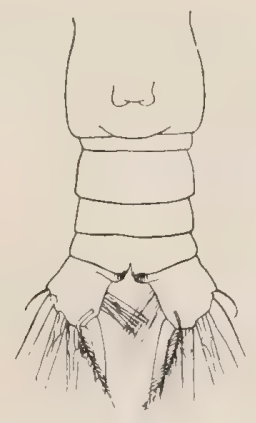

b

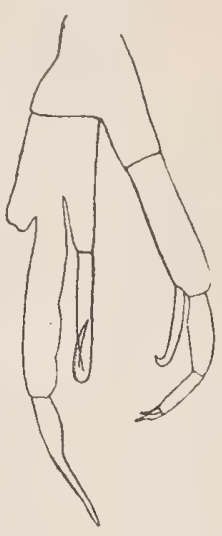

C

Fig. 52. Euchirella rostrata.

a Kopf $q$, lateral, b Abdomen $q$, ventral, c 5. B. $0^{7}$. a und $b$ nach Giesbrecht, c nach Cleve.

१. Stirn ohne Crista, mit Rostrum; Abdomen symmetrisch. Enp. der 2. Antenne halb so lang wie das Exp.; 2. Gl. des Enp. mit $8+6$ Borsten. 1. Gl. des Basp. des 4. B. mit einer Reihe von 6-7 dreieckigen Lamellen am Innenrande. - $\sigma$. Stirn ohne Crista, mit Rostrum; Innenrand des Exp. des rechten B. glatt. - L. ๆ 2,95-3,1 mm, or $2,5 \mathrm{~mm}$.

Mittelmeer, Atlant. Ozean; Ind. Ozean; Pacif. Ozean.

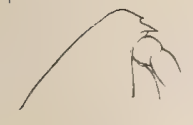

a

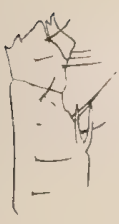

b

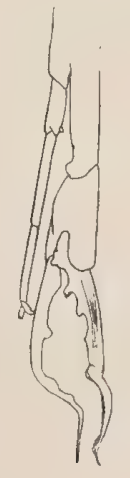

c

Fig. 53. Euchirella pulchra. a Kopf 오, lateral, b Basp. 4. B. , c 5. B. $\sigma^{x}$. Nach Giesbrecht.

\section{Euchirella pulchra Lubbock.}

1856, Undina p. o und C a lanus 1 atus 9 , Lubbock, p. 20, t. 4, 7; p. 10 , t. 2, 11. 1883, Euchaeta pulchra, Brady, p. 63 t. 14, 20 . 1892, E. p., Giesbrecht, p. 233 , t. 15 f. 22,23 ; t. 36 f. 13, 27. 1898, E. p., Giesbr. und Schmeil, p. 36.

ๆ. Stirn mit niedriger Crista und kleinem Rostrum; Gensegm. asymmetrisch, links vor der Segmentmitte stark gewölbt, rechts eingebuchtet. Enp. der 2. Antenne ca. $2 / \overline{5}$ so lang wie das Exp.; 2. Gl. des Enp. mit $6+5$ Borsten. 1. Gi. 
des Basp. des 4. B. mit (1 oder) 2 etwa gleich langen Stacheln am Innenrande, die nicht bis zum distalen Gliedrand reichen. - $\sigma^{7}$. Stirn mit niedriger Crista; 5. B. schlank, Zange des rechten B. etwas kürzer als das Basp. L. क 4,4 mm, of $3,7 \mathrm{~mm}$.

Atlant., Ind. und Pacif. Ozean.

\section{Euchirella curticauda Giesbrecht.}

1888, E. c., Giesbrecht, p. 336. 1892, E. c., Giesbrecht, p. 233, t. 15 f. $3,13,25$; t. 36 f. 19,20 . 1898, E. c., Giesbr. und Schmeil, p. 36.1904 , E. c. var. atlantica, Wolfenden, p. 116.

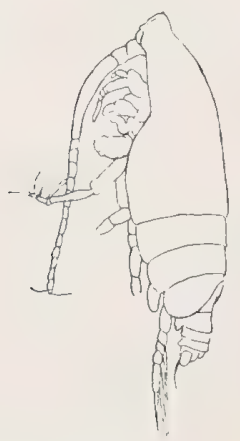

a

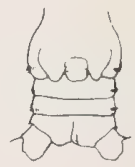

b

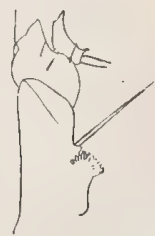

c

Fig. 54. Euchirella curtic a d a.

a $q$ lateral, b Abdomen $q$, ventral, c Basp. 4. B.. . Nach Giesbrecht.

Q. Stirn mit hoher Crista, ohne Rostrum. Abdomen kurz, symmetrisch. Enp. der 2. Antenne 1/4 so lang wie das Exp.; 1. Gl. des Exp. mit einem Zipfel; 2. Gl des Enp. mit $3+2$ Borsten. 1. Gl. des Basp. des 4. B. mit einer Reihe von Zacken (12-13 bei der pacifischen, 6 bei der atlantischen Form) am Innenrande. L. $2,5-3,8 \mathrm{~mm}$. - $\sigma^{7}$ unbekannt.

Atlant. und Pacif. Ozean.

\section{Euchaeta Philippi.}

Q. Kopf mit dem 1. Thsegm. verschmolzen oder mehr weniger deutlich davon getrennt; die beiden letzten Thsegm. verschmolzen; Rostrum 1-spitzig; vor der Oberlippe ein beutelförmiger Anhang. Abdomen 4-gldr.; Gensegm. mehr oder weniger asymmetrisch; Innenrandborste der Furka lang. 1. Antenne mit einzelnen sehr langen, eigentümlich gebogenen Borsten. Äste der 2. Antenne meistens ungefähr gleich lang. 2. Gl. des Basp. der 1. Maxille, zusammen mit 
dem kurzen, stark bewaffneten Enp. ganz nach dem Innenrande übergebogen. Distale Hakenborsten der 2. Maxille länger als die proximalen. Innenrandborste des 1. Gl. der Basp. der B. lang; Exp. des 1. B. 2- (oder 3-) gldr., des 2.-4. B. 3-gldr.; Enp. des 1. und 2. B. 1-, des 3. und 4. B. 3-gldr.; 5. B. fehlt.

$\sigma$. Weicht von obengenannten Merkmalen des 0 in folgendem $a b$ : Kopf mit 1. Thsegm. verschmolzen; Abdomen 5-glar., mit kurzem Ansegm.; Innenrandborste der Furka verkürzt. Mandibel, 1. und 2. Maxille verkümmert. Exp. des 1. B. 3-gldr.; 5. B. lang, beiderseits 2-ästig; linkes Enp. griffel-, rechtes stummelförmig; linkes Exp. 3-, rechtes 2-gldr.

1. Auf der Stirn, vorne an der Basis des Rostrums, ein flacher Höcker 2 Auf der Stirn, vorne an der Basis des Rostrums, ein konischer Vorsprung 7

2. Gensegm. nahezu symmetrisch; in der Mitte des Außenrandes des proximalen G1. am Exp. des 1. B. eine kleine Borste 3 Gensegm. mit asymmetrischen Auswüchsen; die Borste fehit 6

3. Ventralseite des letzten Thsegm. mit langen Haarbüscheln 4 Letztes Thsegm. ohne die Haarbüschel

E. tonsa

4. Genitalöffnung jederseits mit zwei gleich großen Höckern; 2. Außenranddorn des 3. Gl. des 2. Exp. das Ende des Gliedes erreichend; dorsale Borste der Furka nicht länger als die innerste Endborste E. glacialis Genitalöffnung jederseits mit einem Höcker oder mit klappenartigem Vorsprung; 2. Außenranddorn des 3. Gl. des 2. Exp. das Ende des G1. nicht erreichend; dorsale Borste der Furka sehr lang

5. Genitalöffnung jederseits mit einem kegelförmigen Höcker; letztes Thsegm. in eine stumpfe Spitze endigend

E. norvegica Genitalöffnung jederseits mit klappenartigem Vorsprung; letztes Thsegm. gerundet

E. barbata

6. Furka mit 4 etwa gleichlangen Endborsten, dorsale Borste länger und an der Basis viel dicker als jene. Gensegm. links vorne mit einem Knopf

E. acuta

2. Endborste der Furka verlängert, die dorsale Borste so dick wie die Endborsten; Gensegm. rechts vor der Öffnung mit lamellösem Fortsatz und einem Tuberkel auf der Dorsalfläche

E. hebes

7. 1. Antenne überragt das Rumpfende; 2. Endborste der Furka beträchtlich länger als die anderen

E. spinosa

1. Antenne erreicht das Rumpfende nicht; die 4 Endborsten der Furka ungefähr gleich lang

E. marina.

\section{$\left.\sigma^{31}\right)$}

1. Endglied beider B. des 5. Paares mit langem, geradem oder leicht gekrümmtem Pfriem

1) Das ${ }^{7}$ von E. to n s and von E. s p in o s a ist nicht bekannt. 
Endglied des 5. B. links ohne Pfriem, rechts mit stumpfem, kräftigem Haken 3

2. Höcker des Frontalorganes flach

E. acuta

Höcker des Frontalorganes vorspringend

E. marina

3. Gezähnelter Fortsatz am Ende des linken 5. B. mit scharfer Spitze 4 Gezähnelter Fortsatz nicht zugespitzt

4. Gezähnelter Fortsatz des linken 5. B. vor dem Ende verbreitert E. hebes Derselbe nach dem Ende verjüngt

E. norvegica

5. Fortsatz grob gezähnelt, 2 Zähne größer als die übrigen; das Läppchen schmal, am Ende hakenförmig gebogen

E. barbata

Fortsatz quer abgestutzt mit dicht gekerbtem Rande; das Läppchen breiter, am Ende nicht hakenförmig gebogen

E. glacialis.

\section{Euchaeta marina Prestandrea.}

1833, Cyclops marinus, N. Prestandrea, p. 12. 1863, E. prestandreae, Claus, p. 185 , t. 30 f. $8-17.1892$, E. m., Giesbrecht, p. 246, t. 1 f. 10,11 ; t. 15 f. 31,33 ; t. 16 , f. $15-17,22,23,25,29,30,41,46$; t. 37 f. $30,37,38,49$. 1898, E. m., Giesbr. und Schmeil, p. 38, f. 2, 3.

q. Höcker des Frontalorgans vorspringend; Gensegm. ventral gewölbt, asymmetrisch, mit lamellösem Fortsatz rechts von der Genitalöffnung; Furka mit 4 nahezu gleichlangen Endborsten; ihre dorsale Borste an der Basis viel

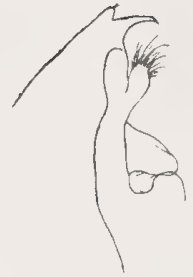

a

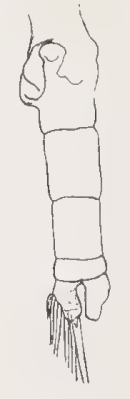

b

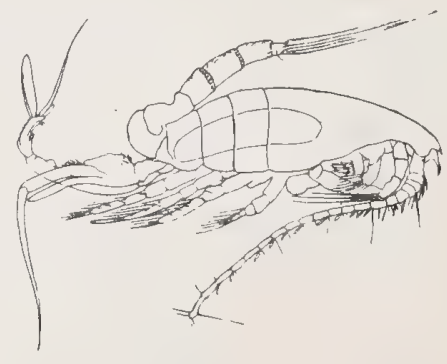

c

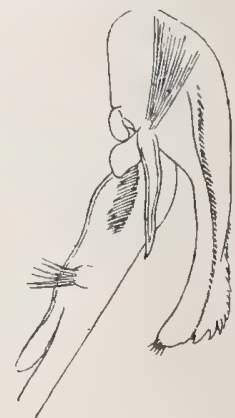

d

Fig. 55. Euchaeta marina. a $q$ Kopf lateral, b $q$ Abdomen ventral, c $\sigma^{\pi}$ lateral, d distaler Teil des linken 5. B. $\sigma^{\top}$. Nach Giesbrecht.

dicker als jene. 1. Antenne erreicht den Hinterrand des auf das Gensegm. folgenden Segm. Außenrand des proximalen Gl, des Exp. des.1: B. leicht konvex; Außenrand und Randdorne des Endgl. des Exp. des 2. B. abweichend; der Dorn seines 2. G1. erreicht nicht die Basis des folgenden Dorns. - $\sigma$. Endgl. des Exp. des linken 5. B. endigt in ein langes Stilett; das 2. Gl. seines Exp. mit grobgezähneltem, nach dem Ende verbreitertem Fortsatz. - L. \& 2,25-3,9 mm, or 3,0-3,2 $\mathrm{mm}$.

Mittelmeer, Atlant., Indischer und Pacif. Ozean. 


\section{Euchaeta acuta Giesbrecht.}

1892, E. a., Giesbrecht, p. 246 , t. 16 f. $6,10,14,18,21,27,39$; t. 37 f. 47, 48, 52. 1898, E. a., Giesbr. und Schmeil, p. 38.

○. Höcker des Frontalorgans flach; Umgebung der Genitalöffnung vorspringend; Gensegm. asymmetrisch und mit einem Knopf vorne links. Furkalborsten wie bei E. marina. 1. Antenne überragt den Thorax wenig. Außen-

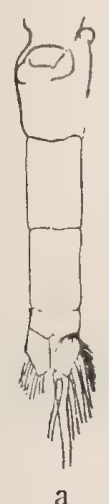

a

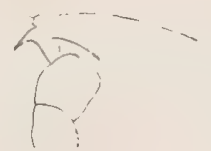

b)

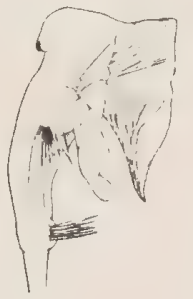

c

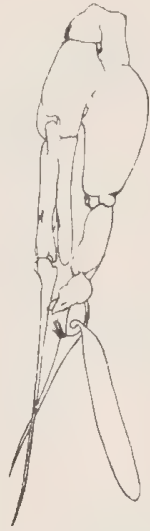

d.

Fig. 56. Euchaeta acuta. a Abdomen , ventral, b Kopf $q$, lateral, c distaler Teil des linken 5. B. Ox, d 5. B. Or, mit Spermatophor. Nach Giesbrecht.

rand des proximalen Gl. des Exp. des 1. B. konkav. Endgl. des Exp. des 2. B. abweichend; Randdorn des 2. G1. reicht fast bis zur Spitze des folgenden Dorns. - J. 5. B. dem von E. marina ähnlich; 2. Gl. des linken Exp. jedoch mit feingezähneltem, pyramidenförmig zugespitztem Fortsatz. - L. † 4,1 mm, of $3,5-4,8 \mathrm{~mm}$.

Mittelmeer, Nord-Atl. Ozean; Ind. Ozean; Pacif. Ozean.

\section{Euchaeta hebes Giesbrecht.}

1888, E. h., Giesbrecht, p. 337. 1892, E. h., Giesbrecht, p. 246 , t. 15 f. 29 , 30 ; t. 16 f. $3-5,20,31,32,38,44$; t. 37 f. $32,33,54$. 1898, E. h., Giesbr. und Schmeil, p. 39, f. 4. 1905, E. h., Gough, p. 336, f. 4-7.

․ Höcker des Frontalorgans flach. Umgebung der Genitalöffnung vorspringend; Gensegm. asymmetrisch, mit lamellösem Fortsatz rechts von der Öffnung und einem Tuberkel auf der Dorsalfläche. 2. Endborste der Furka länger als die anderen, die dorsale Borste so dick wie die Endborsten. 1. Antenne überragt den Hinterrand des Gensegm. wenig. B. ähnlich wie bei 


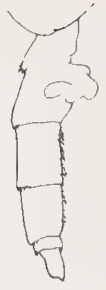

a

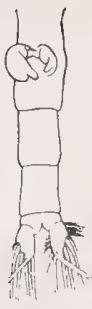

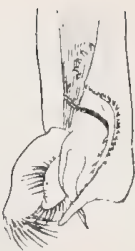

c

Fig. 57. Euchaeta hebes. a Abdomen $O$, lateral, b Abdomen $q$, ventral, $\mathrm{c}$ distaler Teil des linken 5. B. O. Nach Giesbrecht.

E. acuta. - o. Endgl. des linken 5. B. stumpf, ohne Stilett; Fortsatz des 2. Gl. mit einwärts gewendeter Spitze, am Ende breit. - L. ㅇ 2,85-2,95 mm, o $2,75 \mathrm{~mm}$.

Mittelmeer, Gibraltar, Kanal.

\section{Euchaeta spinosa Giesbr.}

1892 , E. s., Giesbrecht, p. 246 , t. 16 f. $12,26,34,47$; t. 37 f. 31,34 , 35, 50. 1898, E. s., Giesbr. und Schmeil, p. 39.

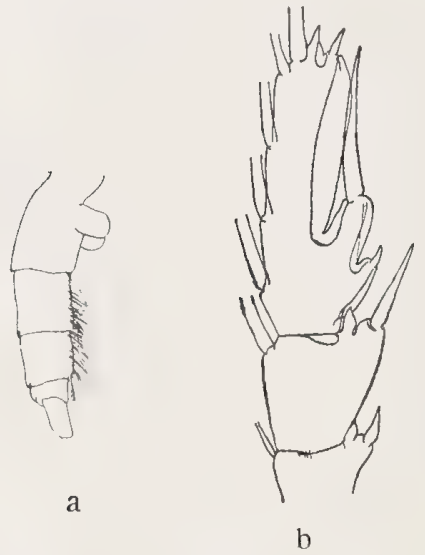

Fig. 58. Euchaeta spinosa.

a Abdomen des $\bigcirc$, von der Seite,

b Außenast des 2. B. Nach Giesbrecht.

q. Höcker des Frontalorganes vorspringend; Gensegm. fast symmetrisch, ventral kaum gewölbt, zu beiden Seiten der Öffnung wulstig. Furkalborsten wie bei $\mathrm{E}$. hebes. 1. Antenne überragt das Rumpfende um mehr als das Endgl. Vordere B. ähnlich wie bei E. hebes; Basp. und Äste der hinteren B. stellenweise mit kurzen Spitzen bedeckt. L. $6 \mathrm{~mm}$. - o unbekannt.

Mittelmeer, Nord-Atl. Ozean; Pacif. Ozean; Ind. Ozean.

\section{Euchaeta norvegica Boeck.}

1872, E. n., Boeck, p. 40. 1875, E. carinata, Möbius, p. 271, t. $7,8$. 1903, E. n., Sars, p. 38, t. 24-26. 1904, E. n., Wolfenden, p. 133, Textf. $13,4,8$ (7?).

Q. Höcker des Frontalorganes flach; Ventralseite des letzten Thsegm. mit langen Haarbüschein; letztes Thsegm. in eine stumpfe Spitze endigend. 
Trib. Gymnoplea.

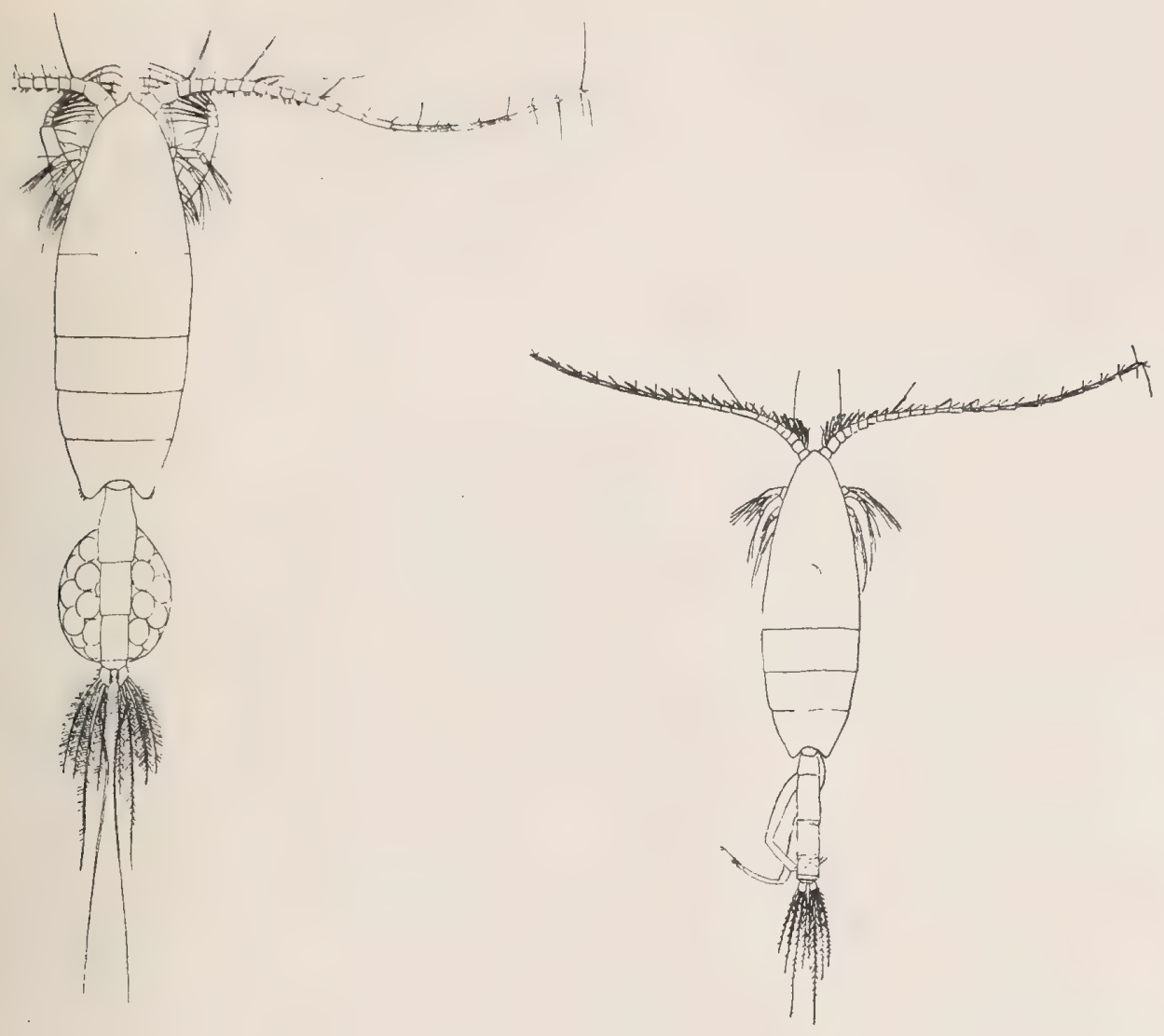

a

b

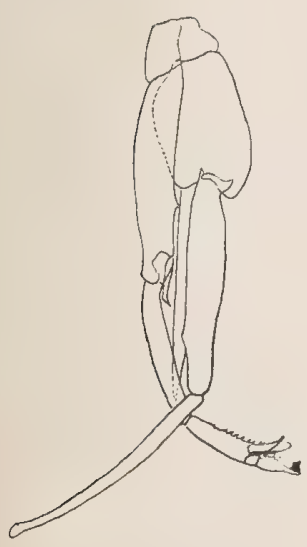

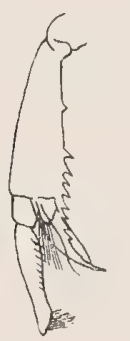

d

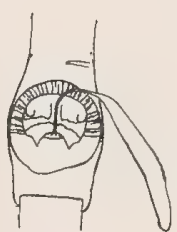

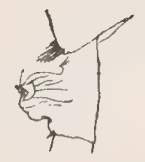

f

Fig. 59. Euchaeta norvegica.

a dorsal, b $\sigma^{\pi}$ dorsal, c 5. B. $\sigma^{\pi}$, d die beiden letzten Außenastgl. des linken B. $\sigma^{\pi}$, stärker vergrößert, e Genitalsegment des + , ventral, f dasselbe, lateral. a-d nach Sars, e und $f$ nach. Wolfenden. 
Gensegm. fast symmetrisch, mit dickem, vorspringendem Wulst an der Ventralfläche; Genitalöffnung jederseits mit einem kegelförmigen Höcker; 2. Endborste der Furka viel länger als die anderen; dorsale Borste der Furka länger, aber dünner als die Endborsten. 1. Antenne etwa so lang wie der Vorderkörper. Außenrand des proximalen Gl. des Exp. des 1. B. konkav, mit kleinem Dorn in der Mitte; Endgl. des Exp. des 2. B. abweichend gebaut.- o . Endgl. des linken 5. B. kurz, stumpf, ohne Stilett, Fortsatz des 2. Gl. gezähnelt, mit scharfer Spitze, nach dem Ende gleichmäßig verjüngt. - L. † 7-11 mm, o $5,5-7 \mathrm{~mm}$.

Nord-Atl. Ozean, Nordmeer, Polarmeer.

\section{Euchaeta glacialis Hansen.}

1886, E. g., Hansen, p. 256 , t. 23 f. $5-5$ k; t. 24 f. $1-1$ d. 1903, E. g., Sars, p. 40 , t. 27 . 1904, E. g., Wolfenden, p. 134, Textf. 1 1,2, 7 (8?).

Verwandt mit E. norvegica, aber hauptsächlich in folgenden Merkmalen abweichend: - $\supsetneq$. Letztes Thsegm. abgerundet. Genitalöffnung jederseits mit zwei gleichgroßen Höckern; dorsale Borste der Furka nicht länger als die

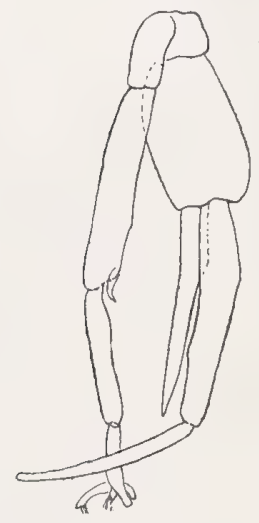

a

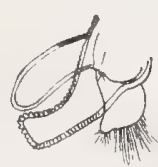

b

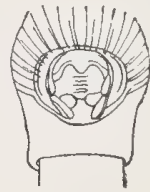

$\mathrm{c}$

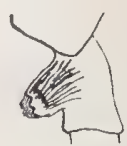

d

Fig. 60. Euchaeta glacialis.

a 5. B. ơ, b distales Ende des 2. Gl. des Außenastes des linken B. stärker vergrößert, c Genitalsegment des $\bigcirc$, ventral, d dasselbe lateral.

a und b nach Sars, c und d nach Wolfenden.

innerste Endborste. - o . Fortsatz des 2. Gl. des linken 5. B. am Ende quer abgestutzt mit dicht gekerbtem Rande; das läppchenförmige Endgl. desselben B. relativ breit, am Ende nicht hakenförmig gebogen. - L. † $10 \mathrm{~mm}$, o $8 \mathrm{~mm}$.

Nord-Atl. Ozean, Nordmeer, Polarmeer. .

\section{Euchaeta barbata Brady.}

1883, E. b., Brady, p. 66, t. 22 f. $6-12$. 1892, E. b., Giesbrecht, p. 246. 1893, E. b., + ? E. hebes var. valida o , Th. Scott, p. 58 , t. 6 f. 17 ; p. 60 , t. 6 f. 20-22. 1898, E. b., Giesbr. und Schmeil, p. 40. 1903, E. b., Sars, p. 41, t. 28 . 1904, E. b., Wolfenden, p. 135, Textf. 1 5, 6, 9. 
Verwandt mit E. norvegica, aber: - $q$. Letztes Thsegm. gerundet; Genitalöffnung jederseits mit klappenartigem Vorsprung; die distalen Reusenborsten der 2. Maxille geringelt, mit kammartigen Spitzen dicht besetzt. 3. Distaler Fortsatz am 2. Gl. des linken 5. B. mit gerundeter Spitze, grob gezähnelt, zwei Zähne größer als die übrigen; das Läppchen schmal, am Ende hakenförmig gebogen. - L. \& $8-12 \mathrm{~mm}$, o bis $10 \mathrm{~mm}$.

Atlant. Ozean, Nordmeer; Ind. Ozean.

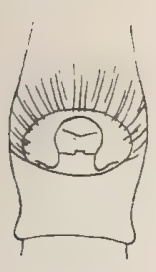

a

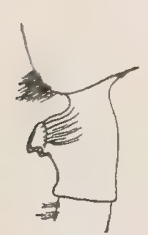

b

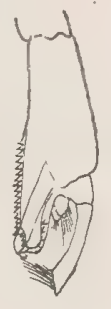

c

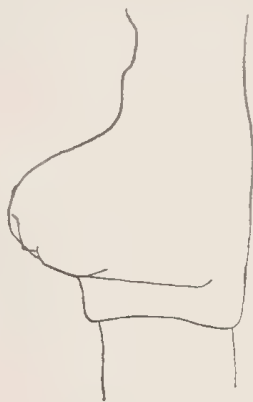

a

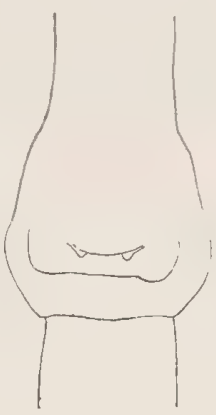

b

Fig. 61. Euchaeta barbata.

a Genitalsegment des $O$; ventral, b dasselbe, lateral, c die beiden letzten Gl. des Außenastes des linken 5. B. $\sigma^{7}$. a und b nach Wolfenden, c nach Sars.

Fig. 62. Euchaeta tonsa.

a Genitalsegment des $\odot$, lateral, b dasselbe, ventral. Nach Giesbrecht.

\section{Euchaeta tonsa Giesbrecht.}

1895 , E. t., Giesbrecht, p. 251 , t. 4 f. 9, 10.1898 , E. t., Giesbr. und Schmeil, p. 40.

Q. Verwandt mit E. norvegica, aber: - Haarbüschel am letzten Thsegm. fehlend; Form des Gensegm. und dessen ventralen Wulstes abweichend; Außenrandlobus der Maxille mit 9 Borsten; Fiederung der Schwimmfüße nicht gefärbt; mittlerer Außenranddorn des Exp. des 2. B. über die Basis des endständigen Dorns hinausreichend. L. 5-6 mm. - $\sigma^{\top}$ unbekannt.

Nord-Atl. Ozean; Pacif. Ozean.

Anm. Euchaeta hessei Brady ist nach Giesbrecht keine einheitliche Art; wenigstens teilweise ist sie mit Euchirella rostrata Cls. identisch. Thompson $(1903$, p. 18) gibt an, ein unreifes männliches Exemplar dieser fraglichen Art in einem Planktonfang des nördlichen Atl. Ozeans gefunden zu haben.

Für die Beschreibung von E. ocean a Thompson siehe Anhang.

\section{Phaenna Claus.}

Vorderrumpf breit, beim $\bigcirc$ kugelig; Rostrum 2-spitzig. Kopf vom 1. Thsegm. gesondert; die beiden letzten Thsegm. unvollkommen verschmolzen; 
Abdomen des $q$ 4-, des of 5-gldr.; Ansegm. und Furka kurz. 1. Antenne mit gesondertem Endgl., beim o 24-, beim or rechts 18-, links 19-gldr., mit kurzen, weichen Borsten. Außenast der hinteren Antennen 6-gldr., länger als der Innenast. Mandibellade sehr lang gestreckt, ebenso der Kaulobus der 1. Maxille, deren Exp. nur 5 Borsten trägt. Schläuche der 2. Maxille z. T. mit Pinselspitzen. Mundteile des or verkümmert, jedoch an der 2. Maxille 4 pinselförmige Schläuche. Exp. des 1.-4. B. 3-gldr.; Enp. des 1. B. 2-, des 2. B. 2-, des 3. und 4. B. 3-gldr.; proximales Basalgl. des 4. B. ohne Innenrandborste; Hinterfläche der Innenäste mit Dörnchen bewehrt. 5. B. fehlt dem Weibchen, beim $\sigma^{\top}$ beiderseits einästig, das linke 5-, das rechte 4 -gldr.

\section{Phaenna spinifera Claus.}

1863, P. s., Claus, p. 189 , t. 31 f. $1-7.1892$, P. s., Giesbrecht, p. 293, t. 5 f. 3 ; t. 12 f. $1-8,35-37$; t. 37 f. $17-21.1898$, P. s., Giesbr. und Schmeil, p. 50 .

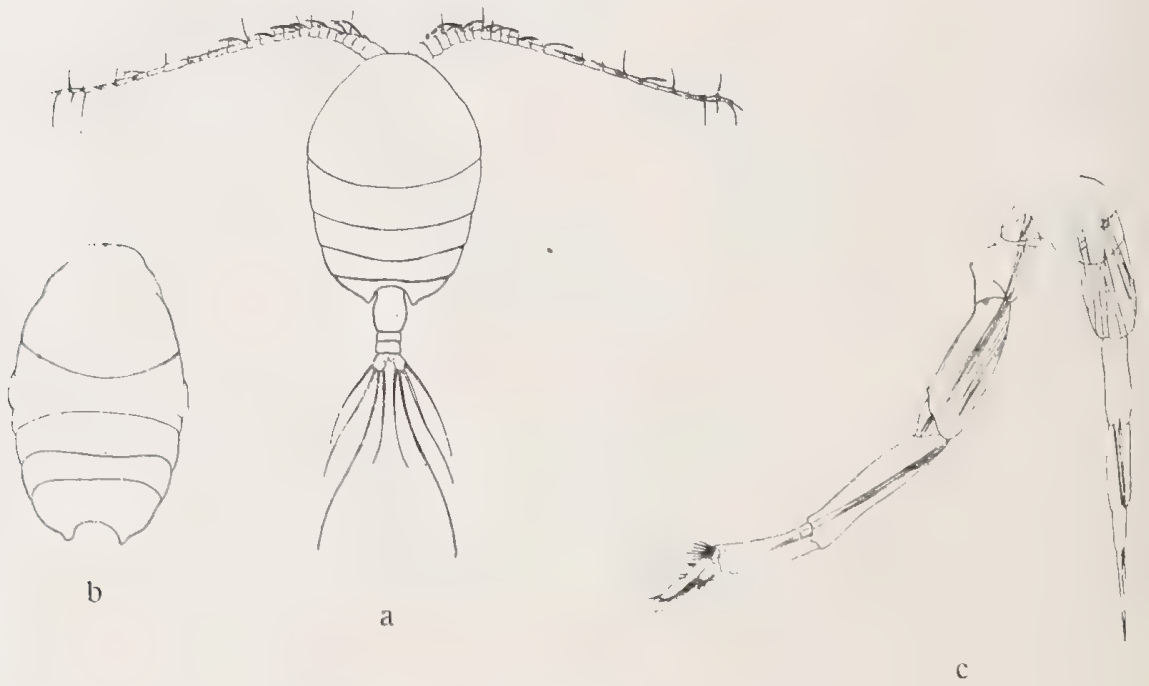

Fig. 63. Phaenna spinifera.

a $q$ dorsal, b Vorderkörper $\sigma$, dorsal, c 5. B. o. Nach Giesbrecht.

7. Vordere Antennen ungefähr bis zum Ende der Furka reichend; Vorderkörper etwa $4 \mathrm{mal}$ so lang und $5 \mathrm{mal}$ so breit wie dẹ Hinterkörper. - $\mathrm{o}^{7}$. 3. Gl. des linken 5. B. sehr kurz; rechtes B. kürzer als das linke, griffelförmig, in eine scharfe Spitze auslaufend. - L. \& 1,8-2,1 mm, o $1,8 \mathrm{~mm}$.

Mittelmeer, Attant., Ind. und Pacif. Ozean.

Anm. Für die Beschreibung von Phaenna zetlandica Th. Scott siehe Anhang. 


\section{Pseudophaenna G. O. Sars.}

Vorderkörper oval; Rostrum ungeteilt, mit gerundeter Spitze; Kopf mit 1. Thsegm., 4. mit 5. Thsegm. verschmolzen. Abdomen beim 4-, beim $^{7}$ 5-gldr., symmetrisch. 1. Antenne beim of 23-, beim or 19-gldr. Exp. der 2. Antenne 6-gldr, viel länger als das schwach entwickelte Enp.; Exp. der 1. Maxille mit nur 2 Borsten; Schläuche der 2. Maxille z. T. mit Pinselspitzen; Mundgliedmaßen des $\sigma^{-1}$ stark verkümmert oder umgeformt. Exp. des 1.-4. B. 3-gldr.; Enp. des 1. B. 1-, des 2. B. 2-, des 3. und 4. B. 3-gldr.; Hinterseite der Enp. ohne Dörnchenreihen; 5. B. beim \& 3-gldr., beim ot beiderseits mehrgliedrig.

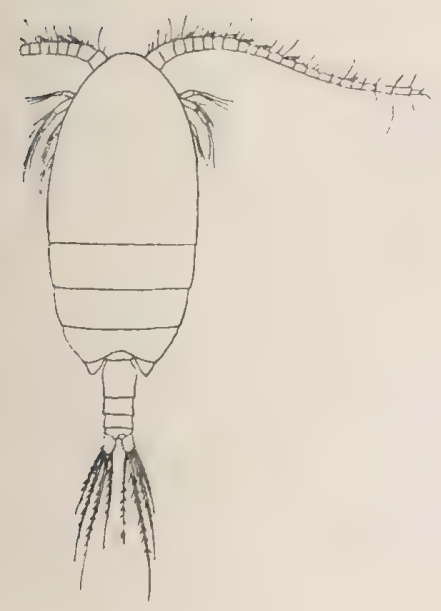

a

Fig. 64. Pseudophaenna typica.

a $q$ dorsal, b 5. B. q, c 5. B. o. Nach Sars.
Pseudophaenna typica G. O. Sars.

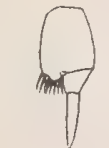

b

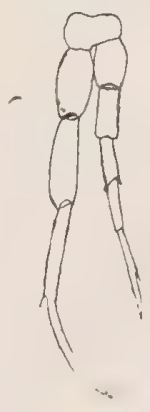

c
1903, P. to, Sars, pr 44 t. 29,30 .

૧. 1. Antenne kaum länger als der Vorderkörper; Exp. der 2. Ant. mehr als 2 mal länger als das Enp., dessen proximales Gl. stark verkürzt ist. 5. B. klein, 3-gldr., Basalgl. breit, mit Dörnchenreihe am inneren Ende, die 2 letzten Gl. unbewehrt, das mittlere sehr kurz, Endgl. gerade, fingerförmig. 万. 5. B. sehr schlank, rechts 5-gldr., das vorletzte Gl. des linken B. erreichend. - L. $+1,6 \mathrm{~mm}$, o $1,4 \mathrm{~mm}$.

Norwegische Küste, immer nur dicht über dem Boden.

\section{Xanthocalanus Giesbrecht.}

Vorderkörper oval; Rostrum mit zwei (selten fehlenden) Fäden; Kopf mehr oder weniger vollkommen vom 1. Thsegm. getrennt; 5. Thsegm. mit dem 4. Thsegm. verwachsen oder frei; Abdomen beim 9. 4-, beim ot 5-gldr, symmetrisch; Ansegm. und Furka kurz. 1. Antenne beim $q$ 23-25-gldr., beim o mit reduzierter Gliederzahl und vermehrten Aesthetasken; Exp. der 2. Ant. 6-gldr., ungefähr so lang wie oder länger als das Enp. Mandibellade lang und schmal; Kaulobus und 2. Gl. des Basp. der 1. Maxille gestreckt; Exp. mit 8-10 Borsten. Schläuche der 2. Maxille z. T. mit Pinselspitzen. Mundgliedmaßen des $\sigma^{\top}$ stark verkümmert oder umgeformt. Exp. des 1.-4. B. 
3-gldr.; Enp. des 1. B. 1-, des 2. B. 2-, des 3. und 4. B. 3-gldr. Proximales Basalgl. des 4. B. mit Innenrandborste; Hinterfläche der Enp. mit Dörnchenreihen. 5. B. beim o 2-oder 3-gldr., beim of rechts fehlend oder vorhanden, links einästig, 5-gldr.

Anm. 'Für die Bestimmung der Arten wird auf die Speziesbeschreibung und auf die Figuren hingewiesen. Besonders zu beachten ist die Bedornung der Schwimmfüße, die aber nur mit Hilfe der Figuren aus den Originalarbeiten richtig zu würdigen ist.
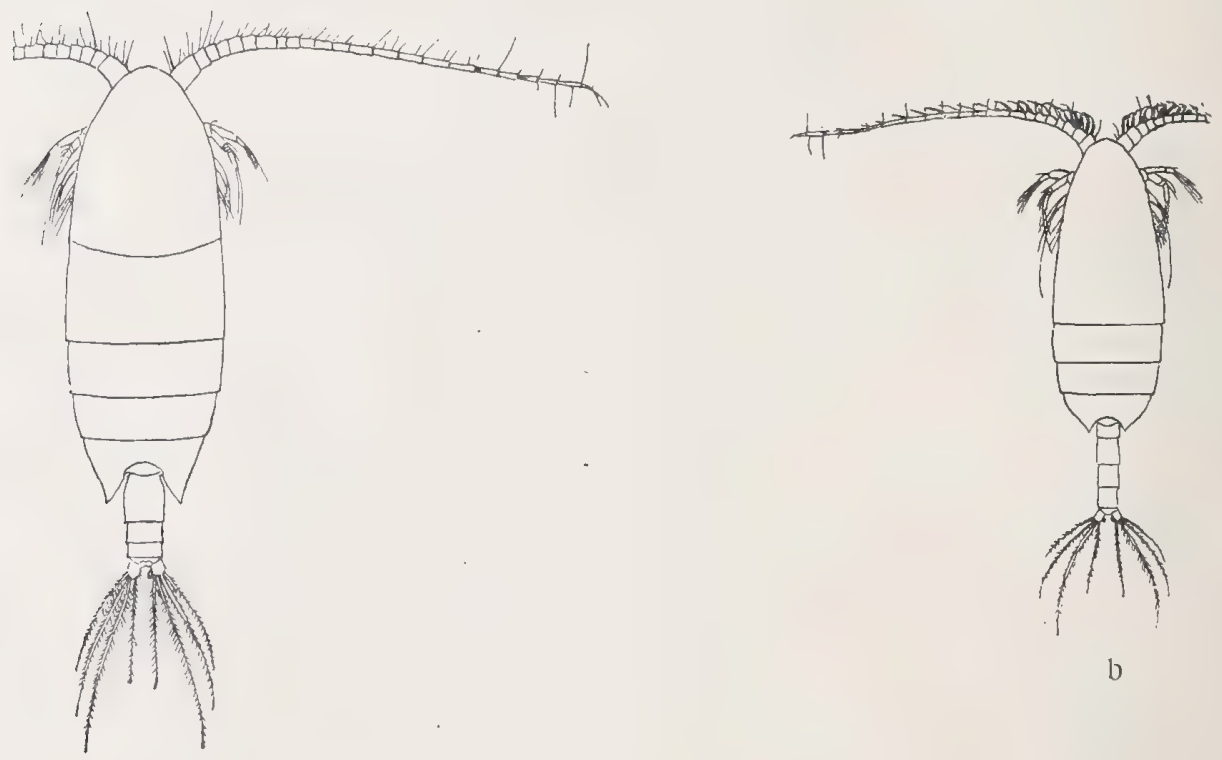

$\mathrm{b}$

a
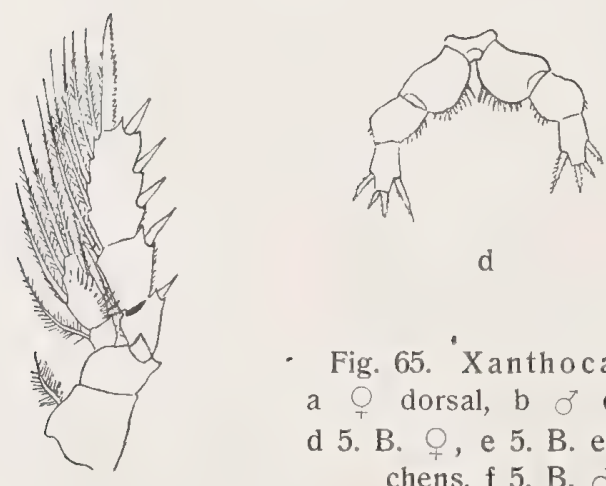

d

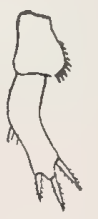

e

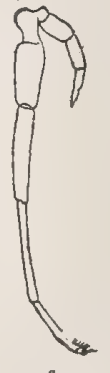

-Fig. 65. 'Xanthocalanus borealis. a $\bigcirc$ dorsal, b $\sigma$ dorsal, c 2. B. $\square$, d 5. B. 7 , e 5. B. eines unreifen Weibchens, f 5. B. ${ }^{r}$. Nach Sars.

1. Xanthocalanus borealis G. O. Sars.

1900, X. b., Sars, p. 49, t. 11 (das q unreif!). 1903, X. b., Sars, p. 46, t. $31,32.1905$, X. b., Farran, p. 38 , t. 8 f. $14-17$. 
Q. Kopf unvollkommen vom 1. Thsegm. getrennt; 4. und 5. Thsegm. verschmolzen; letztes Thsegm. in eine Spitze verlängert, die die Mitte des Gensegm. erreicht; Abdomen ungefähr 1/4 der Länge des Vorderkörpers. 1. Antenne 24-gldr., bis an das Ende des Gensegm. reichend. 5. B. 3-gldr.; Innenrand des 1. Gl. dicht bedornt; 2. Gl. in der Mitte deutlich angeschwollen und an der Innenseite schwach bedornt, bisweilen mit distalem Außenranddorn die beiden Endgl. ungefähr gleich lang, schmäler, mit 2 end- und 2 seitenständigen, kurzen und bewimperten Borsten. - 7 . Seitenloben des letzten Thsegm. weniger vorragend. Mundgliedmaßen relativ wenig reduziert. 5. B. beiderseits entwickelt, links mit sehr schmalen mittleren Gliedern; rechtes bis an das Ende des 2. Gl. des linken reichend. - L. o $3,5 \mathrm{~mm}$, o $2,5 \mathrm{~mm}$.

Nord-Atl. Ozean, Nordmeer, Polarmeer.

Anm. Mrázek (1902, p. 522) ist der Ansicht, daß die Sarssche Form mit X. hirtipes Vanhöffen (1897, I, p. 282, f. 18) identisch ist.

Die Gliederung und die Bedornung des 5 . B. beim $q$ scheinen auch bei reifen Individuen Schwankungen unterworfen zu sein (siehe: Farran, 1905, p. 39, t. 8 f. 14-16), Bei unreifen und nicht völlig ausgewachsenen $\rho$ ist die Form des 5 . B. bedeutend abweichend.
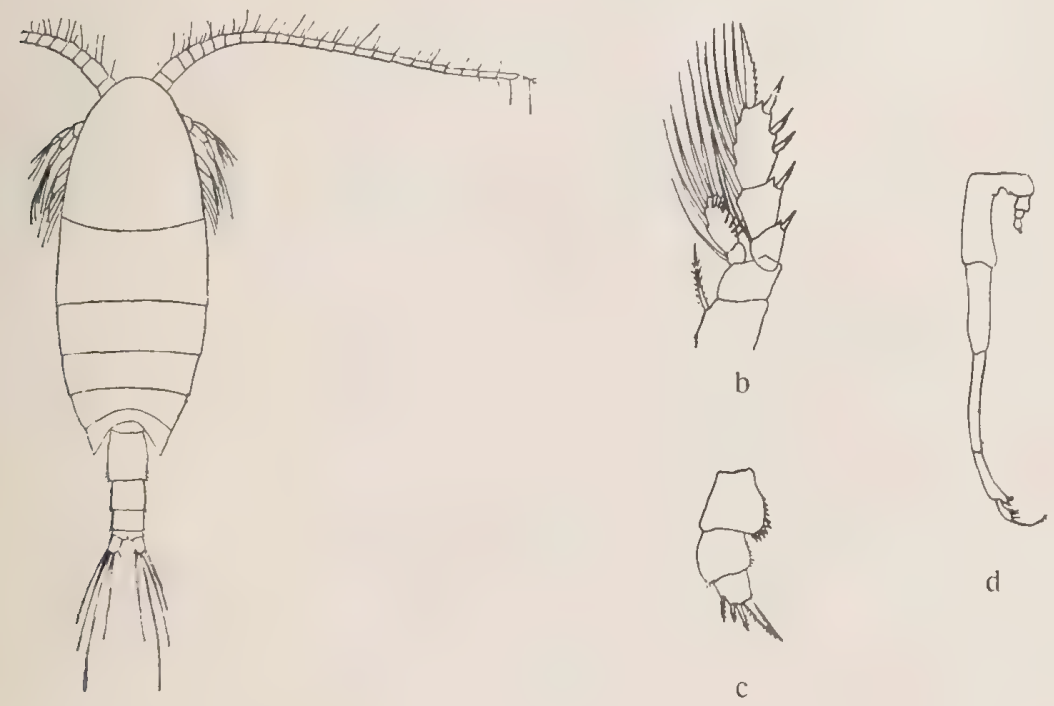

Fig. 66. Xanthocalanus propinquus.

a $q$ dorsal, b 2. B. $q$, c 5. B. $ๆ$, d 5. B. $0^{\pi}$. Nach Sars.

\section{Xanthocalanus propinquus G. O. Sars.}

$190 \$$, X. p., Sars, p. 20 , t. 33.

Mit X. borealis nahe verwandt: - O. 5. Thsegm. deutlich vom 4. Thsegm. getrennt, die Spitzen des 5. Thsegm. kürzer, etwa die Mitte des 
Gensegm. erreichend; Abdomen $1 / 3$ der Länge des Vorderkörpers. 1. Antenne bis an das Ende des 2. Abdsegm. reichend. 5. B. 3-gldr., 1. Gl. an der Innenseite mit Dörnchenreihe; 2. Gl, nicht geschwollen, Endgl. kürzer als das vorhergehende, mit einer schrägen Reihe von 4 Borsten, deren innerste bei weitem die größte ist. - $\sigma^{\top}$. Rechtes 5. B. kaum die Mitte des 1. Gl. des linken B. erreichend. - L. q $1,75 \mathrm{~mm}, 0$ ?

Norwegische Küste.

\section{Xanthocalanus greeni Farran.}

1905, X. g., Farran, p. 39, t. 8 f. $1-13$.

Q. Die beiden letzten Thsegm. verschmolzen; 5. Thsegm. nur wenig nach hinten verlängert, nicht zugespitzt. Vorderkörper 5 mal so lang wie das

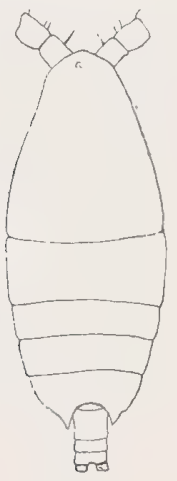

a

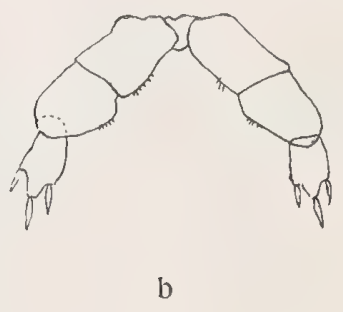

Fig. 67. Xanthocalanus greeni. a $q$ dorsal, b 5. B. $\bigcirc$, c 2. B. $ๆ$. Nach Farran.

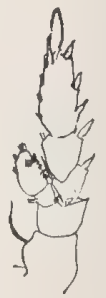

Abdomen. 1. Antenne 24-gldr., wenig länger als der Körper. 1. Innenrandlobus der 1. Maxille mit langen, rasiermesserförmigen Borsten. 5. B. 3-gldr., 3. Gl. mit 1 End- und 2 Seitendornen; Innenränder des 1. und 2. Gl. mit sehr kleinen Dörnchen. L. 6,0 $\mathrm{mm}$ (vielleicht nicht völlig ausgewachsen!). o unbekannt.

Nord.-Atl. Ozean.

\section{Xanthocalanus pinguis Farran.}

1905 , X. p., Farran, p. 39 , t. 8 f. $18-24$; t. 9 f. $1-6$.

Q. Kopf unvollkommen vam 1. Thsegm. gesondert; die beiden letzten Thsegm. getrennt; 5. Thsegm. über die Mitte des Gensegm. verlängert, mit stumpfendigenden Spitzen. Vorderkörper $3 \frac{1}{2} \mathrm{~m}$ mal so lang wie das Abdomen. 1. Antenne 23-gldr, kurz, nicht über das 4. Thsegm. ragend, 5. B. 3-gldr., 
1. Gl. mit feinen Dörnchen am Innenrande; 2. Gl. distal am Außenrande mit wenigen Dörnchen; das Endgl. länger und schmäler als das vorhergehende, mit fein bestachelter Oberfläche und 2 End- und 2 Seitendornen. L. $4,5 \mathrm{~mm}$. - or unbekannt.

Nord-Atl. Ozean.

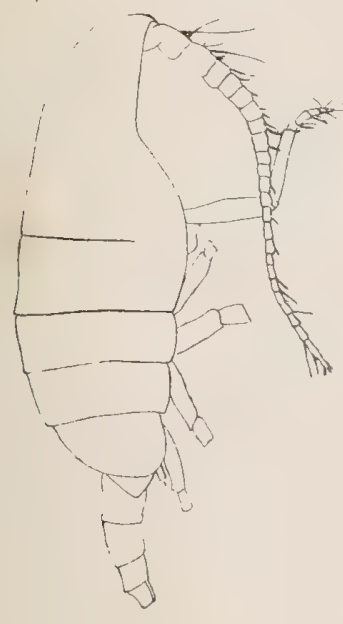

a

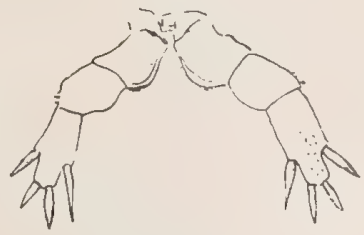

b

Fig. 68.

Xanthocalanus pinguis.

a q lateral, b 5. B. ?.

Nach Farran.

5. Xanthocalanus obtusus Farran.

1905, X. 0., Farran, p. 40, t. 9 f. $10-19$.

१. Kopf vom 1. Thsegm., 4. vom 5. Thsegm. gesondert; 5. Thsegm. kurz, stumpf. Vorderkörper $3^{1 / 2}$ mal so lang wie das Abdomen. 1. Antenne 24-gldr., die Mitte des Gensegm. erreichend; 5. B. 3-gldr., 1. Gl. mit Bewehrung von

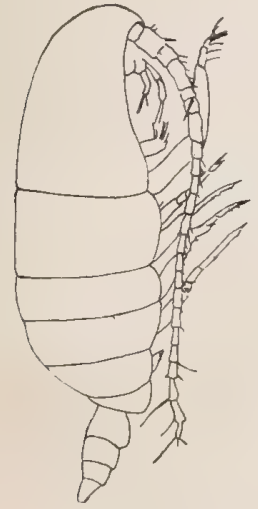

a

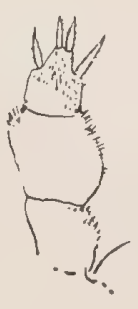

b

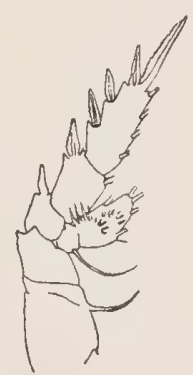

c

Fig. 69. Xanthocalanus obtusus.

a $q$ lateral, b 5. B. $ๆ$, c 2. B. . Nach Farran. 
groben Dörnchen distal an der Innenseite, 2. Gl. das größte, geschwollen, mit Dörnchen an der Innen- und Außenseite; 3. Gl. mit 2 End- und 2 Seitendornen und mit fein bestachelter Oberfläche. L. 2,4 mm. - o unbekannt.

Nord-Atl. Ozean.

\section{Xanthocalanus cristatus Wolfenden.}

1904, X. c., Wolfenden, p. 119 , t. 9 f. $18,19$.

ๆ. Kopf vom 1. Thsegm. gesondert; Kopf drei-

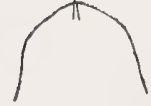

a

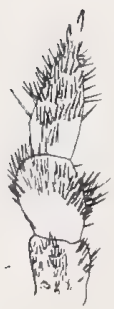

b

Fig. 70 .

Xanthocalanus

cristatus.

a Kopf $ᄋ$, dorsal,

b 5. B. \&, Nach

Wolfenden.

eckig, mit Crista. 1. Antenne das Ende der Furka erreichend; Enp. der 2. Maxille mit 7 kurzen, dicken, pinselförmigen Schläuchen und einem langen, dünnen, wurmförmigen Anhang. 5. B. 3-gldr., 1 Gl. so hoch wie breit; 2. Gl. länger als breit; 3. Gl. $2^{1 / 2}$ mal so lang wie breit, distal mit zwei dornförmigen (nicht artikulierten) Fortsätzen und einem äußeren Randdorn; alle Gl. dicht mit gemischten langen und kurzen Dornen bewehrt. L. $5 \mathrm{~mm}$. - o unbekannt.

Nord-Atl. Ozean.

\section{Xanthocalanus subagilis Wolfenden.}

1904, X: s., Wolfenden, p. 118, t. 9 f. 17, 32.

O. Exp. der 1. Maxille mit 9 Borsten; Enp. der 2. Maxille mit 6 oder 7 pinselförmigen Schläuchen und 2 wurmförmigen Anhängen. 5. B. 3-gldr.; 1. Gl. das längste und breiteste, mit starken Zähnen am Rande; 2. Gl. mit einem Borstenbündel am distalen Rande; 2. G1. mit bedornter Oberfläche, länger als breit, mit 3 Dornen, von welchen die innerste die größte ist. Abdomen gar nicht behaart. -
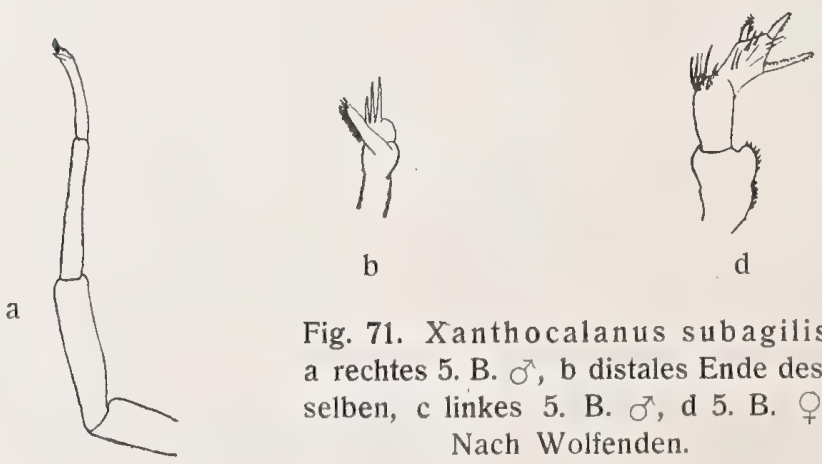

Fig. 71. Xanthocalanus subagilis. a rechtes $5 . \mathrm{B}$. O ${ }^{7}$, b distales Ende desselben, c linkes 5. B. $\sigma^{7}$, d 5. B. .

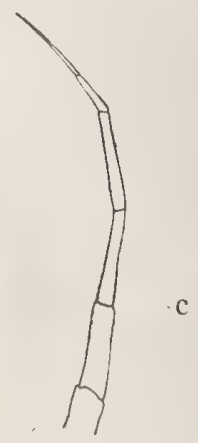
Nach Wolfenden. 
o. Rechtes 5. B. 4-gldr., mit Endläppchen, linkes 5. B. fast gleich so lang, 5-gldr., mit langem, spitzem, nadelförmigem Endgl. - L. ㅇ 2,6 mm, o 2,3 mm. Nord-Atlant. Ozean (w. von Irland).

Anm. Nach Wolfenden (1. c.) sieht die Art X. agilis Giesbr. sehr ähnlich.

\section{Xanthocalanus simplex Aurivillius.}

1898, X. s., Aurivillius, p. 33, f. 7.

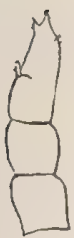

Fig. 72 . Xanthocalanus simplex.

5. B. Aurivillius.

9. Proximales Basalg1. des 1. B. mit einer kammähnlichen Borstenreihe, distales mit zwei getrennten Borstenbüscheln auf der Innenseite. 2. G1. des Enp. des 2. B. in der Seitenfläche 2 Stachelgruppen aus je 3 Stachelchen; 2. Gl. des Enp. des 3. B. mit 5 Stachelchen in der Oberfläche, 3. Gl. desselben Enp. mit 3 Stachelchen. 5. B. 3-gldr.; das Endgl. mit einem schwachen und unvollständigen Sutur in der Mitte; 1. Gl. nach innen nicht hervorstehend, nur mit einem kurzen Börstchen; 2. Gl. von der Länge des ersten, unbewaffnet; Endgl. ungefähr doppelt so lang wie das 2. Gl., mit einem kurzgefiederten Dörnchen nach außen in dem undeutlichen Mittensutur; am Ende zwei ungleich große ähnliche Dörnchen und nahe demselben an der Innenseite einige feine Börstchen. L. ? - o unbekannt.

Skagerak.

\section{Xanthocalanus fragilis Aurivillius.}

1898, X. f., Aurivillius, p. 32, f. 4-6.

9. 1. und 2. Gl. des Exp. des 1. B. auf der Innenseite lang und dicht behaart; Basalgl. desselben Exp. auf der Innenseite mit langen und dichten

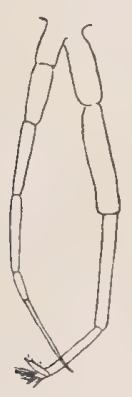

a

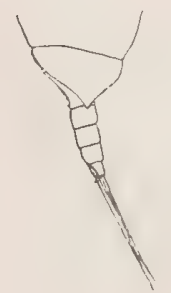

b

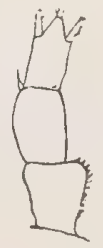

c

Fig. 73. Xanthocalanus fragilis.

a 5. B. $\sigma^{7}$, b hintere Körperteile $q$, lateral, c 5. B. $q$. Nach Aurivillius. 
Haaren in einer langen Kammreihe; 2. Gl. des Enp. in der Seitenfläche mit $4+3$ Stachelchen; 2. Gi. des Enp. des 3. B. mit 7 Stachelchen in der Oberfläche, 3. Gl. mit 4. 5. B. 3-gldr.; die Sutur zwischen dem 2. und 3. Gl. doch nicht deutlich quer über dem Fuße. 1. Gl. auf der Innenseite mit zwei zusammenlaufenden Stachelgruppen, beide ungefähr von derselben Länge bei adulti; sämtliche Stacheln etwa gleich groß. 2. Gl. mit 2 kurzen Außenranddornen am Ende. 3. Gl. in eine dreieckige Spitze auslaufend mit einem Fndstachel und einem Basalstachel an jeder Seite, sämtlich fast von derselben Länge. - o . 3. Gl. des Enp. des 4. B. mit 4 großen Stacheln an der Hinterfläche. 5. B. 5-gldr., das 4. Gl. des linken B. mit einem behaarten Höcker am Ende; 5. Gl. mit kurzen, feinen Börstchen; rechtes B. fast so lang wie das linke, 6-gldr. mit undeutlicher Sutur zwischen dem 5. und 6. Gl. und langem, nadelförmigem Endgl. - L. $2,5 \mathrm{~mm}$.

Skagerak.

\section{Xanthocalanus giesbrechti Thompson.}

1903, X. g., Thompson, p. 22, t. 4 f. $1-9$.

7. Rostrum fehlend; Vorderkörper 5-gldr., 1. Ant. 25-gldr. 5. B. 3-gldr., Innenrand des 1. und 2. Gl. bedornt; Endgl. das längste, rechts mit 4, links mit 3 Dornen. Furkaläste viereckig, fast 2 mal so lang wie breit. L. $3,5 \mathrm{~mm}$. - o unbekannt.

Nord-Atlant. Ozean.

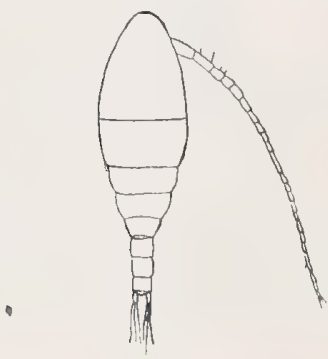

Fig. 74. Xanthocalanus giesbrechti.

a $q$ dorsal, b 2. B. $\bigcirc$, c 5. B. $\bigcirc$. Nach Thompson.

Anm. Obgleich die Beschreibung recht dürftig, die Figuren sehr mangelhaft sind, scheint doch aus beiden so viel hervorzugehen, daß die Thompson'sche Art mit keiner der oben beschriebenen Spezies identisch ist.

Anm. Von den folgenden Xanthocalanus-Arten sind auch die männlichen Tiere beschrieben worden: $X$. borealis, propinquus, fragilis, subagilis, typicus und agilis; die 2 letzten Arten gehören aber nicht zum nordischen Plankton. Farran (1905, 
p. 41 , t. 9 f. $7-9$; t. 11 f. 11) erwähnt ein dem Genus Xanthocalanus zugehörenden $\sigma^{*}$, das er aber mit Sicherheit zu keiner der bekannten Arten bringen konnte (Fig. 75 L. 2,2-2,27 mm). Es stimmte fast genau überein mit (?) Phaenna zetlandica $\sigma^{7}$ von Scott (1902, p. 453 t. 24 f. 5-7), das wahrscheinlich ebenfalls auf dieses Genus zu beziehen ist.

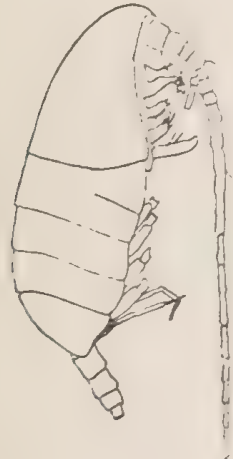

a
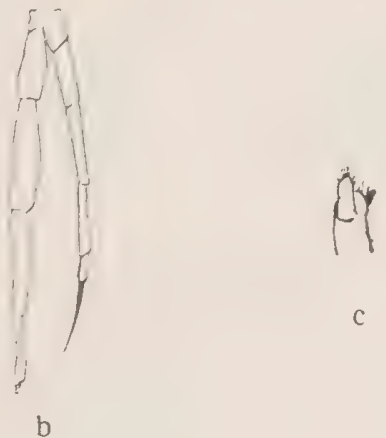

C

Fig. 75. Xanthocalanus sp. ơ.

a lateral, b 5. B., c Endgl. desselben. Nach Farran.

Onchocalanus G. O. Sars.

Verwandt mit Xanthocalanus, aber in folgenden Merkmalen abweichend; - Rostrum eine Chitinlamelle mit zwei Spitzen am Ende; 5. Lobus der 2. Maxille mit einer sehr starken, spitzen und nach vorne umgebogenen Klaue: Maxillipeden schlank; B. mit zahlreichen feinen Dörnchen an der Hinterfläche der Exp. und Enp. - Reife ठั ठั unbekannt.

\section{Onchocalanus chelifer Thompson.}

1903, Scolecithrix c., Thompson, p. 21, t. 5 f. $1-9$ (unreifes $\sigma^{7}$ ). 1905, Scolecithrix c., Farran, p. 36, t. 7 f. 18, 19 ( $($ ).

․ Stirn mit Crista; Rostrum viereckig mit 2 kurzen Spitzen; Vorderkörper 5-gldr., Kopf frei, die beiden letzten Thsegm. verschmolzen. Enp. der 2. Max. mit 5 sensorischen Anhängen. Die Extremitäten scheinen im allgemeinen denen des ơ ähnlich zu sein. 5. B. 3-gldr., nach dem Xanthocalanustypus gebaut, zu jeder Seite mit zerstreuten Haaren, Endgl. länger als die anderen Gl., nach dem Ende regelmäßig verjüngt, an der Spitze mit drei kurzen Dornen. - o'. (unreif!) Oberer Teil des Kopfes abgeplattet; Exp. der 2. Ant. 11/4 mal so lang wie das Enp. Endsäge der Exp. des 2.-4. B. nur wenig schmäler als das Endgl, mit einer distalen sekundären Zähnchenreihe. 5. B. asymmetrisch, Nord. Plankton. 
jederseits 4-gldr.; Endgl. mit 3 apikalen Dornen, von denen der mittlere der größte ist, und einer lateralen Dorn. L. q $7 \mathrm{~mm}$, ơ (unreif!) $6 \mathrm{~mm}$.

Nord-Atlant. Ozean.

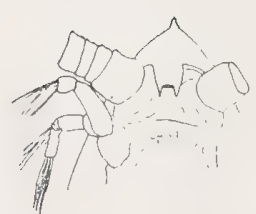

a

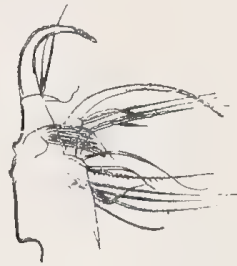

b

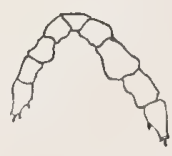

c

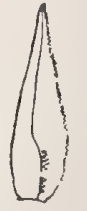

d

Fig. 76. Onchocalanus chelifer.

a Kopf $\bigcirc$, ventral, b 2. Maxille $\bigcirc$, c 5. B. eines unreifen $\sigma^{x}$, d Enddorn des Exp. des 2. B. $\sigma^{2}$. a und b nach Farran, c und d nach Thompson.

Anm. Thompson (1. c.) beschrieb das ơ dieser Art nach zwei unreifen Exemplaren. Farran (l. c.) fand zwei $q$, die er zu der Thompson'schen Art stelite.

\section{Brachycalanus Farran.}

Weicht von Xanthocalanus in folgenden Merkmalen ab: - . Rostrum breit und kurz, vorne quer abgestumpft (wahrscheinlich mit Fäden an den Ecken); 1. Antenne viel kürzer als der Vorderkörper, 24-gldr., an der Basis sehr dick, distal verjüngt; Kopf vom 1. Thsegm., 4. vom 5. Thsegm. gesondert. - $\sigma^{7}$ unbekannt.

\section{Brachycalanus atlanticus Wolfenden.}

1904, Xanthocalanus a., Wolfenden, p. 119, t. 9 f. 24, 25, 33.1905 , B. a., Farran, p. 41 , t. 10 f. $1-14$.

9. 1. Antenne nicht über das 3. Thsegm. reichend. Ansegm. stark verkürzt; Schwimmfüße mit Dörnchen bewehrt; 5. B. 3-gldr, 1. Gl. breiter als lang, der Innenrand mit dicht gestellten Dörnchen bewehrt; 2. Gl. kurz, 3. Gl. $2 \mathrm{mal}$ so lang wie breit, mit 2 starken End- und 2 Seitendornen; alle Segm. oberflächlich mit kurzen Dörnchen besetzt. L. $2-2,5 \mathrm{~mm}$. ơ unbekannt.

Nord-Atlant. Ozean (westl. von Irland). 


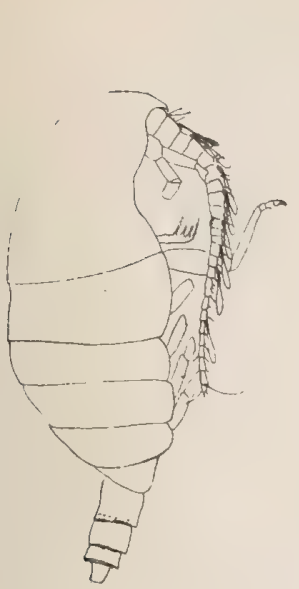

a

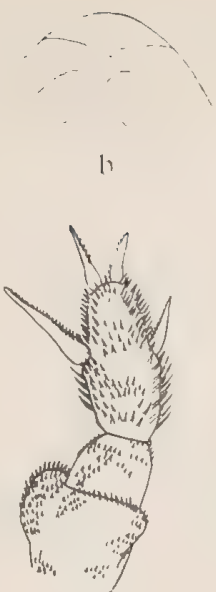

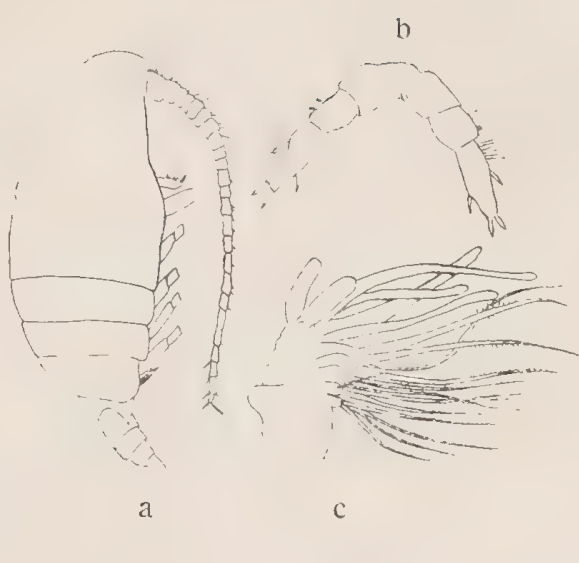

Fig. 78. Oöthrix bidentata.

Fig. 77. Brachycalanus atlanticus.

a $q$ lateral, b Kopf $q$, ventral, c 5. B. 0 .

a und b nach Farran, c nach Wolfenden.

a $?$ lateral, b 5. B. $q$, c 2. Maxille $q$. Nach Farran.

\section{Oöthrix Farran.}

Von Xanthocalanus in folgenden Merkmalen abweichend: $-q$. Rostrum eine breite, vorn quer abgestutzte Platte bildend; Kopf vom 1. Thsegm., 4. vom 5. Thsegm. getrennt; 5. Thsegm. beiderseits in zwei gleich große spitze Zacken endigend; die 2 Endschläuche der 2. Maxille kurz und wurstförmig; Schwimmfüße ohne Dörnchen an der Hinterseite.

\section{Oöthrix bidentata Farran.}

1905 , O. b., Farran, p. 42 , t. 10 f. $15-18$; t. 11 f. $1-10$.

․ 1. Antenne 24-gldr, bis an das 5. Thsegm. reichend; 5. B. 3-gldr., 2. und 3. Gl. am Außenrande behaart, 3. Gl. lang und schmal, mit 2 Endund 2 Randdornen. L. $3 \mathrm{~mm}$. - $\sigma^{7}$. Unreife ot sind wahrscheinlich von Farran beobachtet worden; das unentwickelte symmetrisch gebildete 5 . B. be$\mathrm{saß}$ 2-gldr. Exp. mit 3 terminalen und 1 marginalen Dorn am Endgl. und kurze 1-gldr. Enp. L. $3,35 \mathrm{~mm}$.

Nord-Atl. Ozean.

Undinella G. O. Sars.

Kopf vom 1. Thsegm. getrennt; Rostrum groß, nach unten gebogen, mit zwei kurzen Fäden; 5. Thsegm. mit dem vorletzten verschmolzen, beim $\bigcirc$ nach hinten verlängert; Abdomen beim $q$ 4-, beim of 5 -gldr., Ansegm. in beiden 
Geschlechtern stark verkürzt. 1. Antenne beim $q$ 24-, beim o 23-gldr.; Exp. der 2. Antenne viel länger als das Enp., 6-gldr.; Mandibel schlank mit relativ kleinem Exp.; Enp. der 1. Maxille gegliedert und vom 2. Gl. des Basp. getrennt, Außenrandlobus klein. 2. Maxille mit relativ kurzen Borsten, die distalen kaum in sensorische Anhänge umgeformt. Maxillipeden sehr schlank. Mundgliedmaßen des or nicht umgeformt. Exp. des 1.-4. B. 3-gldr.; Enp. des 1. und 2. B. 1-, des 3. und 4. B. 2-gldr.; Hinterfläche unbedornt. 5. B. vorhanden, beim o symmetrisch, 3-gldr, beim of asymmetrisch, groß, linkes B. mit langem, griffelförmigem Enp.

\section{Undinella oblonga G. O. Sars.}

1900, U. O., Sars, p. 52, t. 12, 13.

. Letztes Thsegm. nach hinten breit-dreieckig verlängert, fast bis an die Genitalöffnung reichend; Hinterkörper halb so lang wie der Vorderkörper, die mittleren Abdsegm. fast so lang wie das Gensegm. 1. Antenne etwas länger als der Vorderkörper. 5. B. 3-gldr. (mit verschmolzenen basalen Gl.), 2. Gl. viel schmäler als das Basalgl., beide völlig glatt; 3. Gl. fast so lang
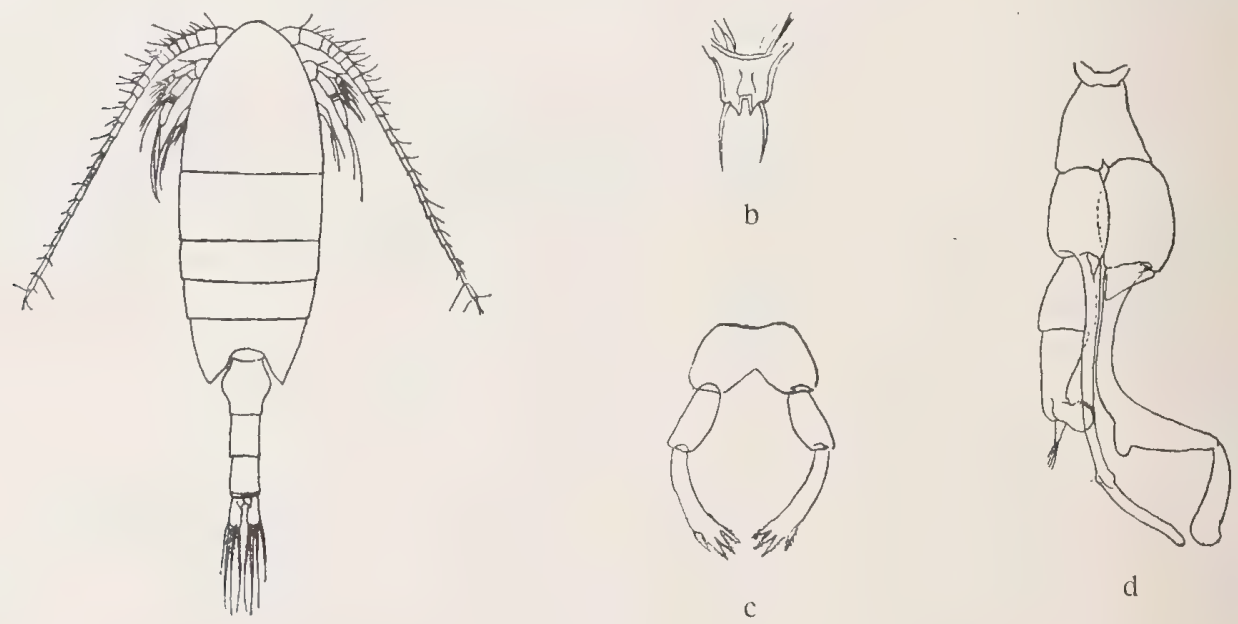

Fig. 79. Undinella oblonga.

a $q$ dorsal, b Rostrum $q$, c 5. B. $q$, d 5. B. 万. Nach Sars.

wie die beiden proximalen Gl. zusammen, schmal, nach dem Ende etwas verbreitert und dort mit 4 kurzen, fingerförmigen, fein gezähnten Dornen versehen, von denen der äußerste ein wenig unterhalb des distalen Randes eingelenkt ist, die drei terminalen aber nicht mit dem Gl. artikulieren. - o ${ }^{7}$. 1. Antenne die Mitte des Abd. erreichend; 5. B. bis an die Furka reichend, mit verschmolzenen 1. Basalgl.; rechtes B. mit 2-gldr. Exp., dessen 1. Gl. stark ge- 
bogen und $2 \mathrm{mal}$ so lang wie das keulenförmige Endgl. ist; Exp. des linken B. 3-gldr.; 2. Gl. distal am Innenrande mit einem dicken Vorsprung; 3. Gl. klein mit einem Haarbüschel an der Spitze; Enp. des linken B. schmal, fast zweimal so lang wie das Exp. und das Ende des rechten B. erreichend. L. ㅇ $3 \mathrm{~mm}$, o $2,5 \mathrm{~mm}$.

Arktischer Ozean.

\section{Scolecithrix.}

Q. Vorderrumpf ellipsoidisch; Kopf mit dem 1. Thsegm. verschmolzen; 2 Rostralfäden; Abd. 4-gldr.; Ansegm. gewöhnlich kurz; 1. Ant. 19-24-gldr., die beiden Endgl. verschmolzen oder getrennt; 2. Ant. mit 6-gldr. Exp.; Kauteile der Mandibel und der 1. Maxille ziemlich schwach; Enp. der 1. Max. meistens ungegliedert und mit dem 2. Gl. des Basp. verschmolzen. Distale Borsten der 2. Maxille in dünnhäutige Schläuche verwandelt, die entweder wurmförmig sind, z. T. auch in Pinsel endigen oder Keulenform haben können; diese Schläuche sind beim $q$ und $\sigma^{7}$ in der Mehrzahl vorhanden. Exp. des 1.-4. B. 3-, Enp. des 1. B. 1-, des 2. B. 2-, des 3. und 4. B. 3-gldr.; die Äste mit oft zahlreichen Stacheln und Spitzen besetzt. 5. B. 1-ästig, 1- bis 3-gldr., selten fehlend. - $\sigma^{7}$. Abd. 5-gldr. Kopfgliedmaßen denen des $q$ meistens ähnlich, zuweilen in eigentümlicher Weise umgeformt. 5. B. beiderseits 1-ästig (rechtes B. zuweilen sehr kurz) oder links 2-, rechts 1-ästig oder beiderseits 2-ästig.

\section{$\left.Q^{1}\right)$}

1. 5. B. fehlt

S. danae

5. B. vorhanden, meistens symmetrisch

2. 5. B. winzig, asymmetrisch

S. bradyi

5. B. symmetrisch

3. 5. B. 1-gldr., blattförmig

5. B. 2- bis 3-gldr.

4. Von den beiden Innenrandborsten des 5. B. entspringt die proximale oberhalb der Mitte des Randes, das Ende des Fußes weit überragend; Vorderkopf in seitlicher Ansicht sehr hoch gewölbt, breit abgestumpft S. minor Die proximale Innenrandborste des 5. B. nicht hinter der Mitte eingepflanzt, das Ende des Blattes nicht überragend; Vorderkopf in lateraler Ansicht nach vorne verjüngt

5. 1. Gl. des Exp. des 1. B. mit Randdorn; Randdorn des 1. Gl. des Exp. des 2. B. nicht hakig verlängert S. ovata 1. Gl. des Exp. des 1. B. ohne Randdorn; Randdorn des 1. Gl. des Exp. des 2. B. hakig verlängert

S. dentata (siehe auch S. similis Wolfenden).

1) $\mathrm{Da}$ die Männchen von vielen Arten nicht bekannt sind, wird nur eine $\mathrm{Be}-$ stimmungstabelle für die Weibchen gegeben. 
6. 5. B. 3-gldr.; Stirn mit Crista

S. frontalis

5. B. 2-gldr.; Stirn mit oder ohne Crista

7. 5. B. mit 3 Dornen

5. B. mit 2 Dornen

8. Stirn mit Crista

Stirn ohne Crista

9. Enddorn des 5. B. länger als der Innenranddorn; 2. Gl. des Enp. des 2. B. mit 2 großen proximalen und drei kleineren distalen Dörnchen an der Hinterfläche

S. echinata

Enddorn des 5. B. kürzer als der Innentanddorn; 2. Gl. des Enp. des 2. B. mit 2 Gruppen von mehreren Dörnchen an der Hinterfläche $S$. brevicornis (Siehe auch $S$. major.)

10. Stirn mit Crista

S. securifrons

Stirn "ohne Crista

11. 1. Ant. den Vorderkörper nicht überragend

S. auropecten

1. Ant. wenigstens das Ende der Furka erreichend oder dasselbe überragend

12. Maxillipede ohne sensorischen Anhang am 1. Gl.

S. obtusifrons

Maxillipede mit pinselförmigem, sensorischen Anhang

$S$. atlanticus.

\section{Scolecithrix danae Lubbock.}

1856, Undina d., Lubbock, p. 15, t. 9. 1883, S. d., Brady, p. 57, t. 17. 1892 , S. d., Giesbrecht, p. 265 , t. 13 , f. $4,9,14,17$; t. 37 f. 6.1898 , S. d., Giesbr. und Schmeil, p. 42.
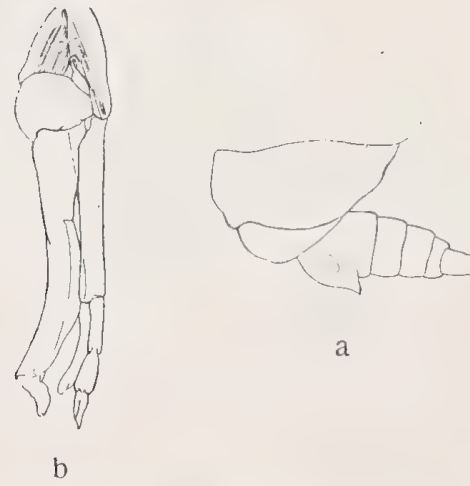

a

Fig. 80. Scolecithrix danae. a Hinterer Teil des $Q$, lateral, b 5. B. o'. Nach Giesbrecht.
Q. Die beiden letzten Thsegm. getrennt; die mittleren beiden Abdsegm. breiter als lang; Ansegm. kurz; Gensegm. mit ventralem, schaufelförmigem Vorsprung. 1. Ant. mit verschmolzenen Endgl. überragt den Hinterrand des Vorderrumpfes nur wenig. Exp. der 2. Ant. $1 \frac{2}{7}$ mal so lang wie das Enp. 2. Gl. des Basp. der 1. Max. mit 5, Enp. mit 6, Exp. mit 5 Borsten. 2. Max. mit wurmförmigen Schläuchen. 1. G1. des Basp. des 4. B. ohne Innenrandborste. 1. Gl. des Exp. des 1. B. mit Randdorn. 5. B. fehit. - . . Mundteile nicht verkümmert. 5. B. links 2-, rechts 1-ästig; Endgl. des rechten 5. B. ganz kurz. - L. o $2,2 \mathrm{~mm}$, o $2-2,15 \mathrm{~mm}$.

Mittelmeer, Atl., Ind. und Pacif. Ozean. 


\section{Scolecithrix bradyi Giesbrecht.}

1888, S. b., Giesbrecht, p. 337. 1892, S. b., Giesbrecht, p. 266, t. 4 f. 7 ; t. 13 f. 1, 3, 7, 11, 21, 28; t. 37 f. 1, 2, 9. 1898, S. b., Giesbr. und Schmeil, p. 42 .

Q. Die beiden letzten Thsegm. unvollkommen getrennt; Lateralplatten des letzten rechts länger als links. Mittlere Abdsegm. breiter als lang, Ansegm. so lang wie die vorhergehenden Segm.; Gensegm. asymmetrisch, Furka doppelt so lang wie breit. 1. Ant. mit verschmolzenen Endgl., erreicht den Hinterrand
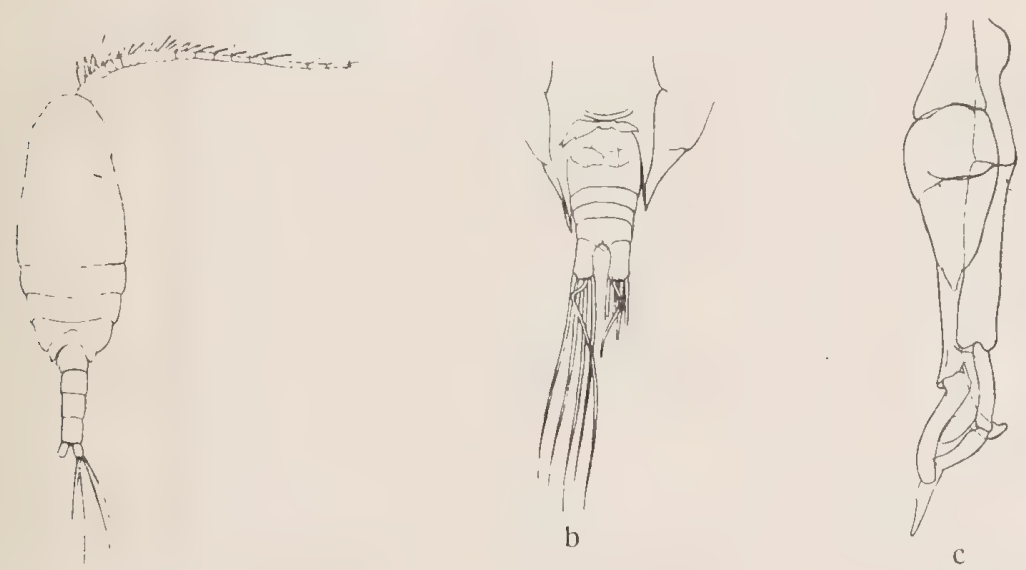

Fig. 81. Scolecithrix bradyi.

a $\sigma^{\nearrow}$ dorsal, b hinterer Teil des $q$, ventral, c 5. B. $\sigma^{\rtimes}$. Nach Giesbrecht.

des Thorax nicht. Exp. der 2. Ant. länger als das Enp. 2. G1. des Basp. der 1. Max. mit 5, Enp. mit 6, Exp. mit 4 Borsten. 2. Maxille mit wurmförmigen Schläuchen. 1. Gl. des Basp. des 4. B. ohne Innenrandborste; 1. Gl. des Exp. des 1. B. ohne Randdorn. 5. B. winzig, asymmetrisch. - o. Mundteile nicht verkümmert. 5. B. links 2-, rechts 1-ästig; Endgl. des rechten 5. B. gegabelt. - L. ㅇ $1,1-1,3 \mathrm{~mm}$, of 1,35--1,42 $\mathrm{mm}$.

Mittelmeer, Atl., Ind. und Pacif. Ozean.

\section{Scolecithrix dentata Giesbrecht.}

1892 , S. d., Giesbrecht, p. 266, t. 13 f. $12,20,33$; t. 37 f. $13,14.1898$, S. d., Giesbr. und Schmeil, p. 44.

q. Die beiden letzten Thsegm. verschmolzen bis auf jederseits eine Kerbe am Lateralrande, die beiden mittleren Abdsegm. wenigstens so lang wie breit und viel länger als das Ansegm. 1. Antenne mit verschmolzenen Endgl, überragt den Hinterrand des Thorax nur wenig. Exp. der 2. Ant. länger als 
das Enp. 2. Gl. des Basp. der 1. Maxille mit 5, Enp. mit 7, Exp. mit 7 Borsten. 2. Maxille mit wurmförmigen Schläuchen. 1. Gl. des Baṣp. des 4. B. mit kurzer,

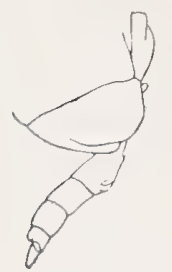

a

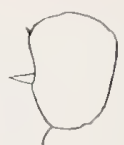

b

Fig. 82. Scolecithrix dentata.

a Hinterer Teil des $\bigcirc$, lateral,

b 5. B. Q. Nach Giesbrecht.

reicht. L. 1,3-1,45 mm. - o unbekannt.

Mittelmeer; Nord-Atlant. Ozean.

\section{Scolecithrix ovata Farran.}

1905, S. o., Farran, p. 37 , t. 6 f. $13-18$; t. 7 f. $1-5$.

Verwandt mit dentata: - $\bigcirc$. Vorderkörper regelmäßig oval in dorsaler und seitlicher Ansicht; die beiden letzten Thsegm. verschmolzen; Abdomen

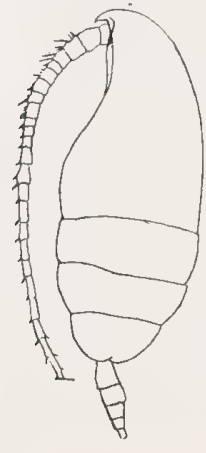

a

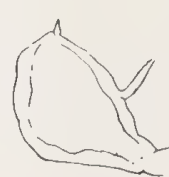

b

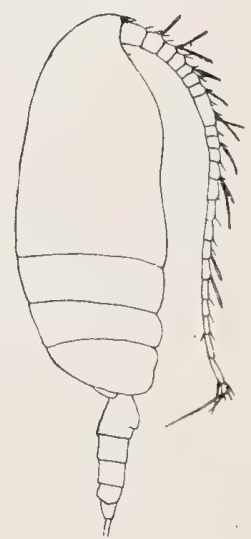

Fig. 83. Scolecithrix ovata.

a 9 lateral, b 5. B. $\bigcirc$. Nach Farran.

Fig. 84. Scolecithrix similis Wollenden. a \& lateral. Nach Wolfenden.

schlank, ungefähr $1 / 4 \mathrm{mal}$ so lang wie der Vorderkörper. 1. Ant. 23-gldr., bis an das 2. Abdsegm. reichend; Exp. der 2. Ant. $1 \frac{1}{3}$ mal so lang wie.das Enp.; Exp. der 1. Maxille mit 5 (?) Borsten; 2. Maxille mit wurmförmigen Schläuchen. 
1. G1. des Basp. des 4. B. mit kurzer, nackter Innenrandborste. 1. Gl. des Exp. des 1. B. mit Randdorn; Randdorn des 1. Gl. des Exp. des 2. B. nicht hakig verlängert; 5. B. 1-gldr., breit-oval mit schmaler Basis; Innenrand mit zwei weit auseinander stehenden Dornen, die obere ganz kurz, die untere länger, aber den distalen Rand des B. nicht erreichend. L. ㅇ 2,3 mm. ot unbekannt.

Nord-Atl. Ozean.

5. Scolecithrix similis Wolfenden (non Scott!).

1904, S. s., Wolfenden, p. 119, t. 9 f. $5,6$.

Verwandt mit dentata: - $\bigcirc$. Vorderkörper von der Seite gesehen oval, nach vorne verschmälert; die beiden letzten Thsegm. verschmolzen; 5 . Th. abgerundet. 1. Antenne das Ende des Vorderkörpers kaum überragend. 5. B. 1 -gldr., 2 mal so lang wie breit, mit sehr kurzem Innenranddorn unterhalb der Spitze und einem längeren Dorn gleich unterhalb der Mitte des Innenrandes, der das Ende des B. nicht erreicht. L. $q$ 1,5 $\mathrm{mm}$. - o unbekannt.

Nord-Atlant. Ozean, Faröerkanal.

\section{Scolecithrix minor Brady.}

1883, S. m., Brady, p. 58 , t. 16 f. 15 , 16 ; t. 18 f. $1-5$. 1898, S. m., Giesbr. und Schmeil, p. 46. 1903, Scolecithricella m., Sars, p. 55, t. 37, 38.

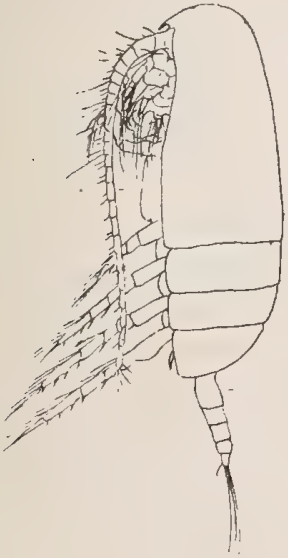

a

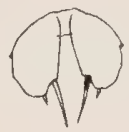

b)

Fig. 85.

Scolecithrix minor.

a q lateral, b 5. B. q, c 5. B. 7 .

Nach Sars.

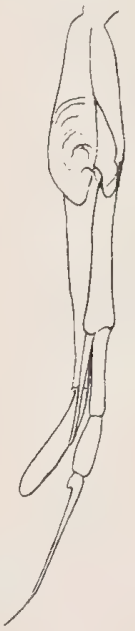

q. Vorderkörper von der Seite gesehen nach vorne fast nicht verschmälert, hoch gewölbt; die beiden letzten Thsegm. verschmolzen; 5. Thsegm. stumpfeckig abgerundet. 1. Antenne mit verschmolzenen Endgl., ungefähr so lang 
wie der Vorderkörper. Exp. der 2. Antenne länger als das Enp.; 2. Maxille mit wurmförmigen Schläuchen. 1. Gl. des Basp. des 4. B. mit kurzer, nackter Randborste; 2. Gl. des Basp. des 3. und 4. B. mit spitz endendem Innenrande; 1. G1. des Exp. des 1. B. ganz ohne, des 2. B. mit hakigem Randdorn, der länger als die übrigen Randdorne ist; 5. B. 1-gldr., breit oval, am Innenrande unterhalb der Spitze mit zwei ungleichen, ziemlich dicht nebeneinander gestellten Dornen, von denen der distale sehr kurz, der proximale etwa halb so lang wie das Blatt ist und das Ende desselben weit überragt. - $\sigma^{7}$. 5. B. schlank, über das Ende der Furka hinausreichend, links 2-, rechts 1-ästig; Endgl. des rechten 5. B. ein dünnes und langes Blatt, des linken pfriemenförmig. L. क 1,4, o $1,4 \mathrm{~mm}$.

Ind. Ozean; Golf von Guinea, Nord-Atl. Ozean, Nordmeer; ? Polarmeer.

\section{Scolecithrix römeri Mrázek.}

1902, S. r., Mrázek, p. 513, t. 6, Textf. 11-13.

Nach der Beschreibung und den Abbildungen Mrázeks zu urteilen, scheint mir diese Art sehr nahe verwandt, wenn nicht identisch mit S. minor. Das 5. B. des $q$ weicht in sehr geringem Grade von demjenigen der letztgenannten Art $a b$, während das 5 . B. des $\sigma^{7}$ nur relativ etwas länger zu sein scheint. L. क $1,6, \sigma^{7} 1,5 \mathrm{~mm}$.

Polarmeer.

\section{Scolecithrix auropecten Giesbrecht.}

1892, S. a., Giesbrecht, p. 266, t. 13 f. $8,18,22,27$; t. 37 f. $3,10$. 1898, S. a., Giesbr. und Schmeil, p. 45, f. 7.

-. Die beiden letzten Thsegm. verschmolzen; Lateralecken des letzten Thsegm. in einen stumpfen Fortsatz auslaufend. Die letzten 3 Abdsegm. breiter als lang und ungefähr gleich lang. 1. Ant. mit verschmolzenen Endgl., reicht nicht bis zum Hinterrande des Vorderrumpfes. Exp. der 2. Ant. kürzer und dünner als das Enp. 2. Gl. des Basp. der 1. Maxille mit 6, das gut ausgebildete Enp. mit 9, das Exp. mit 10 Borsten. 2. Maxille mit wurmförmigen Schläuchen. 1. Gl. des Basp. des 4. B. mit gefiederter Innenrandborste; Innenrand des 2. G1. des Basp. des 2.-4. B. endigt in einen runden Vorsprung. Randdorn des 1. Gl. des Exp. des 1. B. den distalen Rand des folgenden Gl. überragend; Randdorne der beiden ersten Gl. des Exp. des 2. B. länger als die des 3. Gl.; Endgl. der Enp. überall ohne apikale Spitze am Innenrande. 5. B. 2-gldr, mit einer apikalen Borste und einer längeren proximalen davor. L. $q 1,8 \mathrm{~mm}$. - $\sigma^{\pi}$ unbekannt.

Mittelmeer, Nord-Atlant. Ozean; Rotes Meer. 


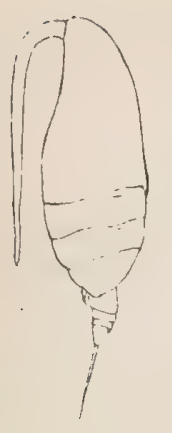

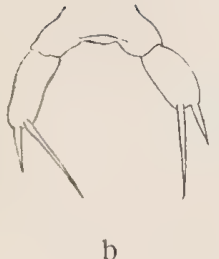

b
Fig. 86. Scolecithrix auropecten. a $\bigcirc$ lateral, b 5. B. ๆ. Nach Giesbrecht.

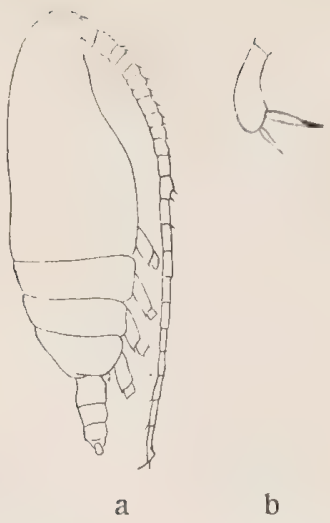

Fig. 87. Scolecithrix obtusifrons. a lateral, b 5. B. Nach Farran.

9. Scolecithrix obtusifrons G. O. Sars.

1905, Amallophora o., Sars, Nr. 26, p. 22. 1905, S. emarginata, Farran, p. 36 , t. 7 f. $6-17$.

․ Körper nach vorne zu nur wenig verschmälert; die beiden letzten Thsegm. unvollkommen verschmolzen, sodaß eine Kerbe im Lateralrande übrig bleibt; Abdomen ungefähr 1/4 mal so lang wie der Vorderkörper; Ansegm. sehr kurz; Gensegm. nur wenig länger als breit; 3. und 4. Abdsegm. etwas breiter als lang. 1. Antenne 23-gldr., das Ende der Furka um ein weniges überragend. Exp. der 2. Ant. etwas länger als das Enp. 2. Maxille mit kurzen, keulenförmigen (?), sensorischen Anhängen. Maxilliped ohne sensorischen Anhang am 1. Gl. 1. Gl. des Exp. des 1. B. mit kurzem Randdorn. 5. B. 2-gldr. mit innerer und apikaler Borste, die erstere ungefähr 2 mal so lang wie die letztere. L. † 4,3 mm. - o unbekannt.

Nord-Atlant. Ozean.

\section{Scolecithrix atlanticus Wolfenden.}

1904, S. a., Wolfenden, p. 120.

‥ 1. Antenne 23-gldr., länger als der Körper.. 2. Antenne mit nahezu gleich langen Ästen. 2. Maxille mit pinsel- und wurmförmigen Anhängen. Maxilliped mit pinselförmigem, sensorischem Anhang. 5. B. 2-gldr., das distale das längere, mit gerundetem Ende, mit kurzer, kräftiger, apikaler und dicker, 2 mal längerer Borste am Innenrande. L. † 3,95 mm. - o unbekannt.

Nord-Atlant. Ozean. 


\section{Scolecithrix securifrons Th. Scott.}

1893, S. s., Th. Scott, p. 47 , t. 4 f. $40-56$; t. 5 f. 1. 1898, S. s., Giesbr. und Schmeil, p. 49. 1904, S. s., Wolfenden, p. 120, t. 9 f. 12-15. 1905, Scottocalanus s., Sars, Nr. 40, p. 1.

ๆ. Stirn mit ziemlich hoher Crista. Die beiden letzten Thsegm. getrennt; das letzte mit spitzen, postero-lateralen Ecken. 1. Ant. 23-gldr., länger als der Körper. Exp. der.2. Ant. 11/2 mal so lang wie das Enp. 2. Maxille mit wurmförmigen Schläuchen. 1. Gl. des Basp. des 4. B. mit gefiederter Innenrand-
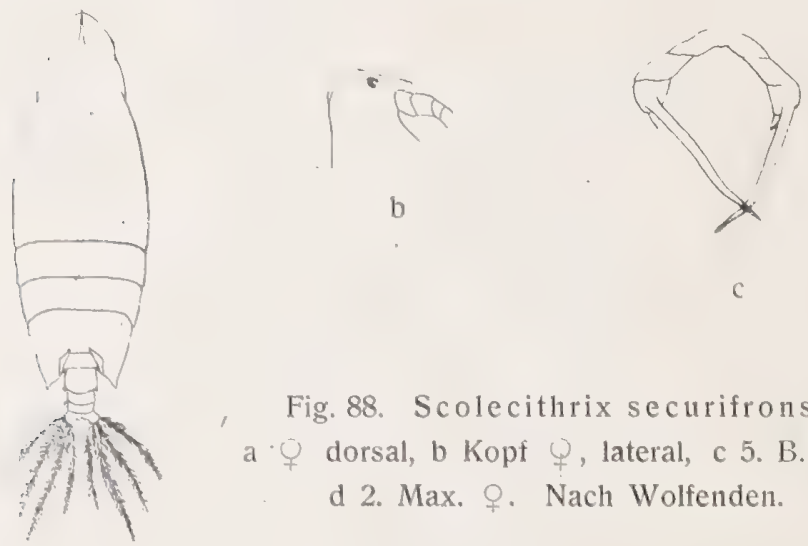

Fig. 88. Scolecithrix securifrons. a. dorsal, b Kopf $q$, lateral, c 5. B. . d 2. Max. ‥ Nach Wolfenden.

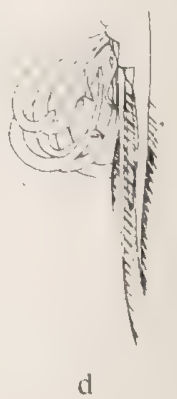

a

borste; 1. Gl. des Exp. des 1. B. mit kleinem Außenranddorn. 5. B. Ź-gldr., mit einer krummen Borste und einem kleinen Dorn am Ende. - $\sigma^{\pi}$. Letztes Thsegm. mit runden Ecken. 1. Ant. 19-gldr. Mundteile nicht verkümmert. Beide B. des 5. Paares 2-ästig; rechtes Enp. lang, gekrümmt; linkes Enp. sehr klein; linkes Exp. 1-gidr. - L. ca. 4 mm.

Atlant. Ozean (Golf von Guinea bis Faröerkanal).

Ammerkung. Nach Wolfenden gleicht das von ihm erwähnte Tier der S. securifrons Scott genau, weicht aber durch die Gliederung der 1. Antenne, sowie. durch das Vorkommen von geknöpften sensorischen Anhängen, welche der Scott'schen Spezies zu fehlen scheinen, $a b$.

12. Scolecithrix magna Th. Scott.

1893, S. (Amallophora) m., Th. Scott, p. 55, t. 4 f. $5-9$. 1895, S. cristata, Giesbrecht, p. 252 , t. 2 f. $6-8$; t. 3 f. $1-5$. 1898, S. cristata, Giesbr. und Schmeil, p. 48, f. 8. 1900, Scaphocalanus acrocephalus, Sars, p. 36, t. 7, 8, 9. 1903, Amallophora m., Sars, p. 51, t. 34, 35. ? 1903, S. cristata, Thompson, p. 21 , t. 3 f. $1-5$.

o. Stirn mit schmaler, ziemlich langer Crista. Die beiden letzten Thsegm. verschmolzen; die beiden mittleren Abdsegm. ungefähr gleich lang, doppelt 
so lang wie das Ansegm. Furka kurz, so lang wie breit. 1. Antenne 22-gldr., mit verschmolzenen Endgl., überragt den Hinterrand des Vorderrumpfes wenig. Exp. der 2. Ant. ca. $5 / 7$ so lang wie das Enp. 2. Gl. des Basp. der 1. Max. mit 4, Enp. mit 9, Exp. mit 7 Borsten. 2. Maxille mit z. T. geknöpften Schläuchen. 1. Gl. des Basp. des 4. B. mit gefiederter Innenrandborste; 1. Gl. des Exp. des 1. B. ohne, 2. Gl. mit winzigem, 3. Gl. mit kräftigem Randdorn;

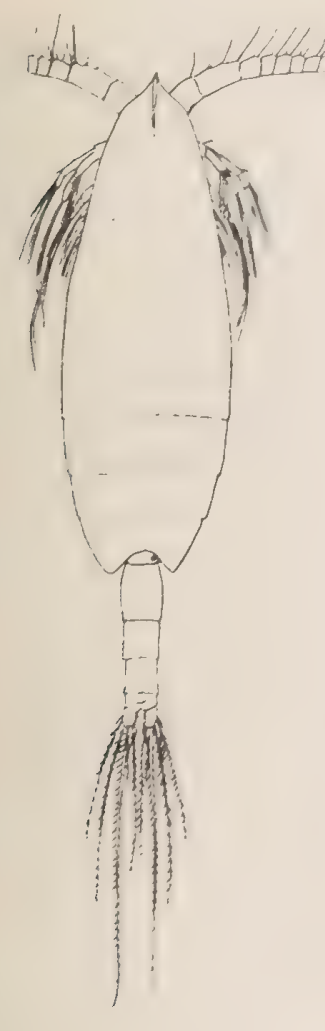

a

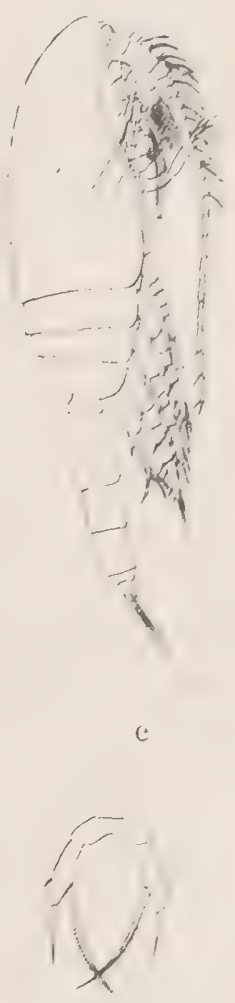

Fig. 89. Scolecithrix magna.

a $q$ dorsal, b Kopf $q$, lateral, c 5. B. ๆ, d Enp. 4. B., e đ lateral, f 5. B. ठँ.

Randdorn des 2. G1. des Exp. des 2. B. etwas länger als der (gekrümmte) des 1. und der des 3. Gl.; letztes Gl. des Enp. der B. nirgends mit Spitze am Ende des Außenrandes. 5. B. 2-gldr., mit 3 Borsten am Endgl., die am Außenrande ist länger als die an der Spitze; die am Innenrande ist winzig. - ${ }^{7}$. Stirn ohne Crista; 5. B. kaum über die Mitte des Abdomens reichend; linkes 5. B. mit schmal zylindrischem 2. Basalgl. und 2 fast gleich langen, 3-gldr. Ästen; rechtes 5. B. mit pfriemenförmigem Enp., das so lang wie das 2. Basp. ist, und mit 3-gldr. Exp. - L. ๆ 4,5-5 mm, o $4,5 \mathrm{~mm}$.

Pacif. Ozean; Atlant. Ozean (Golf von Guinea bis Nordmeer); Polarmeer. 


\section{Scolecithrix brevicornis Sars.}

1900, S. b., Sars, p. 46, t. 10. ? 1902, S. b., Th. Scott, t. 25 f. 1 , $2\left(\sigma^{\top}\right) .1903$, Amallophora b., Sars, p. 53, t. 36.

q. Kopf ohne Crista; Vorderkörper $2 \frac{1}{2}$ mal so lang wie das Abdomen. Die beiden mittleren Abdsegm. weniger als doppelt so lang wie das Ansegm.;
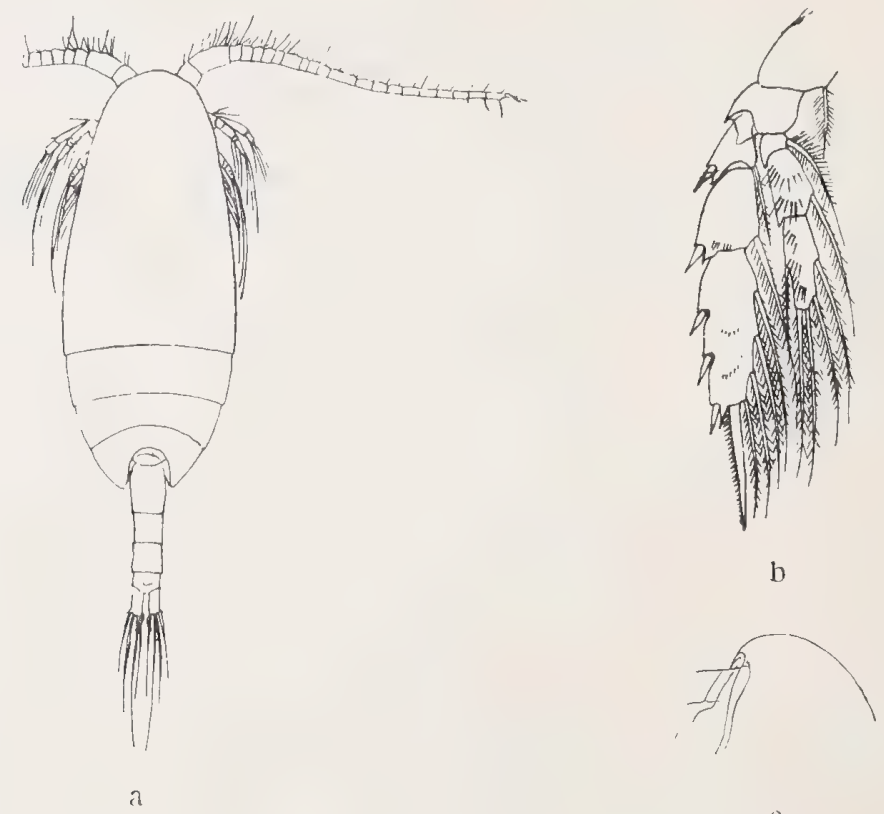

Fig. 90. Scolecithrix brevicornis.

a

Furka 2 mal so lang wie breit. 1. Antenne 22-gldr.; kürzer als der Vorderrumpf; Mundgliedmaßen und $1-4$. B. denen von S. magna sehr. ähnlich; 5. B. wie bei S. magna, nur kleiner. L. ca. $2 \mathrm{~mm}$. - o unbekannt (S. oben).

Shetlandinseln, Nordmeer, Polarmeer.

Anmerkung. Wolfenden (1904, p. 137) fand im Nord-Atlant. Ozean eine ScolecithrixArt, die mit Ausnahme der Gestalt der sensorischen Anhänge an den 2. Maxillen und den Maxillipeden übrigens in jeder Hinsicht genau mit S. brevicornis übereinstimmte. Die sensorischen Anhänge waren nicht amalliform, sondern borsten- (pinsel-) förmig.

\section{Scolecithrix echinata Farran.}

1905, S. e., Farran, p. 37 , t. 4 f. $15-18$; t. 5 f. $12-17$.

Verwandt mit brevicornis: - $\odot$. Kopf ohne Crista. Die beiden mittleren Abdsegm. nur wenig länger als das Ansegm.; Furka 11/2 mal so lang 
wie breit. 1. Antenne das Ende des Vorderrumpfes nicht ganz erreichend; 22-gldr.; 2. Antenne wie bei S. magna, mit etwas längerem Enp.; Enp. der 1. Maxille mit 7 Borsten. 1.-4. B. wie bei brevicornis, aber mit abweichender Bedornung der Enp. des 2-4. B. 5. B. 2-gldr., in Gestalt mit der von S. brevicornis übereinstimmend, aber mit längerem Apikaldorn, den die innere Borste nicht überragt. L. $\uparrow 1,92 \mathrm{~mm}$. - o unbekannt.

\section{Nord-Atlant. Ozean.}

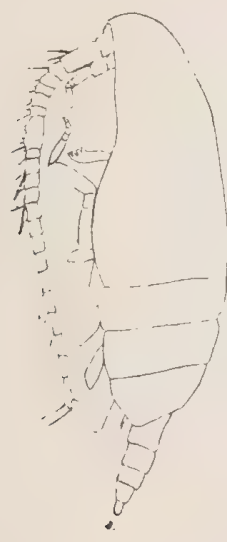

a

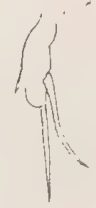

b

Fig. 91. Scolecithrix echinata.

a q lateral, b 5. B. q. Nach Farran.

Fig. 92. Scolecithrix major. 5. B. $\%$. Nach Th. Scott.

\section{Scolecithrix major Th. Scott.}

1893, S. m., Th. Scott, p. 52, t. 3 f. $24-26$; t. 5 f. 44,45 . 1898, S. m., Giesbr. und Schmeil, p. 47.

․ Die beiden letzten Thsegm. verschmolzen; mittlere Abdsegm. etwa so lang wie breit. 2. Maxille mit wurmförmigen Schläuchen. 5. B. 2-gldr., dem von cristata ähnlich. L. † $3 \mathrm{~mm}$. - o unbekannt.

Golf von Guinea, Nord-Atl. Ozean.

\section{Scolecithrix frontalis Giesbrecht.}

1895, Lophothrix f., Giesbrecht, p. 254 , t. 2 f. $1-5,9-12.1898$, S. f., Giesbr. und Schmeil, p. 49, f. 10.

૧. Stirn verlängert, mit schmaler Crista; die beiden letzten Thsegm. verschmolzen. Die beiden mittleren Abdsegm. breiter als lang, das letzte wenig kürzer als das vorletzte. 1. Antenne 24-gldr., mit getrennten Endgl., ungefähr das Rumpfende erreichend. Exp. der 2. Antenne etwa so lang wie 
das Enp., mit verkürzten Endgl.; 2. Gl. des Basp. der 1. Maxille mit 5, Enp. mit 8, Exp. mit 9 Borsten; 2. Maxille mit pinselförmigen Schläuchen. 1. Gl. des Basp. des 4. B. mit gefiederter Innenrandborste; 1. Gl. des Exp. des 1. B. ohne Randdorn. 5. B. 3-gldr., Endgl. mit 3 dicken Borsten, kaum länger als die anderen Gl. L. $q 6,6 \mathrm{~mm}$. - o unbekannt.

Pacif. und Nord-Atlant. Ozean.

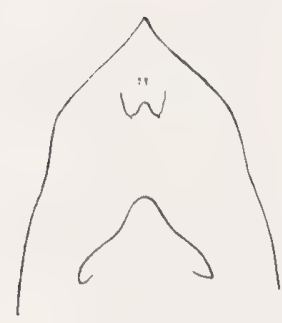

a

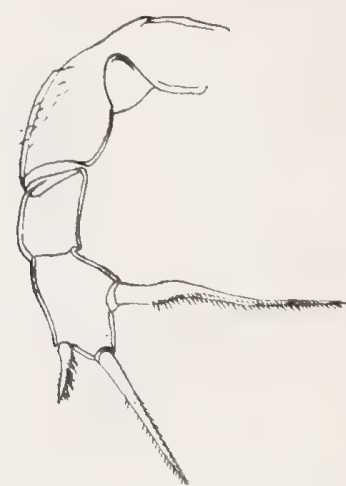

b

Fig. 93.

Scolecithrix frontalis. a Kopf 9 , ventral, b 5. B. 9 . Nach Giesbrecht.
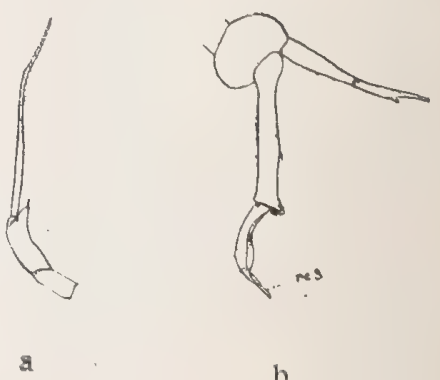

b
Fig. 94. Scolecithrix persecans. a 5. B. ?,

b rechtes 5. B. $\sigma^{\top}$. Nach Esterly.

\section{Scolecithrix persecans Giesbrecht.}

1895 , S. p., Giesbrecht, p. 253, t. 3 f. $6-12$. 1898, S. p., Giesbr. und Schmeil, p. 48, f. 9 . 1905, S. p., Esterly, p. 166, f. 28 a-e.

․ Kopf mit ziemlich hoher Crista. Die beiden letzten Thsegm. verschmolzen. Abdomen symmetrisch. 1. Antenne 23-gldr., bis an das Ende der Furka reichend; Exp. der 2. Antenne wenigstens 11/4 mal so lang wie das Enp.; 2. Gl. des Basp. der 1. Maxille mit 5, Enp. mit 7, Exp. mit 8 Borsten; 2. Maxille mit z. T. pinselförmigen Schläuchen. 1. Gl. des Basp. des 4. B. mit gefiederter Innenrandborste; Mitte des Außenrandes des 1. Gl. des Basp. des 1. und 2. B. mit einem Zähnchen; Außenrand des 2. Gl. des Basp. des 2.-4. B. mit zahnförmigem Vorsprung; Außenranddorn der beiden proximalen Gl. des Exp. des 1. B. kürzer und dünner als der des 3. G1.; 2. Gl. des Enp. des 3. B. mit 3 Dornen an der Hinterseite; Enp. des 4. B. ohne Dörnchen an der Hinterseite; Vorderseite des Exp. des 2.-4. B. ohne Dörnchen; Enddorn des Exp. des 3. B. am Grunde eingekerbt. 5. B. symmetrisch, mit einer sehr langen, kräftigen Borste und einem Dörnchen. - o rechts 17 -gldr, überragt den Hinterrand des Vorderrumpfes. Mundteile nicht 
verkümmert. 5. B. beiderseits 2-ästig; Enp. relativ lang, das rechte ist gerade und üherragt ein wenig das 1. Gl. des 3-gldr. Exp., das linke ist etwa halb so lang als das 1. Gl. des Exp. - L. ₹ $5,3 \mathrm{~mm}$, 万 $4,5 \mathrm{~mm}$.

Pacif. und Nord-Atlant. Ozean.

\section{Diaixis G. O. Sars.}

Kopf mit 1. Thsegm. verschmolzen; die beiden letzten Thsegm. mehr oder weniger deutlich getrennt; Rostrum einfach ohne Fäden. Gliederung des 1.-4. B. wie bei Scolecithrix; 1. G1. des Exp. des 1. B. mit langem, dünnem Randdorn; Enddorn des Exp. des 2.-4. B. grob gezähnelt.

Q. 5. Thsegm. jederseits in eine Zacke verlängert; Abd. 4-gldr; 1. Ant. 24-gldr., mit getrennten Endgl.; Exp. der 2. Ant. viel länger als das Enp., 6-gldr., das Endgl. das längste; 2. Maxille mit wurmförmigen Schläuchen; Maxilliped mit sehr kurzem Enp. 5. B. fehlt. - ऊ. 5. Thsegm. klein und abgerundet; Abd. 5-gidr., mit sehr kurzem Ansegm.; Mundteile von denen des \% abweichend, z. T. verkümmert. 5. B. vorhanden, ein mächtiges Greiforgan darstellend.

\section{Diaixis hibernica A. Scott.}

1896, Scolecithrix h., A. Scott, p. 362 , t. 17 f. $1-19$; t. 18 f. $1-9$. 1898, Scolecithrix h., Giesbr. und Schmeil, p. 47. 1903, D. h., Sars, p. 59, t. 39,40 .

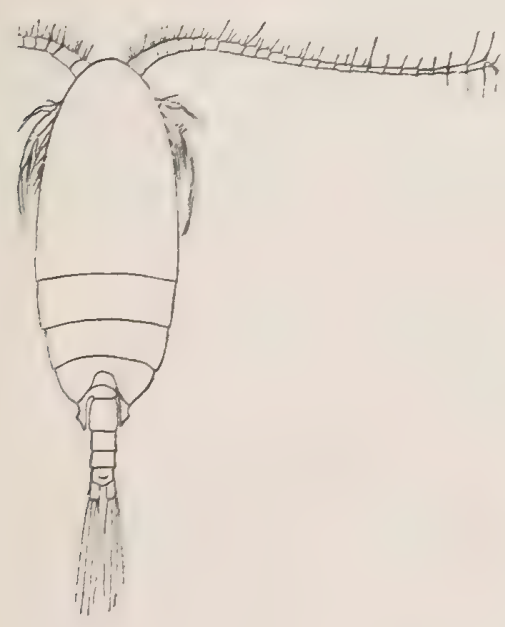

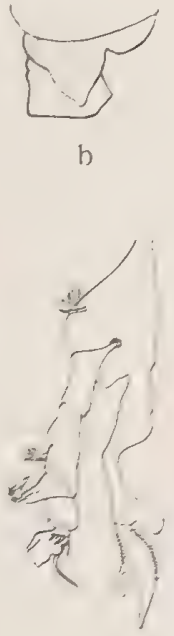

d

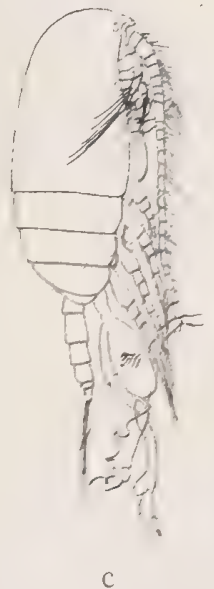

C

Fig. 95. Diaixis hibernica.

a ₹ dorsal, b 5. Thsegm. Q, lateral, c o lateral, d 5. B $\%$. a, b und c nach Sars, d nach Scott. 
7. Fortsätze des 5. Thsegm. an der Spitze nicht hakenförmig nach unten gebogen; 1. Ant. wenigstens das Ende des Gensegm. erreichend; Hinterfläche der Schwimmfüße ohne Dörnchen; proximaler Außenranddorn des Endgl. des 4. B. etwas oberhalb der Mitte eingelenkt. - 7 . 1. Gl. des rechten 5. B. distal stark verbreitert, mit großem, rundem Auswuchs; 2. Gl. breit mit schlankem, fingerförmigem Marginalauswuchs. - L. ㅇ 1,2 $\mathrm{mm}$, or 1,1 $\mathrm{mm}$.

Vor der irischen und schottischen Küste; Kanal; norwegische Küste.

2. Diaixis pygmaea T. Scott.

1899, Scolecithrix p., T. Scott, p. 249, t. 10 f. 1-9.

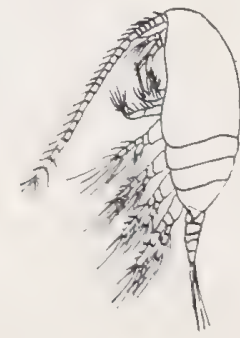

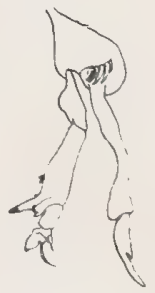

b

Fig. 96. Diaixis pygmaea. a lateral, b 5. B. $\sigma^{\text {r. }}$. Nach Scott.

ๆ. Fortsätze des 5. Thsegm. an der Spitze hakenförmig nach unten gebogen; 1. Ant. das Ende des Vorderkörpers kaum erreichend; Hinterfläche der Schwimmfüße mit Dörnchen; proximaler Außenranddorn des Endgl. des 4. B. unterhalb der Mitte eingelenkt. - $\sigma^{7}$. 1. Gl. des rechten 5. B. distal nur wenig verbreitert, 2. Gl. ziemlich schmal mit sehr kurzem Marginalauswuchs. L. $q 0,95$.

Vor der irischen und schottischen Küste; Kanal.

\section{Stephos T. Scott.}

Kopf mit 1. Thsegm., 4. mit 5. Thsegm. verschmolzen; Kopf ohne Rostrum und Rostralfäden. Abdomen beim $q 5$-, beim o 4-gldr. 1. Ant. in beiden Geschlechtern 24-gldr., mit getrennten Endgl. Exp. der 2. Ant. länger als das Enp., 6-gldr.; Mundteile des ơ denen des $q$ ähnlich; Kaulade der Mandibel wenig verbreitert mit nur wenig abweichendem Außenzahn; 2. Maxille ohne sensorische Anhänge; Maxilliped mit langem Enp. Gliederung des 1.-4. B. wie bei Scolecithrix; 1. Gl. des Exp. des 1. B. ohne Randdorn; 5. B. bei beiden Geschlechtern vorhanden, beim $q$ symmetrisch, 1-ästig, 3-gldr. mit 
dornförmig verlängertem Endgl., beim $\sigma^{7}$ asymmetrisch, beiderseits 4- bis 5-gldr., groß und von kompliziertem Bau.

1. Lateralteile des letzten Thsegm. flügelförmig verbreitert, asymmetrisch, rechter Flügel größer als der linke; Endgl. des 5. B. nicht weit von der Basis mit einem Dörnchen am Außenrande, distaler Teil dornförmig, an der Außenseite fein gewimpert

S. lamellatus Lateralteile des letzten Thsegm. nicht verbreitert, symmetrisch; 5. B. anders gebaut

2. 5. B. symmetrisch

5. B. asymmetrisch

S. fultoni

3. Endgl. des 5. B. ohne Dörnchen, aber distal am Außenrande mit ca. 10 groben Zähnchen

S. scotti

Endgl. des 5. B. distal, mit je einem Dörnchen am Außen- und Innenrande

S. minor.

1. 5. B. rechts mit kurzem, stumpfem Endgl.

S. lamellatus

5. B. rechts mit langem, gekrümmtem Haken am Ende

2. Endgl. des rechten 5. B. ohne Fortsatz an der Basis; linkes B. mit 2 fingerförmigen Anhängen am Ende

S. minor

Endgl. des rechten 5. B. mit Fortsatz an der Basis; linkes Endgl. mit anders gestalteten Endanhängen

3. Endgl. des linken 5. B. mit 2 ganz kurzen Anhängen an der Spitze und einer Reihe von kleinen Anhängen an der Außenseite

S. scotti Endgl. des linken 5. B. mit einer langen, am Ende gegabelten Klaue an der Spitze und einigen langen Anhängen unterhalb derselben

S. fultoni.

\section{Stephos lamellatus G. O. Sars.}

1903, S. 1., Sars, p. 62, t. $41,42$.

Lateralteile des letzten Thsegm. beim $q$ flügelartig verbreitert, deutlich asymmetrisch, rechter Flügel viel größer und breiter als der linke, beim ot symmetrisch und nicht verbreitert; 5. B. beim $q$ mit fast geradem Endgl.; letzteres nicht weit von der Basis mit einem Dörnchen am Außenrande, distaler Teil dornförmig und fein bewimpert am Außenrande; 5. B. $\sigma^{7}$ : rechtes Endgl. kurz, von unregelmäßiger Form, mit mehreren stumpfen, kurz fingerförmigen Anhängen; linkes vorletztes Gl. mit einem starken, dornförmigen Anhang am Innenrande nahe der Basis, und einer Reihe von feinen Dörnchen 
an der Innenseite, linkes Endgl. an der Spitze mit einem Bündel von lanzettförmigen, blattartigen Anhängen. - L. O, ơ $1 \mathrm{~mm}$.

Norwegische Küste.

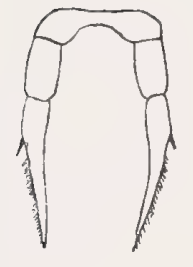

b

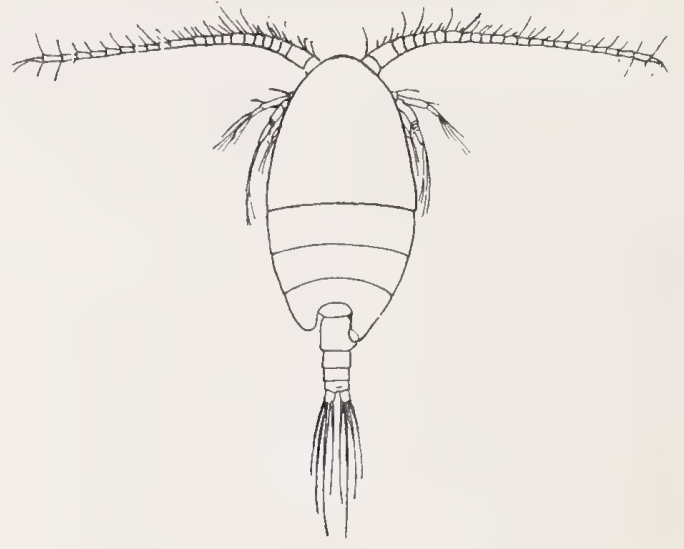

a

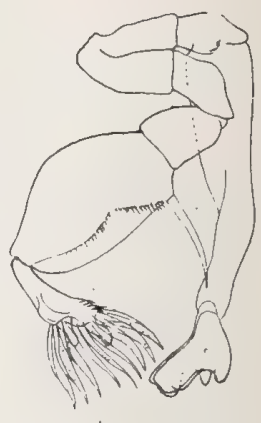

c

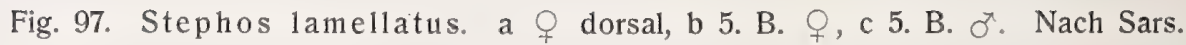

\section{Stephos minor T. Scott.}

1892, S. m., T. Scott, p. 245 , t. 7 f. $1-13$. 1898, Stephus m., Giesbr. und Schmeil, p. 29.

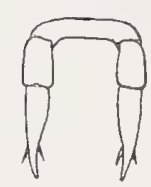

$\mathrm{b}$

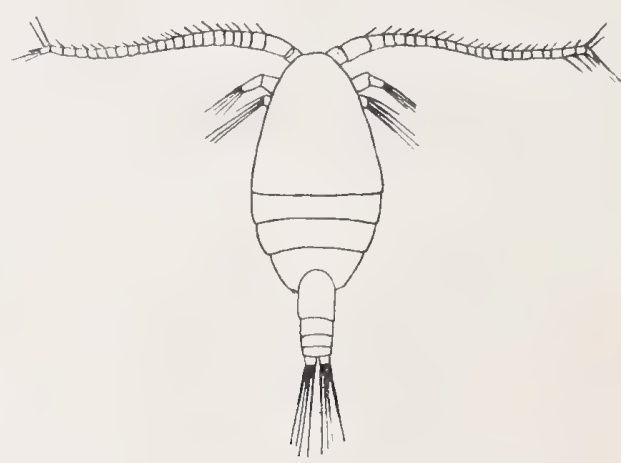

a

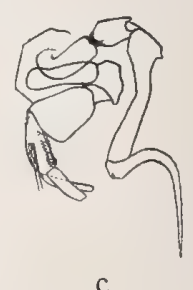

Fig. 98. Stephos minor. a $q$ dorsal, b 5. B. ๆ, c 5. B. 万. Nach Scott.

5. Thsegm. symmetrisch, nicht in Flügeln verlängert. 5. B. beim nach der Spitze gleichmäßig verjüngt mit 2 einander gegenüberstehenden Randdörnchen in der distalen Hälfte; 5. B. des $0^{7}$ rechts mit einem langen, scharf gekrümmten Endhaken ohne Auswuchs an der Basis desselben, links 
mit 2 fingerförmigen Anhängen, die fast so lang wie das Endgl. sind. L. $0,74 \mathrm{~mm}$.

Schottische Küste.

\section{Stephos scotti G. O. Sars.}

1897, S. gyrans (non Giesbrecht!) T. Scott, p. 146 , t. 2 f. 9 ; t. 3 f. 17 , 18. 1903, S. s., Sars, p. 63, t. 43.

5. Thsegm. symmetrisch, nicht flügelartig verbreitert. 5. B. beim $q$ mit leicht gebogenem Endgl., letzteres ohne Dörnchen, aber am Außenrande des distalen Teiles mit ca. 10 groben Zähnen; 5. B. des or rechts mit langem, hakenförmigem Endgl., das an seiner Basis einen fingerförmigen Fortsatz trägt; vorletztes Gl. des linken B. ohne Anhang oder Dörnchenreihe, Endgl. mit einer Reihe von 4 kleinen, blattförmigen Anhängen am Außenrande und 2 kurzen, klauenförmigen Blättchen am Ende. - L. ๆ 0,95 mm, ơ 0,85 mm.

Norwegische und schottische Küste; Kieler Bucht.

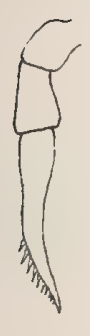

a

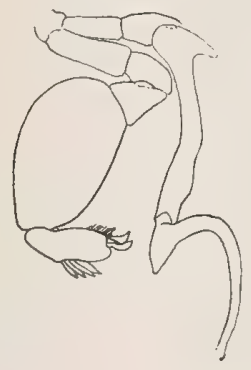

b

Fig. 99. Stephos scotti. a 5. B. 7, b 5. B. C. Nach Sars.

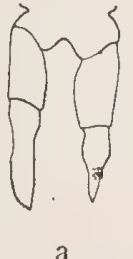

Fig. 100. Stephos fultoni. 5. B. b. 5. B. J. Nach Scott

4. Stephos fultoni T. und A. Scott.

1898 , S. f., T. und A. Scott, p. 185 , t. 10 f. $1-8$; t. 11 f. $1-4.1898$, S. f., T. Scott, p. 266, t. 15 f. $5-16$.

5. Thsegm. nicht flügelartig verbreitert, symmetrisch. 5. B. des $q$ asymmetrisch, Endgl. des rechten (?) B. breit und abgeplattet, distale Hälfte des Außenrandes fein gesägt, Endgl. des linken (?) B. in der unteren Hälfte etwas angeschwollen, in der Mitte plötzlich verschmälert und nach der Spitze hin allmählich verjüngt; 5 . B. des $\sigma^{\top}$ rechts mit langem, hakenförmigem Endgl., das an seiner Basis einen Fortsatz trägt; rechtes Endgl. mit einer starken, dunkel-hornfarbigen, an der Spitze gegabelten Klaue und mehreren längeren, wellig gebogenen Anhängen.

Schottische Küste. 
Parastephos G. O. Sars.

q unbekannt. - $\delta$. Weicht von Stephos in folgendem ab: - Kaulade der Mandibel verbreitert, der Außenzahn groß und klauenförmig; einzelne Äste der Schwimmfüße vom normalen Bau abweichend; Endsäge der Exp. des 2.-4. B. mit gezähneltem Saum.

\section{Parastephos pallidus G. O. Sars.}

1903, P. p., Sars, p. 65, t. 44.

$\sigma^{7}$. 1. Antenne die Mitte des Abdomens erreichend; Exp. des rechten 2. B. und Enp. des rechten 4. B. von der linken abweichend; 5. B. so lang wie das Abdomen, distaler Teil des rechten B. schmal, Endklaue sehr stark
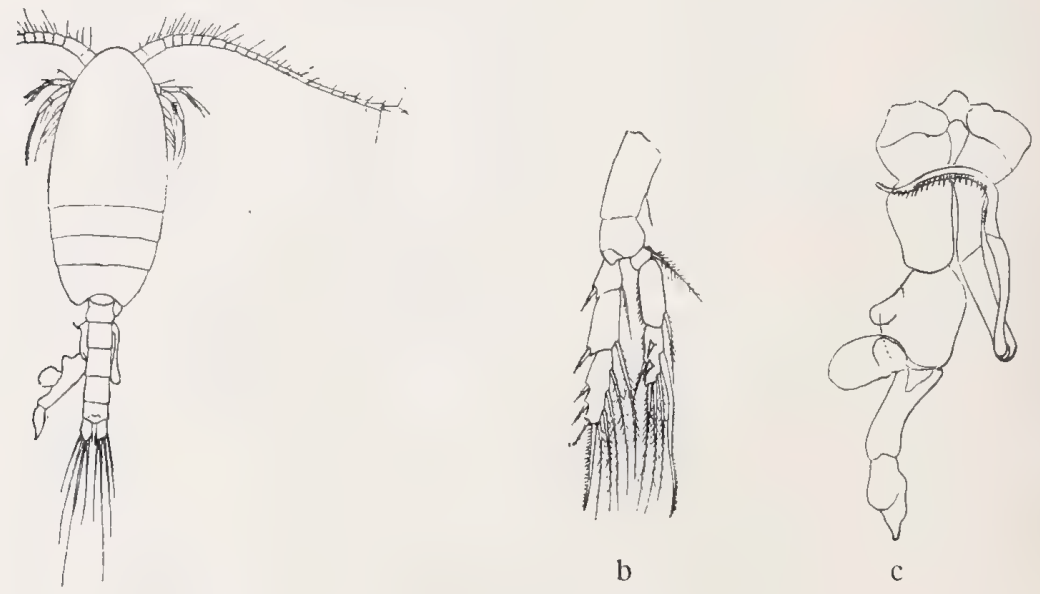

Fig. 101. Parastephos pallidus.

a ơ dorsal, b 4. B. ơ, c 5. B. ơ. Nach Sars.

und gebogen, mit einer regelmäßigen Reihe von Zähnen an der konkaven Seite; linkes B. mit stark verbreitertem drittletztem Gl., dieses am Ende mit ovalem, lamellösem Auswuchs an der Außenseite, Endgl. löffelförmig. - L. ठ 1,9 mm.

Norwegische und schottische Küste.

Tharybis G. O. Sars.

Kopf mit 1. Thsegm. verschmolzen; die beiden letzten Thsegm. getrennt; Kopf mit 2 zarten Fäden. Abdomen beim $\&$ 4,-, beim ơ 5 -gldr., bei beiden mit fast geschwundenem Analsegm. 1. Ant. bei beiden Geschlechtern gleich, 24-gldr, mit getrennten Endgl. Exp. der 2. Ant. viel länger als das Enp., 
6-gldr.; Kaulade der Mandibel kurz und breit, am Kaurande mit 2 starken, zweispitzigen Zähnen und einer dichten Reihe von z. T. befiederten Börstchen; 1. Maxille mit ungewöhnlich großem Kaulobus; 2. Maxille mit pinselförmigen Anhängen. 1.-4. B. wie bei Scolecithrix gegliedert; 1. Gl. des Exp. des 1. B. mit Randdorn; Enddorn des Exp. des 2.-4. B. sägeförmig; 5. B. vorhanden, beim $q$ symmetrisch, 3-gidr., beim of asymmetrisch, links 2-, rechts 1 -ästig.

Tharybis macrophthalma G. O. Sars.

1903, Th. m., Sars, p. 68 , t. $45,46$.

Auge sehr groß, lang-viereckig; 1. Antenne etwa so lang wie der Vorderkörper. 5. B. beim $\bigcirc$ kürzer als das Gensegm., das Endgl. fast 2 mal länger

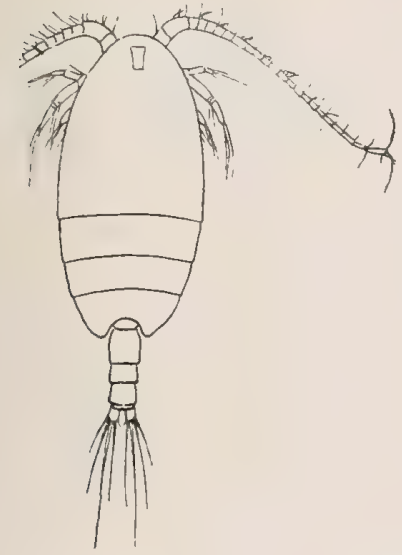

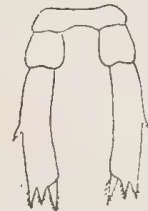

b

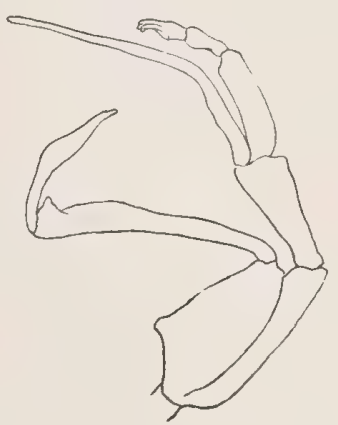

$\mathrm{c}$

Fig. 102. Tharybis macrophthalma.

a 9 dorsal, b 5. B. , c 5. B. 7 . Nach Sars.

als die beiden anderen zusammen, mit zahnförmigem Dorn halbwegs am Außenrande und 3 kurzen, gezähnten Enddornen; 5. B. beim ơ länger als das Abdomen, rechts 1-ästig, 3-gldr., links mit 3-gldr. Exp. und fast doppelt so langem, stilettförmigem Enp. - L. १ $1,4 \mathrm{~mm}$, ơ $1,8 \mathrm{~mm}$.

Norwegische Küste.

\section{Pseudocyclopia T. Scott.}

Kopf mit 1. Thsegm. verschmolzen; 4. vom 5. Thsegm getrennt; Rostrum ohne Fäden. Abdomen beim $q$ 4-, beim o 5 -gldr. 1. Antenne bei beiden Geschlechtern gleich, sehr kurz, 16- bis 20-gldr., mit langem, dickem 1. GI. Exp. der 2. Ant. kürzer als das Enp., 6-gldr.; Mundteile des $\sigma^{7}$ denen des $q$ ähnlich; 2. Maxille ohne sensorische Anhänge. 1.-4. B. wie bei Scolecithrix 
gegliedert; Außenranddorne des Exp. kräftig; Innenrandborste des 1. G1. des Basp. des 3. B. dick, lang, griffelförmig. 5. B. vorhanden, beim $q$ symmetrisch, 3-gldr., beim or asymmetrisch.

1. 1. Antenne 20-gldr., das 1. Gi. noch nicht halb so lang wie die übrigen Gi. zusammen

P. stephoides

1. Antenne 16- oder 17-gldr., das 1 . Gl. über halb so lang wie die übrigen Gl. zusammen

2. Furka doppelt so lang wie breit P. caudata

Furka kaum so lang wie breit

3. Stachel am Innenrande des 1. Gl. des Basp. des 3. B. das Exp. überragend; Endgl. des 5. B. des $q$ mit 3 dünnen, nackten Endborsten

P. minor

Dieser Stachel kürzer als das Exp.; Endgl. des 5. B. des $q$ mit 3 stachelund dornförmigen, dicken Borsten

4. 1. Antenne 16-gldr.

P. crassicornis

1. Antenne 17-gldr.

P. giesbrechti.

\section{Pseudocyclopia crassicornis T. Scott.}

1892, P. c., T. Scott, p. 246 , t. 7 f. $15,16,22-28$. 1898, P. c., Giesbr. und Schmeil, p. 30.

Abdomen ohne Stacheln; Furka kaum so lang wie breit. 1. Antenne 16-gldr.; 1. Gl. $6 / 7$ so lang wie alle übrigen Gl. zusammen. Stachel am Innenrande des 1. Gl. des Basp. des 3. B. fast gerade, das Enp. überragend.

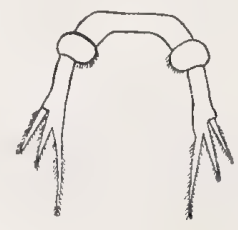

a

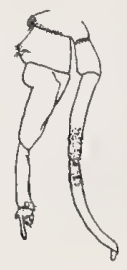

b

Fig. 103. Pseudocyclopia crassicornis. a 5. B. ㅇ, b 5. B. or. Nach Scott.

5. B. des $\bigcirc:$ Mittelgl. geschwollen, viel dicker und kürzer als das Endgl., letzteres mit 2 apikalen, unbeweglichen Stacheln und an der Außenseite mit einem Dorn. 5. B. des $\sigma^{7}$ beiderseits 1-ästig, das rechte (?) nicht viel länger als das andere. - L. 0,66 mm.

Schottische Küste. 


\section{Pseudocyclopia minor T. Scott.}

1892, P. m., T. Scott, p. 247 , t. 8 f. 1, 2, 4-10. 1898, P. m., Giesbr. und Schmeil, p. 30.

2. und 3. Abdsegm. des $\bigcirc$ mit je 2 dorsalen Stacheln; Furka kaum so lang wie breit. 1. Antenne 17 -gldr., 1. Gl. fast $3 / 4$ so lang wie alle übrigen Gl. zusammen, mit einem Häkchen in der Mitte des Vorderrandes. Stachel am Innenrande des 1. Gl. des Basp. des 3. B. gekrümmt, das Exp. überragend.

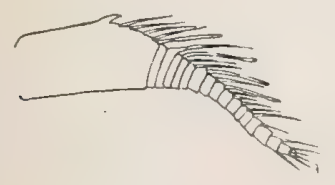

b

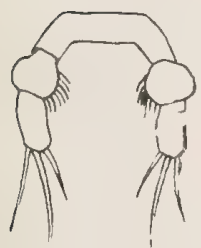

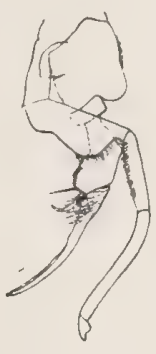

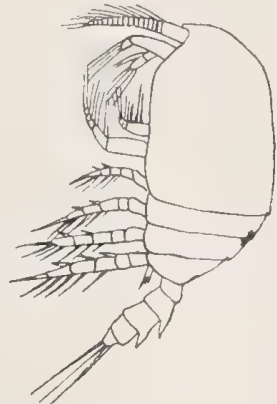

Fig. 104. Pseudocyclopia minor.

a q lateral, b 1. Antenne $q$, c 5. B. q, d 5. B. ơ. Nach Scott.

5. B. des $q$ : Mittelgl, etwas dicker und nicht viel kürzer als das Endgl.; letzteres trägt 3 dünne, nackte, apikale Borsten, von denen die mittlere die längste ist. Das rechte (?) 5. B. des o 2-, das andere 1-ästig. - L. 0,43 mm.

Firth of Forth.

\section{Pseudocyclopia caudata T. Scott.}

1894 , P. c., T. Scott, p. 236 , t. 5 f. $1-8$. 1898, P. c., Giesbr. und Schmeil, p. 30.

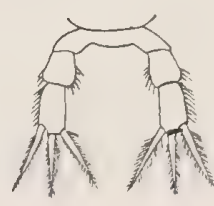

a

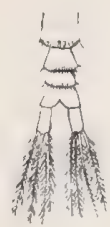

b

Fig. 105. Pseudocyclopia caudata, a 5. B. 오, b Abdomen 우. Nach Scott. 
ๆ. Abdomen ohne Stacheln; Furka etwa doppelt so lang wie breit und fast so lang wie die vorhergehenden beiden Segm. 1. Antenne 17-gldr.; 1. Gl. ca. $7 / 10$ so lang wie die übrigen G1. zusammen. Stachel am Innenrande des 1. G1. des Basp. des 3. B. gebogen, fast bis zum Ende des Exp. reichend. 5. B. des q: Mittelgl. etwas breiter und kürzer als das Endgl., welches 3 dicke, gleich lange, befiederte Borsten trägt. - o unbekannt. - L. O $0,65 \mathrm{~mm}$.

Schottische Küste.

\section{Pseudocyclopia stephoides J. C. Thompson.}

1895 , P. S., J. C. Thompson, p. 96 , t. 6 , f. 1 , 2 ; t. 7 f. $8-14.1898$, P. s., Giesbrecht und Schmeil, p. 31. 1903, P. s., Sars, p. 71, t. 47, 48.

Abdomen ohne Stacheln; Furka etwa so lang wie breit. 1. Antenne 20 -gldr.; 1. Gl. wenig über $1 / 3$ so lang wie die übrigen Gl. zusammen. Innenrandborste am 1. Gl. des Basp. des 3. B. dick und bis zum Ende des Enp.

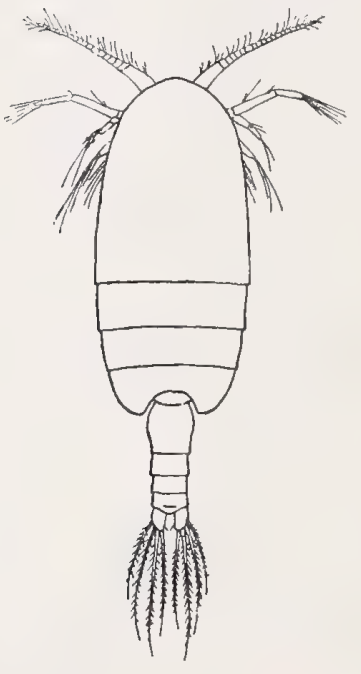

a

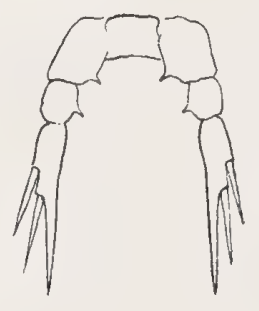

b

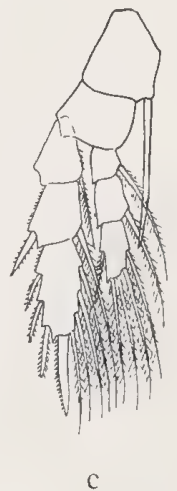

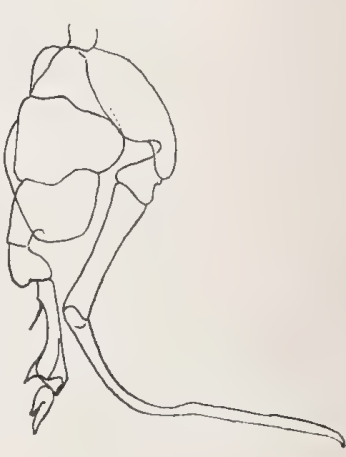

d

Fig. 106. Pseudocyclopia stephoides. a $q$ dorsal, b 5. B. $q$, c 3. B. $q$, d 5. B. ơ. Nach Sars.

reichend, aber nicht stachelförmig; Innenrandborste des 1. und 2. Gl. des Enp. des 4. B. kurz und dick. 5. B. des $Q$ : Innenrand des 1. Gl. und des Mittelgl. mit Stacheln; Mittelgl. viel kürzer, aber nicht breiter als das Endgl.; letzteres läuft in drei Stacheln aus, von denen die äußeren kürzer sind als die inneren. 5. B. des $\sigma^{\top}$ beiderseits 1-ästig; das rechte beträchtlich länger als das linke; sein Endgl. macht nicht weit von seiner Basis einen Bogen und läuft in einen langen, geraden Stachel aus. - L. 1,2 mm.

Liverpool-Bai; Westküste Norwegens. 
5. Pseudocyclopia giesbrechti Wolfenden.

1902, P. g., Wolfenden, p. 370, t. 4.

Abdomen ohne dorsale Stacheln; Furka etwa so lang wie breit. 1. Antennc 17-gldr.; 1. Gl. etwa so lang wie die folgenden dreizehn. Stachel am Innen-
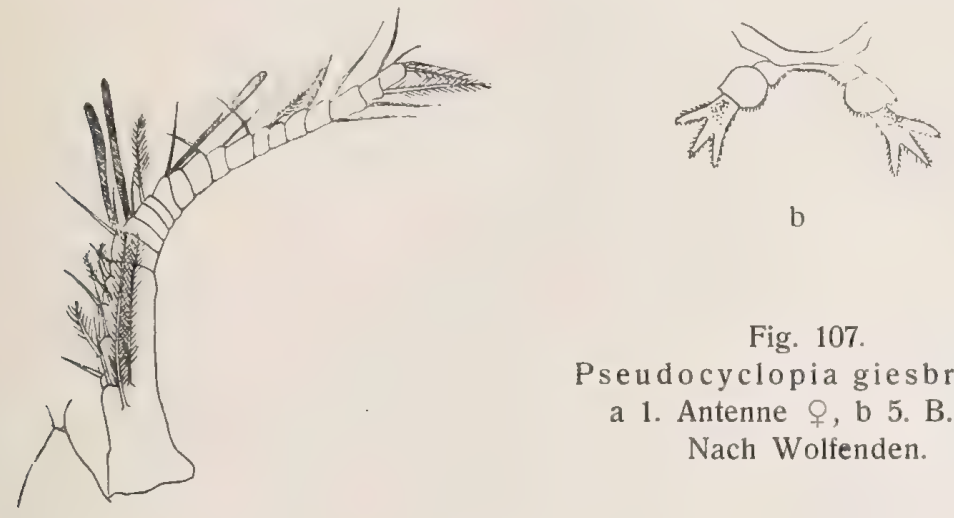

b

Fig. 107.

Pseudocyclopia giesbrechti.

a 1. Antenne 9, b 5. B.

Nach Wolfenden.

a

rande des. 1. G1. des Basp. des 3. B. gebogen und bis zum Ende des Enp. reichend. 5. B. des $\bigcirc$ : Innenrand der Gl. mit Dörnchenreihe: Mittelgl. viel breiter als das Endgl., letzteres mit 3 fingerförmigen, bedornten Stacheln. 万 unbekannt. - L. $\bigcirc 0,72 \mathrm{~mm}$.

Shetlandinseln.

\section{Centropagidae.}

Eine der beiden 1. Antennen des $\sigma^{7}$ in eine Greifantenne umgewandelt durch Ausbildung eines Kniegelenkes zwischen dem 18. und 19. Gl., Verstärkung der Muskulatur in den vorhergehenden Gl. und meist auch Verbreiterung des mittleren Abschnitts der Antenne. 5. B. des $q$ entweder den vorhergehenden ähnlich (Innenrandborste des 2. Gl. des Exp. von auffälliger Form) oder zurückgebildet, fehlt aber niemals. Die sekundären Genitalcharaktere des $\sigma^{T}$ beschränken sich fast stets auf den Bau des Rumpfes (besonders des Abdomens), der 1. Antennen und des 5. B. - Kopf vom Thorax fast stets getrennt, die beiden letzten Thsegm. meistens verschmolzen. Rostrum mit 2 meist schlaffen Fäden. Abdomen des $ᄋ$ 3- oder 4-, selten 2-gldr., des or 5-gldr., letzteres selten mit stark verkürztem Ansegm; Genitalöffnung des $\sigma^{7}$ auf der der Greifantenne gegenüberliegenden Seite. 1. Antenne des $\bigcirc$ meistens 23- bis 25-, selten weniger als 20-gldr.; Endabschnitt der Greifantenne (distal vom Knie- 
gelenk) 2-4- (5?)-gldr. Exp. der 2. Antenne 5- bis 8-gldr., selten unter $2 / 3$ so lang wie das Enp., sein Endgl. länger (meistens um vieles) als die vorhergehenden Gl. Die Mundgliedmaßen entweder denen der Calanidae ähnlich oder mit folgenden Abweichungen: Enp. der Mandibeln rückgebildet, Zähne der Kaulade vermindert; die beiden distalen Innenrandloben und Enp. der 1. Maxille rückgebildet, ihr Exp. verlängert; proximale Borsten der 2. Maxille mit den Loben rückgebildet, distale lang, zuweilen haken- oder sensenförmig, Enp. rückgebildet. 1.-4. B. meistens mit 3-gldr. Exp. und Enp.; Enddorn des Exp. des 2.-4. B. sägeförmig. Enp. des 5. B. des on entweder 3-gldr. oder rudimentär oder fehlend; die Exp. bilden gewöhnlich Hacken oder Scheren; das B. ist stets auf beiden Seiten vorhanden.

\section{Centropages Kröyer.}

૧. Kopf vom Thorax, vorletztes vom letzten Thsegm. getrennt; Rostrum mit 2 schlaffen Fäden. Abdomen 3-gldr., mit asymmetrischem Gensegm. 1. Antenne 24-gldr. (24. mit 25. Gl. verschmolzen). Exp. der 2. Antenne länger als das Enp.; Mandibel und 1. Maxille ähnlich wie bei Calanus. Distale Reusenborsten der 2. Maxille hakenförmig, viel dicker und länger als die proximalen. 1. Gl. des Basp. des Maxillipeden mit starken Vorsprüngen am Innenrande, von denen die beiden mittleren bestachelte Borsten tragen. 1.-4. B. mit 3-gldr. Exp. und fast stets auch 3-gldr. Enp.; Endgl. des Exp. des 2.-4. B. mit 3 Außenranddornen. 5. B. den vorhergehenden B. ähnlich; am Innenrande des 2. Gi. des Exp. ein kräftiger Dornfortsatz. $-\sigma^{7}$. Abdomen 5-gldr., zuweilen mit fast geschwundenem Ansegm. und daher augenscheinlich 4-gldr. Greifantenne rechts, 21 - oder 22-gldr., Endabschnitt (distal vom Kniegelenk) 3- oder 4-gldr. Enp. und das rechte, zangenförmige Exp. des 5. B. des $\sigma^{\pi}$ 3-gldr., linkes Exp. 2-gldr.

1. Am Vorderrande des 1., 2. und 5. G1. der 1. Antenne je eine Zacke 2 Diese Glieder ohne Zacke

2. 1. Antenne des $\bigcirc$ überragt das Rumpfende um etwa die beiden Endgl.; proximaler Zangenhaken des Greiffußes des $\sigma^{\pi}$ nicht länger als der distale

C. typicus 1. Antenne des $q$ erreicht das Rumpfende nicht; proximaler Zangenhaken des Greiffußes des $\sigma^{\nearrow}$ länger als der distale C. chierchiae.

\section{Centropages typicus Kröyer.}

1848-49, C. t., Kröyer, p. 588, t. 6.1892 , C. t., Giesbrecht, p. 303, t. 2 f. 4 ; t. 17 f. 48,$49 ;$ t. 18 f. $4,9,22$; t. 38 f. 9,10 . 1863, Ichthyophorba denticornis, Claus, p. 199, t. 35.1898 , C. t., Giesbr. und Schmeil, p. 54, f. 11 . 1903, C. t., Sars, p. 75 , t. $49-51$. 
Auge an der Ventralseite gewölbt; letztes Thsegm. mit Seitenhaken, beim $\sigma^{\top}$ nicht ganz symmetrisch; Ansegm. des $\sigma^{7}$ verkümmert. Außenrandborste der Furka kurz, nahe am Randende pfriemartig, beim $\sigma^{7}$ fiederlos. Gensegm. des $q$ mit 4 dornartigen Borsten, vorletztes Abdsegm. rechts mit knopfförmigem Fortsatz. 1. Antenne überragt das Rumpfende etwa um die letzten beiden Gl.; Vorderrand des 1., 2. und 5. Gl. mit einer Zacke. Mittlere

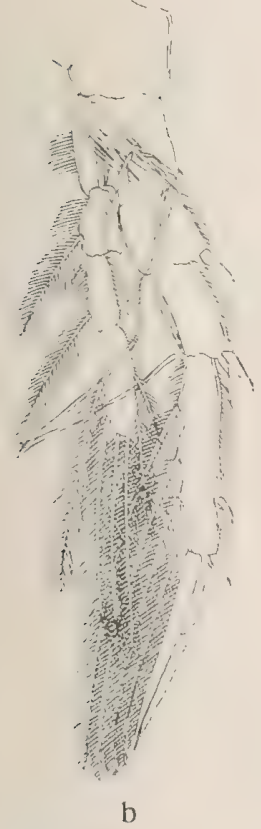

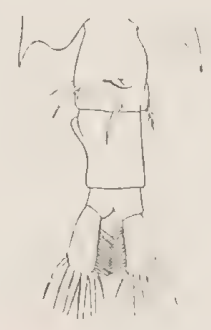

a

Fig. 108.

Centropages typicus.

a Abdomen 9 , ventral,

b 5. B. 우, c 5. B. $0^{7}$.

Nach Giesbrecht.

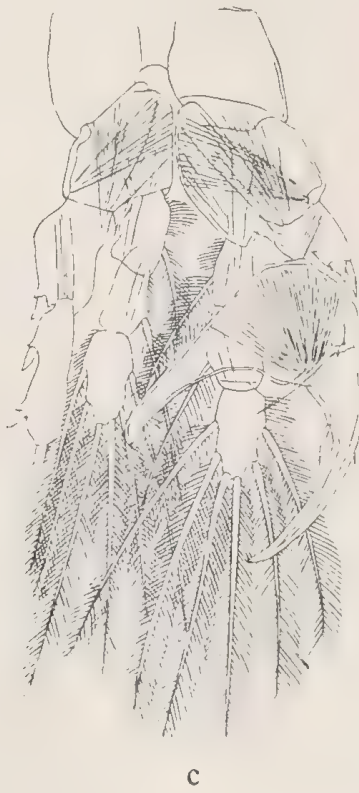

G1. der Greifantenne breit, 15. Gl. mit kleiner, 16. mit größerer Zacke am Vorderrande. Exp. der 2. Antenne wenig länger als das Enp. Äste der Mandibel etwa gleich lang. Enddorn der Exp. der B. fein gezähnelt; Dorn am Innenrande des mittleren Gl. des Exp. des 5. B. beim $q$ dick, länger als das Glied, abstehend. Mittleres Gl. des Exp. des rechten 3. und 4. B. beim ot mit vergrößertem Außenranddorn. Zange am rechten 5. B. des ot gedrungen, distaler Haken länger als der proximale; Randdorne am Endgl. des linken Exp. kurz. - L. 1,6-2 mm, or 1,4-1,9 $\mathrm{mm}$.

Mittelmeer, Atlant. Ozean (zwischen $6^{\circ}$ südl. und $62^{\circ}$ nördl. Breite).

\section{Centropages chierchiae Giesbrecht.}

1889, C. c., Giesbrecht, p. 811. 1892, C. c., Giesbrecht, p. 304, t. 17 f. $38,39,45$; t. 18 f. 5 ; t. 38 f. 3, 7. 1898, C. c., Giesbr. und Schmeil, p. 55.

Verwandt mit C. typicus; aber: die 4 Dornborsten am Gensegm. des o und das folgende Segm. anders geformt; 1. Antenne erreicht das Rumpfende 
nicht; Zacken am 15. und 16. G1. der Greifantenne gleich weit vorspringend; Außenranddorn am 2. Gl. des Exp. des .3. B. des or nicht verlängert; Innenranddorn am 2: Gl. des Exp. des 5. B. des $q$ schlanker; distaler Haken der Zange des 5. B. des or stärker gebogen, am Innenrande seines Endstückes geriefelt. - L. क 1,8-1,9 mm, o $1,65-1,75 \mathrm{~mm}$.

Meerenge von Gibraltar, Atlant. Ozean; Ind. Ozean.

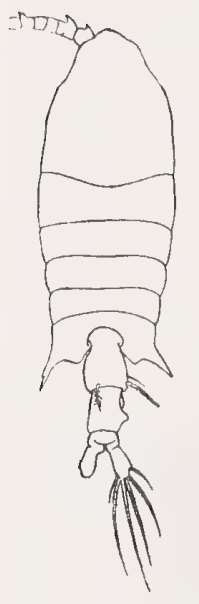

a

Fig. 109. Centropages chierchiae.

a $q$ dorsal, b Exp. des rechten 5. B. $0^{\text {r }}$ Nach Giesbrecht.
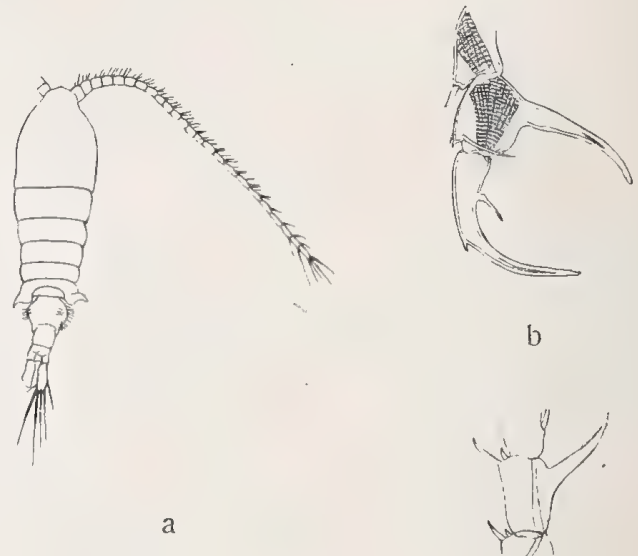

b

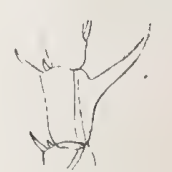

c
Fig. 110. Centropages hamatus. a $\bigcirc$ dorsal, b rechtes Exp. des 5. B. ơ, c 2. Gl. des Exp. des 5. B. ․ Nach Giesbrecht.

\section{Centropages hamatus Lilljeborg.}

1853, Ichthyophorba hamata, Lilljeborg, p. 185, t. 21, 26.1863 , Ichthyophorba angustata, Claus, p. 199, t. 35. 1892, C. h., Giesbrecht, p. 304 , t. 17 f. 51 ; t. 18 f. 3 ; t. 38 f. 23.1898 , C. h., Giesbr. und Schmeil, p. 56.1903 , C. h., Sars, p. 76 , t. 52.

Seitenhaken des letzten Thsegm. beim $q$ asymmetisch, rechter seitwärts gerichtet; vor der Genitalöffnung des $\bigcirc$ ein Stachel; proximale Antennengl. ohne Zacken am Vorderrande; 15. und 16. Gl. der Greifantenne ohne Zacken am Vorderrande; Enddorn der Exp. der B. grob gezähnelt; 3. Außenranddorn des Endgl, des rechten Exp. des 4. B. (nicht der Randdorn des 2. Gl.) beim $\sigma^{T}$ verlängert; Innenranddorn am 2. Gl. des Exp. des 5. B. des nicht länger als das Gl. 1. Gl. des Enp. desselben B. mit normaler Borste; 
proximaler Zangenhaken am rechten 5. B. des or nicht länger als der distale, mit gerader Spitze. - L. क 1,3-1,5 mm, o $1,1-1,3 \mathrm{~mm}$.

Atlant. Ozean (zwischen $41^{\circ}$ und $70^{\circ}$ nördl. $\mathrm{Br}$.), bis in die Ostsee vordringend.

\section{Limnocalanus G. O. Sars.}

․ Segmentierung des Rumpfes wie bei Centropages: Rostralfäden kurz. Abdomen 3-gldr., symmetrisch; Furka wenigstens 6 mal so lang wie breit, mit langer Außenrandborste. 1. Antenne 25-gldr., mit sehr kurzem Endgl. Gliedmaßen des Kopfes ähnlich wie bei Centropages, aber Maxp. gestreckter. 1.-4. B. mit 3-gldr. Exp. und Enp.; Endgl. der Exp. des 1.-4. B. mit 2 Ayßenranddornen. 5. B. ähnlich wie bei Centropages. - $\sigma^{7}$. Ansegm. nicht verkürzt. Greifantenne rechts. Rechtes Exp. des 5. B. ohne Zange, mit rudimentärem oder fehlendem 3. Gl., übrigens wie bei Centropages.

\section{Limnocalanus grimaldi De Guerne.}

1886, Centropages g., De Guerne, p. 1. 1897, L. g., Sars, p. 39, t. 4 f. 1-18. 1898, L. g., Giesbr. und Schmeil, p. 59, f. 12.

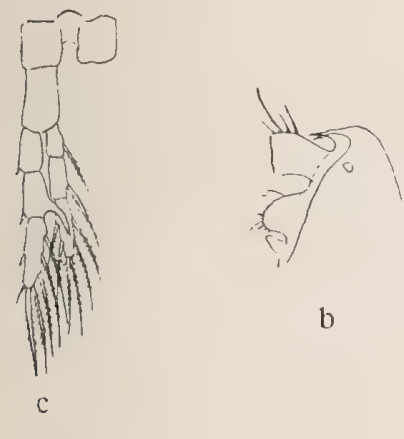

Fig. 111.

Limnocalanus grimaldi.

a $q$ dorsal, b Kopf $q$, lateral, c 5. B. ㅇ, d 5. B. ox. Nach Sars.

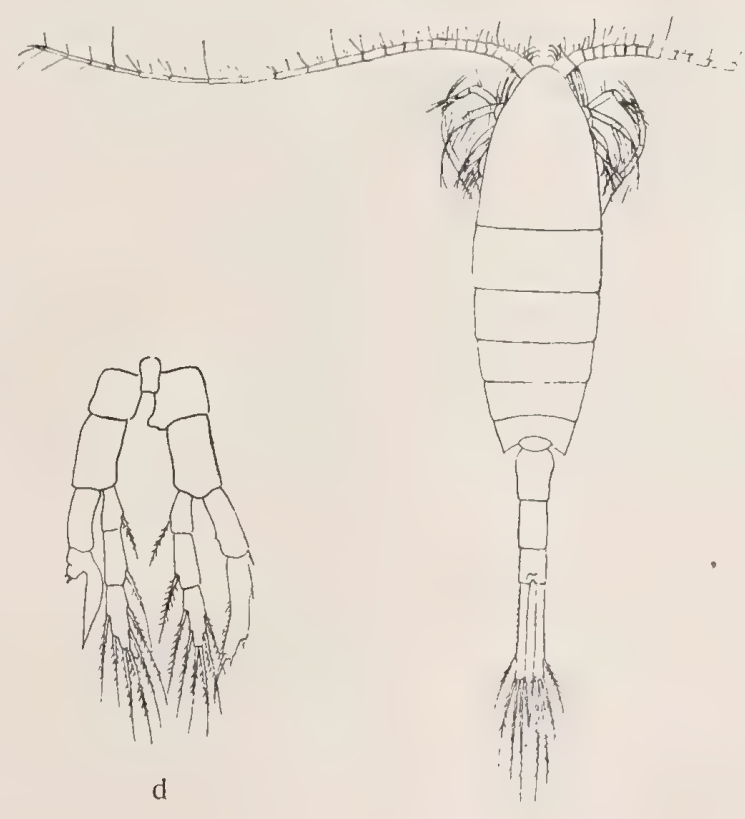

a

Vorderkopf an der Dorsalseite nicht aufgetrieben; Seitenteile des letzten Thsegm. dreieckig, hinten zugespitzt; Abdomen und Furka mit Dornenbesatz; 1. Antenne reicht bis zum Hinterrande des Ansegm. Dornfortsatz am 2. Gi. 
des Exp. des 5. B. des $q$ innen und außen mit sehr feinen Dörnchen. Rechtes 5. B. des $\sigma^{7}: 3$. Gi. des Exp. undeutlich abgegrenzt, rudimentär; 2. Gl. innen zu einem geraden Fortsatz ausgezogen; linkes 5. B. des $\sigma^{7}$ : Exp. 2-gldr., etwas länger als das Enp. - L. $ๆ 2,8 \mathrm{~mm}$, o $2,5 \mathrm{~mm}$.

Östl. Teil der Ostsee, Karisches Meer, Spitzbergen, Kaspisches Meer, Mündung des Jana in Ost-Sibirien.

Isias Boeck.

१. Kopf vom Thorax getrennt; die beiden letzten Thsegm. verschmolzen; Rostralfäden dünn und schlaff. Abdomen 3-gldr, nahezu symmetrisch. Furka ca: 4 mal so lang wie breit. 1. Antenne 24-gldr., die beiden letzten Endgl. verschmolzen. Mundgliedmaßen denen von Centropages ähnlich, aber die distalen Borsten der 2. Maxille nicht verlängert. 1.-4. B. mit 3-gldr. Exp. und Enp.; Endgl. des Exp. des 2.-4. B. mit 3 Außenranddornen. 5. B. mit 3-gldr. Exp. und 1-gldr. Enp.; 2. Gl. des Exp. mit dornförmiger Innenrandborste. - $\sigma^{7}$. Abd. 5-gldr., mit asymmetrischem 3. Segm.; Ansegm. nicht verkürzt; Greifantenne rechts; 5. B. mit 2-gldr. Exp. rechts ohne Enp., links mit rudimentärem, 1-gldr. Enp.

\section{Isias clavipes Boeck.}

1864, I. c., Boeck, p. 18. 1888, I. bonnieri, Canu, p. 91, 228, t. 16-18. 1892 , I. c., Giesbrecht, p. 323 , t. 19 f. $31-37$; t. 38 f. $30-32 . \quad 1898$, I. c., Giesbr. und Schmeil, p. 62. 1903, I. c., Sars, p. 79, t. 53, 54.
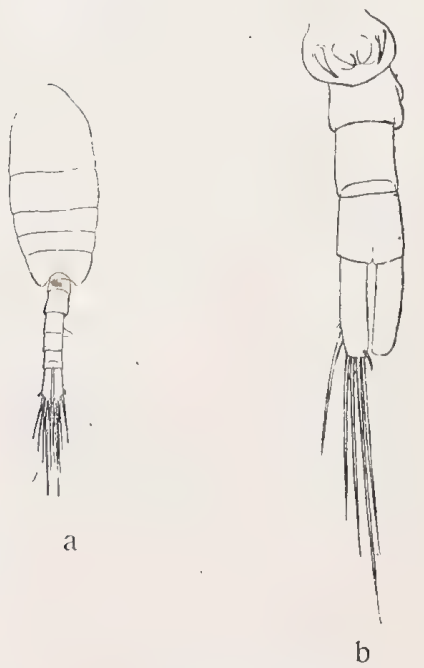

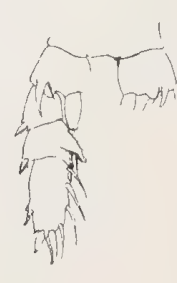

c

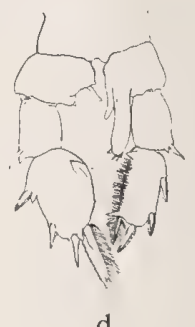

d

Fig. 112. Isias clavipes. a $\sigma^{\top}$ dorsal, b Abdomen $\circ$, ventral, c 5. B. + , d 5. B. O. Nach Giesbrecht.

Letztes Thsegm. abgerundet; 1. Antenne etwa so lang wie der Vorderkörper; 3. Abdsegm. des $\sigma$ rechts mit konischem Fortsatz; 5. B. des $q$ mit 
Enp. des 1. B. 2-, des 2--4. B. 3-gldr., aber mit aufgehobener Artikulation zwischen den beiden proximalen Gl.; Endgl. des Exp. des 2.-4. B. mit 3 Außenranddornen. 5. B. rudimentär, beiderseits 3-gidr. - $\sigma^{7}$. Greifantenne rechts; 5. B. links 4-gldr., zangenförmig, rechts 3 -gldr., hakig.

\section{Temora longicornis Baird.}

1792, Cyclops I., Müller, p. 116. 1850, T. finmarchic’a, Baird, p. 228. 1863, T. 1., Claus, p. 195 , t. 34 . 1882, Halitemora 1., Giesbrecht, p. 154, t. 2, 3, 5-11. 1885, T. 1., Poppe, p. 176, t. 4 f. 10-19. 1892, T. 1., Giesbrecht, p. 329 , t. 17 f. 15,16 . 1898, T. 1., Giesbr. und Schmeil, p. 101.1903 , T. 1., Sars, p. 97, t. 65,66 .

Seitenecken des letzten Thsegm. abgerundet. Furka symmetrisch, keine Borste so lang wie die Furka; 2. Endborste an der Basis leicht verdickt;

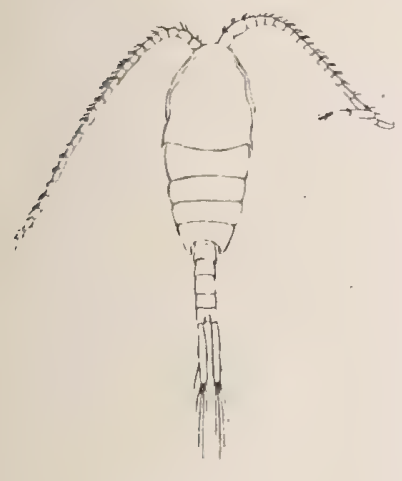

a

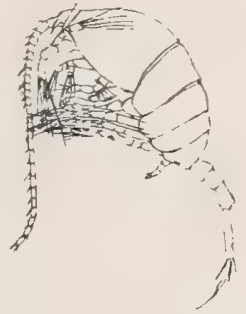

b
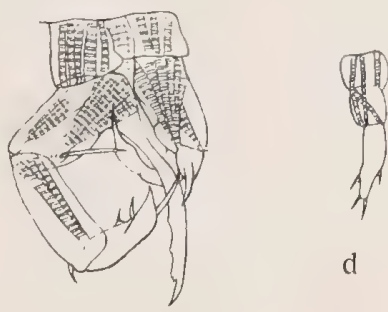

Fig. 113. Temora longicornis.

a dorsal, b lateral, c 5. B. ơ, d 5. B. ㅇ․ Nach Giesbrecht.

Außenborste nahe dem Randende. Das Gl. vor dem Kniegelenk der Greifantenne mit Reibkamm, das vorhergehende nicht. 2. B. auch beim ot symmetrisch. Innerer Dorn am Endgl. des 5. B. des $q$ etwas kürzer als die beiden apikalen; Endgl. des linken 5. B. des ơ unregelmäßig stabförmig. - L. † $1-1,5 \mathrm{~mm}$, ơ $1-1,35 \mathrm{~mm}$.

Ostsee, nördl. Atlant. Ozean (West- und Ostküste), Island; Ind. Ozzean.

\section{Temorites G. O. Sars.}

१. Kopf vom Thorax getrennt; letztes mit vorletztem Thsegm. verschmolzen; Stirn ohne Rostrum und Rostralfäden. Abdomen 4-gldr., symmetrisch; Furka sehr kurz mit reduzierter Borstenzahl. 1. Antenne 24-gldr.; 2. Antenre mit ungefähr gleich langen Ästen. 1. Maxille mit rudimentärem Enp.; Exp. mit Basp. verschmolzen. 2. Maxille mit stark verlängerten distalen Borsten. Exp. des 1.--4. B. 3-gldr.; Enp. des 1. B. 2-, des 2.-4. B. 3-gldr. Endgl. Nord. Plankton. 
des Exp. des 2.-4. B. mit 3 Außenranddornen. 5: B. symmetrisch, 3-gldr., mit langen, schmalen Endgl. - $\sigma^{7}$. Greifantenne rechts; 5. B. beiderseits 4-gldr., 3. Gl. des rechten 5. B. blattförmig, 4. Gl. klauenförmig.

\section{Temorites brevis G. O. Sars.}

1900, T. b., Sars, p. 100 , t. $30,31$.

Körper kurz und gedrungen; Abd. ungefähr 1/3 so lang wie der Vorderkörper; Ansegm. kürzer als die übrigen Abdsegm. Furka mit 3 langen, gefiederten Borsten und einer kurzen, ungefiederten Innenborste. 1. Antenne un-
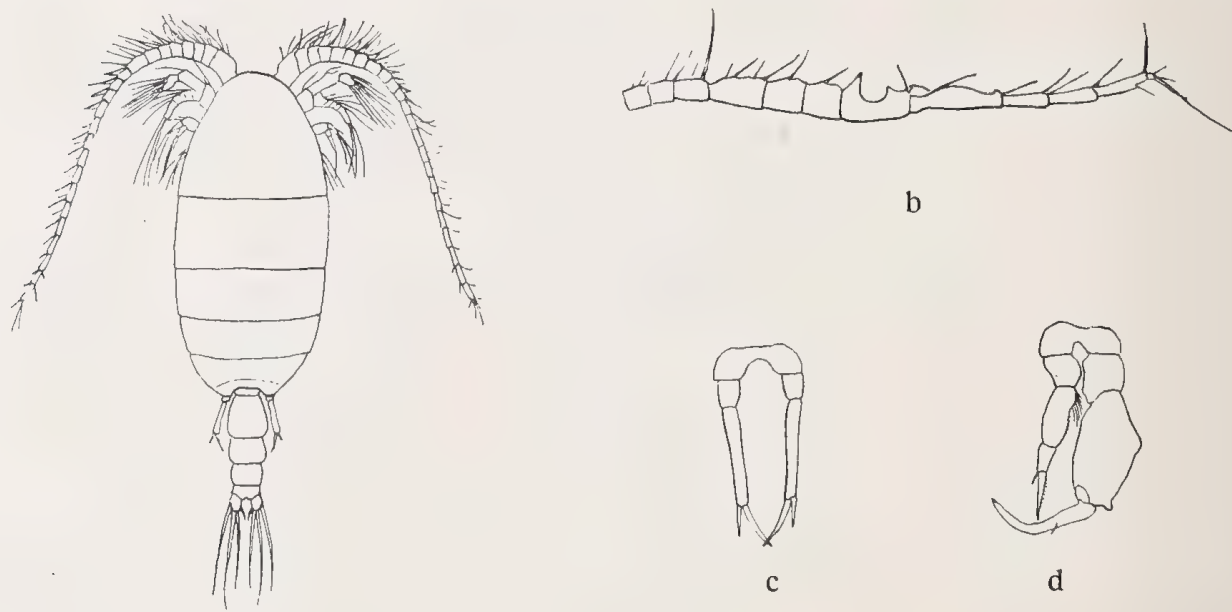

b

a

Fig. 114. Temorites brevis.

a $q$ dorsal, b distaler Teil der Greifantenne $\sigma^{x}$, c 5. B. ㅇ, d 5. B. $\sigma^{\top}$. Nach Sars.

gefähr so lang wie der Vorderkörper. 2. Basp. des 3. und 4. B. am distalen Außenrande mit hakigem Dorn (der am 3. B. besonders groß ist); Außenranddorn am 1. und 2. Gl. des Exp. des 1. B. fehlend; 5. B. des $q$ und $\sigma^{7}$ vergl. Fig. 114 . - L. \% $1,1 \mathrm{~mm}$, o $1,05 \mathrm{~mm}$.

Arktisches Meer.

\section{Eurytemora Giesbrecht.}

q. Kopf vom Thorax, letztes vom vorletzten Thsegm. getrennt; Rostralfäden klein. Abd. 3-gldr.; Furka symmetrisch. 1. Antenne 24-gldr. Exp. der 2. Antenne länger als das Enp.; Mandibel, 1. und 2. Maxille ungefähr wie bei Temora; Maxp. verkürzt mit verbreitertem 2. Basp. Exp. des 1.-4. B. 3-gldr.; Enp. des 1. B. 1-, des 2--4. B. 2-gldr.; Endgl. des Exp. des 2.-4. B. mit 2 Außenranddornen. 5. B. 4-gldr.; vorletztes Gl. mit Dornfortsatz am 
Innenrande; Endgl. knopfförmig. 1 Eiballen. - $\sigma^{7}$. Greifantenne rechts; linkes 5. B. 4-gldr., nicht zangenförmig, das Endgl. distal verbreitert; rechtes 5. B. 4- oder 5-gldr., das Endstück klauenförmig.

1. Vorletztes Gl. des 5. B. mit 1 Außenranddorn E. velox Dasselbe mit 2 Außenranddornen

2. Innenranddorn des vorletzten Gl. des 5. B. fast senkrecht zur Längsachse des Gl. abstehend, glatt oder fast glatt

Derselbe distal gerichtet, mit dicken Spitzen besetzt

E. herdmani

3. Dorsalfläche der Furka ohne Dornbesatz

E. gracilis Dieselbe mit Dornbesatz

4. Gensegm. (dorsal betrachtet) bis zur Mitte sich stark verbreiternd, dann plötzlich verschmälert

E. affinis und hirundoides

Derselbe in der Mitte dick, nach hinten allmählich verschmälert E. hirundo

1. 2. und 3. Gi. des Exp. des rechten 5. B. verschmolzen

E. velox und herdmani

2. Der proximale Teil des verschmolzenen Abschnittes aufgeschwollen

Derselbe nicht aufgeschwollen

E. affinis und hirundo E. gracilis.

\section{Eurytemora velox Lilljeborg.}

1853, Temora v. $q$, Lilljeborg, p. 177, t. 20 f. 2-9. 1853, Cyclopsina lacinulata, Fischer, p. 86 , t. 2 f. $4--17,34$. 1878, Temora v., Brady, p. 56, t. 6 f. $1-5$. 1878, Temora Clausii, Hoek, p. 23, t. 4, 5, 1881, Temorella clausii, Claus, p. 490, t. 2 f. $1-7$. 1889, E. lacinulata, De Guerne et Richard, p. 82 , f. 44, 45. 1891, E. clausii, Brady, p. 40, t. 13 f. $1-5$. 1896, E. lacinulata, Schmeil, p. 109, 181, t. 12 f. 1 -8. 1898, E. v., Giesbr. und Schmeil, p. 102 , f. 23.1903 , E. v., Sars, p. 100, t. $67,68$.

Letztes Thsegm. des $q$ mit 2 großen, nach hinten gerichteten Flügeln, welche mit einer scharfen Spitze enden; Außenränder dieses Segm. stark s-förmig gebogen; Sinneshärchen der Flügel stark. Gensegm. des $\bigcirc$ auf der ventralen Seite mit einer stark ausgeschweiften Chitinduplikatur; Deckel der Genitalöffnung langgestreckt und abgerundet. Furka des $\bigcirc$ dorsal mit Dornenbesatz. 1. Antenne kaum bis zum Ende des Vorderrumpfes reichend. 5. B. des $\bigcirc:$ 1. Gl. des Exp. mit einem Außenranddorn; klauenförmiger Fortsatz kurz und distalwärts gerichtet. Rechtes 5. B. des $0^{7}$ : die beiden letzten Gl. des Enp. nicht (oder nur in beschränktem Maße) miteinander verschmolzen; 
2. G1. nicht aufgeschwollen; linkes 5. B. des $\sigma^{7}$ : Exp. am Ende mit 2 (undeutlichen) Lappen. - L. क 1,3-2 mm, of 1,2-1,5 mm.

Brack- und Süßwasserart. Ostsee, Deutschland, Rußland, Schweden, Norwegen, Kaspisches Meer, England, Holland, Frankreich.

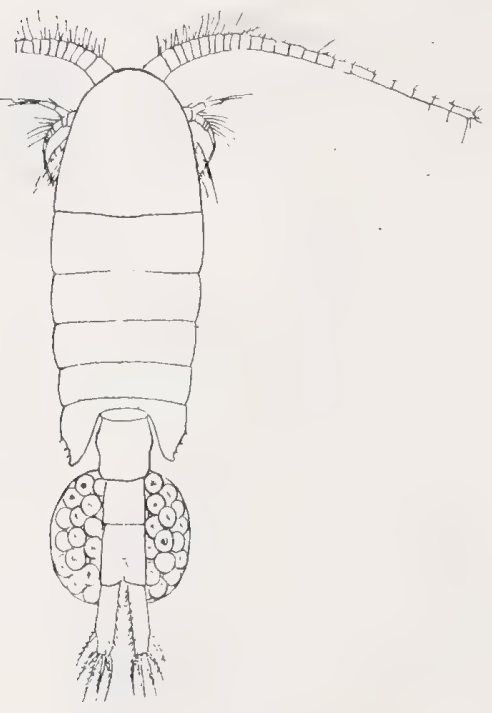

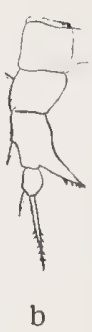

b

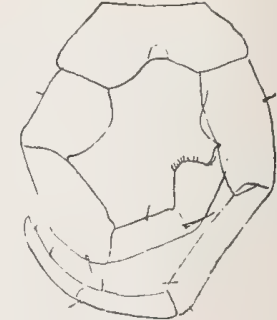

C

Fig. 115. Eurytemora velox. a ㅇ dorsal, b 5. B. 우, c 5. B. ㅈ. Nach Sars.

2. Eurytemora herdmani Thompson u. A. Scott.

1897, E. h., Thompson u. A. Scott, p. 78, t. 5 f. 1, 8, 10.

Letztes Thsegm. des $q$ mit sehr großen, schräg nach hinten und auswärts gerichteten, zugespitzten Flügeln, Außenränder derselben s-förmig gebogen. Gensegm. des $q$ jederseits mit einem starken, nach hinten gerichteten Buckel. 1. Antenne etwa so lang wie der Vorderrumpf. 5. B. des : : 1. Gl.

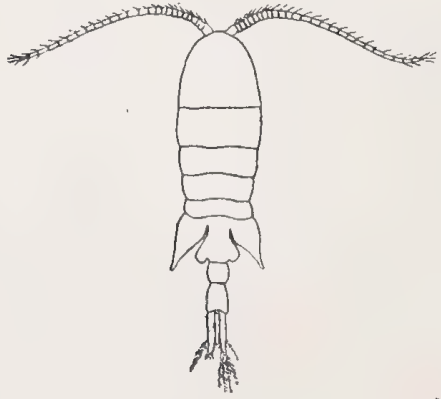

a

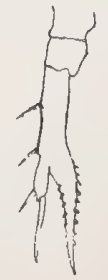

b

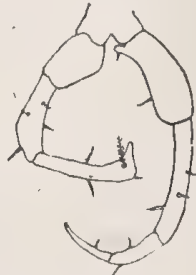

C

Fig. 116. Eurytemora herdmani.

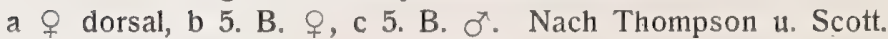


des Exp. langgestreckt, mit 2 dünnen Außenranddornen, sein Klauenfortsatz dick, lang, distalwärts gerichtet, mit dicken Spitzen besetzt; Endgl. doppelt so lang wie breit. 5. B. des $\sigma^{\top}$ dem von E. velox ähnlich, seine Glieder, besonders die des linken B. gestreckter. - L. 1,6 mm.

Golf von St. Lorenz, Narragansett Bay.

\section{Eurytemora affinis Poppe.}

1880, Temora affinis, Poppe, p. 55, t. 3. 1881, Temorella a., Claus, p. 491 , t. 2 f. 8 - 14. 1889, Eurytemora a., Guerne et Richard, p. 84, Textf. 46, 47. 1896, E. a., Schmeil, p. 114,181 , t. 8 f. 11 ; t. 11 f. $1-11$. 1888, Temorella a. var. hispida, Nordquist, p. 53 , t. 5 f. $1,6,7,10$; t. 6 f. $4,5$. 1892, T. a. und T. a. var. hispida, Canu, p. 13, t. 1.

Letztes Thsegm, des $\sigma^{\pi}$ ohne, des $\bigcirc$ mit 2 großen nach außen gerichteten, zugespitzten Flügeln, Außenränder fast geradlinig; Sinneshärchen sehr stark. Gensegm. des $q$ bis etwa zur Mitte sich stark verbreiternd, sodann plötzlich enger und nach dem hinteren Ende zu wieder breiter werdend, ventrale Partie stark erhöht; Erhöhung hinter dem Vorderrande beginnend, sich verbreiternd, wieder schmäler werdend und vor dem Hinterrande endend; Deckel der Geschlechtsöffnung des $q$ spitz. Ansegm. und Furka beim $q$ und $\sigma^{T}$ auf der dorsalen Seite mit Dornenbesatz; Furka des † 5-7 mal, des or etwa 7 mal so lang wie breit. 1. Antenne in der Regel nur wenig über den Vorderrand des 2. Thsegm. reichend. Enddorn des Exp. des 2.-4. B. schmal, so lang wie das Endgl. 5. B. des $ᄋ: 1$. Gl. des Exp. außen mit 2 Dornen; klauenförmiger Fortsatz groß, etwas distalwärts gerichtet. Rechtes 5. B. des ơ: die beiden letzten Gl. des Exp. miteinander verschmolzen, im proximalen Teile aufgeschwollen. Exp. des linken 5. B. am Ende in 3, eine kleine Grube umschließende Lappen gespalten. - L. 1-1,65 mm.

In den Flußmündungen und Brackwasserbuchten von Nordwest-Europa (Elbe, Weser, Jade-Busen, Ems, Rhein, Seine), Küsten von Schweden, England und Schottland, Kaspisches Meer; Golf von St. Lorenz?

\section{Eurytemora affinis var. raboti J. Richard.}

1897 , E. a. var. r., Richard, p. 196, f. 1-3.

Gensegm. des $q$ jederseits mit einem großen, zweilappigen Auswuchs. - L. ㅇ 2,3, o $2 \mathrm{~mm}$.

Im Brackwasser. Spitzbergen.

\section{Eurytemora hirundoides Nordquist.}

1888, Temorella affinis var. h., Nordquist, p. 48 , t. 4 f. $5-11 ;$ t 5 , f. 5 . 1896, E. affinis var. h., Giesbr. und Schmeil, p. 104. 1903, E. h., Sars, p. 102, t. 69. 
Verwandt mit E. affinis, aber: - Laterale Teile des letzten Thsegm. des $q$ spitz oder abgerundet, kürzer als bei E. affinis; Gensegm. des $q$ etwas abweichend gebaut; 1. Antenne etwa so lang wie der Vorderrumpf. L. $0,8-1,15 \mathrm{~mm}$.

Ostsee, norwegische Küste, Zuidersee; atlant. Küste von Nord-Amerika.

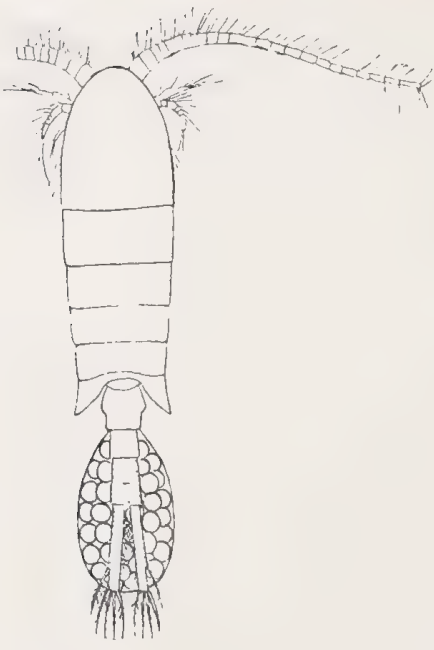

a

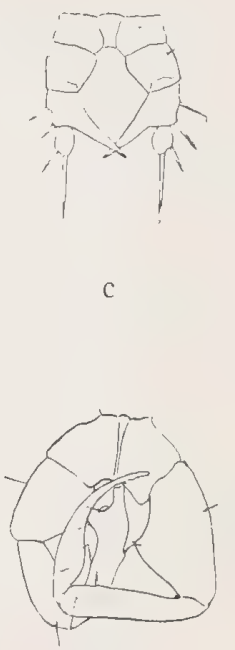

d

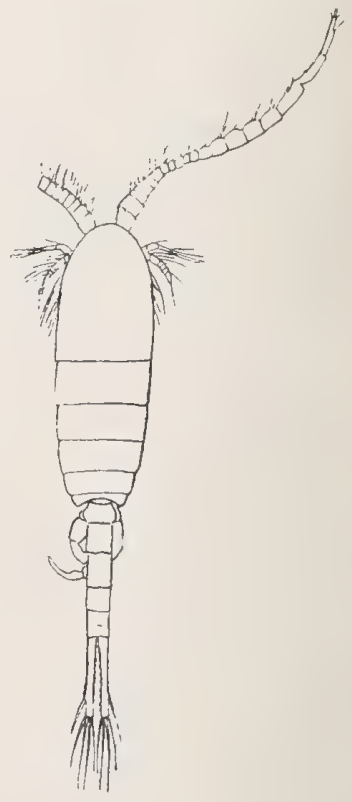

b

Fig. 117. Eurytemora hirundoides.

a o dorsal, b o dorsal, c 5. B. ․, d 5. B. o․ Nach Sars.

\section{Eurytemora hirundo Giesbrecht.}

1881, E. h., Giesbrecht, p. 258. 1882, E. h., Giesbrecht, p. 152, t. 2 f. $1,7,12,19$; t. 3 f. 3,10 ; t. 5 f. 17 ; t. 6 f. 8,20 ; t. 7 f. 5,22 ; t. 8 f. 21 , $33,39,40$; t. 9 f. 1,31 ; t. 10 f. 5,38 ; t. 11 f. 3 . 1898, E. h. Giesbr. und Schmeil, p. 104.

Verwandt mit E. affinis, aber: - Letztes Thsegm. des $q$ stark nach hinten verlängert, abgerundet. Gensegm. des $q$, von der Dorsalseite gesehen, in der mittleren Partie verdickt, nach hinten gleichmäßig enger werdend. 1. Antenne des $q$ etwa bis zum Ende des Vorderrumpfes reichend. - L. $q$ 1,4 , का $1,2 \mathrm{~mm}$.

Wéstlicher Teil der Ostsee.

6. Eurytemora gracilis G. O. Sars.

1898, Temorella g., Sars, p. 336 , t. 8 f. $8-18$. 
Verwandt mit E. affinis, aber: - Laterale Teile des letzten Thsegm. des o lang und schmal. Ansegm. und Furka auf der dorsalen Seite ohne
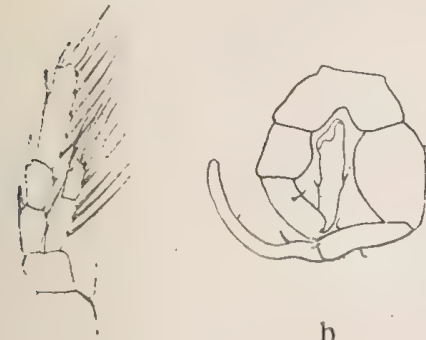

b Dornenbesatz. 1. Antenne etwa so lang wie der Vorderkörper. Enddorn des Exp. des 2. - 4. B. kürzer als das Endgl., messerförmig, in der Mitte verbreitert. 5. B. des $\bigcirc$ dem von E. affinis ähnlich. Rechtes 5. B. des $\sigma^{7}$ : die beiden letzten Gl. des Exp. miteinander verschmolzen, aber im proximalen Teile nicht aufgeschwollen. - L. \& 1,4, o $1,25 \mathrm{~mm}$.

Mündung des Janaflusses in Sibirien.

a

Fig. 118. Eurytemora gracilis:

Pleuromamma Giesbrecht.

a 2. B. ㅇ, b 5. B. ơ. Nach Sars.

. Kopf vom Thorax getrennt; letztes mit vorletztem Thsegm. verschmolzen; Rostrum mit 2 gefiederten Fäden. Dunkel pigmentierter Chitinknopf seitlich am 1. Thsegm., an der Basis des Maxillipeden; Mundgliedmaßen denen von Calanus ähnlich; Maxilliped mit langgestrecktem Enp. Abd. 3-gldr. 1.-4. B. mit 3-gldr. Exp. und Enp.; 1. Gl. des Enp. des 2. B. mit konkavem Innenrande und Häkchen an demselben an beiden Enp.; Exp. des 3. B. mit tiefer Kerbe am Außenrande des 1. Gl.; Endgl. des Exp. des 2.--4. B. mit 3 Außenranddornen. 5. B. rudimentär, symmetrisch, beiderseits 2-4-gldr. - $\sigma^{7}$. Abd. 5-gldr., zuweilen asymmetrisch; Greifantenne rechts oder links; meistens nur an einem des Enp. des 2. Beinpaares ein Häkchen; 5. B. beiderseits 5-gldr., ein Greifbein, aber ohne eigentliche Zange.

1. Stirn in einen spitzen Fortsatz verlängert P. xiphias Stirn ohne diesen Fortsatz

2. Proximale Gl. der 1 . Antenne mit 2 größeren, 1 krummen und 1 geraden Zacke $P$. abdominalis Diese Gl. mit kleinen Zacken

3. 5. B. am Ende mit Borsten P. robusta

5. B. am Ende mit 3 Zinken

4. Ventralfläche des Gensegm. flach $P$. borealis Ventralfläche des Gensegm. geyölbt

P. gracilis.

1. Stirn in einen spitzen Fortsatz verlängert P. xiphias Stirn ohne diesen Fortsatz 
2. Abdomen asymmetrisch

P. abdominalis Abdomen symmetrisch

3. Enp. des 2. B. beiderseits mit Ausschnitt und Haken

P. robusta Enp. des 2. B. nur an einem B. mit Ausschnitt und Haken

\section{4}

4. Vorderrand des Gl. vor dem Kniegelenk der Greifantenne geriefelt P.gracilis Derselbe nicht deutlich geriefelt fluted $P$. borealis.

\section{Pleuromamma abdominalis Lubbock.}

1856, Diaptomus a., Lubbock, p. 22, t. 10. 1863, Pleuromma abdominale, Claus, p. 197, t. 5, 6. 1892, Pleuromma a. und Pleuromma a. var. abyssalis, Giesbrecht, p. 347,356, t. 5 f. 8 ; t. 32 f. $3,5,13,22$, $25-30$; t. 33 f. $43,44,48,49,52.1898$, P. a. und P. a. var. abyssalis, Giesbr. und Schmeil, p. 109.

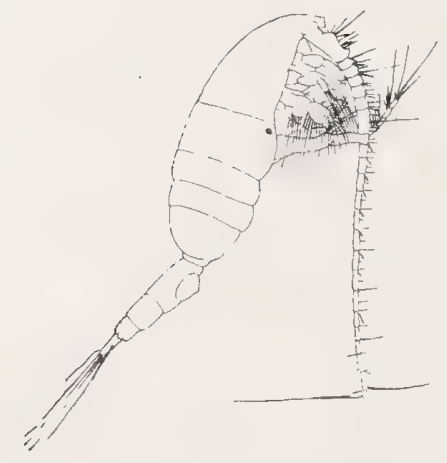

a

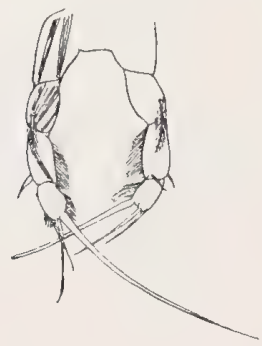

d

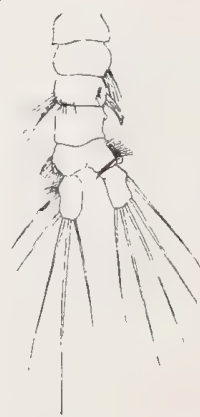

b

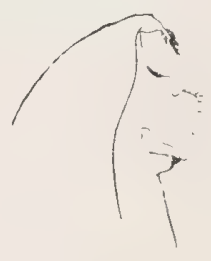

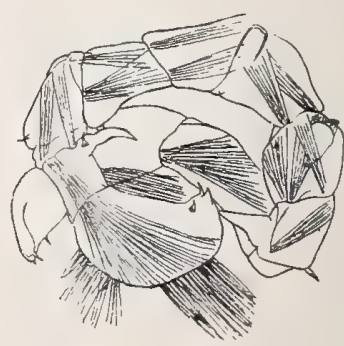

c

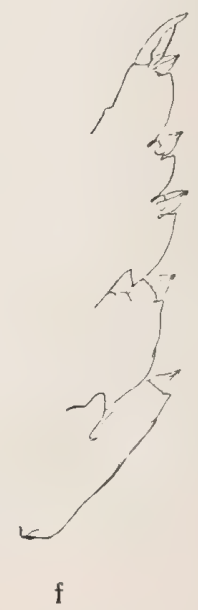

f

૧. Pigmentknopf links oder rechts. Proximale GI. der 1. Antenne mit mehreren kleineren und 2 größeren (1 geraden, 1 krummen) Zacken am 
Vorderrande. 5. B. 4-gldr., mit 3 apikalen Borsten. - o. Pigmentknopf links. Abdomen asymmetrisch. Proximale Gl. der 1. Antenne nur mit kleinen Zacken. Greifantenne rechts; die beiden mittleren Gl. ihres mittleren Abschnittes lang und schmal. 1. Gl. des Enp. des 2. B. nur links mit Ausschnitt und Haken; 3. und 4. B. von dem des $\bigcirc$ abweichend. Endgl. des linken

5. B. verbreitert. - L. $\subsetneq 2,4-3 \mathrm{~mm}$, o $^{7} 2,75-3,5 \mathrm{~mm}$.

Mittelmeer, Atlant. Ozean (26 $6^{\circ}$ S. Br. - Faröerkanal); Pacif. Ozean; Rotes Meer, Ind. Ozean.

\section{Pleuromamma xiphias Giesbrecht.}

1889, Pleuromma x., Giesbrecht, p. 25. 1892, Pleuromma x., Giesbrecht, p. 347 , t. 32 f. 14 ; t. 33 f. $42,45,50$. 1898, P. x., Giesbrecht und Schmeil, p. 109.

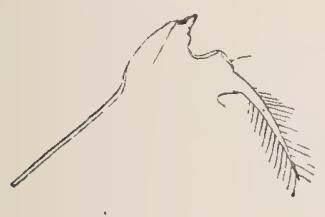

a

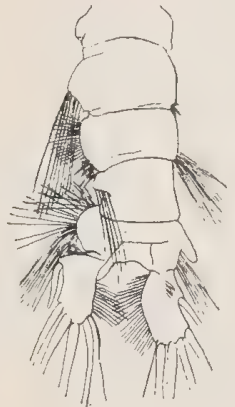

c

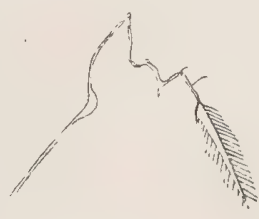

$\mathrm{b}$

Fig. 120. Pleuromamma xiphias.

a Kopf $\mathcal{O}$, lateral, b Kopf $\sigma^{\nearrow}$, lateral, c Abdomen $\sigma^{\nearrow}$, dorsal. Nach Giesbrecht.

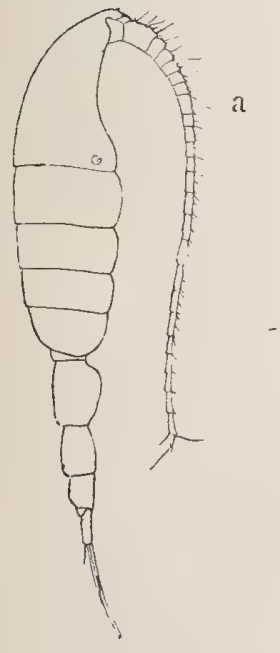

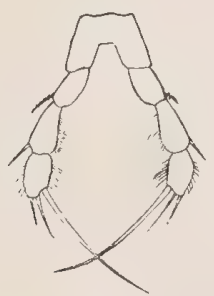

b

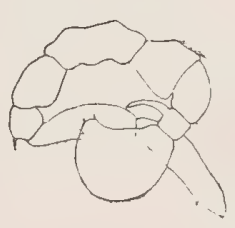

C

Fig. 121. Pleuromamma robusta.

a $q$ lateral, b 5. B. ㅇ, c 5. B. $0^{x}$. Nach Sars. 
Verwandt mit P. abdominalis, aber die Stirn vor dem Rostrum in einen starken, spitzen Fortsatz verlängert. - L. क 4,1-4,5 mm, ơ 4-4,3 mm.

Atlant. Ozean, Nordsee; Pacif. und Ind. Ozean.

\section{Pleuromamma robusta Dahl.}

1893, Pleuromma robustum, Dahl, p. 105. 1898, P. r., Giesbr. und Schmeil, p. 110.1903 , P. r., Sars, p. 115 t. $78,79$.

Zacken an den Antennengl. klein. Abdomen des or symmetrisch. 1. Gl. des Enp. des 2. B. bei $q$ und $\sigma^{r}$ beiderseits mit Ausschnitt und Haken. 5. B. des q 4-gldr., mit 3 apikalen Borsten. - L. q 3-4,3 mm, o 3-4 mm.

Atlant. Ozean.

\section{Pleuromamma gracilis Claus.}

1863, Pleuromma gracile, Claus, p. 197, t. 5. 1892, Pleuromma g., Giesbrecht, p. 347 , t. 5 f. 7 ; t. 32 f. $6,18-20$; t. 33 f. $41,47.1898$, P. g., Giesbr. und Schmeil, p. 110.
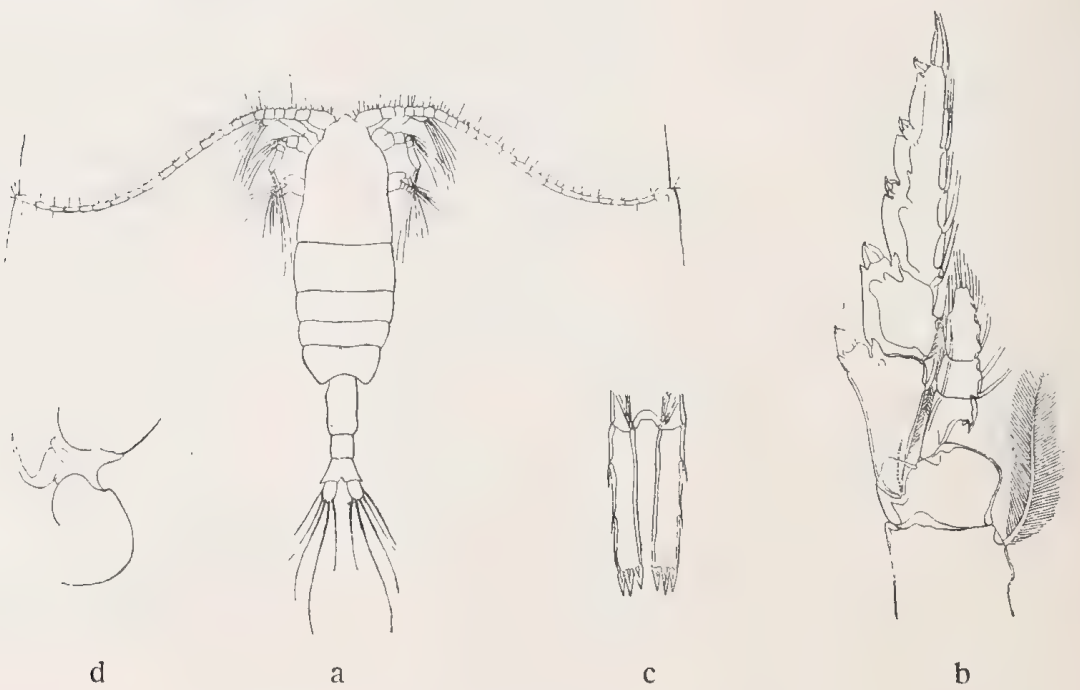

Fig. 122. Pleuromamma gracilis.

a $q$ dorsal, b 2. B. $q$, c 5. B. $q$, d Mittelteil des Exp. des rechten 5. B. o־. Nach Giesbrecht.

7. Pigmentknopf rechts. Proximale Gl. der 1. Antenne nur mit kteinen Zacken am Vorderrande. Ventralfläche des Gensegm. gewölbt. 5. B. 2-gldr., mit 3 apikalen Zacken. - $\sigma^{7}$. Pigmentknopf rechts. Abdomen symmetrisch. Zacken an der 1. Antenne und am 3. und 4. B. wie beim $q$. Greifantenne links; die beiden mittleren Gl. ihres mittleren Abschnittes höchstens doppelt 
so lang wie breit. 1. Gl. des Enp. des 2. B. nur rechts mit Ausschnitt und Haken. Endgl. des rechten 5. B. verbreitert, - L. 1-2 mm.

Mittelmeer, Atlant., Pacif. und Ind. Ozean.

\section{Pleuromamma borealis Dahl.}

Zacken an der 1. Antenne, apikale Zacken am 5. B. des q, Ausschnitt und Haken des 1. G1. des Enp. des 2. B. wie bei P. gracilis, aber Ventralfläche des Gensegm. des $q$ flach; genikulierende Gl. der Greifantenne ohne Zähnchen. - L. 2-2,5 mm.

Atlant. Ozean.

\section{Metridia Boeck.}

Nahe verwandt mit Pleuromamma; aber: der laterale Pigmentknopf fehlt; die B. (besonders das 2. Paar) in beiden Geschlechtern stets übereinstimmend.

1. Furka weniger als doppelt so lang wie breit; 1. Antenne das Rumpfende erreichend

M. brevicauda

Furka $2-3$ mal so lang wie breit

Furka ca. $4 \mathrm{mal}$ so lang wie breit (nicht ganz symmetrisch), $11 / 3$ mal so lang wie das Analsegment

Furka ca. 5 mal so lang wie breit, 2 mal so lang wie das Analsegment.

M. princeps

2. Letztes Thsegm. beiderseits in eine kleine Spitze ausgehend; Vorderkopf hoch gewölbt; 5. B. 3-gldr. (das 4. Gl. undeutlich oder gar nicht vom 3. Gl. getrennt)

M. lucens Letztes Thsegm. beiderseits abgerundet; Vorderkopf flach gewölbt; 5. B. 4-gldr.

M. longa

3. 5. B. beiderseits 2-gldr.; jedes Endgl. mit 2 apikalen Borsten M. venusta

5. B. links 3-gldr. mit 3 Endborsten, rechts undeutlich 3-gldr. mit 2 Endborsten

M. normani.

1. Furka wenigstens 4 mal so lang wie breit (etwas asymmetrisch); 1 . Antenne das Rumpfende fast erreichend oder dasselbe überragend

Furka weniger als doppelt so lang wie breit, symmetrisch; 1. Antenne das Rumpfende erreichend

M. brevicauda

Furka höchstens 3 mal so lang wie breit, symmetrisch; 1. Antenne das 4 . Abdsegm. nicht erreichend

2. Rechtes 5. B. ohne dornförmigen Anhang am Innenrande des 3. G1. M. normani Rechtes 5. B. mit langem Anhang am Innenrande des 3. Gi. M. princeps 
3. Vorderkopf flach gewölbt; beide Endgl. des 5. B., besonders des rechten, distal verschmälert

$M$. longa

Vorderkopf hoch gewölbt; beide Endgl. des 5. B. distal nur wenig verschmälert

M. lucens.

\section{Metridia longa Lubbock.}

1854, Calanus longus, Lubbock, p. 127, t. 5. 1864, M. armata, Boeck, p. 14 . 1892, M. 1., Giesbrecht, p. 339, t. 33 f. 9, 13, 20, 23, 27, 34, 38. 1898, M. 1., Giesbr. und Schmeil, p. 106. 1900, M. 1., Sars, p. 99, t. 19. 1902, M. 1., Th. Scott, p. 454, t. 22 f. $1-4$. 1903, M. 1., Sars, p. 112 t. $75,76$.

१. Vorderrumpf wenig über $1 \frac{1 / 2}{2}$ mal so lang wie der Hinterrumpf; Seitenecken des letzten Thsegm. abgerundet; Gensegm. etwa so lang wie die gleich langen letzten beiden Segm. zusammen; Furka etwas länger als das

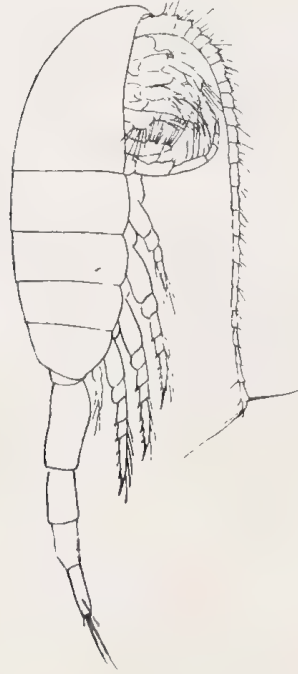

a

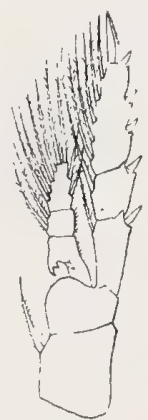

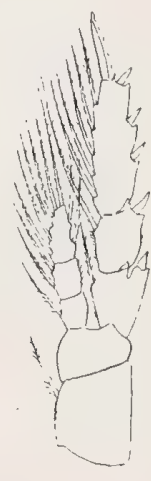

c

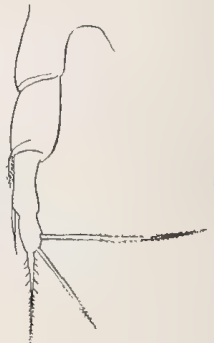

d

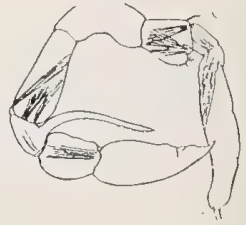

Ansegm. und fast $8 \mathrm{mal}$ so lang wie breit. 1. Antenne reicht kaum bis zum Hinterrand des Gensegm.; Zacken am Grundgl. schwach. Enddorn des Exp. des 4. B. halb so lang wie das Endgl. 5. B. 4-gldr.; Endgl. mit 3 langen, gefiederten Borsten. - $\sigma^{7}$. 5. B. rechts mit langem Dorn, links ohne Dorn am Innenrande; die beiderseitigen Endgl., zumal das rechte, distal verschmälert. - L. क 4,1-4,3 mm, o $3,5-3,7 \mathrm{~mm}$.

Nord-Atlant. Ozean; Polarmeer; Ind. Ozean.

\section{Metridia lucens Boeck.}

1864, M. 1., Boeck, p. 14. 1873, Paracalanus hibernicus, Brady and Robertson, p. 126. 1878, M. armata, Brady, I, p. 42. 1892, M. hibernica, 
Giesbrecht, p. 340 , t. 33 f. 2, 12, 16, 22, 28, 36, 39. 1894, M. 1., Dahl, p. 11. 1898, M. 1., Giesbr. und Schmeil, p. 106. 1903, M. 1., Sars, p. 113, t. 77.

Q. Vorderrumpf ca. $1 \frac{2 / 3}{3}$ mal so lang wie der Hinterrumpf; Seitenecken des letzten Thsegm. ein wenig zugespitzt; Kopf in Lateralansicht hoch gewölbt. Gensegm. etwas kürzer als die beiden letzten Segm. zusammen; Ansegm. 3/4 so

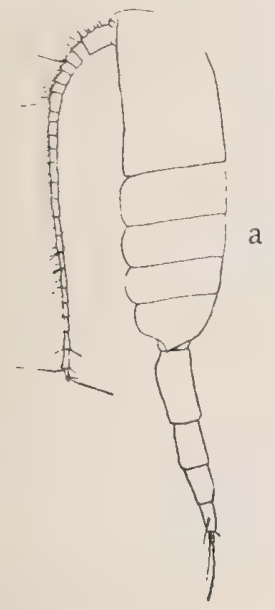

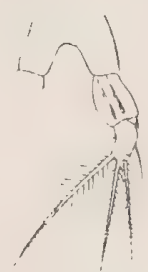

b

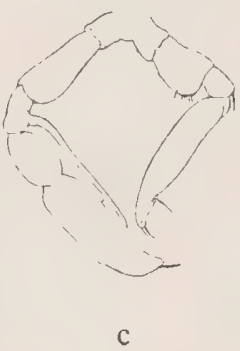

Fig. 124. Metridialucens.

a ㅇ lateral, b 5. B. ㅇ, c 5. B. o

a nach Sars, $b-c$ nach Giesbrecht.

lang wie das vorhergehende Segm.; Furka etwas kürzer als das Ansegm., doppelt so lang wie breit. 1. Antenne ähnlich wie bei M. $10 \mathrm{nga}$. Enddorn des Exp. des 4. B. wenig über $1 / 4$ so lang wie das Endgl. 5. B. 3-gldr., mit längeren Borsten am Endgl. - $\sigma^{7}$. 5. B. ähnlich wie bei M. longa, aber die Endgl. distal weniger verschmälert. - L. † 2,5-2,9 mm, ơ $2-2,3 \mathrm{~mm}$.

Mittelmeer, Nord-Atlant. Ozean bis Island; Pacif. Ozean; Golf von Suez.

Anm. Diese Art gehört zu den wenigen Copepodenarten, welche an dem Leuchten des Meeres Anteil nehmen und zwar in hervorragender Weise. Nach Farran (1903, p. 12) soll sie z. B. an der irischen Küste im Frühjahr das Meerleuchten hauptsächlich verursachen.

\section{Metridia normani Giesbrecht.}

1892, M. n., Giesbrecht, p. 340 , t. 33 f. 1, 6, 24, 25, 30. 1898, M. n., Giesbr. und Schmeil, p. 107. 1904, M. n., Wolfenden, p. 125, t. 9 f. 39, 40.
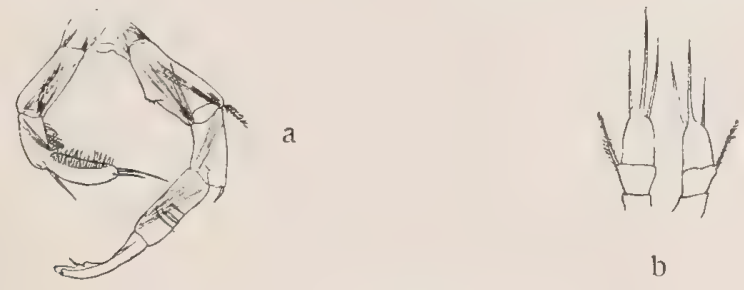

Fig. 125. Metridia normani.

a 5. B. 5, b 5. B. 오 a nach Giesbrecht, b nach Wolfenden. 
१. 1: Antenne das Rumpfende erreichend; Zacken der Basalgl. lang, gerade. Gensegm. kürzer als die zwei yorhergehenden Segm. zusammen; Furka länger als das Ansegm., ca. $4 \mathrm{mal}$ so lang wie breit, rechts kürzer als links. Enddorn des Exp. des 4. B. halb so lang wie das Endgl. 5. B.: linkes B. länger als das rechte und 3 -gldr. mit 3 Endborsten, von welchen die mittlere am längsten ist, und eine lange, äußere Randborste am 2. Segm.; rechtes B. undeutlich 3-gldr., mit 2 Endborsten von gleicher Länge. - $\sigma^{7}$. Beide B. des 5. Paares ohne Dorn am Innenrande. - L. † 2,5 mm, o $2,6 \mathrm{~mm}$.

Nordatlant. Ozean.

\section{Metridia venusta Giesbrecht.}

1889, M. v., Giesbrecht, p. 24. 1892, M. v., Giesbrecht, p. 340, t. 33 f. 7, 17, 29. 1898, M. v., Giesbr. und Schmeil, p. 107. Non 1903, M. v., Thompson, p. 24, t. 6 f. 1, 2 (= Hederorhabdus spec.)

q. Vorderrumpf wenig über $1 \frac{1 / 2}{2}$ al so lang wie der Hinterrumpf. Rechter Furkalzweig etwas kürzer als der linke. Gensegm. etwas kürzer als die beiden letzten Segm. zusammen; Ansegm. $2 / 3$ so lang wie das vorhergehende Segm.; Furka $1 \frac{1}{3}$ mal so lang wie das Ansegm. und 4 mal so lang wie breit. 1. Antenne erreicht das Rumpfende; Zacken am Grundgl. lang. 5. B. 2-gldr., asymmetrisch. - o unbekannt. - L. O 2,9-3,3 mm.

Nord-Atlant. Ozean; Pacif. Ozean.

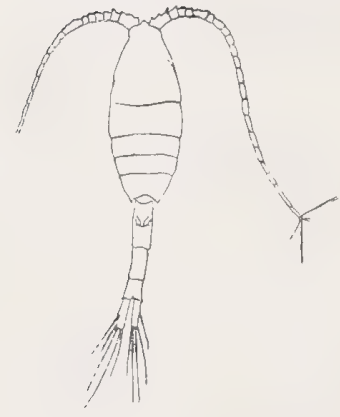

a

Fig. 126. Metridia venusta. a o dorsal, b 5. B. ㅇ. Nach Giesbrecht.

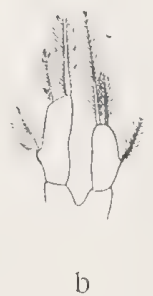

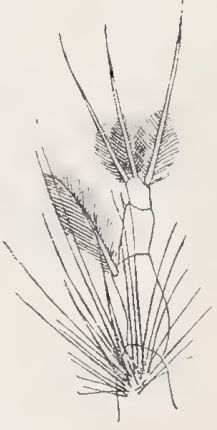

a

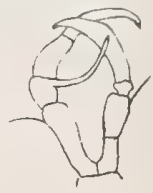

b

Fig. 127. Metridia princeps. a 5. B , b 5. B. ơ. a nach Giesbrecht, b nach Thompson.

\section{Metridia princeps Giesbrecht.}

1889, M. p., Giesbrecht, p. 24. 1892, M. p., Giesbrecht, p. 340, t. 33 f. 3, 18, 35, 40. 1898, M. p., Giesbr. und Schmeil, p. 107. 1903, M. p., Thompson, p. 24, t. 6 f. 3,4 . 
․ Vorderrumpf wenig über $1 \frac{1}{2}$ mal so lang wie der Hinterrumpf. Gensegm. länger als die letzten beiden Abdsegm. zusanmen; Ansegm. halb so lang wie das vorhergehende Segm.; Furka 2 mal so lang wie das Ansegm. und $5 \mathrm{mal}$ so lang wie breit. 1. Antenne überragt das Rumpfende. Enddorn des Exp. des 4. B. kaum 1/3 so lang wie das Endgl. 5. B. 4-gldr., am 1. Gl. ein Büschel langer Haare. - $\sigma^{7}$. Furka sehr wenig asymmetrisch; 5. B. beiderseits 4-gldr. (?), beide Endgl. lang und kräftig, distal verschmälert. — L. q $8,5 \mathrm{~mm}$, के $6,5 \mathrm{~mm}$.

Atlant. Ozean; Pacif. Ozean.

\section{Metridia brevicauda Giesbrecht.}

1889, M. b., Giesbrecht, p. 24. 1892, M. b., Giesbrecht, p. 340 , t. 33 -f. $5,10,11,14,21,32.1898$, M. b., Giesbr. und Schmeil, p. 108.

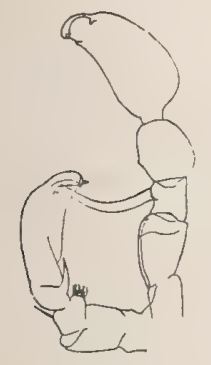

a

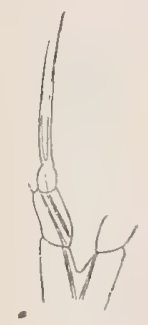

b

Fig. 128.

Metridia brevicauda. a 5. B. or, b 5. q. Nach Giesbrecht.

†. Vorderrumpf über $2 \mathrm{mal}$ so lang wie der Hinterrumpf. Gensegm. etwas kürzer als die beiden letzten gleich langen Abdsegm. zusammen. Furka so lang wie das Ansegm. und weniger als doppelt so lang wie breit. 1. Antenne reicht bis zum Rumpfende; Zacken am Grundgl. schwach. Enddorn des Exp. des 4. B. halb so lang wie das Endgl. 5. B. 3-gldr., mit 2 längeren Borsten am Endgl. - $\sigma^{7}$. Rechtes 5. B. mit kurzem, linkes mit langem Dorn am Innenrande. - L. क 2,1-2,2 mm, o $1,5-1,65 \mathrm{~mm}$.

Nord-Atlant. Ozean; Pacif. Ozean.

\section{Lucicutia Giesbrecht.}

Q. Kopf breit und vom Thorax getrennt; die beiden letzten Thsegm. verschmolzen. Rostralfäden fein und weich. Abdomen 4-gldr., meistens symmetrisch. Furka symmetrisch. 1. Antenne 24-gldr. Exp. der 2. Antenne 8-gldr.; Mandibel mit schwacher Lade; die folgenden Mundgliedmaßen Calanidenähnlich; Außenlobus der 1. Max. mit 5 Borsten; Enp. 2-gldr., mit dem Basp. artikulierend; Exp. groß, oval. Enp. des 1. B. 2- oder 3-gldr.; alle übrigen Enp. und die Exp. des 1.-4. B. 3-gldr.; Endgl. des Enp. des 2. B. mit 5 oder 6 Borsten; 2. Gl. des Basp. des 1. B. zuweilen mit röhrenartigem Fortsatz; 5. B. den vorhergehenden ähnlich, mit pfriemförmiger Innenrandborste am 2. Gl. des Exp. - đ. Greifantenne links. 5. B. ein schwimmbeinähnliches Greifbein, rechts mit 2-, links mit 3-gldr. Exp. und Enp.; Endgl. des rechten Exp. hakig und gegen das proximale Gl. beweglich. 
1. Enp. des 1. B. 3-gldr.

2. Rumpflänge über $5 \mathrm{~mm}$ (Gensegm. asymmetrisch)

Dieselbe unter $3 \mathrm{~mm}$

3. 1. Antenne überragt die Mitte der Furka; Enddorn des Exp. des 5. B. halb so lang wie das Endgl.

L. flavicornis

1. Antenne kaum länger als der Rumpf; Enddorn des Exp. des 5. B. 3/4 so lang wie das Endgl.

L. curta

1. Antenne überragt das Rumpfende um 3-4 Gl.

L. longicornis

4. Enddorn des 5. B. so lang wie das Endgl.

L. longiserrata

Derselbe höchstens halb so lang

5. 1. Antenne erreicht das Ende der Furka

1. Antenne überragt das Rumpfende wenigstens um die 4 letzten Gl.

L. atlantica.

1. Enp. des 1. B. 3-gldr.

$\left.\sigma^{7} \cdot{ }^{2}\right)$

Enp. des 1. B. 2-gldr.

2. Rumpflänge über $5 \mathrm{~mm}$

Dieselbe unter $2,5 \mathrm{~mm}$

L. clausi

1. Antenne reicht nicht bis zum Rumpfende

- Antenne überragt das Rumpfende un die 3

4. Enp. des rechten 5. B. kürzer als das 1. Gl. des Exp.; 2. Basp. des rechten 5. B. ohne Innenranddorn

L. clausi

Enp. des rechten 5. B. so lang wie das 1. Gl. des Exp.; 2. Basp. des rechten 5. B. mit kräftigem Dorn am Innenrande

L. magna.

\section{Lucicutia flavicornis Claus.}

1863, Leuckartia f., Claus, p. 186, t. 32 . 1892, Leuckartia f., Giesbrecht, p. 358, t. 5 f. 4 ; t. 19 f. $2,3,15-17,21,23,29,38$; t. 38 f. $38,40$. 1898, L. f., Giesbr. und Schmeil, p. 111. Non 1883, Leuckartia f., Brady, p. 50.

†. Ansegm. kürzer als das vorhergehende Segm.; 2. Endborste der Furka dick und doppelt so lang wie das Abdomen. 1. Antenne überragt die Mitte der Furka; proximale Gl. mit dicken Aesthetasken. Enp. des 1. B. 3-gldr.; Enḍdorn des 2.-4. B. höchstens halb so lang wie das Endgl.; Enp. des 5. B. reicht fast zum distalen Rande des 2. Gl. des Exp.; 1. Gl. des Exp. viel kürzer als das 3. Gl., welches doppelt so lang wie der Enddorn ist. - $-\sigma^{\top}$. Endstück der Greifantenne (distal vom Kniegelenk) etwas länger als die 5 vorhergehen-

1) Das $\&$ von L. magna ist unbekannt (s. Anmerkung bei L. atlantica).

$\left.{ }^{2}\right)$ Die Männchen von L. atlantica (s. Anmerkung), curta und longiserrata sind unbekannt. 
den Gl. zusammen. Enp. des rechten 5. B. gestreckt, mit 5 apikalen Borsten. - L. O 1,4-1,75 mm, of $1,35-1,7 \mathrm{~mm}$.

Mittelmeer, Atlant. Ozean; Pacif. Ozean; Ind. Ozean.

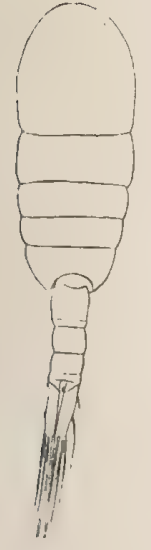

a

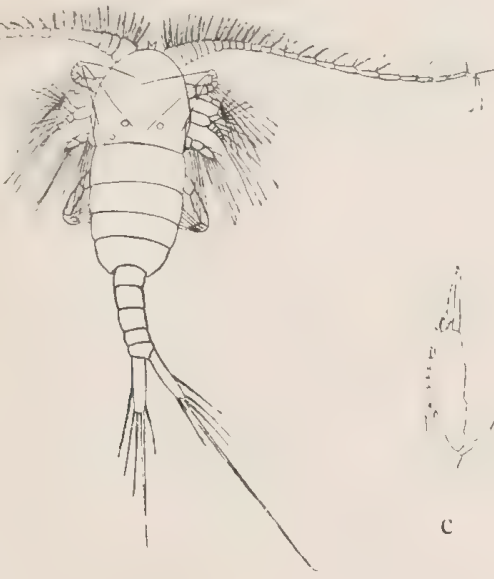

b

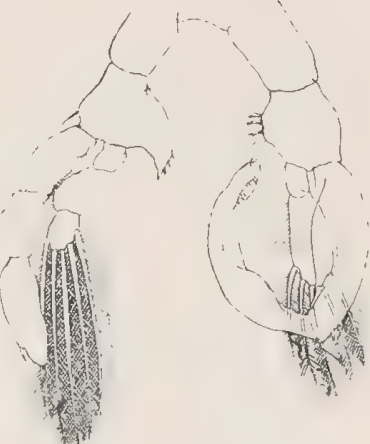

d

Fig. 129. Lucicutia flavicornis.

a $q$ dorsal, b o dorsal, c distaler Teil des Exp. des 5. B. \&, d. 5. B. o . Nach Giesbrecht.

\section{Lucicutia longicornis Giesbrecht.}

1889, Leuckartia l., Giesbrecht, p. 812. 1892, Leuckartia l., Giesbrecht, p. 359 , t. 19 f. 7,30 ; t. 38 f. 39.1898 , L. 1., Giesbr. und Schmeil, p. 111.

Verwandt mit L. flavicornis; aber die 1. Antenne überragt das Rumpfende um die $3-4$ letzten Gl, bei $q$ und $\sigma^{x}$ mit relativ längeren Gl. des distalen Abschnittes. - L. ㅇ 1,8-2 mm, or 1,8 mm.

Atlant. und Pacif. Ozean.

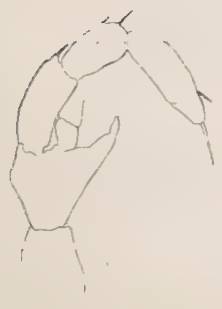

b

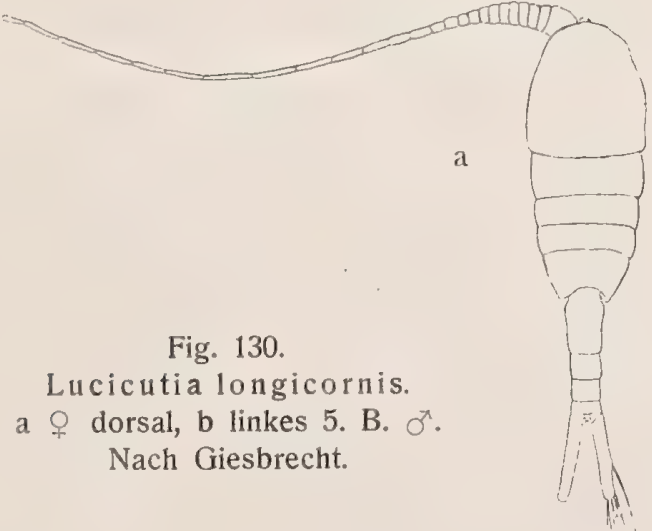

VIII 8 


\section{Lucicutia grandis Giesbrecht.}

1895, Leuckartia g., Giesbrecht, p. 258, t. 4 f. 4 ( ( $)$. 1904, L. g. (vel nov. sp.), Wolfenden, p. 121 , t. 9 f. 37,38 (q).

१: 1. Antenne das Rumpfende um die 4 letzten Gl. überragend. Gensegm. asymmetrisch. Alle Exp. und Enp. 3-gldr. - o . Ansegm. so lang wie das vorhergehende Segm. 1. Antenne reicht fast bis zum Rumpfende; Endstück

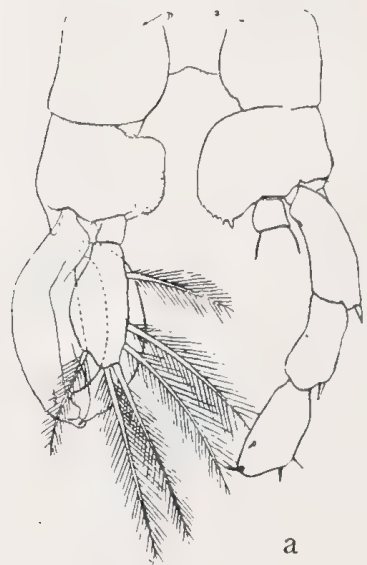

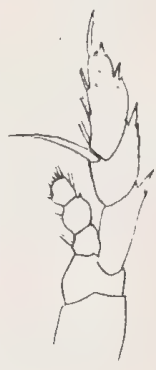

b

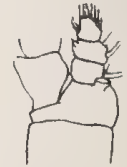

c

Fig. 131. Lucicutia grandis.

a 5. B. $O^{7}$, b 5. B. Q, c. 1. B. ․ a nach Giesbrecht, $b$ und $c$ nach Wolfenden.

der Greifantenne etwas kürzer als die 5 vorhergehenden Gl. Enp. des 1. B. 3-gldr. Enp. des rechten 5. B. dem von L. cla usi ähnlich; innere Hälfte des distalen Gl. des Basp. beider B. wulstig. - L. \& $6,5 \mathrm{~mm}$, or $6 \mathrm{~mm}$.

Nordatlant. Ozean (Wolfenden); Pacif. Ozean (Giesbrecht).

Anm. Ob das von Wolfenden kurz beschriebene Weibchen (I. c) wirklich zu dieser Art, von der Giesbrecht nur das Männchen kannte, gehört, ist unsicher.

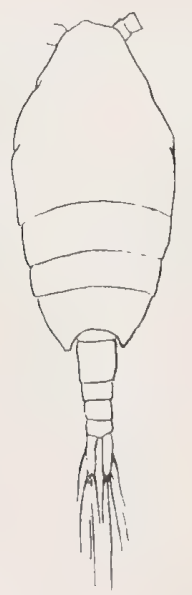

Fig. 132 .

Lucicutia curta.

a $q$ dorsal, b 5. B. $q$. Nach Farran.

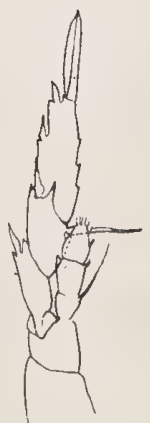

b 


\section{Lucicutia curta Farran.}

1905, L. c., Farran, p. 44, t. 12 f. $1-7$.

Q. Verwandt mit L. flavicornis und longicornis. 1. Antenne etwas länger als der Körper, mit kleinen und undeutlichen Aesthetasken. 1. B. mit 3-gldr. Enp. 5. B.: Außenranddorn des Exp. länger und schlanker als bei L. flavicornis; Enddorn ${ }^{3} / 4$ so lang wie das Endgl. L. $2,4 \mathrm{~mm}$. - $\sigma^{7}$ unbekannt. Nordatlant. Ozean.

Anm. Die Art unterscheidet sich von L. longicornis durch die größere Länge, den kräftiger gebauten Körper mit kürzerem Abdomen, die kürzeren Furkaläste und Antennen.

\section{Lucicutia clausi Giesbrecht.}

1889, Leuckartia clausii, Giesbrecht, p. 812. 1892, Leuckartia clausii, Giesbrecht, p. 359 , t. 19 f. 5, 6, 12-14, 24, 27; t. 38 f. 37.1898 , L. c., Giesbr. und Schmeil, p. 111.

ๆ. Seitenrand des Kopfes öfters mit einer Zacke jederseits; Ansegm. länger als das vorhergehende Segm.; 2. Endborste der Furka kürzer als das Abdomen. 1. Antenne erreicht das Rumpfende; Aesthetasken dünn. Enp. des 1. B. 2-gldr. Enp. des 5. B. reicht lange nicht bis zum distalen Rande des

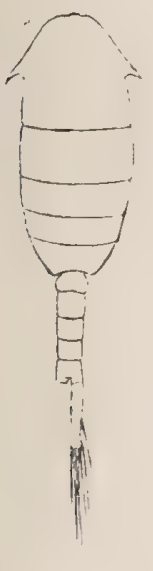

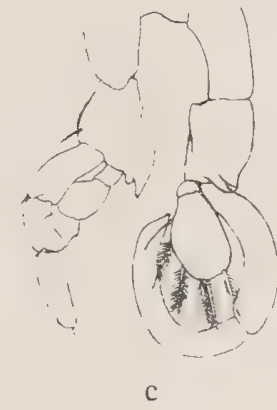

Fig. 133. Lucicutia clausi.

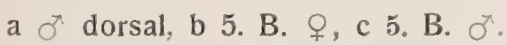
Nach Giesbrecht.
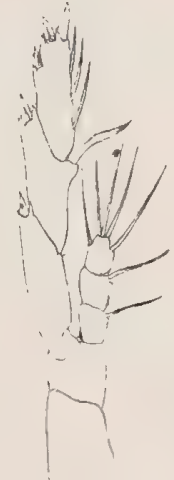

b

2. Gl. des Exp.; 1. G1. des Exp. wenigstens so lang wie das Endgl., welches wenigstens $3 \mathrm{mal}$ so lang wie der Enddorn ist. - $\sigma^{7}$. Distales Stück der Greifantenne etwas kürzer als die 5 vorhergehenden Gl. Enp. des 5 . rechten B. blattförmig, oval, mit 6 Borsten, die z. T. am Innenrande sitzen. - L. \& $1,9-2,05 \mathrm{~mm}, \sigma^{7} 1,8-1,85 \mathrm{~mm}$.

Mittelmeer, Nordatlant. Ozean; Pacif. Ozean. 


\section{Lucicutia longiserrata Giesbrecht.}

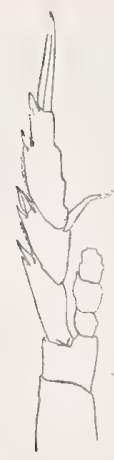

Fig. 134. Lucicutia 10 ngiserrata 5 . B. 9 .

Nach Giesbrecht.

1889, Leuckartia 1., Giesbrecht, p. 813. 1892, Leuckartia 1., Giesbrecht, p. 359 , t. 19 f. 18,25 ; t. 38 f. 36.1898 , L. 1., Giesbr. und Schmeil, p. 112.

Q. 1. Antenne überragt mit dem Endgl. das Rumpfende; mit dicken Aesthetasken. Enp. des 1. B. 2-gldr. Enddorn des Exp. am 3. und $4 \mathrm{~B}$. halb so lang wie das Endgl., am 5. B. fast so lang wie dasselbe. L. 2,2 $\mathrm{mm}$. o unbekannt.

Nordatlant. Ozean; Pacif. Ozean.

\section{Lucicutia atlantica Wolfenden.}

1904, L. a., Wolfenden, p. 121. 1905, L. a., Farran, p. 44 , t. 13 f. $5-10$.

१. 1. Antenne das Körperende um die letzten 4 Gl. überragend. 1. B. mit 2-gldr. Enp.; Enddorn des 2: B. 1/3 so lang wie das Endgl. 5. B.: Enddorn 1/2 so lang wie das Endgl., der Rand nicht gezackt wie bei L. flavicornis, die dicke Innenrandborste fast $2 / 3$ so lang wie das Endgl. und am distalen Ende leicht gesägt. L. $3,5 \mathrm{~mm}$. - o unbekannt.

Nordatlant. Ozean.

Anm. Wolfenden hat diese Form provisorisch als neue Art beschrieben, obgleich es sich vielleicht nur um das Weibchen von L. magna, von welcher Art er nur das Männchen gefunden hat, handelt.
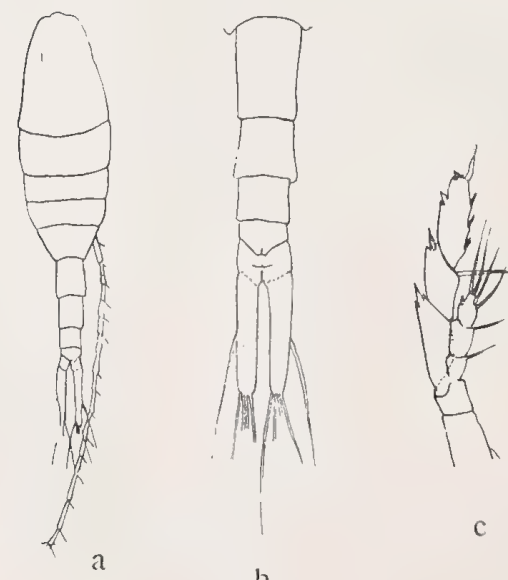

Fig. 135. Lucicutia atlantica. a $q$ dorsal, b Abd. $q$, dorsal, c 5. B. ㅇ. Nach Farran.

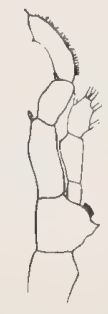

a

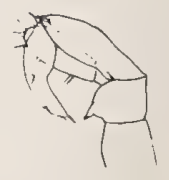

b

Fig. 136. Lucicutia magna. a Linkes, b rechtes 5. B. $\sigma^{r}$. Nach Wolfenden. 


\section{Lucicutia magna Wolfenden.}

1904, L. m., Wolfenden, p. 121, t. 9. f. 35, 35 a.

q unbekannt (s. Anmerkung). - o . 1. Antenne das Rumpfende um $1 \frac{1}{2}$ Gl. überragend. Enp. des 1. B. 2-gldr. Rechtes 5. B. mit kräftigem dornartigem Anhang an der Innenseite des 2. Basp. und mit 2-gldr. Exp.; linkes 5. B. mit 3-gldr. Exp. und Enp. L. 3,54 mm.

Nordatlant. Ozean.

Anm. Das zu dieser Art gehörige Weibchen ist vielleicht die oben beschriebene L. atlantica.

\section{Isochaeta Giesbrecht.}

Verwandt mit Lucicutia; Vorderrumpf im Verhältnis zum Abdomen länger, ellipsoidisch, nach vorne verjüngt; Enp. des 1. B. 3-gldr.; 2. GI. des Basp. des 1. B. mit röhrenartigem Fortsatz; Endgl. des Enp. des 2. B. mit 4 Borsten; Enp. des 5. B. 2-gldr.

\section{Isochaeta longisetosus Thompson.}

1903, I. 1., Thompson, p. 26, t. 7 f. $1-9$.

Q. Mit den Merkmalen der Gattung und einer Länge von $3,5 \mathrm{~mm}$. ơ unbekannt.

\section{Nordatlant. Ozean.}

Anm. Isochaeta ovalis Giesbrecht, der Typus der Gattung, die von Giesbrecht im pacifischen, nacher von Sars auch im atlantischen Ozean gefunden ist, weist nur eine Länge von $1,5 \mathrm{~mm}$ aut.

\section{Heterorhabdus Giesbrecht.}

Q. Kopf vom 1. Thsegm. getrennt; vorletztes und letztes Thsegm. verschmolzen. Rostralfäden schlaff. Abdomen 4-gldr.; linker Furkalast größer als der rechte, mit dem Ansegm. nicht genikulierend, mit ungemein langer 2. Endborste. 1. Antenne 25-gldr. Äste der 2. Antenne etwa gleich lang. Mandibellade mit 3-4 Zähnen; Enp. viel dünner als das Exp. Distale Innenrandloben und Enp. der 1. Maxille sehr zurückgebildet; Exp. lang und rechteckig, mit 5 apikalen Borsten. Proximale Loben und Enp. der 2. Maxille und die Borsten an ihnen sehr verkümmert; distale Loben mit starken Hakenborsten. Enp. des Maxillipeden mit kurzen Borsten. 1.-5. B. mit 3-gldr. Enp. und Exp.; Endgl. des Exp. des 3. B. meistens abweichend, breit, oval. Innenrandborste des 2. GI. des Exp. des 5. B. säbelförmig. - $\sigma^{7}$. Greifantenne links. Endgl. des 5. B. beiderseits 3-gldr.; Exp. meistens hakig, rechts mit Fortsätzen am 2. Gl. des Exp. und am 2. Gl. des Basp. eine unvollkommene Zange bildend. 
1. Endgl. des Exp. am 3. B. wie am 2. und 4. gebaut; Innenrandborsten am 1. und 2. Gl. des Enp. des 5. B. beim o verdickt Endgl. des Exp. des 3. B. breit, oval; Innenrandborsten am 1. und 2. Gl. des Enp. des 5. B. beim $q$ nicht verdickt

2. 1. Antenne überragt das Rumpfende wenig H. grimaldi 1. Antenne überragt das Rumpfende um die 8-9 letzten Gl. 3

3. Länge $3 \mathrm{~mm}$

H. longicornis Länge $5 \mathrm{~mm}$

H. major

4. 1. Gl. des Basp. des Maxillipeden in der Mitte des Innenrandes mit dünner Borste

1. Gl. des Basp. des Maxillipeden daselbst mit auffallend dicker, langer Borste

5. Körper kurz und gedrungen; Hinterkörper kaum $1 / 3$ so lang wie der Vorderkörper

H. compacta

Körper schlank; Hinterkörper über halb so lang wie der Vorderkörper

6. Rostralpapille mit hervortretender Spitze

H. vipera

Dieselbe ohne die Spitze

H. spinifrons

7. 1. Antenne und 5. B. symmetrisch gebaut

Eine der 1. Antennen ein Greiforgan; 5. B. asymmetrisch

8. Borste am 2. Gl. des Enp. des 5. B. viel dünner und kürzer als die Borsten des 3. Gl.

Borste des 2. Gl. des Enp. des 5. B. kaum dünner und kürzer als die Borsten des 3. Gl.; 1. Gl. des Exp. des 5. B. ohne Innenrandborste 10

9. Innenrandborste des 1. Gl. des Exp. des 5. B. dick H. clausi 우 Dieselbe fehlt

H. (abyssalis $\bigcirc)$ ?

10. Von den 3 distalen Hakenborsten der 2. Maxille ist eine kürzer und dünner als die beiden anderen; am vorhergehenden Lobus 2 lange Hakenborsten und 1 kleine dünne Borste H. norvegicus $q$

Die 3 distalen Hakenborsten der 2. Maxille etwa gleich lang und stark; die dünnere von den 3 Borsten des vorhergehenden Lobus über halb so lang wie die beiden anderen

H. papilliger o

11. 2. Gl. des rechten Exp. des 5. B. stark verbreitert oder mit großem Fortsatz; am Innenrande des 2. Gl. des rechten Basp. ein steifer, aufrecht stehender Fortsatz oder ein kürzerer Anhang

Das 2. Gl. des rechten Exp. hat nur einen (behaarten) Vorsprung, das 2. Gl. des rechten Basp. abẹr einen langen, wurstförmigen, gebogenen und distal verbreiterten Fortsatz.

12. 2. Gl. des rechten Exp. am proximalen Teil des Innenrandes mit großem Fortsatz; am Innenrande des 2. Gl. des rechten Basp. ein kürzerer Anhang H. papilliger $\sigma$ Innenrand des 2. Gl. des rechten Exp. in seiner ganzen Länge stark ver- 
breitert; 2. Gl. des rechten Basp. mit langem, steifem, aufrecht stehendem Anhang

H. longicornis o

13. Distale Borste am Endgl. des rechten Exp. des 5. B. überragt das Gliedende wenig

H. norvegicus $\sigma^{7}$

Dieselbe ragt um mehr als ihre Hälfte über das Gliedende hinaus 14

14. Innenrand des 2. Basp. des linken 5. B. mit behaartem Vorsprung; 1. Antenne überragt das Rumpfende nur wenig

H. clausi o Innenrand des 2. Basp. des linken 5. B. konvex und in seiner ganzen Ausdehnung behaart; 1 . Antenne überragt das Rumpfende um die 3-4 letzten Gl.

H. abyssalis $\sigma^{\pi}$.

\section{Heterorhabdus spinifrons Claus.}

1863, Heterochaeta s., Claus, p. 182, t. 32 . 1892, Heterochaeta s., Giesbrecht, p. 372 , t. 20 , f. 1, 3, 11, 16, 19, 31; t. 39 f. $42,43,51,52,54$. 1898 , H. s., Giesbr. und Schmeil, p. 114 . Non 1883, Heterochaeta s., Brady, p. 49.

Stimpapille in eine Spitze verlängert. 1. Antenne überragt das Rumpfende um die 4-5 letzten Gl. Unter den 3 distalen Hakenborsten der 2. Maxille ist eine kürzer und dünner als die beiden anderen; am vorhergehenden Lobus 2 lange Hakenborsten und 1 kleine dünne Borste. Am Ende des Innenrandes

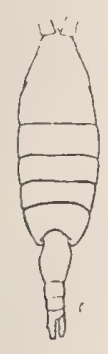

a

b

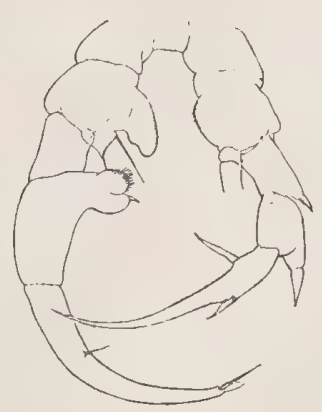

C

Fig. 137. Heterorhabdus spinifrons.

a $\Varangle$ dorsal, b Kopf $q$, lateral, e 5. B. $\sigma^{\top}$. Nach Giesbrecht.

des 1. Gl. des Basp. des Maxp. eine Stachelborste, etwa $1 / 4$ so lang wie die lange, dicke Borste an der Randmitte. Endhaken beider Exp. des 5. B. des $\sigma^{\pi}$ relativ länger als bei papilliger, der linke über doppelt so lang wie die beiden proximalen Gl. des Exp. zusammen; rechtes B. am 2. Basp. mit stark vorspringendem, lamellösem Auswuchs; 2. Gl. des Exp. proximal mit großem Fortsatz; Enddorn wie bei norvegicus. - L. \& 3-3,4 mm, o 2,95-3,4 mm.

Mittelmeer, Atlant. Ozean; Pacif. Ozean; Ind. Ozean. 


\section{Heterorhabdus papilliger Claus.}

1863, Heterochaeta papilligera, Claus, p. 182, t. 32 . 1892, Heterochaeta papilligera, Giesbrecht, p. 372 , t. 20 f. 4, 7, 10, 15, 17, 23, 35, 36; t. 39 f. 40, 53. 1898, H. p., Giesbr. und Schmeil, p. 114.

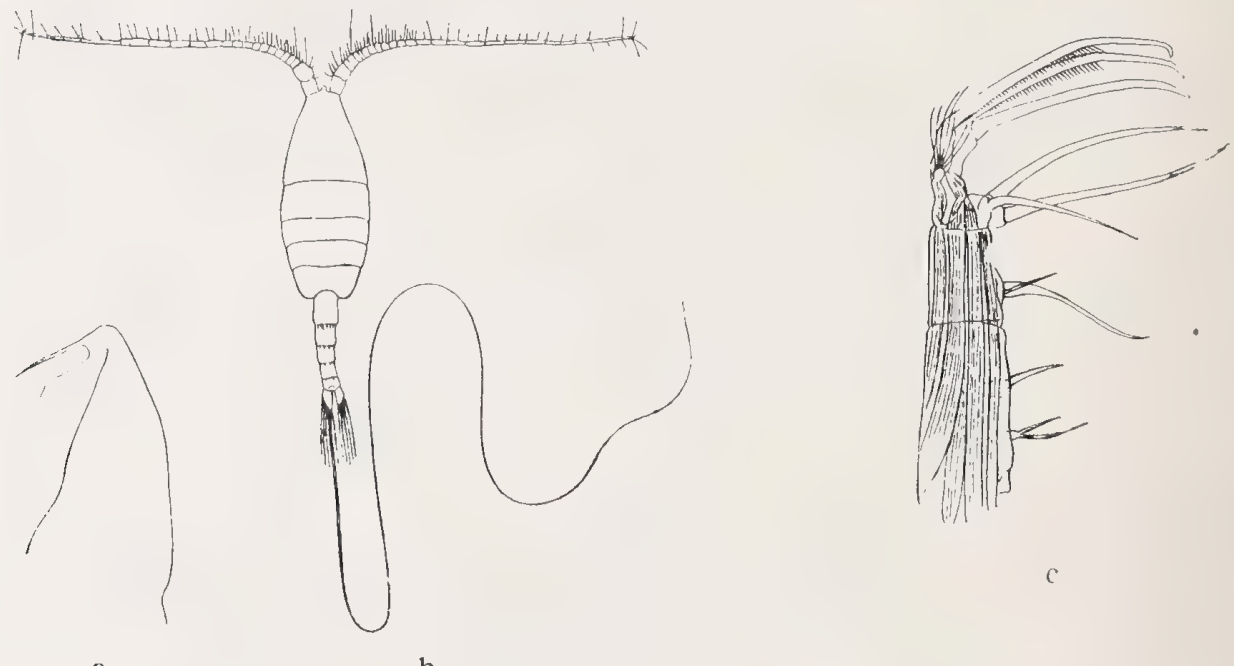

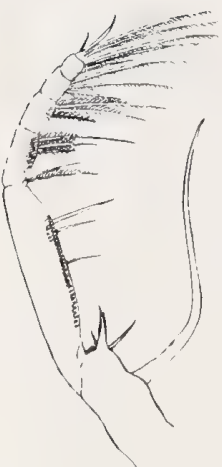

d

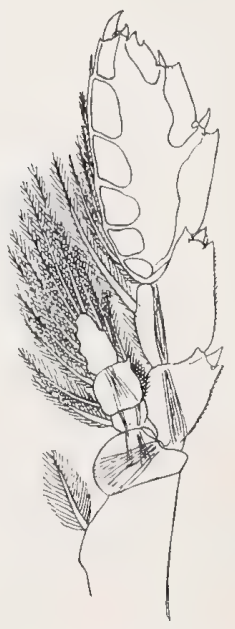

e

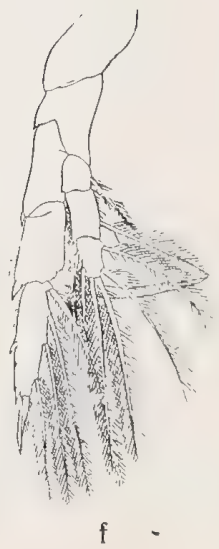

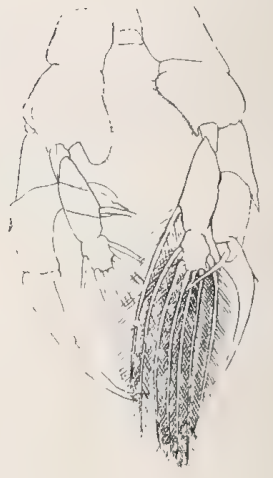

g

Fig. 138. Heterorhabdus papilliger.

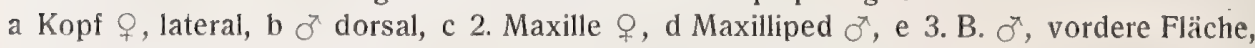
f 5. B. q, g 5. B. ơ. Nach Giesbrecht.

Stirnpapille zugeschärft, aber ohne Spitze. 1. Antenne reicht etwa bis zum Rumpfende. Die 3 distalen Hakenborsten der 2. Maxille etwa gleich lang und stark; die dünnere von den 3 Borsten des vorhergehenden Lobus über 
halb so lang wie die beiden andern. Am Ende des Innenrandes des 1. Gl. des Basp. des Maxillipeden eine Stachelborste, etwa 1/s so lang wie die lange, dicke Borste an der Randmitte. Endhaken beider Exp. des 5. B. des or relativ kürzer als bei spinifrons, der linke wenig länger als die beiden proximalen Gl. des Exp. zusammen; 2. Basp. des rechten B. mit stark vorspringendem, lamellösem Auswuchs; 2. Gl. des Exp. desselben B. proximal mit großem Fortsatz; Enddorn des rechten Exp. wie bei norvegicus. - L. $\bigcirc$ 1,85-2,1 mm, o $1,8-2 \mathrm{~mm}$.

Mittelmeer, Atlant. Ozean; Pacif. Ozean; Ind. Ozean.

\section{Heterorhabdus norvegicus Boeck.}

1872, Heterochaeta norvegica, Boeck, p. 40. 1894, Heterochaeta norvegica, Dahl, p. 80. 1898, H. n.; Giesbr. und Schmeil, p. 115. 1899, Heterochaeta angulata, Aurivillius, p. 32, f. 4, 5. 1900, Heterochaeta norvegica, Sars, p. 79 , t. $23.1903, H$. n., Sars, p. 118, t. $80,81$.

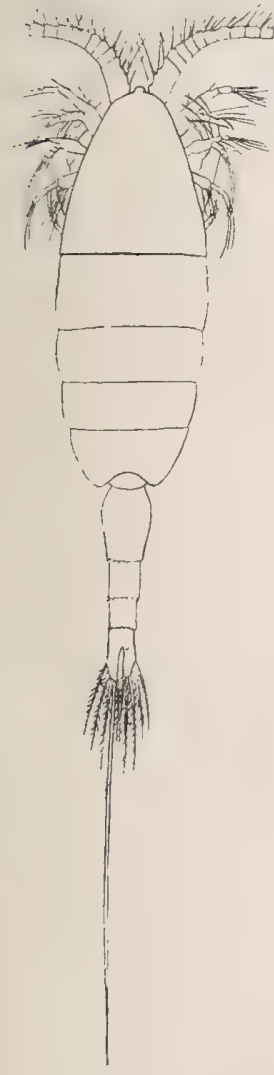

a

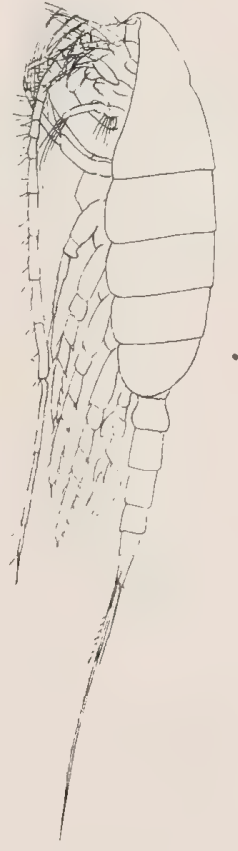

b

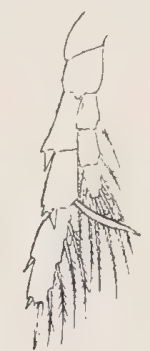

c

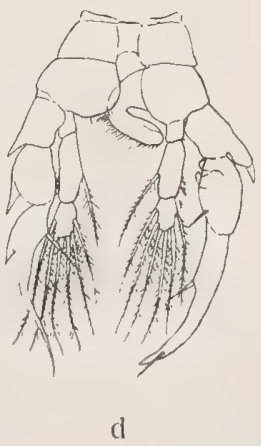

Fig. 139. Heterorhabdus norvegicus.

a $q$ dorsal, b o lateral, c 5. B. ㅇ, d 5. B. $\sigma^{\pi}$. Nach Sars. 
Verwandt mit papilliger; aber: - Unter den 3 distalen Hakenborsten der 2. Maxille ist eine kürzer und dünner als die beiden anderen; am vorhergehenden Lobus 2 lange Hakenborsten und 1 kleine, dünne Borste. Rechtes 5. B. des $\$$ mit großem, gebogenem, vurstförmigem Anhang am 2. Basp. und einem kurzen Vorsprung am 2. Gl. des Exp.; nicht weit vor dem Ende des letzten Gl. des rechten Exp. sitzt eine Borste, die das Gliedende nur um die Länge des Endstückes des Gl. überragt. - L. ㅇ $4,2 \mathrm{~mm}, \sigma^{\top} 3-4 \mathrm{~mm}$.

Nord-Atlant. Ozean, Polarmeer.

\section{Heterorhabdus clausi Giesbrecht.}

1889, Heterochaeta c., Giesbrecht, p. 812. 1892, Heterochaeta c., Giesbrecht, p. 372 , t. 20 f. 2, 28, 37, 38. 1898, H. c., Giesbr. und Schmeil, p. 115. Non 1902, H. c., Wolfenden, ( $=$ H. norvegicus).

Verwandt mit papilliger; aber: - 1. Antenne überragt das Rumpfende etwas; apikale Borsten der 2. Maxille länger, 5. B. des $q$ mit dünnen, kurzen Borsten an den beiden proximalen Gl. des Enp. und mit dicker, hakiger Innen-

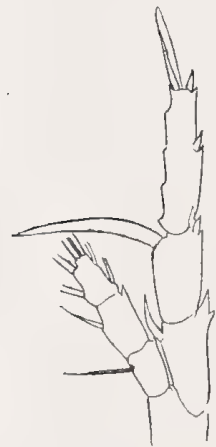

a

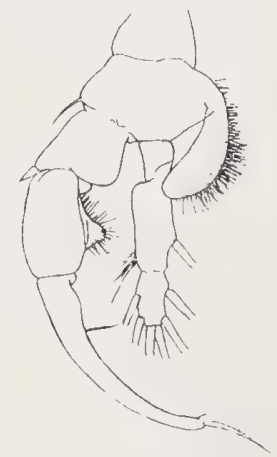

$\mathrm{b}$

Fig. 140. Heterorhabdus clausi. a 5. B. \&, b rechtes 5. B., c linkes 5. B. o7. Nach Giesbrecht.

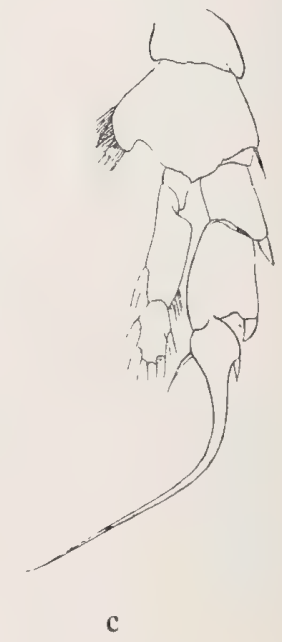

randborste am 1. Gl. des Exp. Endgl. des linken Exp. des 5. B. des o nur an der Basis verdickt mit kurzer Innenrandborste; 2. Gl. des Basp. des rechten B. mit keulenförmigem Anhang; 2. Gl. des rechten Exp. mit mäßig großem, behaartem Auswuchs; die distale Borste am Endgl. des rechten Exp. des 5. B. ragt weit über das Gliedende-hinaus; 2. Gl. des linken Basp. am Innenrande mit behaartem Vorsprung. - L. \& $2,4 \mathrm{~mm}$, o $2,2-2,4 \mathrm{~mm}$.

Atlant. Ozean; Pacif. Ozean; Ind. Ozean. 


\section{Heterorhabdus abyssalis Giesbrecht.}

1889, Heterochaeta a., Giesbrecht, p. 812. 1892, Heterochaeta a., Giesbrecht, p. 373 , t. 19 f. 4 ; t. 20 f. $29,30.1898$, H. a., Giesbr. und Schmeil, p. $116\left(\sigma^{\prime}\right)$. ? 1905, H. a., Farran, p. $45(q)$.

Verwandt mit papilliger; aber:- $\bigcirc$. 1. Antenne überragt das Rumpfende um etwa die 3 letzten Gl.; die innere von den 3 distalen Borsten von der 2. Maxille ist kürzer als die beiden anderen; die dünnere von den

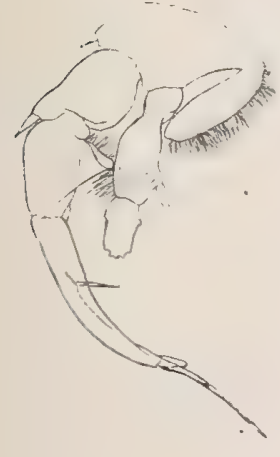

Fig. 141.

Heterorhabdus abyssalis. a rechtes 5 . B., b linkes 5. B. Ø. Nach Giesbrecht.

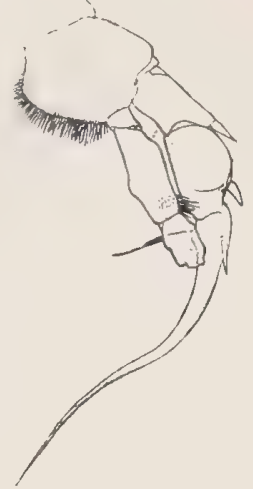

b

3 Borsten des vorhergehenden Lobus ist über halb so lang wie die beiden anderen. Innenrandborste des 1. und 2. Gl. des Enp. des 5. B. sehr dünn, wie bei clausi, aber der hakige Dorn am Innenrande des 1. Gl. des Exp. dieses B. fehlt. - $\sigma^{7}$. 5. B. ähnlich wie bei clausi, aber der ganze Innenrand des 2. Gl. des Basp. des linken B. behaart. - L. क 2,4 mm, ơ 2,75 mm.

Atlant. Ozean; Ind. Ozean.

Anmerkung. Nach Farran (1. c.) gehören die von ihm erwähnten Weibchen wahrscheinlich zu dieser Art.

\section{Heterorhabdus vipera Giesbrecht.}

1889, Heterochaeta v., Giesbrecht, p. 812. 1892, Heterochaeta v., Giesbrecht, p. 373 , t. 20 f. $5,6,12,13,18,20,27,32,33$; t. 39 f. 41.1898 , H. v., Giesbr. und Schmeil, p. 116.

Hinterkörper über halb so lang wie der Vorderkörper. 1. Antenne reicht kaum bis zum Hinterrande des Ansegm. Linke Mandibellade mit sehr langem, ventralem Zahn. Unter den distalen Borsten der 2. Maxille sind nur 2 dick und hakig. Borste an der Randmitte des 1. GI. des Basp. des Maxillipeden von gewöhnlicher Form. Endgl. beider Exp. des 5. B. des ot nicht stilettförmig, sondern breit, gedrungen. - L. ㅇ 2,8 $\mathrm{mm}$, o $2,6-3,7 \mathrm{~mm}$.

Atlant. und Pacif. Ozean. 


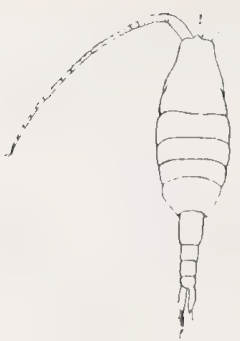

2

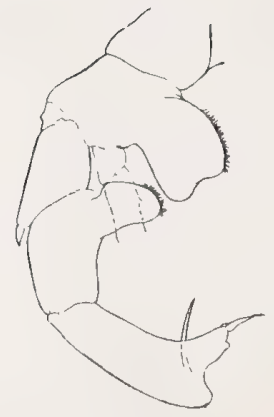

d

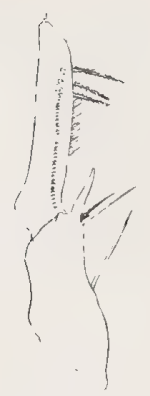

h

Fig. 142 .

Heterorhabdus vipera.

a $q$ dorsal, b Basp. des Maxillipeden $q$, c 5. B. 오, d rechtes 5 . B., e linkes 5. B.

Nach Giesbrecht.

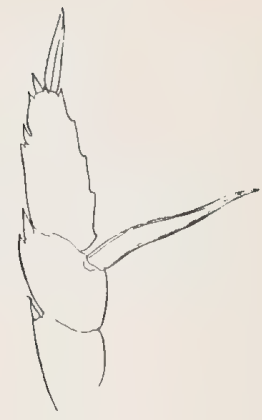

C

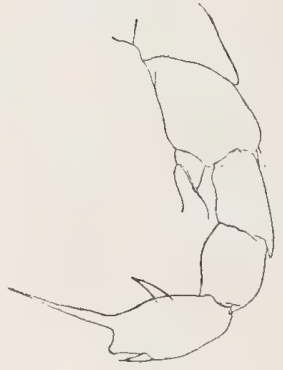

7. Heterorhabdus compactus G. O. Sars.

1900, Heterochaeta compacta, Sars, p. 83, t. 24, 25.

Verwandt mit vipera; aber: - $ๆ$. Körper kurz und gedrungen; Hinterkörper kaum $1 / 3$ so lang wie der Vorderkörper. 1. Antenne ungefähr so lang

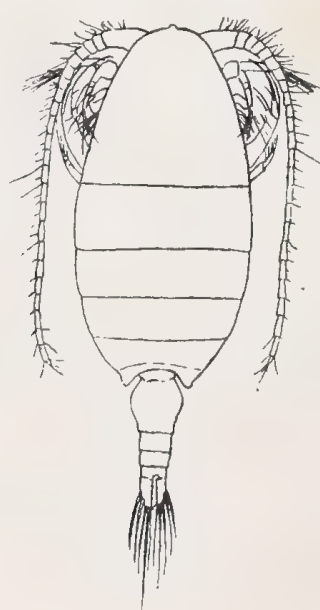

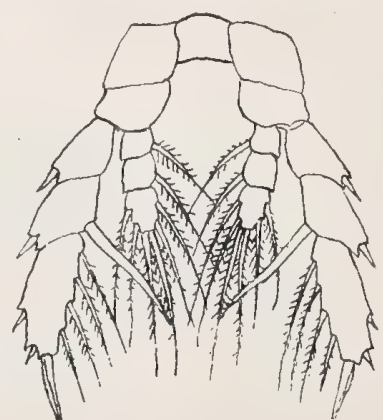

b

Fig. 143. Heterorhabdus compactus. a \& dorsal, b 5. B. ㅇ. Nach Sars. 
wie der Vorderkörper. Ventraler Zahn der linken Mandibellade nur wenig länger als der der rechten. L. 3,35 mm. - o unbekannt.

Polarmeer.

8. Heterorhabdus Iongicornis Giesbrecht.

1889, Heterochaeta 1., Giesbrecht, p. 812. 1892, Heterochaeta 1., Giesbrecht, p. 373 , t. 20 f. $14,21,25,26$; t. 39 f. 44.1898, H. 1., Giesbr. und Schmeil, p. 116. 1902, Heterochaeta zetesios, Wolfenden, p. 367. 1904, H. 1., Wolfenden, p. 124, t. 9 f. 34. 1905, H. 1., Esterly, p. 186, f. 40.

1. Antenne überragt das Rumpfende beim ${ }_{+}$um die $8-9$, beim $\sigma^{\top}$ um die 4 letzten Gl. Zähne der Mandibellade (beim ?) ungefähr gleich dick und fast gleich weit von einander entfernt. 2. Maxille mit zahlreichen distalen Borsten, worunter aber nur 1 bis 2 Hakenborsten. 1. Gl. des Basp. des

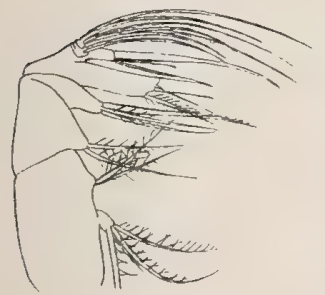

b $\not$

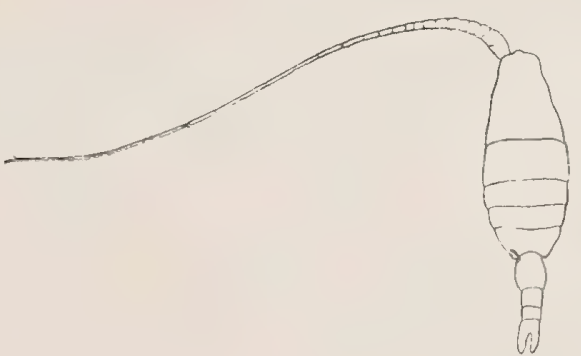

a.

Fig. 144. Heterorhabdus longicornis.

a $q$ dorsal, b 2. Maxille 9 , vordere Fläche, c 5. B. ㅇ. Nach Giesbrecht.

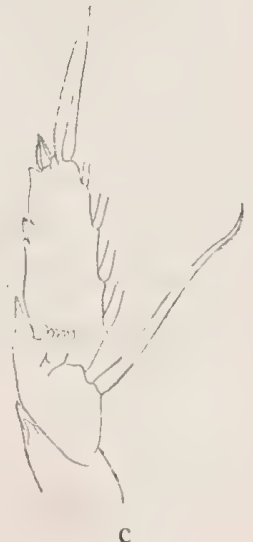

c

Maxillipeden mit Borsten von gewöhnlicher Form. Endgl. des Exp. des 3. B. den der andern Paare ähnlich. Säbelborste des 2. Gl. des Exp. des 5. B. des \& schlank; Innenrandborste des 1. und 2. Gl. des Enp. desselben B. verdickt. Rechtes 5 . B. des of am 2 . Basp. mit einem steifen, aufrecht stehendem Fortsatz; Innenrand des 2. Gl. des Exp. desselben B. in seiner ganzen Ausdehnung lamellös verbreitert; proximaler Teil des Endgl. des linken Exp. des 5. B. verdickt, in einen langen Dorn sich fortsetzend. - L. $\quad 3 \mathrm{~mm}$, o $3-3,5 \mathrm{~mm}$.

Atlant. Ozean; Pacif. Ozean.

9. Heterorhabdus grimaldi J. Richard.

1893, Heterochaeta g., J. Richard, p. 151. 1898, H. g., Giesbr. und Schmeil, p. 117. 
१. Stirn abgerundet, ohne Vorsprung. 1. Antenne überragt nur wenig das Rumpfende. Endgl. des Exp. am 3. B. wie am 2. und 4. B. gebaut. Innenranddorn am 2. Gl. des Exp. des 5. B. schwach, am Ende behaart; die Innenrandborsten des 1. und 2. G1. des Enp. sind in Dornen von gleicher Länge und Dicke umgewandelt, am proximalen Teile gefiedert, am distalen ebenfalls behaart. L. $10,2 \mathrm{~mm}$. - o unbekannt.

Atlant. Ozean (bis etwa $50^{\circ} \mathrm{N}$. Br.).

\section{Heterorhabdus major Dahl.}

1894, Heterochaeta m., Dahl, p. 79.

Verwandt mit $10 \mathrm{ngicornis,}$ aber über $5 \mathrm{~mm}$.

Atlant. Ozean.

\section{Heterorhabdus grandis Wolfenden.}

1904, H. g., Wolfenden, p. 120 , t. 9 f. 36.

. 1. Antenne länger als der Körper; Mandibel ohne lange, gebogene Zähne; Maxilliped ohne den langen Dorn; 5. B. mit 2 kräftigen Dornen am distalen Rande des 2. Gl. des Exp., in derselben Lage wie die Dornen desselben Gl. bei longicornis. L. $6,6 \mathrm{~mm}$. - o unbekannt.

Nord-Atlant. Ozean.

Anmerkung. Es ist vielleicht dieselbe Art wie H. major Dahl, von welcher jedoch keine Beschreibung veröffentlicht ist (Wolfenden, 1. c. p. 121).

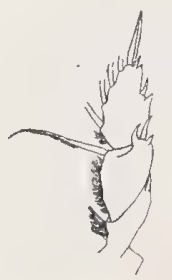

Fig. 145. Heterorhabdus grandis. 5. B. ․ Nach Wolfenden.

Mesorhabdus G. O. Sars.

Weicht von Heterorhabdus in folgendem ab:- Kauloben der Mandibel mit 3 oder 4 dünnen Zähnen und genau von gleicher Form. Loben der 2. Maxille gut entwickelt; von den*Borsten sind nur 2 in klauenförmige Dornen umgewandelt.

Mesorhabdus brevicaudatus Wolfenden.

1905, Heterorhabdus b., Wolfenden. 1905, M. annectens, Sars, Nr. 40, p. 9 . 
ๆ. 1. Antenne etwas länger als der Vorderkörper. Exp. der 2. Antenne kaum halb so lang wie das Enp. Zähne der Mandibelkaulade dünn und alle von gleicher Form. Die 2 Hakenborsten der 2. Maxille ganz glatt. L. 3,35 mm. - or unbekannt.

Nord-Atlant. Ozean.

\section{Haloptilus Giesbrecht.}

․ Kopf vom Thorax getrennt; 4. und 5. Thsegm. verschmolzen. Rostralfäden schlaff. Abdomen 4-gldr., symmetrisch; Furka und Gliedmaßen mit langen, prachtvoll gefiederten Borsten versehen. 1. Antenne 25-gldr. Enp. der 2. Ant. viel länger als das Exp. (bei den $\sigma^{7}$ ist der Größenunterschied zuweilen geringer als bei den $q$ ) ). Mandibel mit zweizinkiger Kaulade und gestrecktem Exp. und Enp.; distale Innenrandloben und Enp. der 1. Maxille klein, Exp. verlängert: 2. Maxille gestreckt, mit kleinen Loben; distale Borsten nicht viel länger als die proximalen. Enp. des Maxp. dick. 1.-5. B. mit 3-gldr. Exp. und Enp. 2. Gl. des Exp. des 5. B. mit zipfelförmiger Innenrandborste oder ohne solche; letzte Innenrandborste des Endgl. des Exp. des 5. B. gewöhnlich verlängert. $-\delta$. Kopf anders geformt als beim $ᄋ ; 1$. Antenne kürzer und reichlicher mit Aesthetasken versehen als beim $q$; Greifantenne links. Mundteile gewöhnlich schwächer gebaut als beim ๆ. 5. B. mit hakigem Endgl. beider Exp., ohne Zange, rechts und links sehr ähnlich.

1. Stirn abgerundet mit medianer Papille oder ohne solche 2 Stirn in einen spitzen Zipfel verlängert

2. 1. Antenne überragt das Rumpfende um die 9-10 letzten Gl.; beide Hakenborsten des 5 . und 6 . Lobus der 2. Maxille dünn H. longicornis 1. Antenne überragt das Rumpfende um die 4-6 letzten Gl.; beide Hakenborsten des 5. und 6. Lobus der 2. Maxille gleich dick und kräftig

H. ornatus

3. Darm vorne ohne Blindsack; Ovidukte erreichen die Mundgegend nicht

H. acutifrons

Darm vorne mit Blindsack; Ovidukte reichen bis in die Stirne $H$. spiniceps.

$$
\sigma^{7} \text {. }
$$

1. Darm an der hinteren Kopfgrenze blind geschlossen H. longicornis Darm mündet wie gewöhnlich im Analsegment

2. Enp. der 1. Maxille mit 3 oder 4 Borsten; Spermatophoren lang und dick

H. fertilis

Enp. der 1. Maxille mit 2 Borsten; Spermatophoren kurz

3. Spermatophore dick; Endgl. des rechten Exp. des 5. B. mit bestachelter, proximaler Außenrandborste

H. ornatus

Spermatophore dünn; Endgl. des rechten Exp. des 5. B. mit glattem, proximalem Außenranddorn

H. spiniceps. 


\section{Haloptilus Iongicornis Claus.}

1863, Hemicalanus 1., Claus, p. 179, t. 29. 1892, Hemicalanus 1., Giesbrecht, p. 384 , t. 1 f. 4 ; t. 2 f. 13 ; t. 27 f. $3,8-10,29$. 1898, H. 1., Giesbrecht und Schmeil, p. 118. 1903, H. 1., Sars, p. 121, t. 82 ; t. 83 f. 1.

Stirn abgerundet, mit medianer Papille, Darm ohne vorderen Blindsack, hinten blind im Thorax endigend. Ovidukte reichen nicht bis zur Mundgegend; Spermatophoren ziemlich lang und dick. 1. Antenne überragt beim ${ }_{q}$ das

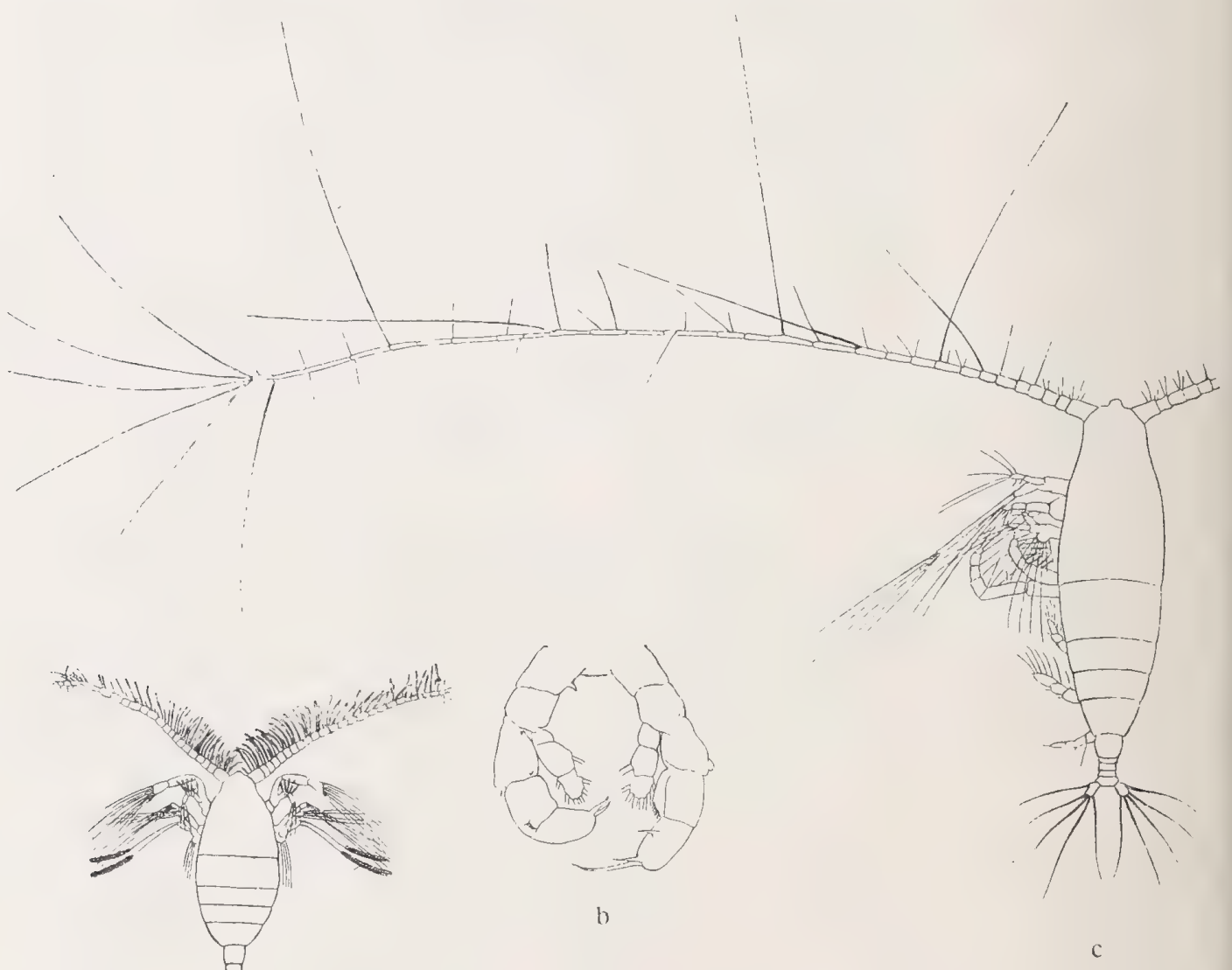

Fig. 146. Haloptilus longicornis. a $\sigma^{7}$ dorsal, b 5. B. $\sigma^{7}$, c 우 dorsal. Nach Giesbrecht.

Rumpfende um die 9-10 letzten Gl. und erreicht es beim $\sigma^{7}$. Enp. der 1. Maxille 2-gldr., mit 5 Borsten. Beide Hakenborsten des 5. und 6. Lobus der 2. Maxille dünn. Endgl. der beiden Exp. des 5. B. des o schwächlich bewaffnet, nur die Endborste des rechten ziemlich lang und dick. - L. $2,1-2,5 \mathrm{~mm}$, o $1,2 \mathrm{~mm}$.

Mittelmeer, Atlant. Ozean; Pacif. Ozean; Ind. Ozean. 


\section{Haloptilus acutifrons Giesbrecht.}

1892, Hemicalanus a., Giesbrecht, p. 384 , t. 3 f. $11 ;$ t. 27 f. $12 ;$ t. 42 f. 12, 20. 1898, H. a., Giesbrecht und Schmeil, p. 119, f. 27b. 1900, Hemicalanus spinifrons, Sars, p. 95, t. 28.1903, H. a., Sars, p. 122, t. 83 f. 2.

१. Stirn zugespitzt. Darm ohne vorderen Blindsack; Ovidukte reichen nicht bis zur Mundgegend. 1. Antenne überragt das Rumpfende etwa um die 7 letzten Gl. Enp. der 1. Maxille 1-gldr., mit 4 Borsten. Hakenborste des 5. Lobus der 2. Maxille dicker als die des 6. L. 2,6-3,2 mm. - o unbekannt.

Mittelmeer, Nord-Atlant. Ozean; Polarmeer.

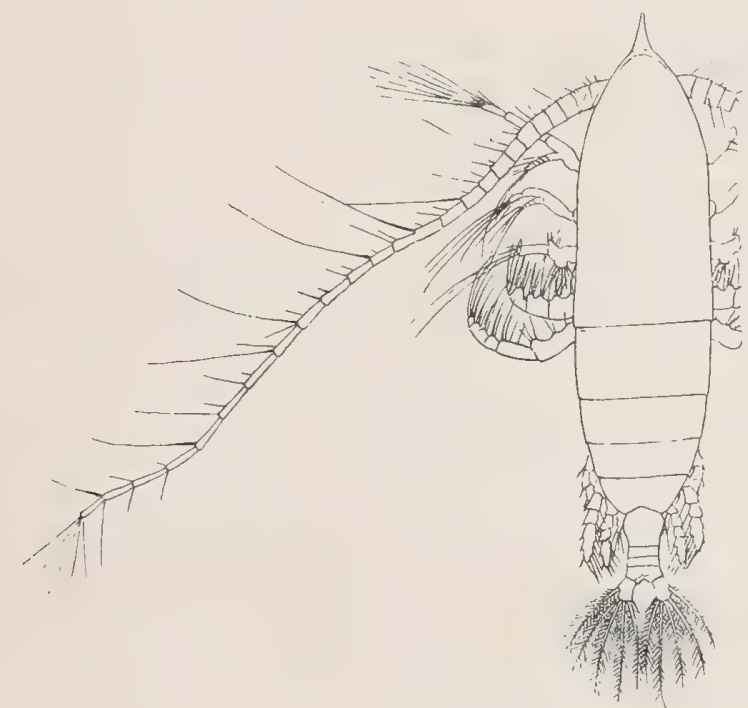

Fig. 147. Haloptilus acutifrons. \& dorsal. Nach Sars.

\section{Haloptilus fertilis Giesbrecht.}

1892, Hemicalanus f., Giesbrecht, p. 384 , t. 27 f. 2,36 ; t. 42 f. 5 . 1898, H. f., Giesbrecht und Schmeil, p. 119.

q unbekannt. - $\sigma^{7}$. Stirn abgerundet. Darm mit vorderem Blindsack; Spermatophore lang und dick. 1. Antenne reicht etwa bis zum Rumpfende; mittlere Gl. der Greifantenne stärker verbreitert als bei den anderen Arten. Enp. der 1. Maxille 1-gldr., mit 3 Borsten. Die beiden Hakenborsten des 5. und 6. Lobus der 2. Maxille gleich dick und kräftig. Endgl. des rechten Exp. des 5. B. mit kräftiger Endborste und bestachelter, proximaler Außenrandborste; Mittelgl. des rechten Exp. mit stark vorspringendem Innenrande. L. 3,2 mm.

Mittelmeer, Nord-Atlant. Ozean (?)

Anm. Farran (1905, p. 45) glaubt, Männchen dieser Art im Atlant. Ozean westlich von Irland gefunden zu haben. 


\section{Haloptilus ornatus Giesbrecht.}

1892, Hemicalanus 0., Giesbrecht, p. 384, t. 27 f. 1, 6, 7, 21, 38; t. 42 f. 1, 9, 19. 1898, H. o., Giesbr. und Schmeil, p. 120, f. 28.

Stirn dreieckig, vorne rund. Darm vorne mit Blindsack; Ovidukte reichen bis in die Stirn; Spermatophore kurz und dick. 1. Antenne überragt beim $q$ das Rumpfende um die $4-5$ letzten Gl. und erreicht es beim $\sigma^{7}$. Enp. der

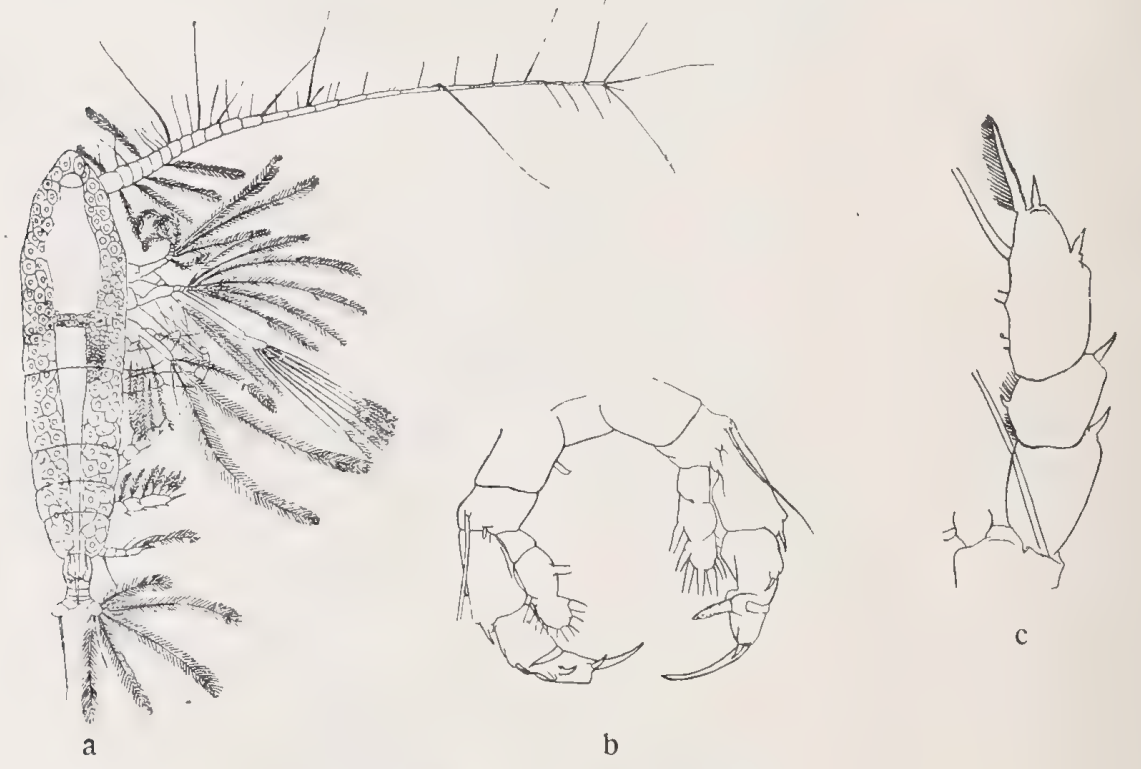

Fig. 148. Haloptilus ornatus.

a $ᄋ$ dorsal, b 5. B. ๙, c Exp. 5. B. ․ Nach Giesbrecht

1. Maxille 1-gldr., mit 2 Borsten. Die beiden Hakenborsten des 5. und 6. Lobus der 2. Maxille gleich dick und kräftig. Endgl. des rechten Exp. des 5. B. des of mit kräftiger Endborste und bestachelter, proximaler Außenrandborste. - L. O . 4,6-4,8 mm, ơ $2,75-3,05 \mathrm{~mm}$.

Mittelmeer, Nord-Atlant. Ozean.

\section{Haloptilus spiniceps Giesbrecht.}

1892, Hemicalanus s., Giesbrecht, p. 384 , t. 27 f. 6,40 ; t. 42 f. 3,8 , 10, 11. 1898, H. S., Giesbrecht und Schmeil, p. 120.

Stirn beim o zugespitzt, beim ot abgerundet. Darm mit vorderem Blindsack; Ovidukte reichen bis in die Stirn; Spermatophore kurz und dünn. 1. Antenne, 1. und 2. Maxille wie bei H. ornatus; 1. Gl. des Exp. der 2. Antenne und Enddorn der B. von geringerer Länge. Endgl. des rechten 
Exp. des 5. B. des $\sigma^{7}$ mit glattem, proximalem Außenranddorn. - L. $q$ $3,8-4,3 \mathrm{~mm}$, o $2,55 \mathrm{~mm}$.

Mittelmeer, Nord-Atlant. Ozean.

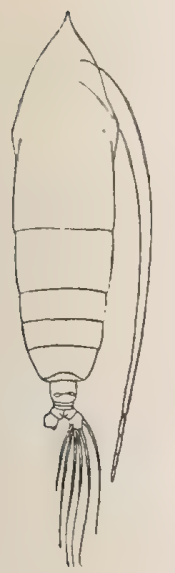

a

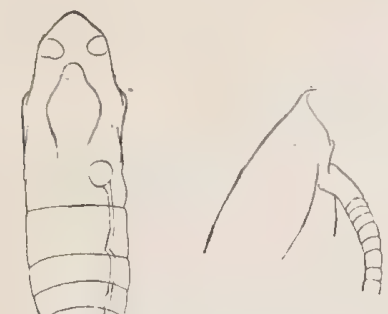

c

Fig. 149. Haloptilus spiniceps.

a q dorsal, b $\sigma^{\pi}$ dorsal, c Kopf ${ }^{\circ}$, lateral, d 5. B. $\sigma^{\pi}$. Nach Giesbrecht.

\section{Augaptilus Giesbrecht.}

ๆ. Kopf vom 1. Thsegm. getrennt; 4 . und 5. Thsegm. verschmolzen; Rostralfäden dünn. Abdomen 3-gldr., mit meist nicht ganz symmetrischem Gensegm. 1. Antenne 25-gldr. Exp. der 2. Antenne relativ länger als bei Haloptilus, aber selten länger als das Enp.; Mandibel zuweilen 1-ästig und mit stilettförmiger Kaulade; Enp. der 1. Maxille fehlt, meist auch die distalen Innenrandloben, und zuweilen sind auch die proximalen Loben des Innen- und Außenrandes sehr verkümmert; 2. Maxille mit rudimentären, proximalen Loben und mit eigentümlichen Anhängen an den distalen Borsten. 1.-4. B. mit 3-gldr. Exp. und Enp.; Exp. der B. mit z. T. verkümmerten Außenranddornen. 5. B. meistens mit 3-gldr., selten mit 2-gldr. Exp. und Enp.; Innenrandborste am 2. Gl. des Exp. meistens vorhanden und pfriemförmig; 3. Innenrandborste des Endgl. des Exp. nicht verlängert. - $\sigma$. 1. Antenne weniger verkürzt als bei Haloptilus; Greifantenne links oder rechts. 5. B. mit 3-gldr. Ästen; mittleres G1. des Exp. des 5. B. meistens mit eigentümlich geformtem Fortsatz am Innenrande.

$$
\text { †. }
$$

1. 1. Antenne reicht nicht bis zum Abdomen

A. rattrayi

1. Antenne deutlich länger als der Vorderkörper

A. gibbus

2. Kopf dorsal mit Buckel

Kopf dorsal ohne Buckel

3. Mittleres Abdsegm. höchstens halb so lang wie das Ansegm.

Mittleres Abdsegm. länger oder nur wenig kürzer als das Ansegm. 
4. Endborste des Exp. des 5. B. fast 2 mal so lang wie das Endgl; Endgl. des Enp. des 5. B. mit 1 Außenrandborste (5 Borsten im ganzen) A.palumboi Endborste des Exp. des 5. B. höchstens nur wenig länger als das Endgl.; Endgl. des Enp. des 5. B. mit 2 Außenrandborsten (6 Borsten im ganzen) 5

5. Exp. der 2. Antenne 8-gldr., wenig über halb so lang wie das Enp. und viel dünner

Exp. der 2. Antenne 5-gldr, etwas länger als das Enp.

A. squamatus ${ }^{1}$ )

6. 1. Antenne bis zum Ende der Furka reichend

A. zetesios

7. Äste des 5. B. 2-gldr.

Äste des 5. B. 3-gldr.

A. magnus

. Exp. der 2. Ant. 5-gldr., ungefähr so lang wie das Enp.; Mandibel 1-ästig 9

Exp. der 2. Ant. 8-gldr., nicht halb so lang wie das Enp.; Mandibel 2-ästig

A. filigerus

9. Kaulade der Mandibel lamellenförmig; Exp. der 1. Maxille mit 3, Innenlobus mit 1 Borste

A. longicaudatus

Kaulade der Mandibel stilettförmig; Exp. der 1. Maxille mit 2, Innenlobus mit 2 Borsten

A. glacialis.

$0^{7}$.

1. Exp. der 2. Ant. 8-gldr., nicht halb so lang wie das Enp.; Mandibel 2-ästig

A. filigerus

Exp. der 2. Antenne wenigstens $3 / 4$ so lang wie das Enp; Mandibel 1 -ästig 2

2. Furka kürzer als die beiden vorhergehenden Segm. zusammen; Exp. der 2. Antenne $3 / 4$ so lang wie das Enp.

A. hecticus

Furka wenigstens "so lang wie die beiden vorhergehenden Segm. zusammen; 2. Antenne mit fast gleichen Ästen

3. Kaulade der Mandibel lamellenförmig; Exp. der 1. Maxille mit 3, 1. Innenlobus mit 1 Borste; Enddorn des Exp. des rechten 5. B. rechts länger als links

A. longicaudatus

Kaulade der Mandibel stilettförmig; Enp. der 1. Maxille und 1. Innenlobus mit 2 Borsten; Enddorn des Exp. des 5. B. beiderseits etwa gleich lang

A. glacialis.

\section{Augaptilus squamatus Giesbrecht.}

1889, A. S., Giesbrecht, p. 814. 1892, A. s., Giesbrecht, p. 400, t. 28 f. $12,18,22,25,34$; t. 39 f. 38.1898 , A. s., Giesbrecht und Schmeil, p. 121.

Q. Abdomen kurz; Ansegm. wenigstens doppelt so lang wie das vorhergehende Segm. 1. Antenne überragt das Rumpfende um die 8 letzten Gi. Exp. der 2. Antenne 8-gldr, wenig über halb so lang wie das Enp. und viel dünner. Mandibel 2-ästig. 1. Maxille: Außenlobus mit 9, 1. Innenlobus mit

1) Siehe auch A. laticeps. 
11, 2. und 3. Innenlobus mit je 2, Exp. mit 7 Borsten. Längenverhältnis des 1. und 2. Gl. des Basp. und des Enp. des Maxillipeden wie $8: 6: 7$. 5. B. dem von filigerus ähnlich, Außenrandborste des 2. Basalgl. kürzer, wenn auch das Ende des Exp. merklich überragend. L. $6,8 \mathrm{~mm}$. - $\sigma^{7}$ unbekannt. Atlant. und Pacif. Ozean.

\section{Augaptilus laticeps G. O. Sars.}

1905, A. 1., Sars, Nr. 40, p. 11.

Verwandt mit squamatus, aber: - . . Vorderer Körperteil viel weniger verbreitert in der Mitte. Kopf länger als der Thorax und vorne breit gerundet. L. $7,6 \mathrm{~mm}$. - o unbekannt.

\section{Augaptilus filigerus Claus.}

1863, Hemicalanus f., Claus, p. 179. 1892, A. f., Giesbrecht, p. 400, t. 3 f. 3 ; t. 27 f. 34 ; t. 28 f. $10,13,14,20,26,27,36$; t. 29 f. 26 ; t. 39 f. 49 . 1898, A. f., Giesbrecht und Schmeil, p. 121.

ㅇ. Gensegm. nicht ganz symmetrisch, doppelt so lang wie die beiden folgenden (etwa gleich langen) Segm. zusammen; Furka so lang wie das Ansegm., wenig länger als breit. 1. Antenne überragt das Rumpfende um die 8 letzten Gl.; Exp. der 2. Antenne 8-gldr., nicht halb so lang wie das Enp. Mandibel 2-ästig, mit griffelförmiger Kaulade. 1. Maxille: Außenlobus mit 6, 1. Innenlobus mit 8, 2. und 3. Innenlobus (beide winzig) mit je 1, Exp. mit 10 Borsten.
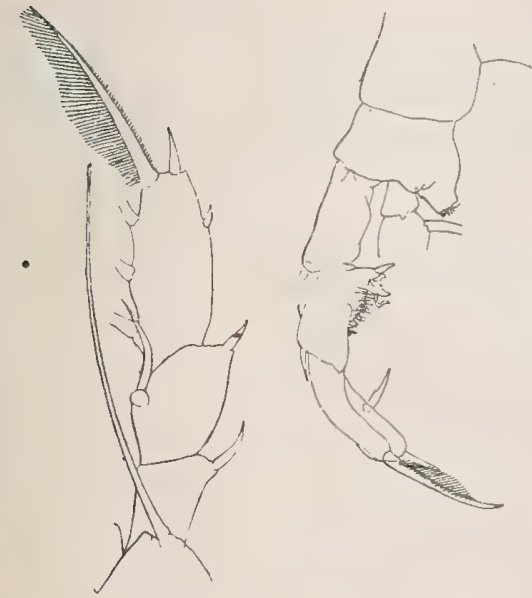

ฉ

Fig. 150. Augaptilus filigerus. a 5. B. \&, b 5. B. o?. Nach Giesbrecht.

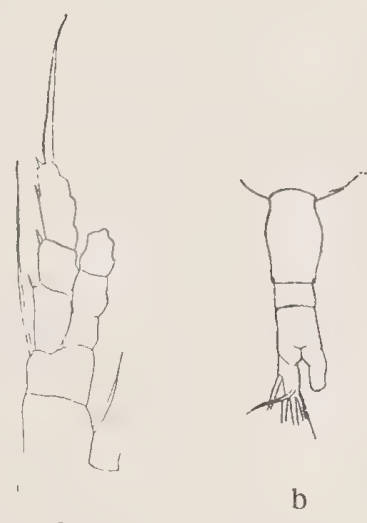

Fig. 151.

Augaptilus palumboi. a 5. B. ㅇ, b Abdomen , dorsal. Nach Giesbrecht. 
Längenverhältnis des 1. und 2. GI. des Basp. und des Enp. des Maxillipeden wie bei squamatus. Außenrandborste des 2. Basalgl. des 5. B. doppelt so lang wie das Exp.; Enddorn des Exp. des 5. B. länger als das Endgl. - o . Greifantenne links; 5. B. vergl. Fig. 150. - L. q 4,9 mm, of 4,1-4,5 mm.

Mittelmeer, Atlant. Ozean.

\section{Augaptilus palumboi Giesbrecht.}

1889, A. palumbii, Giesbrecht, p. 813. 1892, A. p., Giesbrecht, p. 400, t. 27 f. 32 ; t. 28 f. 15,17 ; t. 39 f. $39,50.1898$, A. p., Giesbrecht und Schmeil, p. 122. ? 1903, A. p., Thompson, p. 28, t. 1 f. 1, 2. (o imm.)

१. Abdomen gestreckt; Ansegm. wenigstens doppelt so lang wie das vorhergehende Segm. 1. Antenne überragt das Rumpfende um die beiden letzten GI. Exp. der 2. Antenne 8-gldr., 7/9 so lang wie das Enp. Mandibel 2-ästig; Kaulade lamellenförmig. 1. Maxille: Außenlobus mit 1, 1. Innenlobus mit 9, 2. mit 1, Exp. mit 4 Borsten; 3. Innenlobus rudimentär. Maxilliped ähnlich wie bei A. filigerus. - (Reifes) o unbekannt. - L. $q 2,25 \mathrm{~mm}$, o (unreif) $4 \mathrm{~mm}$. (?)

Nord-Atlant. Ozean (?); Pacif. Ozean.

Anm. Thompson (l. c.) meinte ein einzelnes, unreifes, männliches Exemplar einer Augaptilus-Art zu dieser Art bezielen zu können. Es ist aber sehr fraglich, ob seine Bestimmung richtig war.

\section{Augaptilus rattrayi Th. Scott.}

1893, A. r., Th. Scott, p. 36 , t. 2 f. $25-37$. 1898, A. r., Giesbrecht und Schmeil, p. 122.

१. Gensegm. viel länger als die folgenden beiden Segm. zusammen; Furka kurz. 1. Antenne erreicht kaum das 3. Thoraxsegm. Exp. und Enp. der 2. Antenne etwa gleich lang. Mandibel 2-ästig; Kaulade lamellenförmig. 1. Maxille: Außenlobus mit 5, proximaler Innenlobus mit 2, Exp. mit 3 Borsten. Endgl. des Maxillipeden dünn, relativ lang, mit dünnen, nicht hakigen Borsten. Exp. und Enp. der B. kurz, mit relativ breiten Gl. Rumpf und der größte Teil der Gliedmaßen dicht mit kurzen Härchen bedeckt. L. 4,9 $\mathrm{mm}$. or unbekannt.

Atlant. Ozean.

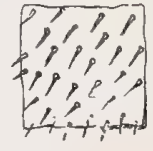

a

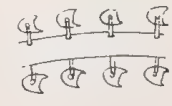

b

Fig. 152. Augaptilus rattrayi.

a Teil des Integuments, b Stück einer Borste des Maxillipeden $q$ mit Anhängen. Nach Scott. 


\section{Augaptilus necticus Giesbrecht.}

1889, A. h., Giesbrecht, p. 814. 1892, A. h., Giesbrecht, p. 400, t. 1 f. 3 ; t. 27 f. 30 ; t. 28 f. $5,9,16,33,37$; t. 29 f. 18 ; t. 39 f. 45 . 1898, A. h., Giesbr. und Schmeil, p. 122.

Gensegm. des symmetrisch, doppelt so lang wie die beiden folgenden (etwa gleich langen) Segm. zusammen; Furka so lang wie das Ansegm., wenigstens $3 \mathrm{mal}$ so lang wie breit. 1. Antenne des $O$ überragt das Rumpfende um die 6 letzten Gl.; Greifantenne des $\sigma^{\pi}$ rechts. Exp. der 2. Antenne
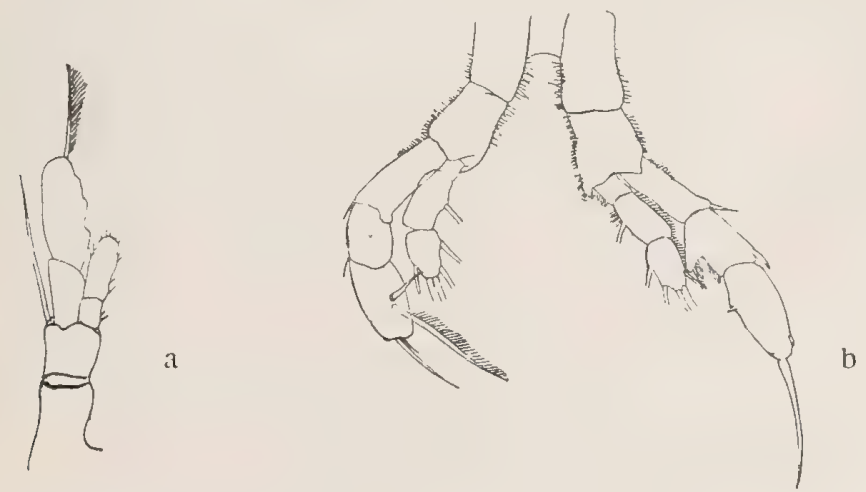

Fig. 153. Augaptilus hecticus. a 5. B. q, b 5. B. o. Nach Giesbrecht.

3/4 so lang wie das Enp. Mandibel 1-ästig; Kaulade griffelförmig. 1. Maxille: Außenlobus mit 3, 1. Innenlobus (schmächtig) mit 4, Exp. mit 5 Borsten; 2. Innenlobus fehlt, 3. Innenlobus rudimentär. Längenverhältnis der 3 Abschnitte des Maxillipeden wie $7: 6: 5$. Exp. des 5. B. des . 2-gldr.; 5. B. o vergl. Fig. 153. - L. ₹ $2,45-2,75 \mathrm{~mm}, o^{7} 2,4 \mathrm{~mm}$.

Mittelmeer, Atlant. Ozean; Pacif. Ozean.

\section{Augaptilus longicaudatus Claus.}

1863, Hemicalanus 1., Claus, p. 129, t. 29. 1892, A. 1., Giesbrecht, p. 400 , t. 27 f. 31 ; t. 28 f. $11,19,23,31,35,38$; t. 29 f. 22 ; t. 39 f. 37,48 . 1898, A. 1., Giesbrecht und Schmeil, p. 123.

Gensegm. des $q$ nicht ganz symmetrisch, länger als die beiden folgenden (etwa gleich langen) Segm. zusammen; Furka ebenfalls länger als diese, ca. 5 mal so lang wie breit. 1. Antenne des $q$ überragt das Rumpfende um die 4 letzten G1.; Greifantenne des $\sigma^{\nearrow}$ links. Exp. der 2. Antenne 5-gldr., wenig kürzer als das Enp. Mandibel 1-ästig; Kaulade lamellenförmig. 1. Maxille: Außenlobus mit 2, Exp. mit 3 Borsten; 1. Innenlobus rudimentär mit 1 Borste, 2. und 3. 
Innenlobus fehlen. Längenverhältnis der 3 Abschnitte des Maxillipeden wie $6: 8: 5$. 5. B. ठ vergl. Fig. 154. - L. क $3,7-3,85 \mathrm{~mm}$, o $3,2-3,35 \mathrm{~mm}$.

Mittelmeer, Atlant. Ozean; Pacif. Ozean.

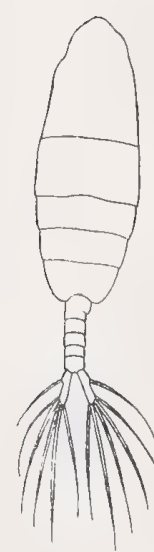

a

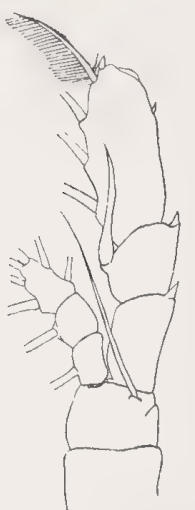

b
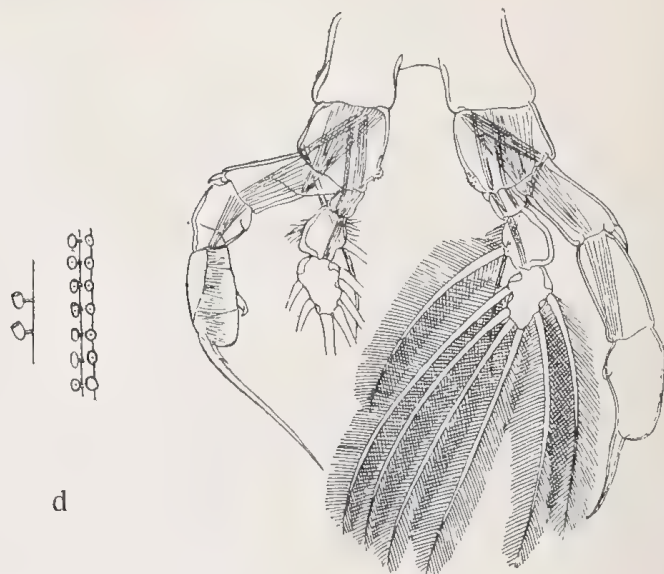

c

Fig. 154. Augaptilus longicaudatus.

a o dorsal, b 5. B. , c 5. B. ơ, d Stück einer Knopfborste aus der 2. Maxille. Nach Giesbrecht.

\section{Augaptilus glacialis G. O. Sars.}

1900, A. g., Sars, p. 88, t. 26, 27.

Gensegm. des q symmetrisch, etwas länger als die beiden folgenden Segm. zusammen; Furka so lang wie die 2 letzten Abdsegm. zusammen. 1. Antenne des $q$ überragt das Rumpfende um die 5 letzten Gl.; Greifantenne des or links. Exp. der 2. Antenne 5-gldr., ungefähr so lang wie das Enp. Mandibel 1-ästig; Kaulade stilettförmig. 1. Maxille: Außenlobus mit 2, Exp. mit 2 Borsten; 1. Innenlobus mit 2 Borsten. 5. B. des $\bigcirc$ mit 3-gldr. Ästen; Außenrandborste des 2. Basalgl. so lang wie das Exp.; Innenrandborste des 2. Gl. des Exp. wenig kürzer als das Endgl.; 5. B. o' vergl. Fig. 155. L. $q 4,8 \mathrm{~mm}, \mathrm{O}^{\top}$ ?

Polarmeer.

\section{Augaptilus zetesios Wolfenden.}

1902, A. z., Wolfenden, p. 369, t. 3.

१. Gensegm. länger als die beiden folgenden Segm. zusammen; mittleres Segm. halb so lang wie das Ansegm. 1. Antenne viel länger als der Körper. Exp. der-2. Antenne 5-gldr., etwas länger als das Enp. Mandibel 1-ästig, mit stilettförmiger Kaulade. 1. Maxille mit 1 Innen- und 1 Außenrandlobus; beide 
Trib. Gymnoplea.

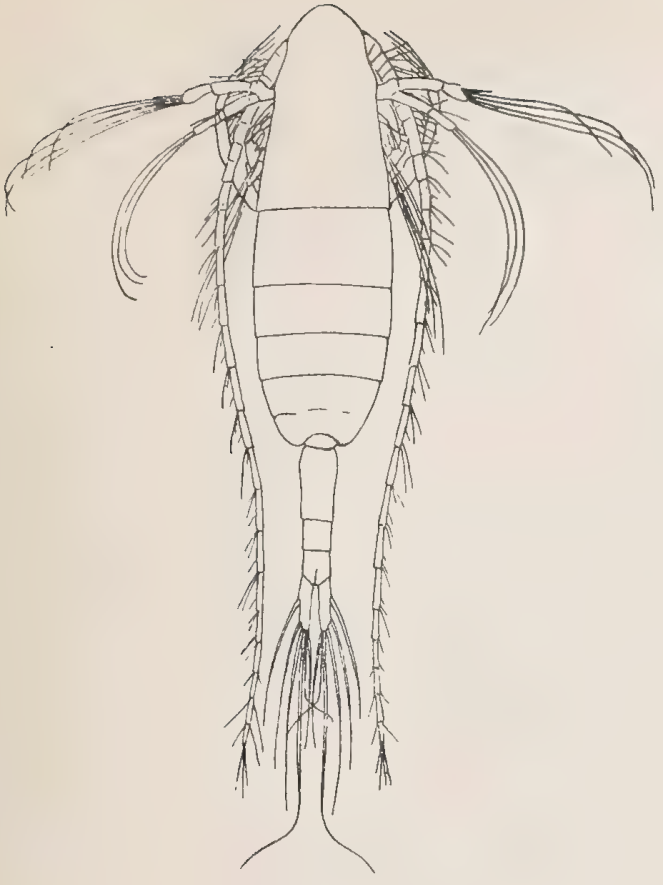

a

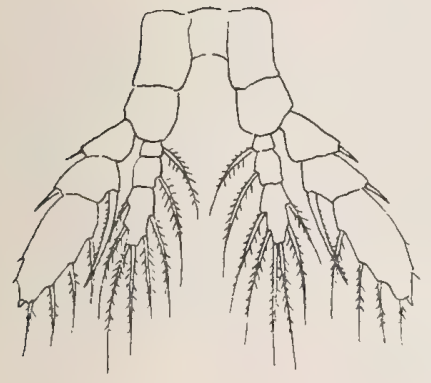

c

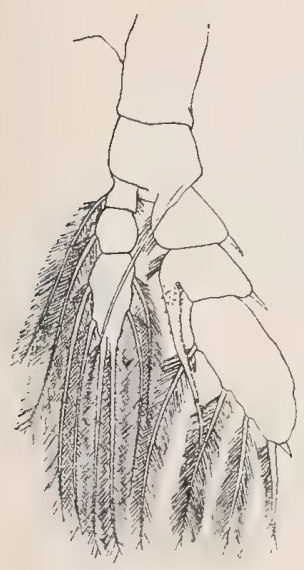

Fig. 156.
Fig. 155.

Augaptilus glacialis.

a $q$ dorsal, b $\sigma^{\top}$ lateral, c 5. B. . , d 5. B. or. Nach Sars.

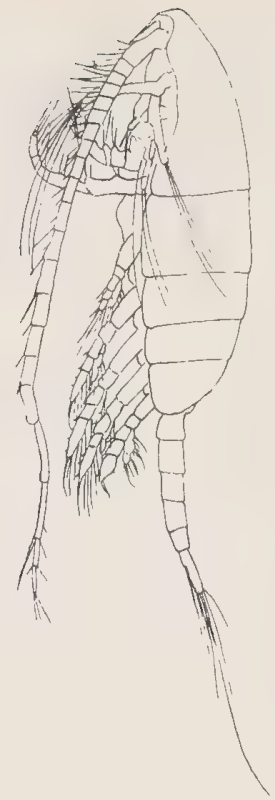

b

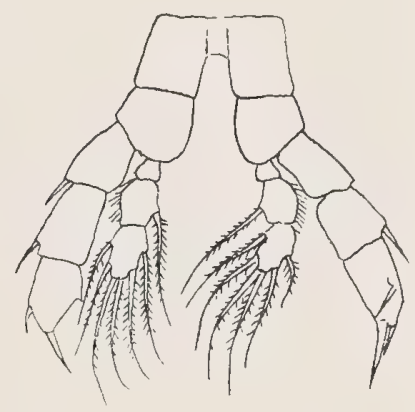

d Augaptilus zetesios. a 5. B. ㅇ, b Innenrandborste des 2. Gl. des Exp. desselben B. Nach Wolfenden.

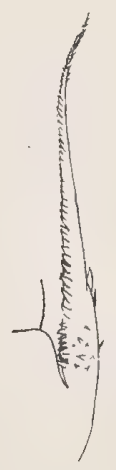


Loben und das Exp. mit 2 Borsten. Distale Borsten des Maxp. mit denselben Anhängen wie die 2. Maxille. Innenrandborste des 2. Gl. des Exp. des q fast das Ende des Exp. erreichend, an der Basis dick und mit einer Reihe kräftiger Zähne am Innenrande; Endborste kürzer als das Endgl. L. 4,7 mm. o unbekannt.

Nord-Atlant. Ozean.

10. Augaptilus magnus Wolf̀enden.

1904, A. m., Wolfenden, p. 122.

†. Gensegm. länger als das übrige Abdomen; 2. Abdsegm. so lang wie oder etwas länger als das Ansegm.; Furkalzweige sehr kurz. 1. Antenne das Ende der Furka erreichend. Mandibel 2-åstig. 2. Antenne mit -fast gleich langen Ästen. 1. Maxille: Außenlobus mit 5, Exp. mit 2, 2. Innenlobus mit 1 Borste. Basalgl. des Maxp. gleich lang, Enp. viel kürzer. 5. B. mit 3-gldr. Exp. und Enp. L. $7 \mathrm{~mm}$. - o unbekannt.

Nord-Atlant. Ozean.

\section{Augaptilus gibbus Wolfenden.}

1904, A. g., Wolfenden, p. 122. 1905, A. g., Sars, Nr. 40, p. 16.

O. Kopf dorsal mit Buckel. Gensegm. so lang wie die beiden folgenden Segm. zusammen; Ansegm. länger als das mittlere Abdsegm.; Furka länger als das Ansegm. 1. Antenne nicht ganz so lang wie der Körper. Exp. der 2. Antenne nicht halb so lang wie das Enp. Mandibel 2-ästig. Die beiden Basalgl. und das Enp. des Maxillipeden gleich lang. 1. Maxille: Außenlobus mit 3, Exp. mit 4, 1. Innenlobus mit 6 Borsten. L. $2,75 \mathrm{~mm}$. - o unbekannt.

Atlant. Ozean.

\section{Paraugaptilus Wolfenden. ${ }^{1}$ )}

ๆ. Abd. 4-gldr. Rostralfäden dünn. 1. Antenne 21-gldr., links etwas länger als rechts. Enp. der 2. Antenne viel länger als das Exp. Mandibel 1-ästig. 1. Maxille mit stark reduzierten Innenloben. 2. Maxille mit sehr langen, distalen, mit augaptiloiden Anhängen besetzten Borsten. Borsten des Maxp. z. T. mit denselben Anhängen. 1--4. B. mit 3-gldr. Exp. und Enp. 5. B. symmetrisch, jederseits eine unvollkommen 2 teilige, blattförmige Platte bildend.

\section{Paraugaptilus buchani Wolfenden. ${ }^{1}$ )}

1904, P. b., Wolfenden, p. 123 , t. 9 f. $44,45$.

1) Sars (1907, Nr. 101, p. 26) erwähnt die einzige Art dieser Gattung als zum Genus Arietellus gehörig: Paraugaptilus buchani Wolfenden = Arietellus buchani Wolfenden. 
ㅇ․ Gensegm. 2 mal so lang wie das nächste Abdsegm; dieses so lang wie jede der beiden folgenden Segm.; Furka nicht ganz 2 mal so lang wie breit, mit 4 Endborsten (deren längste so lang wie das Abdomen ist); 5 . Thsegm. jederseits dorsal in einen kurzen, stumpfen Dorn endend. 1. Antenne beiderseits kürzer als der Körper. Exp. der 2. Antenne etwa halb so lang wie das Enp. 5. B. mit einer Randborste und einer längeren Endborste bewehrt. L. $3,25 . \mathrm{mm}$. - o unbekannt.

Nord-Atlant. Ozean.

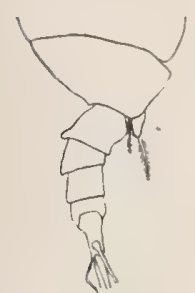

a
Fig. 157.

Paraugaptilus

$$
\text { buchani. }
$$

a Hinterer Teil des Körpers des , lateral, b 5. B. ㅇ.

Nach Wolfenden.

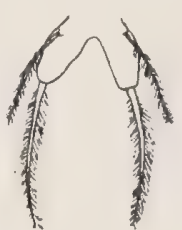

b

Arietellus Giesbrecht.

१. Kopf vom Thorax getrennt; letztes mit vorletztem Thsegm. verschmolzen. Stirn mit Fortsatz; Rostralfäden dünn. Abdomen 4-gldr. 1. Antenne 20-gldr. Enp. der 2. Antenne gestreckt, länger als das Exp. Mandibel 1-ästig, ohne Enp. Enp. und 3. Innenlobus der 1. Maxille fehlt; Exp. lang und eigentümlich gedreht. 2. Maxille mit kleinen Loben und verlängerten, distalen Borsten; die distalen Borsten der 2. Maxille und des Maxillipeden mit Spitzen und Schüppchen besetzt. 1.-4. B. mit 3-gldr. Exp. und Enp. 5. B. nicht schwimmbeinähnlich, 3-gldr. - $\sigma^{7}$. Greifantenne links. 5. B. ohne Zange; Basp. 2-gldr., Exp. 3-, Enp. 1-gldr.

\section{Arietellus setosus Giesbrecht.}

1892, A. s., Giesbrecht, p. 415 , t. 29 f. $1,3-7,9-13,21$; t. 39 f. $34-36$. 1893, Rhincalanus aculeatus, Th. Scott, p. 31 , t. 2 (o juv.) 1897, A. s., Giesbrecht, p. 254. 1898, A. s., Giesbrecht und Schmeil, p. 124. 1905, A. S., Esterly, p. 189, f. 42 .

Stirn mit spitzem Fortsatz; letztes Thsegm. in eine kräftige Spitze verlängert. 5. B. ○ : 2. Gl. das dickste, mit Außenrandborste, am distalen Ende des Innenrandes 2 Borsten; Endgl. dünn, mit einer pfriemförmigen Borste an der Spitze; 5. B. o vergl. Fig. 158. -- L. \& 4,6, o $4,25 \mathrm{~mm}$.

Mittelmeer, Atlant. Ozean; Pacif. Ozean. 


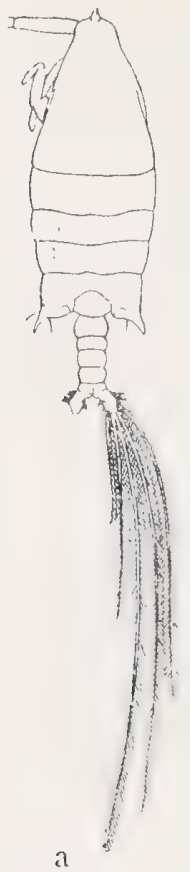

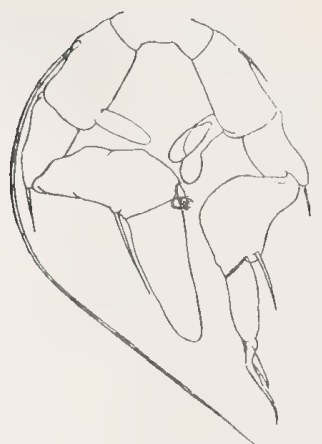

1)

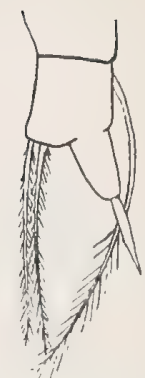

Fig. 158. Arietellus setosus. a 3 dorsal, b 5. B. o, c 5. B. a, b nach Giesbrecht, c nach Esterly.

Paramisophria Th. Scott.

q. Kopf vom Thorax getrennt; die beiden letzten Thsegm. verschmolzen. Rostrum klein mit 2 winzigen Fäden. Abdomen 4-gldr., symmetrisch; äußere Endborste und Seitenborste der Furka lang. 1. Antenne 21-gldr., kurz, von ungleicher Länge. Enp. der 2. Antenne länger als das Exp. Mandibel 1-ästig. 1. Maxille mit mehreren Borsten am Kaulobus; Außenlobus mit Fiederborsten, Innenloben und Enp. rudimentär. 2. Maxille mit kräftigen distalen Borsten und verkümmerten proximalen Loben. 1.-4. B. mit 3-gldr. Exp. und Enp. 5. B. nicht schwimmbeinähnlich, symmetrisch, 3-gldr.; 2. Gl. am Innenrande mit distalem Fortsatz. - $0^{7}$. Greifantenne links. 5. B. asymmetrisch, 1-ästig, beiderseits 5-gldr., rechts mit dornförmigem, links mit spatelförmigem Endgl.

Paramisophria cluthae Th. Scott.

1897, P. c., Th. Scott, p. 147 , t. 2 f. $3-8$; t. 3 f. $13-16$. 1898, P. c., Giesbrecht und Schmeil, p. 123. 1903, P. c., Sars, p. 128, t. 86, 87.

․ 1. Antenne etwa so lang wie der Kopf, links nur wenig länger als rechts. 5. Thsegm. mit einem subdorsalen, gerade nach hinten gerichteten und spitzen Fortsatz. 5. B.: Innerer Fortsatz des Mittelgliedes klein, mit 1 Dörnchen und 1 Borste an der Spitze; Endgl. 2 mal so lang wie die beiden anderen zusammen mit 2 End- und 4 Außenranddornen. - $\sigma^{\top}$. Greifantenne links, mit unvollkommen 2-gldr. kurzem Endstück. 5. B.: Vorletztes Gl, beider- 
seits verbreitert; rechtes Endgl. lang, dornförmig mit 2 Dörnchen an der Basis, linkes Endgl. breit mit 3 Stacheln am Ende. - L. o 1,2-1;4 mm, o 1,1 mm.

Schottische und norwegische Küste.

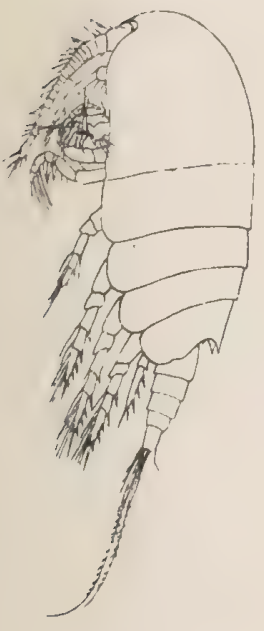

a
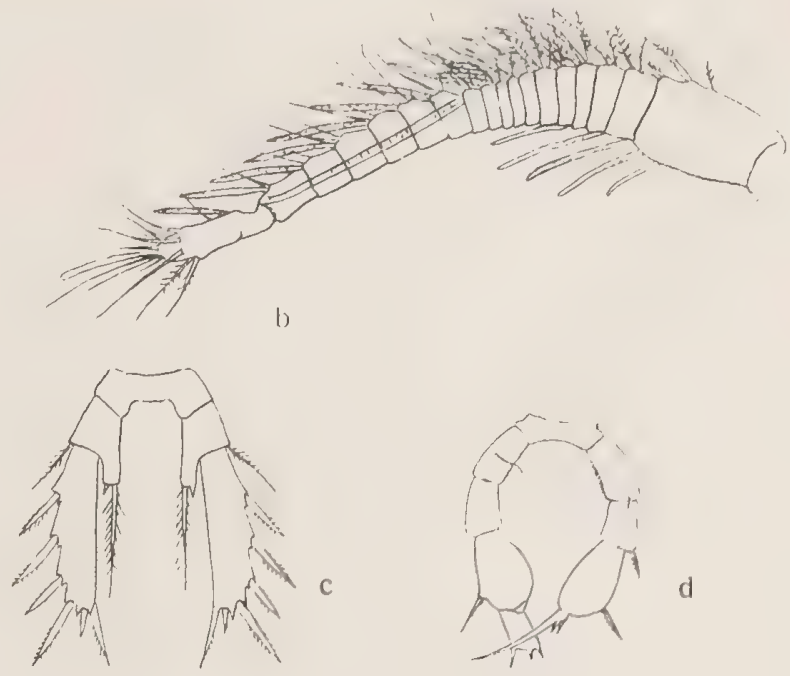

Fig. 159. Paramisophria cluthae.

a $q$ lateral, b Greifantenne ox, c 5. B. q, d 5. B. ox. Nach Sars.

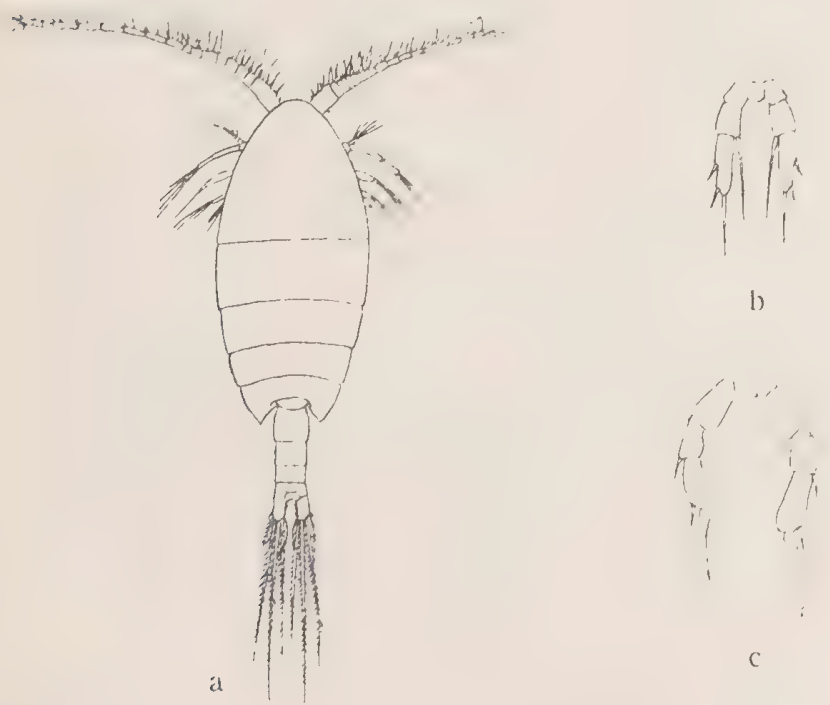

Fig. 160. Scottula inaequicornis

a $q$ dorsal, b 5. B. $q$, c 5. B. o7. Nach Sars. 
Scottula G. O. Sars.

Verwandt mit Paramisophria, weicht aber hauptsächlich in folgenden Merkmalen $a b:-q$. Rostrum mit 2 geraden Fäden. Äußere Endborste der Furka kurz, Seitenborste winzig. 1. Antenne länger, links merklich länger als rechts, links 20-gldr., rechts 19-gldr. 1. Maxille mit rudimentärem Kaulobus (dieser nur mit 2 Borsten); Enp. gänzlich fehlend. 5. B. 3-gldr., 2. Gl. an der Innenseite ohne Fortsatz. - $\sigma^{7}$. 5. B. beiderseits mit klauenförmigem Endgl., nur. wenig asymmetrisch.

Scottula inaequicornis G. O. Sars.

1903 , S. i., Sars, p. 125 , t. 84,85 .

Mit den Merkmalen der Gattung. $\left.{ }^{1}\right)$ - L. $ๆ ~ 1,1 \mathrm{~mm}$, o $0,86 \mathrm{~mm}$.

Norwegische Küste.

\section{Phyllopus Brady.}

․ Kopf vom Thorax getrennt; letztes mit vorletztem Thsegm. verschmolzen. Abdomen 4-gldr., Gensegm. asymmetrisch. 1. Antenne 24-gldr. Enp. der 2. Antenne gedrungen, viel kürzer als das Exp. 1. Maxille: 1. Innenlobus sehr groß, die andern Innenloben und das Enp. klein, Außenlobus knopfförmig, borstenlos, Exp. normal. 1. Gl. des Basp. des Maxillipeden kurz und breit. 1.-4. B. mit 3-gldr. Exp. und Enp. 5. B. nicht schwimmbeinähnlich,

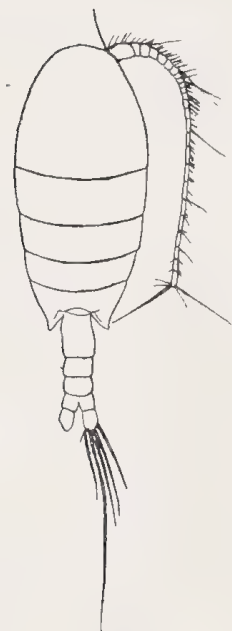

a

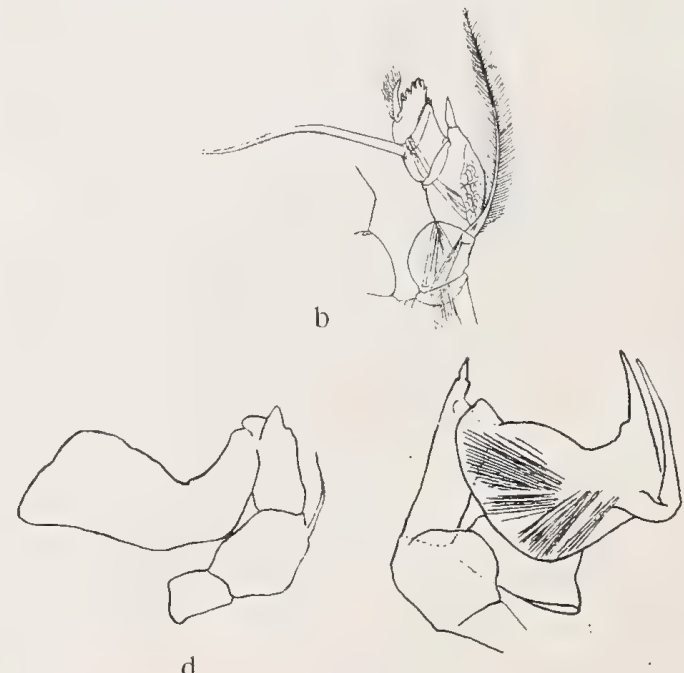

$\mathrm{e}$

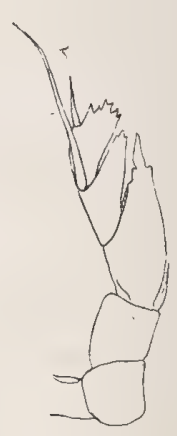

c

Fig. 161. Phyllopus bidentatus.

a $q$ dorsal, b 5. B. q, c 5. B. O var., d rechtes 5. B. ơ, e linkes B. ơ a, b nach Giesbrecht, c-e nach Farran.

1) Die von Sars (1905) beschriebene Scottula obyssalis hat $5 \mathrm{~mm}$ Länge. 
mit 3-gldr. Exp., ohne Enp.; 2. Gl. des Exp. mit dicker, langer Innenrandborste, 3. Gl. verkürzt, am distalen Rande gezähnelt. - o Greifantenne links. 5. B. mit 2-gldr. Basp. und 3-gldr. Exp., links mit blattförmigem Enp., rechts ohne Enp.; Gl. der Exp. z. T. groß und breit.

\section{Phyllopus bidentatus Brady.}

1883 , P. b., Brady, p. 78 , t. 5 . 1892, P. b., Giesbrecht, p. 419, t. 18 f. 25,33 ; t. 38 f. 35.1898 , P. b., Giesbrecht und Schmeil, p. 124. ? 1903, P. b., Thompson, p. 29 , t. 3 f. $6-9$. 1904, P. b., Wolfenden, p. 124, t. 9 f. 16. 1905, P. b., Farran, p. 45, t. 11 f. $12-21$.

‥ 1. Antenne den hinteren Rand des Thorax erreichend; 5. Thoraxsegm. symmetrisch oder ein wenig asymmetrisch, gerundet oder in eine Spitze verlängert. 5. B.: 2. G1. des Basp. mit Außenrandborste, die länger oder kürzer ist und bisweilen ganz fehlen kann; Innenrandborste des 2. Gl. des Exp. dick, lang, s-förmig gebogen, fiederlos, mit feinen Spitzen besetzt; distaler Rand des 3. Gl. gekerbt. - o. 5. B. vergl. Fig. 161. - L. \& 2,4-3 mm, o 2,25 mm. Atlant. und Pacif. Ozean.

Anmerkung. Die Form des letzten Thoraxsegmentes und der Bau des 5. B. beim q (auch beim $\sigma^{7}$ ?) sind nach den Mitteilungen einiger Beobachter ziemlich großen Schwankungen unterworfen. Falls es sich bei genauerem Studium nicht herausstellt, daß es sich um melirere Arten handelt, hat man in dieser Species ein Beispiel von einem ungewöhnlich hohem Grade der Variabilität unter den Copepoden.

\section{Pseudocyclopidae.}

Kopf vom Thorax getrennt; Abdomen des 9 4-, des 0 5-gldr.; Genitalöffnungen des $q$ seitlich; Genitalöffnung des o links; Ansegm. verkürzt; Rostrum 1-spitzig. 1. Antenne kurz, aber wenigstens 15-gldr., die rechte des $\sigma^{7}$ eine genikulierende Greifantenne. Exp. der 2. Antenne mit reduzierter Gliederung. Die folgenden Gliedmaßen calanidenähnlich; distale Stücke der 1. Maxille gestreckt, Borsten des Außenrandlobus reduziert; Enp. des Maxillipeden kurz, undeutlich gegliedert. 1.-4. B. mit 3-gldr. Exp. und Enp. 5. B. des $q$ den vorhergehenden B. ähnlich, mit reduzierten Borsten; 5. B. des o ein Greifapparat (ohne Zange) mit verkümmerten Enp.

\section{Pseudocyclops Brady.}

Letztes Thsegm. vom vorletzten getrennt, dorsal verkümmert. Abdomen symmetrisch. 1. Antenne 15-18-gldr, den hinteren Kopfrand nicht erreichend. 
Greifantenne des of mit schwach verdicktem mittleren Abschnitt und 4-gldr. Endabschnitt. 5. B. des of mit 3-gldr. Exp. und 2- oder 3-gldr. Enp.; 5. B. des ơ asymmetrisch, mit komplizierten Anhängen am Ende der Exp.

\section{Pseudocyclops obtusatus Brady and Robertson.}

1873, P. o., Brady and Robertson, p. 128, t. 8. 1878, P. o., Brady, p. 84, t. 12 f. $1-13.1898$, P. 0., Giesbrecht und Schmeil, p. $126 . \quad 1903$, P. o., Sars, p. 131, t. 88 .

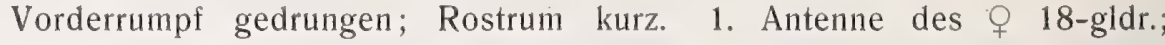
Greifantenne-17-(18-)gldr.; die das Endstück bildenden 4 letzten Gl. der Greifantemne gleich breit. Enp. des 5. B. des $\bigcirc$ 3-gldr.; Endgl. des Exp. mit 4 kleinen Fiederborsten am Innenrande. 5. B. des $\delta$ rechts mit 2 langen, stark gebogenen Endhaken und großem, blattförmigem, apikal gekerbtem Enp. — L. ᄋ 0,8 , o $0,7 \mathrm{~mm}$.

Schottische und norwegische Küste, Polarmeer; Ind. Ozean.

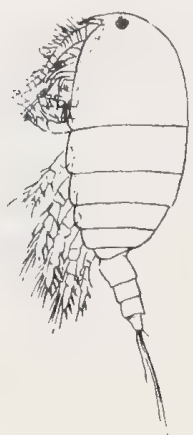

a

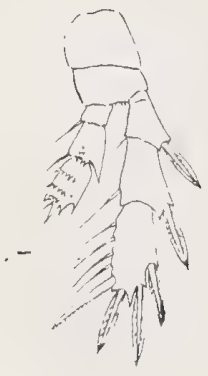

b

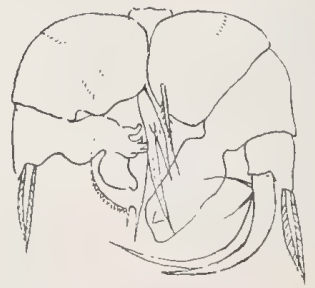

c

Fig. 162. Pseudocyclops obtusatus.

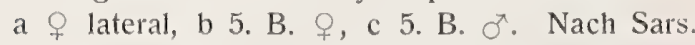

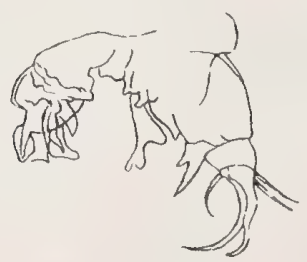

Fig. 163. Pseudocyclops crassiremis. 5. B. ơ. Nach.Brady.

\section{Pseudocyclops crassiremis Brady.}

1872 , P. c., Brady, p. 7 , t. 2 f. $1-8$. 1878, P. c., Brady, p. 82 , t. 7 f. 1,2 ; t. 12 f. 14. 1898, P. c., Giesbrecht und Schmeil, p. 126. 
q. unbekannt. - 5. 1. Antenne 17-gldr.; die 3 letzten Gl. der Greifantenne viel schmäler als das vorhergehende G1. 5. B. rechts mit 2 kurzen Endhaken und kleinem, in 2 Zacken endigendem Enp. L. 0,66 mm.

Britische Küste.

\section{Candaciidae.}

Kopf vom Thorax getrennt, die beiden letzten Thsegm. verschmolzen. Rostrum rückgebildet. Abdomen des $\bigcirc$ 3-, des ơ 5-gldr., letzteres mit linker Genitalöffnung. 1. Antenne 23- oder 24-gldr.; die rechte 1. Antenne des $\sigma^{7}$ ist eine Greifantenne. 2. Gl. des Basp. der 2. Antenne mit dem 1. Gl. des Enp. zu einem dicken Gl. verschmolzen; Exp. dünn, mit langem 2. Gl. und sehr kurzen Endgl. 2. Gl. des Basp. der Mandibel dick, Äste kurz, Kaulade mit wenig Zähnen. 2. Innenrandlobus der 1. Maxille sehr lang, 1. Lobus, Exp. und Enp. klein, 3. Innenrandlobus und Außenrandlobus fehlen. 2. Maxille ohne Loben; distale Borsten stark, sensenförmig. Maxilliped calanidenähnlich, aber sehr klein und schwach. 1.-4. B. mit 3-gldr. Exp. und 2-gldr. Enp. 5. B. beiderseits 1-ästig, 3-oder 4-gldr.

\section{Candacia Dana.}

Letztes Thsegm. meist mit zugespitzten Lateralecken, beim ö öfters asymmetrisch. Vorderkopf rechteckig; Rostralfäden durch 1 oder 2 kleine Höcker ersetzt. Abdomen des o 3-, des $\sigma^{7} 5$-gldr. Gensegm. bei $q$ und $\sigma^{7}$ öfters asymmetrisch. 1. Antenne des ${ }^{\circ} 23$ - oder 24-gldr.; proximale Gl. dick, mit zackigem Vorderrande; Endabschnitt der Greifantenne des o 5- oder 6-gldr. Mandibel mit zweizinkiger Kaulade. 1. Maxille mit langen, steifen, apikalen Borsten; der 2. Innenrandlobus, überragt das Basp. und das damit artikulierende Enp. 1. Gl. des Basp. des Maxillipeden mit wenigen Borsten; 2. G1. des Basp. und Enp. verkürzt. 1.-4. B. mit schmalen Enp.; Außenrand der Exp. gezähnelt. 5. B. 1-ästig, beim o 3-gldr., beim o links 4-, rechts 3-gldr. und in eine Löffelzange oder eine Borste endigend.

$$
\text { O }, 0
$$

1. 5. Thsegm. mit abgerundeten Ecken

C. rotunda

5. Thsegm. mit zugespitzten Lateralecken

2. Endborste des Exp. des 2.-4. B. über halb so lang wie das Endgl.; der dicke, proximale Teil der 1 . Antenne 7 -gldr.

C. norvegica Endborste des Exp. des 2.--4. B. weniger als halb so lang wie das Endgl.; der dicke, proximale Teil der 1. Antenne 6-gldr.

C. armata. Nord. Plankton. 


\section{Candacia armata Boeck.}

1872, Candace a., Boeck, p. 39. 1878, Candace pectinata, Brady, p. 49, t. $8,10.1892$, Candace pectinata, Giesbrecht, p. 424, t. 4 f. 3 ; t. 21 f. 2,12 ; t. 22 f. $9,17,18,31,43-46$; t. 39 f. $1,21,22,24,25$. 1898, C. pectinata, Giesbrecht und Schmeil, p. 128. 1903, C. a., Sars, p. 135, t. 91.

Letztes. Thsegm. des q zu großen, hinterwärts gerichteten Spitzen verlängert; das des $\sigma^{\text {t }}$ leicht asymmetrisch. Gensegm. und das folgende Segm. beim $\bigcirc$ asymmetrisch, letzteres rechts nach hinten zu gewölbt; Gensegm. des or rechts mit einffachem, spitzem Auswuchs. 1. Antenne des o 23-gldr., das Gensegm. nicht überragend; proximales Gl. der beiden genikulierenden Gl. der 1. Antenne beim ơ relativ kurz, braunfarbig, mit grober Zähnelung. Endborste der Exp. des 2.-4. B. kaum die halbe Länge des Endgl. erreichend. Endgl. des 5. B. des $q$ lang, klauenförmig, mit kleinen Außenrandzacken, ohne Innenrandborsten; vorletztes Gl. des rechten 5. B. des of mit breiter Lamelle. L. क 1,95-2,7 mm, o $1,7-2,7 \mathrm{~mm}$.

Mittelmeer, Atlant. Ozean; Ind. Ozean.

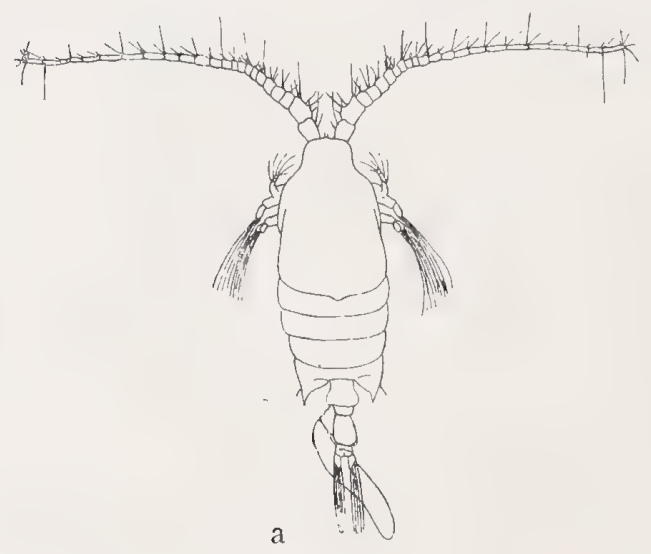

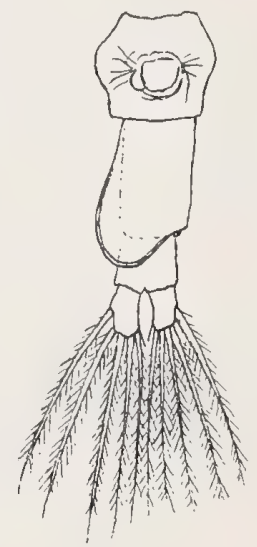

b

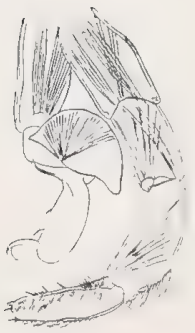

d

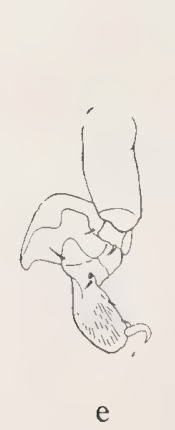

e
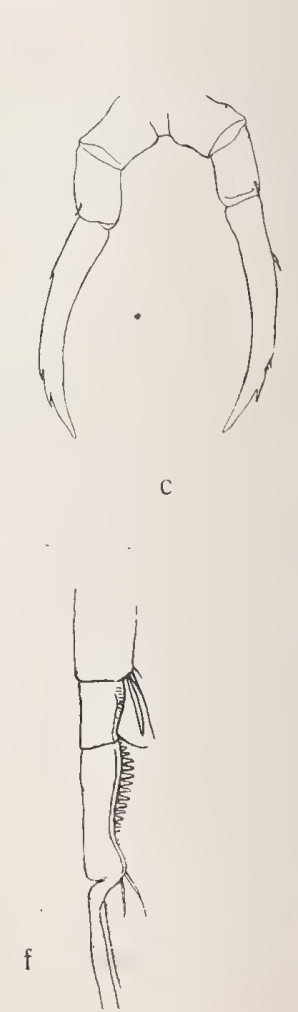

Fig. 164. Candacia armata.

a $q$ dorsal, b Abdomen $q$, ventral, c 5. B. $q$, d 5. B. or, vordere Fläche, e rechtes 5. B. $\sigma^{r}$, hintere Fläche, $f$ Teil des Greifantennes mit den genikulierenden Gl. a, c-e nach Giesbrecht, b und f nach Sars. 


\section{Candacia norvegica Boeck.}

1864, Candace n., Boeck, p. 11. 1898, C. n., Giesbrecht und Schmeil, p. 131. 1903, C. n., Sars, p. 134, t. $89,90$.

Letztes Thsegm. des $q$ mit einer kurzen, schräg auswärts gerichteten Spitze, das des $\sigma^{\pi}$ reclits mit einem dunkelfarbigen, gebogenen Haken. Gensegm. des $q$ symmetrisch, beiderseits mit einem Dorn; das des ot rechts mit einem

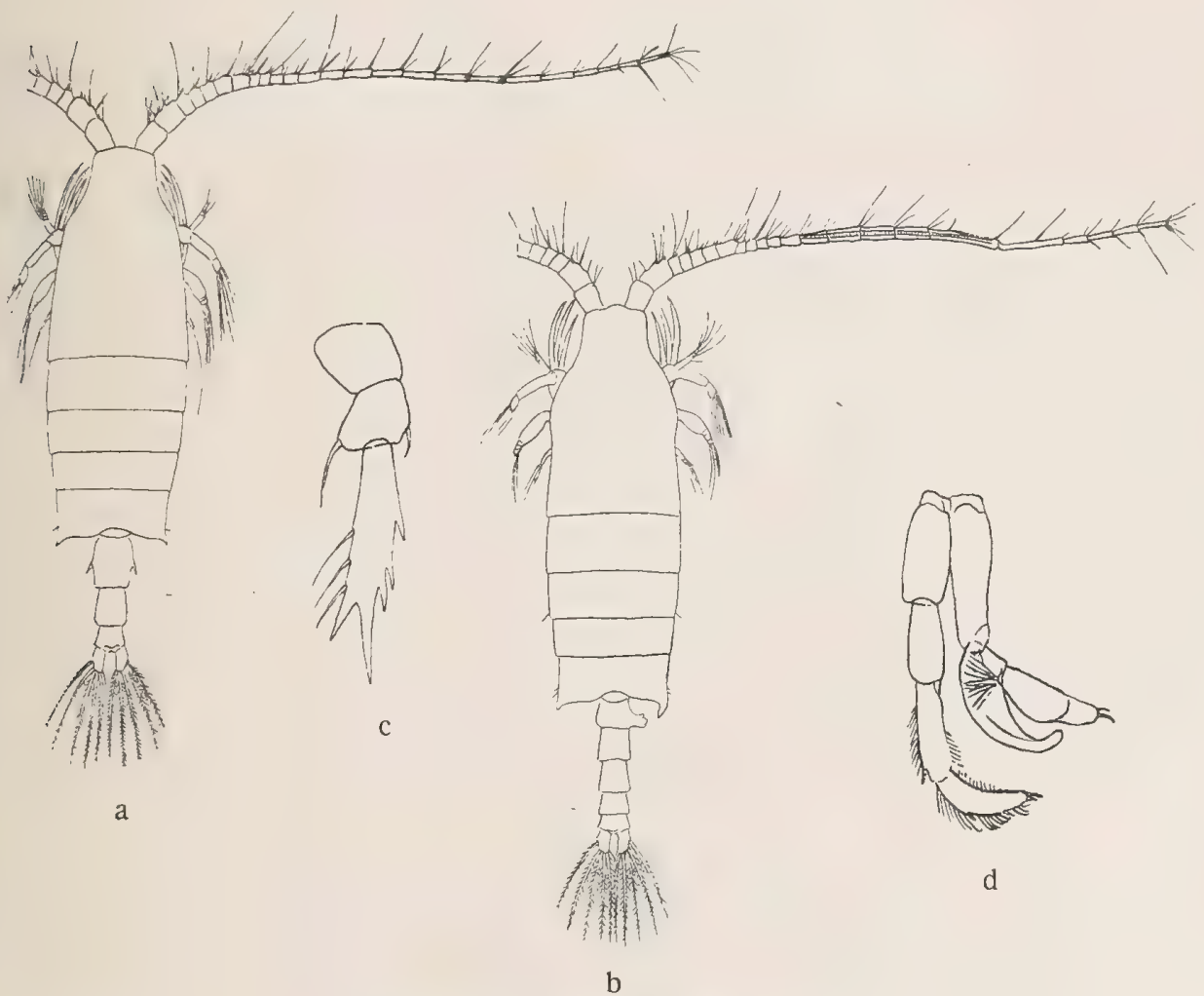

Fig. 165. Candacia norvegica.

a f dorsal, b o dorsal, c 5. B. \&, d 5. B. ơ. Nach Sars.

an dem Ende gekerbten Höcker. 1. Antenne des $q$ 24-gldr., das Ende der Furka erreichend; proximales Gl. der beiden genikulierenden Gl. der Greifantenne lang (7 mal so lang wie dick), mit feiner Zähnelung. Endborste der Exp. des 2.-4. B. länger als das halbe Endgl. Endgl. des 5. B. des $\bigcirc$ mit 2 apikalen Stacheln, am Innenrande mit 3 kurzen Borsten, am Außenrande mit 3 Zähnen; vorletztes G1. des rechten 5. B. des $\sigma^{7}$ mit relativ schmalem Anhang. - L. + und $\sigma^{7} 3,2 \mathrm{~mm}$.

Nord-Atlantischer Ozean. 
3. Candacia rotunda Wolfenden.

? 1864, Candace elongata, Boeck, p. 39. 1904, C. r., Wolfenden, p. 118, t. 9 f. 10, 11. 1905, Sars, C. obtusa, Nr. 40, p. 23.

q. Letztes Thsegm. abgerundet; Gensegm. symmetrisch. 1. Antenne 24-gldr. 5. B.: Endgl. schmal und lang, mit 4 Dornen, von denen die innere apikale die längste ist; Innenrand unbewehrt. L. $3,2-3,5 \mathrm{~mm}$. - $\sigma^{\top}$ unbekannt.

Nord-Atlantischer Ozean.
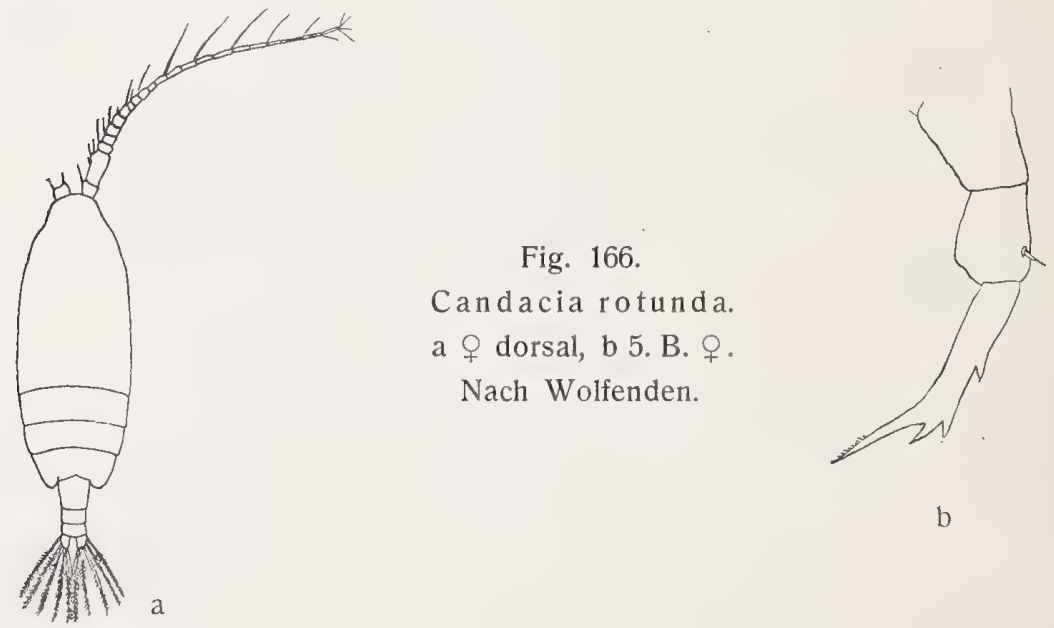

\section{Pontellidae.}

․ Kopf vom Thorax getrennt, an der Mitte seiner Seitenränder zuweilen ein Haken; die beiden letzten Thsegm. meistens verschmolzen. Rostrum gegabelt, öfters mit 2 starken Zinken endigend, zuweilen mit linsenförmig verdickter Basis, selten fehlend. Auge meistens groB, zuweilen mit 1 oder 2 Paar dorsalen Cuticularlinsen und einer ventralen Linse. Abdomen 1-3-gldr., oft asymmetrisch. 1. Antenne 16-24-gldr. Exp. der 2. Antenne mit verkürzten oder rudimentären Endgl. (letztes Gl. seiten länger als die vorhergehenden); proximales Gl. des Enp. mit dem Basp. mehr oder weniger verschmolzen. Mandibel 2-ästig (Exp. zuweilen klein), Kaulade mit 5-7 Zähnen. 1. Maxille mit großem 1. Gl. des Basp. und relativ kleinem, zuweilen rückgebildetem Exp. und Enp.; 2. Innenrandlobus. öfters umfangreich. 2. Maxille mit langen, kräftigen Reusenborsten, die am proximalen Abschnitte zuweilen rudimentär werden. 1. Gl. des Basp. des Maxillipeden groB, mit lappigem Innenrande und langen Borsten; 2. Gl. des Basp. und Enp. relativ kurz, Enp. oft mit 
reduzierter Gliederung. Exp. des 1.-4. B. 3-gldr.; Enp. des 1. B. 2- oder 3-, des 2.-4. B. 2-gldr. 5. B. rudimentär, Basp. 1- oder 2-, Exp. 1- oder 2-, Enp. 1-gldr. oder fehlend. - $\sigma^{7}$. Sekundäre Genitalcharaktere im Bau des Abdomens, der 1. Antenne, des 5. B., zuweilen auch der Augen, des Rostrums und des 5. Thsegm. Abdomen 5-gldr., mit linker Genitalöffnung. Die rechte 1. Antenne ist eine Greifantenne mit 2-4-gldr. Endabschnitt. 5. B. beiderseits 3- oder 4-gldr., selten mit Rudiment von Enp.; rechts mit zuweilen unvollkommener, zuweilen kräftiger Zange.

\section{Labidocera Lubbock.}

q. Kopf meistens ohne Seitenhaken; 1 Paar dorsaler Augenlinsen, die beim $q$ kleiner sind als beim $\sigma^{7}$; Ventralauge birnförmig; Rostrum ohne Linsen, mit kurzen, spitzen Zinken; letztes Thsegm. mit dem vorletzten verschmolzen, seitlich in eine Spitze oder einen Zipfel ausgehend, nicht immer symmetrisch. Abdomen 2- oder 3-gldr.; Gensegm., zuweilen auch Furka, asymmetrisch. 1. Antenne 23-gldr. Mandibellade mit 5-7 hakigen, spitzen Zähnen. 2. Gl. des Basp. der 1. Maxille nach außen übergebogen, ca. doppelt so lang wie der 2. Innenlobus. 2. Maxille gedrungen und besonders an der distalen Hälfte mit langen Reusenborsten beșetzt. Maxilliped 6-gldr., mit winzigen Endgl. Enp. des 1.-4. B. 2-gldr. 5. B. beiderseits aus einem 2-gldr. Basp., 1-gldr. Exp. und 1-gldr. Enp. bestehend; Enp. zuweilen sehr rudimentär. - $\sigma^{7}$. Dorsale Augenlinsen größer als beim $q$, in der Mittellinie zusammenstoßend. Ansegm. kurz; Gensegm. symmetrisch. Rechte Antenne ein Greiforgan mit 4-gldr. Endabschnitt und ziemlich stark verdickten, mittleren Gl. 5. B. beiderseits 4-gldr., rechts ohne Enp., links zuweilen mit rudimentären Enp.; die beiden Endgl. des rechten B. bilden eine kräftige Zange.

\section{\%, ठ․}

1. Stirn mit ventralwärts gekrümmtem Haken; Kopf ohne Seitenhaken

L. acuta

Stirn ohne Crista und Haken, abgerundet; Kopf mit Seitenhaken 2

2. 1. Antenne symmetrisch; Abdomen 3-gldr. 3

Rechte Antenne ein Greiforgan; Abdomen 5-gldr. 4

3. Abdomen ohne Stacheln, Gensegm. dorsal gewölbt L. wollastoni $q$ Abdomen mit Stacheln und Zacken L. kröyeri o

4. Letztes Thsegm. symmetrisch

Letztes Thsegm. asymmetrisch, rechts mit doppelter Spitze; vorletztes Gl. der Zange des 5. B. mit Doppelzacke in der Randmitte L. kröyeri $\sigma^{7}$.

\section{Labidocera wollastoni Lubbock.}

1857, Pontella w., Lubbock, p. 406, t. 10, 11. 1863, Pontella helgolandica, Claus, p. 208, t. $36,37.1878$, Pontella w., Brady, p. 73, 
t. $10 \mathrm{~A}$ f. $1-12$. 1892, L. w., Giesbrecht, p. 445 , t. 4 f. 14; t. 23 f. 5, 6, 9, $17,18,20,25,37$; t. 41 f. $1,12-14,21,22,27.1898$, L. w., Giesbrecht und Schmeil, p. 135. 1903, L. w., Sars, p. 142, t. 95, 96.

Kopf mit Seitenhaken; letztes Thsegm. symmetrisch, beiderseits in einen zugespitzten Zipfel ausgehend. Abdomen des $\bigcirc$ 3-gldr.; Gensegm. und Furka fast symmetrisch, jenes mit dorsalem Wulst. Das Gl. vor dem Kniegelenk der Greifantenne länger als das vorhergehende; 2. Gl. hinter dem Gelenk länger

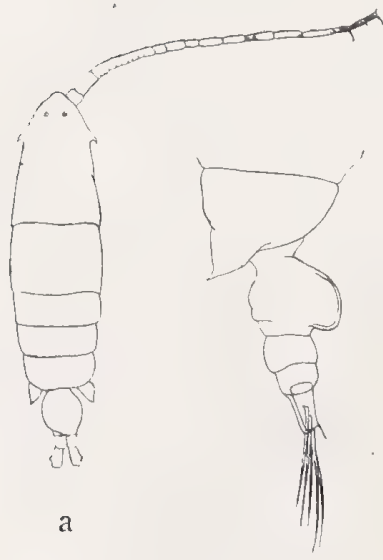

b $\mathrm{c}$

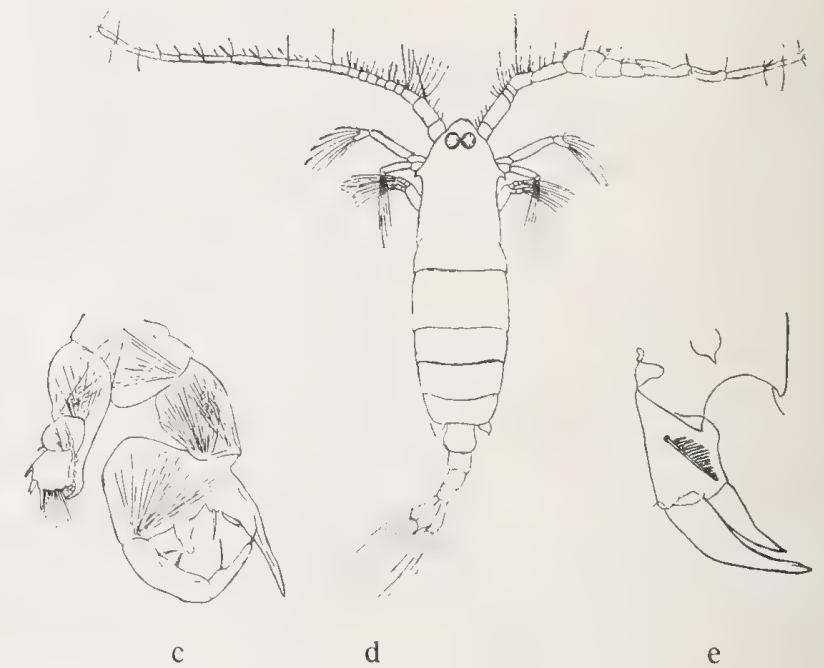

Fig. 167. Labidocera wollastoni.

a $O$ dorsal, b Abdomen $q$, lateral, c 5. B. or, d o dorsal, e 5. B. 옹 Nach Giesbrecht.

als das 3., mit langem Fortsatz am Ende des Vorderrandes. Exp. und Enp. des 5. B. des q klauenförmig, Enp. wenig kürzer als Exp. Linkes 5. B. des $\sigma^{7}$ mit langem, das runde Endgl. überragendem Rudiment von Enp.; Fortsatz an der proximalen Ecke des breiten, vorletzten Gl. des rechten B. lang und spitz; an der Randmitte dieses Gl. ein kürzerer Fortsatz. - L. $\bigcirc 2,2-2,4 \mathrm{~mm}$, of $2,2-2,3 \mathrm{~mm}$.

Mittelmeer, Nord-Atlant. Ozean.

\section{Labidocera acuta Dana.}

1849, Pontella a., Dana, p. 30. 1892, L. acutum, Giesbrecht, p. 445, t. 23 f. $15,44,46$; t. 25 f. 31,33 ; t. 41 f. $10,19,20,28,29,40$. 1898, L. a., Giesbrecht und Schmeil, p. 134.

Kopf ohne Seitenhaken, mit Crista, die nach vorne in einen Haken endigt; Seitenteile des letzten Thsegm. scharf zugespitzt, beim of symmetrisch, beim 
ot rechts mit gedrehter Spitze. Abdomen des $q$ 3-gldr., Gensegm. asymmetrisch, hinten mit ventralen Fortsätzen; Furka fast symmetrisch, ihre Borsten z. T. an der Basis aufgebläht. Das Gl. vor dem Kniegelenk der Greifantenne viel länger als das vorhergehende; Reibleiste am Gl. hinter dem Gelenk dünn, nicht verlängert. Exp. des 5. B. des $q$ etwas variabel, mit relativ großen Spitzen am Außenrande; Enp. klauenförmig, $1 / 2-1 / 3$ so lang wie das Exp. Endhaken der Zange des 5. B. breit, blattartig, wie auch der Anhang an der gegenüberliegenden Ecke des vorletzten GI. - L. \& 3,05-3,4 mm, or 2,8-3,3 mm.

Atlant. Ozean; Pacif. Ozean; Rotes Meer, Ind. Ozean.

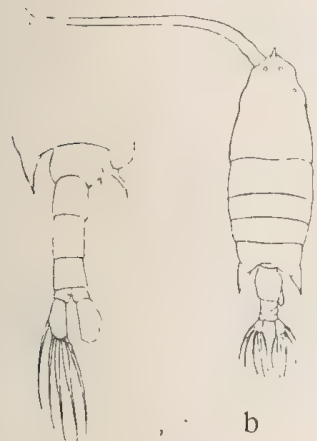

a

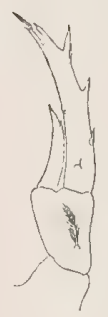

C

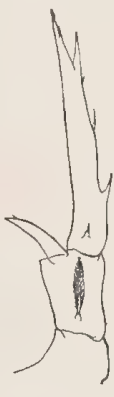

d

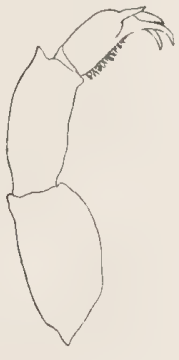

e

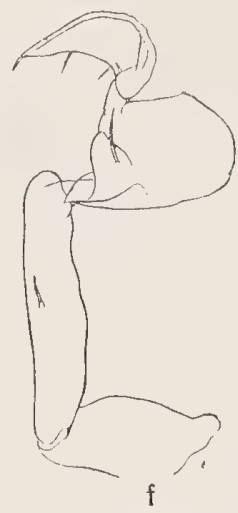

Fig. 168. Labidocera acuta.

a 5. Thsegm. und Abdomen or, dorsal, b $q$ dorsal, c 5. B. , d 5. B. $q$ var., e linkes 5. B. $\sigma^{\pi}$, f rechtes 5. B. or. Nach Giesbrecht.

\section{Labidocera kröyeri Brady.}

1883, P ontella k., Brady, p. 93, t. 39. 1892, L. k., Giesbrecht, p. 446, t. 23 f. 13,38 ; t. 25 f. 30 ; t. 41 f. $6,11,39.1898$, L. k., Giesbrecht und Schmeil, p. 135.

Kopf mit Seitenhaken; Seitenteile des letzten Thsegm. zugespitzt, beim $q$ symmetrisch, beim $\sigma^{7}$ rechts in 2 Spitzen endigend. Abdomen des $q 3$-gldr.; Ansegm. kurz; Gensegm. und folgendes Segm. mit (z. T.) mehrspitzigen Haken und Zacken; Furka ziemlich symmetrisch. Gl. vor dem Kniegelenk der Greifantenne mit einigen starken Zacken und viel länger als das vorhergehende Gl., das einen stark gebogenen Haken am Vorderrande hat; das Gl. hinter dem Gelenk viel länger als das folgende. Exp. des 5. B. des $q$ klauenförmig. Enp. kaum halb so lang wie das Exp., in 2 Spitzen endigend. Handgl. der Zange des 5. B. des $\sigma^{7}$ mit kurzem, 2-spitzigem Fortsatz an der Randmitte. - L. ๆ 2,4-2,5 mm, of 1,95-2,05 $\mathrm{mm}$.

Nord-Atl. Ozean (bei Irland); Pacif. Ozean. 


\section{Pontella Dana.}

Q. Kopf mit Seitenhaken; 1 Paar dorsale Augenlinsen und eine Rostrallinse vor dem Ventralauge; die ersteren sexuell nicht verschieden, letztere beim o zuweilen dünner als beim $\sigma^{\Im}$; Rostrum mit gedrungenen Zinken. Letztes Thisegm. vom vorletzten getrennt, meist mit spitzen Lateralecken, zuweilen asymmetrisch; Abdomen 2- oder 3-gldr., asymmetrisch. 1. Antenne 24-gldr. Mandibellade mit 7 spitzen Zähnen. 1. und 2. Maxille wie bei Labidocera, aber 2. Gl. des Basp. der 1. Maxille nur $1 \frac{1}{2}$ mal so lang wie der Innenrandlobus. Maxilliped 7-gldr. Enp. des 1. B. 3-, des 2.-4. B. 2-gldr.; 5. B. ähnlich wie bei Labidocera. - $\sigma^{7}$. Rostrallinse des Ventralauges zuweilen dicker als beim $\bigcirc$, dorsale Linsen wie beim $\bigcirc$. Letztes Thsegm. anders geformt als beim $q$, meistens symmetrisch. Abdomen symmetrisch. Rechte 1. Antenne ein Greiforgan mit 2-gldr. Endabschnitt. 5. B. ähnlich wie bei Labidocera, aber das linke ohne Rudiment eines Enp.

\section{Pontella lobiancoi Canu.}

1888, Pontellina 1., Canu, p. 102, t. 8, 9. 1892, P. I., Giesbrecht, p. 461, t. 24 f. $4,42,44$; t. 40 f. $9,10,28,30,31$; t. 42 f. 31 . 1893, P. (Iva) 1., Claus, p. 273, t. 4 f. $1-4.1898$, P. 1., Giesbrecht und Schmeil, p. 143.
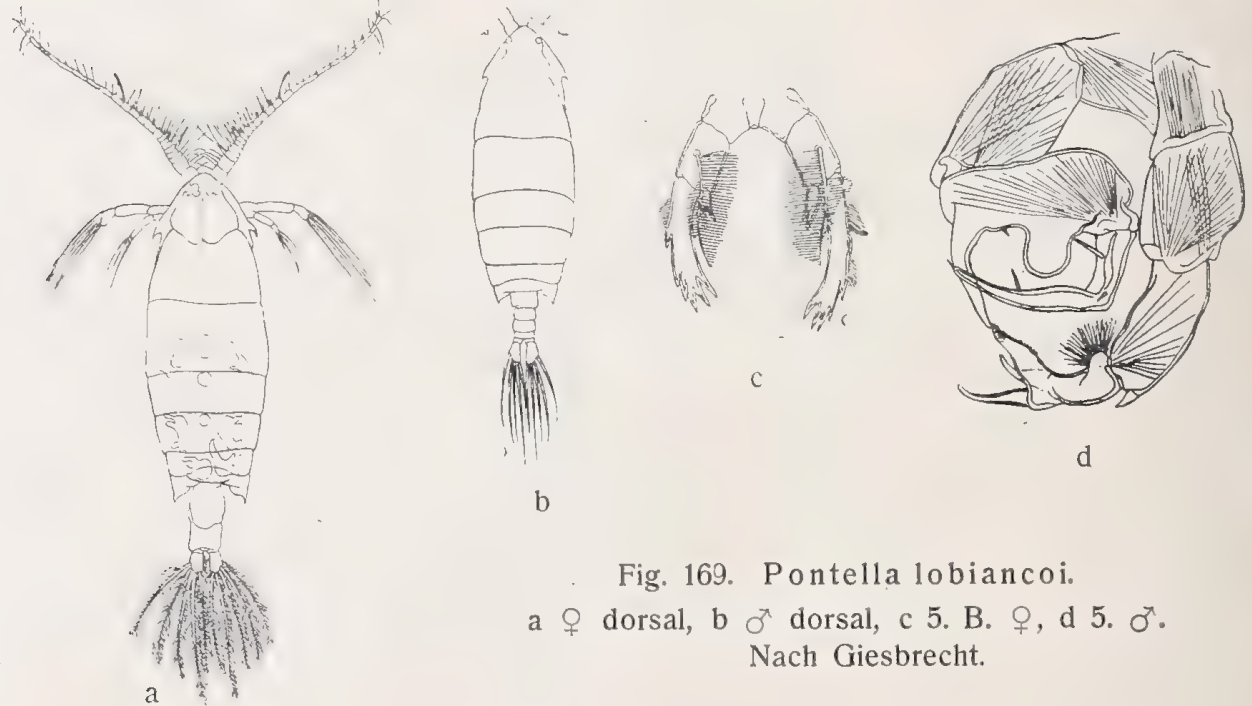

Fig. 169. Pontella lobiancoi.

a $q$ dorsal, b o dorsal, c 5. B. ㅇ, d 5. ơ. Nach Giesbrecht.

Letztes Thsegm. beiderseits zugespitzt, beim $ᄋ$ symmetrisch, beim $\sigma^{\pi}$ rechts verlängert. Abdomen des $\bigcirc$ 3-gldr., mit leichter Asymmetrie der beiden vorderen Segm. und der Furka. Mittelstück der Greifantenne des ov oval; Endstück etwa so lang wie die. 3 Gl. vor dem Kniegelenk, von denen das distale neben einer feingezähnelten Reibleiste einen Vorsprung in der Mitte 
des Vorderrandes hat. 5. B. des $\%$ : Exp. mit 6 Zacken, am Ende und Außenrande, Enp. klein. 5. B. des o: einer der apikalen Fortsätze des linken B. breit, mit feinen Spitzen besetzt; vorletztes Gl. des rechten 5. B. mit breitem, rundem Fortsatz an der Randmitte; Endhaken vor der Mitte knieförmig gebogen. - L. ㅇ 3,95-4,2 $\mathrm{mm}$, o $3,3-3,75 \mathrm{~mm}$.

Mittelmeer, Engl. Kanal.

Anmerkung. Pontella inermis Brady ist ein unreifes $\sigma^{x}$ und es werden vermutlich ebenfalls junge Tiere sein, welche Tompson mit diesem Namen für Liverpool Bai aufführt (Giesbrecht, 1892, p. 467).

\section{Anomalocera Templeton.}

O. Kopf mit Seitenhaken; 2 Paar dorsale Augenlinsen; Rostrum ohne Linse, mit kräftigen Zinken. Letztes Thsegm. vom vorletzten getrennt, symmetrisch, beidersetts zugespitzt. Abdomen 3-gldr., asymmetrisch. 1. Antenne 20-gldr., Mandibellade mit 7 spitzen Zähnen. 2. Gl. des Basp. der 1. Maxille kaum so groß wie der 2. Innenrandlobus, 1. Maxille, 2. Maxille, Maxilliped und 1.-4. B. im übrigen ähnlich wie bei Pontella. 5. B. mit 2-gldr. Basp., 2-gldr. Exp. und 1-gldr. Enp. - $\sigma^{t}$. Ventralauge stärker vorspringend als beim $q$. Letztes Thsegm. asymmetrisch. Abdomen asymmetrisch. Greifantenne wie bei Pontella. 5. B. wie bei Pontella, aber Zange des rechten B. weniger kräftig.
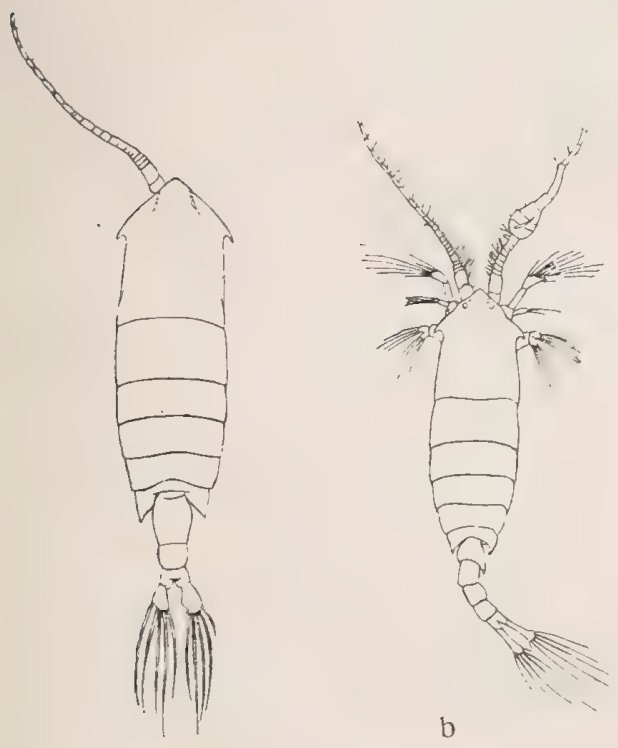

b
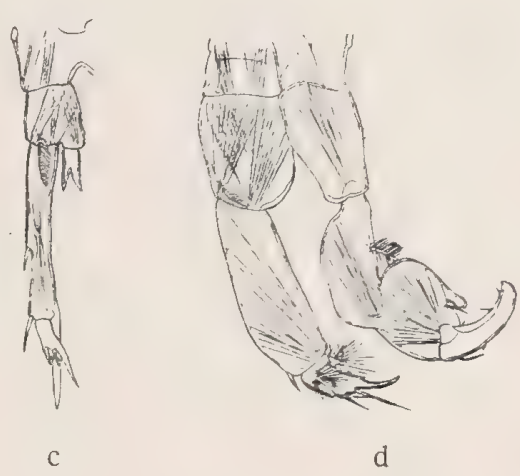

Fig. 170.

Anomalocera patersoni.

a $q$ dorsal, b o dorsal, c 5. B. 우, d 5. B. ơ. Nach Giesbrecht.

\section{Anomalocera patersoni Templeton.}

1837, A. patersonii, Templeton, p. 34,118, t. 5.1863 , Irenaeus p., Claus, p. 206, t. 37.1878 , A. p., Brady, p. 75 , t. 11 f. $1-14$; t. 10 f. $13,14$. 
1892, A. p., Giesbrecht, p. 479 , t. 3 f. 9 ; t. 25 f. $1,3,15,16,19,37,38$; t. 40 f. 43-48, 54. 1898, A. p., Giesbrecht und Schmeil, p. 145. 1903, A. p., Sars, p. 139, t. $92-94$.

Letztes Thsegm. des or rechts mit hakigem Fortsatz, Gensegm des or rechts mit Auswuchs. Gensegm. des $\bigcirc$ am Hinterrande mit griffelförmigem Fortsatz, rechter Furkalzweig beim $q$ größer als der linke. Ventralauge des q flach gewölbt, beim ơ keulenförmig verlängert. - L. ๆ 3,2-4,1 mm, o $3-4 \mathrm{~mm}$.

Mittelmeer, Schwarzes Meer, Atlant. Ozean; Pacif. Ozean (?); Ind. Ozean.

\section{Parapontella Brady.}

. Kopf ohne Seitenhaken; Auge nicht vorgewölbt, ohne Cuticularinsen; Rostralfäden dünn. Letztes Thsegm. mit dem vorletzten verschmolzen, symmetrisch. Abdomen 3-gldr., symmetrisch. 1. Antenne 19-gldr. Endgl. des Exp. der 2: Antenne länger als die vorhergehenden Gl. Kaulade der Mandibel gestreckt; 2. Gl. des Basp. stabförmig; Exp. klein, proximal von der Mitte des Basp. ansitzend. 1. Maxille gestreckt; Exp. und Enp. kurz, nicht artikulierend; Außenrandlobus nur durch 4 kurze Borsten angedeutet. Proximale Loben der 2. Maxille sehr reduziert. Maxilliped 4-gldr. Enp. des 1. B. 3-, des 2.-4. B. 2-gldr. 5. B. mit 2-gldr. Basp. und 1-gldr. Exp. und Enp. - o . Letztes Thsegm. und Abdomen asymmetrisch. Greifantenne mit 2-gldr. Endabschnitt. 5. B. beiderseits 3-gldr., rechts mit Rudiment eines Enp.

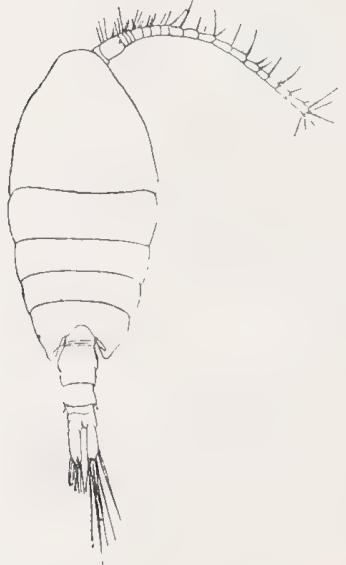

a
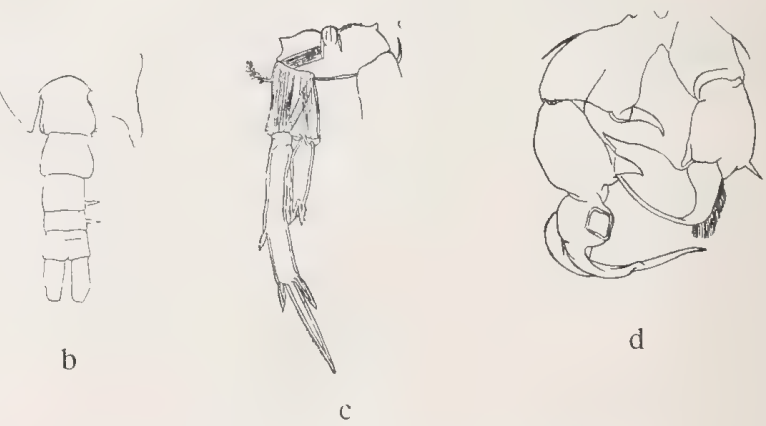

d

Fig. 171. Parapontella brevicornis.

a $O$ dorsal, b Abdomen ơ, dorsal, c 5. B. . , d 5. B. ot. Nach Giesbrecht.

\section{Parapontella brevicornis Lubbock.}

1857, Pontellina b., Lubbock, p. 407, t. 11 . 1878, P. b., Brady, p. 69 , t. 9 f. $1-16.1892$, P. b., Giesbrecht, p. 501, t. 31 f. $17-19,27-30,33-35$; 
t. 38 f. 41, 43, 45, 46. 1898, P. b., Giesbrecht und Schmeil, p. 150. 1903, P. b., Sars, p. 145 , t. 97,98 .

1. Antenne den Hinterrand des 2. Thsegm. erreichend. Hinterrand des 2. Abdsegm. des $q$ mit 2 subdorsalen Dornen; 3. und 4. Abdsegm. des o jedes auf der rechten Seite mit einem kleinen Vorsprung. Letztes Thsegm. beim of mit abgerundeten Ecken, beim of rechts merklich nach hinten verlängert. - L. O $1,37-1,6 \mathrm{~mm}, \sigma^{7} 1,25-1,55 \mathrm{~mm}$.

Mittelmeer, Atlant. Ozean (Europa).

\section{Acartia Dana.}

‥ Kopf ohne Seitenhaken; Auge nicht vorgewölbt, ohne Cuticularlinsen; Rostralfäden fein oder fehlend. Letztes Thsegm. mit dem vorletzten verschmolzen, zuweilen beiderseits in eine Spitze ausgehend, symmetrisch. Abdomen 3-gldr., symmetrisch. 1. Antenne 17-gldr., mit knotigen Vorsprüngen an den Ansatzstellen der langen Borsten. Enp. der 2. Antenne mit sehr gestrecktem Endgl.; Exp. kurz. Kaulade der Mandibel schwach; Exp. und Enp. undeutlich gegliedert; Exp. artikuliert in der Mitte des 2. Gl. des Basp. Exp. und Enp. der 1. Maxille rückgebildet, Exp. durch 2 Borsten vertreten; Außenrandlobus mit langen Borsten. 2. Maxille mit langen Reusenborsten, auch an den proximalen Loben. Maxilliped 4-gldr. Exp. des 1.-4. B. 3-gldr., Enp. 2-gldr. 5. B. 1-ästig, 2- oder 3-gldr.; Endgl. fast stets klauen- oder pfriemförmig; außen am Basp. eine dünne, meist lange Fiederborste. - $\sigma^{7}$. Greifantenne rechts, nicht stark umgeformt, mit nur schwach verdickten Mittelgl. 5. B. 1-ästig, rechts 4-, links 3-gldr.

1. Endgl. des 5. B. gestreckt blattförmig, mit einer langen, geschweiften Borste am Innenrande und 3 kurzen Borsten am Außen- und Endrande

Endgl. des 5. B. borsten-, pfriem- oder klauenförmig

A. forcipata

. Lateralecken des letzten Thsegm, beiderseits in eine spitze Zacke endigend 3 Dieselben abgerundet, zuweilen mit 1- oder mehreren kleinen Spitzen besetzt 4

3. Dorsaler Hinterrand der beiden vorderen Abdsegm. mit feinen Spitzen besetzt; nur das 1. Gl. der 1. Antenne mit dickem Dorn

A. danae Dorsaler Hinterrand der beiden vorderen Abdsegm. mit je 2 Stacheln (die des Gensegm. kleiner als die des folgenden Segm); 1. Antenne unbedornt

4. Furka und mittlere Borsten geschwollen

A. centrura Furka und ihre Borsten nicht geschwollen

A. discaudata

5. Rostralfäden vorhanden

A. bifilosa

Rostralfäden fehlen 
6. Endklaue von der verdickten Basis an allmählich verjüngt, dick, kürzer als die Fiederborste; letztes Thsegm. am Seitenrande mit einigen (4-5) kurzen Zähnchen A. clausi Enđklaue dünn, in der Mitte leicht gebogen, länger als die Fiederborste; letztes Thsegm. am Rande, außer mit einigen seitlichen Härchen, mit einem subdorsal sitzenden, kleinen Dorn

A. longiremis.

1. Seitenecken des letzten Thsegm. beiderseits in eine spitze Zacke endigend; Lateralstacheln des 2. und Dorsalstacheln des 3. und 4. Abdsegm. ungefähr gleich groß

A. centrura Seitenecken des letzten Thsegm. abgerundet, zuweilen mit einer oder mehreren kleinen Spitzen besetzt

2. Rostralfäden vorhanden; Endgl. des rechten 5. B. gedrungen, mit konvexer Mitte des AuBenrandes; 2. Gl. des rechten 5. B. in der Mitte des Innenrandes gekerbt

A. bifilosa Rostralfäden fehlen

3. Vorletztes Gl. des rechten 5. B. mehrfach länger als breit, mit schmalem Vorsprung etwas distal von der Mitte des Innenrandes; drittletztes Gl. desselben B. ohne Vorsprung

A. discaudata Vorletztes Gl. des rechten 5. B. etwa 2 mal so lang wie breit, mit einem Vorsprung am Innenrande, der an der Basis wenigstens halb so breit wie das Gl. lang ist; drittletztes Gl. desselben B. distal mit rundem Vorsprung 4

4. Auf der Dorsalseite des letzten Thsegm, nahe am Hinterrande beiderseits ein kleiner Dorn

A. longiremis Daselbst sitzen einige $(3-5)$ kurze, dreieckige Zähnchen

A. clausi.

\section{Acartia clausi Giesbrecht.}

1889, A. clausii, Giesbrecht, p. 25. 1889, A. longiremis, Bourne, p. 147, t. 11 f. 4-6. 1890, A. clausii, Canu, p. 476, t. 24 f. $1-5.1892$, A. clausii, Giesbrecht, p. 507, t. 30 f. $2,4,13-15,17,28,36,37$; t. 42 f. 32 ; t. 43 f. 3 , 5, 14. 1898, A. c., Giesbrecht und Schmeil, p. 152, f. 30, 31. 1903, A. c., Sars, p. 150 , t. 101.

Rostralfäden fehlen; letztes Thsegm. seitlich abgerundet, am Rande mit 4-5 dreieckigen Zähnchen. Abdsegm. mit kleinen Spitzen an den dorsalen Hinterrändern, ohne Stacheln bei $q$ und $\sigma$. 1. Antenne des $q$ unbedornt, erreicht den Hinterrand des Gensegm. Mittelgl. des 5. B. des $q$ quadratisch; Endklaue über doppelt so lang wie das Gl., kräftig; Fiederborste beträchtlich länger als die Endklaue. 5. B. des ơ vergl. Fig. 172. - L. ๆ 1,15-1,22 mm, o $1-1,07 \mathrm{~mm}$.

Mittelmeer, Schwarzes Meer, Atlant. Ozean; Ind. Ozean. 


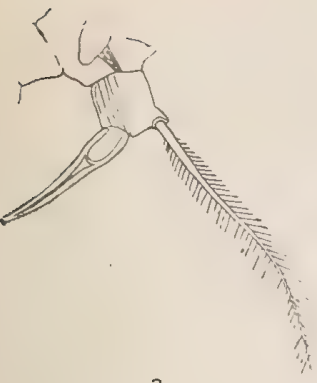

a

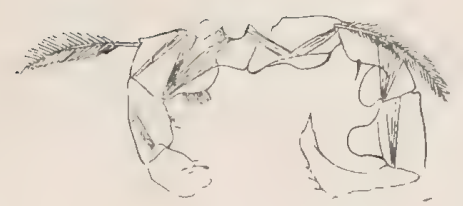

b

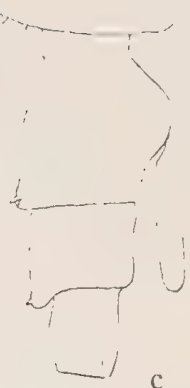

Fig. 172. Acartia clausi.

a 5. B. ㅇ, b 5. B. $\sigma^{\top}$, c Abdomen mit Spermatophor 오. a und b nach Giesbrecht, c original.

\section{Acartia longiremis Lilljeborg.}

1853, Dias 1., Lilljeborg, p. 181, t. 24 . 1882, Dias 1., Giesbrecht, p. 146 , t. 3 f. 7, 21. 1892, A. 1., Giesbrecht, p. 507, t. 43 f. 17, 25. 1898, A. 1., Giesbrecht und Schmeil, p. 153. 1903, A. 1., Sars, p. 149, t. 99, 100.

Verwandt mit clausi, aber: - Letztes Thsegm. beiderseits, außer mit einigen seitlichen Härchen, mit einem mehr dorsal sitzenden, kleinen Dorn; Gensegm.

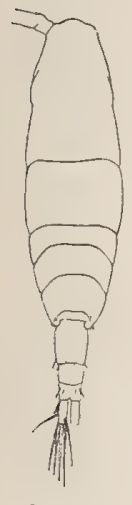

a

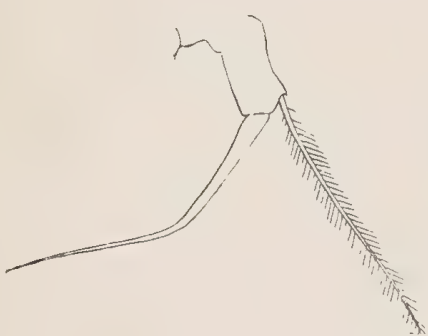

b

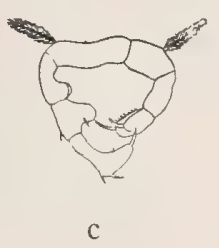

Fig. 173. Acartia longiremis.

a $q$ dorsal, b 5. B. + , c 5. B. $\sigma^{7}$, d 5. Thsegm. und Abdomen mit Spermatophor $q$. a - c nach Giesbrecht, d original.

und Ansegm. des $\uparrow$ mit einigen ziemlich langen, steifen Härchen; 5. B. des O mit gestreckterem Mittelgl. und viel längerer und dünnerer Endklaue; letztere ca. $4 \mathrm{mal}$ so lang wie das Mittelgl. und länger als die Fiederborste; rechtes 5. B. des ot relativ kürzer als bei clausi, mit mehr gedrungen gebauten distalen Gl. - L. † 0,98-1,25 mm, ơ $0,9-1 \mathrm{~mm}$.

Mittelmeer, Nord-Atlant. Ozean; Polarmeer. 


\section{Acartia discaudata Giesbrecht.}

1881, Dias discaudatus, Giesbrecht, p. 257. 1882, Dias discaudatus, Giesbrecht, p. 148, t. 3 f. 4, 22, 23. 1892, A. d., Giesbrecht, p. 507, t. 43 f. 2, 24. 1898, A. d., Giesbrecht und Schmeil, p. 153. 1903, A. d., Sars, p. 152, t. 102.

Rostralfäden fehlen; letztes Thsegm. seitlich abgerundet. Abdomen bei $q$ und $\sigma^{\top}$ ohne Stacheln; Ansegm. des $q$ verbreitert; Furka des $q$ aufge-

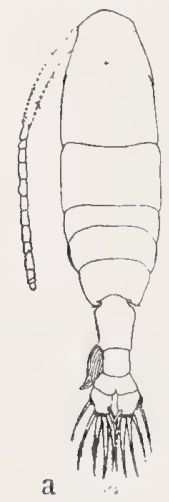

Fig. 174 .

Acartia discaudata.

a $q$ dorsal, b 5. B. o․

Nach Giesbrecht.

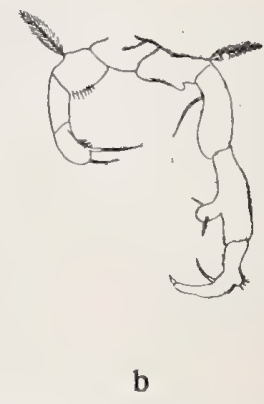

bläht, mit kurzen, z. T. verdickten Borsten. 1. Antenne des $\nmid$ unbedornt, erreicht den Hinterrand des Gensegm. nicht. 5. B. des $q$ ähnlich wie bei clausi, aber mit dünnerer Endklaue. Rechtes 5. B. des ot relativ länger als bei clausi; besonders das vorletzte Gl, gestreckter und mit dünnerem Fortsatz am Innenrande; Endborsten des linken 5. B. länger. … L. O $1-1,2 \mathrm{~mm}$, ơ $0,9-1,1 \mathrm{~mm}$.

Ostsee, Nordsee, Engl. Kanal, Irische Küste, S.-W.-Küste Norwegens, Faröerkanal.

\section{Acartia bifilosa Giesbrecht.}

1881, Dias bifilosus, Giesbrecht, p. 257. 1882, Dias bifilosus, Giesbrecht, p. 147, t. 2 f. 18 ; t. 3 f. $6,18,20.1885$, Dias interm edius, Poppe, p. 173, t. 5 f. 13, 21, 22. 1892, A. b., Giesbrecht, p. 507, t. 43 f. 15, 23; A. intermedia, ibid. p. 508. 1898, A. b. + var. intermedia, Giesbrecht und Schmeil, p. 153, 154.

Rostralfäden vorhanden; letztes Thsegm. seitlich abgerundet. Abdomen bei $q$ und on ohe Stacheln. Gensegm. und folgendes Segm. des $q$ mit sehr feinen Spitzen auf der Dorsalfläche. 1. Antenne des $q$ unbedornt,

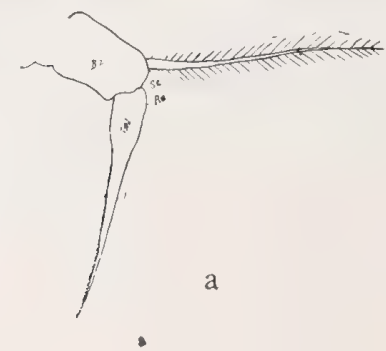

Fig. 175

Acartia bifilos a.

a 5. B. q, b 5. B. ơ.

Nach Giesbrecht.

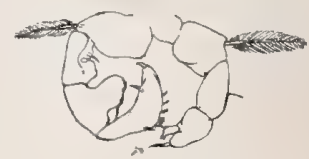

b 
erreicht nicht den Hinterrand des Gensegm. Mittelgl. des 5. B. des q länger als breit; Endklaue über doppelt so lang wie das Gl., ziemlich gerade, pfriemförmig, etwa so lang wie die Fiederborste. 5. B. des $\sigma^{\text {T }}$ ähnlich wie bei clausi, aber 2. Gl. des rechten B. von anderer Form und Endgl. des linken B. ein kleiner, dicker Anhang. - L. \& 1-1,1 mm, o $1-1,1 \mathrm{~mm}$.

Ostsee, Nordsee, Nord-Atlant. Ozean bis $63^{\circ}$ N. B.

Anmerkung. Dias intermedius Poppe halte ich für völlig identisch mit Acartia bifilosa. Giesbrecht erwähnt sie 1898 als Varietät von bifilosa und hebt als den einzigen Unterschied hervor, daß die Endklaue des.5. B. beim $q$ der Varietät kürzer ist und eine stärkere aufgetriebene Basis hat. Nach meinen Beobachtungen an dem 5. B. (ㅇ) der in dem Zuidersee zahlreich vorkommenden Individuen von A. bifilos a ist das genannte B. in dieser Hinsicht ziemlich variabel.

\section{Acartia danae Giesbrecht.}

1889, A. d., Giesbrecht, p. 26. 1892, A. d., Giesbrecht, p. 508 , t. 30 f. 1, 23 ; t. 43 f. 8. 1898, A. d., Giesbrecht u. Schmeil, p. 154.

. Rostralfäden vorhanden. Letztes Thsegm. beiderseits in eine Zacke verlängert. Abdomen ohne Stacheln, aber mit feinen Spitzen am dorsalen Hinterrande der beiden vorderen Segmente. Gensegm. höchstens so lang wie das folgende. 1. Antenne erreicht das Rumpfende; 1. Gl. mit ziemlich langem und dickem Dorn. Mittelgl. des 5. B. länger als breit; Endklaue noch nicht 2 mal so lang wie das Gl, hinter der Mitte mit starken Zacken; Fiederborste ca. 3 mal so lang wie die Endklaue. L. 1,16 mm. - o unbekannt.

Atlant. Ozean (bis $49^{\circ}$ N. Br.); Pacif. Ozean.
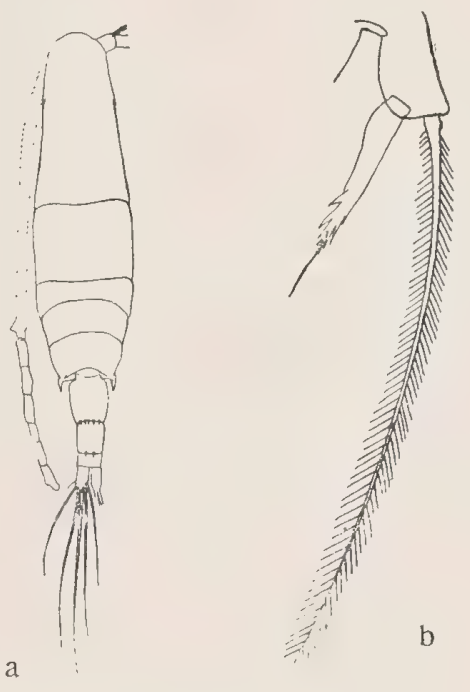

Fig. 176. Acartia danae. a t dorsal, b 5. B. ․ Nach Giesbrecht.

\section{Acartia centrura Giesbrecht.}

1889, A. c., Giesbrecht, p. 25. 1892, A. c., Giesbrecht, p. 508, t. 30 f. 26,31 ; t. 43 f. 9, 16. 1898, A. c., Giesbrecht und Schmeil, p. 155.

Rostralfäden vorhanden. Letztes Thsegm. beiderseits in eine Zacke verlängert, neben der ein kleiner Dorn sitzt. 1. und 2. Abdsegm. des $q$ jederseits mit einem Stachel auf der Dorsalfläche (das vordere Stachelpaar ist kleiner als das hintere); beim or sitzen am 2. Segm. beiderseits 3, an den beiden folgenden Segm. beiderseits je 1 Stachel. 1. Antenne des $q$ unbedornt, überragt etwas den Hinterrand des Gensegm. Mittelgl. des 5. B. des $q$ länger 
als breit; Endklaue etwa doppelt so lang wie das Gl., mit stark verdickter Basis und dünnem, borstenartigem Endstück; Fiederborste länger als die

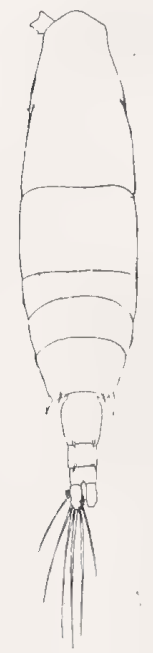

a

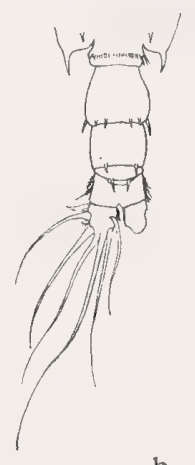

b

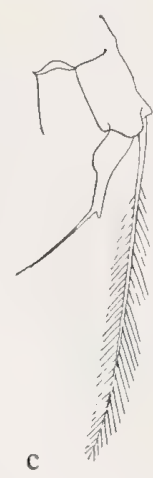

c

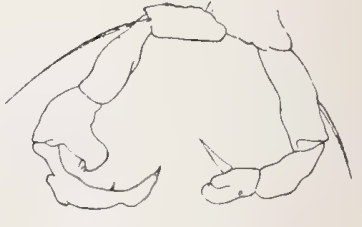

d

Fig. 177. Acartia centrura.

a $q$ dorsal, b Abdomen $\sigma^{x}$, dorsal, c 5. B. $q$, d 5. B. $\sigma^{\pi}$.

Nach Giesbrecht.

Endklaue. 1. Gl. des linken 5. B. des o viel gestreckter als bei A. clausi, Endgl. mit einem ziemlich langen Stachel; 2. Gl. des rechten 5. B. ohne Fortsatz am Innenrande; Fortsatz am folgenden Gl. länger und Endgl. von anderer Form als bei A. clausi. - L. ᄋ 1,2-1,24 mm, ơ $1,03 \mathrm{~mm}$.

Nord-Atlant. Ozean; Rotes Meer, Ind. Ozean.

7. Acartia forcipata Thompson und Scott.

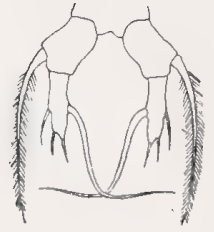

Fig. 178.

Acartia forcipata.

5. B. ㅇ.

Nach Thompson und A. Scott
1897, A. f.. Thompson und A. Scott, p. 81, t. 7 f. 3, 10. 1898, A. f., Giesbrecht und Schmeil, p. 156.

q. Letztes Thsegm. seitlich abgerundet, beiderseits mit einem kleinen dorsalen Dorn versehen. Abdomen ohne Stacheln. Endgl. des 5. B. nicht klauenförmig, sondern gestreckt blattförmig, ca. $3 \mathrm{mal}$ so lang wie breit, mit 3 dünnen Borsten am Außen- und Endrande und einer langen, dicken, geschweiften Borste in der Mitte des Innenrandes. L. $2,35 \mathrm{~mm}$. - of unbekannt.

Golf von St. Lorenz.

Anm. Acartia laxa Dana und Acartia denticornis Brady, welche beide von Thompson und A. Scott (1897) für den Nord-Atl. Ozean erwähnt werden, sind nach Angabe von Giesbrecht (G. und Schmeil, 1898) species incerti generis.

Paracartia T. Scott.

Im allgemeinen wie Acartia gebildet, aber die sexuellen Differenzen stärker markiert: 
Letztes Thsegm. des $q$ flügelförmig verbreitert; Gensegm. des $\bigcirc$ seitlich verbreitert; Furka des $q$ breit, ein wenig asymmetrisch, beiderseits eine der Endborsten in einen kräftigen Dorn verwandelt. Abdomen des $\sigma^{\pi}$ sym-
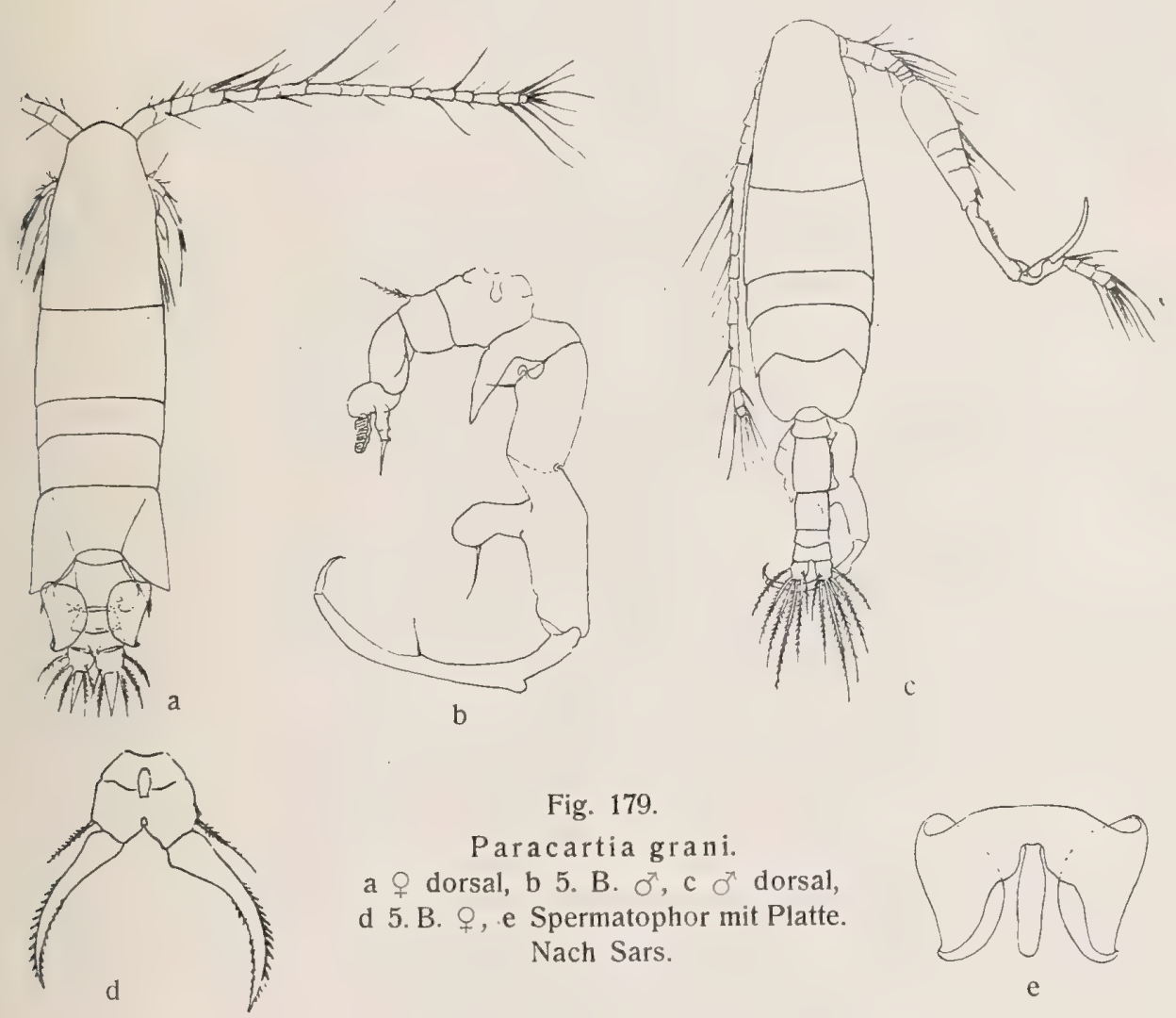

Fig. 179.

Paracartia grani. a o dorsal, b 5. B. ơ, c o dorsal, d 5. B. , , e Spermatophor mit Platte. Nach Sars.

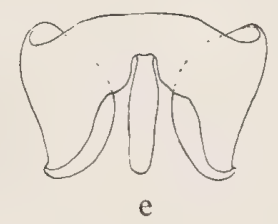

metrisch, mit normal entwickelten Endborsten der Furka. Der am Gensegm. des $\$$ befestigte Spermatophor von einer dünnen Platte begleitet. Greifantenne des $\sigma^{7}$ rechts, viel stärker umgeformt als bei Acartia, mit breit geschwollenen Mittelgl. 5. B. des $q$ mit verschmolzenen Mittelgl. und klauenförmigem Endgl.

\section{Paracartia grani G. O. Sars.}

1904, P. g., Sars, n: o 4, p. 1, t. 1-4.

Rostralfäden vorhanden. Letztes Thsegm. beim $q$ flügelförmig verbreitert, beim $\delta$ abgerundet. Abdomen des $q$ kurz mit stark verbreitertem Gensegm. und asymmetrischer Furka; letztere beiderseits mit 1 Endborste auffallend verdickt. Abdomen beim $q$ und of ohne Stacheln. 1. Antenne des $q$ ungefähr so lang wie der Vorderkörper, unbedornt. Mittelgl. des 5. B. des q miteinander verschmolzen; Endklaue kräftig, gebogen, rechts etwas größer als Nord. Plankton, 
links; Fiederborste dünn und kurz. Rechtes 5. B. des or fast 3 mal so lang wie das linke, am Innenrande des 1. Gl. mit spitzem, am Innenrande des 3. Gl. mit abgerundetem Fortsatz; sein Endgl. bildet einen sehr langen, dünnen Haken; linkes 5. B. des o ohne Innenrandfortsätze, vorletztes Gl. mit einer zarten, gerippten Platte und schmalem Endgl. Spermatophor schmal, flaschenförmig; die Begleitplatte beiderseits aufwärts gebogen. - L. $1 \mathrm{~mm}$.

Norwegische Westküste.

\section{Tortanus Giesbrecht.}

Q. Kopf ohne Seitenhaken. Auge groß, nicht vorgewölbt, ohne Cuticularlinsen. Rostrum fehlt; vor der Oberlippe eine hufeisenförmige, gefranzte Lamelle. Letztes Thsegm. mit dem vorletzten verschmolzen oder davon gesondert, symmetrisch. Abdomen 2- oder 3-gldr., asymmetrisch, öfters um die Längsachse gedreht. 1. Antenne der von Acartia ähnlich. Exp. und Enp. der 2. Antenne etwa gleich lang; Endgl. des Exp. rudimentär. Exp. und Enp. der Mandibel artikulieren am Ende des gestreckten 2. Gl. des Basp. 1. Maxille besteht nur aus dem 1. Gl. des Basp. und den stark bewaffneten beiden proximalen Innenrandloben. 2. Maxille mit ganz reduzierten proximalen Loben. Maxilliped 3-gldr. Exp. des 1.-4. B. 3-gldr., Enp. des 1. B. 2- oder 3-gldr., des 2--4. B. 2-gldr. 5. B. 1-ästig, 2- oder 3-gldr., nicht immer symmetrisch. - $\sigma^{7}$. Abdomen 5-gldr, symmetrisch oder asymmetrisch. Greifantenne mit stärker verdickten Mittelgl. als bei Acartia; 5. B. dem von Acartia ähnlich, aber kräftiger.

\section{Tortanus discaudatus Thompson-und A. Scott.}

1897, Corynura discaudata, Thompson und A. Scott, p. 80 , t. 6 f. $1,10,11$; t. 7 f. 1,2 . 1898 , T. d., Giesbrecht und Schmeil, p. 158.1900 , C. bumpusii Wheeler, p. 184 , f. 21 .

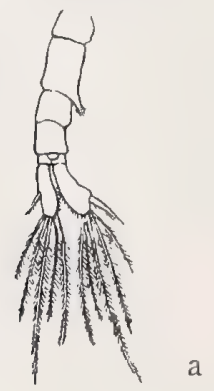

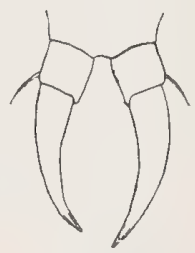

$\mathrm{b}$

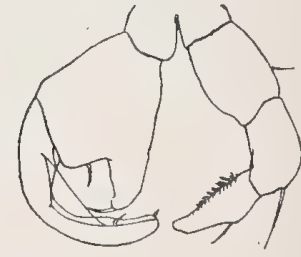

C

Fig. 180. Tortanus discaudatus.

a Abdomen des ơ, dorsal, b 5. B. Q, c 5. B. ơ. Nach Thompson und A. Scott.

9. 4. und 5. Thsegm. verschmolzen, letzteres jederseits mit nach außen gebogener Spitze. Abdomen 3-gldr., Gensegm. so lang wie die beiden folgen- 
den, fast gleich langen Segm. zusammen; 2. Segm. mit einem Büschel feiner Börstchen auf der rechten Seite; Ansegm. asymmetrisch, mit der Furka verschmolzen. Furkaläste gleich lang; rechts mehr als 2 mal so breit als links, mit behaartem Innenrand und verdickter Außenrandborste. 1. Antenne die Basis der Furka erreichend. Enp. des 1. B. 3-gldr. 5. B. etwas asymmetrisch, mit glattrandigem Endgl. - $\sigma^{7}$. Grenze zwischen 4. und 5. Thsegm. nicht deutlich; letzteres mit abgerundeten Ecken. Abdomen 5-gldr.; 1. Segm. etwas asymmetrisch, mit einem runden Vorsprung auf der linken Seite; 2. Segm. mit spitzem Vorsprung auf der rechten Seite; Furka asymmetrisch, rechts länger als links; rechte Außenrandborste länger und breiter als die linke. 5. B. jederseits 3-gldr., rechts eine starke Zange bildend, Mittelgl. stark verbreitert mit Auswuchs, Endgl. gebogen und an der Innenseite ausgehöhlt. - L. $q^{\circ}$ $2,25 \mathrm{~mm}$, o $1,8-2 \mathrm{~mm}$.

Ostküste Nord-Amerikas. 


\section{Trib. Podoplea.}

Rumpf so in Vorder- und Hinterrumpf geteilt, daß die Grenze vor das 5. Thsegm. fällt ${ }^{1}$ ); 1. Segm. des Hinterrumpfes (5. Thsegm.) fast stets mit rudimentärem Beinpaar; die im folgenden Segm. liegenden Genitalorgane beim $q$ und $\delta$ stets mit symmetrisch gelegenen Mündungen; weder das letzte $B$. des Vorderrumpfes, noch das rudimentäre Beinpaar beim $\sigma^{7}$ ein Kopulationsorgan; beide oder keine der 1 . Antennen beim $\sigma^{7}$ genikulierend. Gliedmaßen des Kopfes mit reduzierter Gliederung und verminderten bis fehlenden Fiederborsten, aber mit Haken- und Klammerborsten. Herz fast immer fehlend.

1) Während bei den Gymnoplea, auch in den Fällen, wo das 5. B. und mit diesem auch das 5. Thsegm. reduziert sind, die Grenze zwischen Vorder- und Hinterkörper mit demjenigen zwischen Thorax und Abdomen zusammenfällt, wird bei den Podoplea das letzte (5.) Thsegm. dem Hinterkörper zugerechnet. Da die ursprüngliche Zahl der Körpersegmente (ohne die Furka) bei Gymnoplea und Podoplea dieselbe ist, nämlich 11, von denen 1 dem Kopf, 5 dem Thorax und ebensoviele dem Abdomen entfallen, folgt daraus, daß der Vorderkörper der Podoplea ein Segment weniger zählt als Kopf und Thorax zusammen, der Hinterkörper aber ein Segment mehr als das Abdomen. Durch die Verschmelzung einiger Segmente des Podopleakörpers kann sowohl die Gliederzahl des Vorderkörpers (Kopf + 1. -4 . Thsegm.) wie die des Hinterkörpers (5. Thsegm. + 1. -5. Abdsegm.) verringert werden, und in solchen Fällen der Verwachsung werden nur diese Zahlen in den Diagnosen aufgeführt. Ein 5-gldr. Hinterkörper bei einem PodopleaRepräsentanten ist also nicht vollzählig und Verschmelzung von 2 Segmenten zu einem muB stattgefunden haben, während ein 5-gldr. Vorderkörper bei demselben vollzählig zu nennen ist.

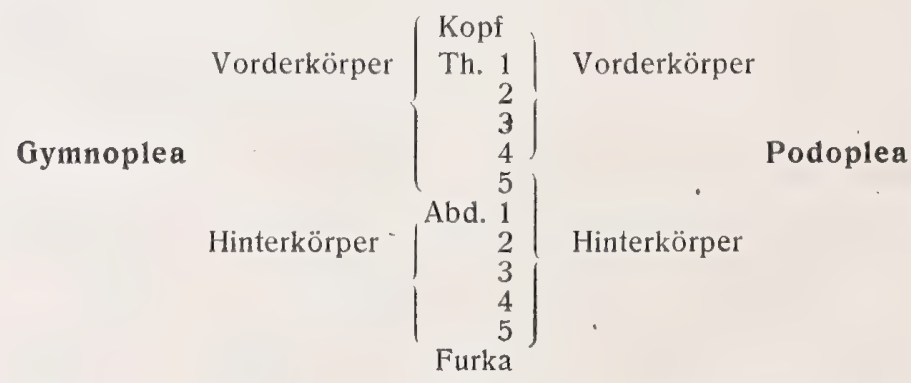


Trib. Podoplea.

\section{Mormonillidae.}

Rumpf gestreckt; weibliche Genitalöffnung ventral. 1. Antenne des $q$ lang, aber aus sehr wenigen Gliedern zusammengesetzt. Kopfgliedmaßen vom Gymnoplea-Typus. Exp. und Enp. der B. 3-gldr. oder mit reduzierter Gliederzahl. 5. B. fehlt beim

\section{Mormonilla Giesbrecht.}

․ Vorderkörper 5-, Hinterkörper 4-gldr.; Rostrum fehit; Furka gestreckt. 1. Antenne lang, 3- (bis 4-) gldr., mit sehr langen Borsten. 2. Antenne mit 7- oder 8-gldr. Exp. Mandibel mit gezähnelter Kaulade und 1-gldr. Exp. und Enp. 1. Maxille mit kleinen Innenrandloben, relativ großem Exp. und rudimentärem 1. Außenlobus. 2. Maxille und Maxilliped mit langen, stacheligen Hakenborsten, erstere mit 4 Loben und 3-gldr. Enp., letztere 2-gldr., mit rückgebildetem Enp. Exp. des 1.-3. B. 2- oder 3-gldr., des 4. B. 2-gldr.; Enp. des 1. B. 1- bis 3-gldr., des 2. B. 1- oder 2-gldr., des 3. und 4. B. 1-gldr. - o unbekannt.

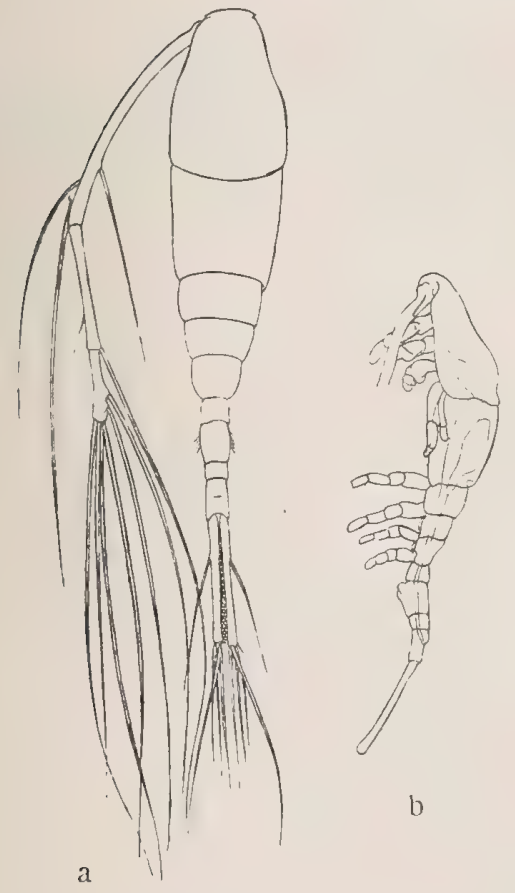

Fig. 181.

Mormonilla phasma. a o dorsal, b o lateral. Nach Giesbrecht.

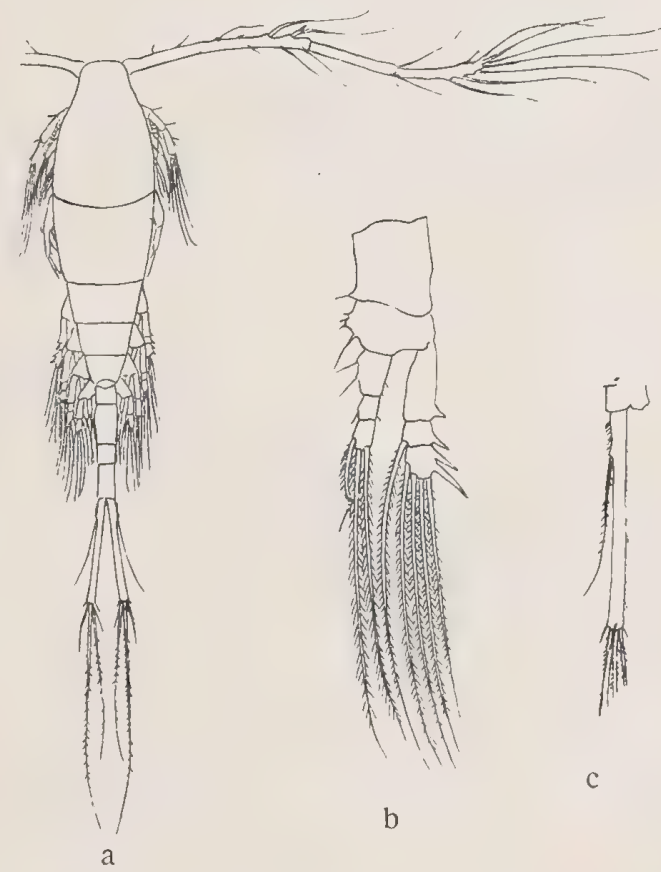

Fig. 182.

Mormonilla polaris.

a q dorsal, b 1. B. 우, c Furkalast. Nach Sars. 


\section{Mormonilla phasma Giesbrecht.}

1891, M. p., Giesbrecht, p. 474. 1892, M. p., Giesbrecht, p. 532, t. 43 f. $28-32,34-41$. 1893, M. p., T. Scott, p. 64 , t. 7 f. $11-21$.

․ Außenrandborste der Furka teilt den Außenrand nach dem Verhältnis $3: 5$. Letztes Gl. der 1 . Antenne $3 / 5$ so lang wie das mittlere. Die längeren Borsten am 1. Gl. des Maxillipeden nicht dicker als die übrigen Borsten; Endgl. mit 6 längeren Borsten. Exp. des 1. und 2. B. 2-, Enp. 1-gldr. L. 1,58-1,73 mm. - o unbekannt.

Atlant. Ozean; Pacif. Ozean.

\section{Mormonilla polaris G. O. Sars.}

1900, M. p., Sars, p. 120, t. 34 .

. Außenrandborste der Furka teilt den Außenrand nach dem Verhältnis $1: 5$. Letztes Gl. der 1. Antenne etwa so lang wie das mittlere. Die längeren Borsten am 1. Gl. des Maxillipeden auffällig verdickt und bestachelt; Endgl. mit 5 längeren Borsten. Exp.. des 1.-3. B. 3-gldr., Enp. des 1. B. 3-, des 2. B. 2-gldr. L. $1,38 \mathrm{~mm}$. - o unbekannt.

Arktisches Meer.

Anmerkung: Wolfenden (1905, p. 16) erwähnt eine neue Art als M. atlantica (q). Nach der Beschreibung, die nicht von Abbildungen begleitet ist, weicht sie nur in einem der aufgeführten Merkmale von $M$. polaris ab, nämlich durch die 4-gldr. 1. Antenne. L. 1,2-1,3 mm. Der Fundort ist der Atlant. Ozean bei Irland.

\section{Cyclopidae.}

Vorderkörper deutlich vom Hinterkörper abgesetzt. 1. Antenne des $q$ kürzer oder länger als das 1. Körpersegment, 6- bis vielgldr.; beim $\sigma^{\top}$ beide zu Greiforganen umgewandelt. 2. Antenne 2- bis 4-gldr., ohne Exp. Mandibel mit Exp. und Enp. oder fast nur aus der Kaulade bestehend. Exp. und Enp. des 1.-4. B. 3-gldr. oder mit reduzierter Gliederzahl. 5. B. rudimentär, bei Weibchen und Männchen fast immer gleich gebaut. Herz fehlend. 2 dorsal angeheftete Eiersäckchen.

\section{Oithona Baird.}

Vorderkörper 5-gldr.; Hinterkörper beim $\bigcirc$ 5- (1. und 2. Abdsegm. verschmolzen), beim or 6-gldr.; Stirn beim $q$ meist in einen spitzen schnabelförmigen Fortsatz endigend, beim $\sigma^{\pi}$ stumpf. 1. Antenne beim $\bigcirc$ mit langen Borsten, ohne Aesthetasken; beim $\sigma^{\text {T }}$ sind beide doppelt genikulierende Greif- 
organe, mit einem Aesthetask am Ende. 2. Antenne 2-gldr, mit undeutlicher Zweiteilung des 2. Gl.; Exp. fehlt. Mandibel gestreckt, mit kleinem, 1-gldr. Enp., 4-gldr. Exp. und gezähnelter Kaulade. 1. Maxille mit 1-gldr. Exp. und Enp. 2. Maxille und Maxilliped schlank, mit kräftigen, stacheligen Borsten, letzterer mit 2-gldr. Enp. 1.-4. B. mit 3-gldr. Exp. und Enp.; beim or meist mit etwas abweichender Beborstung; 5. B. ganz rudimentär, jederseits auf 2 Borsten reduziert.

1. Stirn endigt in einen spitzen Schnabel, der nach vorne vorspringt und in der Dorsalansicht sichtbar ist; 1 . Antenne über das Gensegm. weit hinausreichend 2 Stirn in der Dorsalansicht stumpf endigend oder abgestutzt (ohne Schnabel oder mit ventralwärts gebogenem, nur in der Lateralansicht sichtbaren Schnabel); 1. Antenne über das Gensegm. nicht hinausreichend 3

2. Endgl. des Exp. des 1. B. mit 2 Außenranddornen; Außenrandborste des 2. G1. des Basp. des 2. B. lang, gegen das Ende nicht verdickt O. plumifera Endgl. des Exp. des 1. B. mit 3 Außenranddornen; Außenrandborste des 2. G1. des Basp. des 2. B. gegen das Ende hin verdickt

O. setigera (Vergl. Anm. S. 168).

3. Stirn (in Lateralansicht) abgestutzt; 1. Antenne das Ende des Thorax nicht überragend; Endgl. des Exp. des 1.-4. B. mit 3 Außenrandborsten O. nana Stirn mit einem kleinen, spitzen, ventralwärts gebogenen, nur in der Lateralansicht sichtbaren Schnabel; 1. Antenne die Geschlechtsöffnungen erreichend; Endgl. des Exp. des 1.-4. B. resp. mit 2, 1, 1 und 0 Außenranddornen

O. similis

o'.

1. 1.-3. B. mit 3 Außenranddornen am Endg1. des Exp.; mittlere Ábdsegm. beträchtlich länger als breit

O. nana

1. B. mit 2 Außenranddornen am Endgl. des Exp.; mittlere Abdsegm. kaum länger als breit

2. Gensegm. oval; 2. und 3. B. mit 2 Außenranddornen am Endgl. des Exp.

O. similis

Gensegm. mehr kubisch; 2. und 3. B. mit 3 Außenranddornen am Endgl. des Exp.

O. plumifera.

\section{Oithona plumifera Baird.}

1843, O. p., Baird. 1863, O. spinirostris, Claus, p. 105, t. 11 f. $4-9$. 1892 , O. p., Giesbrecht, p. 537 , t. 4 f. $10 ;$; t. 34 f. $12,13,22,25,27,28,32$, $33,44-47$; t. 44 f. $1,7,12-15$.

१. Stirn endigt in einen spitzen, zwar etwas ventralwärts gebogenen, aber in der Dorsalansicht sichtbaren Schnabel; Furka kürzer als das Ansegm., fast $3 \mathrm{mal}$ so lang wie breit, ihre Außenrandborste ca. 3 mal so lang wie die Furka. 
1. Antenne reicht etwa bis zum Hinterrande des 4. Abdsegm. 2. Gl. des Basp. der Mandibel mit 2 Hakenborsten. Enp. der 1. Maxille mit einem winzigen Börstchen. Exp. des 1. B. mit 1, 1, 2, des 2. mit 1, 0, 2, des 3. mit 1, 0, 1 (längeren), des 4. mit $0,0,1$ (längeren) Außenranddornen; außerdem noch ein verkümmerter, proximaler Außenranddorn am Endgl. des 3. und 4. B. $0^{7}$. Stirnschnabel fehlt. Mittlere Abdsegm. nicht oder kaum länger als breit; Ansegm. etwa so lang wie jedes der beiden vorhergehenden Segm. Proximales G1. des distalen Abschnittes der 1. Antenne mit einem halbkreisförmigen Vorsprung am Innenrande. Endgl. des Exp. des 1. und 4. B. mit 2, des 2. und 3. B. mit 3 Außenranddornen. - L. O $1-1,5 \mathrm{~mm}$, o $0,75-1 \mathrm{~mm}$.

Mittelmeer, Nord-Atlant. Ozean; Polarmeer; Pacif. Ozean; Rotes Meer, Ind. Ozean.
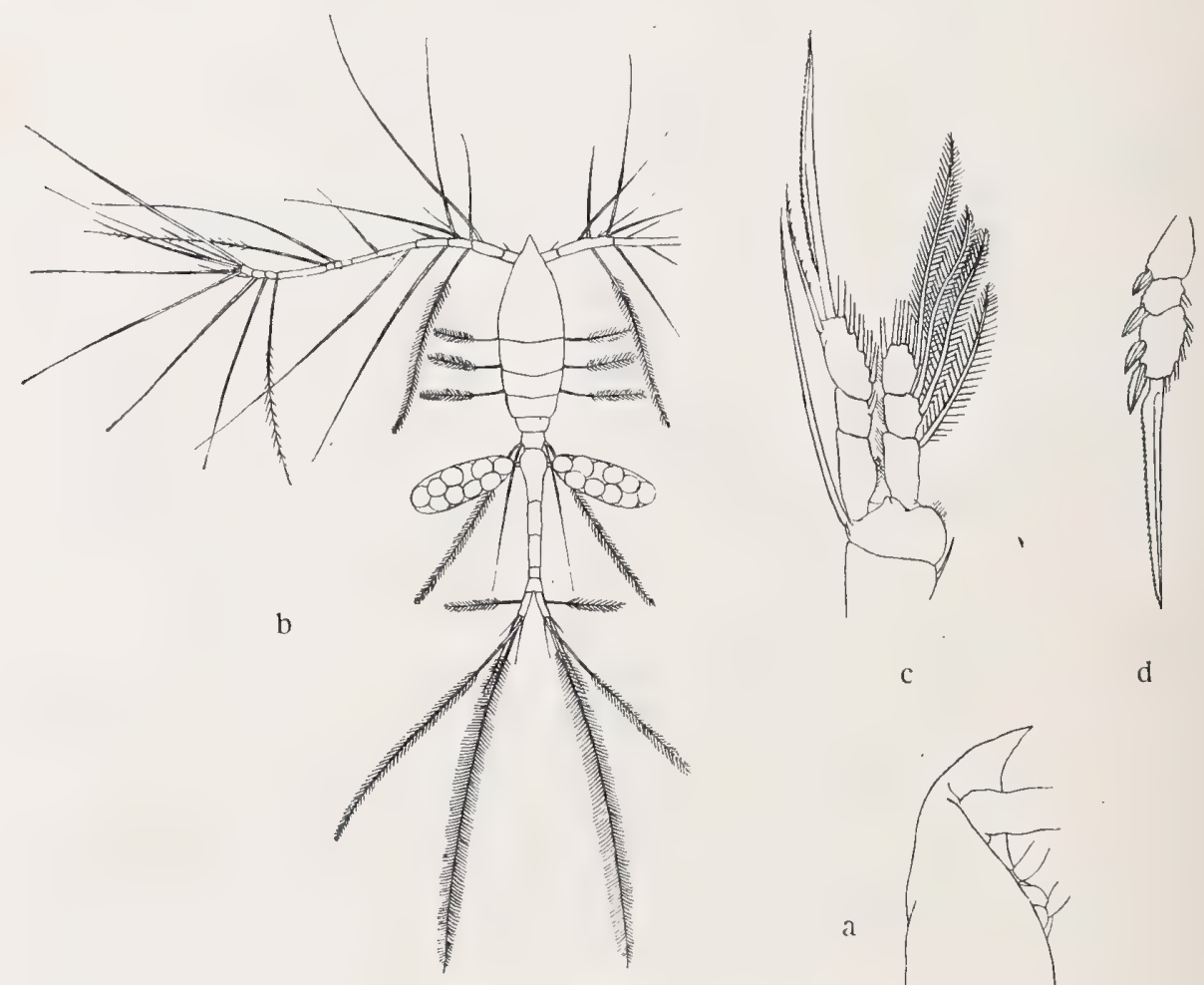

Fig. 183. Oithona plumifera.

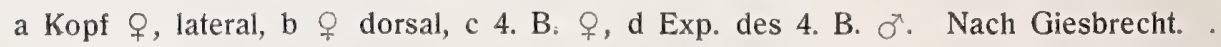

Anmerkung. T. Scott erwähnt das Vorkommen einer Oithona-Spezies, welche sich seiner Angabe gemäB (vergl. 20. Ann. Rep. F. B. Scotl, pt. 3) durch ein allmählich verjüngtes, nicht wie bei O. plumifera plötzlich verjüngtes Rostrum unterscheiden soll und die er O. (?) setiger Dana nennt.

Für O. setigera Dana führt Giesbrecht (1892, p. 548) folgende Merkmale an: 
․ Verwandt mit plumifera, aber: Stirnschnabel etwas stärker nach vorne gerichtet; Enp. der Mandibeln mit 4 Borsten; Enp. der 1. Maxille mit längerer Borste; AuBenrandborste des 2. Gl. des Basp. der B., besonders des 2. B., (Fig. 184) gegen das Ende angeschwollen; Endgl. des Exp. des 1. B. mit 3 Außenrandborsten; 1. Gl. des Exp. des 1.-4. B. mit kurzer Innenrandborste (dieselbe fehit bei O. plumifera $q$ am 2.-4. B.) L. $1,5-1,6 \mathrm{~mm}$.

\section{Oithona similis Claus.}

1864, O. pygmaea, Boeck. 1866, O. s., Claus, p. 14.1882 , O. spinirostris, Giesbrecht, p. 139, t. 2-12. 1891, O. s., Giesbrecht, p. 475.1892 , O. s., Giesbrecht, p. 537 , t. 34 f. $18,19,21,36-39$; t. 44 f. $3,5,8-11$.

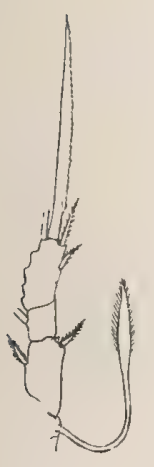

a

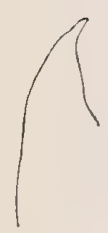

b

Fig. 184. Oithona setigera.

a Exp. des 2. B. \&, b Kopf 우, lateral. Nach Giesbrecht.

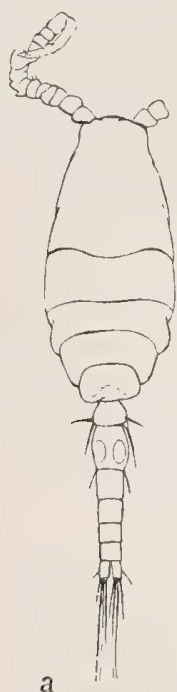

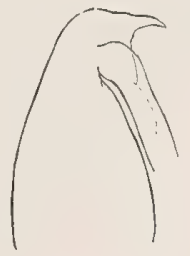

b

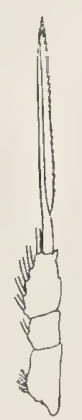

C

Fig. 185. Oithona similis.

a $\sigma^{2}$ dorsal, b Kopf $q$, lateral, c Exp. des 4. B. ‥ Nach Giesbrecht.

q. Stirn endigt in einen hakigen, spitzen, ventralwärts unter rechtem Winkel abstehenden und daher in der Dorsalansicht nicht sichtbaren Schnabel; Genitalöffnungen nur wenig vor der Mitte des Gensegm. gelegen; Furka kürzer als das Ansegm., ihre Außenrandborste etwa so lang wie die Furka selbst, weit nach vorne stehend. 1. Antenne reicht kaum bis zu den Genitalöffnungen. 2. Gl. des Basp. der Mandibel mit 2 Hakenborsten. Enp. der 1. Maxille mit einem winzigen Börstchen. Exp. des 1. B. mit 1, 1, 2, des 2. und 3. B. mit $1,0,1$, des 4. B. mit $0,0,0$ AuBenranddornen. - $0^{7}$. Stirnschnabel fehit. Längenverhältnis der 3 letzten Abdsegm. wie $10: 8: 9$; mittlere Abdsègm. nicht länger als breit. Proximales Gl. des distalen Abschnittes der 1. Antenne mit einem halbkreisförmigen Vorsprunge am Innenrande. Exp. des 1.-4. B. mit 1, 1, 2 Außenranddornen. - L. क 0,73-0,96 mm, ठै $0,59-0,7 \mathrm{~mm}$. 
Mittelmeer, Atlant. Ozean, Ostsee; Polarmeer; Pacif. Ozean; Ind. Ozean, Rotes Meer; Antarkt. Ozean.

\section{Oithona nana Giesbrecht.}

1863, O. helgolandica, Claus, p. 105, t. 11 f. $11-13$. 1892, O. n., Giesbrecht, p. 538 , t. 4 f. 8 ; t. 34 f. $10,11,20,24,26,34,35,42$; t. 44 f. $2,4,6$.

. Stirn stumpf abgestutzt, ohne Schnabel; Genitalöffnungen weit nach vorne gelegen, auf $1 / 4$ der Länge des Gensegm.; Furka so lang wie das Ansegm., ihre Außenrandborste etwa so lang wie die Furka selbst, gerade vor der Mitte der Länge stehend. 1. Antenne reicht nicht über das 3. Thsegm. hinaus. 2. Gl. des Basp. der Mandibel mit 1 Hakenborste. Enp. der 1. Maxille mit

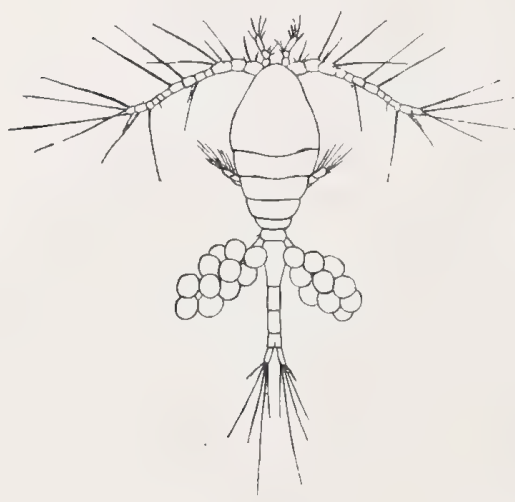

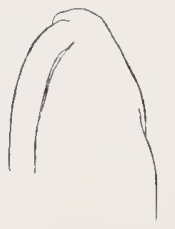

b

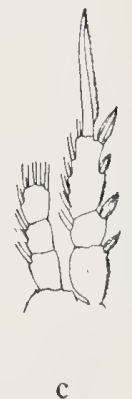

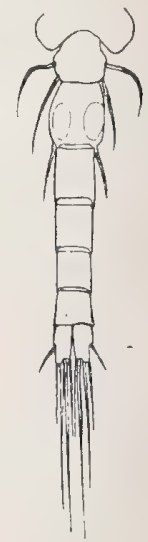

d

Fig. 186. Oithona nana.

a $q$ dorsal, b Kopf $q$, lateral, c 4. B. $q$, d Abdomen ơ, dorsal. Nach Giesbrecht.

4 Borsten. Exp. des 1.-3. B. mit 1, 1, 3, des 4. B. mit 1, 1, 2 Außenranddornen. - $\sigma^{7}$. Stirn wie beim $q$. Relatives Längenverhältnis der 3 letzten Abdsegm. wie 10:9:6; mittlere Abdsegm. länger als breit. Proximales Gl. des distalen Abschnittes der 1. Antenne ohne runden Vorsprung. Exp. des 1.-3. B. mit 3, des 4. B. mit 2 Außenranddornen. - L. $q 0,5-0,65 \mathrm{~mm}$, o $0,48-0,57 \mathrm{~mm}$.

Mittelmeer, Schwarzes Meer, Ostküste des Nord-Atlant. Ozeans; Rotes Meer, Ind. Ozean; Westküste Nord-Amerikas.

Bemerkung. Mit dieser Art ist identisch O. minuta Kriczagin, aber nicht O. minuta T. Scott (1893, Golf von Guinea).

\section{Cyclopina Claus.}

Vorderkörper 5-gldr.; Hinterkörper beim o 5-gldr., beim o7 6-gldr. Stirn ohne Schnabel. 1. Antenne 10- bis 26-gldr., am Endgl. mit dünner 
Aesthetask; beim 0 sind beide doppelt genikulierende Greiforgane. 2. Antenne 4-gldr, ohne Exp. Mandibel groB, mit 2-gldr. Enp., undeutlich 3-gldr. Exp. und gezähnelter Kaulade. 1. Maxille mit 1-gldr. Exp. und Enp. 2. Maxille schlank, mit kräftigen Borsten. Enp. des Maxillipeden wenigstens 3-gldr. 1.-4. B. mit 3-gldr. Exp. und Enp.; Exp. des 2. B. beim of mit abweichender Beborstung. 5. B. 2-oder 3-gldr., beim ${ }_{+}$und $\sigma^{\pi}$ (stets?) etwas abweichend gebaut.

Bemerkung. Von den 5 marinen Genera (Cyclopina, Oithona, Pterinopsyllus, Hemicyclops, Euryte), welche die Familie der Cyclopiden neben dem Genus Cyclops umfaBt, gehört nur das Genus Oithona zum Plankton und seine Arten halten sich zum Teil auf dem hohen Meere, zum Teil in der Nähe der Küsten auf. Die Arten der übrigen 4 marinen Genera findet man fast ausschließlich zwischen Pflanzen und Tieren und im Detritus der Meeresküsten (Giesbr. 1901, p. 39). Cyclopina litoralis Brady jedoch ist wenigstens im südlichen Teile der Nordsee ein zwar spärlicher, aber ziemlich regelmäßiger Bestandteil des neritischen Planktons, im Gegensatz z. B. von C. gracilis, die in solcher Weise niemals vorkommt. Sie nimmt also eine vermittelnde Stellung zwischen eupelagischer und litoraler (benthonischer) Lebensweise ein und ich habe sie meinen eigenen Erfahrungen gemäß unter die Planktonarten mit aufgenommen. Zur besseren Unterscheidung dieser Art führe ich die Unterscheidungsmerkmale der anderen nordischen Arten ihr gegenüber kurz an.

\section{Cyclopina litoralis Brady.}

1872, Cyclops 1., Brady, p. 5 t. 2.1878 , C. 1., Brady, t. 92 , t. 15 f. $1-9$. 1901, C. 1., Giesbrecht, p. 43 , t. 3 f. $1-13$.

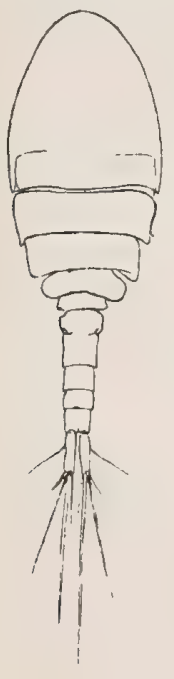

a

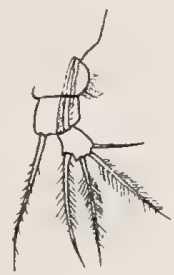

b

Fig. 187.

Cyclopina litoralis.

a $q$ dorsal, b 5. B. 우.

Nach Giesbrecht.

ㅇ. Außenrandborste der Furka das Ende derselben überragend; Länge der Furka sehr variabel; Furka im ganzen mit 6 Borsten. 1. Antenne 18-gldr., etwa bis zum 1. B. reichend; 3. Gl. bei weitem das längste, viel länger als breit. Endgl. der 2. Antenne über doppelt so lang wie das vorletzte Gl. Mittelgl. des Enp. des 1. B. mit nur 1 Borste, Endborste des Exp. desselben 
B. von ähnlicher Form wie an den folgenden B.; Borste des 1. Gl. des Enp. des 2., 3. und besonders des 4. B. verdickt; der distale der beiden Außenranddornen des Endgl. des 4. B. durch eine s-förmige Borste ersetzt. Endgl. des 5. B. wenig länger als breit, mit 4 Randborsten, von denen die innere kürzer und dünner als die anderen ist. L. 0,55-0,8 mm. - o unbekannt.

Mittelmeer, N.-W.-Europäisches Küstengebiet.

Bemerkung. Die übrigen 4 bekannten nordischen Arten weisen folgende Merkmale auf:

2. Cyclopina elegans, T. Scott. (1894, p. 237, t. 5. 1901, Giesbrecht, p. 44, t. 3 f. 14-22). $\quad$. Außenrandborste der Furka sehr kurz; Furka im ganzen mit 7 Borsten. Von der 18-gldr. 1. Antenne sind das 3. und 9. Gl. die längsten; ersteres nicht so lang wie breit. Endgl. der 2. Antenne etwa $1 \frac{1 / 3}{3}$ so lang wie das vorletzte Gi. Mittelg1. des Enp. des 1. B. mit 2 Borsten; Endborste des Exp. desselben B. eine Fiederborste; 1. Gl. des Enp. an keinem B. mit verdickter Borste, dagegen die beiden Innenrandborsten des 2. GI. des Enp. und die proximale Innenrandborste des 3. Gl des Enp. des 4. B. verdickt und zugleich verkürzt; beide Außenranddornen des Endgl. des Exp. des 4. B. dornförmig. Endgl. des 5. B. etwa doppelt so lang wie breit, am Außen- und Endrande mit 4 Borsten, von denen die innere verkürzt und verdickt ist. L. $0,8 \mathrm{~mm}$. - Das Männchen wird von Scott erwähnt. - Schottische Küste, Mittelmeer.

3. Cyclopina longicaudata, T. Scott $(1901$, p. 240 , t. 17 f. $5-14)$. 9 . Furka im ganzen mit 7 Borsten, mit sehr kurzer Außenrandborste. 1. Antenne 26-gldr., ca. so lang wie das Kopfsegm. Endgl. der 2. Antenne etwa so lang wie das vorletzte. Mittelgl. des Enp. des 1. B. mit 2 Borsten, Endborste des Exp. desselben B. von ähnlicher Form wie an den folgenden B. Endgl. des 5. B. mehr als 2 mal so lang wie breit, mit 5 Borsten, von denen 2 am Außenrande, 2 apikal und 1 distal am Innenrande stehen. L. 1,56 mm. - or unbekannt. - Shetlandsinseln (in einem Schellfischmagen).

4. Cyclopina gracilis, Claus $(1863$, p. 104, t. 10 f. 10.1878 , Brady, p. 93 , t. 24 B f. $1-9$; t. 91 f. 10, 11. 1882, Giesbrecht, p. 137, t. 2, 4, 11. 1901, Giesbrecht, p. 45). Q. 1. Antenne 10- oder 11 -gldr., das 6. Gl. $2 \frac{1}{2}$ mal so lang wie das 3. und 7. Gl. Endgl. der 2. Antenne über doppelt so lang wie das vorletzte Gl. Enp. an keinem B. mit verdickten Borsten. Endgl. des 5. B. mit 3 Borsten am Ende, von denen die äußere ein größerer, die innere ein kleinerer Dorn ist. L. 0,45-0,65 mm. $\sigma^{\top}$ unbekannt. - Mittelmeer, Schwarzes Meer, Europäische Küsten des Atlant. Ozeans, Ostsee; Polarmeer.

5. Cyclopina schneideri, T. Scott (1903a, p. 6, t. 1 f. 1-6). . Weicht von gracilis ab durch die 12-gldr. 1. Antenne mit 6 statt 5 kleinen Endgl. L. $1 \mathrm{~mm}$. - $\sigma^{\pi}$ unbekannt. - Küste von Finmarken.

\section{Harpacticidae.}

Körper cylindrisch oder bisweilen abgeplattet oder seitlich zusammengedrückt. Kopf meistens mit 1. Thsegm. verschmolzen; Stirn gewöhnlich in ein großes, bewegliches Rostrum verlängert. Hinterkörper nur in wenigen 
Fällen deutlich vom Vorderkörper abgesetzt. Hinterkörper beim † 5-gldr., 1. und 2. Abdsegm. gewöhnlich ohne deutliche Sutur verbunden; beim $\sigma^{7}$ 6-gldr., mit getrennten 1. und 2. Abdsegm. Von den Endborsten der Furka gewöhnlich nur die 2 mittleren gut entwickelt. Auge einfach oder fehlend. 1. Antenne kurz, mit wenigen (selten mehr als 8) Gl. 2. Antenne mit 2- bis 4-gldr. Hauptast und 1-4-gldr. Nebenast (Exp.) Mandibel mit stark gezähnter Kaulade; Enp. und Exp. anwesend oder rückgebildet. 2. Maxille gut entwickelt, mit gewöhnlich mehreren verlängerten Loben. Maxilliped meistens mit kräftiger, hakenförmiger Endklaue, bisweilen aber nicht zu einem Greiforgan umgebildet. 1. B. meistens von den folgenden B. abweichend gebaut und zu Greiforganen umgewandelt. 2.-4. B. stets schwimmbeinähnlich; 5. B. blattförmig, 1- oder 2-gldr., mit starken Randborsten oder -dornen. Herz fehlend. Eiersack meistens einfach, selten doppelt, stets ventral angeheftet.

\section{Microsetella Brady und Robertson.}

Rumpf schlank, nach vorne und hinten verjüngt, Vorderkörper 4-gldr., mit breiter Randduplikatur; Hinterkörper beim o 5-, beim o7 6-gldr.; Rostrum unbeweglich; Furka kurz, mit sehr langen Borsten. 1. Antenne beim $q$ 5-gldr., mit großem Aesthetasken am 3. Gl.; beim o 6 -gldr. und beiderseits genikulierend (zwischen dem 4. und 5. GI.), mit Aesthetasken am 4. und 7. Gl. 2. Antenne 3-gldr. mit dünnem, aber ziemlich langem, 3-gldr. Exp. Folgende Mundgliedmaßen klein; Mandibel mit großem 2-gldr. Enp. und sehr kleinem Exp. 2. Maxille mit 2 breiten Gl. des Basp. und rudimentärem Enp. Maxilliped 3-gldr.; Mittelgl. groß, Endgl. (Enp.) klein mit 3 Borsten. 1.-4. B. mit 3-gldr. Exp. und Enp., Enp. länger als Exp.; 5. B. beim ๆ rudimentär, blattförmig, 2-gldr.; 1. Gl. an der Innenseite mit lobenförmigem Fortsatz, der 2 Borsten trägt; 2. Gl. mit 4 Borsten; 5. B. beim ot kleiner und mit z. T. reduzierten Borsten. Ein Eiersack.

\section{Microsetella norvegica Boeck.}

1864, Setella n., Boeck, p. 281. 1873, M. atlantica, Brady und Robertson, p. 130, t. 9 f. 11-16. 1880, Ectinosoma atlanticum, Brady, II, p. 13, t. 38 f. $11-19$. 1883, Ectinosoma atlanticum, Brady, p. 100, t. 4 f. 10-14. 1887, Ectinosoma atlanticum, Möbius, p. 116, t. 7 f. 17-23. 1887, Ectinosoma atlanticum, De Guerne, p. 344, t. 6 f. 1-6. 1891, M. brevifida, Giesbrecht, p. 476. 1892, M. atlantica, Giesbrecht, p. 550, t. 44 f. 33, 34, 36, 39, 40, 42, 44, 45. 1903 a, M. n., Sars, p. 44, t. 24.

Furka jederseits mit 4 Endborsten, die innere und äußere derselben kurz; die äußere der beiden mittleren so lang wie der Hinterkörper, die innere so lang wie der Rumpf. Innere Borste des 1. Gl. des 5. B. des $q$ weniger als halb so lang wie die benachbarte (es kommen übrigens Abweichungen in dem relativen Längenverhältnisse der Borsten dieses B. vor). - L. $\subsetneq 0,35-0,53 \mathrm{~mm}$, o $0,33-0,42 \mathrm{~mm}$. 
Mittelmeer, Nordsee, Atlant. Ozean; Polarmeer; Pacif. Ozean; Rotes Meer, Ind. Ozean; Antarkt. Ozean.

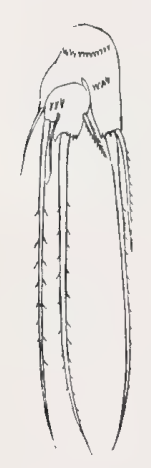

a

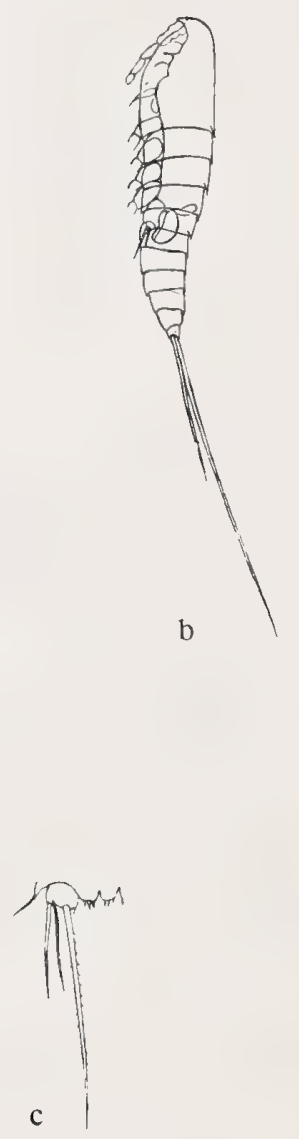

Fig. 188. Microsetella norvegica. a 5. B. $q$, b ot lateral, c 5. B. $0^{x}$. Nach Giesbrecht.
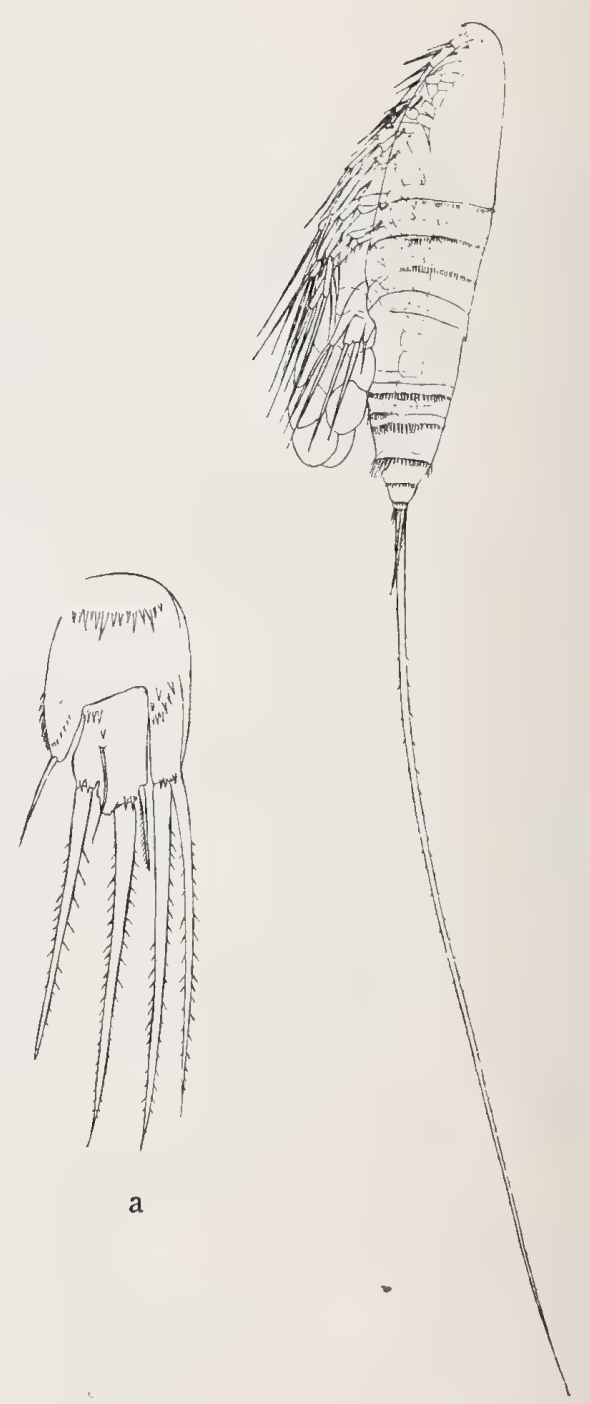

b

Fig. 189. Microsetella rosea.

a 5. B. ㅇ, b o lateral. Nach Giesbrecht.

\section{Microsetella rosea Dana.}

1852, Canthocamptus roseus, Dana, p. 1189. 1891, M. r., Giesbrecht, p. 476 . 1892, M. r., Giesbrecht, p. 550, t. 44 f. $32,35,37,38,41,43,46,48,49$. 
O. Furka mit jederseits 3 Endborsten, von denen die äußere winzig, die mittlere weniger als halb so lang wie das Abdomen, die innere fast doppelt so lang wie der Rumpf ist. Innere Borste des 1. Gl. des 5. B. kaum kürzer

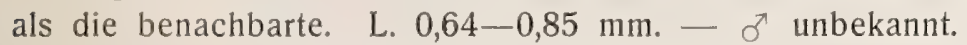

Mittelmeer, Atlant. Ozean; Rotes Meer, Ind. Ozean; Pacif. Ozean.

\section{Halithalestris G. O. Sars.}

Körper verlängert, fast zylindrisch, mit etwa gleich breitem Vorder- und Hinterkörper, welch letzterer groß und breit ist; Vorderkörper 5-gldr., Hinterkörper beim ${ }^{\circ} 5$-, beim $\sigma^{7}$ 6-gldr.; Furkaläste sehr lang und divergierend. 1. Antenne des $\$$ 9-gldr., des or beiderseits genikulierend. 2. Antenne mit 2-gldr. Hauptast und 2-gldr. Exp. Mandibel mit 1-gldr. Exp. und Enp. Maxilliped klauenförmig, 3-gldr., mit sehr breitem Handgl. und kräftigem Endhaken. 1.-4. B. mit 3-gldr. Exp. und Enp., mit Ausnahme des Enp. des 2. B. des $\sigma^{7}$, das 2-gldr. ist. Äste des 1. B. fast gleich lang, Mittelgl. des Exp. und 1. Gl. des Enp. stark verlängert; Endgl. des Exp. kurz mit hakenförmigen Endborsten; die 2 distalen Gl. des Enp. sehr kurz, Endgl. mit 2 hakenförmigen Borsten. 5. B. 2-gldr., blattförmig, beim $q$ viel größer als beim ơ'. Ein Eiersack.

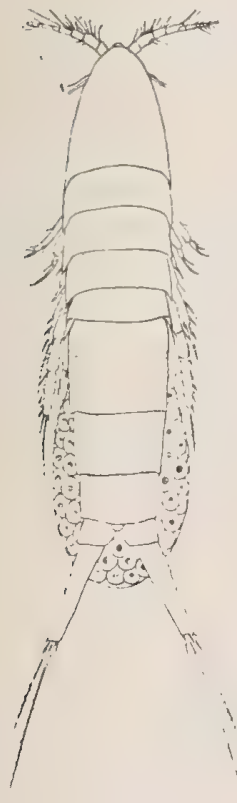

a

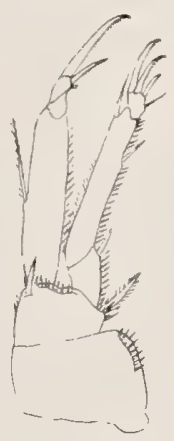

b

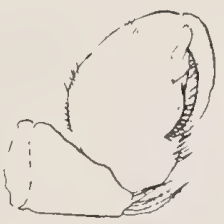

d

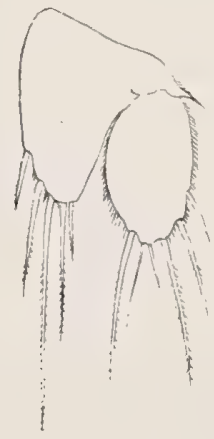

C

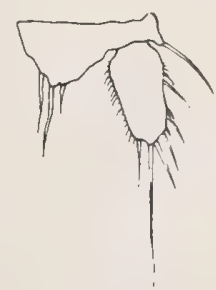

e

Fig. 190. Halithalestris croni.

a $q$ dorsal, b 1. B. $q$, c 5. B. $q$, d Maxilliped $q$, e 5. B. $\sigma^{7}$.

a-d nach Sars, e nach Brady. 


\section{Halithalestris croni Kröyer.}

1845, Harpacticus c., Kröyer, pl. 43, f. 3 a-n. 1880, Thalestris serrulata, Brady, II, p. 133, t. 59 f. 2--11. (o ). 1903 a, H. c., Sars, p. 118 , t. $72(q)$.

. Kopfsegm. kaum länger als die 3 folgenden Segm. zusammen; Rostrum kurz. Gensegm. groß, ca. so lang wie breit; Ansegm. kurz und tief gespalten. Furka sehr lang, fast halb so lang wie das Abdomen, Außenrand mit 3 oder 4 Zähnchen; die längere der beiden Endborsten kürzer als das Abdomen. 1. Antenne kürzer als das Kopfsegm. Maxilliped kräftig gebaut, Handgl. sehr breit, fast halbkreisförmig, der fast gerade Innenrand mit einer gebogenen Reihe von starken Dörnchen, Endklaue kräftig und gebogen. Das kurze Endgl. des Exp. des 1. B. mit 4 Borsten, von denen die 3 distalen klauenförmig sind; von den beiden Endklauen des Enp. ist die innere 2 mal so lang wie die äußere. 5. B. kaum bis zur Mitte des Gensegm. reichend; 2. Gl. oval, mit 6 Randborsten, von denen 2 ziemlich lang sind; Innenlobus des 1. Gl. dreieckig, über die Mitte des 2. Gl. hinausreichend, mit 5 Randborsten, von denen die mittlere die längste ist. Eiersack sehr groß. - $\sigma^{\top}$. Innenlobus des 1. Gl. des 5. B. breit dreieckig, mit 3 Endborsten; 2. Gl. mit mehreren Randborsten, von denen 1 längere apikal gestellt ist. Letztes Abdsegm. sehr kurz, Furka ca. 3 mal so lang wie dasselbe. - L. ๆ 2,3 mm, o $1,7 \mathrm{~mm}$.

Britische Inseln, Norwegische Küste; Arktisches Meer.

\section{Euterpe Claus.}

Vorderkörper 4-gldr.; Hinterkörper beim o 5-, beim of 6-gldr.; Stirn zugespitzt. 1. Antenne beim $\bigcirc 7$-gldr. und beiderseits in ein 5-gldr. genikulierendes Greiforgan umgewandelt (4. mit 5., 6. mit 7. Gl. verschmolzen). 2. Antenne 3-gldr., mit 1-gldr. Exp. Mandibel klein, mit 1-gldr. Exp. und Enp. Maxilliped sehr dünn, 2-gldr., mit gefiedertem Endhaken. 1. B. mit 2-gldr., 2. -4. B. mit 3-gldr. Exp. und Enp. 5. B. rudimentär, 1-gldr., blattförmig, beim $\sigma^{7}$ länger und mit weniger Borsten als beim $q$. Ein Eiersack.

\section{Euterpe acutifrons Dana.}

1852, Harpacticus a., Dana, p. 1192. 1863, E. gracilis, Claus, p. 110 , t. 14 f. 1-13. 1880, E. gracilis, Brady, II, p. 22, t. 40. f. 1-16. 1891, E. a., Giesbrecht, p. 475 . 1892, E. a., Giesbrecht, p. 555, t. 44 f. $16-31$.

Rumpf in der Seitenansicht s-förmig gekrümmt; Stirn in einen umfangreichen, unbeweglichen, schnabelartigen, scharf zugespitzten Fortsatz ausgehend. Furka mit 2 längeren Endborsten, von denen die innere fast so lang wie der Hinterkörper ist. 1. Antenne des $q$ den hinteren Rand des 1. Segm. nicht erreichend. Vorletztes Gl. der 1. Antenne des ot verdickt und vermittelst des 
3. Gl. an die ebenfalls verdickten 1. und 2. Gl. rechtwinklig angefügt; das Gelenk befindet sich zwischen dem 4. und 5. Gl., welch letzteres die. Form eines am Grunde verdickten Hakens hat. 5. B. des $q$ jederseits eine rechteckige Platte bildend und ohne die Borsten fast bis zum Hinterrand des

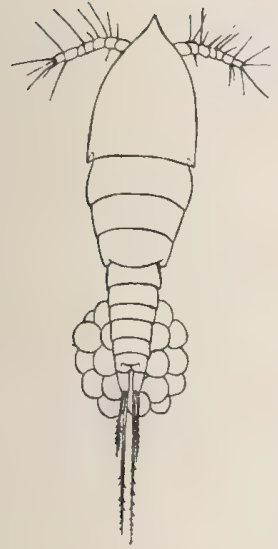

a

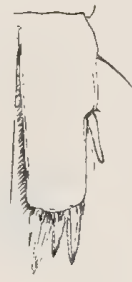

b

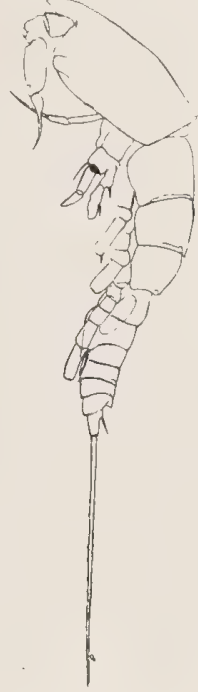

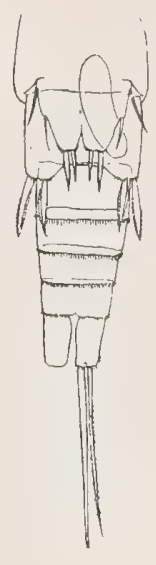

d

Fig. 191. Euterpe acutifrons.

a $q$ dorsal, b 5. B. $q$, c $\sigma^{\pi}$ lateral, d Hinterkörper $\sigma^{\varkappa}$, ventral. Nach Giesbrecht.

2. Abdsegm. reichend, am Ende mit 4 stiletförmigen Borsten, am Außenrande mit 2 Borsten ( 1 dünneren und 1 dickeren) in der Mitte und 1 dünnen proximal gestellten Borste; 5 . B. des o jederseits mit 2 Endborsten. - L. ๆ $0,5-0,75 \mathrm{~mm}$, o $0,5-0,56 \mathrm{~mm}$.

Mittelmeer, Nordsee, Küsten des Atlant. Ozeans; Rotes Meer, Ind. Ozean.

\section{Setella Dana.}

Rumpf lang und dünn, komprimiert; Vorderkörper 4-gldr., Hinterkörper beim ㅇ 5-, beim of 6-gldr.; Rostrum beweglich. Furka griffelförmig mit sehr langen Borsten. 1. Antenne 8-gldr., beim $\sigma^{\top}$ mit Gelenk zwischen dem 5. und 6. G1. 2. Antenne kurz, 2-gldr., ohne Spur eines Exp. Folgende 3 Gliedmaßen winzig; Mandibel und 1. Maxille beide 1-gldr., ohne Exp. und Enp. 2. Maxille mit 2 Loben und kurzem Endhaken. Maxilliped länger und kräftiger, 2-gldr., mit Endhaken. 1.-4. B. mit sehr schmalen und langen Ästen. Exp. des 1.-4. B. 3-gldr.; beim $q$ Enp. des 1. B. 2-, des 2.-4. B. 3-gldr.; beim or Enp. des 1. und 2. B. 2-, des 3. und 4. B. 3-gldr.; 5. B. rudimentär, 2-gldr., gestreckt blattförmig. 


\section{Setella gracilis Dana.}

1852, S. g., (+ tenuicornis + longicauda + crassicornis + aciculus) Dana, p. 1198, t. 85. 1860, S. tenuis, Lubbock, p. 9, t. 29. 1863, S. messinensis, Claus, p. 137, t. 21. 1883, S. g., Brady, p. 108, t. 50 f. $1-10$. 1892 , S. g., Giesbrecht, p. 559 , t. 1 f. 12 ; t. 45 f. $1-15$.
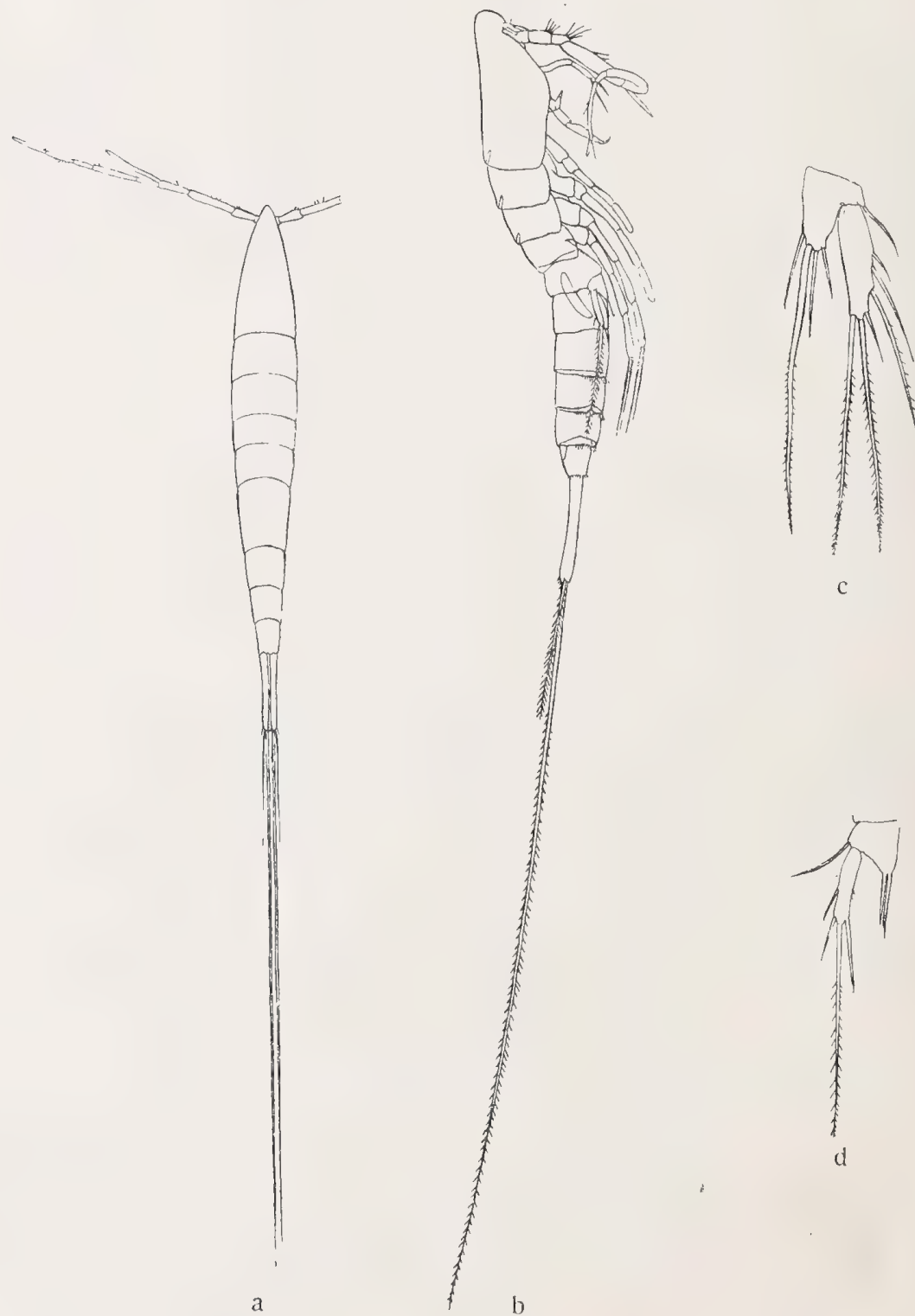

Fig. 192. Setella gracilis.

a $q$ dorsal, b $\sigma^{r}$ lateral, c 5. B. ㅇ, d. 5. B. $\sigma^{\nearrow}$. Nach Giesbrecht. 
1. Antenne des $q$ reicht etwa bis zum Vorderrande des Gensegm.; 4. Gl. der 1. Antenne des ơ stark verlängert und verdickt, Endgl. leicht hakig gebogen. 1. und 2. B. des $\sigma^{7}$ im Bau von denen des $q$ abweichend. 5. B. 2-gldr., beim $q$ mit 4 Borsten am Innenlobus des 1. Gl. und 6 Borsten am gestreckten 2. Gl.; beim or resp. mit 2 und 4 Borsten. - L. o 1,4-1,5 mm, o $1,16-1,3 \mathrm{~mm}$.

Mittelmeer, Atlant., Pacif. und Ind. Ozean.

\section{Clytemnestra Dana.}

Vorderkörper 4-gldr.; Hinterkörper des $q$ 5-, des of 6-gldr.; die posterolateralen Ecken der Segmente des abgeflachten Vorderleibes in Zipfel verlängert; Furka kurz. 1. Antenne 7-bis 8-gldr., mit kurzen Borsten und langen Aesthetasken, beim $\sigma^{7}$ beiderseits zu Greiforganen entwickelt. 2. Antenne 3-gldr., mit rudimentärem, durch 1 oder 2 Borsten vertretenem Exp. Mandibel auf 1 stiletförmige, 2-zinkige Kaulade reduziert; 1. und 2. Maxille ebenfalls stark rückgebildet. Maxilliped 2-gldr., dünn, gestreckt, mit Endhaken. 1. B. mit 1-gldr. Exp. und 3-gldr. Enp.; 2.-4. B. mit 3-gldr. Exp. und Enp. 5. B. rudimentär, 2-gldr., verlängert, beim $\sigma^{\top}$ nicht oder fast nicht abweichend.

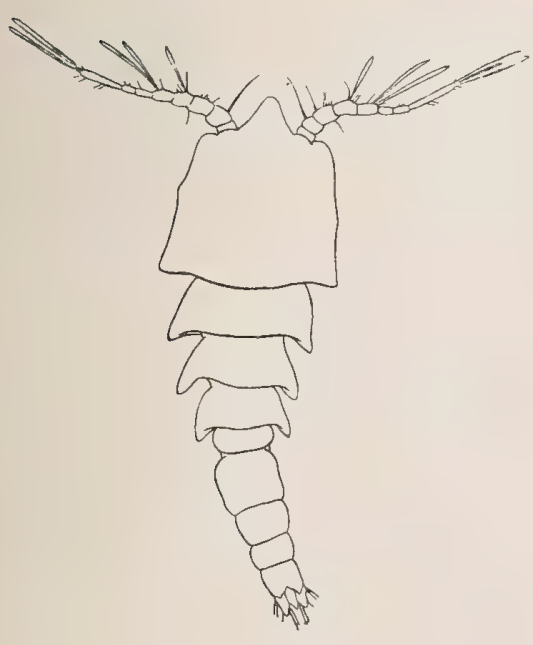

a

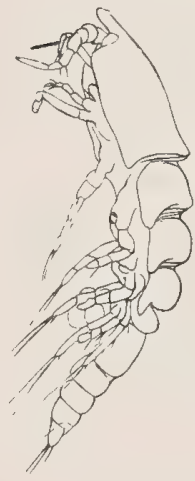

b

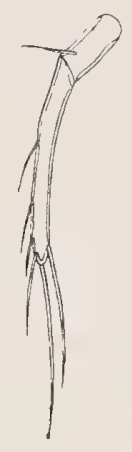

c

Fig. 193. Clytemnestra scutellata.

a 우 dorsal, b 우 lateral, c 5. B. $0^{x}$. Nach Giesbrecht.

\section{Clytemnestra scutellata Dana.}

1852, C. S., Dana, p. 1194, t. 83. 1891, Goniopelte gracilis, Claus, p. 151, t. 1, 2. 1891, C. hendorffi ( + var. quinquesetosa) Poppe, p. 132, 
t. 1. 1892, C. s., Giesbrecht, p. 566, t. 1 f. 9 ; t. 45 f. $16-18,21,23,24$, $27-30,32,34-38$.

Furka doppelt so lang wie breit (bei var. quinquesetosa relativ breiter), ihre beiden längeren Borsten gefiedert und beim o länger als beim $\bigcirc$. 1. Antennen in beiden Geschlechtern 8-gldr.; letztes G1. beim $\bigcirc$ doppelt so lang wie das vorletzte; drittletztes, stark verkürztes Gl. beim or mit lanzettförmigem Dorn. Exp. der hinteren Antennen durch 2 Borsten ersetzt; 2. Gl. des Basp. des 1. B. mit gefiederter Außenrandborste, Exp. desselben mit 4 Borsten; Exp. des 2. B. mit 0, 1, 3 Außenrandborsten; 2. und 3. Gl. des Enp. des 3. B. gleich lang. 5. B. $1 \frac{1 / 2}{2}$ mal so lang wie das Exp. des 4. B., mit 6 (5 bei der var. quinquesetosa Poppe) Borsten am Endgl., von denen die beiden längeren endständigen beim $\sigma^{\gamma}$ kürzer als beim ${ }_{\uparrow}$ sind. -- L. $†$ 1,05-1,2 $\mathrm{mm}$, or $1,07-1,3 \mathrm{~mm}$.

Mittelmeer, Atlant. Ozean; Pacif. Ozean; Ind. Ozean.

\section{Clytemnestra rostrata Brady.}

1883, Goniopsyllus rostratus, Brady, p. 107, t. 42. 1890, Sapphir rostratus, Car, p. 263, t. 14 . 1892, C. r., Giesbrecht, p. 566, t. 45, f. 19 , $20,22,25,26,31,33$.

Furka höchstens so lạg wie breit, ihre Borsten nicht gefiedert und in beiden Geschlechtern gleich. 1. Antennen in beiden Geschlechtern 7-gldr.; letztes Gl. beim o $5 \mathrm{mal}$ so lang wie das vorletzte; ein lanzettformiger

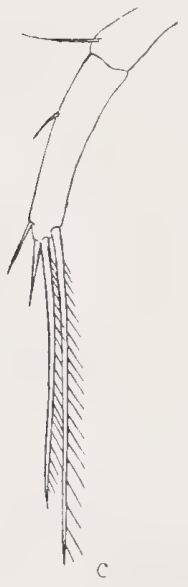

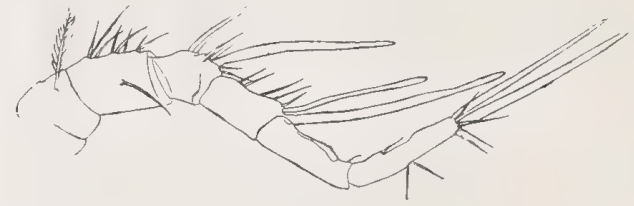

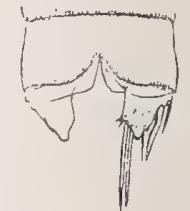

b

Fig. 194. Clytemnestra rostrata Brady.

a 1. Antenne or, b letztes Abdsegm. und Furka $q$, c 5. B. ․

Nach Giesbrecht.

Dorn am drittletzten G1. beim of fehlt. Exp. der 2. Antenne durch 1 Borste ersetzt; 2. G1. des Basp. des 1. B. ohne Außenranddorn, Exp. desselben mit 3 Borsten; Exp. des 2. B. mit 1, 1, 2 Außenrandborsten; 2. und 3. Gl. des 
Enp. des 3. B. länger als das 3. G1. 5. B. so lang wie das Exp. des 4. B., mit 5 Borsten am Endgl., die bei $q$ und $\sigma^{7}$ gleich lang sind. - L. $\bigcirc$ 0,6-1 mm, of $0,87 \mathrm{~mm}$.

Mittelmeer, Atl. Ozean; Pacif. Ozean; Ind. Ozean.

\section{Aegisthus Giesbrecht.}

Q. Rumpf vorn breiter, nach hinten verjüngt; Vorderkörper 5-gldr., Hinterkörper 5- oder 6-gldr. (1. und 2. Abdsegm. nur dorsal oder ringsum getrennt.) Stirn in einen Stachel ausgehend. Die beiden kurzen Furkalzweige und ihre beiden ungemein langen Borsten in der Mittellinie verschmolzen. 1. Antenne 6-bis 7-gldr. mit langen Aesthetasken am 3.Gl. 2. Antenne schmal, 3-gldr., mit kurzem, 1-gldr. Exp. Mandibel fast nur aus der Kaulade bestehend, Kauende beilförmig, gezähnelt. 1. Maxille ohne Exp., Enp. und Außenrandlobus. 2. Maxille gestreckt, am 5. Innenrandlobus mit dicker Hakenborste. Maxilliped klein, schwächlich, 2-gldr. 1.-4. B. mit breiten Basp. und 3-gldr. Exp. und Enp. (am 1. B. sind die beiden letzten Gl. des Exp. und Enp. nicht immer deutlich getrennt); 5. B. rudimentär, lang gestreckt, 1-gldr.; ein relativ großes 6. B. vorhanden. - o unbekannt.

1. Stirnschnabel kurz; 1. Antenne 7-gldr. mit 3 kurzen Endgl.

2. Vordere Segm. des Vorderkörpers mit netzförmigen Chitinrippen; Hinterrand der Abdsegm. nicht gezähnelt A. aculeatus Vordere Segm. des Vorderkörpers ohne netzförmige Chitinrippen; Hinterrand des 2.-5. Abdsegm. gezähnelt A. spinulosus

3. 2.-4. Thsegm. ohne Spitzenreihen am Hinterrande; Endborste des 5. B. ca. halb so lang wie das 5 . B.

A. atlanticus 2.-5. Thsegm. mit kräftigen Spitzenreihen, Endborsten des 5. B. ca. $3 / 4$ so lang wie das 5. B.

A. mucronatus.

\section{Aegisthus aculeatus Giesbrecht.}

1891, A. a., Giesbrecht, p. 476. 1892, A. a., Giesbrecht, p. 573, t. 46 f. $44,45,50$; t. 49 f. $1,4.5 .7-9,11$.

Q. Stirnfortsatz kurz; vordere Segmente des Vorderkörpers mit netzförmig geordneten Rippen im Chitin; Spitzenkränze am Hinterrande der Segmente des Vorderkörpers schwach, am Hinterkörper gar nicht vorhanden. 1. und 2. Abdsegm. auf der Dorsalseite getrennt; Furkalborsten doppelt so lang wie der 
Rumpf. 1. Antenne 7-gldr, am Ende des Innenrandes des 1. Gl. ein kurzer Vorsprung, des 2. Gl. ein 'längerer Dorn. Haken der 2. Maxille von unregelmäßiger Form. 1. B. mit 3-gldr. Exp. und Enp.; Außenranddorn des 1. Gl. des Exp. und besonders des 2. Gi. des 1. B., 'sowie Innen- und Außenrandborsten des Basp. desselben B. lang. Endborste des 5. B. weniger als halb so lang wie das Bein. 6. B. mit 1 ziemlich langen Borste. L. $1,85 \mathrm{~mm}$. - o unbekannt.

Atlant. Ozean; Pacif. Ozean.

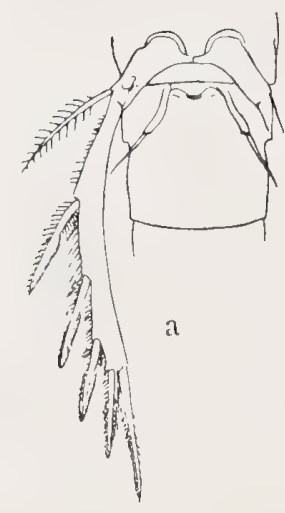

Fig. 195. Aegisthus aculeatus.

a Vorderer Teil des Hinterkörpers mî́ 5. und 6. B. ㅇ, ventral, b $q$ lateral. Nach Giesbrecht.

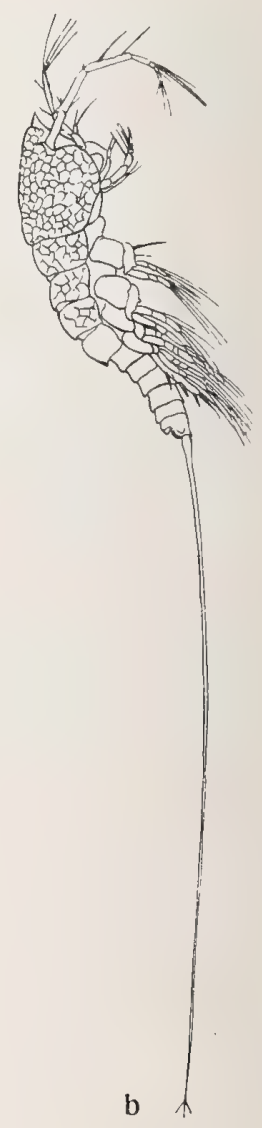

\section{Aegisthus spinulosus Farran.}

1905 , A. s., Farran, p. 46 , t. 12 f. $8-14$; t. 13 f. $1-4$.

Verwandt mit aculeatus, aber: - - 1. und 2. Abdsegm. auch ventral getrennt; Vorderkörper ohne netzförmige Chitinrippen; Hinterrand des 2.-.-4. Thsegm. und des 2.-5. Abdsegm. gezähnelt; Furkalborsten nur wenig länger als der Rumpf. 5. B. dem von aculeatus ähnlich, aber die Beborstung nur 
zum Teile bekannt. 6. B. mit 2 kurzen, fast gleich langen Borsten. L. 1,74 mm. - o unbekannt.

Nord-Atlant. Ozean.

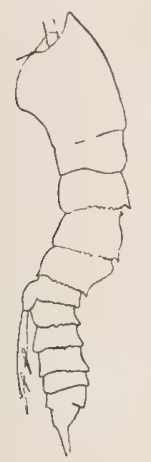

a

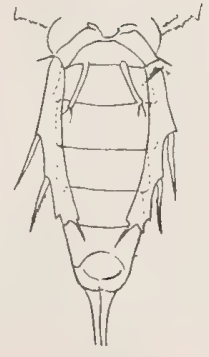

b

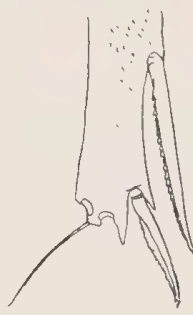

c

Fig. 196. Aegisthus spinulosus.

a ㅇ lateral, b Abdomen , ventral, c Ende des 5. B. (2 Apikalborsten fehlen). Nach Farran.

\section{Aegisthus mucronatus Giesbrecht}

1891, A. m., Giesbrecht, p. 476. 1892, A. m., Giesbrecht, p. 573, t. 46 f. $46-49,51 ;$ t. 49 f. $2,3,6,10$.

‥ Stirnfortsatz lang; Chitin der Rumpfsegmente ohne netzförmige Rippen; am dorsalen Hinterrande des 2.-5. Thsegm. und der Abdsegm. kräftige Spitzenreihen; 1. und 2. Abdsegm. auf der Dorsalseite getrennt; Furkalborsten über $3^{1} / 2$ mal so lang wie der Rumpf. 1. Antenne 6-gldr.; am Ende des Innenrandes des 1. Gl. ein längerer, proximal gebogener Fortsatz, des 2. G1. ein

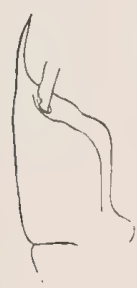

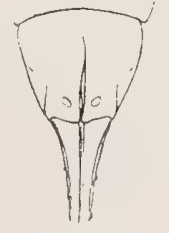

b

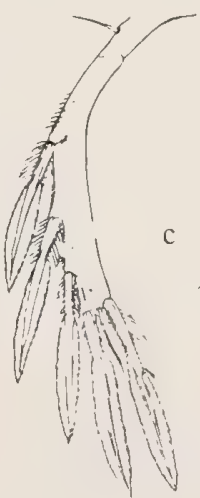

Fig. 197. Aegisthus mucronatus.

a. Kopf q, lateral, b Furka $q$, ventral, c 5. B. \&. Nach Giesbrecht. 
kurzer Dorn. Haken des Maxillipeden von regelmäßiger Form. Exp. und Enp. des 1. B. 2-gldr. 1. und 2. Gl. des 1. B. mit kurzem Außenranddorn; (der des 2. Gl. viel kürzer als das Endgl.). Basp. des 1. B. mit verkürzter Außenund Innenrandborste. Endborste des 5 . B. fast $3 / 4$ so lang wie das Bein; 6. B. mit 2 Borsten. L. $2,55 \mathrm{~mm}$. - of unbekannt.

Nord-Atlant. Ozean; Pacif. Ozean.

\section{Aegisthus atlanticus Wolfenden.}

1902, A. a., Wolfenden, p. 364. 1904, A. a., Wolfenden, p. 122.

Verwandt mit mucronatus, aber hauptsächlich in folgenden Merkmalen abweichend: - . . 2.-4. Thsegm. ohne Spitzenreihe am Hinterrande. Furkalborsten $5^{1 / 2}$ mal so lang wie der Rumpf. Am Ende des 2. Gl. der 1. Antenne kein Dorn. Endborste des. 5. B. ungefähr halb so lang wie das (3-gldr.?) Bein. L. $1,45 \mathrm{~mm}$. - o unbekannt.

Nord-Atlant. Ozean.

\section{Oncaeidae.}

Rumpfform im Allgemeinen cyclopsartig. Vorderkörper und Hinterkörper beim $q$ meist 5-gldr. (Kopf vom 1. Thsegm. gewöhnlich getrennt, zwischen Gensegm. und Furka meistens 3 Segm.); keine paarige Augen mit Cuticularlinsen; Furka jederseits mit 6 Borsten; weibliche Geschlechtsöffnungen dorsal, zuweilen stark lateral. 1. Antenne des $q 4$ - bis 6-gldr., zuweilen mit gut entwickelten Aesthetasken, beim ot niemals genikulierend. 2. Antenne 3- oder 4-gldr., Endborsten meist von ungefähr gleicher Länge und Dicke. Mandibeln auf die Kaulade reduziert. 1. Maxille ein borstentragendes Plättchen, meist ohne deutliche Gliederung. 2. Maxille 2-gldr. Maxilliped 4-, selten 3-gldr., mit kräftigem Endhaken; beim of abweichend gebaut und zum Festhalten des $q$ bei der Kopulation dienend. 1.-4. B. mit 3-gldr. Ästen, deren Beborstung im allgemeinen von vorne nach hinten abnimmt; Enp. des 4. B. länger oder wenig kürzer als das Exp. - Sexuelle Eigentümlichkeiten des ot. im Bau des Abdomens und des Maxillipeden, geringere gewöhnlich auch an den 1. Antennen, selten an den 2. Antennen und Mundteilen.

\section{Oncaea Philippi.}

Rumpfform cyclopsartig; Vorderkörper 5-gldr., Hinterkörper des $q$ 5-, des $\sigma^{7} 6$-gldr., bei letzterem mit verkürzten mittleren Gl. und voluminösem Gensegm. Genitalklappen des. $\sigma^{7}$ mit seitlicher Spitze; Furka jederseits mit 
6 Borsten. 1. Antenne beim $q$ 6-gldr., mit langem miftleren Gl.; Aethetasken schwach entwickelt; beim of die 3 kurzen Endgl. zu einem Stück verschmolzen. 2. Antenne 3-gldr.; mit Hakenborste von mittlerer Länge. Mandibel auf die Kaulade reduziert, nicht keilförmig, mit beweglichen kamm- und borstenförmigen Anhängen. Maxilliped 4-gldr. mit kräftigem Endhaken, der beim $q$ am konkaven Rande mit Spitzen besetzt, beim $\sigma^{7}$ glattrandig ist. 1.-4. B. mit 3-gldr. Exp. und Enp.; Endgl. der Enp. schmal und lang, am 4. B. wenigstens $1 \frac{1}{2}$ mal so lang wie das 1. unc 2. Gl. zusammen; 5. B. ein kleines Stäbchen oder Knöpfchen, mit 1 oder 2 endständigen Börstchen.

Zwischen Gensegm. und Ansegm. 2 Segmente; Genitalöffnungen dorsal 1. Antenne mit 3 von einander getrennten, kurzen Endgl. Endhaken des Maxillipeden mit Spitzen am konkaven Rande.

Zwischen Gensegm. und Ansegm. 3 Segmente; Genitalöffnungen ventral, unter zugespitzten Klappen gelegen. Die 3 Endgl. der 1 . Antenne zu einem verschmolzen. Endhaken des Maxillipeden glattrandig.

1. 2. Thsegm. in der Seitenansicht aus der dorsalen Rumpffläche heraustretend

O. conifera

2. Thsegm. nicht aus dem Profil des Rückens heraustretend

2. Mittleres Gi. der 2. Antenne am Innenrande zackig; Leiste am Innenrande des 2. Gl. des Maxillipeden mit dicken Spitzen besetzt O. ornata Mittleres Gl. der 2. Antenne mit glattem Innenrande; Leiste am Innenrande des 2. Gl. des Maxillipeden mit Haaren

3. Gensegm. wenig länger als die beiden folgenden Segm., welche länger als breit sind

O. subtilis

Gensegm. viel länger als die beiden folgenden Segm., welche breiter als lang sind

4. Furka länger als das Ansegm. 5

$\begin{array}{ll}\text { Furka kürzer als das Ansegm. } & 7\end{array}$

5. Die breiteste Stelle des Rumpfes (Dorsalansicht) liegt beträchtlich vor der hinteren Grenze des Kopfes O. venusta

Die breiteste Stelle des Rumpfes liegt wenig vor der hinteren Grenze des Kopfes

6. Furka etwa 4 mal so lang wie breit, Geschlechtsöffnungen nicht weit von der Mitte des Gensegm. gelegen, von einander abgerückt $O$. mediterranea Furka $2-2 \frac{1}{2} \mathrm{mal}$ so lang wie breit, Geschlechtsöffnungen beträchtlich vor der Segmentmitte gelegen, einander genähert O. media

7. Endhaken (und distale Borste) des Mittelgl. des Maxillipeden grob gezähnelt; 5. B. lang, dorsal abstehend

O. notopus

Endhaken des Mittelgl. des Maxillipeden mit Spitzen besetzt; 5. B. kurz

O. minuta.

1) Da die Männchen zum Teil nicht bekannt oder ungenügend beschrieben sind, wird nur eine Bestimmungstabelle für die Weibchen gegeben. 


\section{Oncaea venusta Philippi.}

1843, O. V., Philippi, p. 62, t. 4. 1860, O. pyriformis, Lubbock, p. 11, t. 29. 1866, Antaria coerulescens, Claus, p. 19.

१. Vorderkörper birnförmig (Kopf breit), meist mit granulierter Cuticula; Gensegm. wenig länger als der übrige Teil des Abd., folgende Segm. breiter als lang; Furka wenigstens so lang wie 4. und 5. Abdsegm. zusammen und wenigstens $4 \mathrm{mal}$ so lang wie breit. 2. Antenne, besonders das Enp. sehr

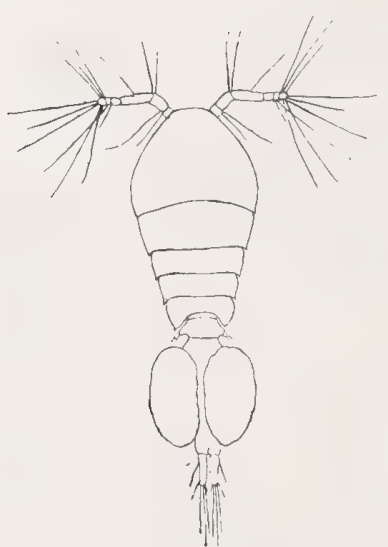

a

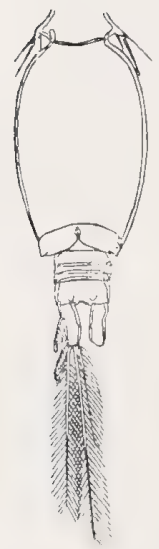

d

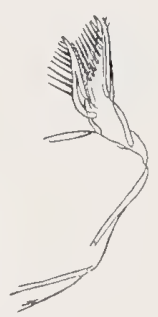

b

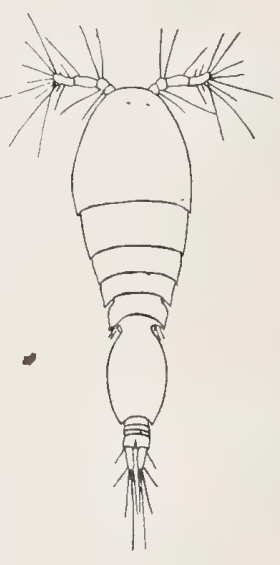

c

Fig. 198. Oncaea venusta.

a $q$ dorsal, b 2. Maxille $q$, c $\sigma^{\top}$ dorsal,

d Abdomen or, ventral, e 4. B. 웅.

Nach Giesbrecht.

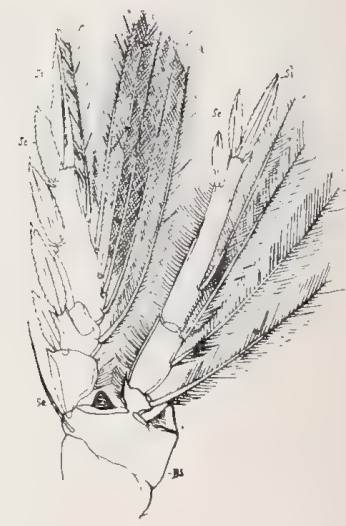

e

gedrungen. Endhaken des Maxillipeden mit einigen Spitzen an der konkaven Seite; die beiden Borsten des 2. G1. des Basp. ziemlich dünn und lang. Exp. der B. mit breit gesäumten, gezähnelten Außenrandborsten; Endgl. des Enp. des 4. B. ohne terminalen Zapfen, mit 3 lanzettförmigen, gezähnelten Borsten im 2.-4. B. - $0^{7}$ mit kurzen Genitalklappen und kurzem, breitem Ansegm. - L. ๆ 1,1-1,27 mm, ठ $0,7-1 \mathrm{~mm}$.

Mittelmeer, Atlant. Ozean; Pacif. Ozean; Ind. Ozean, Rotes und Arab. Meer. 


\section{Oncaea mediterranea Claus.}

1863, Antaria m., Claus, p. 159, t. 30 f. 1-7. 1891, O. m., Giesbrecht, p. 477 . 1892 , O. m., Giesbrecht, p. 591 , t. 4 f. 4 , 16 ; t. 47 f. $8-10,47$.

Verwandt mit venusta; aber: - $\supsetneq$. Vorderkörper schmäler, seine breiteste Stelle weiter nach hinten gerückt. Gensegm. des q relativ länger; 1. Gl. des Basp. der 2. Antenne etwas gestreckter; Endhaken des Maxillipeden mit Zähnchen statt Spitzen und mit kürzeren Borsten am 2. Gl. des Basp. $\sigma^{7}$. Genitalklappe und Ansegm. länger als bei venusta. - L. $q 1-1,3 \mathrm{~mm}$, o $0,7-1,05 \mathrm{~mm}$.

Mittelmeer, Atlant. Ozean; Polarmeer; Pacif. Ozean; Rotes und Arab. Meer, Indischer Ozean.

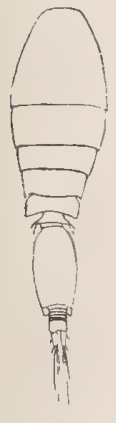

a
Fig. 199. Oncaea mediterranea. a $0^{7}$ dorsal, b Abdomen , dorsal. Nach Giesbrecht.

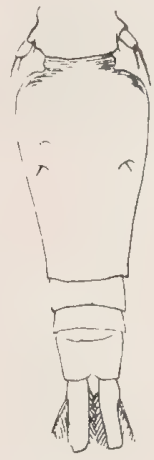

b

3. Oncaea media Giesbrecht.

1891, O. m., Giesbrecht, p. 477. 1892, O. m., Giesbrecht, p. 591, t. 2 f. $12 ;$ t. 47 f. $1,11,29-33,40$.

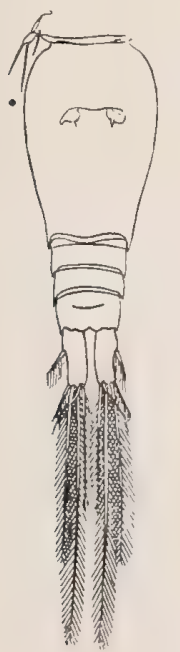

a

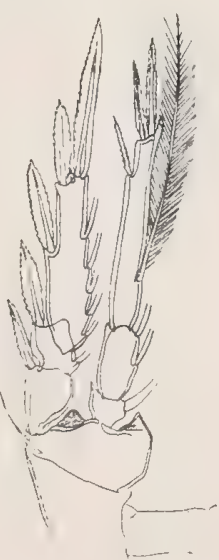

b
Fig. 200. Oncaea media. a Abdomen $q$, dorsal, b 4. B. $q$. Nach Giesbrecht. 
Verwandt mit venusta und mediterranea, aber außer durch die geringere Körperlänge besonders durch die Kürze der Furka (länger als das Ansegm., 2-21/2 mal so lang wie breit) unterschieden; Enp. der 2. Antenne etwas gestreckter. - L. † 0,55-0,82 $\mathrm{mm}$, o $0,6-0,63 \mathrm{~mm}$.

Mittelmeer, Atlant. Ozean; Pacif. Ozean; Rotes und Arab. Meer, Mal. Archipel, Ind. Ozean.

\section{Oncaea minuta Giesbrecht.}

1892, O. m., Giesbrecht, p. 591, t. 47 f. 3, 6, 26, 46, 59. 1905, O. m., Esterly, p. 217, f. 56 .

○. Gensegm. länger als der übrige Teil des Abd., folgende Segm. breiter als lang; Furka kürzer als das 5. Abdsegm., weniger als doppelt so lang wie breit (innere Endborste kürzer als die äußere). 2. Antenne von gedrungenem

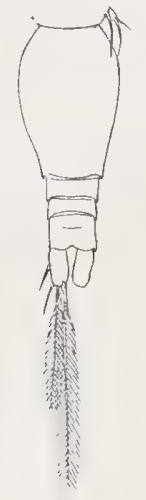

a

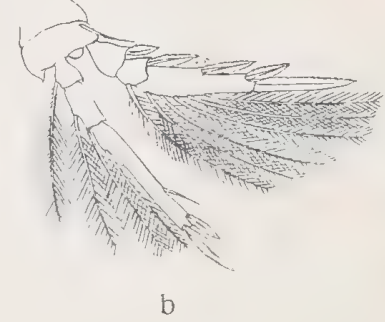

Fig. 201. Oncaea minuta. a Abdomen , dorsal, b 4. B. 오. Nach Giesbrecht.

Bau. Endhaken des Maxillipeden und die distale Borste des 2. Gl. des. Basp. mit groben Spitzen. Exp. der B. mit schmäleren Außenranddornen als bei venusta, Endgl. des Enp. auch am 4. B. mit terminalem Zapfen und mit ungezähneltem, proximalem Außenranddorn. L. 0,46-0,58 mm. - o unbekannt (?).

Mittelmeer, Atlant. Ozean; Pacif. Ozean; Ind. Ozean.

Anmerkung. Aurivillius (1899, p. 29, f. 1-3) bildet ein Oncaea-Männchen ab, das er zu dieser Art gehörig hält.

\section{Oncaea conifera Giesbrecht.}

1891, O. c., Giesbrecht, p. 477. 1892, O. c., Giesbrecht, p. 591, t. 2 f. $10 ;$ t. 47 f. $4,16,21,23,28,34-38,42,55,56.1900$, O. c., Sars, p. 113, t. 32 f. 15,16 . 1902, O. c., Giesbrecht, p. 41, t. 13 f. $7-11.1905$, O. c., Esterly, p. 216 , f. 55. 
†. Mittelstück des 2. Thsegm. aus der Dorsalfläche des Rumpfes heraustretend; Gensegm. so lang wie, oder $11 / 2$ mal so lang wie der übrige Teil des Abd., folgende Segm. breiter als lang; Furka so lang wie, oder etwas länger als das Ansegm., 2-4 mal so lang wie breit, ihre Zweige stark von einander abgerückt. 1. Gl. des Basp. und Enp. der 2. Antenne etwas gestreckter als bei venusta.

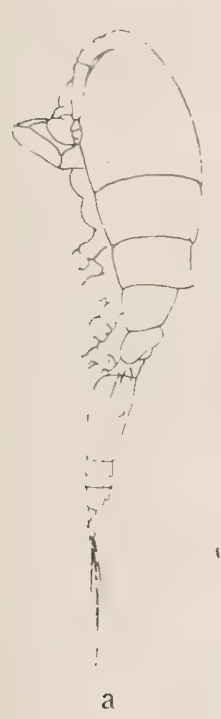

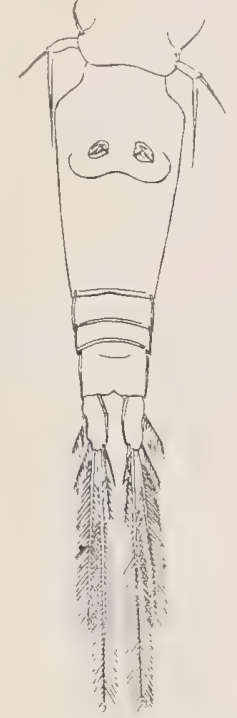

b
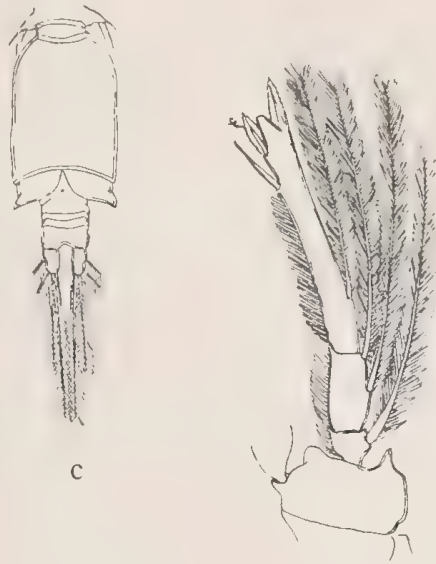

d

Fig. 202. Oncaea conifera.

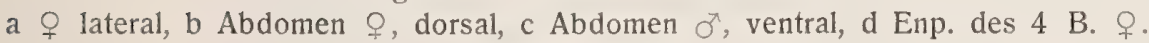
Nach Giesbrecht.

Endhaken des Maxillipeden mit dicken Spitzen besetzt; distale Borste des 2. Gl. des Basp. dicker und länger als die proximale. B. ähnlich wie bei venusta, doch die Zapfen am Ende des Endgl. des Enp. sehr groß und auch im 4. Paare vorhanden, und die daneben befindlichen Lanzettborsten verkürzt. Die längere der beiden Endborsten des 5. B. auffällig dicker als die kürzere oder beide fast gleich dick. - $\sigma^{7}$ mit langen Genitalklappen und kürzerer Furka als bei venusta. - L. O $0,75-1,25 \mathrm{~mm}$, o $0,6-0,8 \mathrm{~mm}$.

Mittelmeer, Atlant. Ozean; Polarmeer; Pacif. Ozean; Antarkt. Ozean; Rotes Meer, Mal. Archipel, Ind. Ozean.

\section{Oncaea notopus Giesbrecht.}

1891, O. n., Giesbrecht, p. 417. 1892, O. n., Giesbrecht, p. 591, t. 47, f. $12,15,45.1900$, O. n., Sars, p. 107, t. 32 f. $1-14$. 1902, O. n., Giesbrecht, p. 41 , t. 13 f. $1-6$.

ๆ. Gensegm. etwas länger als der übrigè Teil des Abd., folgende Segm. breiter als lang; Furka kaum $3 / 4$ so lang wie das Ansegm. und kaum 
doppelt so lang wie breit. 2. Antenne von gedrungener Gestalt. Endhaken des Maxillipeden relativ kurz und gezähnelt, proximale Borste des 2. Gl. des Basp. dünn. B. im Ganzen ähnlich wie bei venusta. 5. B. relativ lang, dorsal abstehend. - o dem von conifera ähnlich. - L. $\bigcirc 0,6-0,95 \mathrm{~mm}, \sigma^{7}$ ?

Polarmeer; Pacif. Ozean; Antarkt. Ozean; Suez-Kanal.

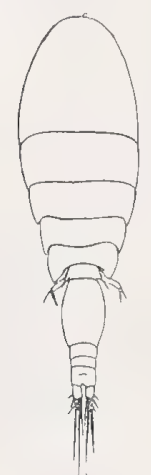

Fig. 203. Oncaea notopus.

a † dorsal, b Hinterkörper $\$$, dorsal. a/nach Giesbrecht, b nach Sars.

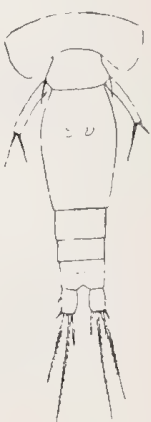

b

\section{Oncaea subtilis Giesbrecht.}

1892, O. s., Giesbrecht, p. 591, t. 47 f. 14, 18, 25, 43, 60.

. . Gensegm. wenig länger als das 4. und 5. Abdsegm. zusammen, diese sowie das Ansegm. länger als breit; Furka kürzer als das Ansegm., 2-21/2

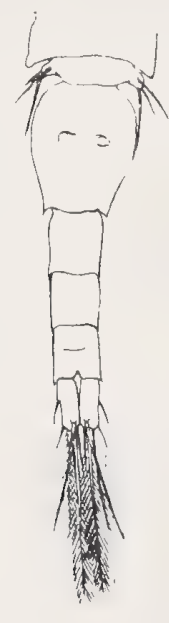

i)

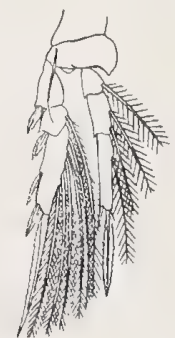

b

Fig. 204. Oncaea subtilis.

a Hinterkörper $ᄋ$, dorsal, b 4. B. 9 . Nach Giesbrecht.

mal so lang wie breit, ihre Zweige einander sehr genähert. 2. Antenne mit sehr gestrecktem Endgl., in dessen proximaler Borstengruppe eine Borste viel länger als die andere ist. Borsten am 2. Gl. des Basp. des Maxillipeden lang, mit Stachelfiedern besetzt. Enp. der B. kaum länger als das Exp., mit 
relativ kurzem Endgl.; die Außenranddornen der Exp. ziemlich schmal und kurz; Endborste des Exp. länger als das Endgl.; terminaler Zapfen am Endgl. des Enp. schwach entwickelt; Endgl. des Enp. des 2.-4. B. ohne proximale Außenrandborste und mit langer endständiger Innenrandborste. L. 0,48-0,5 mm. $\delta$ unbekannt.

Mittelmeer, Atlant. Ozean, Skagerak.

\section{Oncaea ornata Giesbrecht.}

1891, O. o., Giesbrecht, p. 477. 1892, O. o., Giesbrecht, p. 591, t. 44 f. 50,$51 ;$ t. 47 f. $20,24,49,53$.

ๆ. Gensegm. über doppelt so lang wie der übrige Teil des Abd., folgende Segm. breiter als lang; Furka ungefähr so lang wie das Ansegm., kaum doppelt so lang wie breit. 2. Antenne mit gestrecktem Endgl., in dessen proximaler Borstengruppe eine viel dicker als die anderen 3 ist; 2. Gl. des Basp. mit bestacheltem Innenrande. Endhaken des Maxillipeden der Länge nach mit dicken Spitzen besetzt; ähnliche auch am mittleren Teile des Innenrandes vom 2. Gl. des Basp.; distale Borste des 2. Gl. des Basp. lang und

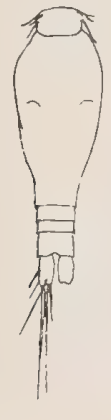

a

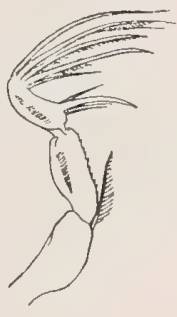

b

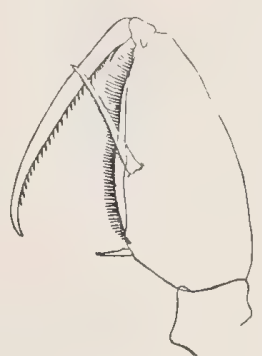

c

Fig. 205. Oncaea ornata.

a Abdomen $q$, dorsal, b 2. Antenne $ᄋ$, c Maxilliped $q$. Nach Giesbrecht.

dick, proximale kurz. Enp. der B. wenig länger als das Exp.; Endgl. des Enp. mit schmalem Zapfen, ohne proximale Außenranddornen und mit langer endständiger Innenrandborste; Außenranddorn des 1. Gl. des Exp. des 1. B. verlängert. 5. B. weiter rückgebildet als bei den anderen Arten, nur noch ein kleines, mit einem Börstchen versehenes Wärzchen und davor ein zweites Börstchen übrig. L. 0,9-1 mm. - o unbekannt.

Engl. Kanal; Pacif. Ozean.

Conaea Giesbrecht.

Verwandt mit Oncaea im Bau des Rumpfes, der Kopfgliedmaßen, der sexuellen Eigentümlichkeiten des $\sigma^{7}$. Aber: - 2. Antenne mit lang gestreck- 
tem Endgl. und sehr langen Hakenborsten. Enp. der hinteren B. kürzer als das Exp. und Endgl. des Enp. des 4. B. nicht länger als das 1. oder 2. Gl. 5. B. jederseits eine Fiederborste.

\section{Conaea rapax Giesbrecht.}

1891, C. r., Giesbrecht, p. 477. 1892, C. r., Giesbrecht, p. 605, t. 48 f. $50-59$.

. Das lange Mittelgl. der 1. Antenne etwa $7 \mathrm{mal}$ so lang wie breit und $21 / 2$ mal so lang wie das proximale und das gleich lange distale Stück der Antenne. Gensegm. so lang wie die übrigen Absegm. und Furka zusammen,

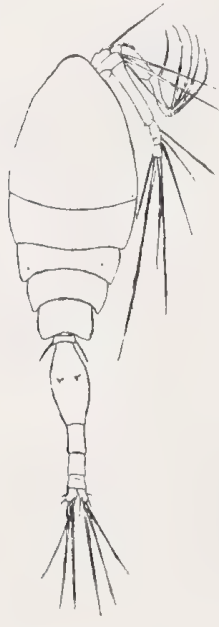

a

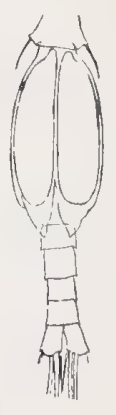

b

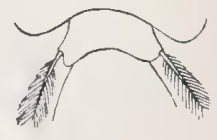

c

Fig. 206. Conaea rapax.

a $\bigcirc$ dorsal, b Abdomen $\sigma$, ventral, c 5. Thsegm. $q$. Nach Giesbrecht.

das auf das Gensegm. folgende Segm. 2 mal so lang wie breit. Exp. des 1. B. mit $1,1,3$, des 2 . und 3. B. mit $1,1,2$, des 4. B. mit 1, 0, 1 Außenranddornen.- $\sigma^{7}$ Sekundäre Sexualcharaktere nahezu dieselben wie im Genus Oncaea. — L. ᄋ $1-1,15 \mathrm{~mm}$, ơ $0,8-0,9 \mathrm{~mm}$.

Atlant. Ozean; Pacif. Ozean.

\section{Lubbockia Claus.}

Rumpf langgestreckt; Vorderkörper 5-gldr., Hinterkörper des o 5-gldr., des $\sigma^{7}$ 6-gldr.; Gensegm. des ơ klein, die folgenden Segm. gestreckt. 1. Antenne des o 4- bis (?) ?-gldr., Aesthetasken schwach entwickelt, beim $\sigma^{7}$ mit verschmolzenen Endgl. und ungemein langer Endborste. Die folgenden 5 Gliedmaßenpaare beim $\delta$ mehr oder weniger rück- oder umgebildet. 2. Antenne des $q$ 3-gldr., mit sehr kurzem mittleren Gl. und kurzen Hakenborsten. Mandibel des $q$ beilförmig, mit verlängerter dorsaler Spitze. 2. Maxille des $q$ dem von Oncaea ähnlich. Maxilliped des $q$ sehr groß, beim $\sigma^{\gamma}$ kleiner und mit stärker gekrümmtem Endhaken. 1.-4. B. ähnlich wie bei Oncaea, aber das Endgl. 
der Enp. weniger gestreckt; hintere Schwimmfüße oft mit mangelhafter oder aufgehobener Artikulation der Astglieder; Exp. des 1. und 2. B. mit 1, 1, 2 oder 1, 1, 3, des 3. und 4. B. mit 1, 1, 2 Außenranddornen; 5. B. stäbchenförmig, mit je 2 lanzettförmigen Borsten.

\section{Lubbockia glacialis G. O. Sars.}

1900, L. g., Sars, p. 114, t. 33.

१. Abdsegm. ohne Spitzenkranz am ventralen Hinterrand; Ansegm. halb so lang wie das vorhergehende Segm. und viel kürzer als die Furka. Das große Gl. des Maxillipeden ohne Stacheln am Innenrande. Endgl. des Exp.

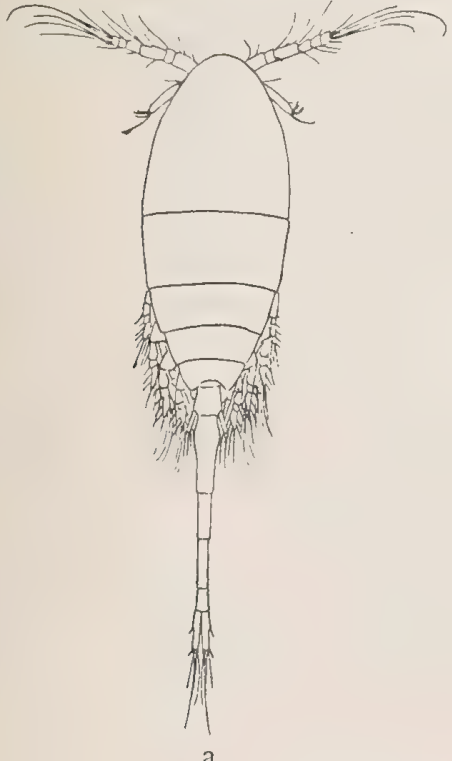

Fig. 207. Lubbockia glacialis.

des 1. und 2. B. mit 3 Außenrandborsten; die längere Borste des 5. B. etwas über die Mitte des Gensegm. hinausreichend, ungefähr 2 mal so lang wie die kürzere. L. 2,45 mm. - ot unbekannt.

Arktischer Ozean.

\section{Lubbockia minuta Wolfenden.}

1905, L. m., Wolfenden.

Q. Furka noch nicht $2 \mathrm{mal}$ so lang wie das Ansegm. (11:8). Enp. der 2. Antenne am Vorderrande mit 1 kurzen proximalen und einer relativ langen Nord. Plankton.

VIII 13 
distalen Borste (bei L. glacialis beide kurz); Endrand mit 6 Borsten (bei L. glacialis mit 8). Gliederung und Beborstung des 1.-4. B. wie bei L. glacialis. L. $1,3 \mathrm{~mm}$. - o unbekannt.

Fundort: ?

\section{Corycaeidae.}

Stirn beim $q$ stets breit, mit zwei stark entwickelten, zuweilen sich berührenden, zuweilen von einander entfernten Chitinlinsen; die Körperform des $\sigma$ von dem des $q$ oft abweichend und seine Augen bisweilen rückgebildet; weibliche Geschlechtsöffnungen dorsal, zuweilen stark lateral. Furka jederseits mit 4 bis 5 Borsten. 1. Antenne 3- bis 6 -gldr., ohne oder mit wenigen, sehr dünnen Aesthetasken, beim or niemals genikulierend. Mundteile des $q$ : 2. Antenne mit wenigstens einem dicken Endhaken am Endgl. Mandibel auf die Kaulade reduziert. 1. Maxille von der Form ovaler oder länglicher Plättchen, mit 3-5 Borsten besetzt. 2. Maxille der mancher Oncaeiden ähnlich. Maxilliped 3-gldr., mit kräftigem Endhaken. 2. Antenne bis 2 Maxille des $\sigma^{7}$ bisweilen rückgebildet oder abweichend gebaut, Maxilliped des $\delta$ stets mit sexuellen Abweichungen, zum Festhalten des $q$ bei der Kopulation dienend. Äste der Ruderfüße 3-gldr., mit Ausnahme vom Enp. des 4. B., das alle Übergänge vom 3-gldr. Ast zu einem aus einer Borste bestehenden Rudiment aufweist; geringe Abweichungen an den Schwimmfüßen beim $\sigma^{\top}$ bisweilen vorhanden.

\section{Sapphirina J. V. Thompson.}

Rumpf deprimiert; dessen Segm. (mit Ausnahme des letzten Thsegm. und des letzten Abdsegm.) beim or blattartig verbreitert, irisierend. Vorderkörper 5-gldr., Hinterkörper beim $\bigcirc$ 5-, beim $\sigma^{\pi} 6$-gldr., aber Gensegm. des $\bigcirc$ dorsal durch eine Linie oft in 2 Stücke getrennt; die mittleren Abdsegm. des $q$ verbreitert. Genitalklappen des $\sigma^{7}$ breit, aber kurz, mit einigen Borsten. Furka blattförmig, mit je 5 Borsten. Die frontalen Augenlinsen berühren sich oder liegen dicht bei einander und sind beim $\delta^{\top}$ vom vorderen Kopfrande oft überwachsen. 1. Antenne 3- bis 5-gldr., bisweilen 6-gldr. beim ơ , ohne Aesthetasken, beim of keine von beiden genikulierend. 2. Antenne 4-gldr., Klammerorgane, mit kurzen Hakenborsten am Endgl. und sonst meist kleinen, dünnen Borsten. Mandibel auf die Kaulade reduziert, beilförmig, mit spitz ausgezogenem dorsalen Ende, beim or bisweilen mehr oder weniger rückgebildet. 1. Maxille ein ovales Plättchen. 2. Maxille 2-gldr.; Endgl. in eine lange Spitze ausgezogen. Endhaken des 3-gldr. Maxillipeden beim of kurz und dick, beim of verlängert und vermittelst eines Zwischengl. mit dem 2. Gl. des Basp. artikulierend. B. mit breiten, im 1.-3. Paare etwa gleich langen Exp. und Enp.; 1.-4. B. mit 3-gldr. Exp. und Enp.; sexuelle Besonderheiten an den Schwimmfüßen nicht allgemein. 5. B. stäbchenförmig, mit 2 Borsten. 


\section{Sapphirina iris Dana.}

1852 , S. i., Dana, p. 1239 , t. 87 f. 1 a-d. 1863, S. salpae, Claus, p. 152 . 1883 , S. gemma, Brady, p. 127 , t. 48 f. $6-8$. 1892 , S. salpae, Giesbrecht, p. 618, t. 2 f. 9 ; t. 52 f. $1,2,18,19,27,45,49$; t. 53 f. 7,23 , 24,$60 ;$ t. 54 f. $9,13,15,16,19,57$.

\%. Furka mehr als $2^{1 / 3}$ mal so lang wie breit, ihr Innenrand stärker konvex als der Außenrand, am Ende des ersteren eine kleine, zuweilen verstreichende Spitze; ihre dorsale Borste sitzt weiter hinten als die Außenrandborste an. 1. Antenne $6 / 7$ so lang wie die 2. Ant., 5-gldr.; 2. Gl. 11/3 mal so lang wie die 3 Endgl. zusammen. Enp. der 2. Antenne $7 / 10$ so lang wie das 2. Gl. des Basp., Endhaken halb so lang wie das 2. Gl. des Enp. Enp. des 4. B. wenig kürzer als das Exp.; Endgl. des Exp. des 4. B. mit Außenrand-

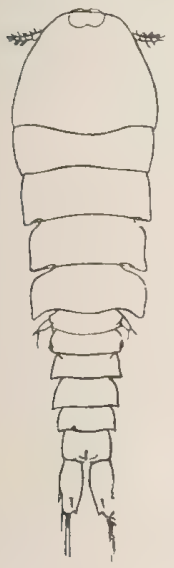

a

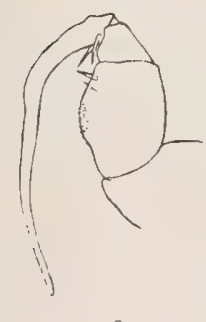

$\mathrm{e}$

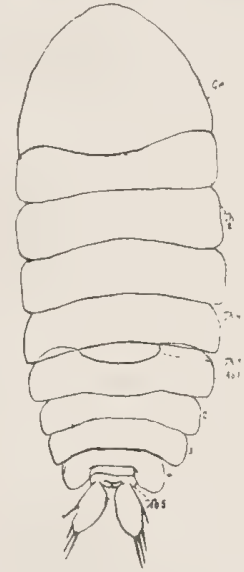

b

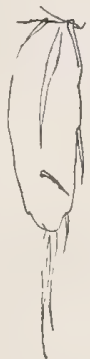

C

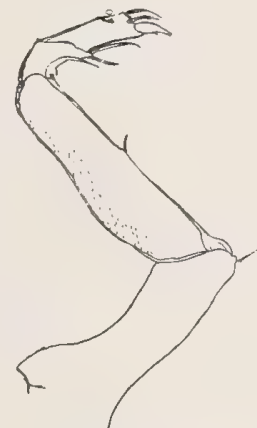

d

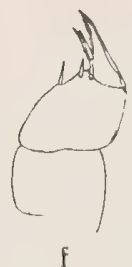

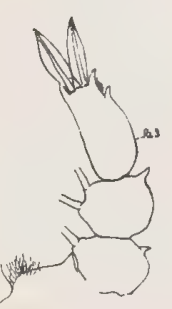

g

Fig. 208. Sapphirina iris.

a $q$ dorsal, b o dorsal, c Furka $q$, dorsal, d 2. Antenne $q$, e Maxilliped $\sigma^{\pi}$, f Maxilliped $q$, g. Enp. des 4. B. ㅇ. Nach Giesbrecht.

borsten; Endgl. des Enp. desselben B. kürzer als das 1. und 2. Gl. zusammen, mit 2 Borsten am Ende. Eiersäckchen rot. - o $\sigma^{7}$. Länge des Rumpfes ca. $2 \frac{1}{2}$ mal so groß wie seine größte Breite; Augenlinsen ventral gelegen, vom Stirnrand überragt. Furka, 1. Antenne, 4. B. ähnlich wie beim 9,2 . Antenne, 
Mandibel, 1. und 2. Maxille etwas abweichend; Endgl. des Enp. des 2. B. mit 3 Lanzettbörstchen und verlängerten Zacken. - L. $ๆ 5,6-7,5 \mathrm{~mm}$, o 5,9-8 mm.

Mittelmeer, Atl. Ozean; Pacif. Ozean; Ind. Ozean.

\section{Sapphirina nigromaculata Claus.}

1863, S. n., Claus, p. 152 , t. 8 f. 5, 6.1892 , S. n., Giesbrecht, p. 619, t. 52 f. $32,35,43$; t. 53 f. $13,26,36,48$; t. 54 f. $6,37,40,68$.

१. Furka doppelt so lang wie breit, mit Spitze am Ende des Innenrandes, ihre dorsale Borste sitzt weiter vorn als die Außenrandborste. 1. Antenne noch nicht $3 / 7$ so lang wie die 2. Ant., 5-gldr.; 2. Gl. kürzer als die 3 Endgl. zusammen. Enp. der 2. Ant. so lang wie das 2. Gl. des Basp., Endhaken $1 / 3$ so lang wie das 2. Gl. des Enp. Enp. des 4. B. noch nicht halb so lang wie das Exp., Endgl. des Enp. etwa so lang wie das 1. oder das 2. G1., mit 2 Borsten
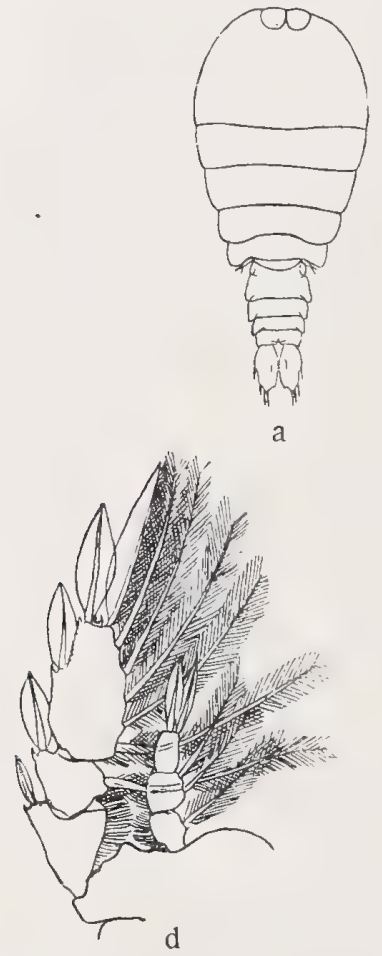

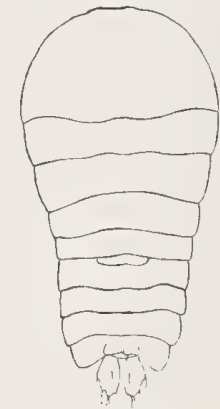

b

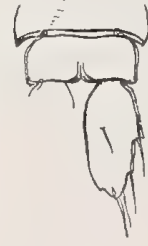

C

Fig. 209 .

Sapphirina nigromaculata. a $q$ dorsal, b of dorsal, c Furka $q$, dorsal, d 4. B. $\%$, e Enp. des 2. B. o Nach Giesbrecht.

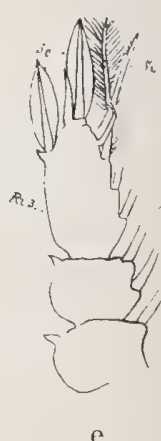

am Ende. - $\sigma^{7}$. Länge des Rumpfes ca. $2 \frac{1}{4}$ mal so groß wie seine größte Breite; Augenlinsen fast bis an den Stirnrand gerückt. Furka, 4. B., 1. Antenne und die folgenden Kopfgliedmaßen wie beim $\$$, nur die 2. Antenne mit geringen Abweichungen. Endgi. des Enp. des 2. B. mit 2 lanzettförmigen Borsten, die 3. einseitig gezähnelt, Zacken verlängert. - L. ๆ 1,9-2 mm, ơ 2,05-2,45 mm. Mittelnieer, Atlant. Ozean; Pacif. Ozean; Rotes Meer, Ind. Ozean. 
Corina Giesbrecht.

. Rumpfform der von Sapphirina ähnlich; Vorderkörper 5-, Hinterkörper 3-gldr.; die Abdsegm. nicht verbreitert und die Furka nicht so blattförmig wie bei Sapphirina. Augenlinsen aneinander stoßend. 1. Antenne 5-gldr., ohne Aesthetasken. 1. Maxille stäbchenförmig. 2. Maxille und Maxilliped klein, mit stark reduzierten Anhängen. Äste der B. schmal; Enp. des 4. B. 2-gldr.; 5. B. jederseits aus 3 Börstchen bestehend. - $\sigma$ unbekannt.

\section{Corina granulosa Giesbrecht.}

1891, C. g., Giesbrecht. 1892, C. g., Giesbrecht, p. 645, t. 49 f. $39-45$; t. 50 f. 53,54 .

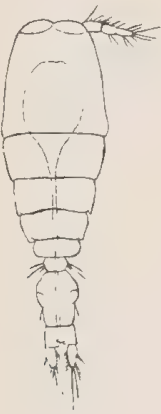

a

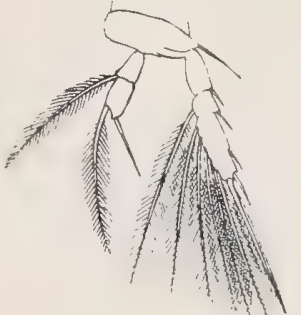

b

Fig. 210. Corina granulosa. a $q$ dorsal, b 4. B. . Nach Giesbrecht.

Q. Vorderkörper fein granuliert. Enp. der B. kürzer als das Exp., am 4. B. nur halb so lang. 5. B. jederseits aus 3 Borsten, die unmittelbar am Segment ansitzen, bestehend. L. 0,68 mm. - o unbekannt.

Atlant. und Pacif. Ozean.

\section{Corycaeus Dana.}

Rumpf walzenförmig; die frontalen Augenlinsen liegen nahe bei einander und berühren sich zuweilen; Vorderkörper 2- bis 4-gldr., Hinterkörper 2- bis 3-gldr.; Lateralteile des 3. und 4. Thsegm. in spitze Zipfel verlängert; 5. Thsegm. sehr kurz. Genitalklappen des or lang, mit je 1 Borste. Furka stabförmig, mit je 4 Borsten (wovon eine distal von der Mitte des Außenrandes). 1. Antenne 6-gldr., mit langem mittleren Gl., mit nackten Borsten und ohne Aesthetasken, beim $\sigma^{\gamma}$ keine von beiden genikulierend. 2. Antenne 4-gldr., mit umfangreichem 2. Basp. und sehr kurzem 1. Gl. des Enp.; 1. und 2. Gl. des Basp. mit je einer langen, dicken Borste und das Enp. mit dicken, gekrümmten Hakenborsten (der Endhaken beim ơ länger als beim $)_{\text {) }}$; Mandibel auf die Kaulade redu- 
ziert, beilförmig, mit beweglichen Anhängen an der Kaulade. 1. Maxille ein ovales Plättchen. Endgl. der 2-gldr. 2. Maxille in einen starken Haken ausgehend. 2. Gl. des Basp. des 3-gldr. Maxillipeden mit 1 Borste am Innenrande; sein Endhaken beim of länger als beim o. 1.-3. B. mit 3-gldr. Exp. und Enp.; 4. B. mit 3-gldr. Exp. und stummelförmigem, doppelt beborstetem oder auf nur 1 Borste reduziertem Enp. oder ganz ohne Enp. Exp. und Enp. gestreckt, Exp. länger als das Enp. 5. B. jederseits aus 2 Börstchen bestehend.

1. Enp. des 4. B. fehlt; ventraler Längskiel vor dem 1. B. schnabelförmig verlängert; Furka wenig über doppelt so lang wie breit, etwa $1 / 3$ so lang wie das übrige Abdomen

C. rostratus Enp. des 4. B. knopfförmig mit 1 oder 2 Borsten; ventraler Längskiel vor dem 1. B. nicht schnabelförmig verlängert

2. Enip. des 4. B. mit 1 Borste Enp. des 4. B. mit 2 Borsten

3. An der hinteren Ecke jeder Genitalöffnung eine Borste (zwei Eiersäckchen); Furka etwa halb so lang wie der übrige Teil des Abdomens C. venustus Genitalöffnungen ohne Borste (ein Eiersäckchen); Furka etwas länger als der übrige Teil des Abdomens

C. speciosus.

1. Abdomen 1-gldr.

C. rostratus Abdomen 2-gldr.

2. Gensegm. vorne mit ventraler Zacke Gensegm. ohne die Zacke, abgerundet; Furka so lang wie der übrige Teil des Abdomens

C. speciosus

3. Gensegm. etwas länger als Ansegm. und Furka zusammen; Enp. des 4. B. mit 2 Borsten

C. anglicus Gensegm. etwa $3 / 4$ so lang wie Ansegm. und Furka zusammen; Enp. des
4. B. mit 1 Borste
C. venustus.

\section{Corycaeus rostratus Claus.}

1863, C. r., Claus, p. 157 , t. 28 f. 5. 1863, C. parvus, Claus, p. 158. 1892 , C. r., Giesbrecht, p. 660 , t. 5 f. 9 ; t. 49 f. $21,28,32$; t. 51 f. $16-18$, 48,52 .

ㅇ. Vorderkörper 2-, Abdomen 1-gldr.; Bauchkiel schnabelförmig verlängert; Furka wenig über doppelt so lang wie breit, etwa $1 / 3$ so lang wie das übrige Abdomen. Die beiden Borsten des 1. und 2. Gl. des Basp. der 2. Antenne etwa gleich lang, mit Stachelfiedern besetzt; Innenrand des 2. Gl. des Basp. am Ende ohne Zacken, nur mit Spitzen besetzt. Endgl. des Exp. des 1.-3. B. mit nur 1 Außenranddorn, Enddorn länger und (wie auch die Außenranddorne) 
gröber gezähnelt als bei den anderen Arten; Enp. des 4. B. gänzlich geschwunden.

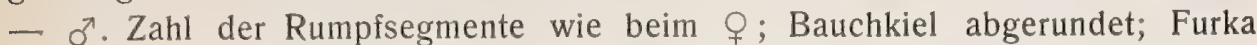
gestreckter als beim ․ - L. q $0,8 \mathrm{~mm}$, o $0,72-0,78 \mathrm{~mm}$.

Mittelmeer, Atlant. Ozean; Ind. Ozean.

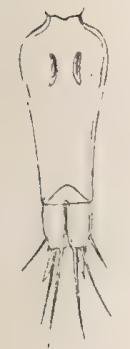

a

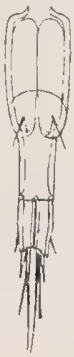

b

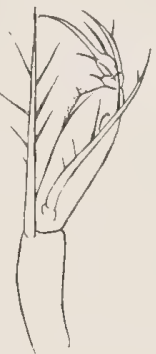

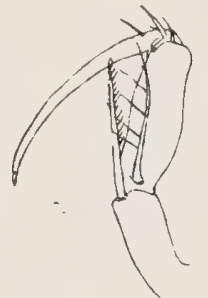

d

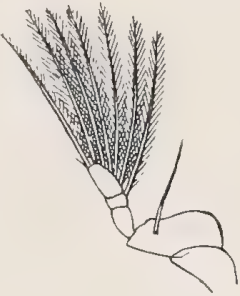

e

Fig. 211. Corycaeus rostratus.

a Abdomen $q$, dorsal, b Abdomen $\sigma^{\pi}$, ventral, c 2. Antenne $q$, d 2. Antenne $\overbrace{}^{\pi}$, d 4. B. $\sigma^{\pi}$. Nach Giesbrecht.

\section{Corycaeus speciosus Dana.}

1852, C. s., Dana, p. 1220 , t. 86 . 1883, C. s., Brady, p. 115 , t. 46 . 1892, C. s., Giesbrecht, p. 660 , t. 51 f. $29,39,40$.

ㅇ. Vorderkörper 4-, Abdomen 2-gldr.; Bauchkiel abgerundet; Furka länger als das übrige Abdomen; Zipfel des 3. Thsegm. so lang wie das Gensegm.

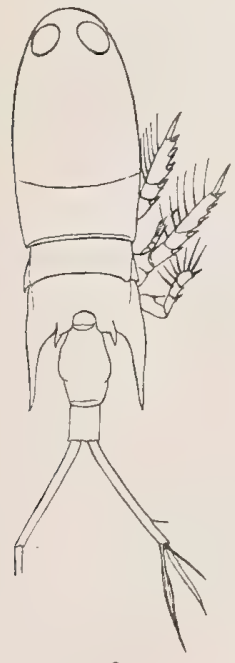

Fig. 212. Corycaeus speciosus. a $q$ dorsal, b or dorsal. Nach Giesbrecht.

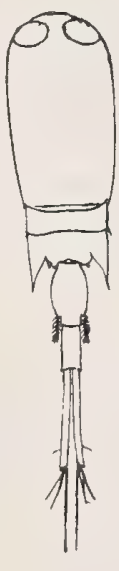

b

Borste des 1. Gl. des Basp. der 2. Antenne wenigstens doppelt so lang wie die des 2. Gl. Endgl. des Exp. des 1.-3. B. mit 3 Außenranddornen; Enp. 
des 4. B. durch eine Fiederborste vertreten. - $0^{7}$. Furka etwa so lang wie das übrige Abdamen. - L. \& 1,9--2,15 mm, o 1,8-1,85 mm.

Atlant. Ozean; Pacif. Ozean; Rotes Meer, Ind. Ozean.

\section{Corycaeus venustus Dana.}

1852 , C. c., Dana, p. 1222 , t. 86 f. 4 a. 1883, C. limbatus, Brady, p. 114 , t. 49 f. $18-22$. Non C. venustus, Brady, 1883 (= C. obtusus Dana). 1892, C. v., Giesbrecht, p. 659 , t. 4 f. 12 ; t. 51 f. $32-34,47$.

૧. Vorderkörper 4-, Abdomen 2-gldr.; Bauchkiel abgerundet; an den Genitalöffnungen eine Borste; Zipfel des 3. Thsegm. mit kurzer Spitze. Furka mehr als 5 mal so lang wie breit, der ganzen Länge nach ungefähr gleich

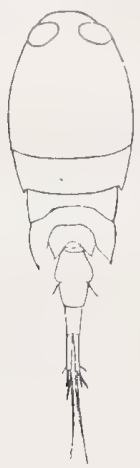

a

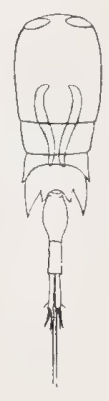

b

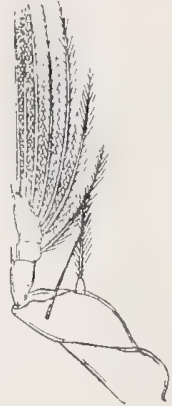

C

Fig. 213. Corycaeus venustus.

a $ᄋ$ dorsal, b ô dorsal, c 4. B. ㅇ. Nach Giesbrecht.

breit; Gensegm. : Ansegm. : Furka $=3: 2: 2$. Borste des 1. Gi. des Basp. der 2. Antenne wenigstens doppelt so lang wie die des 2. Gi. Enp. des 4. B. durch eine Fiederborste vertreten. - $\sigma^{\top}$. Zahl der Rumpfsegmente wie beim $q$; Gensegm. vorne mit medianer ventraler Zacke, etwa $3 / 4$ so lang wie Ansegm. und Furka zusammen. - L. O 0,8-1 mm, or 0,76 mm.

Mittelmeer, Atlant. Ozean; Pacif. Ozean; Ind. Ozean.

\section{Corycaeus anglicus Lubbock.}

1857 , C. a., Lubbock, p. 408 , t. 11 f. $14-17$. 1859, C. germanus, Leuckart, p. 249 , t. 6.1863 , C. germanus, Claus, p. 156 , t. 9 f. $1-4$; t. 24 f. 5 , 6 ; t. 28 f. $1-4$. 1880 , C. a., Brady, III, p. 34 , t. 81 f. $16-19$; t. 83 , f. $11-15$; t. 84 f. $10-14.1898$, C. a., Aurivillius, p. 40, f. 9.1899 , C. a., T. Scott, p. 397 , t. 13 f. $1-14$ (q). 1900, C. a., T. Scott, p. 251 , t. 18 f. 1 (o ).

१. Abdomen 2-gldr., Bauchkiel zugespitzt; an den Genitalöffnungen eine Borste; Zipfel des 3. Thsegm. mit langer Spitze; Furka ungefähr $1 \frac{1}{2}$ mal so 
lang wie das Ansegm. Borste des 1. Gl. des Basp. der 2. Antenne wenigstens doppelt so lang wie die des 2. Gl. Enp. des 4. B. 1-gldr., mit 2 Fiederborsten. - $\sigma^{2}$. Gensegm. vorne mit ventraler Zacke, ca. $1 \frac{1 / 2}{2}$ mal so lang wie das Ansegm. - L. $ᄋ$, o $1-1,1 \mathrm{~mm}$.

Atlant. Küsten von Nordwest-Europa, Nordsee.

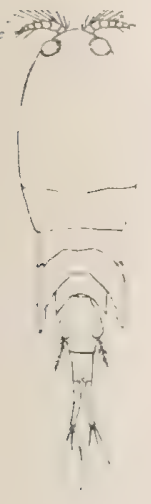

a

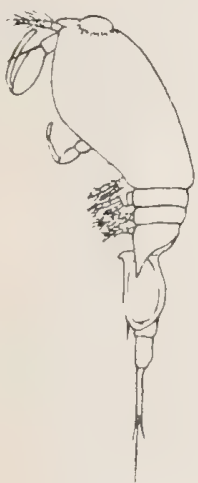

b

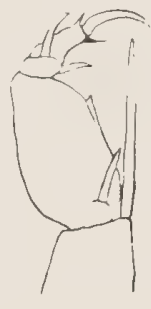

C

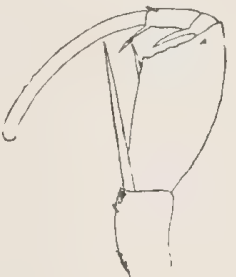

d

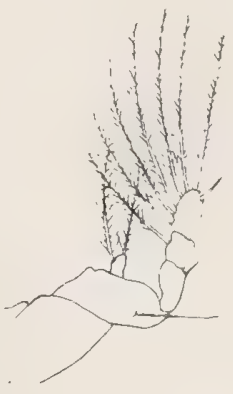

e

Fig. 214. Corycaeus anglicus.

a $q$ dorsal, b $\sigma^{\top}$ lateral, c 2. Antenne $q$, d 2 Antenne $\sigma^{\top}$, e 4. B. $q$. a-c, e nach T. Scott, d nach Aurivillius.

\section{Monstrillidae.}

2. Antennen, Mandibeln, Maxillen und Maxillipeden sowie das Rostrum in beiden Geschlechtern fehlend; beide 1. Antennen des ơ mit Kniegelenk. Das Weibchen trägt die Eier an einem gabeligen, von der Ventralfläche des Genitalsegmentes ausgehenden, borstenartigen Fortsatz, welcher beim of durch einen in 2 Zapfen endigenden Vorsprung ersetzt ist. Abdomen nicht vollzählig gegliedert. - Vom Naupliusstadium an bis zur Zeit der Geschlechtsreife parasitisch. in den Blutbahnen niederer Tiere lebend.

Giesbrecht hat 1892 die damals bekannten Monstrilliden in zwei Gattungen geteilt und für diese die folgenden Diagnosen gegeben:

Thaumaleus Kröyer. Zwischen dem Genitalsegment und der Furka beim o nur 1, beim $\sigma^{x} 2$ Segmente vorhanden; 5. Fußpaar fehlt beim $\sigma^{x}$ (nur von 2 Spezies bekannt) durchaus; Furka jederseits beim $q$ mit 3 , beim $\sigma^{7}$ mit 3 oder 4 Borsten.

१. Vorderkörper 4-gliedrig: Kopf mit 1. Thoraxsegment verschmolzen; Hinterkörper 3-gliedrig. Vordere Antennen 3- bis 4-gliedrig, mit z. T. langen und dicken Borsten, von denen einige verästelt sind. 1.-4. Schwimmfuß mit voluminösem 1. Gl. des Basipoditen und 3-gliedrigen Ästen; 5. Fußpaar rudi- 
mentär. - $\sigma^{\Im}$. Vordere Antennen 5-gliedrig, mit Genikulation zwischen dem 4. und 5. Gliede; die Zipfel am Genitalsegment gestreckt.

Monstrilla Dana. Nahe verwandt mit Thaumaleus, aber: Mund weiter hinten gelegen; zwischen dem Genitalsegment und der Furka sind beim $\sigma^{7}$ 3 Segmente vorhanden, während beim o das erste der 3 auf das Genitalsegment folgenden Segmente nur unvollkommen von diesem getrennt ist und selbst mit ihm verschmelzen kann; das 5. Fuß.paar des $\sigma^{x}$ besteht jederseits aus einer ziemlich langen Borste (oder einem Stummel); die Zipfel am Genitalsegment des $\sigma^{7}$ kurz im Verhältnis zu ihrer gemeinsamen Basis; Furka bei $q$ und $\sigma^{7}$ jederseits mit 5 oder 6 Borsten.

Die Zahl der Spezies hat sich seitdem erheblich vermehrt, wobei aber zu beachten bleibt: erstens, daß nicht in allen Fällen, wo $q$ und $\sigma^{\gamma}$ als zu einer Spezies gehörig aufgefaßt wurden, es sicher feststeht, daß dem auch wirklich so sei, zweitens, daß in manchen Fällen nur eins der Geschlechter erbeutet wurde und drittens, daß in der Beschreibung der Erwähnung derjenigen Merkmale, die Giesbrecht für die Unterscheidung der beiden Genera in den Vordergrund stellte, oft ungenügend Rechnung getragen wurde. Weil man früher die Reichhaltigkeit an Arten dieser Familie nicht ahnte und das Bestreben bestand, neues Material möglichst viel den wenigen, z. T. nur mangelhaft bekannten Spezies einzuverleiben, hat man sich bei den Bestimmungen oft geirrt.

Giesbrecht selber hat später eine Art beschrieben, welche das vermittelnde Glied zwischen beiden Genera darstellt (Monstrilla conjunctiva, 1902).

Malaquin, dessen schöne Arbeit über die Entwicklung der Monstrilliden (1901) weiter unten nochmals Erwähnung finden wird, hat die Zahl der Genera um eins vermehrt und der neu von ihm geschaffenen Gattung den Namen Haemocera beigelegt. Die Diagnose lautet wie folgt:

Haemocera Malaquin. Zwischen. dem Genitalsegment und der Furka beim $q 2$, beim ơ 3 Segmente vorhanden. 5. Fußpaar fehlt beim ơ durchaus. Furka jederseits beim $q$ mit 3 oder 4 , beim $\sigma^{7}$ mit 4 Borsten. 1. Antennen des $q$ 4-, des $\sigma^{7}$ 5-gliedrig. Die Zipfel am Genitalsegment des $\sigma^{7}$ sehr entwickelt.

T. Scott hat 1904 eine Mitteilung über die an der schottischen Küste gefundenen Monstrilliden veröffentlicht. Als Genera werden darin nur Monstrilla und Thaumaleus aufgeführt. ${ }^{1}$ )

Es scheint zurzeit wohl unmöglich, die jetzt bekannten Arten der Monstrilliden in scharf gesonderte Genera zu gruppieren, weshalb ich es vorgezogen habe, die Arten unter dem Namen zu erwähnen, mit welchem sie von den verschiedenen Autoren belegt worden sind. Einer bequemen Übersicht halber sind hier nach der Zahl der Furkalborsten zwei Gruppen unterschieden worden.

1) Die (russisch geschriebenen) Arbeiten von Kriczagin (Copepoda maris nigri nova. Mitt. Nat. Ver. Kiew, 1877) und von Karawaiew (Matériaux pour la faune des Copépodes de la mer noire. Idem 1894) habe ich nicht gesehen. 
Entwicklung der Monstrilliden. Nach Malaquin (1901), der speziell die Entwicklung einer Spezies, $\mathrm{H}$ a e mocera danae, genauer studiert hat, gestaltet sich dieselbe folgendermaßen.

Die Eier werden in einem Eiersack von den frei und pelagisch schwimmenden Weibchen getragen. Der Anfang der Entwicklung findet im Eiersack statt. Die holoblastische Eifurchung endet mit der Bildung eines Nauplius, der sich durch die Hakenform des 3. Gliedmaßenpaares auszeichnet. Nach dem Ausschlüpfen heften sich die Nauplien mit Hilfe der scharfen Mandibelhaken dem Integument des Wirtes an und dringen unter Rücklassung der Cuticula und der Anhänge ins Körperinnere des Wirtes vor, wo sie in der Gestalt einer rundlichen Masse von nur wenig differenzierten Zellen sich einen Weg zu einem der größeren Blutgefäße bahnen. Dort angelangt, umgibt sich der Nauplius mit einer Hülle (Cuticula) und es sprießen am unteren Vorderende zwei lange, ungegliederte Schläuche, die 2. Antennen, hervor, die die Aufnahme der Nahrungsstoffe aus der Blutbahn zu versorgen haben. Der Nauplius durchläuft dann innerhalb der cuticularen Hülle die verschiedenen Stadien der Entwicklung. Nachdem diese vollendet ist, bringt der Parasit durch kräftige Bewegungen seines Körpers, dessen chitinöse Hülle beiderseits spitz ausläuft und außerdem mit Dornen bewehrt ist, die Tegumente des Wirtes und schließlich auch die Hülle selbst zum Platzen. Die embryonalen Tentakeln fallen ab und ihre Anheftungsstellen sind jetzt nur durch eine Narbe gekennzeichnet. Beim Anfang des pelagischen Lebens findet eine Häutung statt, wodurch erst die Borsten der Antennen, der Beine und der Furka zur völligen Entfaltung geraten. Das Äußere der borstentragenden Anhänge wechselt also mit dieser Häutung.

Malaquin weist ferner auf die Schwierigkeiten hin, die mit der Bestimmung der Zugehörigkeit von Weibchen und Männchen einer Art verbunden sind und meint, daß eine Revision der Monstrilliden nur an der Hand eines Studiums des parasitären Aufenthaltes der verschiedenen Arten und unter Zugrundelegung der Beschreibung des or und $q$ einer eine bestimmte Annelidenspezies inficierenden Monstrillidenart sicheren Aufschluß geben kann.

Die Wirte, welche bisher als solche für Monstrilliden erkannt worden sind und sämtlich den Anneliden angehören, sind nach der Angabe Malaquins folgende:

Parasit

Haemocera danae

" filogranarum

" roscovita

Thaumaleus germanicus
Wirt

Salmacyna dysteri

Filograna implexaa

"setosa

Polydora ciliata

"giardi.

$\left.q^{1}\right)$

1. 5. B. 2-lappig mit breitem, beborstetem Innnenlobus

1) Für die Bestimmung des $\sigma^{x}$ vergleiche man die Beschreibungen und Figuren. 
Ein mit einer Borste versehener Höcker am Innenrande des 5. B.

Monstrilla longicornis

(Siehe auch $M$. intermedia Aur.)

5. B. mit unbeborstetem Zipfel oder Höcker am Innenrande oder daselbst ohne Vorsprung

2. Furka mit 6 Borsten

" 3 " 3

Monstrilla grandis

3. Furka mit 6 Borsten (die dorsale Borste meistens kurz)

Thaumaleus rostratus

$$
\text { " "3 oder } 4 \text { Borsten }
$$

4. 5. B. ohne Zipfel am Innenrande, mit 3 Endborsten Monstrilla gracilicauda

5. B. mit 2 Endborsten

5. 5. B. schmal, leicht gekniet; die mittlere der 5 Randborsten der Furka ist etwas kürzer als die etwa gleich langen 2 äußeren und 2 inneren

Monstrilla helgolandica

5. B. breiter und kürzer; von den 5. Randborsten der Furka ist die zweitäußere die kürzeste

Monstrilla anglica (?)

6. Furka mit 3 langen Randborsten

" " 4 . , "

7. 5. B. mit sehr kleinem Innenrandhöcker; Gensegm. schmal

Haemocera filogranarum

5. B. mit ziemlich großem Innenzipfel; Gensegm. breit Monstrilla dubia

8. Zinken der Eigabel am Grunde verschmolzen Thaumaleus longispinosus " " $\quad$ von der Basis an gesondert

9. Innenlobus des 5. B. dem Außenlobus an Länge oder Größe nahezu gleich 10 Innenlobus deutlich, aber klein oder verkümmert und durch einen Höcker vertreten

10. 5. B. kurz und breit mit breit gerundetem Innenlobus

Thaumaleus zetlandicus

5. B. schmäler mit länglich eiförmigem Innenlobus Thaumaleus germanicus

11. Gensegm. etwas kürzer als die beiden folgenden Segmente zusammen; Furka viel kürzer als das große Ansegm.

Haemocera roscovita Gensegm. länger als das. übrige Abdomen (ohne die Furka; wenn das Abdomen 3-gldr. ist, die Furka nicht kürzer als das Ansegm.) 12

12. 5. B. mit ungleich langen Borsten

5. B. mit fast gleich langen Borsten

Thaumaleus rigidus

13. Abdomen 2-gldr.

Thaumaleus thompsoni "3-gldr.

Haemocera danae

A. Furka beim $\bigcirc$ jederseits mit 5 oder 6 Borsten; im letzteren Falle eine derselben (die dorsale) meistens sehr kurz.

\section{Monstrilla Iongicornis J. C. Thompson.}

1890, M. 1., Thompson, p. 119, t. 4 f. 1, 2, 4. (ठ). 1892, M. Iongiremis, Giesbrecht, p. 586, t. 46 f. $10,14,22,37,41$. (q). 1902, M. Iongirem is, 
T. Scott, p. 469, t. 25 f. 3,4 (Q). 1904, M. 1., T. Scott, p. 244, t. 13 f. $1-7\left(0^{7}, \bigcirc\right)$.

ๆ. Integument des Körpers fein granuliert. 1. Antenne ungefähr so lang wie das 1. Körpersegm.; die Abstände des Mundes vom Vorder- und Hinterrand des 1 . Körpersegm. (Kopf +1 . Thsegm.) verhalten sich wie 19:16, die

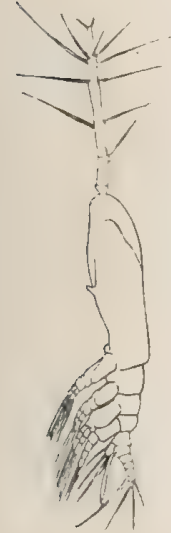

a

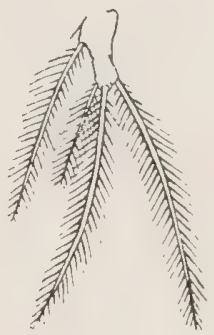

b

Fig. 215. Monstrilla longicornis.

a 우 lateral, b 5. B. 우, c ơ dorsal, d Abdomen $\sigma^{\top}$, ventral.

a-b nach Giesbrecht, c-d nach T. Scott.

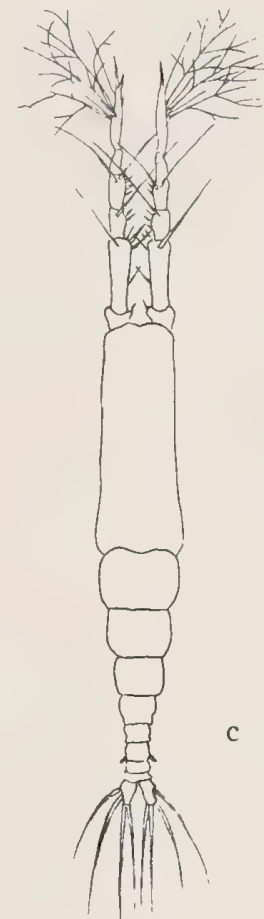

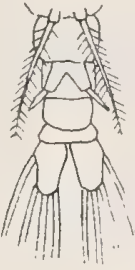

d

Länge des 1. Segm. zu dem 2.-4. Thsegm. zusammen wie 16:13. Abdomen 3-gldr., aber das Gensegm. auf der Dorsalfläche mit deutlicher Segmentation; Furka mit 5 langen Fiederborsten. 1. Antenne sehr undeutlich gegliedert. 2. Gl. des Basp. der B. mit schmaler, abgerundeter Zacke am Innenrande. 5. B. gestreckt, mit einem in der Mitte des Innenrandes vorspringenden und mit 1 längeren Borste bewehrten Höcker; 3 Endborsten, von denen die innere viel kürzer als die beiden andern ist. - $\sigma^{\top}$. 1. Antenne so lang wie das 1 . Rumpfsegm., 6-gldr., das 2. und 6. Gl. etwa gleich lang und viel länger als eines der anderen Gl. Anhänge des Gensegm. schmal und dornförmig; Furka mit 4 langen Fiederborsten. 5. B. eine Fiederborste, die bis zur Furka reicht. L. ᄋ $3-3,1 \mathrm{~mm}$, o $2 \mathrm{~mm}$.

Mittelmeer, britische Inseln.

2. Monstrilla intermedia Aurivillius.

1898, M. i., Aurivillius, p. 40, f. 8 . Non M. intermedia Kriczagin! Verwandt mit M. longicornis: - . 1. Gl. des Exp. des 3. B. mit 1 Innen- 
randborste (diese soll bei $M$. longicornis (fide Auriv.!) fehlen); Außenranddorn des 1. Gl. des Exp. des 3. B. kaum mehr als halb so lang wie der des 3. Gl. (bei M. longicornis sind beide gleich lang). L. $4 \mathrm{~mm}$. - $\sigma^{\text {t }}$ unbekannt.

Skagerak.

Bemerkung. Falls diese Art von M. Iongicornis Thompson (- longiremis Giesbr.) wirklich spezifisch verschieden ist, sind die Unterschiede, wie sie aus der kurzen Beschreibung bei Aurivillius zu entnehmen sind, doch sehr geringfügig. Die Antenne soll relativ kürzer sein, aber nach Scott ist die Antennenlänge bei M. 1ongicornis in gewissem Grade variabel. Giesbrecht erwähnt das Fehlen der Innenrandborste am 1. Gl. des Exp. des 3. B. in der Beschreibung nicht und gibt an: die Zahl der Borsten sei dieselbe wie bei Thaumaleus, wo eine Innenrandborste daselbst vorhanden ist. Die Abbildung des 3. B. bei Giesbrecht (t. 46 f. 37) ist in dieser Hinsicht einigermaßen zweideutig und Aurivillius hat die Befiederung wie oben angegeben aufgefaßt. Der Bau und die Befiederung des 5. B., sowie die Zahl der Furkalborsten stimmen mit M. longicornis genau überein.

Fig. 216. Monstrilla intermedia. 5. B. Q. Nach Aurivillius.

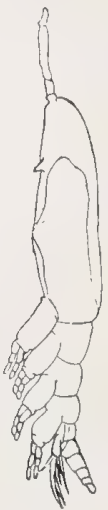

a

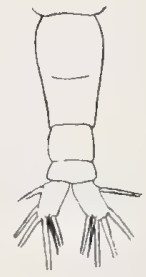

b

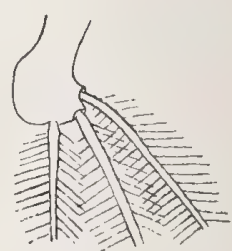

C
Fig. 217. Monstrilla gracilicauda. a $q$ lateral, b Abdomen $q$, dorsal, c 5. B. 우. Nach Giesbrecht.

\section{Monstrilla gracilicauda Giesbrecht.}

1892, M. g., Giesbrecht, p. 586, t. 46 f. 9, 16, 18, 29, 32, 43. (q). 1904, M. g., T. Scott, p. 245 , t. 13 f. $8-10$; t. 14 f. 15 . (q).

‥ Vorderkörper etwa $4 \frac{1}{2}$ mal so lang wie der Hinterkörper. Die Antennenlänge verhält sich $z u$ den Abständen des Mundes vom Vorder- und Hinterrand des 1. Körpersegm. (Kopf +1 . Thsegm.) wie $22: 16: 34$, die Länge des 1. Körpersegm. zu der gesamten Länge des 2.-4. Thsegm. wie 34:23. 1. Antenne undeutlich 4-gldr. Abdomen 3-gldr., mit sehr schwacher Andeutung einer dorsalen Segmentation des Gensegm.; dieses gestreckt und fast 2 mal so lang wie das 5. Thsegm.; Furka mit 6 Borsten, 4 fast gleich langen Endborsten, 1 langen Außenrandborste und 1 dorsalgestellten Borste, die so lang 
wie die Furka ist. 5. B. kurz, nach vorne verbreitert, mit 3 Randborsten. L. 2,7-3,1 mm. - o unbekannt.

Mittelmeer, britische Inseln.

\section{Monstrilla helgolandica Claus.}

1863, M. h., Claus, p. 165, t. 12 f. 15 ; t. 13 f. 9. (क). 1896 a, M. h., Timm, p. 376 , t. 5 f. 1,8 ; t. 6 f. $2,4,6,8$. ().

Q. Die Antennenlänge verhält sich zu den Abständen des Mundes vom Vorder- und Hinterrand des 1. Körpersegm. (Kopf +1 . Thsegm.) wie 30:37:38. Abdomen 3-gldr.; die Länge des 5. Thsegm., der 3 Abdsegm. und der Furka verhält sich wie $10: 15: 6: 5: 6$; Furkaläste mit 6 Borsten; von den 5 Rand-

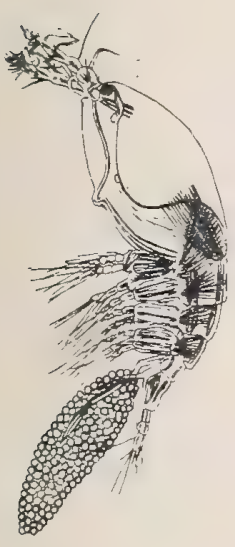

a
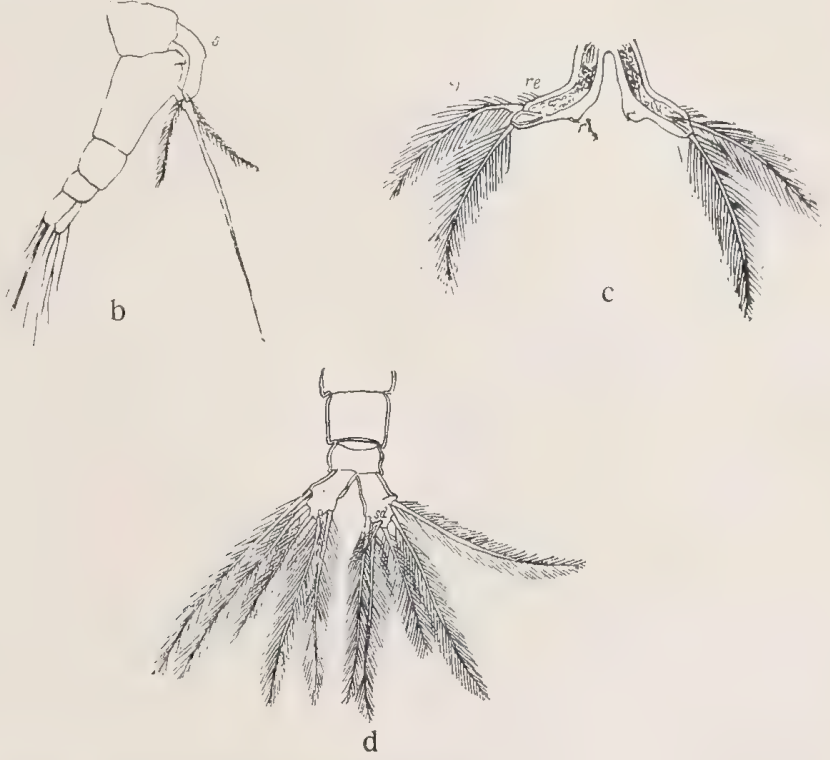

d

Fig. 218. Monstrilla helgolandica.

a $q$ lateral, b 5. Thsegm. und Abdomen $q$, lateral, c 5. B. $q$, d Furka $q$, dorsal. Nach Timm.

borsten sind die 2 äußeren und die 2 inneren gleich lang, die mittlere ist etwas kürzer; die dorsale Borste so lang wie die Furka. 2. Gl. des Basp. der B. ohne Zacke am Innenrande. 5. B. schmal, leicht gekniet, mit 2 Endborsten und einem kleinen Vorsprung an dem ausspringenden Winkel des Knies. L. 1,4 mm. - o unbekannt.

Nordsee, Skagerak, Engl. Kanal.

\section{Monstrilla grandis Giesbrecht.}

1891, M. g., Giesbrecht, p. 476. 1892, M. g., Giesbrecht, p. 586, t. 46 
f. $2,8,11,17,19,24,25,35,39(q$, б) $) 1896$ a, M. g., Timm, p. 378 , t. 5 f. 4. (q).

†. Vorderkörper etwa $31 / 2$ mal so lang wie der Hinterkörper. Der Mund liegt in der Mitte des 1. Segm., welches $1 \frac{1}{2}$ mal so lang wie 2.-4. Thsegm. ist; die Länge der 1. Antennen verhält sich zu der des 1. Körpersegm. wie 10:13. Abdomen 4-gldr. (die beiden Teile des Gensegm. sind noch etwas schärfer als bei longiremis gesondert). Eigabel ungefähr $2 \frac{1}{2}$ mal so lang wie das Abdomen; Furka mit 6 langen Borsten; die dorsale Borste wenigstens so lang wie das Abdomen. 2. Gl. des Basp. der B. ohne Zacke am Innenrande. 5. B. gestreckt, 2-zipfelig; Außenlobus mit 3 langen Borsten, Innenlobus mit 1 äußeren, kurzen und 1 inneren, langen Borste, beide in gleicher Höhe stehend. -

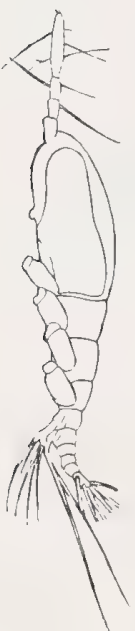

a

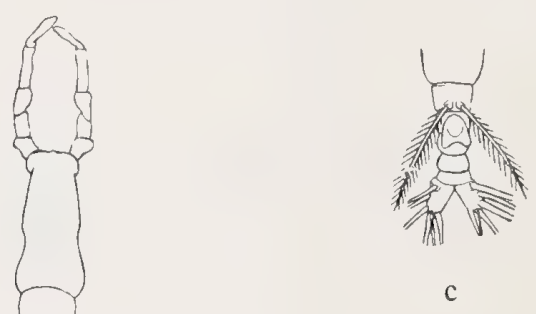

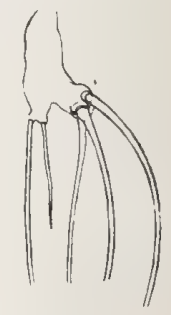

d

Fig. 219. Monstrilla grandis.

a \& lateral, b o dorsal, c Abdomen $\sigma^{\pi}$, ventral, d 5. B. ㅇ․

Nach Giesbrecht.

$\sigma$. Vorderkörper noch nicht 3 mal so lang wie der Hinterkörper. Mund etwas vorider Mitte des 1. Segm. gelegen. 1. Segm. $1^{1 / 3}$ mal so lang wie 2. -4 . Thsegm. 1. Antenne so lang wie das 1. Segm.; die ersten $3 \mathrm{Gl}$. nahezu gleich lang. Auswuchs des Gensegm. in 2 seitlich gekrümmte, kurze Zipfel endigend; Furka mit 6 langen Borsten. 5. B. eine Fiederborste, die das Ende der Furka nahezu erreicht. - L. 2,3-3,75 mm, or 1,7-1,9 mm.

Atlant. Ozean, Nordsee.

Bemerkung: Nach Timm's Angabe (I. c.) hatte das bei M. grandis so charakteristisch ausgebildete 5. B. an seinen beiden weiblichen Stücken genau die Form der Giesbrechtschen Abbildung und wie die Habitusfigur erkennen läßt, stimmt ebenso die relative Länge der Antennen und wahrscheinlich auch die Beborstung der Furka, mit der Beschreibung von Giesbrecht überein.

Die Beschreibung und Figuren von Monstrilla (?) grandis von Brady (1902, t. 4 f. 1-3) habe ich nicht gesehen.

Die von T. Scott (1904, Monstrilla grandis, p. 243 , t. 13 f. 11,$12 ;$ t. 14 f. 9, 11; t. 15 f. 1, 2) an der schottischen Westküste gefundenen Stücke, $1 \sigma^{\top}$ und 2 \%, scheinen 
mir ziemlich stark von der Giesbrechtschen Form abzuweichen, wie aus den folgenden Angaben ersichtlich ist.

1. Antenne des $q^{1 / 4}$ so lang wie der Körper (und kaum halb so lang wie das 1. Körpersegm.); dorsale Borste der Furka bei $q$ und $\sigma^{r}$ sehr kurz (kaum länger als die Furka). 5. B. beim $q$ breiter, Außenlobus mit 3 Borsten, von denen die innere viel

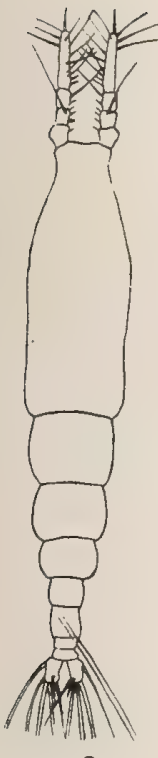

a

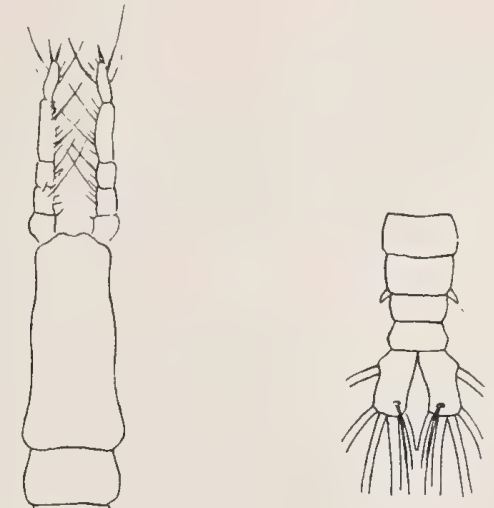

C

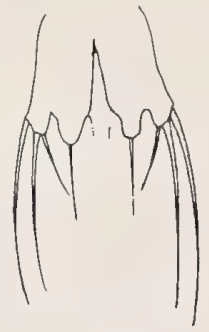

d

Fig. 220. Monstrilla grandis (?)

a 우 dorsal, b ơ dorsal, c Abdomen ơ, dorsal, d 5. B. .

Nach T. Scott.

kürzer als die zwei äußeren ist; Innenlobus mit 1 Borste am Ende, welche so lang wie die innere Borste des Außenlobus ist. Außerdem zeichnet Scott als fraglich vorkommend noch eine zweite, weit proximal am Innenrande dieses Zipfels eingepflanzte Borste. Beobachtet nämlich hat er dieselbe nicht ${ }_{2}$ sondern nur das, was die Basis einer Borste $z u$ sein scheint. L. $\$ 4,25 \mathrm{~mm}, \sigma^{\nearrow} 2 \mathrm{~mm}$. Man vergleiche übrigens die Figuren 219 und 220.

\section{Monstrilla anglica Lubbock.}

1857, M. a., Lubbock, p. 409, t. 10 f. $7,8\left(\sigma^{7}\right)$. 1890, M. a., Bourne, p. 576, t. $37\left(\sigma^{7}\right)$. Non Monstrilla anglica Scott 1904 (Siehe Anm.).

7. Furka mit 6 Borsten, von denen die dorsale etwa halb so lang wie die 5 ungefälır gleich langen Randborsten ist. 5. B. durch einen unbeborsteten Stummel vertreten. L. 1,3 mm (Lubbock). - o unbekannt (Siehe Anm.).

Britische Inseln.

Anmerkung. $\mathrm{Ob}$ die von Bourne gefundenen männlichen Tiere, welche er mit der Art, von der Lubbock auch nur das Männchen untersuchen konnte, identifizieren zu können glaubte, vielleịcht zu einer Art gehörten, von der an andrer Stelle und unter einem andern Namen auch das Weibchen beschrieben ist, läßt sich nicht entscheiden.

Nord. Plankton.

VIII 14 


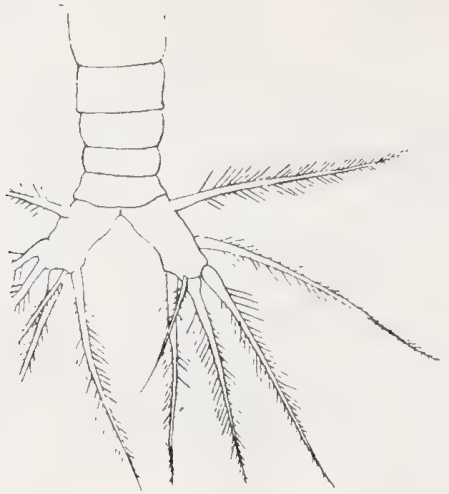

a

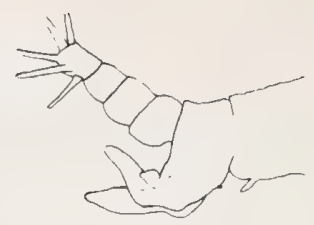

b

Fig. 221. Monotrilla anglica.

a Abdomen ơ, dorsal, b id., lateral. Nach Bourne.

Als Monsitrilla anglica hat Scott das Weibchen einer an der schottischen Küste gefundenen Art beschrieben und abgebildet (1904, p. 246, t. 13 f. 13; t. 14 f. 12-14). Als Synonym führt der Autor selbst die früher von ihm als Monstrilla (?) danae beschriebenen Individuen an (1900, p. 398, t. 13 f. 15-20). 1904 lagen nur Weibchen vor, 1900 sowohl Weibchen wie Männchen; das Vorkommen in dem gleichen Fange und in noch höherem Grade die eigentümliche Beborstung der Furka, die bei $\sigma^{\nearrow}$ und 의 gleich ist, weisen auf die Zugehörigkeit der beiden Geschlechter zu einer und derselben Art hin. Die Männchen weichen aber von Monstrilla anglica or, wie Bourne dasselbe abgebildet,

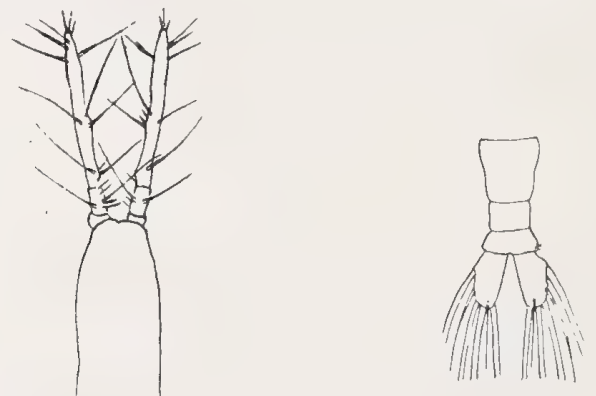

b

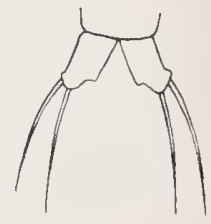

c

Fig. 222. Monstrilla anglica (?)

a $q$ dorsal, b Abdomen $q$, dorsal, c 5. B. $q$. Nach T. Scott.

bedeutend ab. Bei der Scottschen Art ist in beiden Geschlechtern die zweitäußere der 5 Randborsten der Furka auffallend kurz, während Bourne (ebenso wie Lubbock) die Randborsten ungefähr gleich lang abbildet. Weiter zeigt die Figur bei Scott die Bewehrung des 5 . Thsegm. mit 2 langen Borsten, während Bourne dàs 5 . B. als stummelförmig und unbeborstet abbildet. Die Scottsche Art scheint mir daher weder mit Monstrilla anglica noch mit einer der anderen hier beschriebenen Arten identisch zu sein und Anspruch auf einen eigenen Speziesnamen erheben zu können. 
B. Furka mit 3 oder 4 Borsten (die Zahl bei of und $q$ nicht immer gleich).

\section{Thaumaleus longispinosus Bourne.}

1890, Monstrilla longispinosa, Bourne, p. 575 , t. 37 f. $1-4,10$ (q). 1892, T. 1., Giesbrecht, p. 578 , t. 5 f. 10 ; t. 46 f. 1, 4, 6, 12, 13, 23, 30, 38, $42\left(\%, \sigma^{7}\right)$.

†. Vorderkörper ca. $5 \frac{1}{2}$ mal so lang wie der Hinterkörper; Rumpf ungefähr zylindrisch; seine größte Dicke, etwa in der Mitte des 1. Segmentes (Kopf + 1. Thsegm.) gelegen, beträgt etwa $1 / 7$ der Rumpflänge; Kopf +1 . Thsegm. etwa $3 \mathrm{mal}$ so lang wie die 3 folgenden Thsegm. zusammen; Vorderkopf vorne
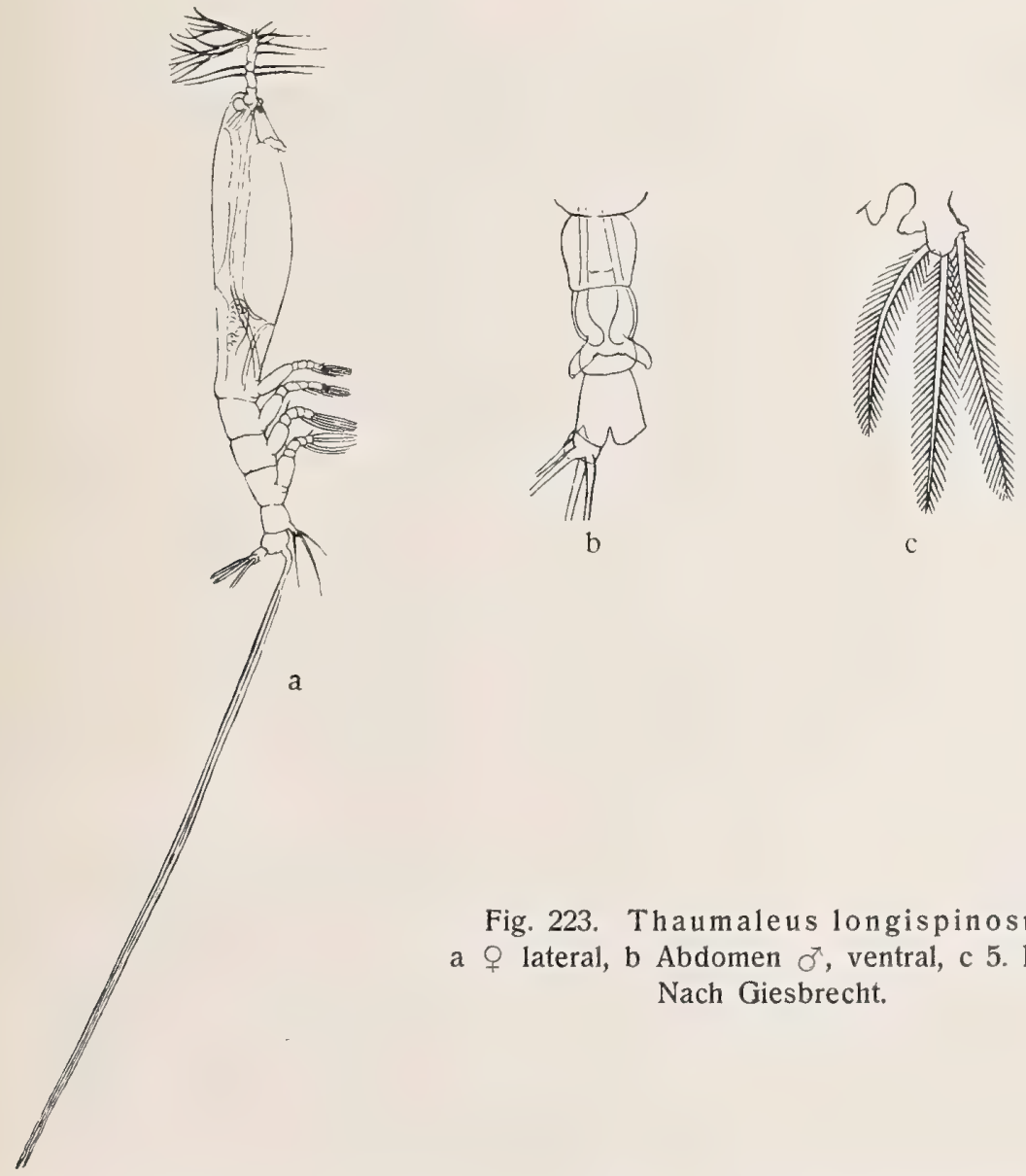

Fig. 223. Thaumaleus longispinosus. a ${ }^{\circ}$ Iateral, b Abdomen $\sigma^{\top}$, ventral, c 5. B. 오. Nach Giesbrecht.

abgerundet, zu beiden Seiten der vorderen Antennen etwas vorspringend. Es verhält sich die Antennenlänge zu den Abständen vom Mund bis zum Vorderund Hinterrand des 1. Segmentes wie $1: 1: 41 / 2$. Abdomen nur aus Gensegm. und Ansegm. bestehend; Eigabel doppelt so lang wie der Rumpf, ihre Zinken am Grunde verschmolzen; Furka jederseits mit 3 dicken Fiederborsten, die 
nicht $1 \frac{1}{2}$ mal so lang wie der Hinterkörper sind. 1. Antennen 4-gldr, sehr gedrungen, das 2. Gl. nicht viel länger als das 3. 5. B. mit zipfelförmigem, innerem Anhang, seine 3 Endborsten ungefähr gleich dick und lang. - ot. Vorderkörper ca. $3 \mathrm{mal}$ so lang wie der Hinterkörper; Kopf +1 . Thsegm. nur etwa $10 / 7 \mathrm{mal}$ so lang wie das 2.-4. Thsegm. zusammen; Entfernung vom Munde bis zum Vorderand des 1 . Segmentes fast halb so groß wie die Entfernung vom Munde bis zum Hinterrand. Zwischen Gensegm. und Ansegm. 1 freies Abdsegm., das kürzer als beide ist. Furka jederseits mit 4 dicken Fiederborsten, die länger als der ganze Hinterkörper sind. 5. B. völlig fehlend. L. क 2,6-3,1 mm, o $1,8 \mathrm{~mm}$.

Mittelmeer; Engl. Kanal.

\section{Thaumaleus thompsoni Giesbrecht.}

1892, T. thompsonii, Giesbrecht, p. 578, t. 46 f. 7, 27, 31, 36, 40 (q, o). ? 1902, T. t., T. Scott, p. 470 , t. 25 f. $5,6$.

․ Hinterkörper $2 / 9$ so lang wie der Vorderkörper; es verhält sich die Antennenlänge: Entfernung von Stirnrand bis Mundöffnung: Entfernung von Mundöffnung bis Hinterrand des 1. Körpersegm. wie $6: 4: 11$; Eigabel nicht halb so lang wie der Rumpf, ihre Zinken gesondert entspringend; Furkaläste mit 3 Borsten. 1. Antenne gestreckt, das 2. Gl. beträchtlich länger als das 3 .

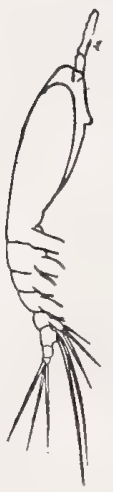

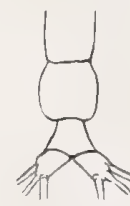

b

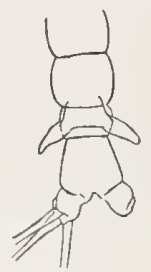

d

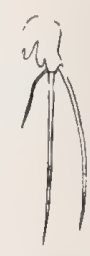

c

Fig. 224. Thaumaleus thompsoni.

a $q$ lateral, b Abdomen $q$, dorsal, c. 5. B. $q$, d. Abdomen ơ, ventral. Nach Giesbrecht.

5. B. mit zipfelförmigem, borstenlosem innerem Anhang, der halb so lang wie der Außenzipfel ist; 3 Endborsten, von denen die innere viel dünner und kürzer als die beiden andern ist. - $\sigma^{7}$. Hinterkörper $2 / 5$ so lang wie der Vorderkörper; das oben angegebene Verhältnis wie $4: 2: 5$. 5. B. völlig fehlend. Furkaläste mit 3 Borsten. - L. $q 0,8-1 \mathrm{~mm}$, o $0,65-0,8 \mathrm{~mm}$.

Küsten von Nordwest-Europa, vom Kanal bis zur westlichen Ostsee und den Shetlandsinseln (?).

Bemerkung. Die von Giesbrecht unter obigem Namen beschriebenen Exemplare aus einem bei Langeland (Ostsee) gemachten Fang sind von Möbius (1887) als Monstrilla danae Clap. aufgeführt worden. 
Ob die von T. Scott (1904, p. 248, t. 14 f. 1-4) als Thaumaleus thompsoni erwåhnte Spezies mit der Giesbrecht'schen Art völlig identisch ist, erscheint mir zweifel-
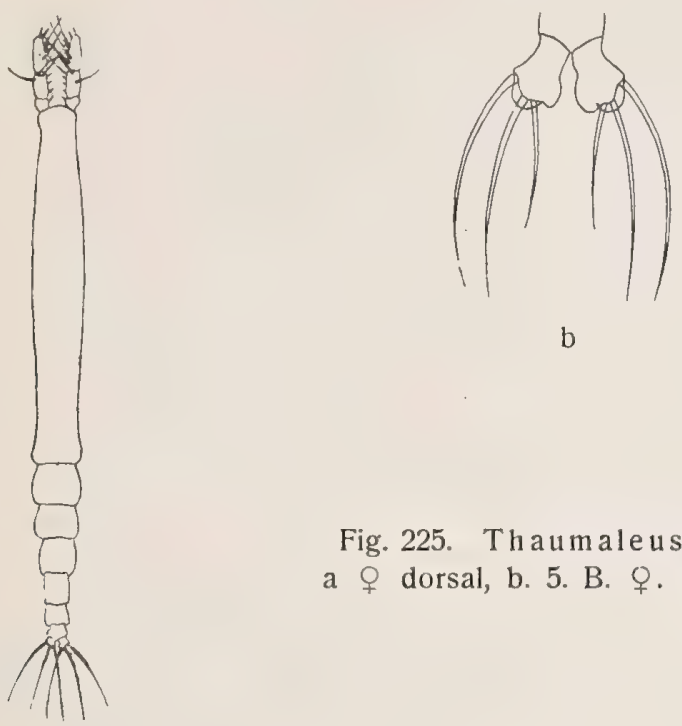

b

Fig. 225. Thaumaleus thompsoni.

a $q$ dorsal, b. 5. B. $q$. Nach T. Scott.

haft.: In einigen Merkmalen, z. B. der relativen Länge der 1 . Antennen (hier $1 / 5$ der Länge des 1. Körpersegm.) und vor allem der Körpergröße weichen beide merklich von einander $\mathrm{ab}$; die Länge des $q$ der Scottschen Art beträgt 4,8 $\mathrm{mm}$ !

\section{Thaumaleus rigidus Thompson.}

1888, Cymbasoma rigidum, Thompson, p. 154 , t. 13 f. $1-4.1892$, T. claparedii, Giesbrecht, p. 381 , t. 46 f. 5, 15, 21, 26. 1904, T. r., T. Scott, p. 248 , t. 13 f. $15-17$; t. 14 f. 19 .

․ Körpergestalt ungefähr wie bei T. longispinosus; es verhält sich die Antennenlänge zu den Abständen des Mundes vom Vorder- und Hinterrand des 1. Segm. wie 2:1:6. Eigabel noch nicht halb so lang wie der Rumpf, ihre Zinken entspringen gesondert; Furkaläste mit 3 Borsten, die doppelt so lang als der Hinterkörper sind. 1. Antennen ziemlich gestreckt, das 2. G1. beträchtlich länger als das 3. 5. B. ohne Zipfel am Innenrande, am Ende mit 3 Borsten von gleicher Länge und Dicke. L. 2,2-2,7 mm. - o unbekannt.

Mittelmeer, Atlant. Ozean, Nordsee.

\section{Thaumaleus germanicus Timm.}

1896 a, T. g., Timm, p. 373 , t. 5 f. $2,3,6,7$; t. 6 f. $1,3,5,7,9-11$. 1901, T. g., Nordgaard, p. 20, t. 1 f. 1, 2. 


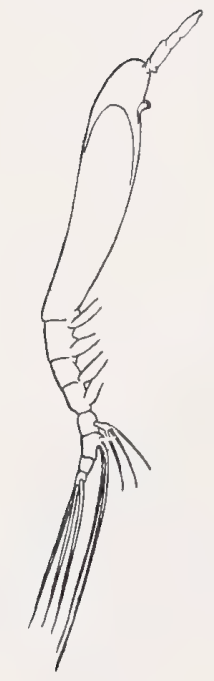

a

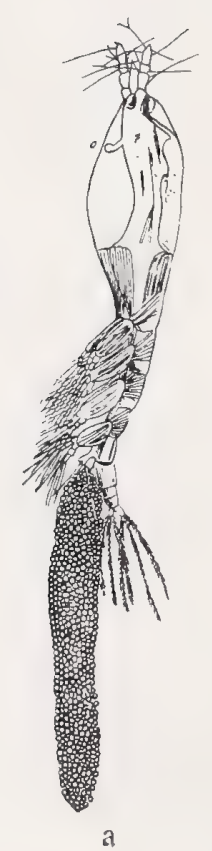

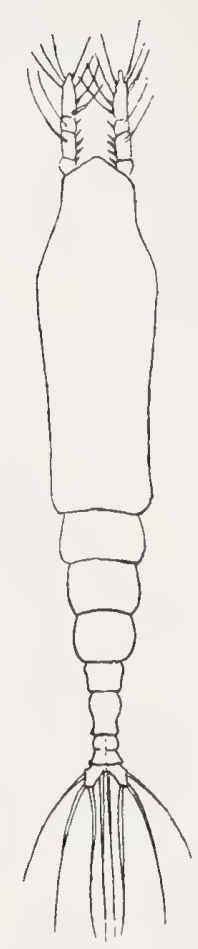

b

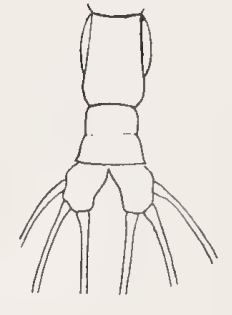

C

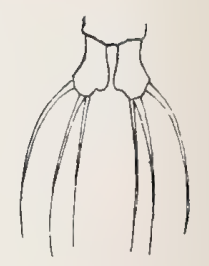

d
Fig. 226. Thaumaleus rigidus.

a \& lateral, b o dorsal, c Abdomen of, dorsal, d. 5. B. 오.

a nach Giesbrecht, $b-d$ nach Scott.

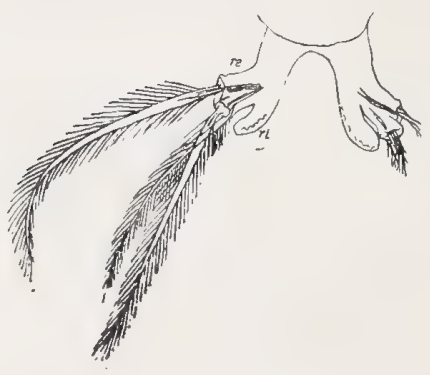

b

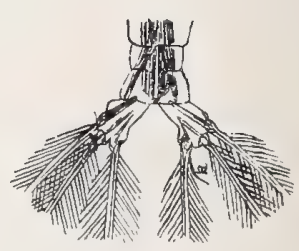

c

Fig. 227. Thaumaleus germanicus.

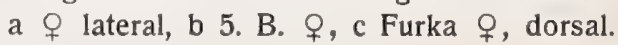

Nach Timm. 
ㅇ. Vorderkörper ca. $4 \frac{1}{2}$ mal so lang wie der Hinterkörper; größte Breite des 1. Segm. 1/5 von der Länge des Rumpfes; Kopf +1 . Thsegm. etwa 2 mal so lang wie die 3 folgenden Thsegm. zusammen. Es verhält sich die Antennenlänge zu den Abständen vom Mund bis zum Stirnrand und Hinterrand des 1. Segm. wie $1: 1: 2^{1 / 2}$. Abdomen nur aus Gensegm. und Ansegm. bestehend, letzteres oft durch eine feine Einschnürung in 2 Teile geteilt; Gensegm. doppelt so lang wie das vorhergehende und das Ansegm. Eigabel von Grund an zweiteilig, halb so lang wie der Rumpf. Furkaläste mit 3 langen Fiederborsten; über der Innenborste ein an Länge die Furka nicht übertreffendes, fiederloses Börstchen. 5. B. mit 2 Loben; der innere länglich eiförmig, den äußern ein klein wenig überragend, ohne Borsten; Außenlobus mit 3 Borsten. L. 2,6-3 mm. - $\sigma^{7}$ unbekannt.

Nordsee, norwegische Westküste, Engl. Kanal. Lebt in Polydora ciliata und Polydora giardi.

\section{Thaumaleus zetlandicus T. Scott.}

1904 , Th. z., T. Scott, p. 249 , t. 13 f. 18,19 ; t. 14 f. $20-22$; t. 15 f. $3,4$.

․ Körper ziemlich stark verlängert; 1. Körpersegm. etwa $1 \frac{1}{2}$ mal so lang wie die übrigen Thorax- und Abdseg. zusammen. Abdomen 3-gldr.;

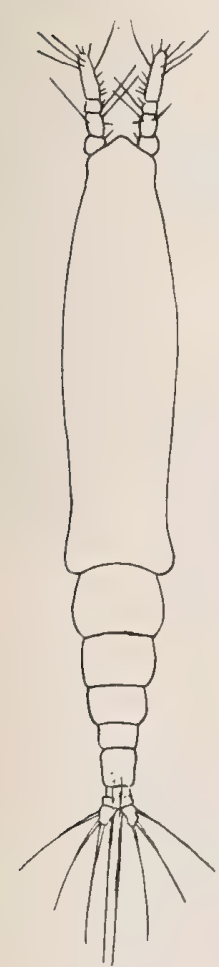

a
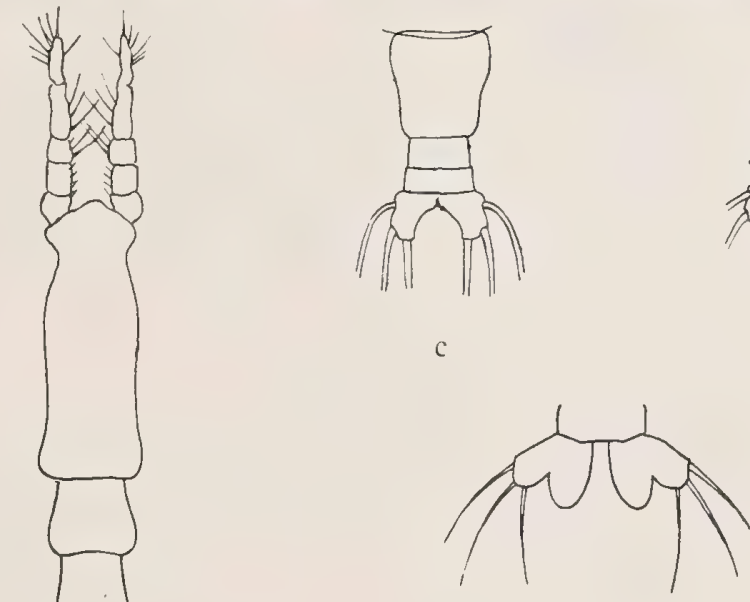

e

Fig. 228. Thaumaleus zetlandicus. a $\Varangle$ dorsal, b or dorsal, c Abdomen q, dorsal, d Abdomen $0^{\pi}$, dorsal, e 5. B.. . Nach T. Scott.

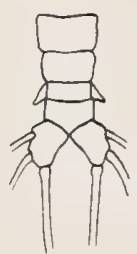

d

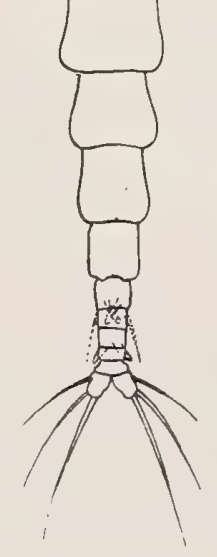

b 
Gensegm. deutlich breiter als das 5. Thsegm. und etwa 2 mal so lang wie die beiden folgenden Abdsegm. zusammen, von denen das letzte etwas länger als das vorhergehende ist; Furka mit 3 langen Borsten. 1. Antenne 4-gldr.; 3. Gl. halb so lang wie das 2., Endgl. etwa so lang wie die 3 anderen zusammen. 5. B. breit-blattförmig, mit zwei fast gleich großen gerundeten Loben; der äußere derselben mit 3 mäßig langen Borsten. - o. 1. Körpersegm. nur wenig länger als die übrigen Thsegm. zusammen. 1. Antenne noch nicht $3 / 4$ so lang als das 1. Körpersegm. Abdomen 4-gldr.; die 2 vorderen Abdsegm. fast gleich lang und schmäler als das letzte Thsegm.; die 2 hinteren zusammen nur wenig länger als das vorhergehende Segm. Furka wie beim $q$ mit 3 Borsten. - L. क 4,8 mm, ơ $2,6 \mathrm{~mm}$.

Shetlandsinseln.

\section{Thaumaleus rostratus $T$. Scott.}

1904, Th. r., T. Scott, p. 250 , t. 14 f. $5-8$.

O. Körper in der Mitte des 1. Segmentes am breitesten, nach vorne gleichmäßig in die stumpfspitzige Stirn verjüngt, nach hinten gleichmäßig verschmälert. Abdomen 3-gldr.; 1. Segm. so lang wie die 2 folgenden zusammen,

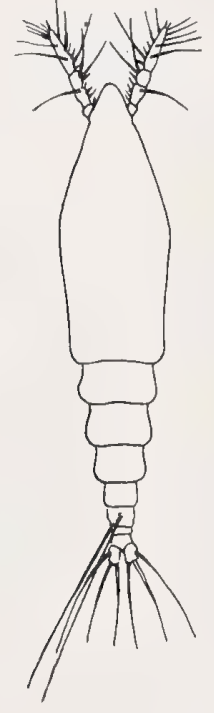

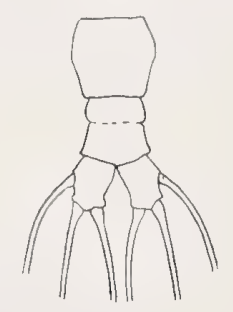

b

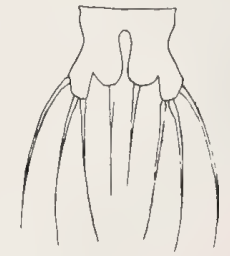

c

Fig. 229. Thaumaleus rostratus.

dorsal, b Abdomen $q$, dorsal, c 5. B. $q$. Nach T. Scott.

2. Gl. sehr klein. Furkaläste mit 3 Borsten. 1. Antenne kurz, 4-gldr.; 3. Gl. ca. $1 \frac{1}{2}$ mal so lang wie das 2 , Endgl. so lang wie die anderen 3 zusammen. 5. B. kurz und blattförmig, mit 2 ungleichen Loben; äußerer Lobus schmäler als der innere und denselben überragend, mit 3 mäßig langen Borsten; innerer Lobus breit-gerundet mit einer einzigen Borste. L. 3,9 mm. - $\sigma^{\top}$ unbekannt. Shetlandsinseln. 
13. Monstrilla dubia T. Scott.

1904, M. d., T. Scott, p. 247 , t. 13 f. 14 ; t. 14 f. $16-18$.

9. 1. Antenne etwa halb so lang wie das 1. Körpersegm., 4-gldr., 2. Gl. doppelt so lang wie das 3. Abdomen 3-gldr.; Gensegm. ungefähr so groß wie das 5. Thsegm. und so lang wie die beiden andern zusammen, von denen das erstere kürzer ist als das zweite. Eigabel weit über die Furkaborste

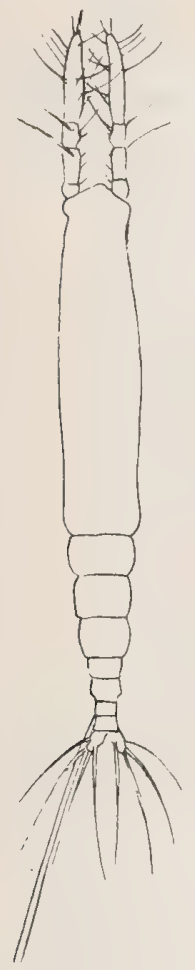

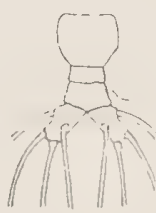

b

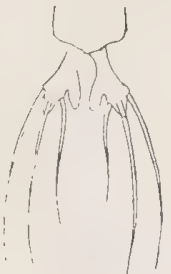

C

Fig. 230. Monstrilla dubia.

à $q$ dorsal, b. Abdomen $q$ dorsal, c 5. B. $q$. Nach T. Scott

hinausreichend. Furka mit 4 langen Borsten. 5. B. 2-lappig; AuBenlobus mit 3 Borsten, von denen die innere die kürzeste ist; Innenlobus kürzer, ohne Borsten. L. $3,3 \mathrm{~mm}$. - o unbekannt.

Schottische Küste.

\section{Haemocera danae Claparède.}

? 1863, Monstrilla d., Claparède, p. 95, t. 16 f. $1-6$ (,,$\left.\sigma^{7}\right) .1901$, H. d., Malaquin, p. 109, Textf. 1, 2; t. 2 f. $1-5\left(\%, \sigma^{7}\right)$.

․ Vorderkörper etwas mehr als 4 mal so lang wie der Hinterkörper; Rumpf ungefähr zylindrisch, Kopf und 1 . Thsegm. etwa 3 mal so lang wie die 3 folgenden Thsegm. zusammen; Vorderkopf vorne abgerundet. 1. Antenne $1 / 3$ so lang wie das 1 . Segm. und ungefähr 2 mal so lang wie der Abstand 
von Stirnrand bis Mund. Abdomen 3-gldr.; Gensegm. länger als das 5. Thsegm. und als die beiden gleich langen. Abdsegm. zusammen, bisweilen mit leichter Einschnürung; Eigabel mit gesonderten Zinken, ca. 2 mal so lang wie der Hinterkörper; Furka mit 3 fast gleich langen Fiederborsten, die wenigstens so lang wie das Abdomen sind. 1. Antenne 4-gldr.; 2. Gl. 2 mal so lang wie das 3. 5. B. mit kleinem Zipfel am Innenrande und 3 ungleich langen Borsten.
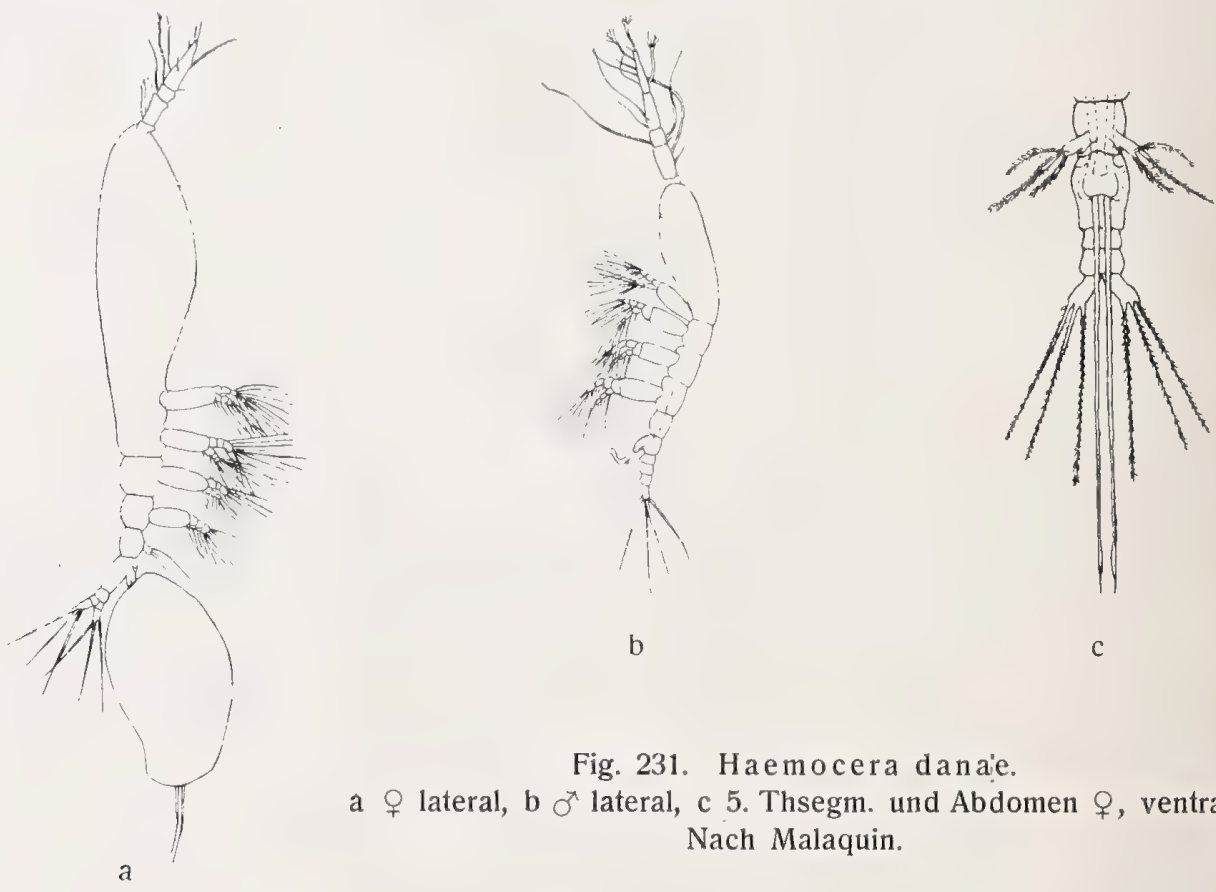

Fig. 231. Haemocera danaie.

a $q$ lateral, b o lateral, c 5. Thsegm. und Abdomen $q$, ventral. Nach Malaquin.

- $\sigma^{7}$. Vorderkörper etwa $3 \mathrm{mal}$ so lang wie der Hinterkörper; Kopf und 1. Thsegm. ${ }^{1} 1 / 2$ mal so lang wie die 3 folgenden Thsegm. zusammen. Es verhalten sich die Abstände vom Mund bis zum Stirnrand und Hinterrand des 1. Segm. wie $1: 3 ; 1$. Antenne ungefähr so lang wie das 1.Segm.; 5. Thsegm. ohne Spur von Gliedmaßen. Abdomen 4-gldr.; die 3 hinteren Abdsegm. ungefähr gleichlang; Furka jederseits mit 4 gleich langen Borsten, die den Hinterkörper an Länge übertreffen. L. ?

Engl. Kanal. Lebt in Salmacyna Dysteri Huxley.

\section{Haemocera filogranarum Malaquin.}

1896, Thaumale us f., Malaquin. 1901, H. f., Malaquin, p. 110, Textf. 3.

१. Abdomen 3-gldr.; Gensegm. so lang wie das 5. Thsegm. und nicht länger als die beiden folgenden Abdsegm. zusammen; Ansegm. nur wenig länger als das mittlere Abdsegm. Eigabel mit gesonderten Zinken, etwa $1 \frac{1}{2}$ mal 
so lang wie der Hinterkörper. Furka so lang wie das Ansegm. mit 4 gleich langen Borsten, die halb so lang wie der Hinterkörper sind. 5. B. mit kleinem Zipfel am Innenrande und 3 fast gleich langen Endborsten. - $\checkmark$. Furka mit 4 Borsten. - L. ?

Engl. Kanal. Der Wirt ist Filograna implexa.

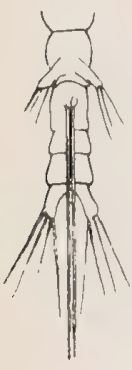

Fig. 232.

Haemocera filogranarum.

5. Thsegm. und Abdomen , ventral. Nach Malaquin.

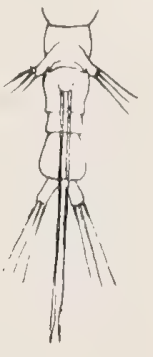

Fig. 233.

Haemocera roscovita.

5. Thsegm. und Abdomen , ventral Nach Malaquin.

16. Haemocera roscovita Malaquin.

1901, H. r., Malaquin, p. 110, Textf. 5.

१. Abdomen 3-gldr.; Gensegm. so lang wie das 5. Thsegm. und etwas kürzer als die beiden folgenden Abdsegm. zusammen, von welchen das Ansegm. 2 mal so lang wie das vorhergehende ist. Eigabel mit gesonderten Zinken, fast $11 / 2$ mal so lang wie der Hinterkörper; Furka viel kürzer als das Ansegm., mit 3 Borsten, die halb so lang wie der Hinterkörper sind. 5. B. mit kleinem Zipfel am Innenrande und 3 gleich langen Endborsten. L. ? - o unbekannt.

Roscoff. Lebt in Filograna setosa Langerhans. 


\title{
Anhang.
}

\author{
I.
}

\section{Megacalanus (Seite 13).}

Seite 13 Zeile 5 von oben: "oder gefiedert" fällt fort.

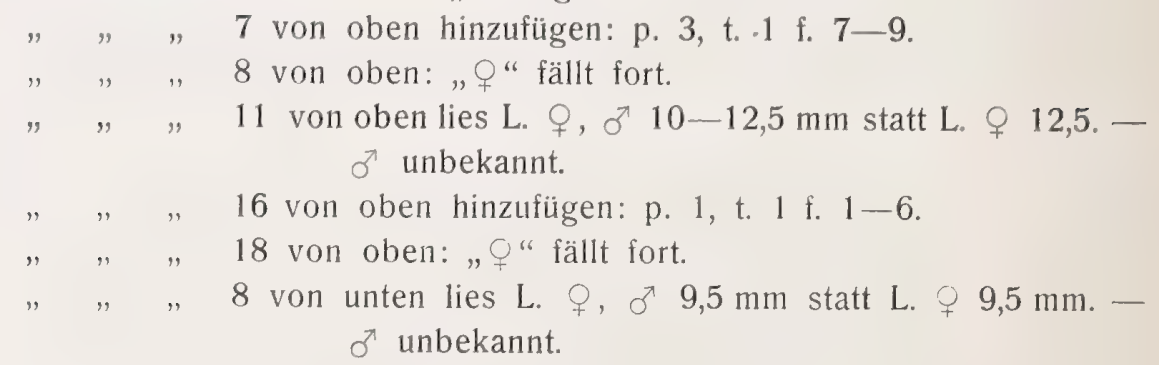

\section{Bradyetes (Seite 32)}

Sars (1907) beschreibt eine neue Art (B. major).

Gaetanus denticulatus Aurivillius (Seite 38).

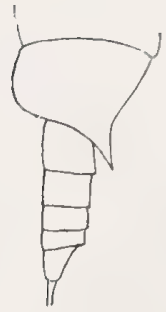

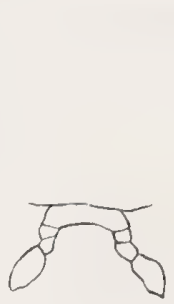

b
Fig. 234. a Hintere Thsegm. und Abdomen 9 von der Seite, b5.B. $ᄋ$. Nach Aurivillius.
1898, G. d., Aurivillius, p. 30 f. $1-3$ (ㅇ)

Unter obigem Namen hat Aurivillius eine Copepoden-Art erwähnt, die aber keine Gaetanus sein kann. Erstens fehlt ihr der dorsale Kopfstachel und zweitens ist ein 5. B. beim $q$ vorhanden. Man vergl. Fig. 234.

Skagerak. 


\section{Euchirella atlantica Wolfenden (Seite 48).}

In seiner letzten Arbeit (1905, p. 48). weist Wolfenden darauf hin; daß die geringere Zahl der Dörnchen am Basipoditen des 4. Beinpaares sich als ein konstantes Merkmal der atlantischen Exemplare herausgestellt hat, weshalb er die früher von ihm als Varietät (E. curticauda var. atlantica) erwähnte atlantische Form zu dem Rang einer selbständigen Art erhebt.

\section{Euchaeta oceana J. C. Thompson (Seite 55).}

1903, E. o., Thompson, p. 19 t. 2 f. $1-9\left(\sigma^{\top}\right)$. o . Vorderkörper 4-gldr. Vorn zwischen den Antennen ein runder zentraler Höcker; Rostrum dorsal sich erhebend und einem zentralen Dorn ähnlich. 1. Antenne 21-gldr.; Borsten kurz, ausgenommen am 3., 8. und 12. Gl. Am 3. bis 8. Glied dreieckige Papillen, 2 an jedem Gl., mit einem feinen Haare im Zentrum.

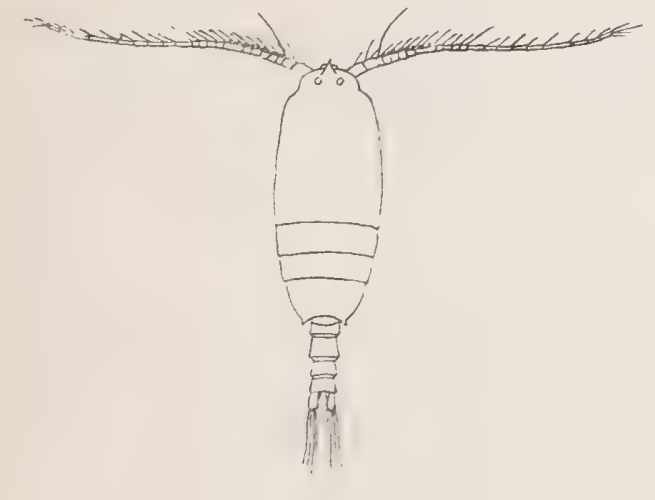

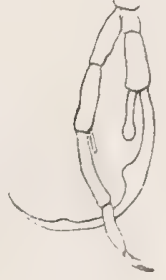

b

Fig. 235. a ठ dorsal, b 5. B. ठॅ. Nach Thompson.

Exp. und Enp. der 2. Ant. fast gleich lang. Mandibel ohne Kaulade. 2. Maxille scheint nicht vorhanden zu sein. Exp. des 1. B. 3-gldr., Enp. des 1.-4. B. resp. 1-, 2-, 3- und 3-gldr. 5. B. lang mit zwei 1-gldr. Enp.; Exp. rechts 3-gldr., mit starkem Dorn am Ende; Exp. links 4-gldr., mit starkem Dorn am Ende, mit feinen Borsten am Innenrande nahe der Spitze und einem kleinen Dorne. L. $6 \mathrm{~mm}$.

\section{Atlant. Ozean.}

Anm. Keins der Exemplare, welche Thompson vorlagen, erschien völlig reif. Die Art ist sicher keine Euchaeta, zeigt aber im Bau des 5. B. Verwandtschaft mit Gaidius und Gaetanus, mit welch' letzterem sie außerdem den Besitz eines Kopfstachels gemein hat. 
Phaenna zetlandica. T. Scott (Seite 56).

1902, P. z., T. Scott, p. 453 t. 22 f. $5-7$.

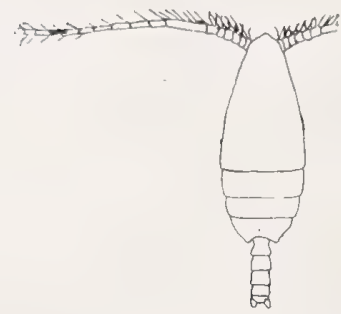

a

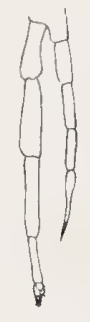

b

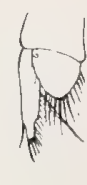

C

Fig. 236. Phaenna zetlandica.

a $\sigma^{x}$ dorsal, b 5. B. $\sigma^{x}$, c Ende des rechten 5. B. $\sigma^{x}$. Nach T. Scott.

T. Scott fand bei den Shetlandsinseln das Männchen einer CopepodenArt, welch' letztere er provisorisch zum Genus Phaenna stellte. Wahrscheinlich handelt es sich um das $\sigma^{\pi}$ irgend einer Xanthocalanus-Spezies. Man vergl. die Figuren.

\section{Cornucalanus Wolfenden (Seite 65).}

Statt der Diagnose von Onchocalanus und der Beschreibung von O. chelifer zu lesen:

Cornucalanus Wolfenden.

Verwandt mit Xanthocalanus, aber davon abweichend durch den großen und starken Endhaken der 2. Maxille und besonders durch die außerordentlich kräftigen, klauenförmigen Anhänge des Enp. des Maxillipeden.

\section{Cornucalanus magnus Wolfenden.}

1903, Scolecithrix chelifer, Thompson, p. 21, t. 5 f. 1-9. 1905, Scolecithrix chelifer, Farran, p. 36, t. 7 f. 18, 19. 1905, C. m., Wolfenden, p. 21, t. 7 f. $1-7$.

ๆ. Kopf mit dorsalem Stachel. Rostrum stark, kurz, zweispitzig; Epistom vorragend. Abdomen kurz, stark behaart. 1. Antenne nicht länger als der Vorderkörper, 24-gldr. 2. Maxille: letzter Lobus mit kräftigem, dickem und langgebogenem Haken nebst 3 Borsten am Ende, von denen eine so lang wie der Haken; Enp. klein, mit 7 (5 ?) sensorischen Anhängen und einem 
langen wurmförmigen Anhang. Maxilliped: proximaler Teil des Innenrandes des 2. Gl. des Basp. gezähnelt, Borsten dieses Gl. sehr kurz; 2. und 3. Gl. des Enp. jedes mit einer starken, gebogenen Hakenborste mit spärlichen Zähnen; 1. Gl. des Basp. mit sensorischem Anhang. Flächen der B., besonders die Enp., mit Dornen versehen. 5. B. klein, nicht deutlich 3-gldr., mit wenigen Randhaaren und einem kurzen Enddorn; Endgl. verjüngt. $-\sigma^{T}$. Kopf ohne Dorsalstachel. 1. Antenne 23-gldr. Mundgliedmaßen rückgebildet; 2. Maxille und Maxilliped ohne Hakenborsten. 5. B. Fig. 237 e. - L. o $8 \mathrm{~mm}$, o $6 \mathrm{~mm}$.

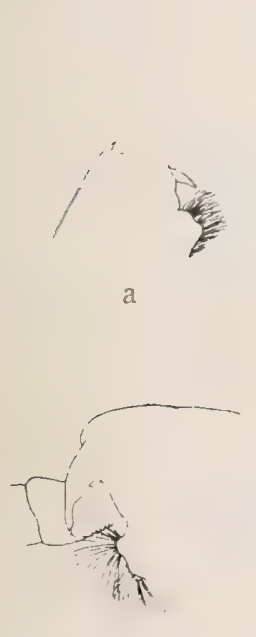

b

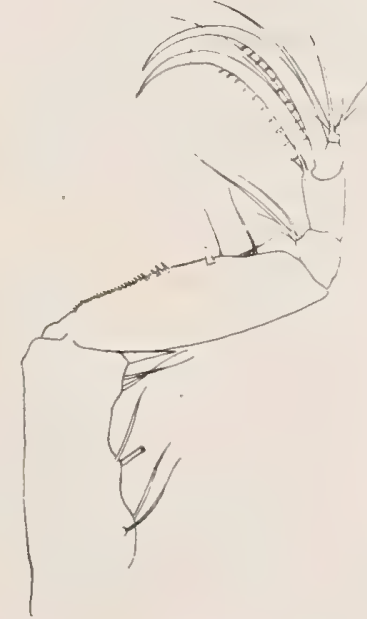

c

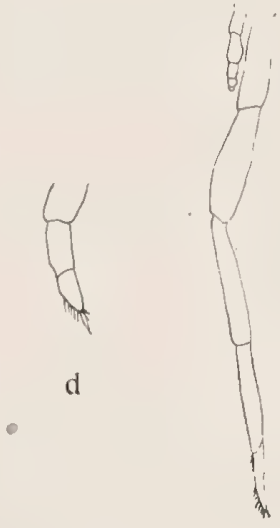

e

Fig. 237. Cornucalanus magnus.

a Kopf $q$, lateral, b Kopf $\sigma^{7}$, lateral, c Maxilliped ㅇ, d 5. B. $q$, e 5. B. $\sigma^{\nearrow}$. Nach Wolfenden.

\section{Cornucalanus simplex Wolfenden.}

1905, C. s., Wolfenden, p. 22.

q. Weicht von C. magnus ab durch das Fehlen des Kopfstachels.

Parastephos pallidus G. O. Sars (Seite 86).

Diese Art ist von T. Scott (1903) nach schottischen Exemplaren, unter denen sich auch Weibchen befanden, kurz beschrieben worden.

$\sigma^{\top}$. Die von Sars erwähnte Asymmetrie der Schwimmbeine des $\sigma^{7}$ scheint einer individuellen Verkümmerung zugeschrieben werden zu können, 
denn. Scott fand diese Eigentümlichkeit nicht bei seinen Exemplaren. Exp. des 2. B. 3-gldr., Enp. 2-gldr.

7. 1. Antenne 24-gldr., gleich lang, spärlich beborstet, mit mehreren kleinen Aesthetasken. 5. B. asymmetrisch, links länger als rechts. L. $2,19 \mathrm{~mm}$.

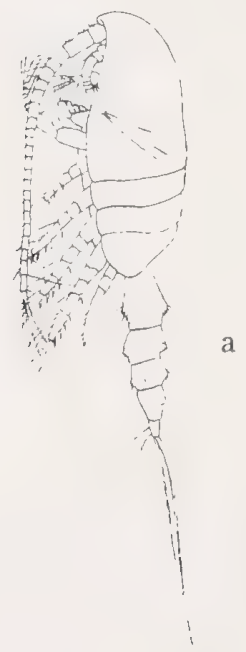

Fig. 238.

Parastephos pallidus a . l lateral, b 5. B. $q$.

Nach T. Scott.

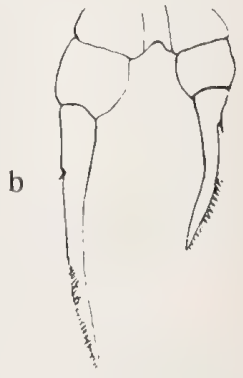

Pseudocyclopia giesbrechti Wolfenden (Seite 91).

Das Männchen dieser Art hat T. Scott gefunden.

$\sigma^{7}$. Vorderkörper 5-gldr, letztes Gl. unter dem vorletzten verborgen; Abdomen schlank, 5-gldr. 1. Antenne kürzer als das 1. Segm., 17-gldr.; Basalglied mehr als halb so lang wie die übrigen Gl. zusammen; diese kurz.

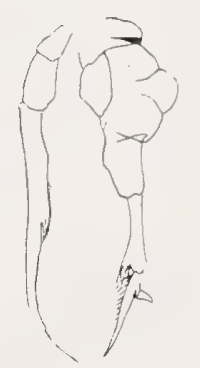

Fig. 239.

Pseudocyclopia giesbrechti.

5. B. $\sigma^{x}$. Nach T. Scott.

2. Antenne: Exp. kaum halb so lang wie das Enp., 5-gldr., 3 und 4 Gl. klein. Übrige Mundteile und die B. wie bei den anderen Arten, aber letztere mehr behaart, besonders die Enp. des 4. B.; Dorn des 1. Gl. des Basp. des 3. B. länger als das Enp. 5. B. verlängert, asymmetrisch; linkes B. lang und schlank (die Figur nach einem am Ende beschädigten [linken] 5. B. hergestellt); rechtes 5. B. proximal verbreitert. L. $0,8 \mathrm{~mm}$.

Schottische Küste. 


\section{Heterorhabdus (Seite 117.)}

Die Bestimmungstabelle dieses Genus ist durch folgende zu ersetzen.

1. 1. Antenne merklich kürzer als der Körper

1. "wenigstens so lang wie der Körper 3

2. Körper kurz und gedrungen; Hinterkörper kaum $1 / 3$ so lang wie der Vorderkörper

H. compactus

Körper schlanker; Hinterkörper über $1 / 2$ so lang wie der Vorderkörper

H. vipera

3. Endgl. des Exp. am 3. B. wie am 2. und 4. gebaut

4. 1. Antenne ca. so lang wie der Körper; Länge $3 \mathrm{~mm}$

H. (Mesorhabdus) brevicaudatus

1. Antenne das Rumpfende überragend; Länge 3-10 $\mathrm{mm}$

5. Länge ca. $10 \mathrm{~mm}$; 1 . Antenne das Rumpfende nur wenig überragend

H. grimaldi

Länge $3-5 \mathrm{~mm} ; 1$. Antenne viel länger als der Körper

6

6. Länge ca. $3 \mathrm{~mm}$

H. longicornis , $5 \mathrm{~mm}$

H. major

7. 1. Gl. des Basp. des Maxillipeden in der Mitte des Innenrandes mit auffallend dicker, langer Borste

Dieses Gl. daselbst mit dünner Borste

8. Rostralpapille mit hervortretender Spitze ohne Spitze

Enp des 5. B viel dünner und kürzer als die Borsten des 3. Gl.

Borste des 2. Gl. des Enp. kaum dünner und kürzer als die Borsten des 3. Gl.

10. Innenrandborste des Exp. des 1. Gl. des Exp. dick und hakig $H$. clausi Dieselbe fehlt

H. abyssalis?

11. Von den 3 distalen Hakenborsten der 2. Maxille ist eine kürzer und dünner als die beiden andern; am vorhergehenden Lobus 2 lange Hakenborsten und 1 kleine dünne Borste
H. norvegicus

Die 3 distalen Hakenborsten der 2. Maxille etwa gleich lang und stark; die dünnste von den 3 Borsten des vorhergehenden Lobus über halb so lang wie die beiden andern

H. papilliger.

1. 1. Antenne höchstens das Ende des Gensegm. erreichend

1. " so lang wie oder länger als der Körper

2. Endgl. des Exp. am 3. B. wie am 2. und 4. B. gebaut $"$ " " 3. " breit, oval

3. Endgl. des Exp. am 3. B. wie am 2. und 4. B. gebaut

H. atlanticus

H. vipera.

" " "3. " breit, oval 
4. Länge ca. $3 \mathrm{~mm}$

H. longicornis

$" \quad \begin{array}{rr}5 & \end{array}$

H. major

H. grimaldi

5. Endgl. des Exp. des rechten 5. B. kurz

H. grandis

" " " $" 5$. "dornförmig

6. Rostralpapille mit hervortretender Spitze Dieselbe ohne Spitze

H. spinifrons

2. Gl. des rechten Exp. des 5. B. mit großem Fortsatz; am Innenrande des

2. Gl. des rechten Basp. ein kürzerer Anhang

H. papilliger

2. Gl. des rechten Exp. des 5. B. mit einem behaarten Vorsprung; 2. G1. des rechten Basp. mit einem langen, lamellösen, distal verbreiterten Fortsatz 8

8. Distale Borste am Endgl. des rechten Exp. des 5. B. überragt das Gliedende wenig

$H$. norvegicus

Dieselbe reicht um mehr als ihre Hälfte über das Gliedende hinaus 9

9. 2. Gl. des linken Basp. am Innenrande mit behaartem Vorsprung H. clausi Der ganze Innenrand des 2. Gl. des linken Basp. behaart H. abyssalis

\section{Heterorhabdus longicornis Giesbrecht (Seite 125)}

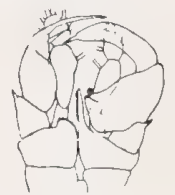

Fig. 240. Heterorhabdus longicornis. 5. B. đ. Nach Wolfenden.

Zu der Beschreibung des ơ (S. 125) wird hier die Abbildung des 5. B. desselben gegeben.

Heterorhabdus grimaldi J. Richard (Seite 125).

Das Männchen dieser Art wird von Wolfenden erwähnt und die 2. Maxille nebst dem 5. B. desselben abgebildet (1905, p. 10, t. 4 f. 3-5). L. $9 \mathrm{~mm}$.

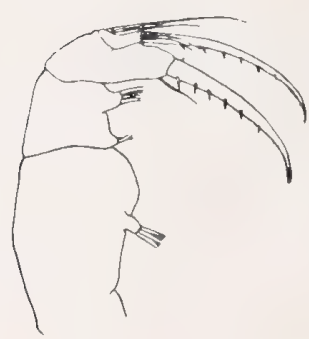

a

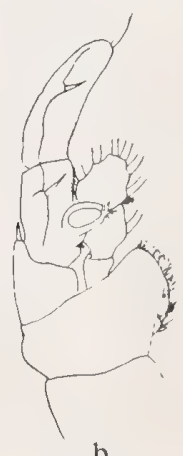

b

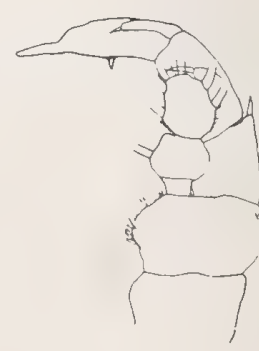

C

Fig. 241. Heterorhabdus grimaldi.

a 2. Maxille $\sigma^{\pi}$, b. rechtes 5. B., c linkes 5. B. $\sigma^{\pi}$. Nach Wolfenden. 
Heterorhabdus major Dahl (Seite 126).

Das Männchen wird noch von Wolfenden erwähnt und das 5. B. desselben abgebildet. (1905, p. 11, t. 6 f. 14.)

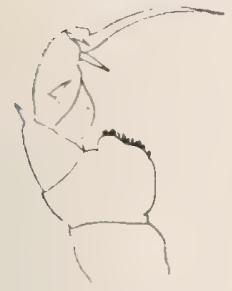

a

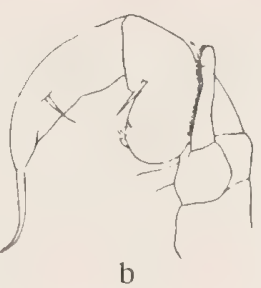

b

万. 1. Antenne sehr lang. 5. Lobus der 2. Maxille verlängert; Hakenborsten ohne Fiedern; 4. Lobus mit 1 dicken Hakenborste, kleiner als die des 5. Lobus; Borsten des Enp. sehr lang. Maxilliped ohne Dorn. 3. B. wie das 4. B. gebaut. 5. B. siehe Fig. 242. L. $4,8 \mathrm{~mm}$.

\section{Heterorhabdus atlanticus Wolfenden.}

1905, H. a., Wolfenden, p. 13.

q unbekannt. - $\sigma^{7}$. Kopf mit frontaler Papille. 1. Antenne das Ende des Gensegm. erreichend. 2. Maxille wie bei longicornis, aber mit kürzerem 5. Lobus. Maxilliped ohne gebogenen Dorn am 1. Gi. des Basp. 3. B. wie das 4. B. gebaut. Rechtes 5. B.: 2. Gi. des Basp. mit kurzem distalen Vorsprung, distale Hälfte des Randes behaart; 2. Gl. des Exp. mit langem, distal behaarten Auswuchs, der eine kurze, ziemlich dicke Borste trägt; 3. Gl. des Exp. kurz und mit sehr dicker, langer Borste am distalen Innenrand; Enp. mit verlängertem 2. Gl. und sehr dicker und langer Innenrandborste. Linkes 5. B.: 2. Gl. des Basp. nur wenig vorspringend, mit behaartem Innenrande; 2. Gl. des Exp. mit 2 langen dünnen Borsten; 3. Gl. des Exp. in einen gebogenen Haken verlängert, der einen halb so langen inneren Dorn trägt; Enp. mit sehr verlängertem 2. Gil, aber ohne Randborsten. L. 3,7 mm.

Das or wird von Wolfenden nur vorläufig als neue Art betrachtet und ist verwandt mit $H$. major und longicornis.

\section{Heterorhabdus grandis Wolfenden (Seite 126).}

Wolfenden hat 1905 die Art zum zweitenmal erwähnt, diesmal auch das or $(1905$, p. 8, t. 4 f. 7,8$)$. Der Beschreibung S. 126 ist folgendes hinzuzufügen:

१. 1. Antenne den Körper um etwa die 4 letzten Gl. überragend; 5. Lobus der 2. Maxille etwas länger als der 4, dessen drei Borsten dünn sind; Borsten 
des Enp. der 2. Maxille lang und dick, z. T. so lang wie die Hakenborste am 6. Lobus. Endgl. des Exp. des 3. B. breiter als der des 2. und 4. B. 万. Für das 5. B. siehe Fig. 243.

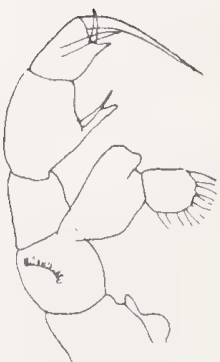

Fig. 243. Heterorhabdus grandis. a linkes, b rechtes 5. B. $\sigma^{\pi}$. Nach Wolfenden.

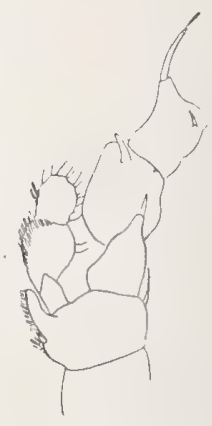

b

Heterorhabdus brevicaudatus Wolfenden (Seite 126).

1905, H. b., Wolfenden, p. 12 , t. 6 f. 15 ; t. 4 f. 1,2 . ? 1905, Mesoshabdus annectens, Sars, Nr. 40, p. 9.

ๆ. Kopf mit Papille, ohne Dorn; Vorderkörper etwas mehr als 2 mal so lang wie das Abdomen. 1. Antenne so lang wie der Körper. Enp. der 2. Antenne 3 mal so lang wie das Exp. 2. Maxille mit Hakenborste und 2 sehr dünnen Borsten am 5. Lobus; 6. Lobus mit ähnlichem, aber schwächerem Haken und 1 einfachen Borste; Borsten des Enp. sehr lang. Maxilliped ohne Dorn. 3. B. nicht verbreitert. 5. B. mit breiter Exp.; 1. Gl. des Exp. ohne Innenrandborste, 2. Gl. mit dünner, gebogener Innenrandborste, die so lang wie das Endgl. ist; Innenrandborste aller $3 \mathrm{Gl}$. ähnlich geformt. L. $3,15 \mathrm{~mm}$.- o unbekannt.

Fig. 244.

Heterorhabdus brevicaudatus.

a $\odot$ dorsal, b 2. Maxille 9 . Nach Wolfenden.
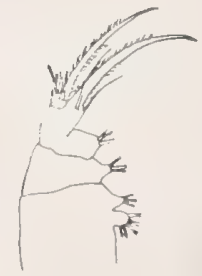
II.

\section{Die Entwicklungsstufen der marinen Copepoden.}

Über die jüngeren Stadien der marinen Copepoden ist zurzeit noch recht wenig bekannt. Die erste Arbeit, die sich systematisch mit dem Studium der Nauplien und Copepoditen mariner Plankton-Copepoden beschäftigt hat, ist die von Oberg (1905) publizierte, in welcher der Autor den von ihm in der Kieler Bucht beobachteten Entwicklungsstufen eine gewissenhafte und ausführliche Beschreibung widmet. Aus dieser Arbeit wird im Folgenden ein Auszug gegeben, so weit es sich um die Beschreibung der Nauplien und die Bestimmung dieser und der Copepoditen handelt. Die Zahl der NaupliusStadien bis inklusive zum ältesten beträgt 6 und ebenso ist die Zahl der Copepodit-Stadien bis inklusive zum reifen Tiere 6. Alle 12 Stadien sind durch Häutungen von einander geschieden. Die einzelnen Stadien scheinen auch in der Zeit zwischen den einzelnen Häutungen in einem gewissen Grade des Wachstums fähig zu sein, denn es treten Exemplare derselben Entwicklungsstufe von recht verschiedener Größe innerhalb derselben Spezies auf. Während die Nauplius-Stadien II-VI im Habitus und auch in der Hauptsache der Bewaffnung von 2. Antenne und Mandibel bei derselben Art sich ziemlich gleich bleiben, tritt nicht nur bei der Häutung nach Stadium VI die tiefeingreifende Änderung der Verwandlung in den Copepoditen auf, sondern auch das Stadium I, auf dem der Nauplius das Ei verläßt, ist von den folgenden recht scharf geschieden, so zwar, daß es schwer fällt, die Nauplius-Stadien I ihrer Zugehörigkeit nach zu erkennen.

\section{Bestimmungstabelle der Plankton-Nauplien der Kieler Bucht.}

1. Mandibel zwischen Basipoditen und Endopoditen mit einem medianwärts gerichteten, daumenförmigen Fortsatz, der drei kräftige divergierende Borsten trägt. Körper in der Aufsicht oval; Furkalbewaffnung bei den älteren aus zwei schilfblattförmigen endständigen Borsten und jederseits noch aus einem Paare feinerer Borsten bestehend

Oithona similis Mandibel ohne den daumenförmigen. Fortsatz; Körper mehr oder weniger gestreckt (trifft nur für Stadium I nicht $z u$ )

2. Endanhänge ${ }^{1}$ ) klein, stachelförmig

" 1) lang, degenförmig bis stangenförmig, der linke länger als der rechte

1) als kräftige, dorsalwärts gerichtete Haken entwickelt

1) Auf Nauplius-Stadium I wird die Furkalbewaffnung jederseits nur durch einen Anhang angedeutet, der jedoch nicht einem bestimmten der späteren Furkalanhänge entspricht, denn es entstehen aus inm immer diejenigen Anhänge zuerst, die zuerst im 
3. Tastborsten nicht auffallend groß. An der zweiten Antenne ist das 1. Gl. des Exp. mit dem 2. verwachsen Acartia bifilosa und longiremis Linke Tastborste als langer Stachel kolossal entwickelt, rechter als kräftige Rute dorsal abstehend. 2. Antenne normal Centropages hamatus

4. Linke Endborste nur wenig länger als die rechte, beide nur wenig länger als die linke Tastborste

Eurytemora hirundo Linke Endborste viel länger als die rechte und als die Tastborsten, zu einem mächtigen Schwebstachel entwickelt. Gestalt schlank und besonders gestreckt

Temora longicornis

5. Groß

Klein

Pseudocalanus elongatus ${ }^{2}$ ) Paracalanus parvus.

\section{Bestimmungstabelle der Plankton-Copepoditen der Kieler Bucht.}

1. 2. Maxille dem Maxilliped ähnlich, gestreckt, Endglieder wenig kleiner als das Mittelglied, verjüngen sich wenig und ganz allmählich. 2. Antenne anscheinend 1-ästig, die übrigen Mundgliedmaßen treten gegen die genannten an Größe zurück. Stirn in einen senkrechten Dorn auslaufend

Oithona similis

2. Maxille typisch, Endglieder eng ineinander geschachtelt, eben nur als Ringelung des kurzen Endabschnittes erkennbar

2. Abstand zwischen 1. und 2. Maxille auffallend groß. Maxilliped kürzer oder höchstens ebenso lang wie diese, neben ihr wenig auffallend, nur ihr 1. Gl. des Basp. gut entwickelt und kräftig bewaffnet 3 Abstand zwischen 1. und 2. Maxille nicht bedeutend, Maxilliped länger als 2. Maxille, in allen Teilen mittelstark bewaffnet

3. Mit Rostralfäden Acartia bifilosa Ohne " (paßt auch für Stadium I von bifilosa, die dann von longiremis nicht zu unterscheiden ist) Acartia longitemis

4. Außenrand des Exp. des 1. B. deutlich mit dolchförmigen, weit abstehenden, oft gesägten Dornen bewaffnet

Bewaffnung am Außenrande des Exp. des 1. B. schwer wahrzunehmen, da sie entweder nur aus Haaren oder gekrümmten Borsten oder eng anliegenden schwachen Dornen besteht

5. Maxilliped die 2. Maxille nur mit dem Endabschnitt überragend; Furkaläste deutlich kürzer als das proliferierende Segment Centropages hamatus Maxilliped die 2. Maxille auch mit dem Mittelabschnitt überragend; Furkaläste fast so lang oder länger als das proliferierende Segment 6

6. In der Aufsicht schlank

Eurytemora hirundo " , " plump Temora longicornis

nächsten Stadium auftreten, also bei Oithona die Außenborsten, bei Centropages die Tastborsten, bei Temora die Endborsten (Oberg, p. 22). Die Tastborsten sind median von den Endanhängen eingepflanzt.

$\left.{ }^{2}\right)$ Vergleiche hierzu auch Calanus finmarchicus (Grobben, 1881). 
7. Exopodit der 2. Antenne überragt den Endopoditen. Tier groß

Pseudocalanus elongatus ${ }^{1}$ )

Exopodit der 2. Antenne so lang wie der Endopodit. Tier klein

Paracalanus parvus.

\section{Pseudocalanus elongatus Boeck.}

Furkalbewaffnung des Nauplius-Stadiums I durch 2 dreieckige Zipfel gebildet, die bereits etwas an die späteren Haken erinnern. Am Ende der 1. Antenne 4 Borsten; mittlerer Abschnitt der 1. Antenne mit 3 Borsten. 2. Gl. des Basp. der Mandibel mit einer kräftigen kurzen Klinge.

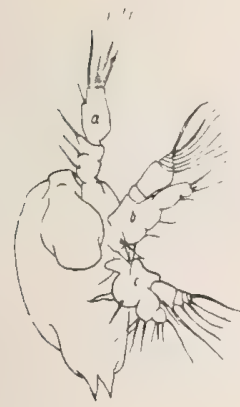

a

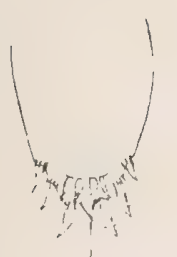

b

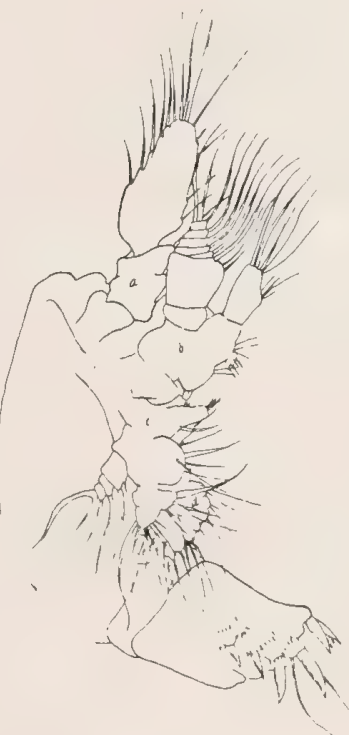

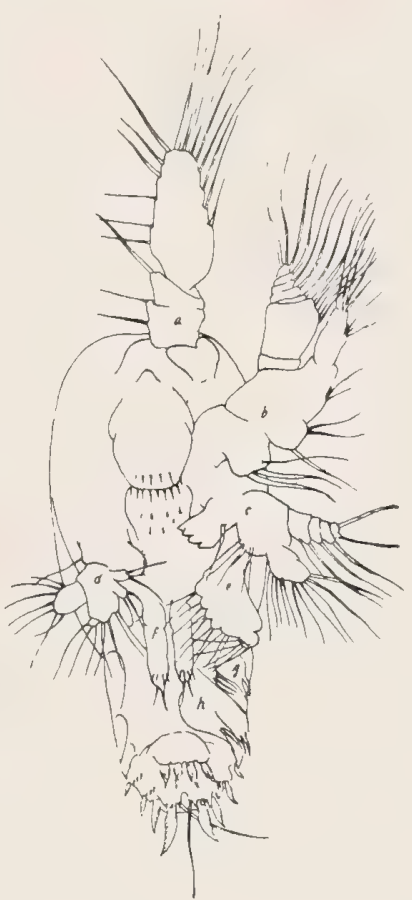

d

Fig. 245. Nauplius von Pseudocalanus elongatus.

a Stadium I, b Hinterleib des Stadiums IV, c Stadium V, lateral, d Stadium VI, ventral. Nach Oberg.

II.-VI. Nauplius-Stadium. Vorderer Abschnitt des Körpers unter dem elliptischen Kopfschilde verborgen; hinterer Abschnitt nur wenig schmäler als der vordere, gegen das Ende leicht verjüngt und plötzlich mit der querabgestutzten Furkalanlage endend. Furkalbewaffnung aus 2 kräftigen Haken und

1) Durch noch ansehnlichere GröBe zeichnen sich die Copepoditen von Calanus fin marchicus aus. 
1 Paar Tastborsten, deren rechte auf die Dorsalseite verschoben ist, bestehend. An den Seiten auf Stadium IV 2, dann 3 Lateralhaken, auf der Ventralfläche eine Querreihe von 2, später 4 Ventralhaken. Segment des Maxillipeden scharf abgesetzt und deutlich vom Kopfschilde geschieden. Auf Stadium VI ist die gesamte Kaubewaffnung reduziert, der Kaurand der Mandibularlade sogar völlig geschwunden und deshalb die Fähigkeit zu Nahrungsaufnahme gering. Die Größenzunahme zwischen dem V. Nauplius- und dem I. Copepoditstadiums ist dementsprechend ebenfalls sehr gering und die Tiere scheinen längere Zeit auf dem VI. Nauplius-Stadium zu verweilen (Puppenstadium).

Länge: Nauplius-Stadium I: 0,173-0,190 mm, N.-St. VI: 0,42 mm: Copepodit-Stadium I: $0,52 \mathrm{~mm}$.

\section{Paracalanus parvus Claus.}

Von dieser Art hat Oberg die 3 jüngsten Nauplius-Stadien nicht beobachtet. Die späteren Stadien weichen von Pseudocalanus ab durch die Größe, das V. Nauplius-Stadium außerdem dadurch, daß das Segment des Maxillipeden mit unter das Kopfschild einbezogen und mit dem Kopfabschnitt verschmolzen

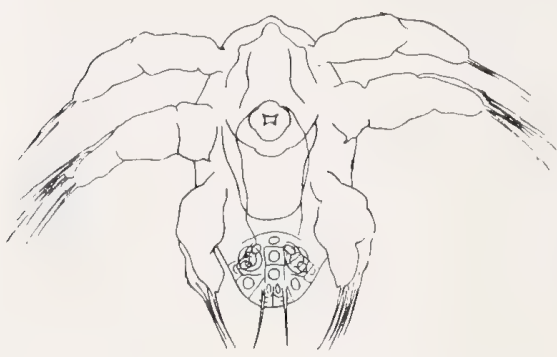

a
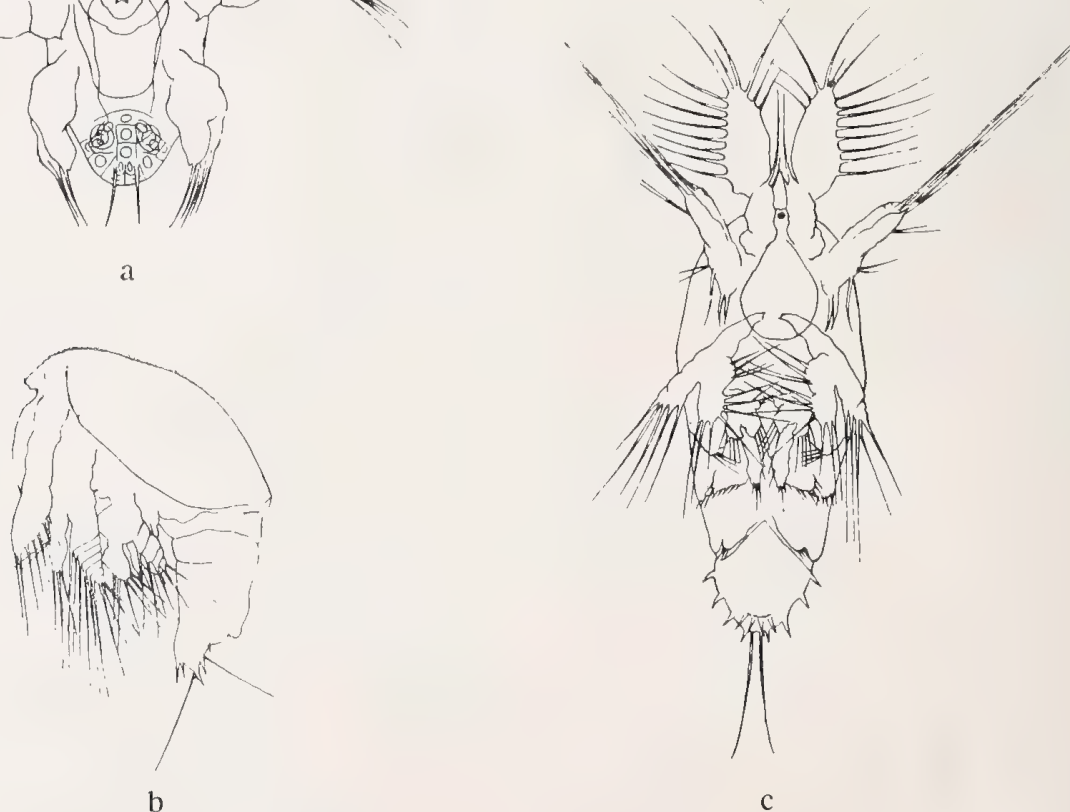

Fig. 246. Nauplius von Calanus finmarchicus.

a Stadium I, ventral, b späteres Stadium, lateral, c Stadium VI, ventral Nach Grobben. 
ist. Beim I. Copepodit-Stadium überragt das Exp. der 2. Antenne das Enp. nicht (wie bei Pseudocalanus).

Länge: N. IV 0,17 mm (Pseudocalanus 0,315), N. VI 0,21 mm, C. I $0,327 \mathrm{~mm}$.

\section{Calanus finmarchicus Gunner.}

Nauplius-Stadium I mit 2 fadenförmigen Anhängen. Die späteren Nauplius-Stadien gleichen denen von Pseudocalanus; genaue Größenangaben fehlen aber.

\section{Centropages hamatus Lilljeborg.}

Nauplius-Stadium I eiförmig. Furkalbewaffnung durch 2 lange starre Fäden gebildet (Tastborstenanlage). Mittleres Gl. der 1. Antenne mit 2 Borsten; Endgl. mit 3 Endborsten. Kauhaken der Mandibel schwach und nach außen gerichtet.

II.-VI. Nauplius-Stadium. Körper sich gleichmäßig rostral- und furkalwärts verjüngend; Vorder- and Hinterleib kaum einen Winkel mit einander bildend.

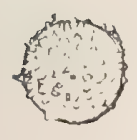

a

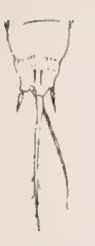

d

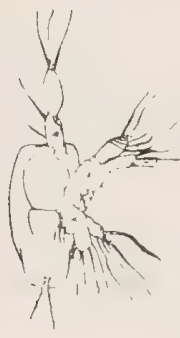

b

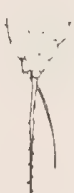

c

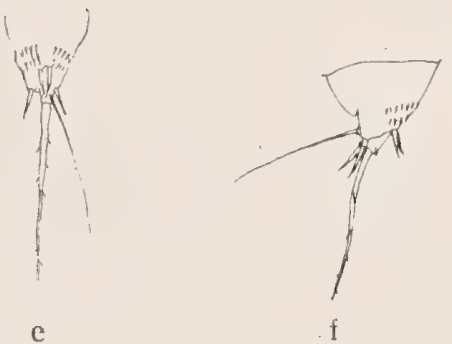

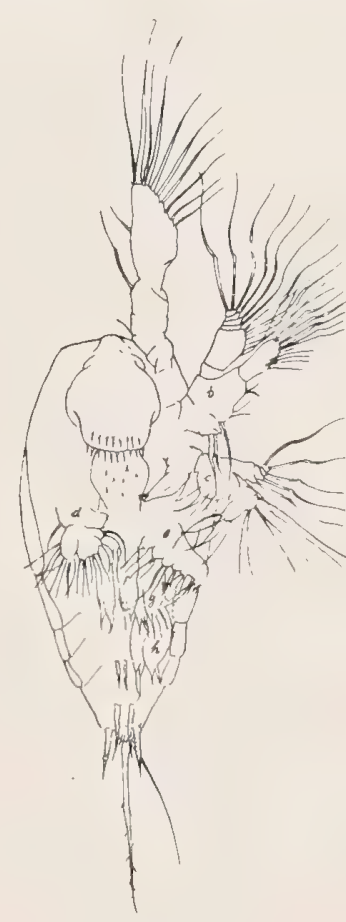

$\mathrm{g}$

Fig. 247. Ei und Nauplius von Centropages hamátus.

a Ei, b Stadium I, ventral, c Furkalbewaffnung Stad. II, ventral, d idem Stad. III, ventral, e idem Stad. IV, ventral, f idem Stad. V, lateral, g Stad. VI, ventral.

Nach Oberg. 
Furkalbewaffnung: linke Tastborste ein gewaltiger Stachel, bei jüngeren Stadien fast so lang wie der Körper; rechte Tastborste kräftig entwickelt und dorsal verschoben. Enddorne schwach, jederseits vor dem langen Stachel stehend, auf Stadium III auftretend: Lateralhaken jederseits durch 2 Börstchenreihen vertreten. Die distalen Ventralhaken auf Stadium III, die schwächlichen proximalen auf Stadium VI auftretend.

Länge: N. I $0,145 \mathrm{~mm}, \mathrm{~N} .0,288-0,345 \mathrm{~mm}$, C. I $0,40 \mathrm{~mm}$.

Temora longicornis O. F. Müller.

Nauplius-Stadium I mit birnenförmigem Körper. Furkalanlage weit nach hinten gewachsen; Furkalbewaffnung aus 2 nicht sehr langen, ziemlich breiten und gleich langen Borsten (Anlage der Endborsten) bestehend, die an ihrem Ursprung weiter auseinander stehen als die 2 Borsten von Centropages. Mittleres Gl. der 1. Antenne mit 3 Borsten, Endgl. mit 3 Borsten. Kaubewaffnung der Mandibel weich und schlaff.

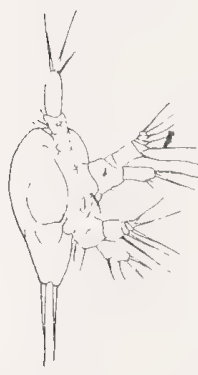

a

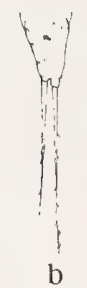

Fig. 248. Nauplius von Temora longicornis.

a Stadium I, ventral, b Furkalbewaffnung Stadium II, ventral, c Stadium IV, ventral, d Furkalbewaffnung Stadium V, lateral.

Nach Oberg.

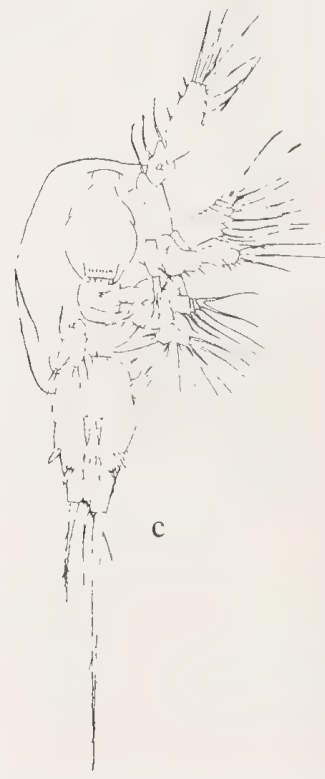

d

II.-VI. Nauplius-Stadium. Hinterkörper vom Vorderkörper scharf abgesetzt, schlanker als dieser. Enddorne zu langen stangenförmigen Borsten ausgewachsen, asymmetrisch, die linke länger als die rechte. Auf Stadium IV und VI sind 2, auf Stadium V 3 Paar Lateralhaken vorhanden. Die 4 Ventralhaken, deren 1. Paar auf Stadium III auftritt, stehen paarweise vor einander. 2 Tastborsten auf Stadium III auftretend, die rechte auf die Dorsalseite verschoben. Dorsalrand des Endgl. der 1. Antenne auf Stadium VI mit 8 Borsten (sonst 6). Kauhaken des 1. Gl. des Basp. der 2. Antenne gleich stark.

Länge: N. I $0,11 \mathrm{~mm}$, N. VI $0,34-0,40$, C. I 0,54 (ohne Furkalborsten). 


\section{Eurytemora hirundo Giesbrecht.}

Von dieser Art sind nur einzelne jüngere Stadien von Oberg beobachtet worden und diese lassen sich von den andern hier beschriebenen Nauplien zur Genüge unterscheiden, wie aus beistehender Figur ersichtlich ist.

Fig. 249.

Nauplius von Eurytemora hirundo Giesbr.

a Stadium I, ventral, b Stadium III, ventral.

Nach Oberg.

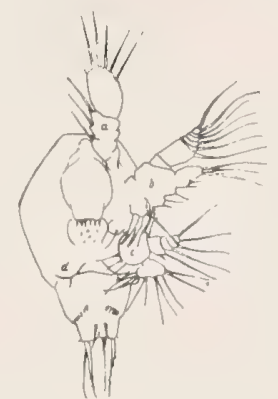

b

Acartia bifilosa Giesbrecht und longiremis Lilljeborg.

Körperform des I. Nauplius-Stadiums elliptisch. Furkalanlage mit 2 mäßig langen, dicht zusammenstehenden Dornen, drehrund und leicht gesägt (Anlage der Tastborsten). Mittleres G1. der 1. Antenne mit 3 Borsten, Endgl. mit 3 Borsten. Bewaffnung der Mundgliedmaßen auffallend zart; Kauhaken der 2. Antenne stumpf und nach außen gekrümmt.

II.-VI. Nauplius-Stadium. Körper gestreckt oval, bis auf Stadium IV ganz bis fast zum After unter dem Kopfschilde verborgen. Maxillipedanlage

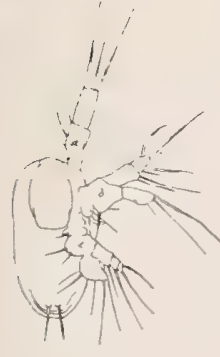

a

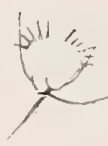

$\mathrm{b}$

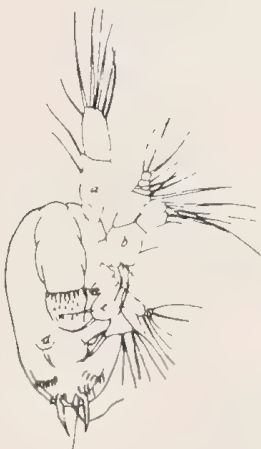

C

Fig. 250. Nauplius von Acartia longiremis und bifilosa.

a Stadium I, ventral, b Furkalbewaffnung Stadium II, ventral, c Stadium III, ventral, d Stadium VI, ventral. Nach Oberg.

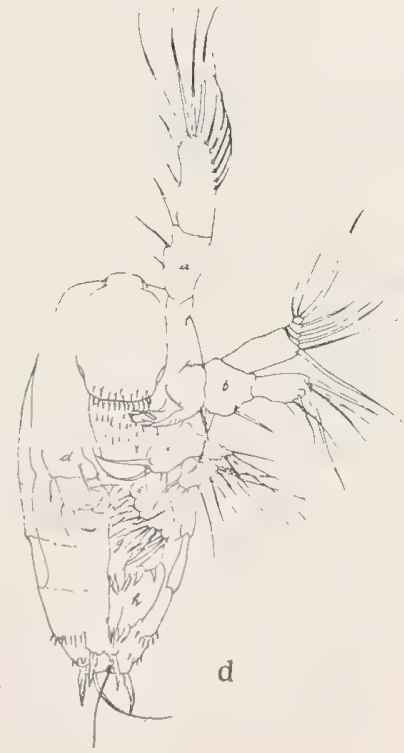


mit ihrem Segment auf Stadium V gut zu erkennen; auf Stadium VI sind die Segm. des 1. und 2. B. und das Ansegm. deutlich getrennt. Endborsten der Furkalanlage Dornen von mäßiger Stärke; Tastborsten gut entwickelt, ähnlich wie bei Centropages inseriert. Statt der Lateralhaken sind 2 Börstchenreihen entwickelt, von denen 1 Paar auf Stadium II, das 2. Paar auf Stadium V auftritt. Stadium IV-VI mit 1 Paar Ventralhaken.

1.-3. Gl. des Exp. der 2. Antenne verschmolzen. 1. Maxille auch noch auf Stadium VI 1-ästig und dann 2-gldr. Maxilliped auf Stadium VI zitzenförmig, klein.

Erst auf dem II. Copepodit-Stadium sind A. bifilosa und longiremis zu unterscheiden durch das Auftreten der Rostralfäden bei der erstgenannten Art.

Länge: N. $10,12 \mathrm{~mm}$, N. VI $0,235-0,312 \mathrm{~mm}$, C. I $0,47 \mathrm{~mm}$.

\section{Oithona similis Claus.}

Das I. Nauplius-Stadium nicht scharf von den späteren Stadien geschieden. I.-VI. Nauplius-Stadium. Körper elliptisch; der After erst auf Stadium V etwas unter dem Kopfschilde hervorrückend; Segmentierung des freien Körperendes auch auf Stadium VI sehr unscharf erkennbar. Furkalbewehrung des

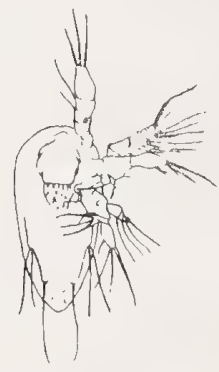

a

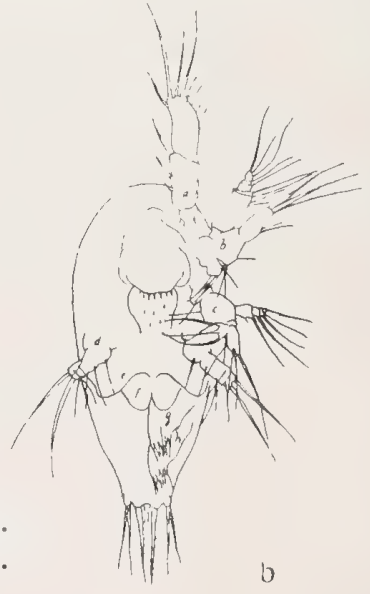

Fig. 251. Nauplius von Oithona similis a Stadium II, ventral, b Stadium VI, ventral. Nach Oberg.

I. Stadiums: jederseits eine sehr lange, dorsal gekrümmte Borste; des III. Stadiums: jederseits ein laterales Borstenpaar; zwischen diesen treten auf Stadium IV ein Paar kräftige Borsten und auf Stadium V noch 1 Paar feine, kurze Medianborsten auf. 1. Antenne auf Stadium I mit 2 Endborsten, später mit 3 Endborsten und dünnen, kurzen dorsalen Randborsten. Mandibel ohne Kaulade am Basp.; Kaulade des Enp. derselben daumenförmig, vom Endabschnitt des Enp. völlig abgegliedert, mit 3 kräftigen, leicht gekrümmten Borsten. 2. Maxille und Maxilliped auf Stadium VI kaum als Wülste erkennbar.

Länge: N. I $0,11 \mathrm{~mm}$, N. VI $0,20-0,23$, C. I $0,37 \mathrm{~mm}$. 


\section{Übersicht der Gattungen.*)}

1. Zwischen den 1. Antennen und dem 1. Schwimmbeinpaar sind keine Gliedmaßen vorhanden Fam. Montrillidae (p. 201). Daselbst sind meistens sämtliche 5, wenigstens aber 2 Paar Gliedmaßen vorhanden

2. Enp. des 3. und 4. B. 3-gldr.

Enp. des 3. und 4. B. 2-gldr.

Enp. des 3. und 4. B. 1-gldr.

Mormonilla

Enp. des 3. B. 3-, des 4. B. 2-gldr. bis fehlend

Enp. des 3. B. 2-, des 4. B. 3-gldr.

Drepanopus

3. Enp. des 1. B. 3-gldr.

Enp. des 1. B. 2-gldr.

Enp. des 1. B. 1-gldr.

4. Kopf mit 2 großen Chitinlinsen, die meist am vorderen Stirnrand liegen, zuweilen auf die Ventralfläche des Kopfes rücken; Abdsegm. mit lateralen Verbreiterungen

Sapphirina

Kopf ohne Chitinlinsen

5. Am 1. Thsegm. in der antero-lateralen Ecke, rechts oder links, ein dunkelbrauner Knopf

Pleuromamma

Dieser Knopf fehlt

6. 1. Gl. des Enp. des 2. B. am Innenrande mit proximalwärts gekrümmten Häkchen

Metridia

Dieses Gl. wie an den übrigen B. gebaut

7. Grenze zwischen Vorder- und Hinterkörper (vergl. p. 164) hinter dem letzten Thsegm. liegend; 2. Antenne 2-ästig, mit meistens 5-bis 7-gldr. Exp., an beiden Ästen mit Fieder-, ohne Hakenborsten

Grenze zwischen Vorder- und Hinterkörper vor dem letzten Thsegm. liegend; 2. Antenne 1-ästig oder 2-ästig, in letzterem Fall mit höchstens 3-gldr. kleinem Exp.; am Ende des Enp. einige ungefiederte gekrümmte oder hakenförmige Borsten

8. Letztes Gl. des Exp. des 3. und 4. B. mit 2 Außenranddornen, von denen der distale am Randende sitzt, und 1 Enddorn

Letztes Gl. des Exp. des 3. und 4. B. mit 3 (selten verkümmerten) Außenranddornen und 1 Enddorn

9. Enddorn am Exp. des 3. und 4. B. mit breitem, glattrandigem Saum; Furka höchstens 2 mal so lang wie breit

Calanus

Enddorn am Exp. des 3. und 4. B. scharf gezähnelt; Furka mehr als 5 mal so lang wie breit

Limnocalanus

*) Diese Tabelle ist im allgemeinen nur für die Bestimmung der in dieser Arbeit aufgeführten nordischen Arten. zusammengestellt worden, sodaß erstens alle nicht im nordischen Plankton vertretene Gattungen daraus fortgelassen sind und zweitens die zur Unterscheidung benutzten Merkmale oft nur für die hier aufgeführten Arten eines Genus, nicht aber für das Genus in seinem ganzen Umfange Geltung haben sollen. 
10. 1. Antenne reicht höchstens bis zum Hinterrand des Kopfes

1. Antenne (jedenfalls die linke) reicht wenigstens bis gegen den Hinterrand des Vorderrumpfes

11. Exp. des 5. B. gegliedert Pseudocyclops Exp. des 5. B. ungegliedert Paramisophria

12. Keine der beiden 1. Antennen zu einem genikulierenden Greifapparat umgewandelt

Eine der beiden 1. Antennen zu einem, bisweilen undeutlichen Greifapparat umgewandelt

13. Exp. und Enp. des 5. B. 3-gldr.

Exp. des 5. B. 3-, Enp. 2-gldr.

Isochaeta

Exp. und Enp. des 5. B. 2 gldr.

Augaptilus

Exp. des 5. B. 3-, Enp. 1-gldr.

Isias 0

5. B. 1-ästig

14. Mittleres Gl. des Exp. des 5. B. mit Dornfortsatz am Innenrande

Centropages $q$

Dasselbe mit pfriem- oder säbelförmiger oder rudimentärer Borste am Innenrande

15. Eine Borste des linken Zweiges der Furka viel länger und dicker als die übrigen Furkalborsten

Furkalborsten symmetrisch

16. Proximale Loben der 2. Maxillen und die Borsten an ihnen sehr verkümmert Heterorhabdus ${ }^{1}{ }^{1}$ )

2. Maxillen mit gut entwickelten proximalen Loben und Borsten

17. 4. und 5. Thsegm. gesondert Mesorhabdus $q$

4. und 5. Thsegm. verschmolzen

Megacalanus ${ }^{2}$

18. Abdomen 3-gldr.; 2. Maxille mit eigentümlichen Anilängen an den distalen Borsten

Abdomen 4-gldr.; 2. Maxille ohne solche Anhänge

Augaptilus $q$

19. Endgl. des Enp. des 5. B. mit 5 Borsten

Endgl. des Enp. des 5. B. mit wenigstens 6 Borsten

Lucicutia 9

Haloptilus

20. 5. B. mit 2-gldr. Basp. und 3-gldr. Exp.

Phyllopus

5. B. aus höchstens $3 \mathrm{Gl}$. bestehend

21. 5. B. unvollkommen 2-teilig mit 1 End- und 1 Randborste Paraugaptilus 9

5. B. 3-gldr.

22. 5. B.: Fig. $158 \mathrm{c}$

5. B.: Fig. $160 \mathrm{~b}$

23. Greifantenne rechts

Greifantenne links

24. Beide Enp. des 5. B. 3-gldr., mit Fiederborsten

Dieselben rudimentär, ohne Fiederborsten

1) Vergl. die Tabelle für dieses Genus im Anhang S. 225.

2) Siehe auch S. 220. 
25. Die beiden Exp. des 5. B. ungleich, rechts mit Zange Centropages o Dieselben ähnlich, ohne Zange

26. Beide Exp. und Enp. des 5. B. 3-gldr.

Beide Exp. des 5. B. 3-gldr., die Enp. rudimentär oder fehlend 29

27. Eine Borste des linken Astes der Furka viel länger und dicker als die übrigen Furkalborsten

Heterorhabdus $\sigma^{71}$ )

Furkalborsten symmetrisch

28. Enp. der 1. Maxille vorhanden; distale Borsten der 2. Maxille mit Spitzen besetzt oder nackt

Haloptilus o

Enp. der 1. Maxille fehlt; distale Borsten der 2. Maxille mit hutpilzförmigen Anhängen

Augaptilus ơ

29. Enp. beiderseits vorhanden, 1-gldr., zipfelförmig

Arietellus o

Enp. nur einseitig vorhanden, groß und blattförmig; Exp. mit z. T. großen und breiten Gl.

Phyllopus o

Enp. beiderseits fehlend; Exp. nur wenig asymmetrisch, mit zylindrischen Gl.

Scottula o

30. Exp. des 1. B. 1-gldr.

Clytemnestra

Exp. des 1. B. 2- oder 3-gldr.

31. Exp. der 2. Antenne 1-gldr.; 5. B. (meistens) 1-gldr., langgestreckt; ein rudimentäres 6 . B. vorhanden

Exp. der 2. Antenne 2- oder 3-gldr.; 5. B. 2-gldr. blattförmig (beim $\sigma^{\text {T }}$ aber viel kleiner als beim $q$ )

Exp. der 2. Antenne fehlt; 5. B. 1- bis 3-gldr., gestreckt, stab- oder knopfförmig oder durch Borsten ersetzt; 6. B. fehlt

32. Exp. der 2. Antenne 3-gIdr.; Furka kurz

Microsetella

Exp. der 2. Antenne 2-gldr.; Furka lang

Halithalestris

33. 2. Maxille und Maxilliped ähnlich gebaut, beide mit langen, stacheligen Borsten versehen

Maxilliped mit wenigen (oder keinen) kurzen Borsten und einem endständigen Haken

34. 5. B. ganz rudimentär, jederseits auf 2 Borsten reduziert

Oithona

5. B. 2- oder 3-gldr.

Cyclopina

35. 5. B. 1-gldr., mit je 2 lanzettförmigen, gesäumten Anhängen am Ende; Rumpf langgestreckt

Lubbockia

5. B. (2-, 1-gldr. oder knopfförmig) mit nackten oder gefiederten Borsten versehen; Rumpf gedrungener

36. Hakenborsten am Endgl. der 2. Antenne von mittlerer Länge; Enp. der hinteren B. wenigstens so lang wie das Exp., sein Endgl. im 4. Paare wenigstens $11 / 2$ mal so lang wie das 1 . und 2 . zusammen

Oncaea Hakenborsten am langgestreckten Endgl. der 2. Antenne sehr lang; Enp. der hinteren B. kürzer als das Exp., sein Endgl. im 4. Paare nicht länger als jedes der beiden proximalen Gl.

Conaea

') Vergl. die Tabelle für dieses Genus im Anhang S. 225. 
37. 2. Antenne 2-ästig, mit mehrgl. Exp.: 1. Antenne mit wenigstens 15 Gl. 38 2. Antenne 1-ästig oder 2-ästig, in letzterem Fall mit kleinem 1-gldr. Exp.;

1. Antenne mit höchstens $9 \mathrm{Gl}$.

38. Enp. an beiden B. des 5. Paares vorhanden, 2- oder 3-gldr., mit Fiederborsten versehen

Lucicutia

Enp. des 5. B. ohne Fiederborste oder fehlend; zuweilen fehlt das ganze

5. B. einer- oder beiderseits

39

39. Furka lang und schmal, wenigstens 6 mal so lang wie breit

Temora

Furka höchstens 3 mal so lang wie breit

40. Mittelgl. des Enp. des 3. und 4. B. mit 1, sein Endgl. mit 5 Borsten 41 Mittelgl. des Enp. des 3. und 4. B. mit 2, sein Endgl. mit 7 Borsten 42

41. Exp. des 1. B. 3-gldr.; 5. B. beim $q$ fehlend, beim $\sigma^{7}$ beiderseits ohne Enp.

Eucalanus

Exp. des 1. B. 2-gldr.; 5. B. beim $q$ beiderseits 3-gldr, beim: $\sigma^{7}$ links mit Enp.

Rhincalanus

42. Enddorn der Exp. der B. skalpellförmig, mit ungezähneltem Saum; ơ ohne Greifantenne

Außenrand des Enddornes des Exp. des 2.-4. B. mit Zähnchen oder Spitzen besetzt; or mit Greifantenne

Temorites

43. Außenrand der Exp. der B. nicht gezähnelt; 5. B. beim † 3- bis 4-gldr., beim or rechts 4 -, links 5 -gldr.

Calocalanus

Außenrand der Exp. der hintereri B. gezähnelt; 5. B. beim q 2-gldr., beim or rechts 2-, links 5-gldr.

Paracalanus

44. Stirn konisch, vorn abgerundet; Rumpf sehr schmal; Exp. der 2. Antenne fehlt

Setella

Stirn zugespitzt; Rumpf breiter; Exp. der 2. Antenne 1-gldr.

45. 5. B. blattförmig, 4-eckig; ein 6. B. fehlt; Furka mit getrennten Ästen und Borsten

Euterpe

5. B. langgestreckt, schmal; ein 6. B. vorhanden; Äste der sehr kurzen Furka sowie ihre beiden ungemein langen Borsten in der Mittellinie verschmolzen

Aegisthus

46. Enp. des 2. B. 3-gldr.

Mecynocera

Enp des 2. B. 2-gldr.

47

Enp. des 2. B. 1-gldr.

47. 1. Antenne kurz, den Hinterrand des 1. Rumpfsegm. nicht erreichend, mit sehr großem 1. Gl.

Pseudocyclopia

1. Antenne denselben überragend

48. Flächen der Äste des 2.-4. B. (besonders der Enp. des 3. und 4. B.) mit größeren Stacheln und Dörnchen

Dieselben ohne Stacheln und Dörnchen

49. Vorderrumpf breit bis kuzelig

Phaenna

$\begin{array}{ll}" \text { gestreckt ellipsoidisch } & 50 \\ & 51\end{array}$

50. 5. B. fehit
5. B. vorhanden (bisweilen winzig, Fig. 81 b). 
51. Endg1. des Exp. des 2.-4. B. mit 5 Innenrandborsten Spinocalanus o " " " " " $\quad 4 \quad, \quad 2$

52. Gensegm. mit ventralem schaufelförmigen Vorsprung Scolecithrix (danae) $q$ ohne solchen Vorsprung

53. Rostrum einfach, ohne Fäden

Diaixis " klein, 2-spitzig

54. Abdomen 5-gldr., oft mit sehr kurzem Ansegm.; 5. B. asymmetrisch, beiderseits 1 - oder 2-ästig und meistens langgestreckt

Abdomen 4-gldr, zuweilen mit sehr kurzem und verdecktem Ansegm.;

5. B. symmetrisch (wenn asymmetrisch, dann winzig) beiderseits 1-ästig, 1 - bis 3-gldr.

55. Endgl. des Exp. des 2.-4. B. mit 5 Innenrandborsten Spinocalanus o

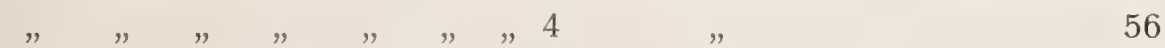

56. 5. B. rechts fehlend oder vorhanden, 1-ästig; links 1-ästig, 5-gldr., mit schmalen Gil.

$\left\{\begin{array}{l}\text { Xanthocalanus } 0^{7} \\ \text { Cornucalanus ot }\end{array}\right.$

5. B. links 2-, rechts 1-ästig oder beiderseits 2-ästig Scolecithrix or

5. B. groß, beiderseits 1-ästig, mit sehr unregelmäßigen Gil. und mehreren Auswüchsen

Diaixis ot

57. 5.B.1-gldr., blattförmig verbreitert oderwinzig und asymmetrisch Scolecithrix ?

5. B. 2-gldr.; von den 2 oder 3 Dornen des Engl. sind 1 oder 2 relativ lang

Scolecithrix $q$

5. B. 3-gldr.

58. Endgl. mit 3 Dornen, von denen 2 merklich länger sind als das Gl.

Scolecithrix (frontalis) 우

Dorne des Endgl. alle kurz (nur selten einer etwas länger als das Endgl.) 59

59. 1. Antenne viel kürzer als der Vorderkörper, an der Basis mit sehr dicken Gl.; alle Gl. des 5. B. mit zahlreichen Dörnchen und Stacheln besetzt Brachycalanus o

1. Antenne meistens länger als der Vorderkörper; wenn kürzer, das 5. B. nur spärlich bestachelt

60. Letzter Lobus der 2. Maxille mit sehr kräftigem langgebogenem Haken; Enp. des Maxillipeden mit 2 starken Hakenborsten Cornucalanus $q$

2. Maxille und Maxilliped mit weniger kräftig gebauten Borsten

\section{Xanthocalanus}

61. Basp. und Exp. im 2. und 3. B. breiter als im 4. B., 2. Gl. des Basp. mit Zacken am distalen Rande

Clausocalanus

2. und 3. B. ohne diese Unterschiede vom 4. B.

62. Außenranddorne des letzten Gl. des Exp. des 3. und 4. B. kammförmig, in tiefen Randkerben sitzend

Ctenocalanus

Dieselben von gewöhnlicher Dornform 63

63. 5. B. symmetrisch oder fehlend 64

5. B. asymmetrisch 83 
64. 5. B. vorhanden 65

5. B. fehlt ${ }^{1}$ ) 70

65. Endgl. des 5. B. klauenförmig oder schmal, fingerförmig 66 " " " anders geformt 69

66. Endgi. des 5. B. klauenförmig, stark gebogen Drepanopus " " " gerade oder leicht gebogen 67

67. 5. B. gedrungen, mit breitem Basalgl. und schmalem fingerförmigen Endgl.

5. B. anders geformt

Pseudophaenna 9

68. 5. B. symmetrisch oder wenn asymmetrisch, die beiden B. fast gleich lang

5. B. links merklich länger als rechts

Stephos 우

Parastephos o

Oothrix of

69. 5. B.: Fig. $78 b$

5. B.: Fig. $102 b$

70. Vorderkopf mit dorsalem Medianstachel

Tharybis ${ }^{\circ}$

Gaetanus ?

ohne solchen Stachel

71. Innenrand des 1. G1. des Basp. des 4. B. mit 5 Dornen

Innenrand des 1. Gl. des Basp. des 4. B. nackt oder behaart 72

72. 5. Thsegm. abgerundet 73

5. " beiderseits in einen spitzen Fortsatz verlängert oder mit einer

Zacke (Fig. 95 a und b). $\quad 76$

73. Gensegm. asymmetrisch Undeuchaeta $q$

" symmetrisch 74

74. Borsten der 5 letzten Gl. der 1. Antenne dick, quer geringelt Bradyetes $q$

Borsten dieser Gl. von gewöhnlicher Form $\quad 75$

75. 1. Gl. des Exp. des 1. B. ohne Außenranddorn; Enp. des 1. B. mit 4 Borsten Microcalanus $ᄋ$

1. Gl. des Exp. des 1. B. mit Außenranddorn; Enp. des 1. B. mit 5 Borsten Pseudocalanus o

76. Rostrum 2-spitzig 77

1-spitzig oder fehlend $\quad 80$

77. Exp. des 1. B. mit 2 Außenranddornen Aetideus $q$

" " $1 ., 3078$

78. Rostrum groß, mit 2 Zinken; Endborste des Exp. des 2.-4. B. mit zahlreichen, dichtgereihten, langen Zähnen Aetideopsis q

Rostrum klein, 2-spitzig $\quad 79$

79. Borsten der 6 letzten Gl. der 1. Antenne dick, quergeringelt Bradyidius $q$ Borsten dieser Gl. von gewöhnlicher Form Pseudaetideus $q$

80. Exp. der 2. Antenne länger als das Enp. $\quad 81$

1) Bei Pseudocalanus ㅇ ist bisweilen ein winziges 5. Beinpaar vorhanden (vergl. Fig. $22 \mathrm{c}$ und d).

2) Nach Wolfenden (1905) gehört diese Art zum Genus Euchirella Giesbr. 
Exp. der 2. Antenne $1 / 2$ so lang wie das Enp.

81. Exp. des 1. B. mit 3 Außenranddornen

82. Enp. der 2. Maxille mit wurmförmigen Schläuchen; 5. Thsegm. mit Zacke oder mit kleiner, aufwärts gebogener Spitze

Diaixis 0

2. Maxille nur mit Borsten bewehrt; 5. Thegm. mit nach hinten gerichtetem spitzem Fortsatz

83. 5. B.: Fig. 100 a.

5. B.: Fig. 238 b.

Chiridius $q$

5. B. anders geformt

84. 5. B. sehr groß und von kompliziertem Bau; Gl. unregelmäßig geformt und

z. T. stark verbreitert, mit mehreren Auswüchsen und Anhängen, aber ohne Enp. (Fig. 95 б, 97-101 ठ)

5. B. anders geformt; Gl. meistens alle schmal; 1 oder 2 Enp. vorhanden oder gänzlich fehlend

85. 1. Gl. des Exp. des 1. B. mit langem, dünnem Randdorn Diaixis o

1. " " " $" 1$. " ohne Randdorn

86. Vorletztes Gl. des linken 5. B. zylindrisch, Endgl. desselben löffelförmig, ohne Anhänge

Parastephos ot

Vorletztes Gl. des linken 5. B. verbreitert, Endgl. desselben mit 2 bis vielen Anhängen

87. 5. B. beiderseits ohne Enp. oder einerseits 1-ästig, anderseits fehlend 88

5. B. einer- oder beiderseits mit mehr oder weniger verkümmertem Enp. 93

88. 2. Maxille mit z. T. sensorischen Anhängen mit Pinselspitzen

2. Maxille nur mit Borsten bewehrt

Pseudophaenna ơ

89. Exp. des 1. B. mit 2 Außenrandborsten

" " 1. " "

90. 5. B. links 5-gldr., rechts fehlend

5. B. beiderseits vorhanden

91. Rostrum fehlend

" vorhandell

Aetideus

Microcalanus o

Chiridius ot

92. Rechtes 5. B. weniger als halb so lang wie das linke oder fehlend

Bradyidius o

Rechtes 5. B. ungefähr so lang wie das linke, mit nadelförmigem Endgl.

Pseudocalanus o $^{1}$ )

93. Rechtes 5. B. mit 3-gldr. Exp. und fast doppelt so langem stilettförmigem

Enp.; linkes B. ohne Enp.

Tharybis o

5. B. beiderseits mit einem Enp., das bisweilen stark verkümmert ist 94

94. Vorderkopf mit dorsalem Medianstachel

Gaetanus $0^{7}$

ohne solchen Stachel

1) Vergl. auch Drepanopus bungei (Fig. 26c), wovon das $\sigma^{\top}$ in ausgewachsenem Zustande nicht bekannt ist. 
95. Letztes Thsegm. abgerundet Undeuchaeta (spectabilis) $\sigma^{7}$ ) beiderseits in eine Spitze verlängert

96. Rostrum 1-spitzig; Exp. des 1. B. mit 2 Außenrandborsten Gaidius o "2-spitzig; " " 1. , , 3. " $\quad 97$

97. Enp. des rechten 5. B. sehr kurz Pseudaetideus o " " 5. "stilettförmig, länger als das" 2. Gl. des Basp. desselben B.

98. Vorderkopf mit medianem Kopfstachel

Bradyidius o

$$
\text { " ohne solchen Stachel }
$$

Gaetanus

99. 5. B. fehlt

5. B. asymmetrisch

100. 5. Thsegm. beiderseits in einen spitzen Fortsatz verlängert

5. " abgerundet oder in stumpfe Zipfel endigend

101. Exp. des 1. B. mit 3 Außenranddornen

" "1. " " 2

102. Rostrum klein, 2-spitzig

„ fehlend

103. Rostrum groß, in 2 dicke Zinken ausgehend " klein, 1-spitzig

104. Innenrand des 2. Gl. des Basp. des 4. B. mit Dörnchen oder Stacheln 105 " 2. " " " 4. " ohne Dörnchen 106

105 Exp. des 1. B. 3-gldr. Undeuchaeta (pustulifera) $\bigcirc^{1}$ ) , " 1. "2-gldr.

108. Exp. des 1. B. mit 2 Außenrandborsten; 5. B. einerseits fehlend Aetideus ${ }^{7}$ Exp. des 1. B. mit 3 Außenrandborsten; 5. B. beiderseits vorhanden

109. Rechtes 5. B. zangenförmig (Fig. 52c, 53c)

Chiridius o Rechtes 5. B. nicht zangenförmig

110. Exp. des linken 5. B. distal mit Zangenapparat (Fig. 49d, Fig. 56d, 59c) 111 5. B. ohne Zangenapparat

111. Enp. der 2. Antenne (meistens) so lang wie das Exp. Euchaeta o Enp. der 2. Antenne über halb so lang wie das Exp. Undeuchaeta (major) $\sigma^{7}$

112. Rostrum 1-spitzig; Exp. des 1. B. mit 2 Außenrandborsten Gaidius or Rostrum 2-spitzig; Exp. des 1. B. mit 3 Außenrandborsten Bradyidius ơ

113. Enp. des 1. B. 3-gldr.

Enp. des 1. B. 2-gldr. 
Kopf mit 1 oder 2 Paar Cuticularlinsen und beiderseits mit einem Randhaken

115. Endgi. des Exp. der 2. Antenne länger als die vorlergehenden Gl.; 5. B. des $q$ mit 1-gldr. Exp. und Enp.

Parapontella

Endgl. des Exp. der 2. Antenne rudimentär; 5. B. des $q$ 1-ästig, 2- oder 3 -gldr.

Tortanus

116. Kopf mit 2 Paar Augenlinsen

Anomalocera Kopf mit 1 Paar Augenlinsen

Pontella

117. Kopf mit 1 Paar Cuticularlinsen

Labidocera Kopf ohne dorsale Augenlinsen

118. Enp. des 5. B. gegliedert Enp. des 5. B. 1-gldr. oder fehlend

119. Maxilliped länger als die 2. Maxille; Furka lang und schmal, wenigstens 6 mal so lang wie breit

Temora Maxilliped kürzer als die 2. Maxille; Furka kurz

120. 1. Gl. des Maxillipeden mit wenigen, kurzen Borsten

Candacia Dasselbe mit langen, bestachelten Borsten

121. Letztes Thsegm. des $q$ flügelförmig verbreitert; Greifantenne des or mit breitgeschwollenen Mittelgl.

Paracartia Letztes Thsegm. abgerundet, zuweilen mit Dörnchen; Greifantenne des Oフ wenig umgeformt, mit nur schwach verdickten Mittelgl.

Acartia

122. Endgl. des 2.-4. B. mit 2 Außenranddornen; 5. B. beim $q$ am vorletzten Gl. mit Dornfortsatz am Innenrande, beim or beiderseits ohne Enp.

Eurytemora Endgl. des 2.-4. B. mit 3 Außenranddornen; 5. B. beim o ohne Dornfortsatz am Innenrande, beim ơ links mit griffelförmigem Enp. Undinella

123. Enp. des 4. B. 2-gldr.

Corina

Enp. des 4. B. 1-gldr. oder stummelförmig oder durch 1 oder 2 Borsten ersetzt

Corycaeus. 


\section{Literaturverzeichnis.}

\section{Aurivillius, C. W. S.}

1896. Das Plankton des baltischen Meeres. Bih. K. Sv. Vet.-Ak. Handl., v. 21.

1898. Vergleichende thiergeographische Untersuchungen über die Plankton-Fauna des Skageraks in den Jahren 1893-1897. K. Sv. Vet.-Ak. Handl., Bd. 30.

1899. Animalisches Plankton aus dem Meere zwischen Jan Mayen, Spitzbergen, K. Karls Land und der Nord-Küste Norwegens. K. Sv. Vet.-Ak. Handl. v. 32. No. 6 .

\section{Baird, W.}

1843. Notes on British Entomostraca. Zoologist (Newman) Vol. I.

1850. Natural History of the British Entomostraca.

\section{Bỏeck, A.}

1864. Oversigt over de ved Norges Kyster jagttagne Copepoder henhörende til Calanidernes, Cyclopidernes og Harpactidernes Familier. Vid. Selsk. Forhandl. Christiania.

1872. Nye Slaegter og Arter af Saltvandscopepoder. Vid. Selsk. Forhandl. Christiania.

\section{Bourne, G. C.}

1889. Report on the pelagic Copepoda collected at Plymouth in 1888 - 89. Journ. Mar. Biol. Ass. (2) Vol. 1.

1890. Notes on the genus Monstrilla Dana. Q. Journ. Micr. Sc. (2) Vol. 30.

\section{Brady, G. S.}

1872. Contributions to the Study of the Entomostraca. No. VII. A List of the non-parasitic marine Copepoda of the northeast Coast of England. Ann. Mag. Nat. Hist. (4) vol. 10.

1878-80. A Monograph of the free and semi-parisitic Copepoda of the British Islands. Vol. I, 1878; Vol. II und III, 1880.

1883. Report on the Copepoda collected by H. M. S. "Challenger" during the years 1873-1876. Challenger Report. Vol. 8.

1891. A Revision of the British Species of fresh-water Cyclopidae and Calanidae.

N. H. Trans. Northumb., Durh., Newcastle. Vol. II.

1902. N. H. Trans. Northumb., Durh., Newcastle, v. 14. 


\section{Brady, G. S. and D. Robertson.}

1873. Contributions to the Study of the Entomostraca. No. VIII. On Marine Copepoda taken in the West of Ireland. Ann. Mag. Nat. Hist. (4) vol. 12.

\section{Breemen, P. J. van.}

1903. Über das Vorkommen von Oithona nana Giesbr. in der Nordsee. Conseil permanent international pour l'exploration de la mer. Publications de Circonstance Nr. 7.

\section{Canu, E.}

1888. Les Copépodes libres marins du Boulonnais. 1. Les Calanidae; 2. Description d'Isias bonnieri. Bull. Scient. France Belg. 19.

1890. Idem 4. Les Calanides pélagiques. Bull. Scient. France Belg. 22.

1892. Etude zoologique de Temorella affinis, Ann. Station Aquicole Boulognesur-Mer, vol. I.

1896. Copepodes. Résult. scient. Camp. Caudan. Ann. Univ. Lyon. 26.

\section{Car, L.}

1884. Ein Beitrag zur Copepoden-Fauna des Adriatischen Meeres. Arch. Naturg. 50. Jahrg.

1890. Ein neues Copepoden-Genus (Sapphir) aus Triest. Arch. Naturg. 56. Jahrg.

\section{Claparède, A. R. Ed.}

1863. Beobachtungen über Anatomie und Entwicklungsgeschichte wirbelloser Tiere an der Küste von Normandie angestellt.

\section{Claus, C.}

1863. Die freilebenden Copepoden mit besonderer Berücksichtigung der Fauna Deutschlands, der Nordsee und des Mittelmeeres.

1866. Die Copepoden-Fauna von Nizza. Schriften Ges. Naturw. Marburg. 1. Suppl.-Heft.

1881. Über die Gattungen Temora und Temorella nebst den zugehörigen Arten. Sitzungsber. Ak. Wien, Math.-Nat. Cl. v. 83.

1891. Über Goniopelte gracilis, eine neue Peltidie. Arb. Zool. Inst. Wien. v. 9. 1893. Über die Entwicklung und das System der Pontelliden. Arb. Zool. Inst. Wien. v. 10.

\section{Cleve, P. T.}

1899. Plankton collected by the Swedish Expedition to Spitzbergen 1898. K. Sv. Vet.-Akad. Handl. v. 32, Nr. 7.

1899. Plankton-Researches in 1897. K. Sv. Vet.-Akad. Handl., v. 32. Nr. 7.

1900. The Plankton of the North Sea, the English Channel and the Skagerak in 1898. K. Sv. Vet.-Akad. Handl., v. 32, Nr. 8.

1900. Geographical distribution of Atlantic Copepoda and their physical conditions. Öfv. Vet.Ak. Förh. Arg. 57. 
1900. Notes on some Atlantic Plankton-organismus. K. Sv. Vet.-Ak. Handl. v. 34. Nr. 1.

1900. The Plankton of the North Sea, the English Channel and the Skagerak in 1899. K. Sv. Vet.-Akad. Handl., v. 34, Nr. 2.

1900. Plankton collected by the Swedish Expedition to Greenland in 1899 . K. Sv. Vet.-Akad. Handl.. v. 34, Nr. 3.

1901. The Seasonal Distribution of Atlantic Plankton Organisms.

1901. Plankton from the Indian Ocean and Malay Archipelago. K. Sv. Vet.-Ak. Handl., v. 35, Nr. 5.

1902. The Plankton of the North Sea and the Skagerak in 1900. K. Sv. Vet.Akad. Handl., v. 35, Nr. 7.

1903. Plankton-Researches in 1901 and 1902. K. Sv. Vet.-Akad. Handl., v. 36, Nr. 8.

1903. Report on plankton collected by Mr. Thorild Wulff. Arkiv Zool. v. 1.

Conseil permanent international pour l'exploration de la mer.

1902 - 1906. Bulletin des résultats acquis pendant les courses (croisières) periodiques. 1906. Catalogue des espèces de plantes et d'animaux observées dans le plankton recueilli pendant les expéditions périodiques depuis le mois d'août 1902 jusqu'au mois de mai 1905. Publications de circonstance, Nr. 33.

\section{Dahl, F.}

1893. Pleuromma, ein Krebs mit Leuchtorgan. Zool. Anz. v. 16.

1894. Leuchtende Copepoden. Zool. Anz. v. 17. p. 10-13.

1894. Ueber die horizontale und verticale Verbreitung der Copepoden im Ocean. Verh. Deutsch. Zool. Gesell. p. 61-80.

1894. Die Copepodenfauna des unterèn Amazonas. Ber. Naturf. Gesell. Freib. v. 8. 1896. Die Verbreitung freischwimmender Tiere im Ocean.

\section{Damas, D.}

1905. Notes biologiques sur les Copépodes de la mer norvégienne. Publications de circonstance, Nr. 22.

\section{Dana, J. D.}

1847-49. Conspectus crustaceorum in orbis terrarum circumnavigatione C. Wilkes, e classe reipublicae foederatae duce, collectorum. Proc. Am. Acad. Arts and Sci. 'v. 1, 2.

1852, 1855. Crustacea. U. S. Exploring Expedition during the years 1838 - 1842 under the Command of Charles Wilkes. v. 13, pt. 2; Atlas (1855).

\section{Esterly, C. 0.}

1905. The pelagic Copepoda of the San Diego Region. Univ. Cal. Publ, Zool. v. 2. Nr. 4.

1906. Additions to the Copepod Fauna of the San Diego Region. Univ. Cal. Publ., Zool. v. 3, Nr. 5. 
Farran, G. P.

1903. Record of the Copepoda taken on the Mackerel Fishing Grounds off Cleggan, Co. Galway, in 1901. Ann. Rep. Fish. Ireland, 1901.

1905. Report on the Copepoda of the Atlantic Slope off Counties Mayo and Galway. Ann. Rep. Fish. Ireland, 1902-03.

\section{Fischer, S.}

1851, 1853. Beiträge zur Kenntnis der in der Umgegend von St. Petersburg sich findenden Cyclopiden. Bull. Soc. Imp. Nat. Moscou. v. 24, 26.

\section{Foster, E.}

1904. Notes on the free-swimming Copepoda of the waters in the vicinity of the Gulf Biologic Station, Louisiana. 2nd Rep. Guif Biol. Stat.

\section{Giard, A.}

1895. Sur l'Ethologie du genre Thaumaleus Kröyer. C. R. Acad. Sc.

1896. Sur le parasitisme des Monstrillidae. C. R. Acad. Sc.

1897. Sur le parasitisme placentaire des Monstrillidae. C. R. Soc. Biol. (10) v. 4.

\section{Giesbrecht, W.}

1881. Vorläufige Mitteilung aus einer Arbeit über die freilebenden Copepoden des Kieler Hafens. Zool. Anz. v. 4.

1882. Die freilebenden Copepoden der Kieler Föhrde. 4. Ber. Comm. Wiss. Unt. Deutschen Meere, Kiel.

1888-91. Elenco dei Copepodi pelagici raccolti dal tenente di vascello G. Chierchia durante il viaggio della R. Corvetta "Vettor Pisani“ negli anni 1882-1885, e dal tenente di vascello F. Orsini nel Mar Rosso, nel 1884. Atti. Accad. Lincei Roma (4) Rend. v. 4, 1888; v. 5, 1889; v. 7, 1891.

1892. Systematik und Faunistik der pelagischen Copepoden des Golfes von Neapel. Fauna u. Flora Golf. Neapel. v. 19.

1895. Reports on the dredging operations off the west-coast of Central America to the Galapagos, to the west-coast of Mexico, and in the Gulf of California, in charge of Alex. Agassiz, carried on by the U. S. F. C. Steamer Albatross, during 1891. XVI. Die pelagischen Copepoden. Bull. Mus. Comp. Zool. Harvard, v. 25.

1896. Über pelagische Copepoden des Rothen Meeres. Zool. Jahrb. Syst. v. 9.

1897. Notizen zur Systematik der Copepoden. Zool. Anz. v. 20. p. 253.

1897. Zur Ontogenese der Monstrilliden. Zool. Anz. v. 20. p. 70.

1901. Mitteilungen über Copepoden. 12. Die litoralen Cyclopiden des Golfes von Neapel. Mitt. Zool. Stat. Neapel, v. 14.

1902. Copepoden. Résultats du Voyage du S. Y. Belgica.

1904. In: Le pesche abissali eseguite da F. A. Krupp col Yacht Puritan. Mitt. Zool. Stat. Neapel. v. 16.

\section{Giesbrecht, W. und O. Schmeil.}

1898. Copepoda. I. Gymnoplea. Das Tierreich. Lief. 6. 


\section{Gran, H. H.}

1902. Das Plankton des norwegischen Nordmeeres. Report on Norwegian Fishery- and Marine-Investigations, v. 2, Nr. 5.

\section{Grobben, C.}

1881. Die Entwicklungsgeschichte von Cetochilus septentrionalis. Wien.

\section{Gough, L. H.}

1905. Report on the Plankton of the English Channel in 1903. International Investigations. Mar. Biol. Ass. Report I. 1902-03.

\section{Guerne, J. de.}

1886. Description du Centropages grimaldii, Copépode nouvean du golfe de Finlande. Bull. Soc. Zool. France, v. 11.

1887. Sur les genres Ectinosoma Boeck et Podon Lilljeborg. Bull. Soc. Zool. France, v. 12.

\section{Guerne, J. de et J. Richard.}

1889. Revision des Calanides d'eau douce. Mém. Soc. Zool. France, v. 2.

\section{Gunner, J. E.}

1765. Nogle smaa rare, mestendelen nye norske Södyr, beskrevene. Skrift Kjöbenh. Selsk., v. 10.

\section{Hansen, H. J.}

1886. Dijmphna-Togtets zoologisk-botaniske Udbytte.

\section{Herrick, C. L. and C. H. Turner.}

1895. Synopsis of the Entomostraca of Minnesota. Rep. Geol. Nat. Hist. Surv. Minnesota, Zool. Ser. 2.

Hoek, P. P. C.

1878. De vrijlevende zoetwater-copepoden der Nederlandsche fauna. Tijdschr. Ned. Dierk. Ver., v. 3.

Jensen, S., A. C. Johansen og J. Chr. L. Levinsen.

De danske Farvandes Plankton i Aarene 1898-1901.

\section{Kojevnikov, G.}

1892. La Faune de la Mer Baltique orientale etc. Congrès international de Zoologie à Moscou, 1892.

\section{Kröyer, H.}

1838. Grönlands Amfipoder. - II. Beskrivelse af nogle Grönlandske Kraebsdyr udenfor Amfipodernen; III. Oversigt af de Grönlandske Kraebsdyr, ledsaget 
af nogle zoologisk-geografiske Bemaerkninger. Danske Vid. Selsk. Nat. Math. Afhandl. v. 7.

1842-45. Crustacés. Voyages de la commission scientifique du Nord en Scandinavie, en Laponie, au Spitzberg et aux Feröe pendant les années 1838, 1839 et 1840 sur la corvette "La Recherche"; herausg. von Paul Gaimard. (Atlas).

1848-49. Karcinologiske Bidrag. Naturh. Tidskrift. Kjöbenh., v. 2.

\section{Leuckart, $\mathrm{R}$.}

1859. Carcinologisches. Archiv Naturg. 25. Jahrg. 1. Bd.

\section{Lilljeborg, W.}

1853. De crustaceis ex ordinibus tribus: Cladocera, Ostracoda et Copepoda, in Scania occurrentibus.

\section{Lohmann, $\mathbf{H}$.}

1904. Eier und sogenannte Cysten der Plankton-Expedition.

\section{Lubbock, J.}

1854. On some Arctic Species of Calanidae. Ann. Mag. Nat. Hist. (2) v. 14. 1856. On some Entomostraca collected by Dr. Sutherland in the Atlantic Ocean. Trans. Entom. Soc. London (2) v. 4.

1857. Descreption of eigth new Species of Entomostraca found at Weymouth. Ann. Mag. Nat. Hist. (2) v. 20.

\section{Malaquin, A.}

1896. Parasitisme et évolution de deux Monstrillidae à l'interieur du système vasculaire des Filogranes et des Salmacynes. Ethologie. C. R. Acad. Sc. 1897. Evolution des Monstrillides. C. R. Ac. Sc.

1900. Nouvelles recherches sur l'évolution des Monstrillides. C. R. Acad. Paris, v. 130.

1901. Le parasitisme évolutif des Monstrillides. Arch. Zool. Exp. (3) v. 9.

\section{Möbius, K.}

1875. Zoologische Ergebnisse der Nordseefahrt 1872. IX. Copepoda und Cladocera. Jahresber. Komm. Unt. Deutsch. Meere Kiel, 2. und 3. Jahrg.

1887. Systematische Darstellung der Thiere des Plankton, gewonnen in der westlichen Ostsee und auf einer Fahrt von Kiel in den Atlantischen Ocean bis jenseits der Hebriden. 5. Ber. Komm. Wiss. Unt. Deutsch. Meere.

\section{Mrázek, A.}

1902. Arktische Copepoden. Fauna Arctica, v. 2.

\section{Müller, O. F.}

1792. Entomostraca seu Insecta testacea quae in aquis Daniae et Norvegiae reperit, descripsit et iconibus illustravit. 


\section{Nordgaard, $\mathbf{O}$.}

1898. Nogle oplysninger om Puddefjorden. Berg. Mus. Aarb. f. 1897.

1899. Undersögelser i fjordene ved Bergen 1897-98. Berg. Mus. Aarb. f. 1898.

1901. Undersögelser i Fjordene ved Bergen 1899. Berg. Mus. Aarb. f. 1900.

1905. Hydrographical and Biological Investigations in Norwegian Fjords. Bergens Museum.

\section{Nordquist, $\mathbf{0}$.}

1888. Die Calaniden Finnlands. Bidr. Känned. Finl. Nat. Folk, Heft 47.

\section{Norman, A. M.}

1903. Copepoda Calanoida, chiefly abyssal from the Faröe Cbannel and other parts of the North Atlantic. Journ. Linn. Soc. London, Zool., v. 29.

\section{Oberg, $M$.}

1905. Die Metamorphose der Plankton-Copepoden der Kieler Bucht. Wiss. Meeresunt. Abt. Kiel. N. F. Bd. 9.

\section{Paulsen, $\mathbf{O}$.}

1906. Studies on the Biology of Calanus finmarchicus in the Waters round Iceland. Meddelelser fra Komm. for Havunders. Plankton. v. 1, Nr. 4.

\section{Pearson, J.}

1905. A List of the Marine Copepoda of Ireland. I. Litoral Forms and Fish Parasites. Fish., Ireland, Sci. Invest., 1904, III.

1906. Idem. II. Pelagic Species. Fish., Ireland, Sci. Invest., 1905, VI.

\section{Philippi, A.}

1843. Fernere Beobachtungen über die Copepoden des Mittelmeeres. Archiv Naturg. 9. Jahrg., 1. Bd

\section{Poppe, S. A.}

1880. Über eine neue Art der Calaniden-Gattung Temora Baird. Abh. Nat. Verh. Bremen, v. 7.

1884. Über die von den Herren Dr. Arthur und Aurel Krause im nördlichen stillen Ocean und Behringsmeer gesammelten freilebenden Copepoden. Arch. Naturg. 50. Jahrg.

1885. Die freilebenden Copepoden des Jahdebusens. I. Abh. Nat. Ver. Bremen, v. 9. 1891. Beitrag zur Kenntnis der Gattung Clytemnestra Dana. Nat. Verh. Bremen, v. 12. 1891. a. Zur Literatur des Genus Monstrilla Dana. Nat. Ver. Bremen, v. 12.

\section{Prestandrea, N.}

1833. Su di alcuni nuovi crostacei del mare di Messina. Effemeridi scient. e litt. per la Sicilia, Palermo, v. 6. 
Redeke, H. C.

1902. Overzicht over de samenstelling van het Plankton der Oosterschelde. Bijlage C. Rapport over de oorzaken van den achteruitgang in hoedanigheid van de zeeuwsche oester door Dr. P. P. C. Hoek.

Redeke, H. C. en P. J. van Breemen.

1903. Plankton en Bodemdieren, in de Noordzee verzameld von 1 - 6 Aug. 1901. Tijdschr. Ned. Dierk. Ver. (2) v. 8.

\section{Richard, J.}

1893. Heterochaeta Grimaldii ' n. sp. Calanide nouveau etc. Bull. Soc. Z. France, v. 18.

1897. Entomostracées recueillies par M. Ch. Rabot à Jan Mayen et au Spitzberg. Bull. Soc. Z. France, v. 22.

\section{Sars, G. 0 .}

1884. In: J. Sparre Schneider. Undersögelser af Dyrelivet i de arktiske Fjorde.

II. Crustacea og Pycnogonida indsamlede i Kvänangs Fjorden 1881. Tromsö Mus. Aarsh., Nr. 7.

1885-86. Crustacea. Norske Nordhavs-Expedition 1876-1878.

1897. Pelagic Entomostraca of the Caspian Sea. Annuaire Mus. Zool. Petersb. 1898. The Cladocera, Copepoda and Ostracoda of the Jana Exped. Annuaire Mus. Zool. Petersb.

1900. Crustacea. The Norwegian North Polar Expedition 1893-1896. v. 1. 1903. An Account of the Crustacea of Norway. IV. Calanoida, Copepoda.

1903a(-06). Idem. V. Copepoda Harpacticoida.

1904. Description of Paracartia Grani G. O. Sars. Berg. Mus. Aarb. f. 1904.

1905. Liste préliminaire des Calanoidés recueillis pendant les campagnes de S.

A. S. le Prince Albert de Monaco. $1^{\text {re }}$ partie. Bulletin du Musée Océanographique de Monaco, Nr. 26; $2^{\mathrm{e}}$ partie. Ibidem, Nr. 40.

1907. Notes supplémentaires sur les Calanoidés de la Princesse Alice. Bulletin de l'Institut Océanographique, Nr. 101.

\section{Schmeil, 0 .}

1892 - 98. Deutschlands freilebende Süßwasser-Copepoden. Bibliotheca Zoologica.

I. Cyclopidae, 1892; II. Harpacticidae, 1893; III. Centropagidae, 1896; Nachtrag, 1898.

1898. Vergl. Giesbrecht und Schmeil.

\section{Scott, A.}

1896. On Scolecithrix hibernica, a new species of Copepod, with some remarks on the distribution of the Crustacea. Ann. Mag. Nat. Hist. (6) v. 18.

1902. On some Red Sea and Indian Ocean Copepods. Trans. Liv. Biol. Soc., v. 16.

- and I. C. Thompson siehe unter Thompson. 
Scott, A. and T.

\section{Scott, $\mathrm{T}$.}

1901. On some Entomostraca collected in the Arctic Seas in 1898 by W. S. Bruce. Ann. Mag. Nat. Hist. (7) v. 8.

1891. Additions to the Fauna of the Firth of Forth. 9. Ann. Rep. Fish. B. Scotl., pt. 3, p. 300.

1892. Idem. 10. Ann. Rep. F. B. Scotl., pt. 3, p. 244.

1893. Idem. 11. Ann. Rep. F. B. Scotl., pt. 3, p. 197.

1893. Report on Entomostraca from the Gulf of Guinea. Trans. Linn. Soc. London, (2) v. 6.

1894. Idem. 12. Ann. Rep. F. B. Scotl., pt. 3, p. 231.

1895. Idem. 13. Ann. Rep. F. B. Scotl., pt. 3, p. 165.

1896. Idem. 14. Ann. Rep. F. B. Scotl., pt. 3, p. 158.

1897. The Marine Fishes and Invertebrates of Loch Fyne. 15. Ann. Rep. F. B. Scotl., pt. 3, p. 107.

1897a. Notes on the Animal Plankton form H. M. S. „Research“. 15. Ann. Rep. F B. Scotl., pt. 3, p. 305.

1898. On the Distribution of Pelagic Invertebrate Fauna of the Firth of Forth and its Vicinity during the Seven Years form 1889 to 1895 , both inclusive. 16. Ann. Rep. F. B. Scotl., pt. 3, p. 153.

1898. Some Additions to the Invertebrate Fauna of Loch Fyne. 16. Ann. Rep. F. B. Scotl., pt. 3, p. 261.

1899. Notes on Recent Gatherings of Microcrustacea from the Clyde and the Moray Firth. 17. Ann. Rep. F. B. Scotl., pt. 3, p. 248.

1900. Notes on some Gatherings of Crustacea collected for the most part on Board the Fishery Steamer "Garland" and examined during the past year (1899). 18. Ann. Rep. F. B. Scotl., pt. 3, p. 382.

1901. Notes on Gatherings of Crustacea, collected for the most part by the Fishery Steamer "Garland" etc., and examined during the year 1900. 19. Ann. Rep. F. B. Scotl., pt. 3, p. 235.

1902. Notes on Gatherings of Crustacea, collected by the Fishery Steamer „Garland“ etc. during the year 1901. 20. Ann. Rep. F. B. Scotl., pt. 3. p. 447.

1903. On some New and Rare Crustacea collected at various times in connection with the Investigations of the Fishery Board for Scotland. 22. Ann. Rep. F. B. Scotl., pt. 3, p. 109.

1903a. Notes on some Copepoda form the Arctic Seas collected by A. M. Norman. Ann. Mag. Nat. Hist. (7) v. 11, p. 4.

1904. Notes on some rare and interesting Marine Crustacea. 22. Ann. Rep. F. B. Scotl., pt. 3, p. 242.

1905. On some New and Rare Crustacea from the Scottish Seas. 23. Ann. Rep. F. B. Scotl., pt. 3, p. 141.

\section{Templeton, $\mathbf{R}$.}

1837. Description of a new Irish Crustaceous Animal. Trans. Entom. Soc. London v. 2. 
Thompson, J. C.

1887. Second Report on the Copepoda of Liverpool Bay. Proc. Biol. Soc. Liverpool, v. 2.

1888. Copepoda of Madeira and the Canary Islands. Journ. Linn. Soc. London, v. 20,1890 .

1890. Monstrilla and the Cymbasomatidae. Trans. Biol. Soc. Liverp., v. 4.

1895. Recent Additions to the Copepoda of Liverpool Bay. Proc. (Trans.) Biol. Soc. Liverp., v. 9.

1896. Revised Report on the Copepoda of Liverpool Bay. Rep. Fauna Liverp. Bay, v. 4.

1896. Free swimming Copepoda from the west coast of Ireland. Trans. Liv. Biol. Soc., v. 10.

1898. Contributions to our knowledge of the plankton of the Faroe Channel Nr. 4. Report on the Copepoda collected by Dr. G. H. Fowler. Proc. Z. Soc. London.

1899. Report on the free swimming Copepoda (1895-98) of Valencia Harbour, Ireland. Proc. R. Irish Acad. (3) v. 5.

1900. Report on two collections of tropical and more northerly plankton. Trans. Liv. Biol. Soc., v. 14.

1903. Report on the Copepoda obtained by Mr. George Murray during the Cruise of the „Oceana“" in 1898. Ann. Mag. Nat. Hist. (7) v. 12.

\section{Thompson, J. C. and A. Scott.}

1897. On the Plankton collected continously during two transverses of the North Atlantic in the summer of 1897. Trans. Biol. Soc. Liverpol., v. 12.

1900. Some recent additions to the Copepoda of Liverpool Bay. Trans. Liv. Biol. Soc., v. 14.

1903. Report on the Copepoda collected by Prof. Herdman at Ceylon in 1902.

\section{Timm, R.}

1896. Die Copepoden und Cladoceren Helgolands. Wiss. Meeresunt., v. 1.

1896a. Copepoden und Cladoceren. Beiträge zur Fauna der südöstlichen und östlichen Nordsee. Wiss. Meeresunt., v. 1.

1905. Copepoden. Jahrb. Wiss. Anst. Hamburg, v. 20, 2. Beiheft.

\section{Vanhöffen, E.}

1895. Das Leuchten von Metridia longa. Zool. Anz.

1897. Die Fauna und Flora Grönlands. Grönland-Expedition der Gesellschaft für Erdkunde zu Berlin, 1891—93.

\section{Wheeler, W. M.}

1899. The freeswimming Copepods of the Woods Hole region. Bull. U. S. Fish. Comm., v. 19. 
Williams, L. W.

1906. Notes on marine Copepoda of Rhode Islands, Amer. Nat., v. 40.

Wolfenden, R. N.

1902. The plankton of the Faröe Channel and Shetland. Journ. Mar. Biol. Ass. (2) v. 6.

1903. On the Copepod sub-family Aetidiinae, with a proposed revision of the classification. Rep. 72. Meet. Brit. Ass. Adv. Sci.

1903a. In: G. H. Fowler. Plankton of the Faröe Channel. Nr. 8. Proc. Zool. Soc. London, v. 1.

1904. Notes on the Copepoda of the North Atlantic Sea and Faröe Channel. Journ. Mac. Biol. Ass. (2) v. 7.

1905. Plankton Studies, Part I, Copepoda. 


\section{Druckfehler.}

Seite 1 Zeile 5 von unten lies des Maxillipeden statt der Maxillipeden.

" 14 " 8 von oben lies ihr statt ihren.

" 41 " 10 von oben lies 1 - gldr. statt 2-gldr.

$" 44$ " 9 von unten hinzuzufügen: 1900, U. s., Sars, p. 59, t. 15, 16.

„56 " 7 von oben lies 2 - statt 1 -.

" 68 " 11 von oben lies U. o. statt U. O.

" 78 " 2 von oben hinter Scott hinzuzufügen: p. 452.

" 96 Zwischen Seite 96 und 97 sind folgende Zeilen ausgefallen:

sehr kurzem Enp.; 5. B. des $\sigma^{7}$ mit verbreiterten Endgl., jedes Endgl. mit 4 Randborsten. - L. O, $\sigma^{\pi} 1,25-1,35 \mathrm{~mm}$.

Mittelmeer, Atlant. Ozean.

\section{Temora Baird.}

q. Kopf vom Thorax getrennt; letztes Thsegm. mit vorletztem verschmolzen; Rostralfäden weich. Abdomen 3-gldr.; Furka lang und schmal, zuweilen asymmetrisch. 1. Antenne 24 -gldr., die beiden letzten Gl. verschmolzen. Exp. der 2. Antenne kaum länger als das Enp. Mundgliedmaßen denen von Calanus ähnlich; distale Borsten der 2. Maxille nicht verlängert. Exp. des 1.-4. B. 3-gldr., aber am 2.-4. B. des $q$ und zuweilen am linken 2. B. des $\sigma^{x}$ mit aufgehobener Artikulation zwischen den beiden proximalen Gl.;

„ 107 Zwischen Zeile 4 und 5 von oben einzuschalten: 1893, P. b., Dahl, p. 105.

" 117 Zeile 3 von unten lies 5. B. beiderseits mit 3-gldr. Exp. und Enp.; Endgl. des Exp. meistens hakig statt Endgl. des 5. B. beiderseits 3-gldr.; Exp. meistens hakig.

"118 Eine neue Bestimmungstabelle für das Genus Heterorhabdus findet sich im Anhange.

"144 Zeile 2 von oben lies $q$ statt $\sigma^{x}$. 


\section{Alphabetisches Register.}

abdominale (Pleuromma)
abdominale var. abyssalis (Pleu-
romma)
abdominalis (Diaptomus)
abdominalis (Pleuromamma)
abyssalis (Heterochaeta)
abyssalis (Heterorhabdus)
abyssalis (Pleuromma abdominale
$\quad$ var.)
abyssalis (Spinocalanus)
Acartia
aciculus (Setella)
acrocephalus (Scaphocalanus)
aculeatus (Aegisthus)
aculeatus (Rhincalanus)
acuspes (Lucullus)
acuta (Euchaeta)
acuta (Labidocera)
acuta (Pontella)
acutifrons (Euterpe)
acutifrons (Harpacticus)
acutifrons (Haloptilus)
acutifrons (Hemicalanus)
acutum (Labidocera)
Aegisthus
Aetideopsis
Aetideus
affinis (Eurytemora)
$\quad$ (Temora)
affinis var. hirundoides (Eurytemora)
affinis var. hirundoides (Temorella)
affinis var. hispida (Temorella)
affinis var. raboti (Eurytemora)
anglica (Monstrilla)
anglicus (Corycaeus)
angulata (Chirundina)
angulata (Heterochaeta)
$\quad$

Pag. angustata (Ichthyophorba)

Pag. annectens (Mesorhabdus)

Anomalocera

arcuicornis (Calanus) 23

arcuicornis (Clausocalanus) 23

Arietellus $\quad 139$

armata (Candace) $\quad 146$

armata (Candacia) $\quad 146$

armata (Euchaeta) $\quad 32$

armata (Metridia) 108, 108

armatus (Aetideus) 30

armatus (Aetidius) 30

armatus (Bradyidius) 31

armatus (Chiridius) $\quad 32$

armatus (Chiridius) 34

armatus (Pseudaetideus) 32

armatus (Pseudocalanus) 30, 31, 35

armiger (Aetidius)

armiger (Gaetanus)

atlantica (Euchirella)

221

atlantica (Euchirella curticauda var.) 48, 221

atlantica (Lucicutia) 116

atlantica (Microsetella) $\quad 173$

atlantica (Mormonilla) 166

atlanticum (Ectinosoma) 173

atlanticus (Aegisthus) 184

atlanticus (Brachycalanus) 66

atlanticus (Eucalanus) $\quad 15$

atlanticus (Gaetanus) $\quad 39,40$

atlanticus (Heterorhabdus) 227

atlanticus (Scolecithrix) 75

atlanticus (Xanthocalanus) 66

attenuatus (Calanus) 16

attenuatus (Eucalanus) 16

Augaptilus . 131

auropecten (Scolecithrix) 74

barbata (Euchaeta) 54

bidentata (Oothrix) 67 
Alphabetisches Register.

VIII 259

bidentatus (Phyllopus)

bifilosa (Acartia)

bifilosa var. intermedia (Acartia)

bifilosus (Dias)

bonnieri (Isias)

boreale (Pleuromma)

borealis (Gaidius)

boreatis (Pleuromamma)

borealis (Xanthocalanus)

Brachycalanus

Bradyetes

Bradyidius

bradyi (Megacalanus)

bradyi (Scolecithrix)

bradyi (Undinopsis)

brevicauda (Metridia)

brevicaudatus (Heterorhabdus)

brevicaudatus (Mesorhabdus)

brevicornis (Bryaxis)

brevicornis (Parapontella)

brevicornis (Pontellina)

brevicornis (Scolecithrix)

brevifida (Microsetella)

brevis (Temorites)

brevispinus (Gaidius)

brevispinus (Chiridius)

Bryaxis

buchani (Paraugaptilus)

buchani (Arietellus)

bumpusii (Corynura)

bungei (Drepanopus)

Calanidae

Calanus

Calocalanus

Candacia

Candaciidae

carinata (Euchaeta)

carinata (Euchirella)

caudani (Gaetanus)

candata (Pseudocyclopia)

Centropages

Centropagidae

centrura (Acartia)

chelifer (Onchocalanus)

chelifer (Scolecithrix)

chierchiae (Centropages)

Chiridius

Chirundina

claparedii (Thaumaleus)

clausi (Acartia)

clausi (Heterorhabdus)

clausi (Lucicutia)

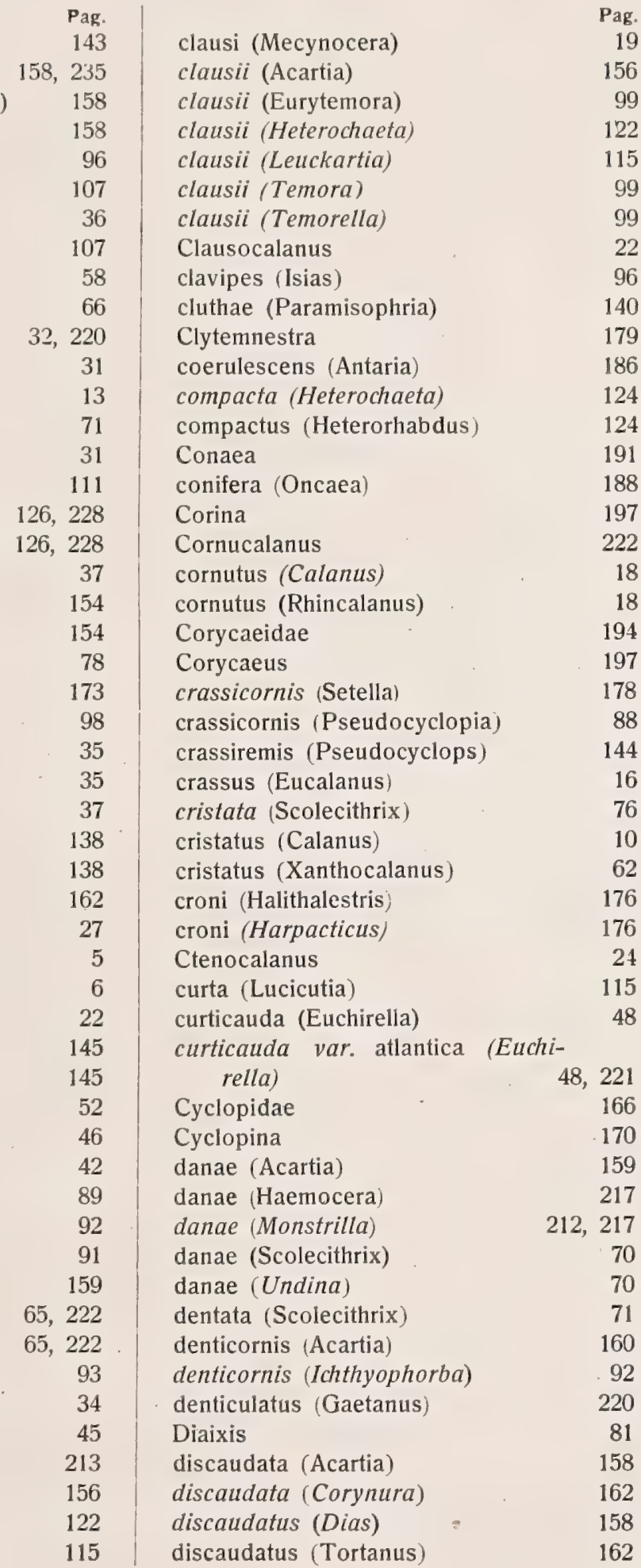


VIII 260

Drepanopus

dubia (Monstrilla)

echinata (Scolecithrix)

elegans (Cyclopina)

elongata (Candace)

elongata (Clausia)

elongatus (Calanus)

elongatus (Eucalanus)

elongatus (Pseudocalanus)

emarginata (Scolecithrix)

Eucalanus

Euchaeta

Euchirella

Eurytemora

Euterpe

fertilis (Haloptilus)

fertilis (Hemicalanus)

filigerus (Augaptilus)

filigerus (Hemicalanus)

filogranarum (Haemocera)

filogranarum (Thaumaleus)

finmarchica (Temora)

finmarchicus (Cälanus)

finmarchicus (Monoculus)

flavicornis (Leuckartia)

flavicornis (Lucicutia)

forcipata (Acartia)

fragilis (Xanthocalanus)

frontalis (Lophothrix)

frontalis (Scolecithrix)

fultoni (Stephos)

Gaetanus

Gaidius

gemma (Sapphirina)

germanicus (Thaumaleus

germanus (Corycaeus)

gibbus (Augaptilus)

giesbrechti (Pseudocyclopia)

giesbrechti (Xanthocalanus)

gigas (Rhincalanus)

glacialis (Augaptilus)

glacialis (Euchaeta)

glacialis (Lubbockia)

gracile (Pleuromma)

gracilicauda (Monstrilla)

gracilis (Calanus)

gracilis (Cyclopina)

gracilis (Eurytemora)

gracilis (Euterpe)

gracilis (Goniopelte)

gracilis (Pleuromamma)

gracilis (Pseudocalanus)
Alphabetisches Register.

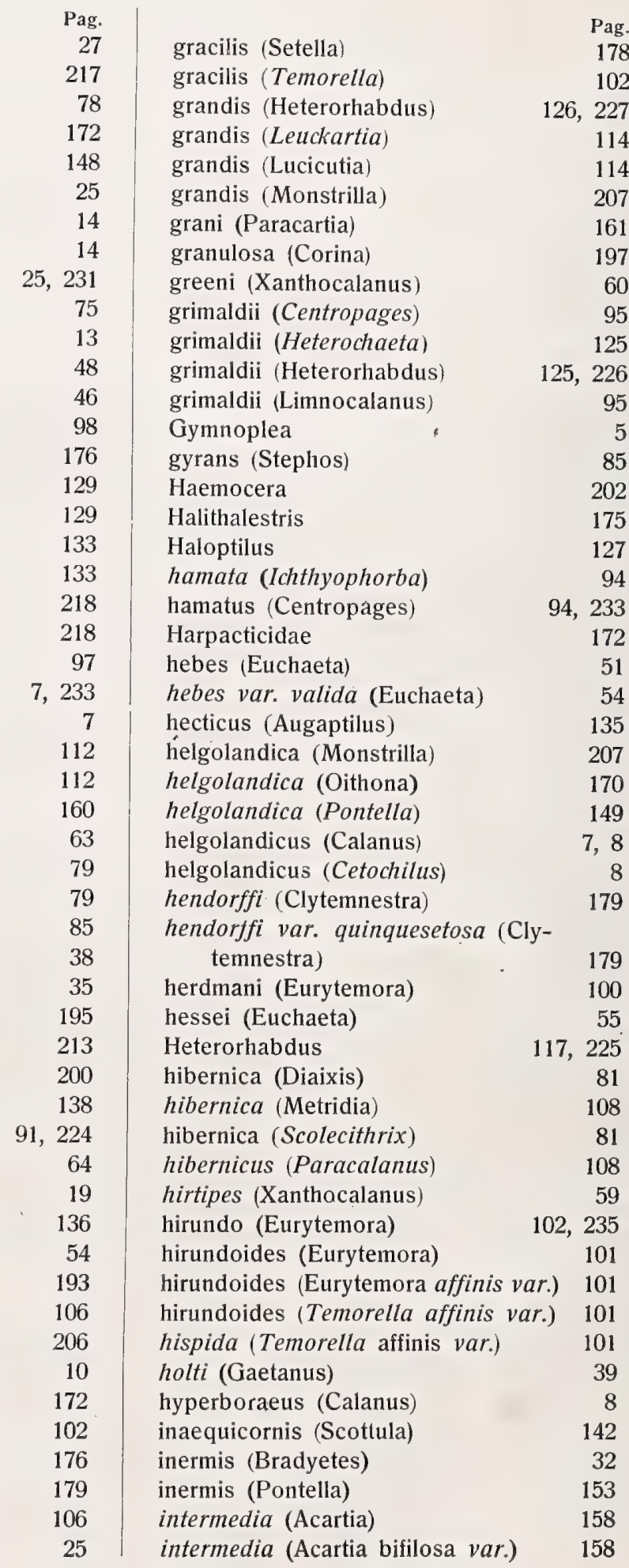


intermedia (Monstrilla)

intermedius (Dias)

iris (Sapphirina)

Isias

Isochaeta

kröyeri (Labidocera)

kröyeri (Pontella)

kruppi (Gaetanus)

Labidocera

lacinulata (Cyclopsina)

lacinulata (Eurytemora)

lamellatus (Stephos)

laticeps (Augaptilus)

latifrons (Gaetanus)

latus (Calanus)

laxa (Acartia)

limbatus (Corycaeus)

Limnocalanus

litoralis (Cyclopina)

litoralis (Cyclops)

lobiancoi (Iva)

lobiancoi (Pontella)

lobiancoi (Pontellina)

longa (Metridia)

longicauda (Setella)

longicaudata (Cyclopina)

longicaudatus (Augaptilus)

longicaudatus (Hemicalanus)

longicornis (Cyclops)

longicornis (Cyclopsina)

longicornis (Halitemora)

longicornis (Haloptilus)

longicornis (Hemiculanus)

longicornis (Heterochaeta)

longicornis (Heterorhabdus)

longicornis (Leuckartia)

longicornis (Lucicutia)

longicornis (Megacalanus)

longicornis (Macrocalanus)

longicornis (Monstrilla)

longicornis (Spinocalanus)

longicornis (Spinocalanus)

longicornis (Temora)

longiremis (Acartia)

longiremis (Acartia)

longiremis (Cetochilus)

longiremis (Dias)

longiremis (Monstrilla)

longiserrata (Leuckartia)

longiserrata (Lucicutia)

longisetosus (Isochaeta)

longispinosa (Monstrilla)

\begin{tabular}{r|lr} 
Pag. & & Pag. \\
205 & longispinosus (Thaumaleus) & 211 \\
158 & longus (Calanus) & 108 \\
195 & Lubbockia & 192 \\
$\subseteq 6$ & lucens (Metridia) & 108 \\
117 & Lucicutia & 111 \\
151 & macrophthalmus (Tharybis) & 87 \\
151 & magna (Amallophora) \\
41 & magna (Lucicutia) & 76 \\
149 & magna (Scolicithrix) & 117 \\
99 & magnus (Augaptilus) & 76 \\
99 & magnus (Cornucalanus) & 138 \\
83 & magnus (Spinocalanus) & 222 \\
133 & major (Gaetanus) & 29 \\
39 & major (Gaidius) & 40 \\
47 & major (Heterochaeta) & 35 \\
& mat & 126
\end{tabular}

$\begin{array}{lr}\text { major (Heterorhabdus) } & 126,227 \\ \text { major (Pseudocalanus) } & 25\end{array}$

major (Scolecithrix) $\quad 79$

major (Undeuchaeta) 43

marina (Euchaeta) $\quad 50$

marinus (Cyclops) 50

mastigophorus (Calanus) 23

Mecynocera 19

media (Oncaea) 187

mediterranea (Antaria) 187

mediterranea (Calanella) 16

mediterranea (Oncaea) 187

Megacalanus $\quad 13,220$

Mesorhabdus $\quad 126$

messinensis (Setella) $\quad 178$

Metridia 107

Microcalanus $\quad 26$

Microsetella $\quad 173$

miles (Gaetanus) $\quad 39$

minor (Bryaxis) 37

minor (Gaetanus) 41

minor (Pseudocyclopia) $\quad 89$

minor (Scolecithricella) 73

minor (Scolecithrix) 73

minor (Stephos) 84

minor (Undeuchaeta) 44

minuta (Lubbockia) 193

minuta (Oithona) 170

minuta (Oithona) 170

minuta (Oncaea) 188

Monstrilla - 202

Monstrillidae 201

Mormonilla 165

Mormonillidae $\quad 165$

mucronatus (Aegisthus) 183

multiserrata (Faroella) 33

nana (Oithona) $\quad 170$ 
VIII 262

nasutus (Rhincalanus) nigromaculata (Sapphirina) normani (Metridia) norvegica (Candace) norvegica (Candacia) norvegica (Euchaeta) norvegica (Heterochaeta) norvegica (Microsetella) norvegica (Setella) notopus (Oncaea) norvegicus (Heterorhabdus) oblonga (Undinella) obtusa (Candacia) obtusatus (Pseudocyclops) obtusifrons (Amallophora) obtusifrons (Chiridius) obtusifrons (Scolecithrix) obtusus (Xanthocalanus) oceana (Euchaeta)

Oithona

Oncaea

Oncaeidae

Onchocalanus

Oothrix

ornata (Oncaea)

ornatus (Haloptilus)

ornatus (Hemicalanus)

ovata (Scolecithrix)

pallidus (Parastephos)

palumbii (Augaptilus)

palumboi (Augaptilus)

papilligera (Heterochaeta)

papilliger (Heterorhabdus)

Paracalanus

Paracartia

Paramisophria

Parapontella

Parastephos

Paraugaptilus

parvus (Calanus)

parvus (Corycaeusi

parvus (Paracalanus)

patersoni (Anomalocera)

patersonii (Anomalocera) patersonii (Irenaeus)

pavo (Calanus)

pavo (Calocalanus)

pectinata (Candace)

persecans (Scolecithrix)

Phaenna

phasma (Mormonilla)
Alphabetisches Register.

\begin{tabular}{|c|c|c|}
\hline $\begin{aligned} \text { Pag. } \\
18\end{aligned}$ & philippi (Euchaeta)1) & \\
\hline 196 & Phyllopus & \\
\hline 109 & pileatus (Gaetanus) & \\
\hline 147 & pinguis (Xanthocalanus) & \\
\hline 147 & Pleuromamma & \\
\hline 52 & plumifera (Oithona) & \\
\hline 121 & $\begin{array}{l}\text { Podoplea } \\
\text { polaris (Mormonilla) }\end{array}$ & \\
\hline 173 & $\begin{array}{l}\text { polaris (Mormonilla) } \\
\text { Pontella }\end{array}$ & \\
\hline 173 & $\begin{array}{l}\text { Pontella } \\
\text { Pontellidae }\end{array}$ & \\
\hline 189 & poppei (Chiridius) & \\
\hline 121 & prestandreae (Euchaeta) & \\
\hline 68 & princeps (Calanus) & \\
\hline 148 & princeps (Megacalanus) & \\
\hline 144 & princeps (Megacalanus) & \\
\hline 75 & princeps (Metridia) & \\
\hline 34 & propinquus (Calanus) & \\
\hline 75 & propinquus (Calanus) & \\
\hline 61 & propinquus (Xanthocalanus) & \\
\hline 221 & Pseudaetideus & \\
\hline 166 & Pseudocalanus & \\
\hline 184 & Pseudocyclopia & \\
\hline 184 & Pseudocyclopidae & \\
\hline 222 & Pseudocyclops & \\
\hline 67 & Pseudophaenna & \\
\hline 191 & pulchra (Euchaeta) & \\
\hline 130 & $\begin{array}{l}\text { pulchra (Euchirella) } \\
\text { pulchra (Undina) }\end{array}$ & \\
\hline 130 & $\begin{array}{l}\text { pulchra (Undina) } \\
\text { pungens (Gaidius) }\end{array}$ & \\
\hline $\begin{array}{r}72 \\
223\end{array}$ & $\begin{array}{l}\text { pungens (Gaidius) } \\
\text { pungens (Gaidius) }\end{array}$ & \\
\hline $\begin{array}{l}223 \\
134\end{array}$ & $\begin{array}{l}\text { pungens (Gaidius) } \\
\text { pusillus (Microcalanus) }\end{array}$ & \\
\hline $\begin{array}{l}134 \\
134\end{array}$ & pustulifera (Undeuchaeta) & \\
\hline 120 & pygmaea (Diaixis) & \\
\hline 120 & pygmaea (Oithona) & \\
\hline 20 & pygmaea (Scolecithrix) & \\
\hline 160 & pygmaeus (Microcalanus) & \\
\hline 140 & pygmaeus (Pseudocalanus) & \\
\hline 154 & pyriformis (Oncaea) & \\
\hline 223 & quinquesetosa (Clytemnestra hen- & \\
\hline 138 & dorffi var.) & \\
\hline 20 & raboti (Eurytemora affinis var.) & \\
\hline 198 & rapax (Conaea) & \\
\hline 232 & rattrayi (Augaptilus) & \\
\hline 153 & Rhincalanus & \\
\hline 153 & rigidum (Cymbasoma) & \\
\hline 153 & rigidus (Thaumaleus) & \\
\hline 22 & robusta (Pleuromamma) & \\
\hline 22 & robustum (Pleuromma) & \\
\hline 146 & roscovita (Haemocera) & \\
\hline
\end{tabular}

1) Species incerti generis; wird für den Nordatlantischen Ozean erwằhnt. 
rosea (Microsetella)

roseus (Canthocamptus)

rostrata (Aetideopsis)

rostrata (Clytemnestra)

rostrata (Euchirella)

rostrata (Undina)

rostratus (Corycaeus)

rostratus (Goniopsyllus)

rostratus (Sapphir)

rostratus (Thaumaleus)

rostrifrons (Calanus)

rotunda (Candacia)

römeri (Scolecithrix)

salpae (Sapphirina)

Scaphocalanus Sapphirina

.76. Schaudinni (Spinocalanus)

schneideri (Cyclopina)

Scolecithrix

scotti (Stephos)

Scottula

scutellata (Clytemnestra)

securifrons (Scolecithrix)

securifrons (Scottocalanus)

serrulata (Thalestris)

Setella

setigera (Oithona)

setosus (Arietellus)

similis (Bradyidius)

similis (Oithona)

similis (Scolecithrix)

similis (Undinopsis)

simillimus (Calanus)

simplex (Cornucalanus)

simplex (Xanthocalanus)

speciosus (Corycaeus)

spectabilis (Undeuchaeta)

spiniceps (Haloptilus)

spiniceps (Hemicalanus)

spinifera (Phaenna)

spinifrons (Hemicalanus)

spinifrons (Heterochaeta)

spinifrons (Heterorhabdus)

spinirostris (Oithona)

Spinocalanus

spinosa (Euchaeta)

\begin{tabular}{|c|c|c|}
\hline $\begin{array}{l}\text { Pag. } \\
174\end{array}$ & spinulosus (Aegisthus) & $\begin{array}{r}\text { Pag. } \\
182\end{array}$ \\
\hline 174 & squamatus (Augaptilus) & 132 \\
\hline 33 & stephoides (Pseudocyclopia) & 90 \\
\hline 180 & Stephos & 82 \\
\hline 47 & streetsi (Chirundina) & 46 \\
\hline 47 & styliremis (Calocalanus) & 22 \\
\hline 198 & subagilis (Xanthocalanus) & 62 \\
\hline 180 & subtilis (Oncaea) & 190 \\
\hline 180 & Temora & 97 \\
\hline 216 & Temorites & 97 \\
\hline 18 & tenuicornis (Calanus) & 11 \\
\hline 148 & tenuicornis (Setella) & 178 \\
\hline 74 & tenuirostris (Aetideus) & 30 \\
\hline 195 & tenuis (Setella) & 178 \\
\hline 194 & tenuispinus (Gaidius) & 36 \\
\hline 29 & tenuispinus (Chiridius) & 36 \\
\hline 172 & Tharybis & 86 \\
\hline 69 & Thaumaleus & 201 \\
\hline 85 & thompsoni (Thaumaleus) & 212 \\
\hline 142 & tonsa (Euchaeta) & 55 \\
\hline 179 & tonsus (Calanus) & 12 \\
\hline 76 & Fortanus & 162 \\
\hline 76 & typica (Pseudophaenna) & 57 \\
\hline 176 & typicus (Centropages) & 92 \\
\hline 177 & Undeuchaeta & 42 \\
\hline 168 & Undinella & 67 \\
\hline 139 & valida (Euchaeta hebes var.) & 54 \\
\hline 32 & vanhöffeni (Chiridius) & 35 \\
\hline 236 & vanus (Ctenocalanus) & 24 \\
\hline 73 & velox (Eurytemora) & 99 \\
\hline 32 & velox (Temora) & 99 \\
\hline 10 & venusta (Metridia) & 110 \\
\hline 223 & venusta (Oncaea) & 186 \\
\hline 63 & venustus (Corycaeus) & 200 \\
\hline 199 & vipera (Haeterochaeta) & 123 \\
\hline 44 & vipera (Heterorhabdus) & 123 \\
\hline 130 & wollastoni (Labidocera) & 149 \\
\hline 130 & wollastoni (Pontella) & 149 \\
\hline 56 & Xanthocalanus & 57 \\
\hline 129 & xiphias (Pleuromamma) & 105 \\
\hline 119 & xiphias (Pleuromma) & 105 \\
\hline 119 & zetesios (Augaptilus) & 136 \\
\hline 169 & zetesios (Heterochaeta) & 125 \\
\hline 28 & zetlandica (Phaenna) & 222 \\
\hline 52 & zetlandicus (Thaumaleus) & 215 \\
\hline
\end{tabular}




\section{Inhaltsübersicht.}

Trib. Gymnoplea

Fam. Calanidae

Centropagidae

Pseudocyclopidae

Candaciidae

Pontellidae

Trib. Podoplea

Mormonillidae

165

Cyclopidae

166

Harpacticidaé

Oncaeidae

Corycaeidae

Monstrillidae

Anhang I (Zusätze)

Anhang II. Die Entwicklungsstufen der marinen Copepoden

Ürbersicht der Gattungen

Literaturverzeichnis

Alphabetisches Register 
WILSON COPEPOD LIBRARY

Smithsonian Institution

Invertebrate Zoology

(Crustacea) 





\section{WILSON COPEPOD LIBRARY}

Smithsonian Institution Invertebrate Zoology

(Crustacea) 


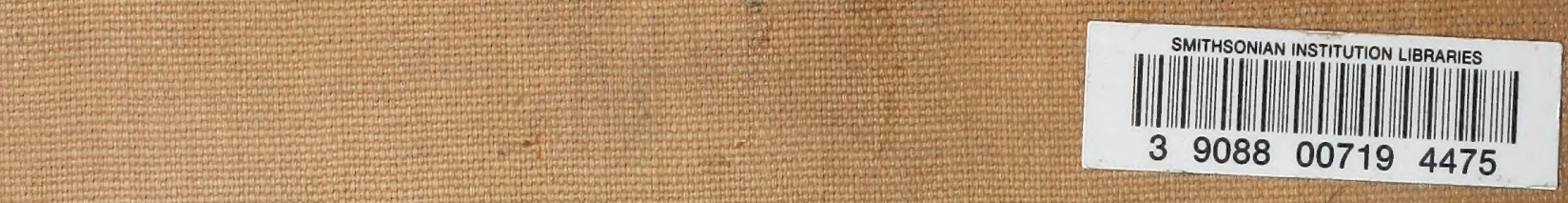

